\title{
Annotated Bibliography of
} LAMPF Research and Development

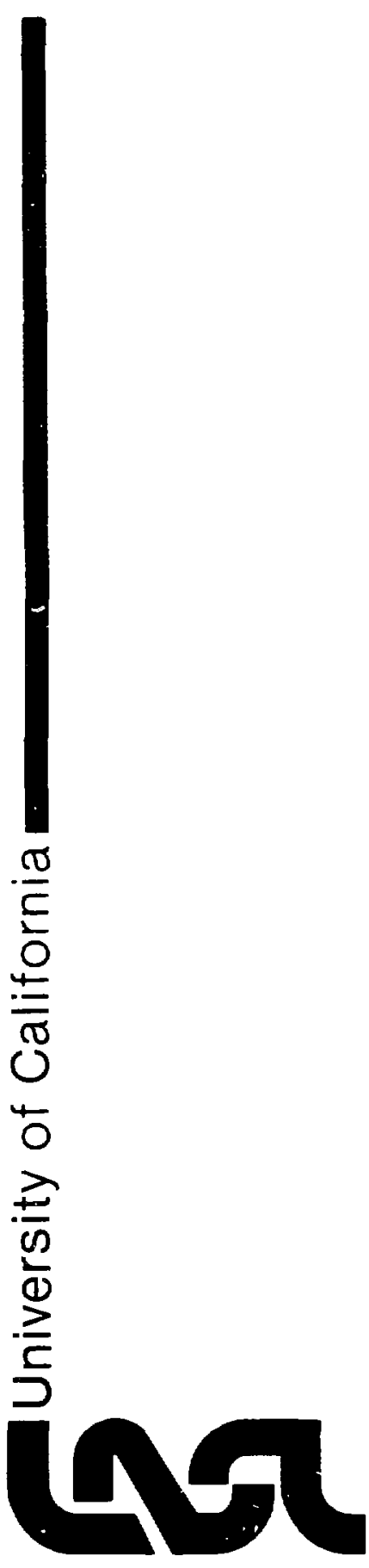




\title{
Annotated Bibliography of LAMPF Research and Development
}

\author{
R. A. Jameson \\ E. U. Roybal
}

\section{- HOFE BLN ONLY}

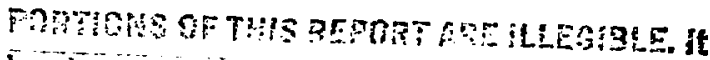

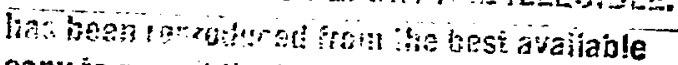
comy oformia the brodest possible availanifty. 
AWNOTATED BIBLTOCRAPIIY OF LAMPF RESEARCH AND DEVELOPMENT

by

R. A. Jameson and E. U. Roybal

\begin{abstract}
A bibliography on published and in-house technical material. written on LAMPF activities since its inception is presented. Subject and author concordances provide cross-reference to detailed citations, which include an abstract and notes on the material. The bibliography resides in a computer database that can be searched for key words and phrases.
\end{abstract}

I. INTRODUCTION

This bibliography brings together, from a variety of sources, reference material pertinent to LAMPF research and development. The following areas are emphasized:

- Experimental physics research program - a complete record is being kept of publications resulting from experiments allocated beam time in the LAMPF experimental areas.

- Accelerator related documents - 1963 to present, coverage of internal technical memos as well as publications.

The bibilography is stored in a computer database, using the Master Control Program developed at Lawrence Livermore Laboratory. The program has search and retrieval capability and can produce output in a variety of formats that can easily be tailored to the needs of the user. Most documents have been annotated with abstracts, key words, and other information. Typical information includes:

$\begin{array}{ll}\text { Record Number } & \text { Date Published } \\ \text { Author } & \text { Conference } \\ \text { Affiliation } & \text { Journal } \\ \text { Title } & \text { Abstract } \\ \text { Sub- and Section Titles } & \text { Subjects } \\ \text { Docuinent Type } & \text { Comments } \\ \text { Report Number } & \text { Notes }\end{array}$

Pages

\author{
References \\ Data Record \\ Proposal Number \\ Channel Name \\ Channel Code \\ Category \\ Cataloging Source \\ Storage Location
}

Searching can be performed on any of this information. 
This report contains four examples of the output capability.

The first section is an alphabetical author listing with the number of occurrences.

The second section is an author concordance, again in alphabetical order, with record number, title, and date published of articles written by each author listed under his name.

The third section is an alphabetical subject listing - a dictionary of key words or phrases - with the number of occurrences.

The fourth section contains publications resulting from experiments that have been run at LAMPF which lists proposal number, record number, title, date published and channel code.

The original documents have also been filmed, and therefore are readily available for reading or copying.

Requests for detailed searches or to read documents should be directed to E. U. Roybal, MP-9, telephone 667-4433, TA-53, Bldg. MPF-6, Room 108 .

\section{CONTRIBUTING PERSONNEL}

$\begin{array}{ll}\text { Word-Processor: } & \text { B. Talley } \\ \text { Compilers: } & \text { M. Bradley } \\ & \text { S. Chavez } \\ & \text { W. Christian } \\ & \text { S. Davison } \\ & \text { R. Pritchard } \\ & \text { C. Watson }\end{array}$




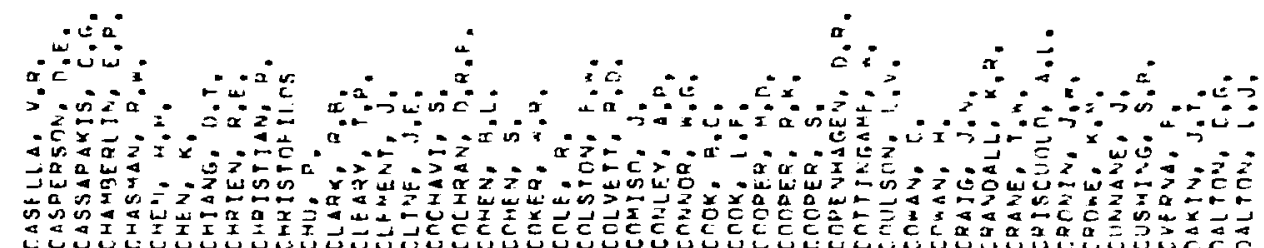

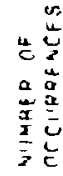

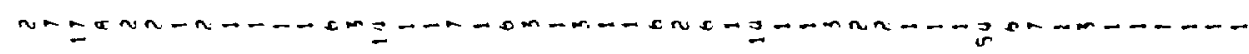

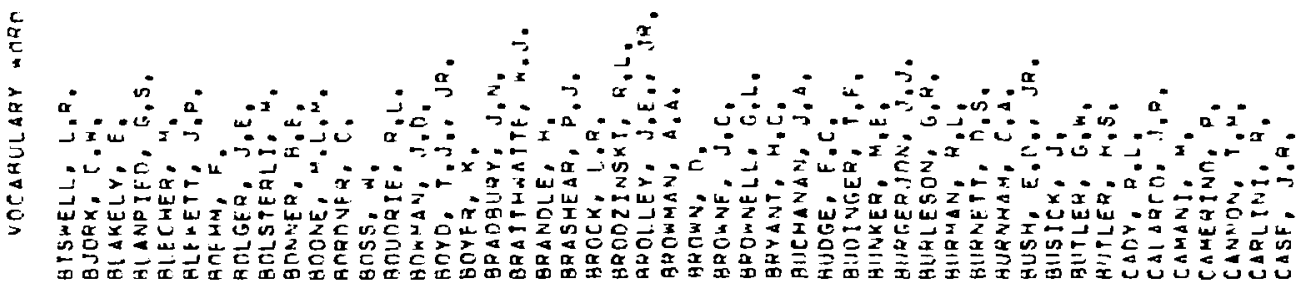

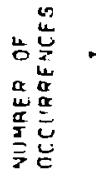

-

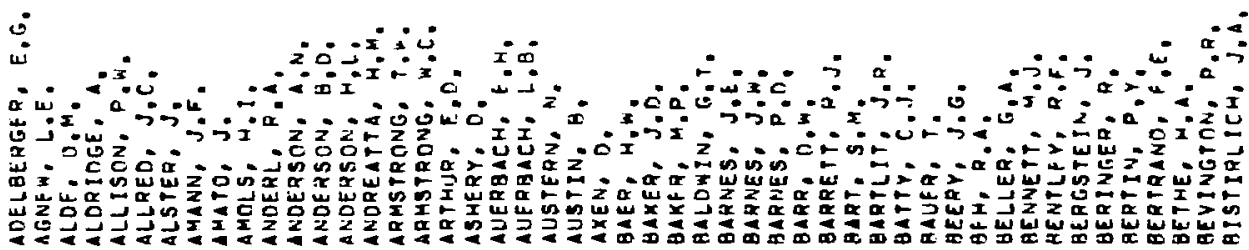

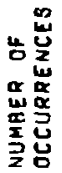

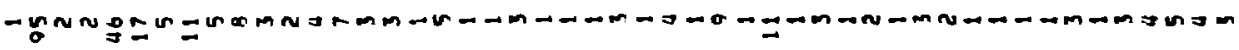




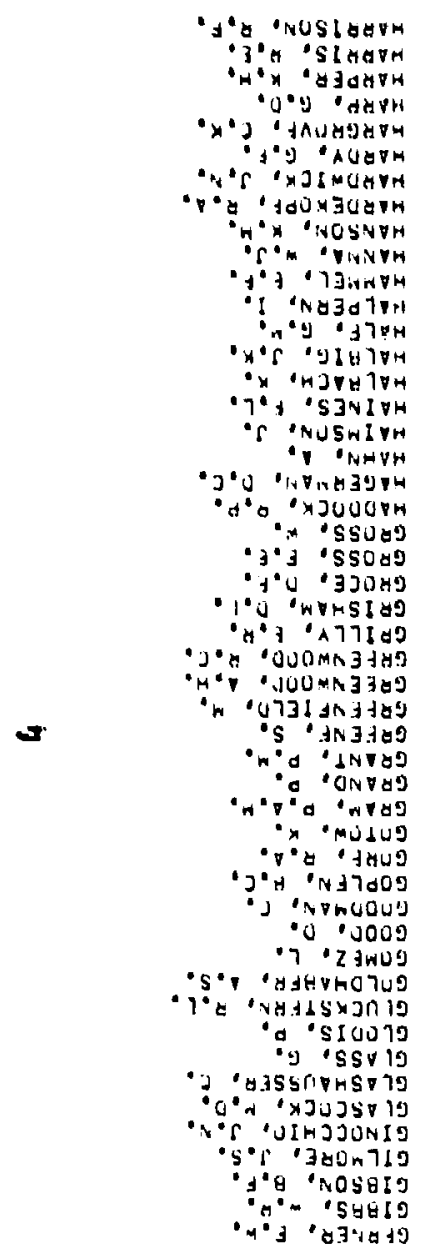

UYUM AEV MOYVJUA

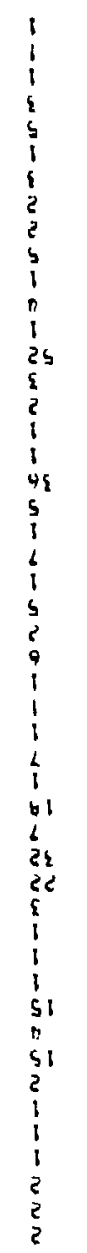

S33n30 3010 IO a Jun

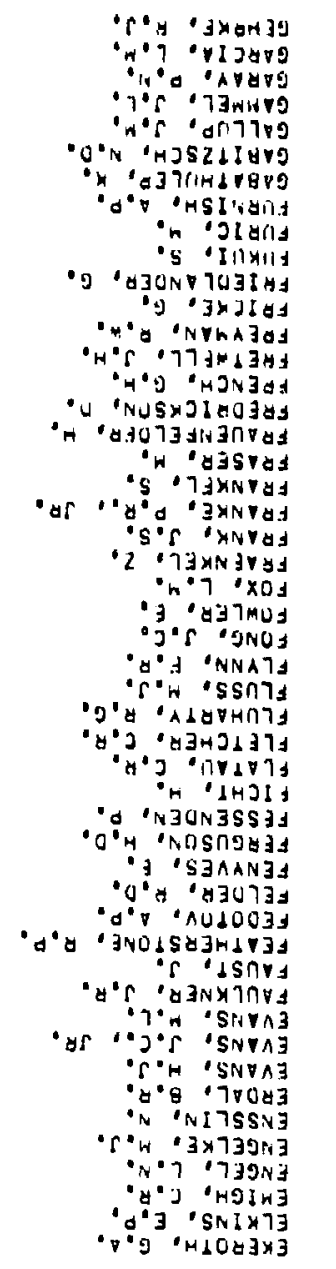

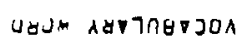

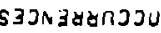
10 B38nfin

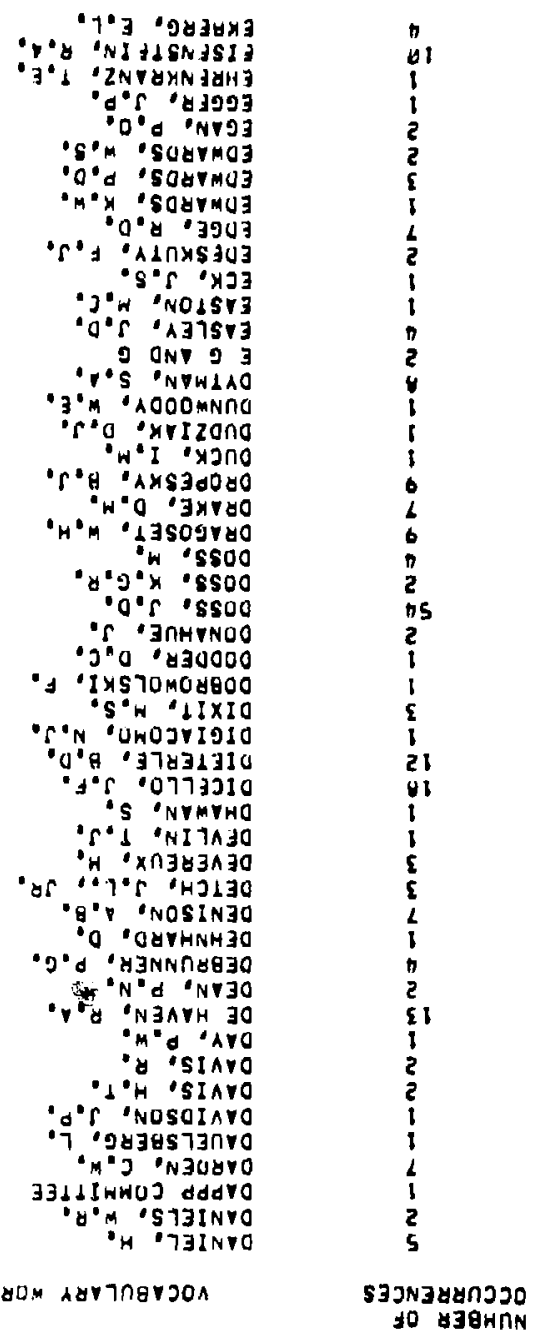




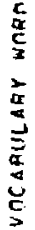

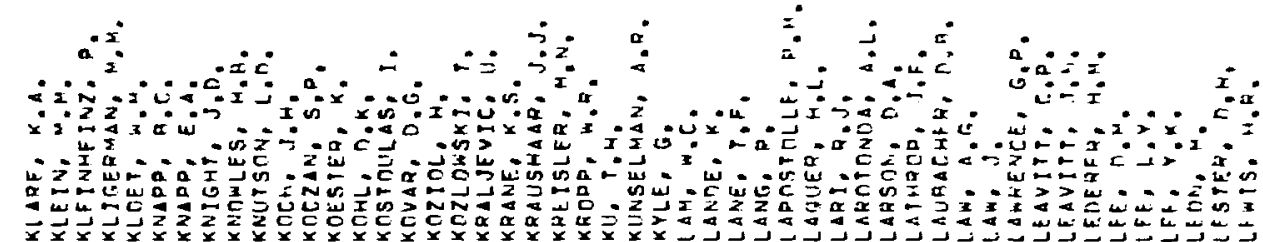

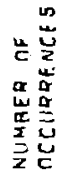

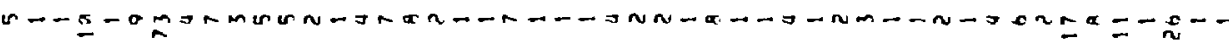

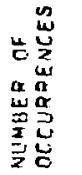

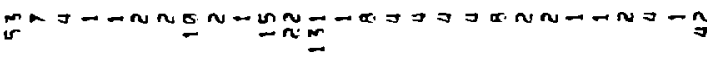

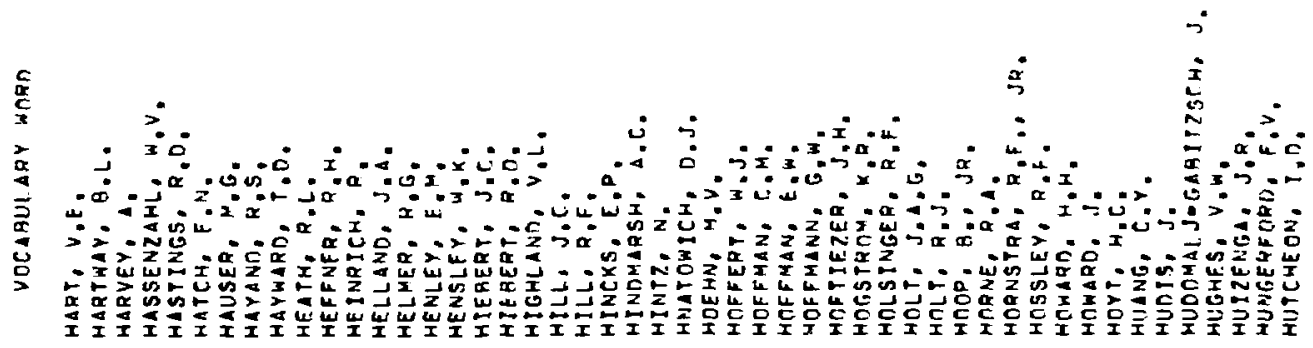

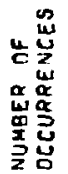




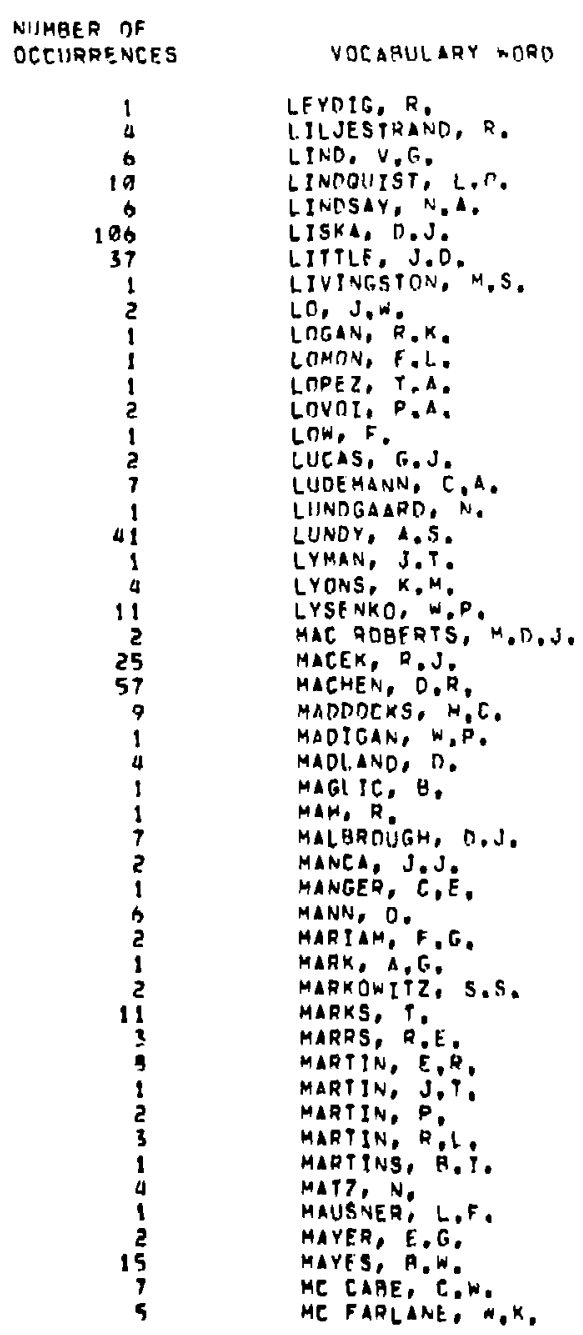

C GUIRE, A.0. MCATAMS, R.

MrCONAFli. j.R.

MEDANIELS, T.K.

HCGILL, J,

MENAUGHTOC:

MEIF $\square, K$,

MENIEL, M.T.

ME TZFER,

MEYER, $5 . A$,

MFYFR, $M, O$

MFYER, $R, E$

MFYER, S.L.

MEYFR-SCHIITIMFISTEH, I MIITER, F

MLICH, C,P.

MILLER, H.F

MILIER, G,

MILIER, R.M

MIlाEस, J,

MILLER, J.F.

MILIER, J, $M$,

MILLER, R. $C$

MILLS, SE

MINOR,

MISCHKF, O.

MOINESTFR, M.A.

MOIP, D.C:

MONAE, C...

MOORE, R. H.

MORRIS, D,I:

Hous, "H.

MP OIVISION

MPG IVISION PITNI

MUELLEH, $O, W$.

MUELLER, D. W.

MUELLFR, R.

MUTCHLEP: $B . s$

NAGA INEE, $k$,

NAGLE, D.K.

Nalvak, $F, j$,

OCCIRREVCHS

nCAMILADY mern

MATOATT7, 7,

NALIPACAN, H,

AFEKFVS, R.

NEGFLF, 3,k.

TE TMETRY, $F, S$

NE WC OM. $F, B$

NEWELL, R, H,

NE WMAPA.

NEWS DN, C.P.

NICKELL, j,

NTESCHATDT, $1,8$.

NISHIKAWA,

NORPYS, A, L.

NORTHCLIFFE: L.T.

NHVAK, J.K.

NIJMANA, RET RA, JA

ACONNELL, J.S

DFTITNE. J.D.

OTADD, A.F.

THL SEN, GS,

OHISEN, S,D.

THNIIMA, $S$,

OחTHOINT,

IRTH, C.J.

TRTH: H.

DTTFSON, N.H.

DACHECOA,

PALFQun, S.D.

PALEYSKY, $N$.

PARYFE. JaA

PARKER, JR。

PASSONS, J.B.

PARTHPTIT, 5 .

pasta.

PaTTÓN, R,

pauletia, ${ }^{\circ}$

PECK. R.A.

DEHL. R.N

PENYROT, J A

PFREZ=MEAIE Z;

PERKINS, R, R. 


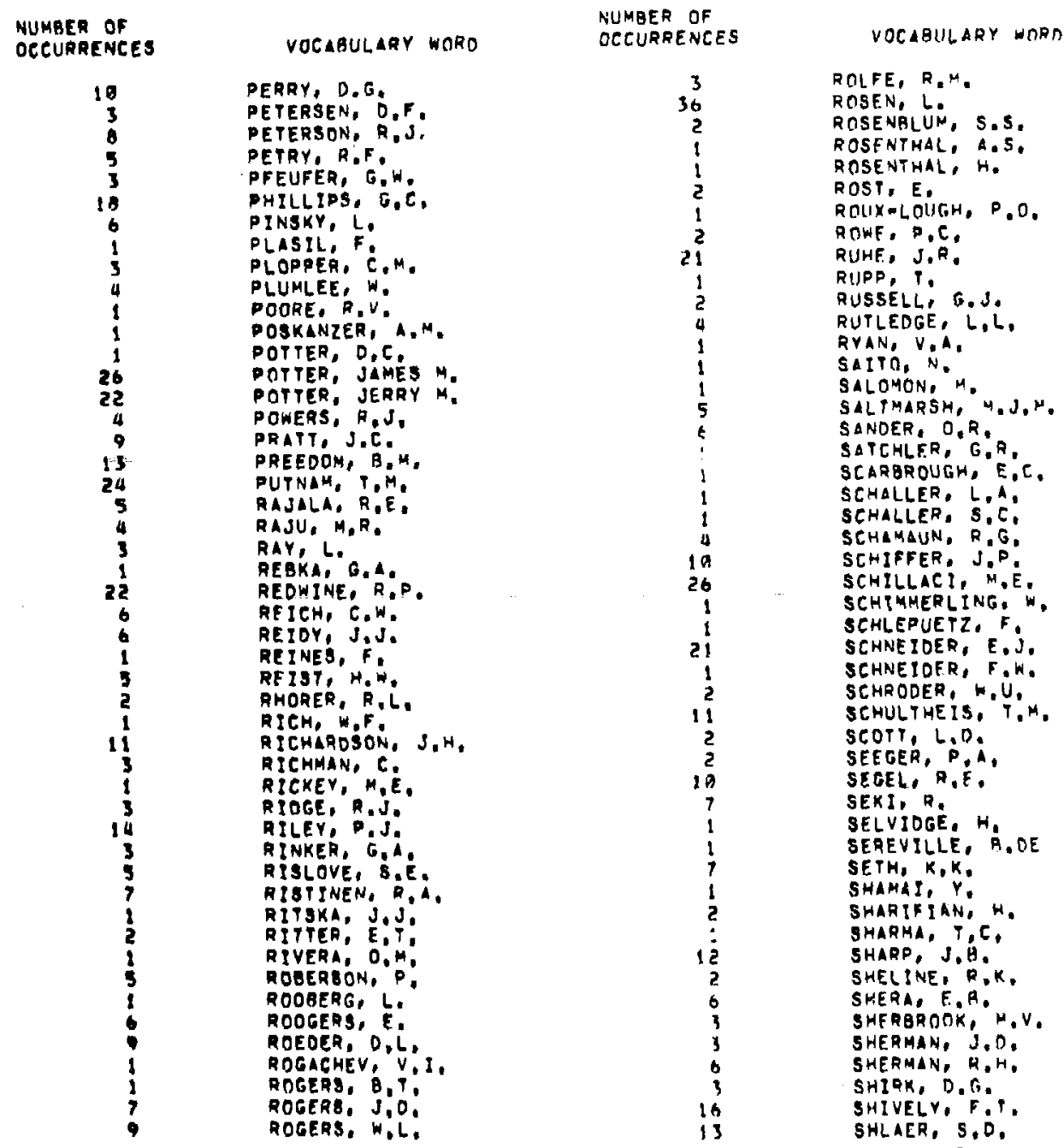

OTCURREVCES

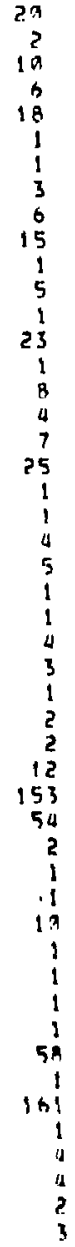

VOCLAUI.ARY WORO

SHLAER, H.J,

SHONK, C.F.

SIMMONDS, 0,0 ,

SIMMONS, $\$ . E$.

STMMONS, M.L.

SKOGLUND, V.J.

SMTTH,

SMITH,

SMITH, J.J.

SMITH,

SMITH, L.E.

SMITH, T.W

SHYDER, J, N

SOBOTTKL, S.E.

SORENSEN, L. $R$.

SOUNER.

SPENCER, I, E

SPENCER, $A, C$

SPERINDE, $J$.

SPHINGER:

STAMBAUGH, R, $R$

STARNEA, JAR:"

STEARNS, R.

STEFFEN, R, M

STEIN,

STE INRERT, E,P.

STEPNENSON, G.J.

STERNHAGEN, C,J.

STEFNHE IH, H, M,

STRASSNER, G.

STRDIK, 0.3 .

STROYINK, M"

STUDEBAKER, J,K,

STUPIN, D, M

SUAZO, G.

SUNIER, J,W

SUTPER, R,J

SWAIN, G.R.

SHaLLNE, E.

SWENSON, D.W.

SWENSCN: W:

TALGA

TALAERT, W,L, JR. 


\begin{tabular}{|c|c|c|c|c|c|}
\hline $\begin{array}{l}\text { Numbeh of } \\
\text { OCCURRENCEs }\end{array}$ & VOCABULARY WORO & $\begin{array}{l}\text { NUMAER OF } \\
\text { OCCURRENCES }\end{array}$ & VOCABULARY WORD & $\begin{array}{l}\text { NUMBER OF } \\
\text { OCCURRENCES }\end{array}$ & YOCABULARY WORD \\
\hline $\begin{array}{r}23 \\
2 \\
1 \\
13 \\
3 \\
2 \\
48 \\
1 \\
19 \\
1 \\
2 \\
1 \\
5 \\
13 \\
2 \\
2 \\
1 \\
2 \\
2 \\
22 \\
2 \\
7 \\
3 \\
7 \\
4 \\
8 \\
1 \\
6 \\
14 \\
36 \\
1 \\
4 \\
4 \\
1 \\
1 \\
1 \\
3 \\
1 \\
10 \\
2 \\
23 \\
2 \\
1 \\
4 \\
4 \\
9 \\
4 \\
1\end{array}$ & 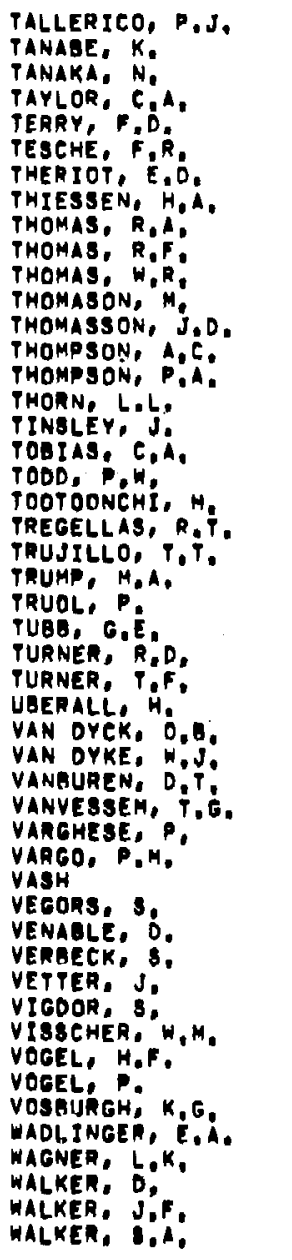 & $\begin{array}{r}18 \\
5 \\
7 \\
11 \\
1 \\
32 \\
2 \\
1 \\
2 \\
2 \\
2 \\
3 \\
9 \\
3 \\
3 \\
1 \\
2 \\
4 \\
3 \\
4 \\
4 \\
13 \\
5 \\
1 \\
2 \\
1 \\
15 \\
1 \\
7 \\
2 \\
2 \\
12 \\
1 \\
4 \\
1 \\
37 \\
3 \\
1 \\
1 \\
1 \\
1 \\
1 \\
1 \\
1 \\
1 \\
1 \\
1 \\
3 \\
6\end{array}$ & 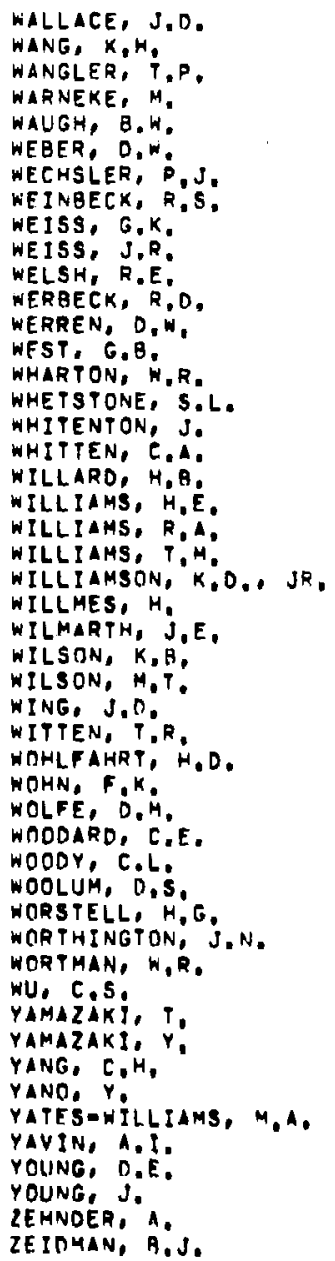 & $\frac{?}{7}$ & $\begin{array}{l}\text { ZIOCK, K. R.H. } \\
\text { ZU PUTLITZ, G. }\end{array}$ \\
\hline
\end{tabular}


ADELBERGER, E. G

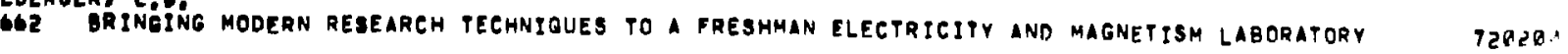

AGNEW, LiE:

2024 LAMPF AS A NEIITRON SOURCE

1637 EXPERMENTAL FACILITIES AT LAMPE

2504 MUON CHANAEL DEVELOPMENTS

3090 MINUTES OF EADC NEETING 750819

3001 MINUTES OF EADC MEETING 7509

3002 MINUTE OF EADC MEETING 751023

3003 MINUTES OF EABC MEETING 751113

3004 MINUTES OF EANC MEETING 751126

3005 MINUTES OF EADC MEETING 951211

3006 MINUTES OF EANC MEETING 951230

3097 MINUTES OF EADC MEETING 760121

3000 MINUTES OF EATC MEETING 960205

3909 MZNUTES OF EANC MEETING 760210

3010 MJNUTES OF EANC MEETING 760394

3011 MINUTES OF EANC MEETING 760311

3012 MINUTES OF EANC MEETING 760318

3013 MINUTES OF EANC MEETING 760325

MINUTES OF EAnC MEE

MINUTES OF EANC MEEP

MINUTSE OF EANC MEETING 760422

MINUTES OF EANC MEETING 760429

MINUTES OF EANC MEETING 760596

MINUTES OF EAnC MEETING 760513

MINUTCS OF EANC MEETING 760520

MINUTES OF EARC MEETING 76052

MINUTES OF EAnC MEET?NG 760693

MINUTES OF EANC MEETING 760619

MINUTES OF EADC MEETING 76 MGI7

MINUTES OF EAnC MEETING 769620

MINUTES OF SOPNT EADC-ADCOLSC MEETING 76070

MINUTES OF EANC MEETING 760788

MINUTES OF CANE MEETING 760713

MINUTES OF EARC MEETING $76 \pi 722$

MINUTES OF EANC MEETING 76月026

MINUTES OF EANE MEETING 760999

MINUTES OF EANC MEETING 769916

MINUTES OF EANC MEETING 760930

MINUTES OF EANC MEETING 761007

MINUTES OF EATC MEETING 761014

MINUTE OF EADC MEETING 76!02!

MINUTE OF EANC MEETING 761026

MINUTES OF EANC MEETING 761194

MINUTE F F EAC MEETING 761111

MINUTE OP EANC MEETING 761118

MINUTE OP EADC MEETING 761292

JEA3 MINUTES OF EANC MEETINE 761216 
AGNEH, LE E TCONTO

3044 MINUPES OF EADC MEET ING 770106

395 MINUTES OF EADC MEET?NG 770113

304 MINUTES OF EADE MEETNG 779

MINUTE OF EANC MEETNG 77a1?

MINUTES OF EANC MEETING T7RzIO

MINUTES OF EADC MEETING T?

MiNUTES OF EADC MEETING 770?2

MINUTES OF EANC MEETING 770303

MINUPES OF EARC MEETING 790310

MINUTES OF EARC MEETING 790317

MINUTES OF EANC MEETING 79032

MINUTES OF EANC MEETING 770407

MINUPE OF EARC MEEY ING 770414

MINUPES OF EANE MEETING 79942

MINUTES OF EARC MEETING 9765 S

MINUTES OF EARC MEETING 77051

MINUTE OF EANC MEETING 770519

MINUTES OF EANC MEETING 77068

MINUTES OF EANC MEETING 770609

MINUTES OF EANC MEETING $7906 !$

MINUTES OF EANE MEETING 79 G62

MiNUTES OF EAAC MEETING 79070

MINUYES OF EANC MEETING T7aT!

MINUTES OF EANC MEETING 77072 !

MINUTES OF EANC MEETING 770818

MINUTES OF EANC MEEYSNG 77082

minures or EAne MEET

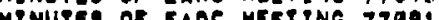

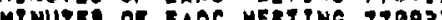

MiNUTE of EAnC MEEP

Minupe of EAnC MEergng 771014

MINUTE OF EACC MEET

MINUTE OF EADC MEETINE T7IOQ

MPNUPE OP EANC MEETING 77IIOS

MINUTES OF EADC HEETING TIIIIO

MINUTES OF EABC MEETING 771 II

MINUTES OF EANC MEETING T7ISOI

MINUTES OF EADC MEETING 771315

MINUTES OF EANC MEETING 771222

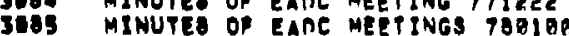

3086 MINUTEB OF EADC meETINGS 700200

3967 MINUTES OF EANC MEETINGS 780380

1476 PAOCEEOINGS OF PHE FIPTM LAMPF USERS MEETING
3539 REVIBEO CYCLE O PLAN

77911.4

77919

97023

779280

$790 ? 1$.

770253

770393

778398

77031

77032

77032

77001

7705

$770<$ ace

77051.

7714516

770 aed

$77048 \mathrm{~s}$

$778+15$

77052

77093

T>a>s?

779025

770091

$7709 ! 5$

71002

771.03

771.18

771 .

$7+1.31$
719

7911 )

Trilia

TiPan

$77120^{2}$

771222

TEPIOA, 7AA113

7 Bम119

TaB. 24

7月0205, 7Aazdo

TAB?द्य

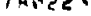

79म3E? TAS3001

7 als241

$72030^{\circ}$

7 inisis 
AGNEW, LOE. (CONTO.)

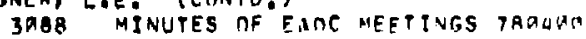

3AOQ MINUTES OF EANC WEFTINGS TRG5G

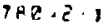

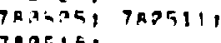

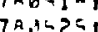

$\triangle L D E, D . M$.

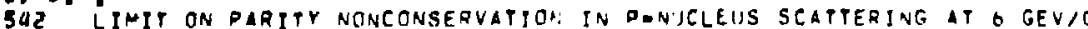

1628 LIMIT ON PARITY VIOLATION IN FONUCLELIS SCATTHETAG AT O GEVIC

75840 in

7 hat! $=$

ALORIDGE, $A$.

S4A MAGNETIC FIELT MFASUREMENTS ON THREE PIJAORUPGLE TRIPLETS

Q71 MAGNETIC MEASIRFMENTS ON BOS TYPEE (a ING AORE) QUADRUOOLE DOUQLET

$7+9117$
7410

ALLISON, $P, W$,

249Q IOAGMEV SPECTROMETER ALVAREZ LiNAC ENERGY ANALYSIS

2719 ACCELERATING STRUCTUAE RESEARCH AT LIS ALANOS

GOS BEAM PROFILE MONITIRING AND ANALYSIS RY TELEVISION

2580 BEAM TRANSPORT SYSTEM IN THE INJECTOR COMPLEX IF LAMPF

49 COCXEROFTEWALTON HIGHEVOLTAGE CONBR

400 COCKCROF TWHALTON INTERLOCKS
52 COCKCROFTOWALTON RFFERENCE ONWER SUPPLY AND CONTROLS

194 COUPLING BETWFEN TRANSVERSE NSCILLATINUS IN LINACS

S79 EMITTANCE SCANNER

FTRUITRY FOR THE INJECTOR AREA

TUBO FORETGN TRAVEI REPORT (ALLISON)

54 H. BEAM TUNEUP

पA7 HE COW VOLTAGF CALIBRATION

55 H. DOME VACUUM GYSTEM

2494 HE ION SOURCE

5! HE ION SOURCE DATA

233 HE ION SOURCE PROGRESS

53 HIGH VOLTAGE OIVIDER SPECIFICATIONN

2497 TBS PROFILE MANITOR - 690012

2498 IBS PROFILE MANTTOR: 699612

1454 INITIAL OPERATION OF THE GEAM TAANSPORT SYSTEM IN THE LAMPF INJECTOR COMDLFX

INVESTIGATION OF OELTATRON POWEQ SUPPLIES FOR DOLARIZEO ION INJECTOR

JOINT HO ION SOURCE DEVELOPMENT PROGRAM

LAMPF HE ION SOURCE DEVELOPMENT

MULTICHANNEL MAMPLE AND HOLO

NOTES ON THE NE INJECTOR

ON PHE FEASTBTLITY OF HO ION ACCELERATION AY LAMPF

OPERATING INSTRUCTIONS FOR HO GEAM CHOPPER

OPERATION OF THE LAHPF 75HAKFV INSECTOR

PERFoRMANCE OF THE LAMPF Ho IN,JECTOR

POLARIZED Mo souRCE FOR LAMPF

60.45

anatis.

$710 \cdot 3 \cdot 19$

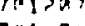

70125

73 म०.

7...

6730

74841 ?

की ? ?

001112
75,2925

$744=1$.

73112

$\operatorname{lopicos} 0$

Bol 697

720 व

7 7.4.

74031

ganalo

00780

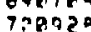

7310.47

75919.

7il

oquzin

popap

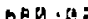

Tallit?

Tlama

7 at?

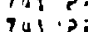


ALLISON, D:W: (CONTD,)

2491 PRECISION YOLTAGE OIVIDER

2501 QUADRUPOLE ABFRRATION CALCULATIONS

2500 REPORT ON TRID TO MIGH VOLTAGE ENGINEERING, LRL, ANO CVCLOTRON GORP, TO TISCUSS HE ICN SOURCES

SOHE CONB IDERATIONS FOR IN BDOGMEV PROTON STORAGE RING

4 SPECIFICATIONA FOR EOUIPMENT DOME

2562 STAPUS OF THE INJECTOR COMPLEX AT LAMPF

234 STATUS OF THE LAMPF HE INJECTOR

412 TENTATIVE SPECIFICATIONS FOR 750 KV HE POWER SIIPPLV AND EQUIPMENT TERMINAL FOR THE LOS ALAMOS HESDN PHYSICS FACILITY

1432 THE BEAM TRANGDORT DESIGN FOR THE LAMPF INJECTOR

1453 THE INJECTOR POMPLEX FOR THE LAMPF ACCELERATOR

B86 TRANGPORT SYSTEM OPTICS

411 VOLTAGE DIVISTON RATIO MFASUREMENTS ON THE COCKCROFYOWALTON HIGHEVOLTAGE GENERATRRS

691113

60997

$7 ! 3: 8 \pi$

7 3popet

70 OQ1

730300

LRED, S.C.

2BO9 ANGULAR OISTRTRUTION FOR PO GOESTO 3HE PI ZERO REACTION AT PPBBAQ NEV

222 FINAL -STATE INTERACTIONS IN PAD BREAKUO AT BRA AND GBQ MEV

IAS1 KINEMATICALLY-COHPIETE STUOY OF DION DRODUCTION BY THE REACTION 1 WYOROGENIPROTON, PIO PROTONJNEUTRON AT BOE MEY

21. NUCLEAR-COULOMB INTERFERENCE IN PI +OR- 16 OXYGEN SCATTERING

1901 DION PRODUETION GY BQR MEV PROTONS FROM G-LITHTUM

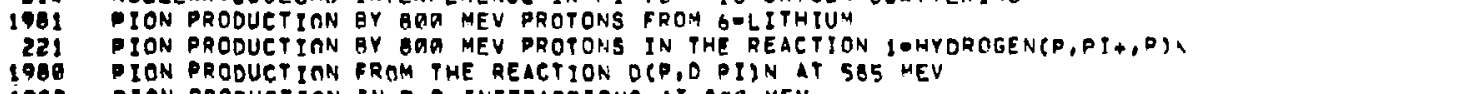

- ION PRODUCTION IN PAP INTERACTIONS AT OAQ MEV

- ION PRODUCTION VIA A D+P GOESTO D+PI OUAST OFREE PRDTONS IN DEUTERTUM

- ionenuclear total CROSS seCTIONS NEAR THE 3/2, 3/2 RESONANCE

PION-NUCLEUS FORWARD SEATPERTHE AMPL ITUDES NEAR THE 3/z. 3/z RESONINCE

- IONONUCLEUS MMALL ANGLE ELASTIC CROSS SECTIONS NEAR THE 3/2, 3/2 RESONANCE

TONONUCLEUS TOTAL CROSS SECTIONS NEAR THE 3/2, 3/2 RESONANCE

AND ORA MEV

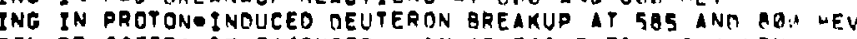

ECSUB PD. SaG MFY

TUNE-UP OF THF LAMPF HIGHENERGY PION CHANNEL

090523

60900 ?

670729

739523

73932 ?

77009.

$7534 \pi:$

769105

$75 \operatorname{ing} \theta$,

$7+1,5:$

756019

Tolitis:

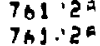

73111 ?

761 . टक

770425

770625

$75010 ?$

960794

750.19

ALBTEh, J,

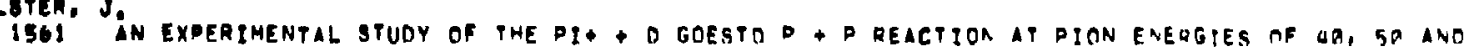

7anapo

794 MEABUREMENTS OF PHE (PION PLUS, PION ZERT) REACTION ON LIGH ELEMENTS IN THE $(3,3)$

7ha1 RESONANCE REGITN

2 TGQ THE DEUTERON BOSTATE AND PHE PI+ + D GOESTO D D REACTION

2421 THE PI+ D GRESTOD + P REACTION AT 49. 5M AND OG MEV

7 (19.25 TUNEUP OF THE LAMPE LOW ENERGY PION CHANNEL

$761+25$
779.90

AMANN, J.F.

2385 COMPUTING IN SUPPORT OF EXPERIMENTS AT LAMPF

150 ELASTIC SCATTERING OF 59 MEV PIT AND ILACAMBON 
AMANN, JiF. (CONTD,)

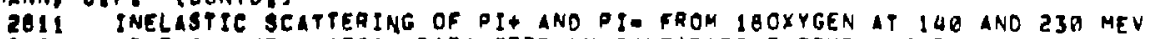

2406 NEUTRON DEFORMATION PARAMETER BY COMPARATIVE STUDY OF PI+ AND PIO INELASTIC SCATTERING

1995 SCATTERING OF 5 G MEV PI+ FROM LIGHT NUELEI

163 STUDIES OF CHARGED PARTIELE EMISSION IN REACTIONS INDUCEO BY 220 AND 5 R MEV PLUSOPIONS

164 STUDIES OF PARTICLE AND GAMMAESPECTRA IN REACTIONS INDUCED QY OQOANEV PRTTONS

2809 THE 12C (PI+.0)IAC 6.3 .9 AND 10B(PI+D)BB(G,S.) TRANSITIONS LT E SUB PI. 5O MEV

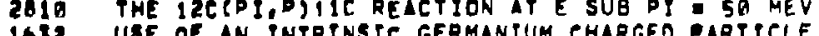

1632 USE OF AN INTRYNSTC GERMANIUM CHARGEO GARTICLE SPECTROMETER AT LAMPF

219 USE OF HIGHAPIIRITY GERMANIUM DETECTORS FOR INTERMEDIATE ENERGY DHYSICS EXPERIMENTS

72. COROSS SECTIONG FOR (PI+,P) REACTIONS ON 9-BERYLLIUM, 12-CIABON, ANO 16-OXYGEN

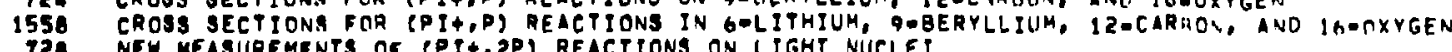
NEW HEASUREMENTS OF (P I+,2P) REACTIONS ON LIGHT NUCLEI

THE LAMPF LOW ENERGY PION LINE

7500.21

AMOLE, H.I:

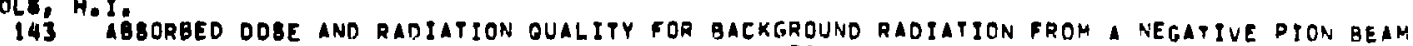

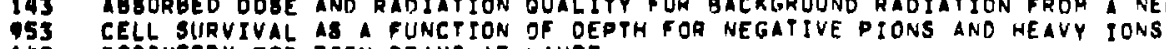

142 DOSIMETRY FOR PION BEAHS IT LAMEF

ESTIMATION OF BIOMED BEAM FLUX GASED ON CURRENT OUTPUT OF HE CM TRANSMISSION CHAMBER

MICRODOSIMETRY IN TISSUE EOUIVILENT FLUID IRRADIATED OY DET NEUTRONS

MICRODOSIMETRY OF NEGATIVE PIONS

1506 WHOLE BODY DOSE TOR PATIENTS TREATED WITH PIONS

710011

74020.

$71092 ?$

710011

ANDERL, R.A.

2067 I34-GARIUM LEVEL SCHEME AS OBSERVEO IN THE OECAY OF 13UMLANTHANUM

1630 DECAY OF 1280 CESIUM

2968 LEVEL BTRUCTURE OF 131-CESIUM AND THE OECAY ENERGY OF 131-BARIUM

$750 \times 0:$

75.154

75851

$750,0 \circ$

75 का1?

75402 ?
750 ?

$760 \mathrm{at}$.

INOLASON, A.N'.

1968 ABgOLUTE DIFTFRENTIAL CROSS SECTION MEASUREMENTS FOR PROTONOPROTON ELASTIC SCATIERTNG AT

1436 BEAM INTENBITY MONITORING FOR THE EXTERNAL PROTON BEAM AT LAMPF

$7 n 114$.

77860\%

7hI A :

760091

$7507 \%$

ANOEROON, D,O:

1968 ABSOLUTE OIFFERENTIAL CROSS SECTION MEASUREMENTS FOR PROTONAPROTON ELASTIC SCATTERING AT

1436 BEAM INTENSITY MONITORING FOR THE EXTERNAL PROTON BEAM AT LAMPF

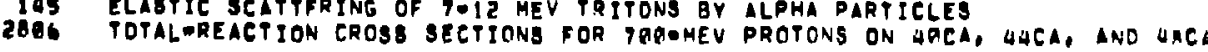

ThPQA

$7507 a^{2}$

$754 \mathrm{H}^{\circ}$

ANDERSON, H.L: 
ANDERSON, H.L. (CONTO':)

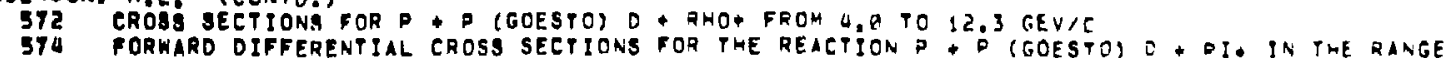

6T2 3.4 PO 12.3 GFYIC 3.4 90 12.3 GFVIC

627 LAMPF DATAEACOUISITION BYSTEM

1542 LIMIT ON PARTTY NONCONGERVATION IN PANUELEUS SEATTERING AT GEVIC

1620 LIMIT ON PARITY YIOLATION IN P.NUCLEUS SCATTERING AT G GEVIE

537 VACUUM POLARITATION IN MUUNIS APOMS

ANDNEATTA, H,M

1724 TESTING PROCEAURE FOR MK II PRINTED CIRCUIT MOTOR STEPPER BOARO O3Y 125708

1725 PESTING PROCERURE FOR MK II B PRINTEN CIRCUIT WOTOR STEPDER POARO 3 TY 55708

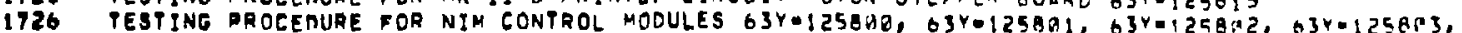
$63 Y \cdot 125807$

ARMSTRONG, P.H.

1926 APPLICATIONS HF COMPUTER PROGRAM TO CALCULATE THE RESPONSE OF GAS PRGPRTIMNAL COUNTERS

9 CALCULATIONG FOR DOSIMETAY AND MICRODOSIMETRY OF NEGATIVE PIONS

960 CHARACTERISTITS DF ENERGY DEDOSITION BY PIONS

ARMSTRONG, W.C.

TO6 PHE EFFECS OF RADIATION ON SUDERCONDUCTING NENT WIRE

Trase:

$792: 9$

onara.

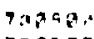

0000 .

700052

140053

$600+11$

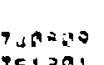

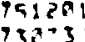

T103p

ARTHUR, E.D.

565 A STUDY OF PHF (PI+ P) IND (PI+, PP) REACTIONS ON SELECTED LIGHT NLELEI

924 CROSS OECTIONR FOR (PI+,PI REACPIONS ON Q-BERYLLIUM, 120EARBCN, AND 10-OXYGE

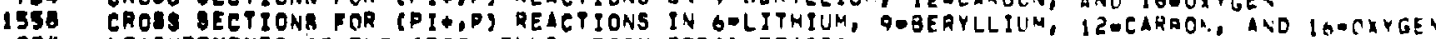

994 MEASUREMENTS OF THE (PION PLUS, PTON ZERO) RELCTION ON LIGNT ELEMEATS IN THE $(3,3)$

NEW MEASUREMENTS OF (DIH, 2P) REACTIONS ON LIGHT NUCLE:

$73+1,5$

718011

$740>0$

ThP 162

71032

ASHERY, D.

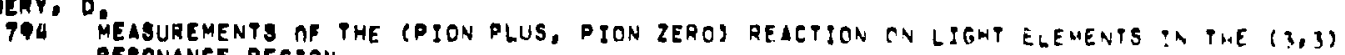

7na11?

GUERBACH, E.H S7I ELASPIC SCATTFRING OF 19,50 ANO I4, SOMEV POLARIZEO PROTONS FROM NICLEI AND TME OPTICAL

$650 \times 20$.

NUERBAEH, L, B:

1627 LIMITS ON PHE OIAECT PRODUCTION OF POSITRONS HY 250. AND BAR-MEV PROTONS

7 TEARCH FOR DIRECT LEPTON PRODUEPION IN PD COLLISIONS AT AOA MEV ANO 250 JE 
AUSTERN, N.

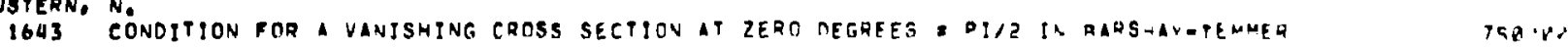

AUSTIN, B.

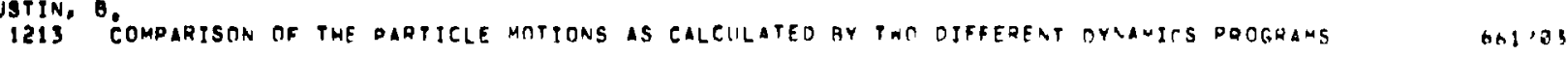

IXEN, D.

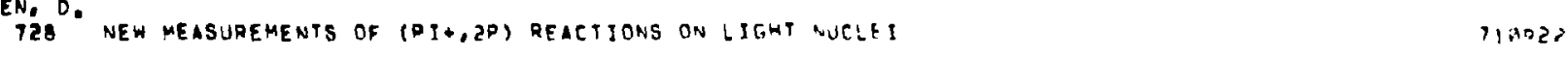

BAER. H.W.

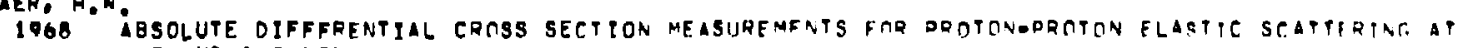
647 ANO BQD MFY

1633 PHOTON SPECTRIIM IN PION CAPTURE ON TRITIUM
2515 PAOIATIVE NETATIVE PIDN CAPTURE IN LIBUIO NEOS

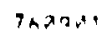

$7 \rightarrow+10$

ALKER, J.D.

2965 GIMMAORAY EMIGSION FROM I3U-CEZIUM AND LEVELS IN 134-LANTHANUM

Thand

BAKER, M.P.

2U1! MASS DEPENDENTE OF PITN ROUILLE CHARGE EXCHANGE

1570 OBSERVATION OF THE PION DOUALE CHARGE EXCHANGE REACTION 18-OXYGEN(DI+,01-)18-NEON

2422 PION DOUBLE-CHARGE EXCHANGE ON TGOOXYGEN ANO IA-OXYGFN

2417 PION NONOANALOG DOURLE CHARGE EXCHANGE IG-OXYGENCPI\$,DI-I16-NE

$77: 5$

7791,

BALDWIN, G.T.

2117 SUMMARY OF REUISFD WIRE SEAN CODE OPERATION

The, s?

GARNES, J,E.

1916 CODE FOR THREE-DTMENSIONAL TREATMENT OLANNING WITH OIONS

142 DOSIMETRY FOR PION BEAMS AT LAMPF

903 INITIAL COMPARATIVE RESPONSE OF NORMAL SKIN AND UNOERLYING TISSLE SURANUADiNG SUPERFiCIAL METABTATIE NDEULES

907 MEASUREMENT OF ONSE OISTRIBUTION OF COLLIMATED NEGATIVE PION BEAMS WITH EILM

1015 MINUYES OF MEFTING ON PIINN TREATMENT COUCM AT UNM CINEEQ RESEARTH AND TREATMENT CENTER

1910 NOTES ON MEETTHG TO DISCUSS THE CONFIGURATION AT THE END OF THE BIOMEDICAL BEAL LINE

1917 SPECIFICATIONA FOR AN ISCDOSE MEASURING INSTRUMENT

Q96 TECHNIOUES FOR VISUALIZING PION TREATMENT PORTS

105 TECHNIOUES FOO VISUALIZING PION TREATMENT PORTS AT LAMPF

BARNES, S.W.

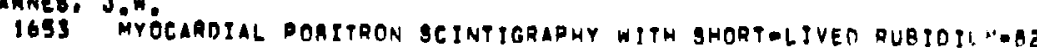

Garnes, P,D. 


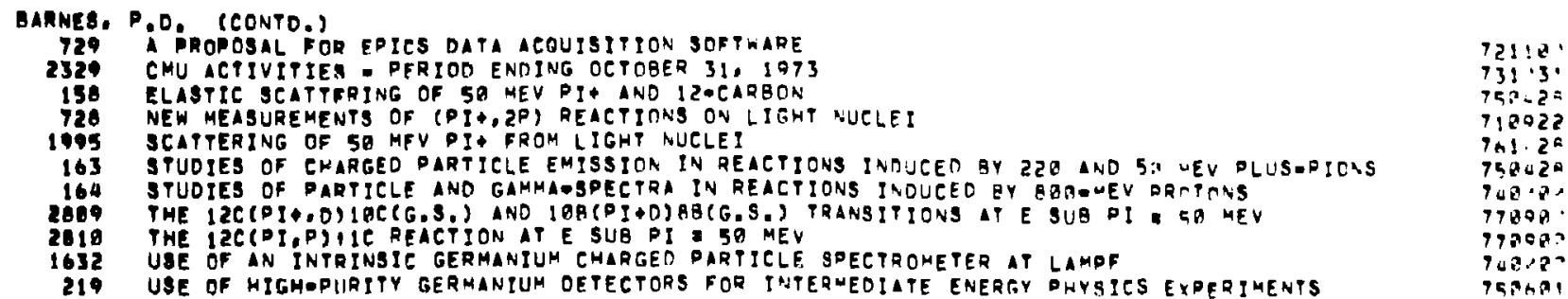

Darpy, C.J:

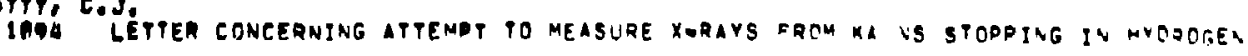

9,469

Buen, $r$.

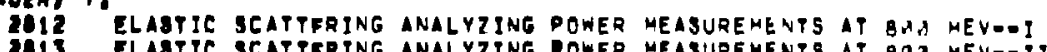

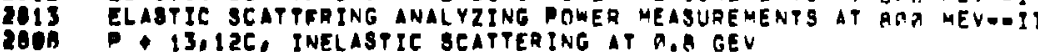

$\operatorname{tans?}$

Tethy, J.G.

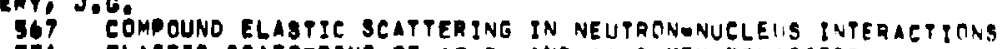

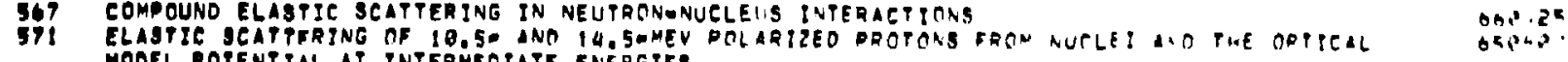

DEH. R'.A.

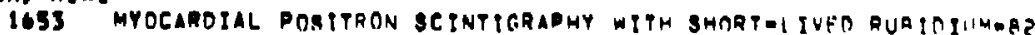


-eller, g.a.

1653 MYÓCARDIAL DOSITRON, SCINTIGRAPHY WITH SHORTELIVEO RUAIOIUMERz

BENAETT, M.J'.

15P MUONIC ISOTODF SHIFTS IN THE EVEN IRON NULLEI

BENTLEY, R.Fi,

BERGETEIN, J',

456 LIMITING BEAM CURRENT TO EXPERIMENTAL AREIS
1523 THE CENTRAL ENNTROL ROOM MANGMACHIVE INTERFACE AT LAMPF

BERINGER, Rí,
2724

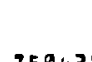

7 thate-

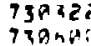

690524

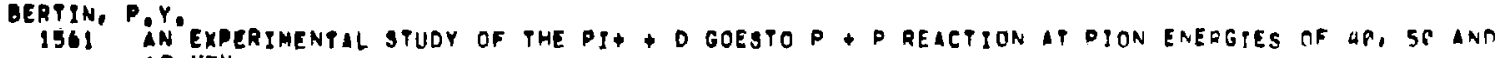

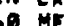

2AGO PHE DEUTERON DESTATE AND THE PI+ + D GOESTO P + P REACTION

750020

$761+25$

BenThund. F,E.

2MOS ELABSTIE SCATTFRING OF POSITIVE PIONS ON 160OXYGEN IT HO, A ANO 49,7 MEV

2UOB IAELASTIC SCATTERING OF PI + MESTNS FROM NUCLEI AT 5 A HEV

1091 MEASUREMENTS OF PION-NUCLEAR ELASTIC SCATTERING AT LOW ENERGIES

zABA PIONONUCLEAR INELABTIC SCATTERING AT 50 mEV

$\operatorname{lip} 310$

int 1 .

QETHE, H. A.

218 DENBE GaRYON MATTER CALCULATIONS WTTH REALISTIC POTENTIALS

1979 EFFECTS OF NUTLELR GROUND STATE CORRELATIONS ON PION SCATTERING

3000 CAULI PRINETPAL AND PION SEATTERING

217 THEORY OF PHE SCATTERING OF OIONS BY NUCLEAR MATTER

BEVINGTON, P.R.

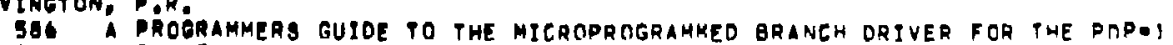

506 ABSOLUTE OIFFRENTIAL CROSS SECTION MEASUREMENTS FOR PROTONOPROTON ELASTIC SCATTERTNG AT

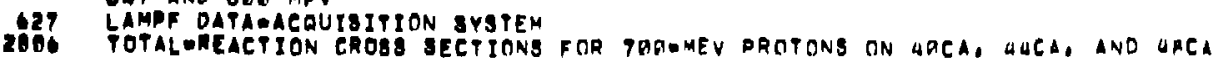

7 4อ०ด?

$70,75,5$

$751,5:$

750,0

อterInLICH, S,A,

Thoops

Thang:

$\operatorname{limanan}$ 
etstgalich, si, i, icontó,',

1633 DHOTON SDECTRIJM IN PION CAPTURE ON PRITIUM

23IS RADIATIVE NEGATIVE PION CAPTUAE IN LIQUIO NEON

2369 RADIATIVE NEGATIVE PION CAPTIRE IN LIOUIO TRITIUM

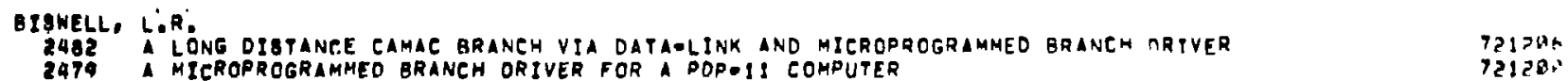

2479 A MICROPROGRAMMED BRANCH ORIVER FOR A POP-II COMPUTER
394 A STANDARD INTERFACE CONEEPT FOR COMPUTER OCONTROLLED DARTICLE ACCELERATORS

394 A STANDARD INTERFACE CONEEPT FOR COMPUTER OCONTROLLED DART ICLE ACCELERATORS

38U DESIGN AND OPFRATION OF A MICROPROGRAMMEO BRANCH DRIVER FOR A PDPOII COMPUPER

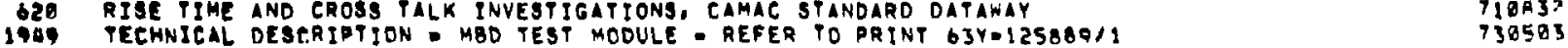

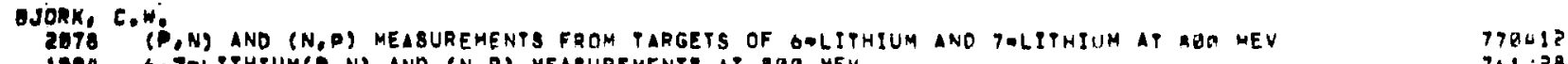

1994 607OLITHIUM(R,N) AND (N,P) MELSUREMENTS AT BBQ HEV

20A A MULTIWIRE PROPORTIONAL CHAMBER SPECTROMETER FOR NUCLEONONUCLEON EXPEQIMENTS 731. P

2418 EXTREME BACK ANGLE ND ELASTIC SCATYERING AY 794 MEV

2497 MEASUREMENT OF (P,N) AND (N,P) REACTIONS ON GOLITMIUM ANO 7-LITHPUM AT BAB MEV

203 LIGHT NUCLEI

1634 NEUPRON 9PECTRA AT 2ERO DEGREE FROM (P,N) REACTIONS ON Q-RERYLLIIIM, IZ-CARAON AND $27-$

Toaras

1635 NEUTRON SPECTRA AT ZERO OEGREE FROM POP ANO DOO COLLISIONS AT GLY LNO RAR WEV INCIOENT

760795 ENERGIES

1969 NEUTRON GPECTAA AT ZERO GEGREE FROM PROYON-PROTON COLLISIONS BETHEEN GLT ANO BRS MEV NEUTRON SPECTRA FROM SPR MEY PROTOHS ON

NEUTRON BPECTRA FROM PAOTON BOMBAROMENT OF DEUTERIUM AT GLT ANO AOA MEV

PRECISTON MEASUREMENT OF NAP CHARGE -EXENANGE CROSS SECTION AT GH MEV

PRECIOION MEAQUREMENT OF NAO CHLRGE -EXCHANGE CROSS SECTION IT GUO MEV

24E DUASILLATIC GHARGE EXCHANGE IN ND GOESTO PNN AT 900 MEV

7 7aras

740.10.

$750.0 \%$

7633 की

$750.10 \%$

7902 (n)

DLaKely, É:

$740590^{\circ}$

OLANDIED, G.8

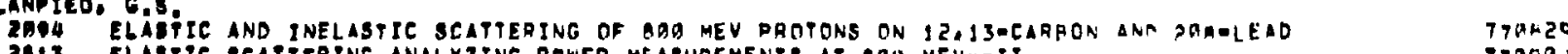

2613 ELASTIC SCATTFATNG ANALYZING POWER MEASUREMENTS IT BQG MEVIEII

2806 P I3,I2C, INELASTIC BCATTERING AT 9.8 GEV

Tpopa

DLECHER, M

ISGI MN EXPERIMENTAL STUOY OF THE PI+ + D GOESTO P + D REACTION AT PION ENERGIES OF UA, SP ANA T5ARBO

2406 INELASTIC SCATTERING OF DI+ MESONS FROM NUCLEI AT 5 P MEV

$771>15$ 
DLECHER, M: CCONTO,

24BU PION-NUCLEAR TNELASTTC SCATTERTNG AT 50 ME

2069 THE DEUTERON RESTATE INO THE PI+ + D GOESTOP + D REACTION

2421 THE PI+ D GOESTOP LP REACTION AT 49 A 59 ANA OP MEV
1543 TUNEUP OF THE LAMPF LOW ENEQGY PION CHANNEL

BLEWETT. S.P:

2293 FOUR POLE MAGNET RINGS FRR FOCUSING BELMS OF CHIRGED PARTICLES
2307 SUMMARY REPORT OF COMMITTEE CONVENEO BY H. KAPLAN, M, D., TO STUOY POSSIBLE PION GENERATORS
TSALAS

BOEHH, F.

141 A PRECISE DETFRMINATYON OF EZ ANO EL HOMENTS IN 165 HOLMIUM FROM MUONIE XODAYS

A PRECISION DFTERMINATION OF THE RADIAL CHARGE PARAMETERS ANC THE QULDOUDOLE HOMENT OF

2410 181-TANTALUM 115 ING MUONIC XORAYS RAOTALS

419 CIRCULAR POLARIZATION DF MUONIC X RAYS ANO ORIGIN OF STRANGE MU MINUS TEPOLARIZLTION IN 771205

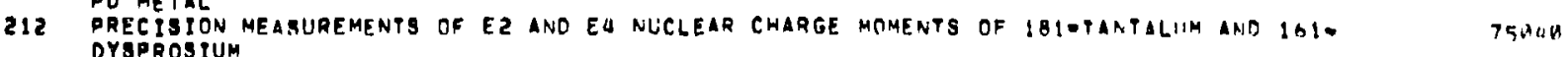

BOLGER, J,E.

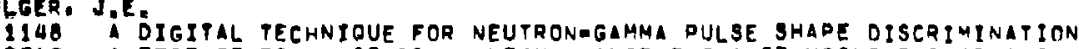

2060 A TEST OF FINAL STATE NUELEON CHARGE EXCHANGE USING THE REACTIONS 13-CARDONTPI+OR-,PI+OR. N) I

1133 COMPARISON OF NEUTRON REMOVAL FROM 7-LITHIUM BY PI +OE- WITH FREE PIONANICLEAN RESULIS

1990 EVIDENCE FOR SPRONG TRANSITIONS TO 5/2- STATES IN AEII INDUCED BY PI+ ON I2-CARBON

1999 MEASUREMENTS OF THE 7-LITHIUMPDI+OR-,DI+OR=N) 6=LITWIUM CROSS SECTIONS COMPAREO TO FRFE PIONANUCLEON RESULTS

2093 PION INOUCEO NUCLEON REMOVAL FROM LIGHT NUCLE!

1992 STUDY OF THE (PIE,N) AND (PI,PI PRIME P) REACTIONS IN LIGHT NUCLEI

OOLBTERLI, M.

2726 ESTIMATES DF MAXIMIJM ENERGY PION PRODUCTION ON NUCLEL, KAON, LAMRDA PRODUCTION ON NUCLET, ANO STRANGENESS VIOLATING LAMBDA PROQUCTION ON NEUTRONS BY BOB MEV PROTONS

769901

$7 n !: 19: 9$

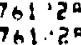

$\log a$

$7+1,5 \cos$

Bhด्ดि:

DONNER, B,E.

2A78 (P,N) AND (N,P) MEASUREMENTS FROM TARGETS OF GOLITHIUM ANO 7-LITHIUM AT MGO MEV

1994 6.7-LITHIUM(P,N) AND (N,P) MEABUREMENTS AT BBO MEV

204 AMULIIWIRE PROPORTIONAL CHAMBER SPECTROMETER FOR NUCLEONONUCLEON FXPERI UENTS

2016 EXTREME BACK ANTLE NO ELASTIC SCATTERING AT T94 MEV

2409 MEASUREMENT OF RP,NS AND (N,P) REACTIONS ON GOLITHIUM AND 9ELITHIUM AT SARO MEV

179 MEASUREMENT OF THE BNDEGREE NEUTRON SPECTRA FROM G49 ANO BME MEV PROTON ROMBAROMENT OF LIGHT NUCLEI

203 MEASUREMENT OF SUB 964 MFV

NOP AND NOD INTERACTEONG AT ENERGIES UP TO SGA MEV 1634 NEUTRON SPECPRA AT ZERA GEGREE FROH (P,N) REACTIONS ON Q-BERYLLIUM, IZACARAON AND 27.
ALUMINUM AT GOT AND GAG MEV 
BOMnER, B.E. CCONTO.)

IG35 NEUTRON SPECYRA AT ZERO DEGREE FROM PAP AND POD COLLISIONB AT GAT AND GOB HEV INEIDENT

EWEREIES

NEUPAON SFECTRA FROM 5 GQ MEV PROPONS ON OABERYLLIUM

NEUPRON GPECTRA FROM PROTON BOMBARDMENT OF OEUTERIUM AT GUT AHD BOG MEV

- ION PRODUCTION IN NEUTRONGPROTON COLLISIONS

NEP CHARGE-EXCHANGE EROSS SECTION AT GET MEV

PRECISION MEARUREMENT OF NGP CHARGEEEXCHANGE EROSS BECTION AT GUP MEV

QUASIELASTIC CHARGE EXCHANGE IN ND GOESTO PNN AT T94 MEV

900705

176101

70040.

$750 \times 9 \%$

751220

$76030 \mathrm{Aa}$

249

$779 \times 2 h$

BOONE, M.LiM.

36 LOCALIZEO HEATIMG AS AN ADJUNCT TO RAOIATION PHERAPY

95. PROGRESS TOWARD USTHG MUEAESTC XORAYS AS A OIAGNOSTIC TOOL

TR THE POTENTSAL OF LOCALIZED HEATING AS AN AOJUNCT TO RADIATION THERAPY

701291

73073.3

$741, A Q ?$

Dononen, $c$

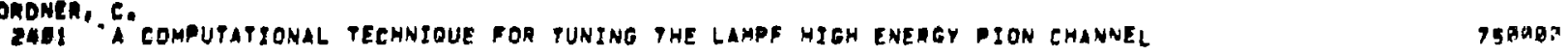

IOSG. W. SPECIFICATIONR FON PRIMARY OR CERTIFIEO GAS MIXTURES

730917

BOUORIE, M.L:

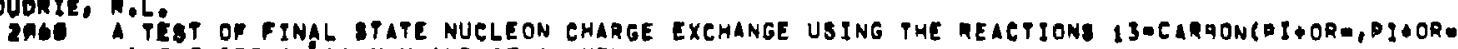
N) 12-CARBON (A.AL MEV AND 15.11 MEV)

2811 INELABTIC SCATTERING OF I AMO P . FROM 180XYGEN AT 140 AND 230 MEV

2919 HEUTAON OEPORMATION PARAMETER GY COMPAOATIVE STUOY OF PI AND P I INELASTIC SEATTERING

2001 PI -MESON INDUEED DROTON REHOVAL FROM O-BERYLLIUM TO THE G. OBI MEV ONE PLIIS STATE OF

2003 TIOM INDUCED MULLEON REMOVAL GROM LIGHT NUCLE!

$779098:$

779189

170000

DOWMAN, J,D.

1547 G MEABUREMENT OF DARITY VIOLATION IN PROTONAPROTON SCATTERING AT 15 MEV

167. CONIZATION CHAMBERS

26S8 LAMPF OEAM NOTBE FORMALISM

15U2 LIMIT ON PAR ITY NONCONGERVATION IN P-NUCLEUS SCATTERING AT G GEV/C

1620 LIMIT ON DARITY VIOLATION IN PONUELEUS SCAPTERING AP G GEVIC

22. TEST OP PARITY CONGERVATION IN PAP SCATTERING

7099.

Goro, T. Jor Jh.

2570 INCMEABED GRANIENTST

638 GAMPF 2 GOAMHZ POWER SOURCES

206 OP IMUM GENERATOR CHARACPERISTICS OF RF AMPLIFIERS FOR MEAVILY BEAMELOADED ACCELERATORS

Q43 PHERHEX A HPGHCURQENT ELEETRON ACCELEATTOR FOR USE IN DYNAMIE RADIOGRAPHY

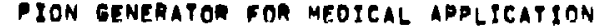

1232 DRELIMINARY OFVELOPMENT OFF PHE LLTERNATING PHASE FOCUSED LINAC STRUETUAE 
DOYO, Tis.s. JR. (CONTR,

TOR 201.25 MHZ ORIFT TUQE LTNAC RF BELLOWO

TUE

741,39

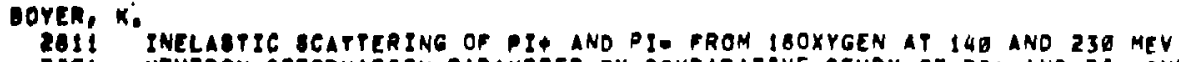

2011 INELABTIC BCATPERING OF PI\$ AND PID FROM 180XYGEN AT 140 ANO 230 MEV 77090 G

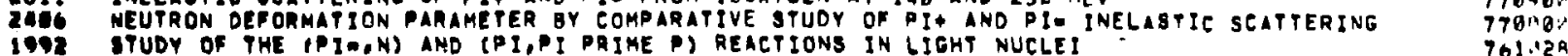

BRAOUURY, J.N:

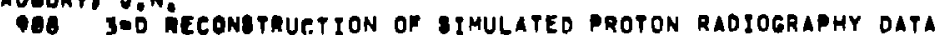

750nou

143 ABgORECD DOSE AMD RADIATION QUALITY BOR BACKGROUNO RADIATION FROM A NEGATIVE PION BEAM TSOAOX

1024 ANNUAL REPERY TO COMGRESS

ABNORMALITIES

912 EVALUATION OF NONDDESTRUCTIVE ANALYSIS TECHNIOUES AT LAMPF

1023 . EXPERIMENT NUMAER IO

1023 - EXPERIMENT NUMBER 195 g INITISAL COMPATATIV LIGHT ION LINACE FOR HEOICAL APPLICATIONB

1. HEDTCAL LINAC OESIGN POSBIBILITIFs

II O

1463 OOBERVATION OF THE NUCLEAR RES ONANCE EFFE

DAMAMETER OF THE LAMPF BIOMEDICAL BEAM

- ION GENERATOR FOR MEOICAL APPLIEATIONB

23.9 PROPOSAL FOA NEVELOPNENT OF AP PON

918 MOLE OF LOB AI AMOS SEIENTIFIC LABORATORY

2071 PECMNOLOGY PROGRAMS AT A MEBON PACTORY TOMOGAPHY

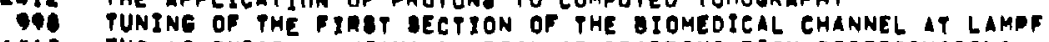

1062 TWOODIMENBIONAL VIQUALIZATION OF BTOPDING DION OISTRIBUTIONE

950 VIBUALIIATION OF SPOPPING PION OIBTRIQUTION

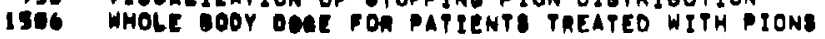

75 की

750.0 .9$.

750116

760.0014

750604

748607

756217

760725

750501

$75000 \mathrm{P}$

$70398:$

7 CH3 30

771 : 7 त

$75060^{\circ}$

76 PQ⿻:

$75029: 9$
$76920 ?$

\section{ORALTHWAITE, H'.J.}

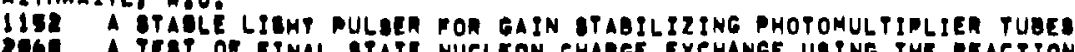

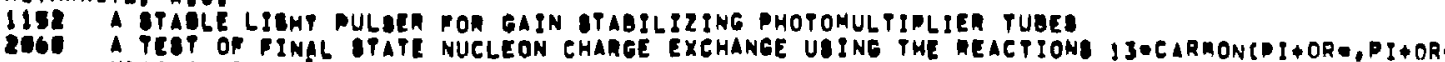

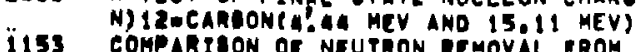

1153 COMPARION OF NEUTMON REMOVAL PROM Y-LITHIUM OY PI +OR- WITH FREE PION-NITLEON RESULTS

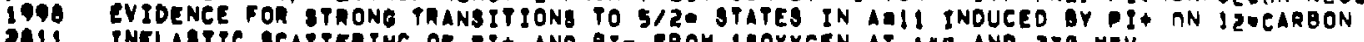

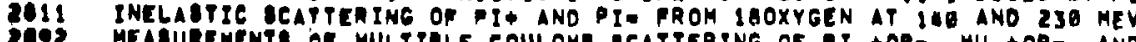

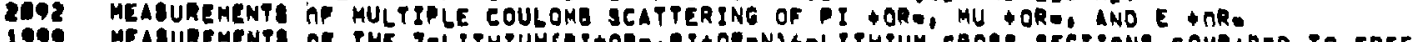

1999 MEA UREMENTS AP PHE T-LITHIUHCPI+ORE,PI+ORONI6OLITHIUM CROSS SECTIONS COUPARED TO FREE

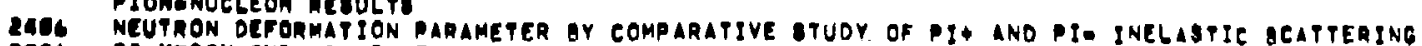

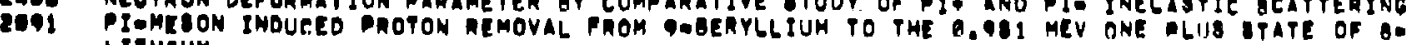

eses GION IMONCLO WUCLEON REMOVAL PAOM LIGHT NUELEI 
BRALTHWAITE, W.J. (CONTD,)

1992 STUOY OF THE (PIA,N) INO (PI,PI PRIME P) REACTIONS IN LIGHT NUELEI

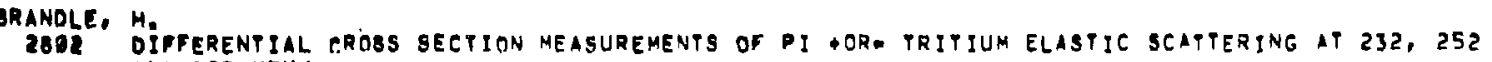

AND 295 MEV/C

2423 MEA 295 MEVTC.

MEAOREMENTS HF THE OIFFERENTIAL CROSS SECTION FOR PION CHIRGE EXCHANGE GN TRITIUN . TTQRZ

2424 MEABUREMENTS AF THE DIFTERENTIAL CROBS SECTION GOR PION CHARGE EXCHANGE ON TRITIUM - TBBIZ3

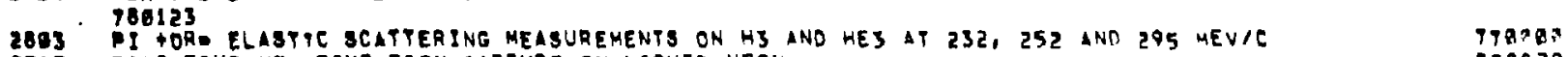

2515 RADIATIVE NEGATIVE PION CAPTURE IN LIGUIO NEON
2589 RADIATIVE NEGATIVE PION CAPTURE IN LIOUIO TRITIUM

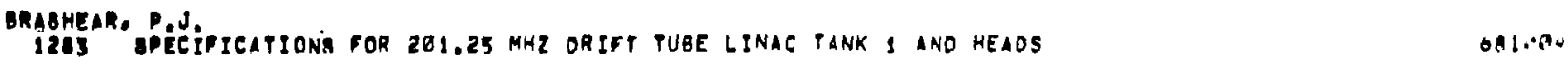

BROCK, L.R:
2710 BTANDANO ORERATING PROCEDURE CITRIC ACIO WASH FOR TUBES

7ดถ :1:

BROOZZNOKI. R:L

659 INTERLABORATORY COMPARISON OF GMALLATION REACTION CROSS SECTIORS FOR IUOA ANO COPPER WITH TAB?B

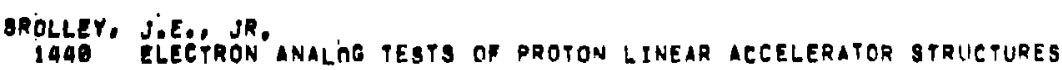

$658 n 8$.

BROWMAN, ÁÁ.

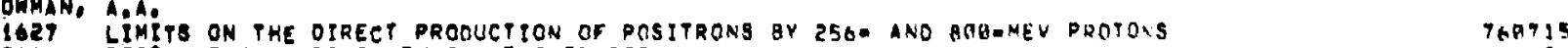

2693 LIMTTS ON THE OIREET PRC

2568 PROPIOSAL CHANAES IN PUNTNG PROCEDURES

2060 REPORT ON MACHINE STUDIES DONE IN OECEMBER OEVELOPMENT PERIOO

2560 REQUEST FOR DFVELOPMENT TIME - 778623

2572 REOUEST FOR OFVELOPMENT TIME - 770100

783 SEARCH FOR OIRECT LEPTON PRODUCTION IN PP COLLISIONS AT BAP MEV AND 250 "EV

1621 OEAREH FOR TMF DIRECT PRDOUCTION OF POSIPRONS BY 250. AND APPEMEV PROTANS

2665 TR AND 201 ALTGNMENT EXPERIMENT

2571 UPGRADE OF WIRE BCANNER

17845

$770 \times 2$.

761229

778 23

77 A

768.20

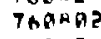

770.2

$7749 ?$

Brown, D.

204 A MULTIMIRE PROPORTIONAL CHAMEER SPECTROMETER FOR NUCLEON-NUCLEON FXPERIUENTS

192 ACRATCH PAD MEMORY OO BUFFERING MULTIWIRE PROP RRTIONAL CHAMBER OATA

731.13

UROANE, J.C. 
RROWNE. J,C, CCONTO.)

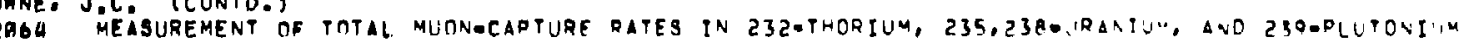

1990 MUON CAPTURE RATES ON ACTINTDE NUCLE?

$7+125$

750,20

BROWNELL, G.L.

1653 MYOCARDIAL DOSTTROY SCINTIGRAPHY WITH SHORTWLIVEO RUBITIUMAE

76020.

245 COLLIDING BFAMS EXPERIMENT AT LAMPF, PHOTOOETACHMENT RESONANCES TN THE H. ION

A MULTIWIAE PROPORTIONAL CHIHBER SPECTROMETER FOR NUCLF ON-NUCLEO EXOEEI AE. TS

ENERGY AND ANGULAR DISTRTAUTION OF NEUTRONS PRODUCED BY BDA MEV DRTTIINADEOTON COLLISIONS

179 MEASUREMENT OF THE P-DEGREE NEUTRON SOELTRA FROM GUT AND SRP MEV PQDTOA OOUA COMENT OF 203 LIGLT NUCLEI

1634 SEUTRON SOECTRA AT ZERO DEGREE FROM (P,N) REACTIONS ON QABERYLLIHM, IZ-CARRTA ANO 270

1634 ALUMINUM AT GAT AND GQA MEV

1035 NEUTRON SPECTRA AT ZERO OEGREE FROM PAP AND DAD COLLISTONS TT GLT ANIS RR: WEV INCIDEAT ENERGIES

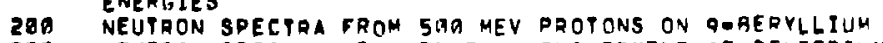

232 NEUTRON SPECTRA FROM PROTON BOMBARDHENT OF DEUTERIUM AT GHT $\triangle$ NAT RAM "EE 2061 OBSERVATION OF RESONANEES NEAR 11 EV IN THE PHOTODETACHMENT CROSS SECTYOA AF THE HE $10 \mathrm{~N}$

1990 PRECISTON MEASIIPEMENT OF NOP CHARGF -EXCHANGE CQOSS SEETION AT G4T MEV

202 PRECISION MEASUREMENT OF NOP CHARGE-EXCHANGE CROSS SECTION AT GUA MEV

\section{BUCHANAN, J.A.}

627 LAHPF DATA-ACAUTBITION SYSTEM

1960 PION PRODUCTION FROM THE PEACTION DIP,O PIIN AT 585 HEV

771115

331.95

754050
750120

$75 p \cdot a-$

T68789

700705

74960.

738.99.

701115

7 hastar

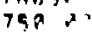

BUDGE, E.C.

TiGs AC OR DC MOTOQ DRIVERS - LAMPF CONTROL SYSTEM

86A ANALOG CONTROI TROM THE OIGITAL COMPUTER

1430 GPOLAR DATA TRANSMYSSION SYSTEM

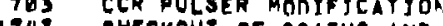

ITUS CHECKOUSE OF SPATUS AND VF PANELS

THO CIURR CE TRANAMTSSION SYSTEM

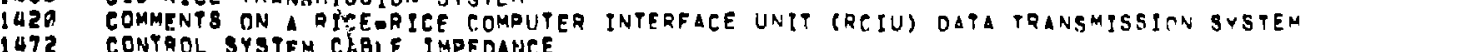

167 CONTRO SYSTEM DE

498

1408 CONTROL BYSTEM INTERLOCK CIRCUTTS

1989 ELECPRON HODEI REMOTE CONTROL. UNIT LOGIC CONNECTOR BOAROS SDECIFICATIOLS

1719 EMITTANCE DATA BYSTEM

718 EMITTANCE DATA IYSTEM PRTDOSAL

EPI TIHING SYBTEM

TaORAs

$761 \cdot 20$

09030

650916

67802 ?

ong.?

6985.

69?

bili.

6ioui:

670 ?

65 a

ancis

$6703 a$ a

b79

करा 1210

621210 
DUDER, E.C: (CONTO.)

176 "EXPERIMENYAL MASTER PULSER SPECIFICATION

FABRICATION RFQUEST NO. 211 MODEL L INTERLOCK ANO FILAMENT CONTROL
FABRICATION RFQUEST NO. $23 !$ MODIFICATIONS TO HVOI565 POWER SUPPLY

HIGH DUTY FACTOR ONESBHOT

INJECTOR CONTAOL RIOM CONSOLE CIACUIT OESIGN

INJECTOR CONYROL ROOM PANEL AND RACK LAYOUT

INSEETOR HIGH VOLTAGE CONTROL

ION SOURCE ARE MODULATOR

ION SOURCE PUI SER (TRANSISTOR SWITCH)

LAMPF ARE MOOULATOR

LAMPF ARE MOOIILATOR MODEL 3 - DESIGN REPORT

LAMPF CONTROL SYSTEM TIMING ANO FAST SHUTDOWN

LAMPF EXPERIMFNTAL STATUS PANEL SPECIFICATION

LAMPF GRAPHIC OISPLAYS

LAMPF I AND C PULSE TRANSMISSION BYSTEM NOISE

LAMPF ION SOLIRCE ARC MODULATOR

LAMPF VIOEO SYOTEM . SIGNAL TRANGMISSION

LIGHT LINK FEABIBILITY

LINEAR LIGHT I INK OATA TRANSHISSION

LOCAL MASTER PULBER - FABRICATION SPECIFICATION

MACHINE STATUS CHASSIS SPECIFICATION

MAGNET POWER

MAGNEY POWER AND CONTROL (Li.F. MODULES) PART I AND II

MAONET OHUNTS (BOME AODITIONAL THOUGHTS

Magren CONTRO CHASSIS SPECIFICATION

MOLT PANEL SDECIFICATION

DOF MODEL PROGRESS REPORT - O51120

MOQULAROR POUER JUPPLY MODEL PROGRESS REPORT " OSIIZ

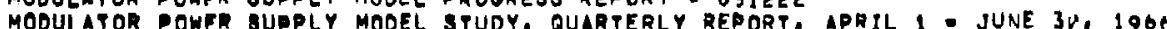

MOOULATOR POWFR SUPPLY MOOEL

NOPE ON OISCIISTONS WITH PQROFESSOR WILLILM HANNA

NOTES ON LOW IEVEL LTE ORIVERS

DEM DATA TRANRMIBS ION SYSTEM USING SPLIT PHASE CODE

PRELIMINARY RFY OESIGN

DROCUREMENT OF A CONTROL COMPUTER FOQ IME LAMPF MOCKUP EXEERTMENT

PROCUREMENT OF A CONTROL COMPUTER FOR THE LAMPE MOCK-UP EXPERTMENT

PULSER TESTS ON MODULATOR POWER SUPOLY MODEL

RACK AND OISPI AY LAYOUT - CCR

RAEK AND EOUTAMENT LAYOUT : ICR

RF MODULE PURNODN SEQUENCE

RICE COMMLND MLISY REOUIRFMENT

RICE-CIU LTNK EE

SAMPLING SCOPF PECHNIOUE FOR VIOEO CHANNELS

SCR ARC MODULATOR

SLEWING INPUT TO ANALOG CONTROL CHANNELS

gOME DEBTGN CONCEPTS PERTAL

SWITCH BOUNCE 3 UPDOESSION

916 TIMING SYBTEM WIRING AND CONTROL SUMMARY

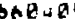

660942

660097

66000 A

$691+92$

668223

651111

662113

689104

$67111 \%$

oxต5a?

60 परद

65122 ?

bhä

obehat

ono711

60031 .

071100

608194

000319

671103

6องร2?

668114

651?

660028

660505

670520

651121

051222

651190

651114

67833 .

606319

GAB110

6อง

Gดिua

onh

65092 .

65and,

6月ais?

67110

651.25

b59.5?

bopagh

G7a?.

690310

$67052^{\circ}$

hnpine 
DUDE, E.C: (CONTO,)

BOS VIOEO OATA AND GINARY COMMAND CIRCUTTS OSII20

1752 VIDEO DATA SYSTEM (LAMPF) O5BQAR
1754 VIDEO BAMPLING

GUDINGER, T'F',

1653 MYÓCARDIAL POAITRON SEINTIGRAPHY WITH SHORT-LIVEO RUBIOIUM-BZ

DUNKER, M.E.

2335 PROPOSAL FOR AN ONGLINE MASS SPECTROMETER FACILITY AT LAMPF TO STUDY PRONUETS OF

73119

194 THE 158-RBIUM GOESTO ISBMOLMIUM DECAY SCHEME AND OCSUB BETAS VALUE

BURGERJON, j,j.

2080 SOLUTION FOR REMTTE HANOLING IN ACCELERATOR INSTALLATIONS

1000 FORCEMREFLCT TNG SERVOMANIPULATOR

2002 INTERIM SOLUTION FOR SERVOMANIPULATOR

2001 BHOCK HAVES IN LAMPF BEAM LINES

$79031 \mathrm{~h}$

Tha! 1 i

760119

QURLESON. G,R:

2011 INELASTIC SCATTERING OF PI+ AND PI- FROM 180XYGEN AT IHA ANO 23B MFV

77009 ?

75 ango

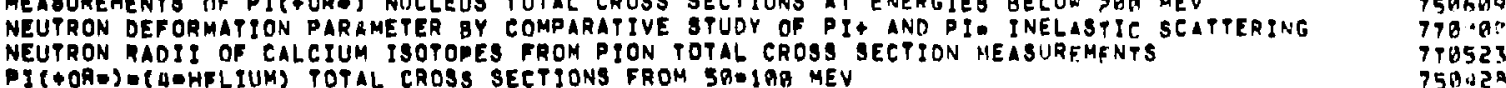

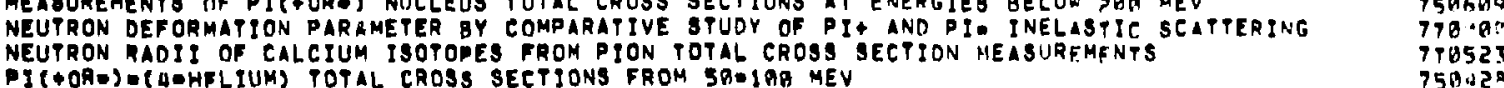
P (\$OA-) - (LOHFLIUM) POTAL CROSS SECTIONS FROM SBOIAB MEV

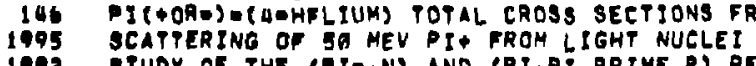

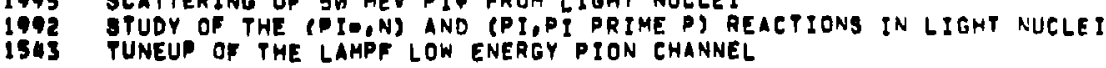

$7 B I: 2 A$

$761+2 n$

$74640 \%$

DURMAN, R:L.

ISG3 R. ABOLUTE CROSA BECTIONS FOR PRODUCTION OF PROMPT NUCLEAR GAMMA RAYS GY FAST PIONS

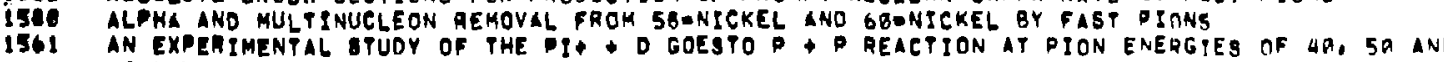
SO MEV

724 CHOES SECTIONA FOR (PI+,P) REACTIONS ON QTBERYLLIUM, IZ-CARBON, AND IGEOYYGEN

1558 CROSS GECTIONA FOR CPI+,QS REACTIONS IN GWLITHIUM, QUBEFYLLIUM, 12-CARQON, AND 16-OXYGEN

343 "LABPIC PIONANUELEUS SCATTERING FOR STUDIES OF THE NUCLEAR SURFACE

ELABTIC SCATTFRING OF POSITIVE PIONS ON 16-OXYGEN AT 4D. O ANO 49,7 MEV

ENEA ETIC CHARGED PARTICLE YIELDS INDUEEO BY PIONS ON COHPLEX NUELEI

- AGT PION INDUCED RROCESGE IN COMPLEX NUCLEI PROJECTILE SYSTEMATICS

159 PAST OION INDIICEO PROCESSES IN COMPLEX NUCLEII SYSTEMATICS IN PRODUET NUCLR

2AIS GAMMAORAY SPUAY OF PIONGINDUCED REACTIONS ON COMPLEX NUCLEI

2006 INELABTIE SCATTERING OF PI+ MESONS FROM NUCLEI AT SU MEV

1993 INTERACTION OF FABP OIONG WITH G2,640NICKEL

7 LAMPF NEUPRTNO FACILITY PROPOSAL

$750: 102$

75 anar

750600

710911

74928 ?

720800

77900 a

17040

$150401^{\circ}$

$75240^{\circ}$

750400

770425

771 ?

7कl 2 a

$71120: 4$
GQ90!? 
BURMAN, R,L, (CONTO,)

1467 LOW ENERGY PINNGNUCLEON AND PION-DEUTERON INTERACTIONS

2411 MABS DEPENOENTE OF PION DOUGLE CHAPGE EXCHANGE

D PONONUCLEAR ELASTIC SCATTERING AT LOH ENERGIES

NEW MEABUREMENTS OF (PI+. 2P) REACTIONS ON LIGHT NUELEI

P PON CHANNEL FOR LAMOF

A

- ION DOURLE =CHARGE EXCHANGE ON 16OOXYGEN AND IB-OXYGEN

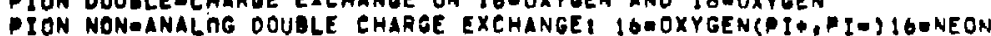

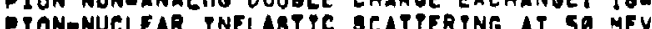

PROPERTIE OF A PROPOSED LONOENERGY GENERAL PURPOSE PION CHANNEL

PROTON GPECTRA PROM PIONOINDUCED REACTIONB

moron greta pa

SYETEMATICS OF PION ANO PROPON INTEAACTIONE WITH NICKEL NUCLIOES

THE LAMPF LOW ENERGY PION LINE

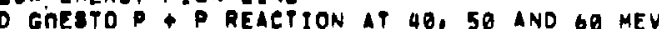

15a3 PUNEUP OF THE LAMPF LOW ENERGY PION CHANNEL

750000

780153

7611429

71905 ?

$\operatorname{690h20}$

770124

778.9 .9

$770+20$

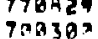

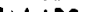

761128

761.125

719094

740.0.

BURNETT. DE.

1969 PHOR IUMEURANIUM F188ION RAOIOGRAPHY

76 ig

DURHHAM, C.

1653 MYOCAROIAL POAITRON BEINTIGRAPHY WITH SHORT-LIVEO RUBIDIUMABZ

76924.

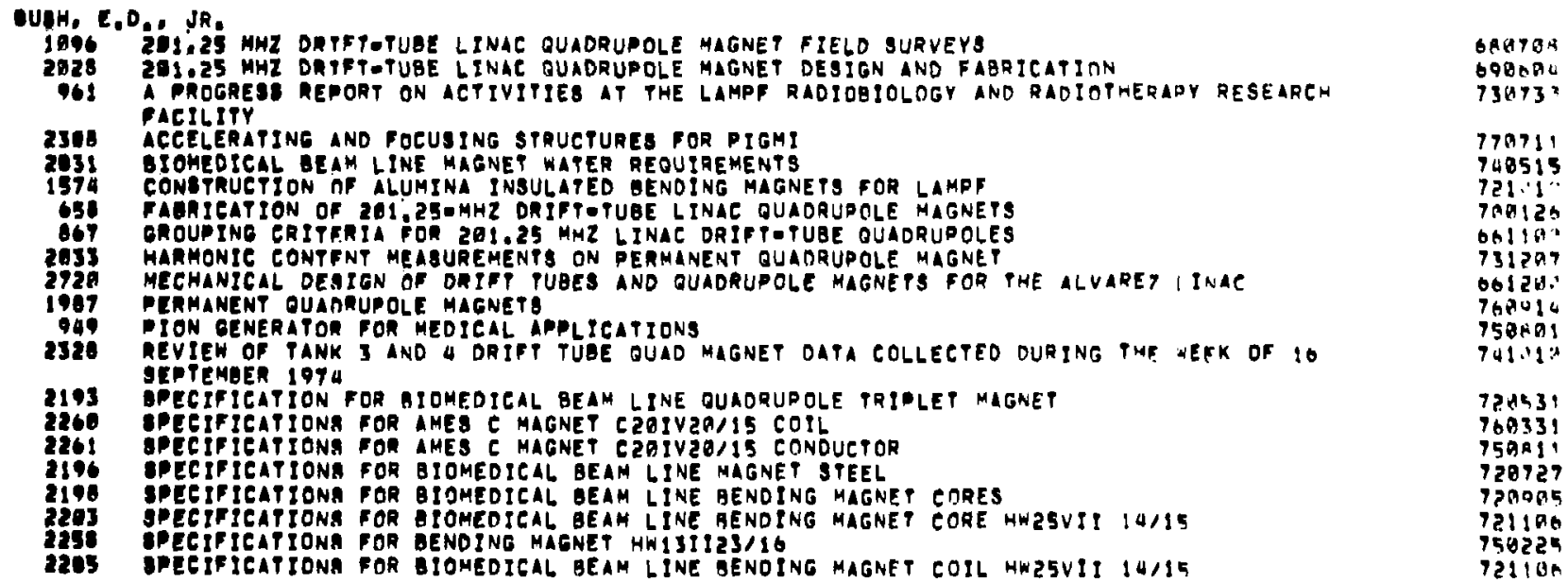


BUSH, E, D, JR, (CONTO,)

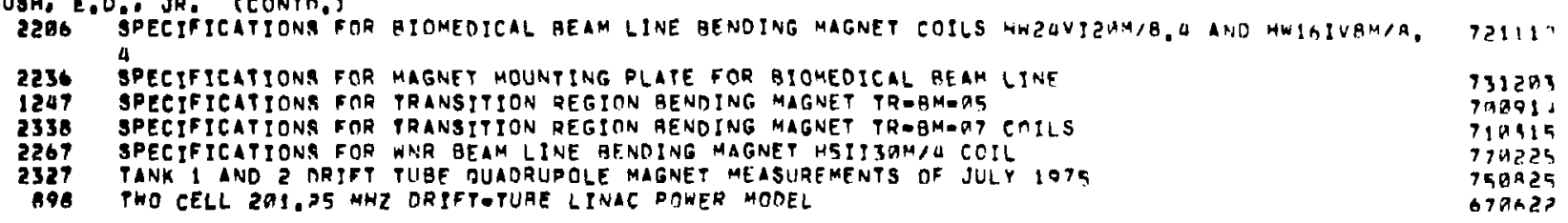

Bugrex, J.

2996 EDA

$7 ! 11=$

BUTLER, G.W.

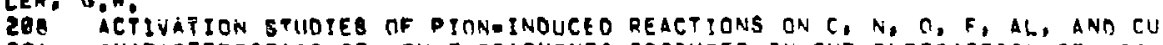

206 CHARACTERISTIPS OF LOW Z FRAGMENTS PROOUCEO IN THE INTERACTION OFF ARPALU PRTTONS WITH

$75 \ln 80$ HARACTEAISTITS OF LOW Z FRAGMENTS PROOUCEO IN PHE INTERACTION OF ABR AEU PRTTONS WITH

$15 \pi \sin 10$

171 EXCITATION FUNCTIONS FOR THE IZ-CARBON(PI+ORA,PI NIII-CARBON REACTIONS OUFF THE REGION OF

153331

1199 REECTIONS OF RAST DIONS WITH 197-GOLD

$\operatorname{Tत19.4}$

AUTLER, $\mathrm{H}, 3$

UID 'COMPUTER-BANED ACEELERATOR CONTROL SYSTEM

5 AN OPERATORS PONSOLE FOR THE LAMPF ACCELERATOR

632 CLARIFICATION OF LAMPF COMPUTER SPECIFICATION NO, MP.1.993

1418 COMPUTER CONTROL OF THE LAMPF ACCELERATOR

624 COMPUTER CONTROL OF PHE LAS ALAMTS LINEAR ACCELERATOR

2583 COMPUTING IN RUPPORT OF EXPERIMENTS AT LAMPF

1961 FLOATING-POINT HARDWARE SPECIF ICATIONS

622 INTERACTION OF A BUNCHED BEAM WITH TRANBVERSE MODES IN RF CAVITIES

613 INTERACTIVE GRAPHIES FOR ACCELERATOR CONTROL

LAA JUSTIFICATION FOR A CONPROL COMPUTFR FOR THE LOS ALANOS MESON PHYSTES FATIL IPY

1405 JUSTIFICATION FOR AOOITIONAL PERIPHERAL DEVICES FOR THE EPA CONTROL COUPIJTE

89. LAMPF CONTROL SYSTEM ANO COMPUTER INTERFACES

992 LAMPF DATA ACOUTSITION AND CONTROL SYSTEM

627 LAMPF DATAEACDUISITITN SYSTEM
1570 LAMPF MASTER TIMER ANO T SUGEZERO

1516 LAMPF SPECIFISATION FOR YWO DIGITAL COMPUTER SYSTEMS

1ABI LAMPF SPECIFICATION FOR DIGITAL CONTROL COMPUTER SYSTEM

2453 MINUTES OF MEFTING ON MAGNEY SETTING

169 MPOI PROGRAMMPNG BTANOARD 1 - FLOWCHARTING

1092 MPOL R ANO D MROGRAM FOR FYOTO

1 1AB MP-1 R AND D PROGRAM FYM69

2451 MPOI SUPPORT FOR PRONUCPION CODES

6BA NATURAL OEBUNCHING OF PHE LINAC GEAM MICROSTRUETURE NOTE ON MU CHANNELS

IIQ1 NOTE ON PION THANBPDRT CHANNELS

2A5U ONOLINE MASS RPNAGE REDUIREMENTS FOR MP OIVISION
2476 PERFORMANCE OF LAMOF COMPUTER CONTROL SYSTEM

$\operatorname{lng} 10 \operatorname{sen}$

19Bम"

6ดR1

6B1?ค1

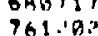

Toanz?

7oम?2?

6ha1日,

691117

$690 \therefore+199$
$60032 ?$

6oha?

66919

70 a

790120

$71 B 110$

6oalith

जकराशू

$669.2 n$

600720

6 की5 द?

770 दे।

650 in

Guसpar

679721

in: 125 
BUPLER. H.S. (CONTO.)

COMPUTER SYSTEM

PROCESS COMPUTER CONTROL OF PHE LAMPF PROTON LINEAR ACCELERATOR

PROCUREMENT OF A CONTROL EAMPUTER FOR THE LAMPF MOCK-UP EXDERIMENT

ROGRAMMING FOR THE MOCKUP

PROGRAMMING PHE BEAM POSITION MONITORS

PROPOSAL FOR THE LAMPF COMPUTER PLUS APPENOIX $A, B, C, O$

PROPOSAL FOR THE LAMPF CONTROL COHPUTER PLUS APPENDIX A G

PHE FAST GINARY COMMAND CARO PREPARED WITH PHE AID OF ROQ HILL, SIU LITTLE, ANO

WAYNE SMIPH

1301 REVIEW OF PME MP-I R AND D PROGRAM FOR 1969

1407

SPECIFICATION FOR DIGITAL CONTROL COHPUTER SYSTEM

INE OIGITAL COMPUTER SYSTEM

162 THE DESIGN OF HIGH-INTENSITY MUON CHANNELS FOR MESON FACILITY

625 THE IMPACT OF COMPUTERS ON THE CONTROL OF PARTICLE ACCELERATORS

1490 TIMING CONBIOERATIONS IN LAMPF CONTROL SYSTEM

626 TRANSVERSE BEAM BLOWWUP IN A STANDING WAVE LINAC CAVITY
iG26 USE OF COMPUTERS FOR CONTROLS

CADY, R.L.

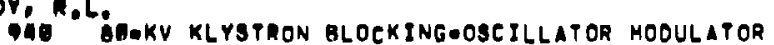

283 FULL POWER OEFRATION OF PHE LAMPF BRS MHZ SYSTEM

2274 HO PROBE CALIARATION IN THE BOSWMHZ ACCELERATOR ILNKS

2356 KLYSTRON TEBT BTAND SOR - RF BYSTEM FOR THE EPA

26. PERPORMANCE OF PULSED GO5-MHZ, 1,25.MW KLYSTRONS INTO MISMATCHED LOACS

2167 PRE-IMSTALLATION TUNTNG PAOCEDURE BS5M MHZ ACEELERATOR TANK SECTIONS

1904 MROCESSING PRACEDURE FOR LPT UG MIGH CURRENT FAILUAES

2232 GPCCIFICATIONE FOR AC DIELECTRIC/INSULATION TEST UNIT

2223 PHE DESIGN ANA PERFORMANCE OF THE LAMPF 1-1/A MH KLYSTRON MONULATOD

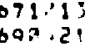

$31: 1$

ด6อ?ด

6ดคำ

60031

671.52

6815a?

68129 ?

$701 \cdot 07$

क्विका

65092

करा द3

65172.1

75 and

(67)

071.23
658796

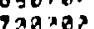

CalAREO, J'R.

156 MEASUREMENTS AF PI(०ORE) NUCLEUS TOTAL CROSS SECTIONS AT ENERGIES RELO. PMR MEV

2081 NEUTHON RADIS OF CALCIUM ISOTOPES FROM PION TOPAL CROSS SECTION MEASIREMFNTS

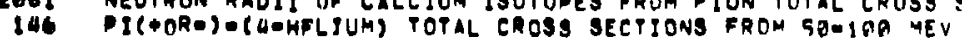

onasel

$\operatorname{limas}$

$\operatorname{rogh} 3$

790005

14009:

T?B? 2 ?

74 คคด

731111

$73 \mathrm{BOPh}$

73 PQ1:

CAMANT, M

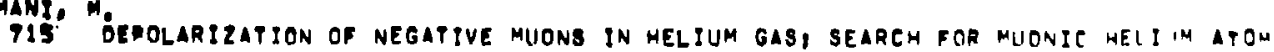

759090

778423

$75002 a$

$74 \sin 13$

CAMEAINO,

550 PHEORY OF INTRRACTION OF MESONS ANO NUCLEONS WITH NUCLEI

71123

CAMHON, T,M, 
CANNON, T.M, (CONTD,)

908 30D RECONBTRUETION OF SIMULATEO PROTON RAOIOGRAPHY DATA 13 OEVELOPMENT OF A PAOTON RAOIOGRAPHIC SYSTEM FOR OIAGNOSIS ANO LOCALIZATION OF SOFT TISSUE

2412 THE APPLICATION OF PROYONS TO COMPUTED TOMOGRAPHY

$750 A B$

75078

$771<a$

CARLINI, R.

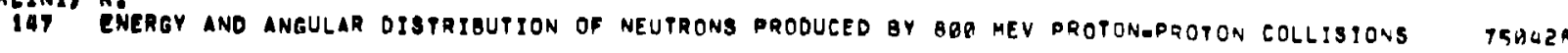

\section{CABE, J.R EABLE TYPES TOR LAMPF}

1530 CABLE TYPES PAR LAMPF

lait MPOI PROCEDURF FOR SUBHITTING INSTALLATION WORK TO PHE ELECTRICAL CRAFTS AT LAMDF

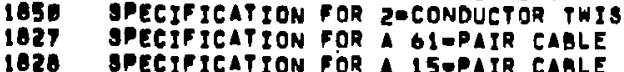

IOa7 BPECIFICATION FOR A CONTROL ROOM CONBOLE, ELECTRONIC RACKS ANO 3 O DEGRE WEDGE SECTIONS

i840 SPECIFICATION FOR A IOAPAIR CAQLE

1075 SPECIFICATION FOR LAMPF POWER SUPPLY CABLES

1022 SPECIFICATION POR FHRE CONOUCTOR. TWISTED TRIPLET, NO, 20 AWG, CARLE, WTTHOUT SHIELD OR

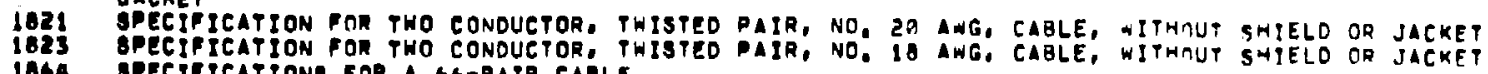

186a GPECIFICATIONA FOR a GGOPAIR CABLE

1926 GPCIFICATIONG FOR CAMAC JOBO CABLE

1006 SPECIFICATIONA FOR LAMPF SIGNAL AND CONTROL CABLE - GOAGZ5

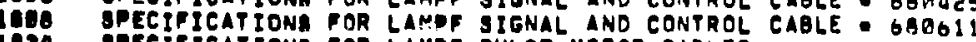

1024 SPECIFICATIONR FOR LAMPF PULBE MOTOR CABLES

1022 SPECIFICATIONR FOR LAMPF BIGNAL AND CONTROL CABLE

1812 SPECIFICATIONA DOR LAMPF RICE I/O CABLES

1057 BPECIFICATIONR FOR IIGNAL ANO CONTROL CABLE

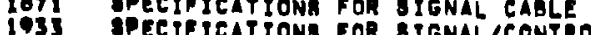

of WIRUNOA COMPUPER PHOGRAM FOR HIRING LARGE FACILITIES

GAOELLA, Vin:

I6IS GPALLATION CHOSS SECTIONS INO THE LAMPF MEOICAL RADIOISOTOPE PROGRAM

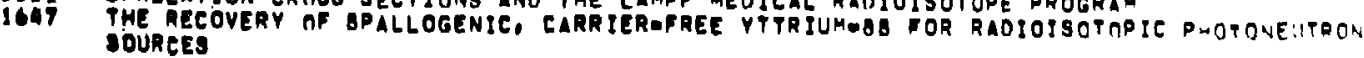

6RO० 14

609525

690924

69103

69015

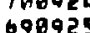

69095

7911

711.914

690325

690327

701200

720027

ongusa

GAPQII

000505

720705

719320

7 เง 31

72005

72128

7 Inp.

750303

75 Andis

CAPPERSON, DEE'.

IGIf A NEW HIEH PAPCIOION MEABUREMENT OF THE MUONgUM HYPEAFINE STRUCTURE INTEMVAL OELTA NU

29. DEPOLARIZATION OF NEGATIVE MUONS IN HELIUM GAS, SEARCH FOR MUONIC HELIIIM ATOM

MUONFUM

MUONIUM FORMATION IN NOBLE GASES ANO NOBLE GAS MIXTURE

I6 GAECISION MEAEUREMENT OP MUONIUM HFS INTERVAL AND MUON MAGNETIC MOMENT 
CABPERBON, D.E, (CONTD,)

169 PRECISION MEAGUREMENT OF 19 \&SUg I/2) MUONIUM HYPERFINE STRUCTURE IATERVAL TELTA NUS PART 1

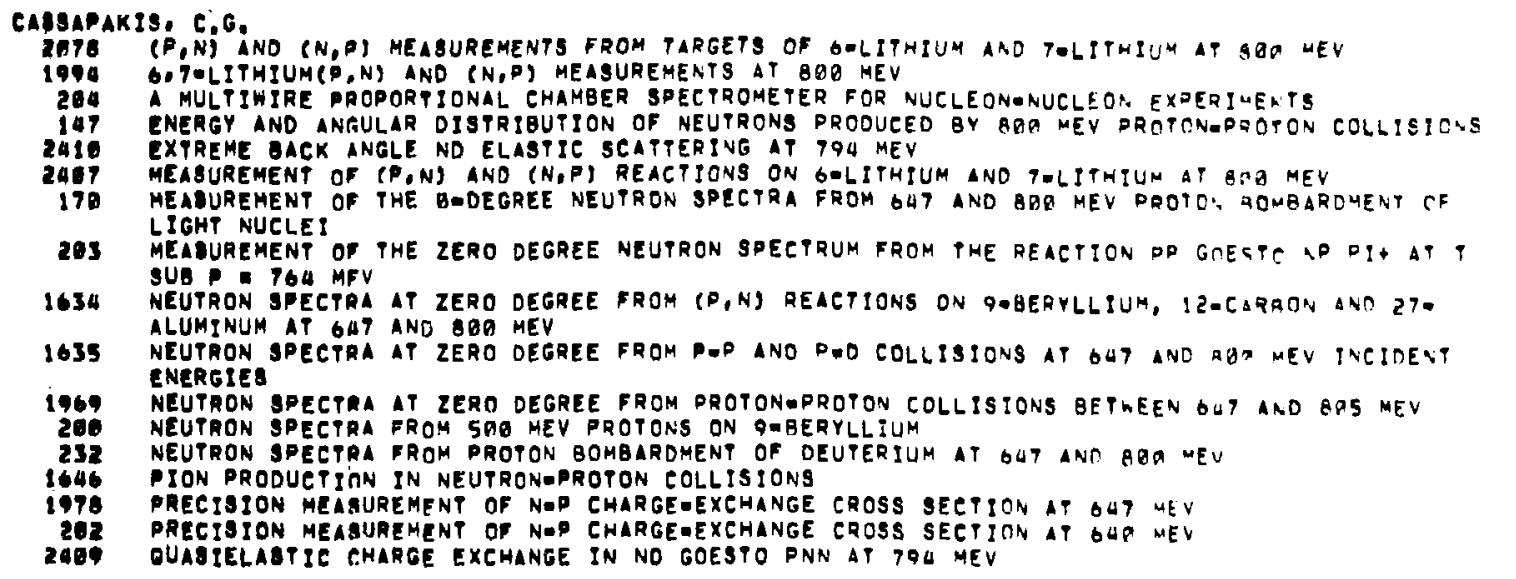

\section{CHAMUERLIN, E,}

2547 IMJECTOR MAGNFT MOOTFICATIONG

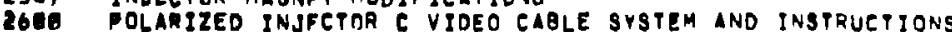

2595 POLARIZED INJFCTOR DOHE OOWER AND LIGHTING

25a9 POLARIZED ION INJECTOR PROGRAM

2546 POLARIZED ION SOURCE (CHAMBERLIN 75001B)

2S44 REYISED LIST OF CONTROLS FOR POLARIZED INJECTOR

TME LAHPF POLARIZEO ION PACILITY G TATUS REPORT

2551 TRIPREPORT G ARGONNE NATIONAL LABORATORY, AUGUST 23-29, 1976 (CHAMBFRLI.)

5012

$75+\cdots$

700705

7 7ด7

778901

740497

750.198

751250

70830.

$750 \times 5$

CHABHAN, R,W.

IA3I GÁNO CLOSING IN AN MLYAREZ TYPE PARTICLE STRUCTURE

1427 ROUND PAGLE DPSCUSSION ON SPACE CHARGE AND RELATED EFFECTS

$760+25$

763494

$76012 !$

760725

75 haik

790310

Thด्वश?

$70005 \%$

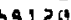

CHEN, $\mathrm{H}_{1} \mathrm{H}_{\mathrm{B}}$

MEASUREMENT OF STOPDED PION DECAYS IN A COPPER BEAM DUMP IT INETRE:T DOCTONS CE TZM

inas,:

17 MEY (A CALIBMATION OF THE LAMPF NEUTRINO BOUREE INTENSITY)

$7112 \%$

CHEN, $K_{\text {, }}$ 
CHEN, K. (CONTO.)

104. INPRANUELEAR CASCAOE STUDIES OF LOW-ENERGY PION-INDUCED NUCLEAR REACTI TVG-DOSSTBLE EFFETS OF THF FINITE LIFETIHE OF THE $(3,3)$ ISOBLR

73मค日:

CHIANG. D.T.

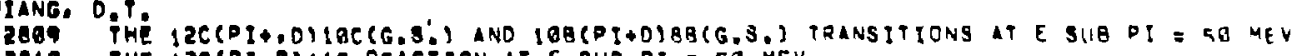

2810 THE LCCDI,PIIIC REACTION ATE SUB PI : SQ MEV

77890.

CHRIEN, R,E.

627 RAMPF DATA-ACQUTSTTION SYgTEM

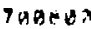

EHRISTIAN, RE

727 30 MAGNETIC FELL COMPUTATIONB AND COMPARISON WITH MEASUREMENTS 7?9, A:

CHRIBTOFILOS

2203 FOUR POLE MAGNET RINGS STR FOCUSING REAMS OF CMARGED PARTICLES

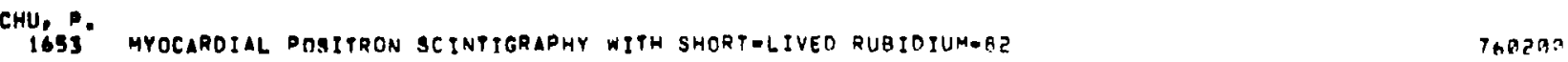

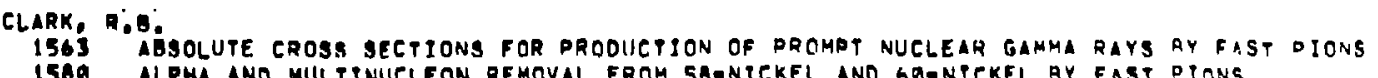

1560 ALPHA AND MULTINUELEON REMOVAL FROH SO-NICKEL ANO 6D-NICKEL BY FAST DITNS

1581 FAST PION INOLICEO PROCESSES IN COMPLEX NUCLES! CROSS SECTION ANO ALPMA QF

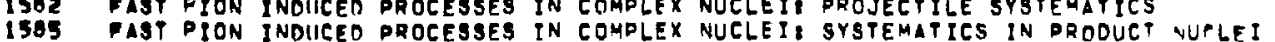

1505 PAST PION INDIICEO PROCESSES IN COMPLEX NUCLEI SYSTEMATICS IN DROOUCT VUPLEI

CLEARY, T,

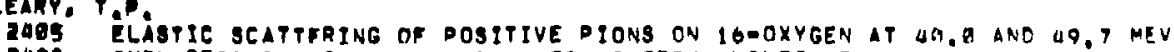

2WOS ELASPE SCATTFRING OF DOSITIVE PIONS ON 16-OXYGEN AT 49.

24R. PNELASTIC SCATPERING OF PP\$ MESONS FROM NUCLE

CLEMENT, J

2BO7 AHGULAR OISTRTBUTION FOR PD GOESTO 3HE PI ZERO REACTION AT TPEBOP MEV

222 FINAL CBTATE INTERACTIONS IN POD HREAKUP AT BAR ANO OQQ ME

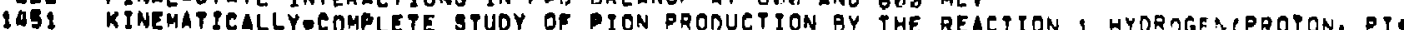
PROPONgNEUTRON AT

1991 ION PRODUCTINN OY BQG MEV PROTONS FRAM 6-LITHIUM

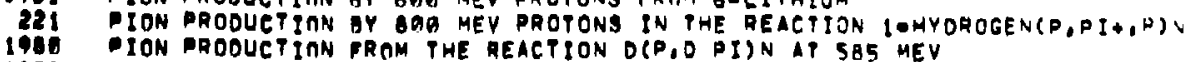

- ION PRODUET INA IN POP INTERACTIONB AT GDB MEV

ION PAOOUCTINN VIA A PAP GOESTO DAPI QUASTFFRE PROTONS IN DEUPERIUM

$756 \times 97$

$150 \operatorname{san}$

$75049=$

$759+8 A$

750140

$778 \cdot 9.9$

771210

770001

75049

70142 .

(i)

this

$301 / 2 a$

7ol 7 . 


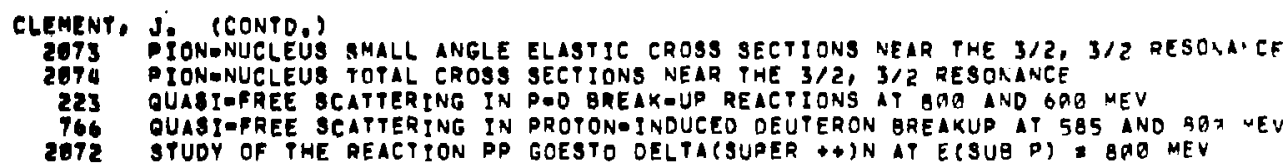

CLINE, J.E:

659: INTERLABORATORY COMPARISON OF GPALLATION REACTION CROSS SECTIDNS FOR IROA AND COPPER WITH

$769 \cdot 9-$ GQBOMEY PROTONS

COCHAVI. 3.

704 MEASUREMENTS AF PHE (DION PLUS, PION ZERO) REACTION ON LIGHT ELEMENTS iN THE $(3,3)$ RESONANCE REGION

96112

COCHRAN, D.R.P.

2429 CROSS SECTIONA FOR PI + NUCLEUS GOESTO PI +OR - ... FOR 740AMEV PROTOAS

117 LAMPF NEUYRINA FACILITY DROPOSAL

347 PRODUCTION OF CHARGED PIONS BY 730 MEY PROTONS FROM WYOROGEN ANO SELECTEN NUCLEI

61 RAOIATION THRTUGH BEAM CHANNELS IN THE PARGET CELL SHIELDING

6ES SHIELDING CONAIDERATIONS FOR THE PROPOSED LOS ALAMOS MESON PHYSICS FACILITY

063 SUMHARY OF BTATUS OF DESIGN OF STOPPED MUON CHANNEL
689 THE RADIATION AND SHIELOING OESIGN FACTORS FOR THE LOS ALAMOS MESON OHYSICS FACILITY

COHEN, O'L L
LIQT

$92110 \mathrm{a}$

71150.

0,975

$651: 15$

of日a

051 C2 $^{2}$

ดดaด1

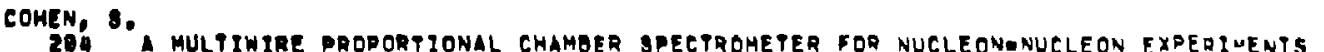

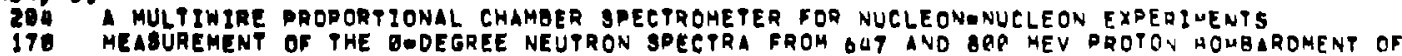

170 MEASUREMENT OF PHE OODEGREE NEUTRON SPECPRA FROM GUT AND BQP HEV DROTON HOMBAROMENT OF

MEABUREMENT OF TH

290 HEUTRON GPECTRA FROM SAO MEV PROTONS ON GOBERYLLIUM

232 NEUTRON SPECTAA PROM PROPON BOMBAROMENT OF OEUPERIUM AT 647 IND BRO NEV

20 DRECISION MEARUREMENT OF NOP CHARGEOEXHANGE CROSS SECTION AT GHG MEV

750.6

740,8 .

750.80

Coken, W.R.

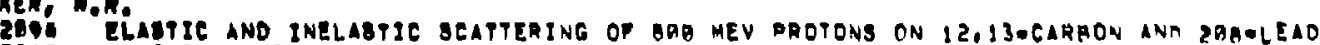

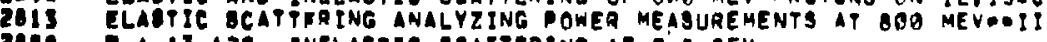

798020

770009

colten. mealunement of Pio elastic scattering 
colston, E.W.

1584 ALIGNAENT PHII OSOPHY, DESIGN AND TECHNIOUES USED AT LAMPF

INETALLATION AND ALIGNMENT OF LAMPF 201.25=MHZ AND DO5.MHZ LINAC IANKS

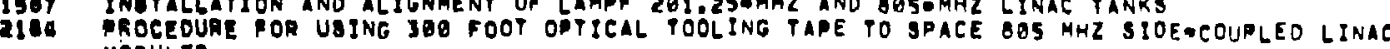
MOOULE:

COLVETY, Mi.

T16 GePi EMISSION FROM ENCAPSULATEO SCURCES OF CALIFORNIUM-232

758909

comino. s.

P13 HEABUREMENT OP THE ALPHAOPARTICLE SPECTRUM RESULTING FROM DIO GADTURE IN CARAON-12 T5OTB?

1459 CLECK AND CALPNDAR FUNCTIONAL DESCRIPTION AND DROGRAMMING INFORMATION

1792 FAGRTCATION RFQUEST NO. 24I ELECTRON MODEL LENS CONTROL

LAMP MAgTer tIMER AND

1190 LAMPF MASTER TIMER DESIGN

159. OECDNDOGENERATION MASTER TIMER

CONNGA. W. G.

930 LOCALIZED HEATING aS AN ADJUNCT TO RADIATION THERAPY

926 PHE POTENTIAL OF LOCALIZEO HEATING AS AN AOJUNET TO RAOIATION PHERAPY

Datran

669923

$71072^{\circ}$

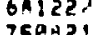

750421

74928

cook, $1 . \mathrm{c}$.

1563 AGOOLUTE CROBR SECPIONg FOR PRODUCTION OF PROMPT NUCLEAR GAMMA RAYS BY FAST PIONS

1300 ALPHA ANO MULTINUCLEON REMOVAL FROM 58-NICKEL. AND GOONICKEL OY FAST PIONG

1301 FAST PION INDUICD PROCESSES IN COMPLEX NUCLEIT CROSS SECTION AND ALPMA REMOYAL

$13 E 2$ FABT PION INOUCED PROCESBES IN COMPLEX NUCLEY PROJECTILE SYSTEMATICS

15 PAST PION INOUCED PROCESSES IN COMPLEX NUCLEI SYSTEMATICS IN PRODUCT NUCLFI

ISG2 BYBTEMATIES OF PION AND PROTON INTERACTIONS WITH NICKEL NUCLIDES

COOK, L,F GHEORY OF INTERACTION OF MESONB AND NUELEONS WITH NUCLE:

$711: 23$

coopen, $M, 0$ :

2HAP COULOMBONUELEAR INTERFERENCE WIPH PIONG

1629 IMPROVEO ANALYAIS OF COULOMB-NUELEAR INTERFERENCE EXPERIMENT FOR PIONS OS 10 OXYGE

$750,10 \%$

75 Hnp

7504 (19.

750.19

758049

778000

76953

764115

7 The3:4

$\operatorname{7an} 123$

LOW ENERGY IOHN ELABTIC SCATTERING FROM LIGHT NUCLEI

2411 MABS DEFENDENCE OF PION DOUBLE CHARGE EXEMANGE

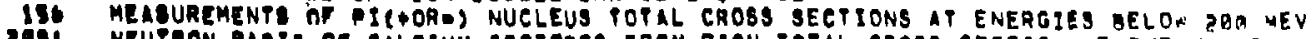

2051 NEUTRON RADII OF CALCIUH ISOTOPES FROM PION TOTAL CAOSS SECTION MEASUREMENTS

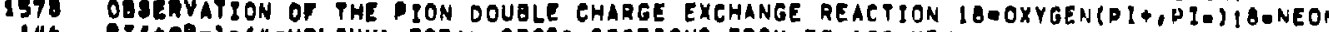

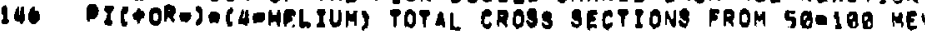

19052

790120

75 मीट 
cogper. M.O. (CONTO.)

2422 ION DOUBLE-CMARGE EXCHANGE ON 16-OXYGEN AND 18-OXYGEN

2417 ION NONGANALHG DOUBLE CHARGE EXCHANGEI 160OXYGEN(PI+,PI-I16-NEON TTB7IE

TA6 PIONANUCLEUS POTAL CROSS SECTIONS

1376 PIMEOOFOFLIGHY MEABUREMENTS IT TKE LAMPF LOWOENERGY PION CHANNEL

COOPER, RaK, LAMPF LINF D FABT OEFLECTOR SYSTEM
2097 PHE LAMP

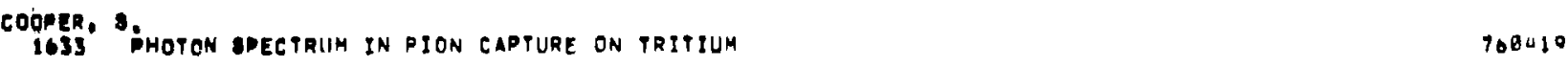

COPENHAGENE ORR:
TQB AN AECURATE MFPHOO FOR THE MEASUREMENT OF HIGH O MICROWAVE RESONATORS

793 AN ACCURATE HFPHDO FOR THE MEASUREMENT OF HIGH Q MICROWAVE RESONATORS
O67 GROUPING CRITFRIA FOR 291.25 MHZ LINAC DRIFTOPUBE QUAORUPOLES
2739 OPERATING CHARACPERISTICB OF A FULL POWER CLOYERLEAF ACEELERATOR TANK

COTIINGAHE, M

2811 INELASTIC SCATTERING OF PI+ AND PI- FROM 180XYGEN AT 148 AND 230 MEV 770987

2919 NEUTMON DEFOAMATION PARAMETER BY COMPAPATIVE STUDY OF PI AND PIO INELASTIE SCATTERING

COULOON, LAY.

210 NUCLEARACOULOMB INTERFERENEE IN PI OQR- 16 OXYGEN SCATTERING

Cowan, 6

TI7 "LAMPF nEUTRINI PACILITY PROPOSAL

759009

2125

COWAN, H.

IIES PROPERTIES OF A PROPOSEO LOWEENERGY GENERAL PURPOSE PION CHANNEL

700646

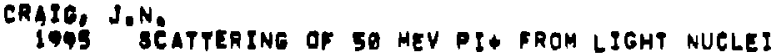

$\ln 1: 2=$

CRANDALL, K,R:

20Z OOS MHZ RFOACEELERATOR SYSTEH

57 OOSTMHZ LINAC DEQIGN MODIFICATIONS DUE TO LENGTH ERRORS

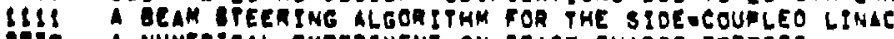

2930 A NUMERICAL EXPERIMENT ON SPACZ-CHARGE EFFECTS

1366 ACCELERATOR DFVELOAMENT OR INVESTIGATION - EXPERIMENT 76015

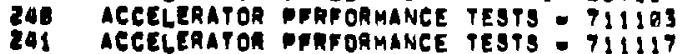


CAANDALL, K.R. (CONTO!)

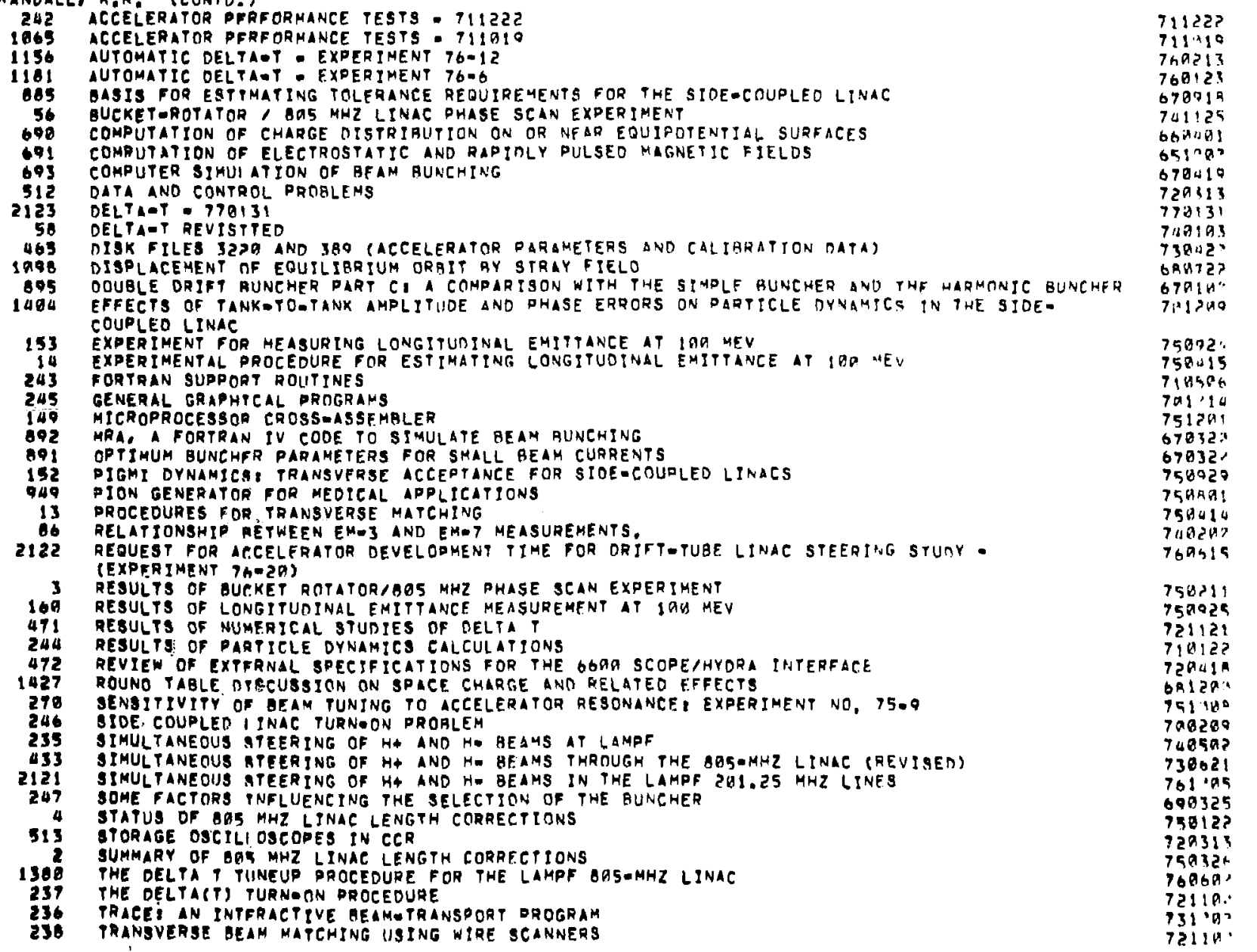

CAANE, T,W: 
CRANE, T.W. (CONPD.)

144! A NEW HIGH PRETISION MEASUREMENT OF THE MUONTUM HYPERFINE STRUCTURE INTEZVAL DELTA NU!

IS DEPOLARIZATION OF NEGATIVE MUONS IN HELIUM GAS, SEARCH

101 MUONIUM FORMATION IN NOBLE GABES AND NOBLE GAS MIXTURES

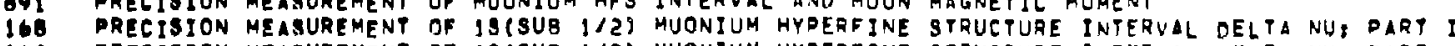

169 PRECISION MEASUREMENT OF ISISUB $1 / 2$ S MUONIUM HYOERINE GTRUCTURE INTERVAL DELTA NUI PART

enIBCUDLo, Aili:

303 A BEAM INHIBIT SYSTEM FOR LAMPF

195 EVALUATION OF COCOR LINE DRIVER AMPLIFIER

FAst protet EOR a II MEV

741 LAMPF FAST PRATECT GYSTEM - OPERATION ANO CHECKOUT

1888 PDOII/20-18M-T29, IV MT CONTROL UNIT PERFORMANCE SPECIFICATION

PERFORHANCE SPECIF ICATION FOR LAMPF VIOEO SYGTEM

VIOEO SYSTEM CONFIGURATIONOPERFORMANCE-MAINTENANCE REPOR

CRONIN, J.W.'
25R3

151:5:

$74051 ?$

740985

77 (1)

$750<26$

2593 CAN

CROWE, K.M.

2633 PHOTON SPECTRIM IN PION CAPTURE ON TRITIUM

2919 RADIATIVE NEGATIVE PION CAPTURE IN LIOUIO NEON

2989 RAOIATIVE NEGATIVE PION CAPTURE IN LIOUIO TRITIUH

710002

690319

710 B13

71:11.

71111?

प11 2द!

CUNAME, Ja

209 NEUTRON BACKGROUND STUDY FOR LAMPF EXPERIMENT NO, 53 , STUDY OF THE NEUTRINO CARTURE CROSS

$690+2 A$ SECTIONS IN CHLORINE-39 WITH MU DECAY NEUTRINOS

Cusming.

2330 2GREA REVERING SWITCH BTUOIES

759509

evenua, F.

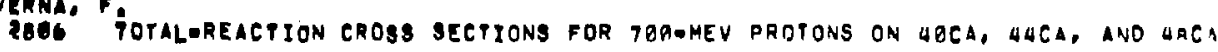

77090 .

DARIN, J.T

6G A AN EXPERIMENTAL STUDY OF OMEGA NEUTAAL DECAYS

$711 \cdot 1 \mathrm{a}$

OALTON, C.G.

1929 REGUEST FON PABRICATION PROPOSAL - LAMPF PHOTOUULTIPLIER TURE RAGES

DALTON, L,J. 
DALTON, L.J. (CONTO.)

2025 gitpon motor acsonance

730613

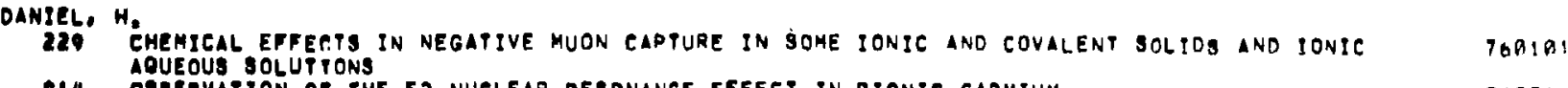

914 OQgeRVATION OP THE E2 NUELEAR RESDNANCE EFFECT IN PIONIC CADMIUM

1003 RECENT EXPERIMENTAL RESULTS OF PHE MESIC CHEHISTRY PROGRAM AT LAMPF

iHo PHODOIMENGIONAL VISUALIZTIION OF STOPPING PION OISTRIBUTIONS

DANIELE, WiR.

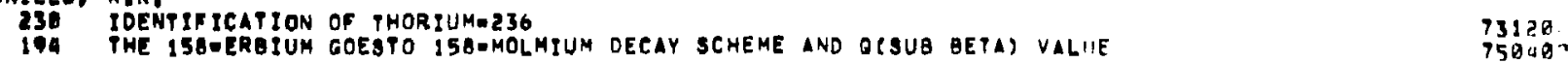

OAMPN COMMPTFE

273 DATA BABE MANAGEMENT/INFORMATION RETRIEVAL

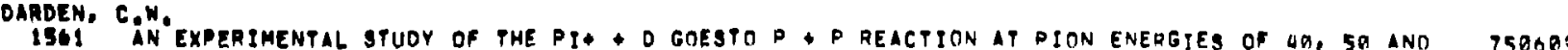

$756 \operatorname{son}$

CLABTIC SCATTFRING OF POSITIVE PIONS ON 16-OXYGEN AT UQ. T ANO UQ, 7 MEV

$779 \sin 3$

718090
71910

790420

761.25

MEADUEMENTS AF IONONUCLEAR ELASTIC SCATTER
PIONGNUCLEAR TNELASTIC SCATTERING AT SO MEV

THE DEUTERON BABTATE AND THE PI+ * DOESTO P P REACTION

THE PI. D GEESTO P P REACTION AT 49, 5A INO $6 B$ MEV

760720

ISWO POLARIZED ION INJECTOR PROGRAM

DAVIOSON, S,P',

140 MUONIE KGRAYS FROK THE TRANSITION NUELEI 194,195,196-PLATINUM 75042

DAVIB, H.T.

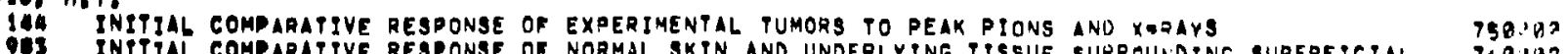

QE3 INITIAL COMPARATIVE RESPONSE OF NORMAL SKIN AND UNDERLYING TISSUE SURRAU.DING SUPERFICIAL TGQIG? HETABPATIC NODULES

DAYgF, "LAMPF NEUTRINO RACILITY DROPOSAL

TH

$75030^{\circ}$ 
DAY, H.

OE HAVEN, R, A:

2485 20GOMHZ OPERATING MANUAL

201,25 MHZ RF

2693 OREAT OHUTDOWN CHANGES IN RF SYSTEMS

2694 PROCEOURE FOR CONDITIONING ALVAREZ TINKS FOR RF

270 PROCEDURE FOR CONDITIONING ALVAREZ TANKS FOR RF II

235! PROPOLED YIOEN CHANNELS TO CCR

2232 SOP - 2BI.25 HHZ IFA ONALINE SETUP
2251 SOP : ALVIREZ TANK RESONANEE CONTROLLERS - TaQ5e3

2230 DOP: ALVAREZ TANK RESONANCE CONTRCLLERS: 93121

2708 STANDARD OPERATING PROCEDURE - REASSEMBLY PROCEDURE FOR 7835 CAYITY

27if BTANDARD OPERATING PROCEDURE - TUNINE 4616,0664 IN MODULE I

2165 STANOAKO OPERATING PROCEOURES (2RI MHZ) MOOULES I, $2,3,4$, AND BUNCHERS
2237 OTANDARD OPERATING PROCEDURE ALAREZ TANK RESONANE CONTROLLER

OEAN, P.N.

OEBRUNAER, P.G.

ISAT A MEASUREMENT OF PARITY VIOLATION IN PROTONAPROTON SCATTERING AT IS MEV

1542 LIMIT ON PARITY NONEONGERVATION IN PONUCLEUS SCATTERING AT G GEYIC

IG28 LIMIT ON PARITY VIOLATION IN P-NUCLEUS SEATPERING AT 6 GEVIC

22. TEST OF PARITY CONSERVATION IN POP SCATTERING

701193

720.9

758695

750612

701100

740500

700593

$7315 ! ?$

751.16

760224

T705ค

31217

OEHNHARO, D.
2811 INELABTIC SCATTERING OF PI. ANO PIO FROM 10OXYGEN AT 140 AND 23 A MEV

ग215日,

7401107

750505

760715

2811 INELASTIC SCATPERING OF PI" AND PID PROM 180 YYGEN AT 140 AND 23 M MEV

770909

OENISON, A:B:

A NEW HIGH PRFTISION MEASUREMENT OF THE MUONIUM HYPEREINE STRUCTURE INPERYAL OELPA NU

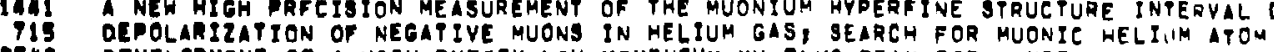

DEVEL OPMENT OF A HIGHAPURITY LOHOMOMENTUM MU PLUS BEAM FOR

MUONIUM FORMATION IN NOBLE GASES AND NOBLE GAS MIXTURES

PRECTOION MEASUREMENT OF MUONIUM HFS INTERVAL ANO MUON MAGNETIC MOMENT

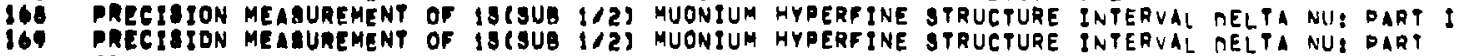
II

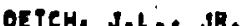

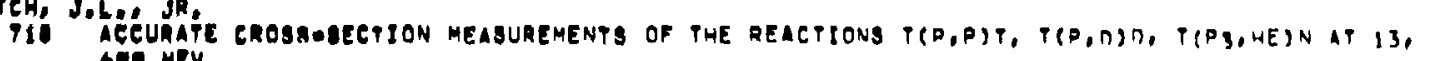

GOG MEV

919 PROTONGPROTON ELABTIC BCATTERING FROM 9.6 TO 13.6 MEV

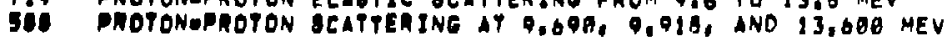

751120

Thange

T०0ดด?

77019 :

750425
750429

71030.

710102
70407 
DEVEREUX, M.

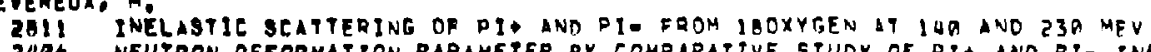

DET

DEVLIN, T.J.

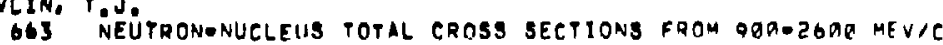

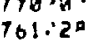

OHAHAN, 3

24Q8 STANDaRo software for camac

$7 ? 1$ PAM

DICELLO. J.F.

919 A VACUUM SYATFM WITH GAS FLOW FOR MICRODOSIMETRY

JI9

91. IPPLICATION OF PARTITLE ACCELERATORS TO MEDICINE

916 AETA EMISSION FROM ENCAPGULATED SOURCES OF CALIFURNIUY-252

223 BIOLOGIEAL EFFECTS OF THE LOS ALAMDS MESON BEAM ON CELLS IN CULTIAF

917 CALCULATIONS FOR DHSIMETRY AND MICRODOSIMETRY DF NEGATIVE PIONS

953 CELL SURVIVAL AS FUNCTION OF OEPTH FOR NEGATIVE PIONS AND HEAVY IONS

142 DOSIMEPRY FOR PION BEAMS AT LAMPF

1455 DOSIMETRY OF DION REAMS

IA23 GAS FLOWMETERS

144 INITIAL COMPARATIVE RESPONBE OF EXPERIMENTAL TUMORS TO OEAK PIONS ANI XORAYS

14U3 INIFIAL EOMPARATIVE PESPONSE OF NORMAL SKIN AND UNDERLYING TISSUE SURRAUDING SIIPERFICIAL MEPAGTATIC NOBILES

Q90 MICRODOSIMETRY OF NEGATIVE PIONS
PBI MICRODOBIMETRY OF NEGATIVE PIONS

PQ1 MICRODOBIMETRY OF NEGATIVE PIONS
I9G SPATIAL OISTRTBUTION OF EFFETIS OF NEGATIVE PIONS ON CUL TUAED HUMAN CELLA

1969 SPATIAL OISTRTBUTION OF EFFECTS OF NEGATIVE PIONS ON CULPUDED HUMAN CELLS
1459 SURVTVAL OF CILPURED MAMMALIAN CELLS TRRADIATEO AT VARIOUS DEPTHS IN TWE LAMPF NEGATIVE

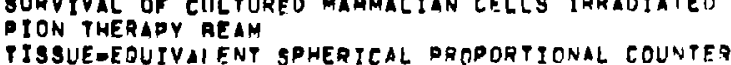

1929 TISSUEEEQUIVAIENT SPHERICAL PROPORTIONAL COUNTEO
1506 WHDLE BOOY DOSE FOR PATIENTS TPEATEO WITH PIONS

OSETERLE. B.0.

204 A MULTIWTRE PROPORTIONAL CHAMBER SPECTROMETER FOR NUCLEONONULLEON FXPERIWENTS

147 ENERGY ANO ANFULAR DISTRIBUTION OF NEUTRONS PRODUCED BY BGO MEV OROTON-PRDTON COLLISTONS

190 MEASUREMENT DF FHE R-DEGQEE NEUTRON SPECTAA FROM GUT AND BQB MEV PROTON VOMBARDMENT HF

203 MEASUREMENT OF THE ZERO DEGREF NEUTRON SPECTRUM FROM THE REACTION DP GIESTH NP PIO AT T SUB $P$. ThU MFV

1634 NEUTRON SPECTRA AT ZERO DEGREE FROM (P,N) REACTIONS ON OOBERYLLIUM, IZOCARHON ANA 270

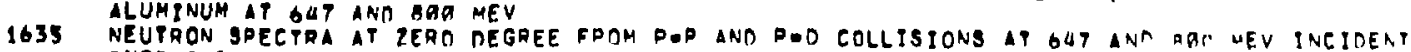
NEUTRON

ENERGIES

232 NEUTRON JPEETQA FROM DOOTON BOMBAROMENT OF OEUTERTUM AT GUT ANC RAQ WEV

206! DESERVATION OF RESANANCES NEAR II EV IN THE PHOTODETACHMENT COOSS SECTIO. OF THE H. ION PION PRODUCPION IN NEUTRONAPROTON COLLTSIONS

750.10

741.90.

750599

75ด7ด्

751291
750592

758411

751001

75 किवर

759 a

759719

$75072 ?$

758704

750000.

731210

$760 \times 8$.

$731 \cdot a=$

150020

759120

750 (1)

76 ด7a

InATG5

74060.

7 ilis

761115
$75122 \%$ 
DIETERLE, B.D. (EONTD:.)

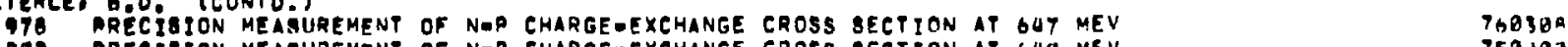

202 RQECTSION MEATUREMENT OF MEP CHARGE-EXCHANGE CROSS SECTION AT GUFR MEV

Digiacomo, Ni.s.

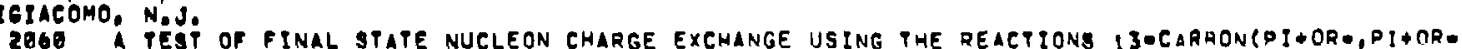
N) Iz-CARQONCU:

Dixis, Mes.

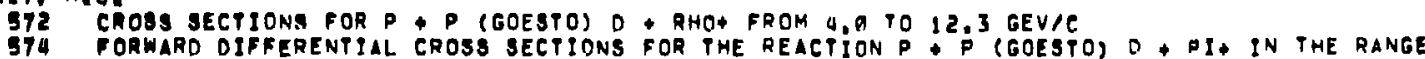
GTh

672 PORWARO OIFFERENTIAL CROSS SECTIONS FOR PME REACTION + P COOSTO) D OI+ IN THE RANGE 3.4 TO 12.3 GEV/C

Doshowolokt, $\mathrm{F}$ 933 INEPIAL COMEARATIVE RESPONBE OF NORMAL SKIN AND UNOERLYING TISSUE SURROUNDING SUPERFICIAL

DOOOER, D,C,

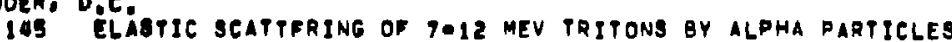

T7a?09

7เด791

6คด7ร.

760149

DONAHUR, J:

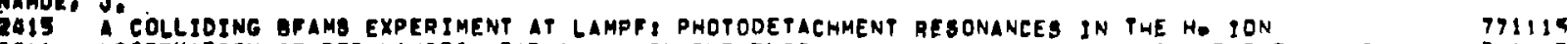

2061 OQBERVATION OF RESONANCES NEAR II EV IN TME PHOTODETACHMENT CROSS BEETION OF THE HE ION ThIIIS

Dosg, J. D,

9ME RoGKY KLYBTRON BLOCKINGAOSCELLATOR MODULATOR

932 A HIGH VOLTAGF ERGENERATIVE PULOE MODULATOR

932 A NEW ELECTROSURGICAL COAOULATING-CUTTING FORCEPS

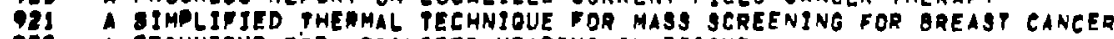

A IIMLITIED THE MAL TECHNIOUE VRA MASS SCREEN

A TECHNIOUE FHR LOEALL

AODIPIONS TO FGB9-1BQ

AN BAS-ME 1-1/4-MW AMPLIFgER FOR ACCELERATOR SERVICE

GACKGROUND PACPO CONEERNING LASL SUPPORT OF TUCSON EFFORTS

BACKGROUND OF LASL TEMOERATUAE DIN

GEAM POBITION MONITOR ELECTFONICS

GIOMED COMBUTFR READOUT OF ELETROMETER CALIBRATION POTENT TOMETER DOSITITN

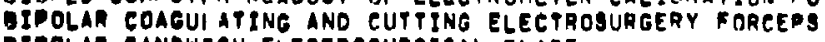

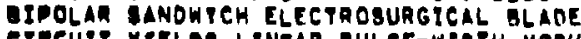

CERCUIT YIELOR LINEAR PULSE-WIOTH MOOULATION

CLINICAL (HUMAN) TRIAL OF LOCALI2ED CURRENT FIELO TUMOR TMERAPY

OETECTION OF MALGGNANT TUMONS GY MEASURE HENT OF BIOELEETRIC POTENTTALS

DOBIMEThY FON BOON BEAMS AT LAMPP

73011 ?

7 a digen

75002 2

759114

6 कीnen

7 TAH 14

759505

74060

94131

72112 ?

TuBดa

921115

359411

Tsasil 
Doga, s.o. (CONTOE)

IO40 ELECPROMETERS

10SO ENGINERT ING A

1041 ENGINEERING ASSISTANCE TO DR, STERLING EOWAROS

642 FAST PROTECTIITN FOR HIGHAPOWER VACUUM PUBES

13. FAULT PROTECTYON CTRCUITRY FOR THE LASL 201,25 -

713 FAULTGPROTECTYON SYSTEM FOR LINAE RF AMPLIFIERS

738 IMPROVING ELECTRIC FIELD RESOLUTION IN RF LESIONS

935 LAMPFOUNM SEHINOL TECHNOLOGY PRANAFER, A REVIEW

1046 LETTER CONCERNING AN RE GENERATOR

10HS LETTER CONCERNING CROWBARS

1042 LETTERS CONEERNING ELECTROSURGERY-INDUEED ECG NOISE

23 LOCALIZEO CURRENT FIELD TUMOR THERAPY

LDCALIZEO CURAENT FIELOS AO A POSSIBLE THERAPEUTIC TECHNIQUE IN CANCER THERAPY

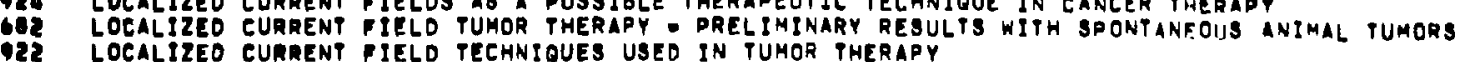

LOCALIZEO CUARENT FIELD TECHNIOUES USED IN TUHOR PHERAP

LOCALIZED HEATING AB AN ADJUNC

1038 MODIFIEATION TO MODEL TA-1I5 TEMPERATURE CONTROLLER POR USE AND EXTERNAL CONTROL EIRCUIT, ANO ALIGNMENT INSTRUCTIONS FOR TRIAC CONTRILLE MODIFICATIONS TO THE HAEFELY COCKEROFT WWALTON BOUNCE

NEH ELECTROSURGICAL DEVICES AND TECHNIQUES

36 NOIOE OFREE ECG DATA DURING ELECTROSURGIGAL PROCEDURES

ERAL EFPCTS OF VHF CANCER THERAPY

141 PULBe GATE

IN GANEER THERAPY

1020 SPECIFICATIONS OOR THE MOUSTON X-Y RECOROER ANO HOOULES

1030 TEMPERATURE OTFFERENCE INTEGRATOR CIREUIT

947 THE BQS MHE RF SYSTEM

31 THE OEOIGN ANR PERTORMANCE OF THE LAHPF 101/4 MW KLYSTRON HODULATOR

THE LAOL COAGIILAPING-CUTTING SURGICAL TOOL

20 THE POTENTIAL OF LOCALIZEO HEATING AS AN ADJUNET TO RADIATION THERAPY

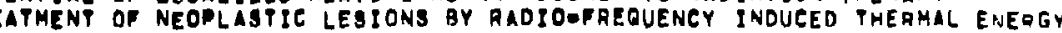

PREA EFFECTS ON LUKEMSA

Q46 TWO CONDITIONA NECESBARY FOR NOISESFRE ECG DATA

758122

$75031 t$

$75031 \%$

741201

730103

. 110

719323

7 मरू०क

7303 कर

750116

6b!?la

72 ด72क

750725

7110?:?

712315

$73001 \%$

720729

741.6

73633

$949 ? 1 ?$

741 as
$730 \times 3 ?$

$0088, K_{1} G_{1} R_{1}$

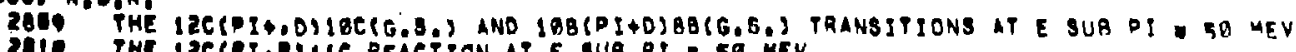

$77840 *$

2AT THE IXC(PI,PIIIC REACTION AT E SUB PI $=50 \mathrm{MEV}$

770090

Doss. $M_{1}$

$130^{\circ}$ ELABPIC SCAYTFRING OF 50 MEV PI+ AND 12-CARBON

1995 BCATPERING OP BQ MEV PIO FROM LIGHT NUGLE

163 GTUDIES OF CHARGED PARTICLE EMISSION IN REACTIONS INDUCED BY 220 AND 5.4 MEV DLUSAPIONS

750429

76 , 12 h

164 PTUDIES OF PAATICLE AND GAMMAOBPECTRA IN REACTIONS INOUCEO BY BOROMEV DROTONS

DNAGOAET, W. H. 
DRAGOAET, H, H, (CONTO')

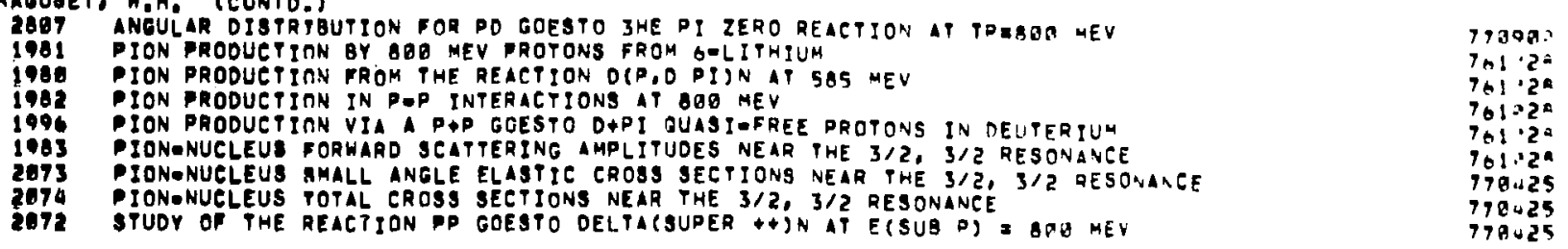

Denakg

I5H3 ABSOLUTE CROSE SECTIONS FOR PRODUCTION OF PROMPT NUCLEAR GAMMA RAYS GY FAST OJONS

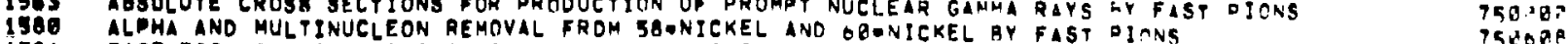

1581 PAST ION INDUCED PROCESSES IN COMPLEX NUCLEIS CROSS SECTION AND ALPHA REMUVAL TALT PON

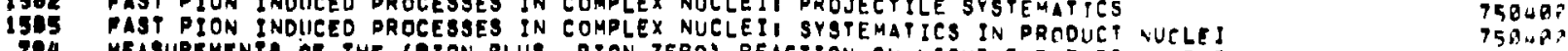

994 MEASUREMENPA HF THE (PION PLUS, PION ZERO) REACTION ON LIGHT ELEMENTS IN THE (3,3)

1362 BYSTEMATICS OP PION AND PROTON INTERACTIONS HITH NICXEL NUCLIOES TEBOH

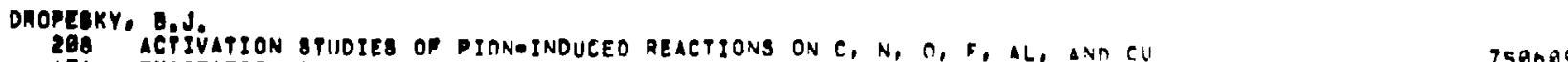

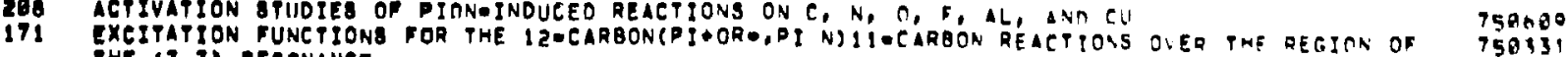

238 THE $(3,3)$ RESTNANCE.

YHOR IUM-23

INFER

2335 PROPOSAL FOR AN ONGLINE MASS SPECTROMETER FACILITY AT LAMPF TO STUDY DZOMJETS OF

1011 REACTIONS INDIICED BY AN INTENSE BRPAMEV DROTON BEAM

231 BPALLATION OF COPPER BY IOA MEV PI(+OR-) ANO 340 MEV DRDTONS

I95 CTATUS REPORT ON PHE INITIAL NUCLEAR CHEMISTRY EXPERIMENTS AT THF CLINTON D, AMOERSON

194 PHE ISQERGIUM GOESTD I58-HOLMIUM DECAY SCHEME AND O(SUR BETA) VALUE

731502

760.8.

731.4

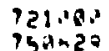

140040

19340

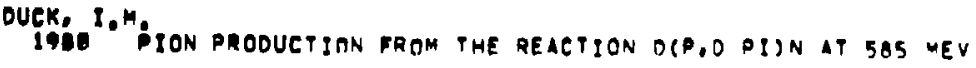

$\ln 1 \cdot 2=$

OUDZIAK, D.S.
IHE NEUTRONE FROM A HIGH ENERGY PROTON BEAM STOB

Taบ14

DUNHOOOY, W.E

723 MAGNETIC MEAGIMEMENTS ON THE LABL PROTOTYPE MAGNE?

OYTMAN, 8.A.

221109 


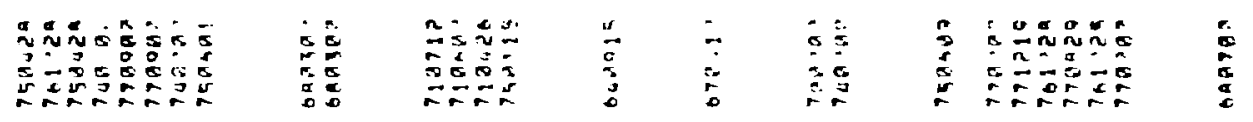

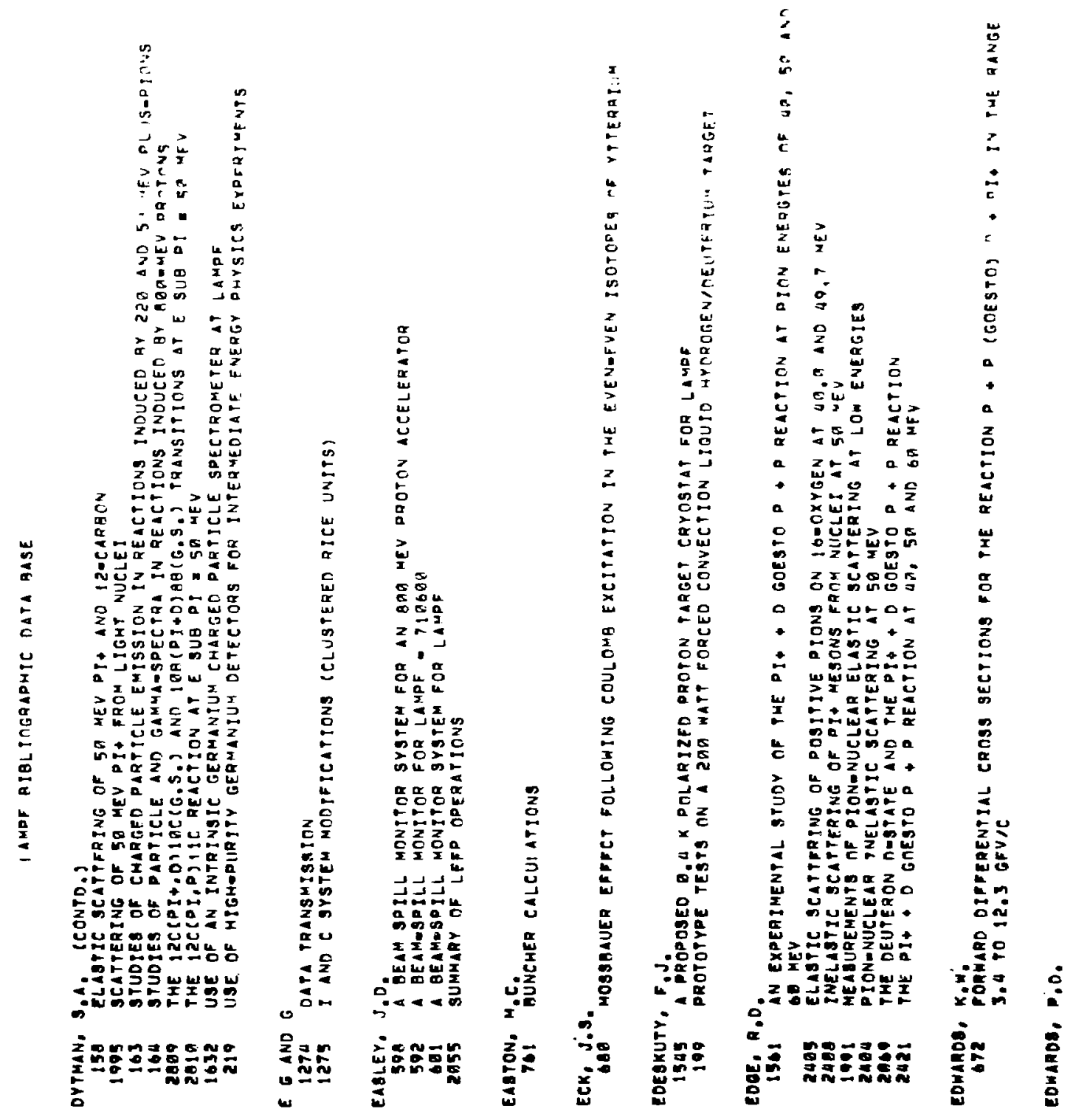


EOMAROA, P.D. CCONTO.)

694 CONSTRUCTION OF LAMPF FACILITIES OR THE CHALLENGE OF HOUSING AN INUENTIO: TAO50?

iscal YeAR 1OGR CONBTRUETION OBLIGATIONS

2727 LAMPF BRIEFING MEETING OF JANUARY 10,1960

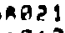

EOWARDS, W's':

932 NEM ELECTROSURGICAL COAGULATINGECUTTING FORCEPS

LOE LOCALIZED CURRENT FIELO TUMOR PHERAPY O PRELIMINARY RESULTS WITH SPONTINFOUS ANIMAL TUMORS

739119

EGAN, 10 .

2548 OEVELOPMENT OF A HGG-PURITY LOH-MOMENTUM MU PLUS BEAM FOR LAMPF

2548 OEVELOPMENT OF HIGHOPURITY LOWOMOMENTUM MU PLUS BEAM FOR LAMPF

770006

EOQER, J.P.ंmpUTER CONTMOL OF EPICS BEAH LINE ANO gPECTROMETER

$72110 ?$

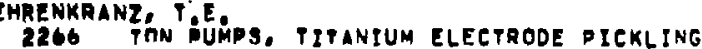

700095

EIBENBTEIN, R'A A.

228 (PI,O) REACTION FOR SMALL INCIOENT PION ENERGIES

729 A PROPOSAL FOR EPICS DATA DCOUISITION SOFTWARE

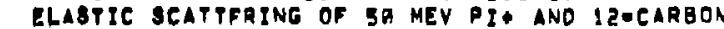

I577 LOW ENERGY DIAN ELASTIC SCATTERING FROM LIGHT NUELES

207. LOW ENERGY INN INUCLEUS SCATTERING

1995 OCATTERING OF 30 MEV PI FROM LIGHT NUCLE

263 STUDIES OF CHARGEO PARTICLE EMISSION IN REACTIONS INOUCED BY $22 A$ AND 5R "EV PLUSOPIOAS

164 STUOIES OF GARTICLE. ANO GAMMAOSPECTRA IN REACTIONS INOUCED BY BGROMEY PRTTANS

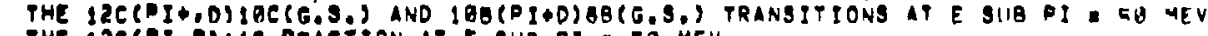

PKE I2C(PI,PIIIC REACTION ATE SUB PI 5A MEV

75one:

72110.

$756.5 \%$

76038 :

77019 ?

$781: 29$

$758+24$

$708.90 \%$

990.0 .1

tKang.

2008 A'BHORT-CUT MFMOTE HANDLING PROPOSAL FOR AREA A AND BEYONO

2004 A BOLUTION TOR REMOTE HANOLING IN ACCELERATOR INGTALLATIONS

T46 MONTPOR - A VERSATILE REMOTE MANOLING SYSTEM

$75,9, a \times 4$

770314

751121

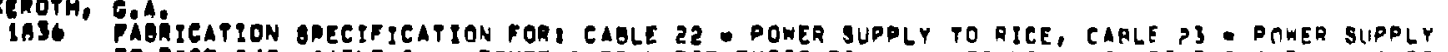

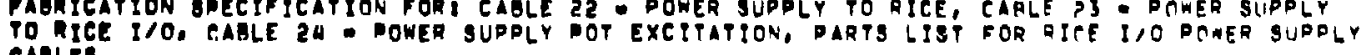

1849 PABRiCATION SPFCIFICATION - LAMPF NIM MODULES, MECMANICAL 1805 PABICATION BPECTFICATION FOR CIU CHASSIS B ANDE, LOCAL CONTROL MONULES, LAMPF MOTOR

$001 \cdot 0$

09191 
EKEROTH, OAA: CCONTO,

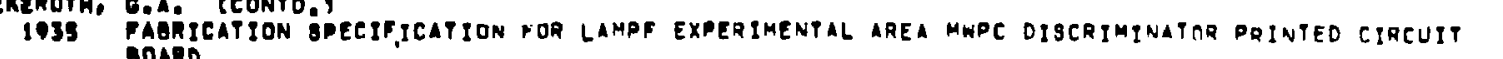

1089 BOARD

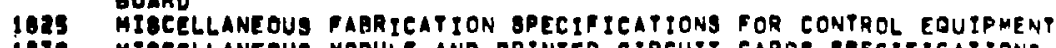

1030 MIBCELLANEOUS MODULE AND PRINTEO CIREUIT CARDS SFECIFICATIONS

1031 MICE 110-TOMRTCE CABLE PARTS LIST

103. TEGP GECIPILATIONS FOR MHPC DIBCR GMINATOR PRINTED CIACUIT BOARO

1040 TEET PFEIFICATIONG FOR PHOTOMULTIPLIER PUBE AASE ASSEMBLY PER 63Y-1495339

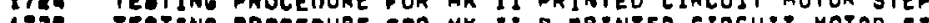

1728 PEBTING PROCEnURE FOR MK II B PRINTEO CIRCUIT MOTOR SPEPPER BOARO 63Y-1258:5

ELKIMB, E,P:

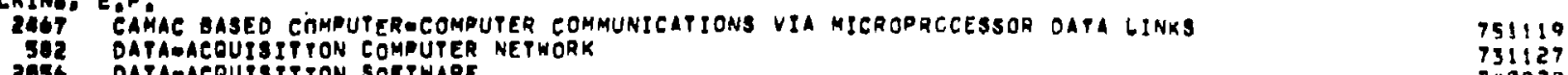

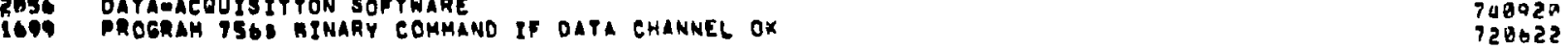

EMIEH, C'R,

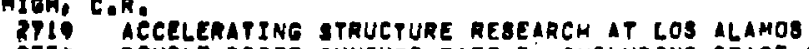

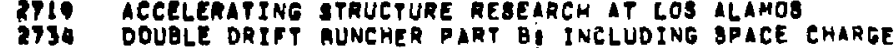

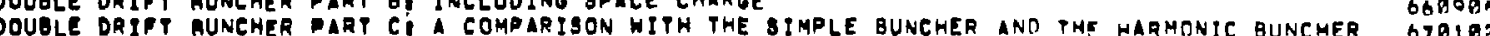

DOUELE ORIFT RUNCHER PART AI WITHOUT SPACE CHARGE

ELETTON ANALOG TESTS OF PROTON LINEAR ACEELEATOR STRUCTURES

INTTIAL OPERATION OF THE GEAM YRANSPORT GYSTEM IN TME LAMPF INJECTOR COMDLEX

670189

$658 \sin$

LAAL EXPERIENOURCE OEVELOPMENT

ODOERYATONS BFMINLR ALULATIONG FOR A DUOPLASMATRON WIPH EXACT PIERCE EXPRACTION

bignin

972070

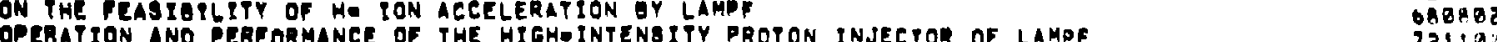

gPERATION ANO PERFORMANCE OF THE HIGHOINTENBITY PROPON INJECTOR OF LAMOF

OPERATION OF THE LAMHF 1380KEV INJECTOR

PART Ai INJEeTor complex, PART OI gON gOUREe test stand

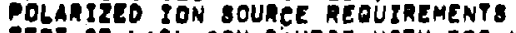

TeET OP LASL TON SOURCE WITK 758=KV AC

TEBT OF LARL TON SOUREE WITM 2MAGKV PIERCE ACEELEQATING COLUMN

THE INJEC TOR C.OMPLEX POR THE LAMPF ACCELERATOR

THE LAOL HIGH.DUTY-FACTOR ION SOURCE

THE BiekeE GenkETRY

bo ThIEK TAREE TRREMGTRAHLUNG PHEORY

$721: 0$

$71008:$

670410

iagoza

60 opa:

ogishas

GAQ52:

$60900 ?$
60129

engel, L.N.

2710 ACEELERATING s TMUCTURE REBEARCH AT LOS ALAMOS

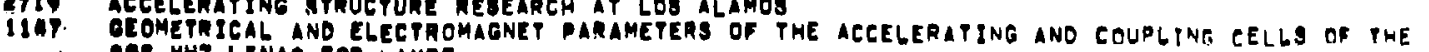

$\operatorname{sog} 6102 n$

i310 INTERPRETATION OF LALA OUPPUT

197. INTEAPRETATION OF LALA OUYPUT

1137 BOLUTLON OF E PIELOB PROM P.TABLES OF THE LALA COOE

TUNING AND PIFLO MEABUREMENT TEEHNIGUES IN A RESONANTLY COUPLED ACCELEQATOR TANK 
-NGELKE, M.S.

399 MNALYSIB OF LAMPF INJECTOR OPERATION FROM MEABUAEMENTS OF BREMSSTRAHLUNG PQOOUCEO RY GACK. STREAMING ELETTRONS

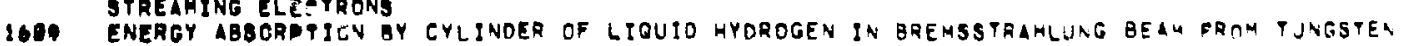
TARGET ATERD OF 25 HEV ELECTRON LINAC ODERATING ATI MA AVERAGE ELIQEEV

1684 GENERATION OF RAOSOACTIVE TIR AND NOXIOUS GASES

596 HIGH INTENSITY ELECTRON ACCELERATOR RADIATION HAZAROS

1613 INJECTOR XORAY RADIATION MEASUREMENTS

628 NEUTRON MEASUREMENT USING PHERMOLUMINESCENT DOSIMETERS

IN TARGET AREA AT EPA

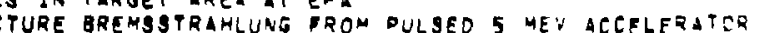

113.26

$608+1$ ?

$07351:$

710.54

7 ingis

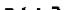

901125

EHESLIN, $N$

731 SIIT SCATTERING IN THE EPICS BEAM LINE

72055

EADAL, OR.

1648 STRONTSUMESO RUBIOIUMOBZ ISOTOPE GENERATOR OR USE IN NUCLEAR MEDICI.E

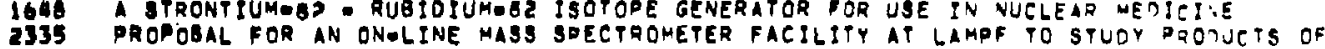

AEACTIONS INOUCEO OY

SFALLATION CRTSS BECTIONS AND THE LAMOE MEDICAL PADIOISOTOPE OROCRAM

1631 SPACTRTYUTH

EVANE, M.J.

372 CROSS SEETIONE FOR \& P (GOESTOS O PHO+ FROM $4 . F$ TO 12,3 GEV/C

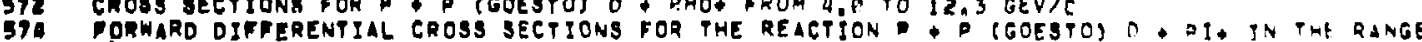

3." TO 12,3 GFV/C

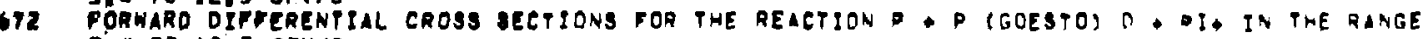
3.4 TO $12.3 \mathrm{GrV} / \mathrm{C}$

EVANQ, S.CA, JR,

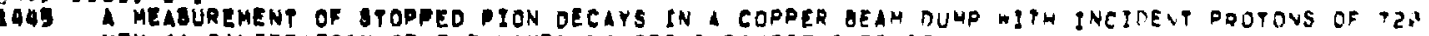

MEV (A CALIBRATION OF THE LAMPF NEUTRINO SOURGE INTENSITY)

209 NEUTRON BACKGROUND STUOY POR LAMPF EXPERIMENT NO, 53: STUDY OF PHE NEUTRINA CADTIAP CRCSS SECPIONE IN CHLORINEWIT WIPH MU DECAY NEUTAINOS

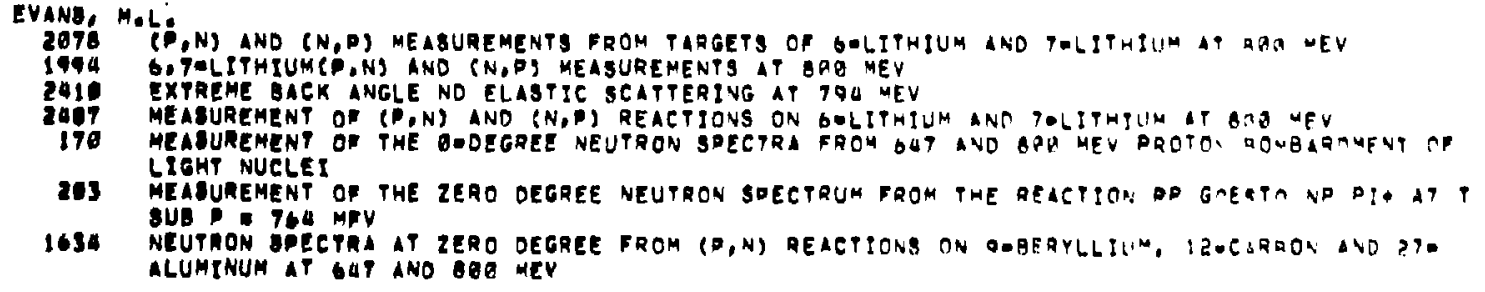


EVANB, M.L: (CONTO,)

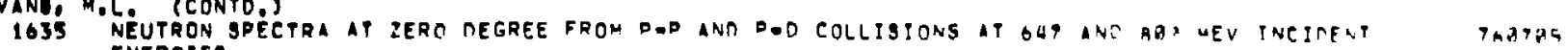
ENERGIES

1969 NEUTRON SPECTRA AP ZERO DEGREE FROM PROTON-PQOTON COLLISIONS BETWEEN OJT AAD GICS MF

232 NEUTRON SPECTRA FRTM PROTON BOMBARDMENP OF DFUTERTUM AP GUT ANO AHA UE

1978 PRECISION MEASUREMENT OF NOP CHARGE -EXCHANGE CROSS SECTION IT GUT MEV

282 PRECISION MEASUREMFNT OF NOP CHARGE - XCHANGE CROSS SECTIOU AT OUA HE

2409 OUASIELASTIC CHARGE EXCHANGE IN ND GOESTO PNN AT TOU MEV

$77 n 14$

$750.0 \%$

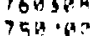

$\rightarrow \tan ^{2}$

FAULKNER, J,R.

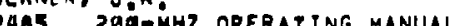

GUG 2 OD-RHE OPERATING MANUAL

638 DAMPF

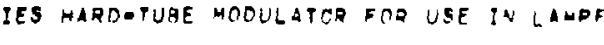

MEOICAL APPLICATIONS

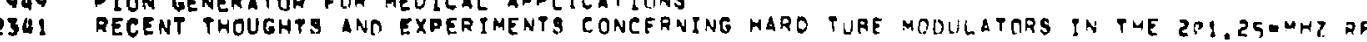
PYgTEMS OF LAMPF

2216 SPECIFICATIONG FOR AN SCR POWER SUPPLY REGULATOR AND FIRINE CIRCUIP

SPECIFICATIONG FOR LINE C G-INCH OCTOPOLE STEERING MAGNET COIL ASSEMQL

2106 STANOARD PROCFDURE FOR DARALLELING 201 MHZ SECTOR TRANSFORMERTRETTEIE, INITS

2097

STANDARD PROCFDURE FOR DARALLELING $20 !$
THE LAMPF LINF D FAST DEFLECTOR SYSTEM

$\operatorname{lin} 21,2$

$00,13: 48$

of 20,5

T9:452:

7301.45

$73+19$

773519.

FAUST, J.

Q59 PROGRESS TOWARD USING MU-HESIC X-RAYS AS A DIAGNCSTIC TOOL

93873

FEATHERSTONE, R,P.

936 FURTHER NOTE ON WUARM BRIDGE PHASE DETECTORS

752 MACHINE RF POWER REQUIREMENTS - 2MR MC SECTION

134 MISCELLINEOUS NOTES ON LINAC CONGTRUCTION

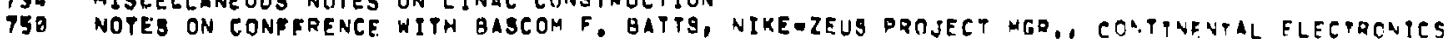
NOTES ON RAOIN-FREQLENCY POHER AMPLIFIERS FOR USE WITH DROTON LINEAR ACCELFAATORS

OPERATING EXPERIENCE WITH THE UNIVERSTTY OF MINNES TTA GB MEY PQOTOA LIEAR ACCELLEATAR

POSSIBLE DESITN PARAMETERS FOR 2 TQ MC/SEC SECTION OF THE LIMPE

153 VOLTAGE GRADIENT IN PROTON LINEAR ACCELEPATOR

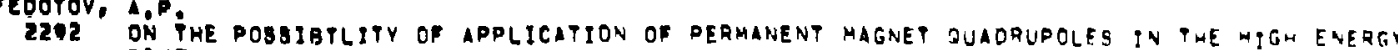
PROTON LINEAR ACCELERATIRS

$63 a+1$,

03171.

os

030,1

$\cos 2 \cos ^{2}$

6.

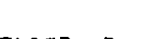

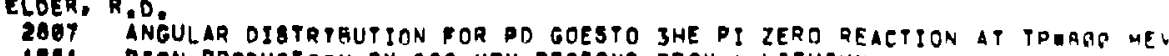

1981 PION PRODUCTINN GY BAQ MEV PROPONS FROY GWLITHIUM

1980 PION PRODUCTION TROM THE REACTION DLP,D PIIN AP 585 MEV

1982 PION PRODUCTION IN PDP INTERACTIONS AT $B B G$ MEV

1996 ION PRODUCTITN VIA A PQP GOESTO D+PI QIJASIOFREE PROTONS IN DEUTERTUU

1983 PION-NUCLEUS FOAWARO SCATTERING AMPLITUOES NEAR PHE 3/2, 3/2 RESONAWCE

2973 PION-NUCLEUS MMALL ANGLE ELASTIL CROSS SECTIONG NEAR THE 3/2, 3/2 RESOAA.CE

2070 PIONANUCLEUS POTAL COOSS SECTIONS NEAR PHE 3/2, 3/2 RESDNAVEE

inases

79609

7n: $5 \mathrm{~A}$

$\ln 1 \cdot 5 \mathrm{~s}$

in!. in $^{2}$

Thl.

ग7मेय 
FELDER, R, D, (CONTD.)

223 QUASIOFREE BCATTERING IN PAD BREAKOUP REACTIONS AT QPB AND DPQ MEV

766 GUAST-FREE SCATTERING IN PROTON-INOUCEO OEUTERON BREAKUO 47585 ANR APA "EV

2072 STUDY OF THE REACTION PP GOESTO DELTA (SUPER ++)N AT E(SUB D) I BOO MEV

FEnYYes, E.

717 LAMPF NEUTRINO FACILITY PROPOSAL

$11>0$

FERGUSON, $\mathrm{H}_{.} \mathrm{O}$ :

2483 200-MHZ OPERATING MANUAL

1551 A GENERAL IONOOPTICAL CDRRECTION ELEMENT

ORT STRUCTURE

L

Thrsis

733913

FEBSENDEN, $:$

728 NEW MEASUREMENTS DF (PI+, 2P) REACTIONS ON LIGNT NUCLE?

$71802 ?$

FICHT: H. PROTOTYPE TESTS ON A 2OG WATT FORCEO CONVECTION LIQULD HYDROGEN/OEUTERTUM TAPGET

740

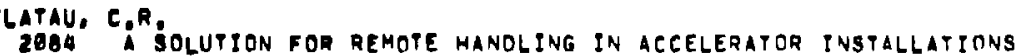

790314

FLETEHER, $C, R$.

210 NUCLEAR-COULOMB INTERFERENCE IN OI TOR- 16 OXYGEN SCATTERING

IO3 OIONGNUCLEAR TOTAL CROSS SECTIONS NEAR THE $3 / 2,3 / 2$ RESONANCE

750402

PLUHARTY, R,G'.

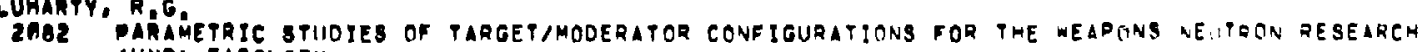
(WNR) FAESLITY

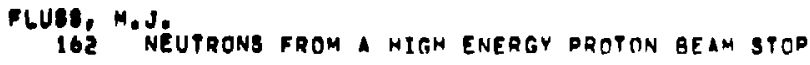

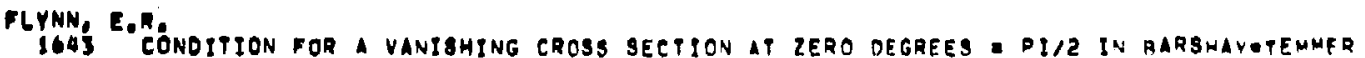

759.0.

FONG. J,e.

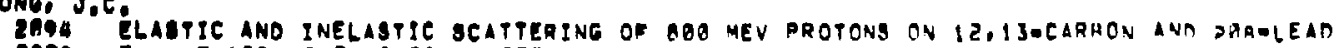

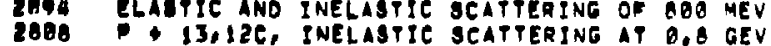

$\operatorname{T7na20}$

Tropas:

Fonten, e. 
FOWLE, E, (CONTO')

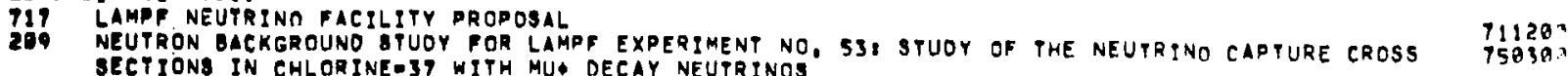

Fox, Lim:

20a A MULTIWIRE PROPORTIONAL CHAMBER SPECTROMETER FOR NUELEONONUCLEON EXPERI"ENTS T31MAT

COAENKEL, 2.

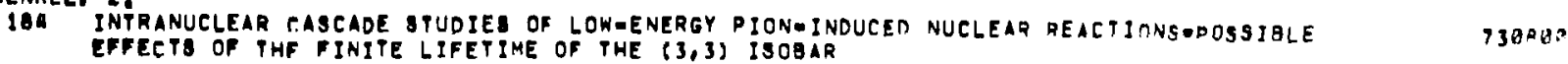

FRANK, J.s.

1627 LIMITS ON THE OIRECT PRISUCTION OF POSITRONS BY 256- ANO BOEOMEV PROTONS

983 SEARCH FOR OIRECT LEPTON PRODUCTION IN PP COLLISIONS AT BOB MEV AND 256 MEV

1621 SEARCH FOR THE DIRECT PRODUCTION OF POSITRONS QY $256=$ AND GBPGMEV DROTONS

1544 PHE ELASTIC STATTERING OF LOW ENERGY PIONS ON PROTONS

1943 TUNEUP OF PME LAMPF LOW ENERGY PION CHANNEL

FRANKE, P.R., JR,

1580 MASSIVE SHIELTING LDAPTED TO REMOTE MAINTENANCE METHOOS

1228 PROPOSAL FOR DESIGN STUDY - AREA EAST COVER

70113.

FRANKEL, $:$ :

2814 VERY HIGH MOMFNTUM COMPONENTS OF NUCLEONS IN NUCLEAR MATTER T790BP

FAABER, M.

629 'SIMULATION OF THE SELGIGA COMPUTER ON HANIAC II (BELMA)

FR $\triangle U E N F E L D E R, H$.

1 1597 A MEABUREMENT OF PARITY VIOLATION IN PROTONDPROTON SCATTERING AT IS MEV

917 LAMPF NEUTRINO FACILITY PROPOSAL

152 LIMIT ON PARITY NONEONBERVATION IN PONUCLEUS SCATTERING AT O GEV/C

62. LIMIT ON PARITY VIOLATION IN PUNUCLEUS SCATTERING AT G GEVIC

TEAT OF PARITY CONBERVATION IN PAP SCATTERING

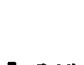

$748 \cdot 9$

71129

769719

741119

FREDRICKSON, D

106 DOUBLE.CHARGE-EXCHANGE AND INELASTIC SCATTERING IN PI- + 3-KELIUM

$940: 07$

FRENCH, G.H,

2604 GEAH DIAGNOSTICS MEABUREMENTS 
FRETHELL, JiH:

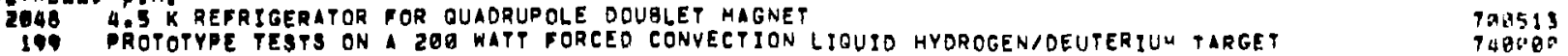

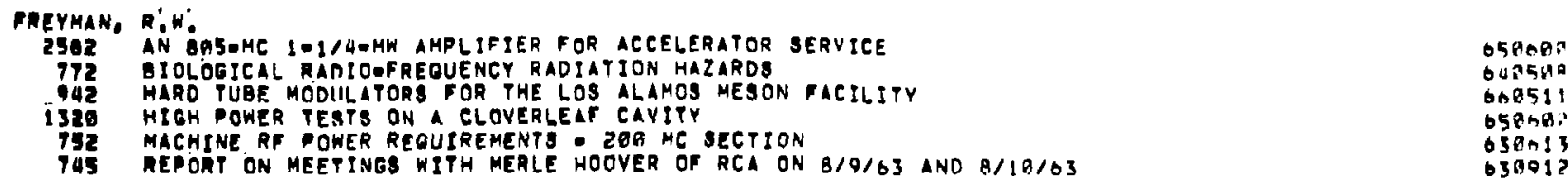

PRICKe, 6.

150 MUONIC ISOTORF SMIFTS IN THE EVEN IRON NUCLEI BYSTEMATICS OF NUCLEAR CHARGE DISTRIBUTIONS IN TRON, COBALT, NICKEL, COPPEA AND ZINE

FAIEDLANDER, G.

208 ACTIVATION STUDIES OF PIONEINDUCEO REACTIONS ON $C, N, O, F, A L, A N D C U$

171 EXCITATION FUNCTIONB FOR THE IZ-CARBON(PI+OR-OFI N)IIIECARBON REACTIONS OVER THE REGION OF T5R3II

184 INTRANUCLEAR CABCADE STUDIES OF LOW-ENERGY PIONGINDUCEO NUCLEAR REACTIONSOPOSSIBLE EFFCTS OF THF. FINITE LIFETIHE OF PHE $(3,3)$ ISOBAR

$73089=$
FUKU1, :
572 EROSS SEETIONA FOR P D (GOESTOS D + RHO FROM U, T TO 12,3 GEV/C
374 FORWARD DIFFERENTIAL CROSB SECTIONS FOR THE REACTION P + P (GOESTO) D P \& IN THE RANGE
$6723.49012,3$ GFV/C 3.4 TO 12.3 GFVIC

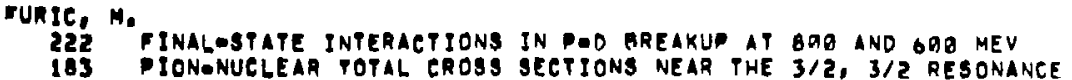

OUAG TOFE

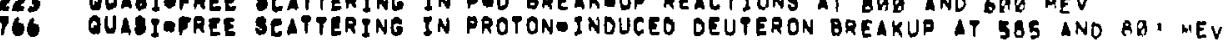

PUNNISH, A:P.

T32 MACHIHE RF DOWER REQUIREMENTS - 290 ME SECTION

700791

73010 :

6*ค7ด.

7506 int

731117

764785

GothuLER, $K$

1561 AN EXPERIMENTAL STUDY OF PHE PI+ D GOESTOP P REACTION AT PION ENEQGTES OF 4R, 5P AND

630513

1561 ON MEV

2069 THE DEUTERON BOSTATE AND THE PI\$ + D GOESTOP + P REACTION

2421 THE DI* D GOESTO \& REACTION AT UQ, SA ANO 60 MEV

$75 \sin i 0$

$7+1.29$ 
GABITZSER, N, O.

2U01 COMPUTATIONAL TECHNIOUF FOR TUNING THE LAMPF MIGH ENERgY PION CHANNEL

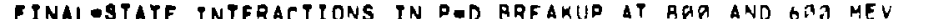

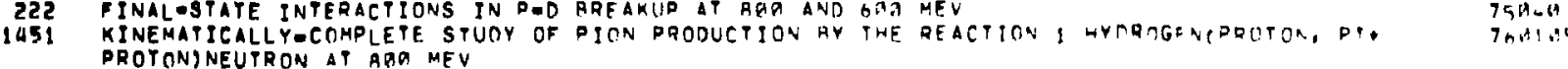

219 NUCLEAR-COULOMB INTERFERENCE IN PI OOR 16 OKYGEN SCATTERING

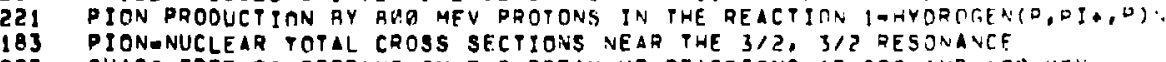

223 OUASIOFREE SCATTERING IN P=D BREAKOUP REACTICNS AT BPR ANR GPA MEV

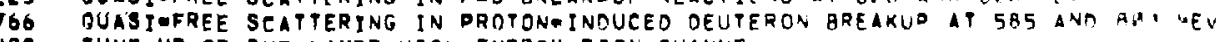

2409 TUNEAUP OF THF LAMPF HIGHOENERGY PION CHANVEL

2585 COMPUTING IN SUDPORT OF EXPERIMENTS AT LAMPE

758 and

75949.

73111 ?

Tod 1040

75 Q 9 ?

GAMMEL, J.L.

370 RETHE-SALPETER SOLITION FOR NUCLEOHWNUCLEON SCATTERING WITH PION EXCMAGG

$\operatorname{Tn} 1 \times 14$

GARAY, PiVity COOLINT SYSTEMS

7ดย1ด:

6,6127

GARCIA, L.M.

$1350^{\circ}$ RICE INO BINARY DATA UNYT CBNU)
1579 SECONO GENERATION CONSOLE INTERFACF UNIT (CIU) AND ASSTCSATEO COLSOLE WALDAEE (DRAFT)

GEHRKE, R.J.

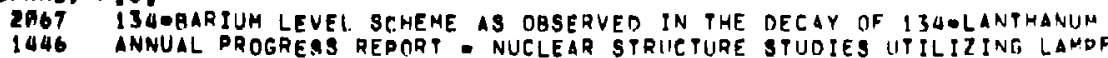

1630 DECAY OF 128-CESTUM

2963 GAMMAORAY EMISSION FROM 1340CERIUM ANO LEVELS IN 1340LANTHAALM

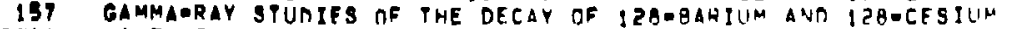

MALF-LIVES OF 1340LANTHANUM AND 1320135-CER IIM

2月GB LEVEL BPRUCTURE OF 131-CESIUN ANO THE DECAY ENERGY OF 131=BARIUM

$7 \log 20$

$13020-$

$\operatorname{lnl} 1,4$

$\operatorname{tingands}$

Thent.

919.19

Tox $>53$

7nI19:

GERNER, E,W

930 LOCALI2EO HEATING AS AN AOJUnCt TO RADIATION THERAPY

126 THE POTENTIAL DF LOCALIZED HEATING AS AN LOJUNT TO RAOIATION THFPAPY

$7112+1$

$741 \cdot p$

GIHDS, $W_{\text {OR. }}$

2016 AN OPTICAL MONEL STUDY OF LOW ENERGY DION SCATTERING FROM 160 YYGFN

2\%69 THE OEIJTERON MESTATE AND THE PI+ D GOESTO $P$ P REACTION

770 op.

$7 n 1 \cdot 25$

GIOSON, B,F.

2016 AN DOPICAL MONEL STUOY UF LOW ENERGY PION SCATTERING ERTM IODXYGFN

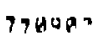


GIBSON, D.F, (EONTO.)

2891 OARITYONONCONAERVING ABYMAETRY IN NOD SELTTERING

GILMORE, J.3.

262 NEUTRONS FROM A HIGH ENERGY DROTON GEAM STOP TUQUIH

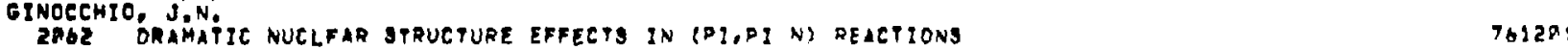

GLABCOCK, M. O;

253 INTERNAL-CONYTRSION COEFFITIENT OETERMINATION OF ODO PARITY FOR THE IUA,QOKEV FIRSTO T5BUAR EXCITED STATE OF QIDRUBIOTUM

GLABHAUSBER, $C$.

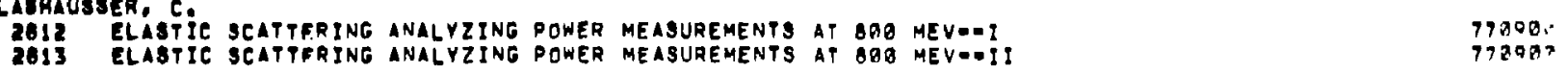

GLAC8: O. (P,NS AND (N,D) MEABUREMENTS FROH TARGETS OF GOLITHIUM ANO TOLITHIUH AT ROQ NEV

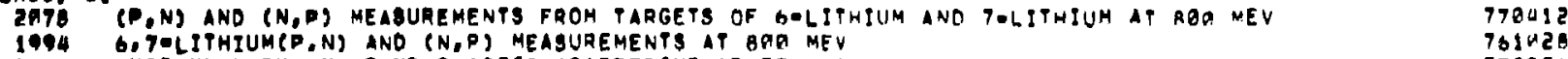

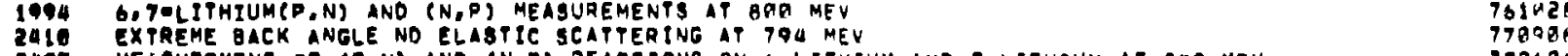

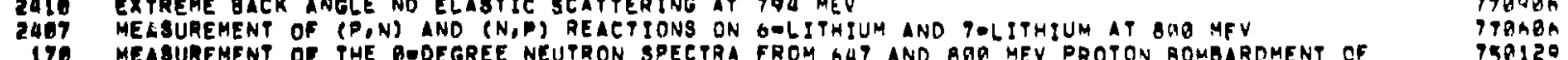

17. MEABUREMENT OF PHE BDDEG

203 MEASUREMENT OF THE ZERO DEGREE NEUTRON SPECTRUM FROM THE REACTION DP GOESTO NP PI+ AT T

758202

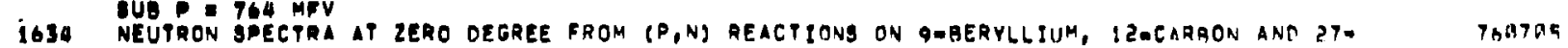

1635 ALUTRON SPECTRA AT ZERO DEGREE FROM POP AND POD COLLISIONS AT GUY AND HBG WEV INCIOENT

7מostan ENERGIES

760745 NEUTAON SPECTRA AT ZERO DEGREE FROM PROTONADROTON COLLISIONB BETWEEN GH AND BHS MEV NEUTRON SPECTRA FROM SAG MEV PROTONS ON QABERYLLIUM

iq6 MEUTRON BPECTRA FROM BROTON BOMBARDMENT OF DEUTERIUM AT 607 AND AOQ LEV

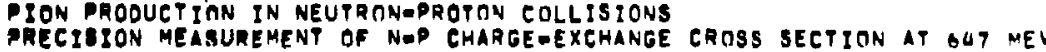

PREC ISION MEABUREMENT OF NAP CHARGE-EXCHANGE CROSS SECTION AT GUT MEV

OUABIELASIT C.HARGE EXCHANGE IN ND GOESTO PNN AT 994 MEV

790991

7400104

751220

751320

750.19.

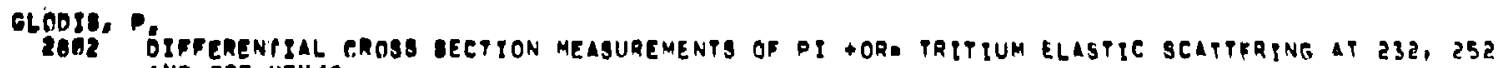

189123 2423 MEAOHREMENTS GF PHE DIFFERENTIAL CROSS SECTION FOR PION CHARGE EXCHANGE TN PRITIIIM.

2U24 MEAGUREMENTS OF PHE OIFFERENTIAL CROSS BECTION FOR PION CHARGE EXCHANGE ON TRITIIIM -

$790 \times 20$

730123

2803 PI TOR. ELABTTC SCATTERING MEASUREMENTS ON H3 AND HES AT 232, 25 ? AND 295 MEVIC 
GLUCKaTERn, R:LL,

143: BAND CLOSING tN AN ALYAREZ TYPE PARTICLE STRUCTURE

T62 CAVITYGBEAM INTERACTIONS
1194 COUPLING BETWEEN PRANSVERSE OSEILLATIONS IN LINACS

1194 COUPLING BETWEEN PRANSVERSE OSEILLATIONS IN LINACS
1G32 EOUIVALENT CIREUIT FOR A PERTURAED MULTIOCELL.STRUCTURE

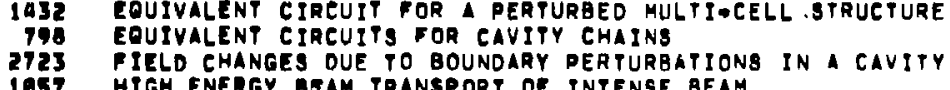

1057 HIGH ENERGY BSAM TRANSPORT OF INTENSE BEAM

INTERACTION OF A BUNCHEO BEAM WITH TRANSVERSE MODES IN RF CAVITIES

2722 NOTE ON PART TCLE MOTI

NOTES OY GLUEKSTERN

ROUND TABLE OTSEUSSION

ION ON SPACE CHARGE AND RELATEO EFFECTS

626 TRANGVERSE BEAM BLOW-UP IN A GANOING WAVE LINAC CAVITY

TRANGYERSE BEAM BLOW-UP IN STANDINGEWAVE LINAES

GOLOHABER, A.S.

37! ELABTIC SCATŸFRING OF 10,50 AND 14.5-HEV POLARIZED PROTONS FROM NUCLEI AND THE OPTICAL MODEL POTENTIAL AT INTERMEDIATE ENERGIES

GOMER, L.

14. INITIAL COMPARATIVE RESPONGE OF EXPERIMENTAL TUMORS TO PEAK DiONS IND XORAYS

$6000,0$.

1620 " LIMIT ON PARITY VIOLATION IN PANUCLEUS SCATTERING AT G GEVIC

$79095 \%$

640821

$670 \times 00$

65019

650722

$680900^{\circ}$

$60018 \%$

667314

659722

609619

$60^{6} 12 B$ A

670329

OOOMAN, C:

205 NEUTRON ENERGY FLUX AND DOSAGE IN THE LAMPF PID BIOMEDICAL CHANNEL

210 NUCLEAR-COULOMG INTERFERENCE IN PI +ORE 16 DXYGEN SCATTERING
103 PIONENUCLEAR TOTAL CROSS SECTIONS NEAR THE $3 / 2,3 / 2$ RESONANCE

050200

OPF, B.C.

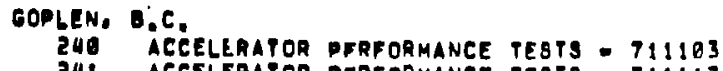

24! ACCELERATOR PPRFORMANCE TESTS: 711117

242 ACCELERATOR PIRFORMANCE TESTS - 711222

1665 ACCELERATOR PFRFORMANCE TEST

25! BEAM MEASUREMFNTS ON THE HIOHOINTENBITY PROTON INJECTOR OF LAMDF

37 BEAM MEABUREMFNYS ON THE FIRST TANK OF LAMPF

253 EEAM PROPERTIFS AT THE COLUMN EXIT - T18410

2512 OEAM PROPERTIFS AT THE COLUMM EXIT - TIBOIL

326 GEAM TRANSOOFT STUDIES ON THE PROTON BEAM LINE IN THE INJECTOR COMPLEX OR LAMPF CHANNELS FOA RF STABILITY GTUOY

473 GUIOELINES FOR OPERATIONS MANUAL - SYSTEMS

325 OPERATION ANO PERFORMANCE OF THE HIGHOIMPENSITY PROTON INJECTOR OF LAMPF

2333 PMASE AND AMPIIPUDE STUDY POR BUNCHERS ANO DRIFY TUEE LINAC

768715

741602

71360 ?

18410

intis

72110.

72113 .

721?12

गरा1\%. 
GOPLEN, B, C, (CONTD.)

43 PHASE SCANS ON MODULES I THROUGH 4 - 738119

248 PIOHESON ABSORPTION ON THE DEUTERON T5EIB::

aTS PROGRESS ON OPERATIONS MANUAL

252 OUADRUPOLE GRAOIENTS IN THE TRANSITION REGION

244 REBULTS OF PARTICLE DYNANICS CALCULATIONS

STMPR OF THFPEA

2069 PHE DEUTERON ROSTATE AND THE PI+ + D GOESTO P P REACTION
A9. TRANSITION REAION SPECTROMETER LINE

72111.

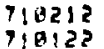

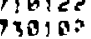

$761 \cdot 25$

750353

GORE, R,A.

1204" A COMPACT DATA ACOUTSITION AND CONTROL TERMTNAL FOR PARTICLE ACCELERATIRS

1991 CALIBRATION INO PESTING PROCEDURE FOR THE WATER FLOW SWITCH (REVISEO)

624 COMPUTER CONTROL OF THE LOS ALAMOS LINEAR ACCELERATOR

1893 DYNAMIE TESTS OF A MAGNET AND PONER SUPPLY

1464 ELCCTMICAL INTERFACING WITM MP - E EQUTPMEN

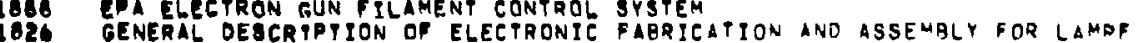

1740 INATALLTION PLANING

1740 INATALLATION PLANNING ERE VACUUM SYSTEM (ADOENDUH NO, 1 )

INGTRUETTON PLANTNG

618 LAMP CONTROL PHILOSOPHY

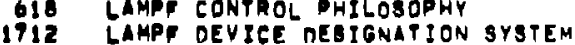

IUIS LAMPF DEVTEE DEQTGNATHON SYSTEM

2472 MYNGCOMPUTER APPLICATIONS AT LAMPF

T799 MIBCELLANEOUS FABRICATION SPECIFICATIONS FOR COMPUTER COMPONENTS

2030 MOQIFICATIONS TO PHE HAEFELY COCKCROFT -WALTON BOUNCER

1681 MOPOR STEPAE GOARD OPERATION

14I0 MPI ENGINEERTNE MANPOWER REVIEW

$1 A 13$ MPOI PROCUREMFNT SCHEOULE FOR LAMPF

1092 MPOI R AND D PHOGRAM FOR FYETB

1609 MPAI R AND D PROGRAM FYO69

1913 ON THE CALIBRATION OF ANALOG DATA CHANNELS

1862 PERFORMANCE GPECIFICATION FOR A 3 PROAMP DE CURRENT REGULATOR IIIS PROPOBAL FOR DATA PRANSMISSION SYSTEM USING STANDARO LOCAL TELEPHONE DAIES (OIAL NETHORK,

PROPOBED LAMPF DEVICE DESIGNATION

REVIEN OP THE MPAI A AND D PRCGAAM FO

SPECIFICATION POR ARC POWER SUPPLY

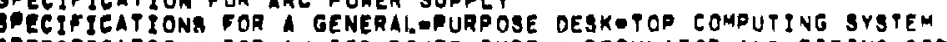

GPECIFICATIONG FOR AN SCR PONER SUPPLY REGULATOR AND FIRING CIRCUIT

TEMPERA PURE CANTROL FOR MAINTAINING RESONANEE OF LINAC TANKS

247 THE UIE OP MINICOMDUTERS IN PARTICLE ACCELERATORS

090303

071.23

$0497 ! ?$

Trats

67palc

090508

090588

071151

071105

orents

orentis

istisis

1351 .

71932

681'23

क० 1 1

009720
0 वas

720?17

909. दू:

$75 \mathrm{Al}^{2} 3$

GRA?

6034 a 9

600227

Tansy

7204!?

OPI?

13116?

Gopon, $K_{\text {. }}$

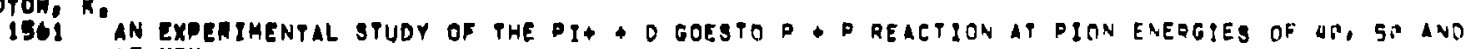

$75 \sin 6$

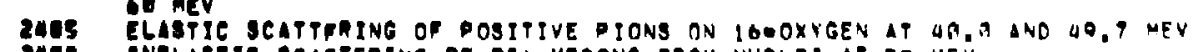

$179 \cdot 1$.

2AIE INELABTIC BEATPERING OF PI, MESONS FROM NUELE: AT SA MEV

171210 
GoTOW, K. (CONTO,)

1991 MEASUREMENTS TF PIONWNUCLEAR ELASTIC SCATTERING AT LOW EVERGIES TOI 20

2WOQ PION-NUCLEAR TNELASTIC SCATTERING AT 5A MEV V PO PEACTION

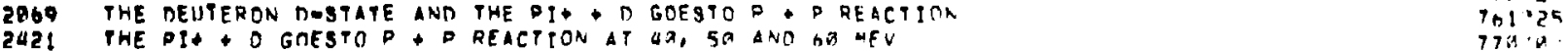

GRAM, P.A.M.

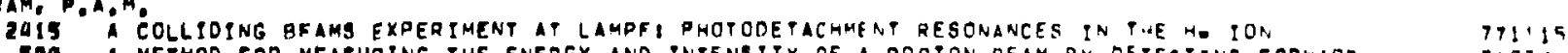

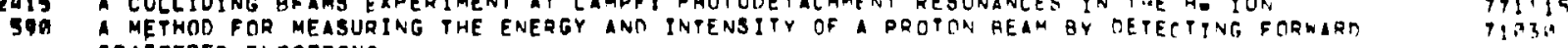

GPI SCATERED ELECTRONS VEROSATILE HIGHEENERGY PION HEAM FACILITY

1174 A PROPOSAL TO MEASIIRE THE PHOTDOTSINTEGRATION OF THE DEUTERON WITM POLARTZFO GAHMA RAYS TOBSIE

AT EPA

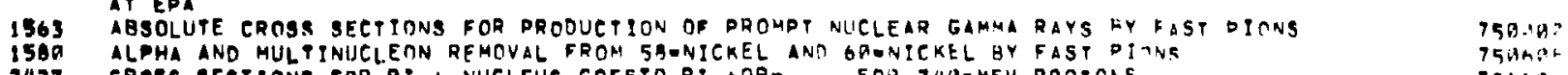

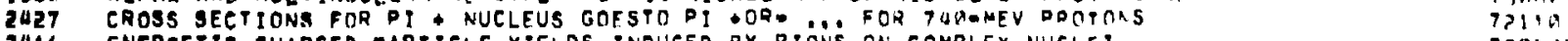

2416 ENERGETIC CHARGED PARTICLE YIELDS INDUCED RY DIONS ON COMPLEX NUCLE ! T7RIA

$15 B 1$ FAST PION INDHCEO PROCESSES IN COMPLEX NUCLEII CROSS SECTION AND ALPNA RFHCVAL TSAIA.

1582 FAST PION INOUCED PROCESSES IN COMPLEX NUCLEY PROJECTILE SYSTEMATICS

1385 FAST PION INDUICE PROCESSES IN COMPLEX NUCLEI: SYSTEMATICS IN PRAOICT "URLFI

2416 GAMMARAY STURY OF PION-INOUCED REACTIONS ON COMPLEX NUCLEI

1903 INTERACTION OF FAST DIONS WITH 62.64-NICKEL PHOTOOETACHMENT CROSS SECTIO. OF THE HE ITA

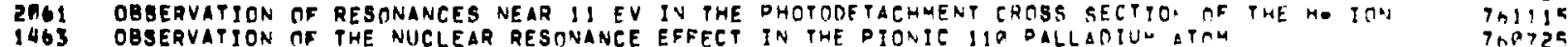

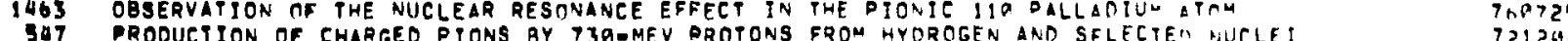

1997 PROTON SPECTR EROM DIONGINDUCED REACTIONS

1562 SYSYEMATICS OF PION AND PROTON INTERACTIONS WITH NICKEI. WUELIOES

GRANO, $P$.

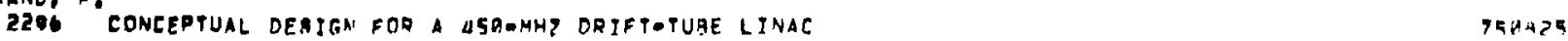

GRANT, P.H.

16U8 A STRONTIUMEAR - RUATOIUMOAZ TSOTORE GENERATOR FOR USE IN NUELEAR LEMTCI.E

1633 MYOCARDIAL POAITRON SCINTIGAAPHY WITH SMORT-LIVED RUAIDIUMABZ

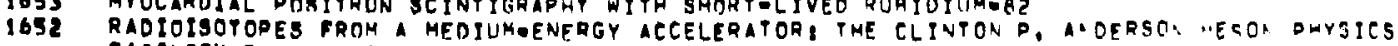
PACILITY RADIOTSOTOPE PROGPAM

165! SPALLATION CRASS BECTIONS ANO THE LAMPF MEOICAL RLOIOISOPODE PROSRAM

165 STRONTIUMAGZ - RUBIDTUMARZ RADIOISOTOPE GENERATOR

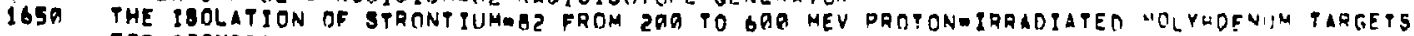
FOR ATOMEDTCAL APQ TCATIONS

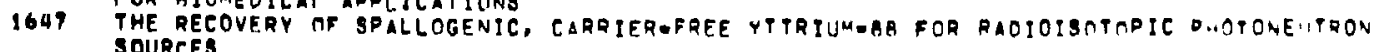

T5ach?

$70 \% 39$.

1203015

750345

760 i. 27

75040,5

GREENE. 8

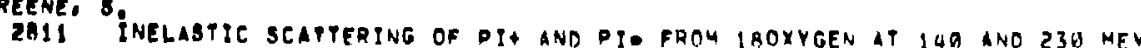

GREENTELO. M. 


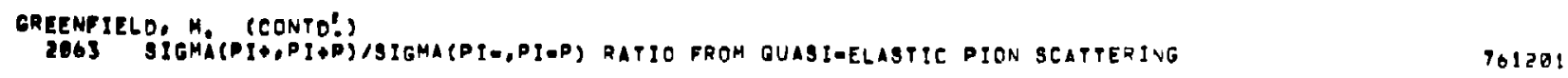

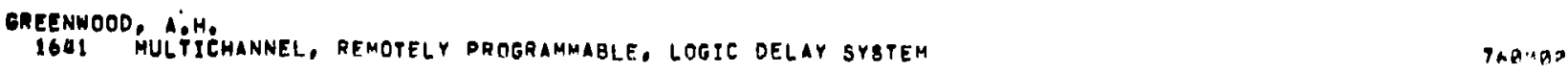

GREENHOOD, R.C.

2969 I34aBARIUM LEVEL SCHEME AS OBSERVED IN THE OECAY OF 134 -LANTHLNUM

14G6 ANNUAL PROGRESS REPORT - NUCLEAR GTRUCTURE GTUDIES UTILIZING LAMPF

1630 DECAY OF 1280 EESIUM

159 GAMHARAY EMTASTON FROM 1340 CERIUM AND LEVELS IN 1340LANTHANUM

OF 126 OBARIUM INO 128-CESTUM

LEVEL STRUCTURE OF 131 -CESIUM AND THE OECAY ENERGY OF 131 -BARIUM

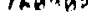

GRILLY, E,R.

1633 PHÓTON SPECTRIIM IN PION CAPTURE ON TRITIUM
2589 RADIATIVE NEGATIVE PION CAPTURE IN LIOUIO TRITIUM

GRISHAM, D:L

2004" BOLUTION FOR REMOTE MANDLING IN ICCELERATOR INSTALLATIONB

1209 AES PARGET MECHANISM ORTVE

2016 AOG HAPP AND POROID EHANGE OUT

1321 GENERAL OROCENURE FOR REMOVING ITEHS FROM LINE A (TARGET MECMANISMS ANT PROFILE MONITORS)

2003 THE EVOLUTION OF PHE LAMPF HIGH POWER PION PROOUCTION TARGET MECHANISMS

76119.

$76038:$

77940

768610

7416000

761109

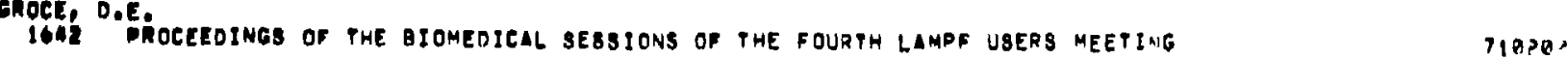

Grose, R.E.

1561 AN EXPERIMENTAL STUDY OF THE PI* + D GOESTO P - P REACTION AT PION ENERGIES OF WA, 5G ANR

60 MEV

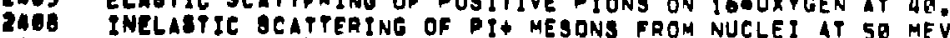

1991 MEABUREMENTS OF DION-NUCLEAR ELASTIC SCATTERING AT LOW ENERGIES

2AOU PIONONUCLEAR TNELASTIC BCATTERTNG AT SO MEV

2AG9 THE DEUTERON DOSPATE ANO THE PIO D GOESTO D. D REACTION

2421 THE PI+ D GRESTOP : D REACTION AT UQR 59 AND GQ MEV

$77931 \%$

7 b! 11

30 a 3

$761+14$

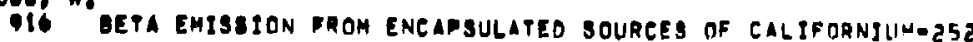

$7 \sin 30$

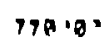

711519

7412

?7कम

761.25
770.19

HADOOCK, Ri.

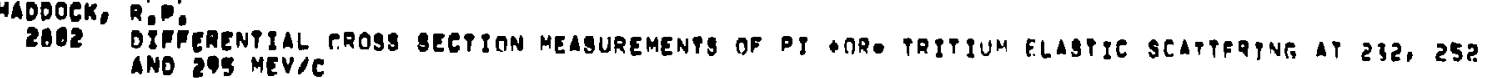

749500

7AB 123 
HADOOCK, RiP: (CONTO,)

2423 MEABUREMENTB OF THE OIFFERENTIAL CROSS SECTION FOR PION CHARGE EXCHANGE ON TRITIUM - TTUAZO 2424 MEASUREMENTS OF THE OTFFERENTIAL CROSS SECTION FOR PION CHARGE EXCHANGE TN TAITIUM -

7 ani2s

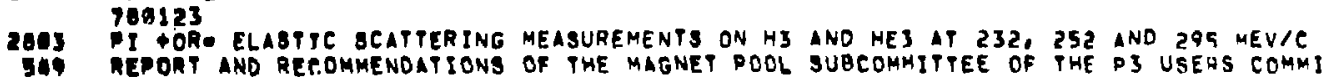

5HA REPORT AND REROMMENOATIONS OF THE MAGNET POOL SUBCOMHITTEE OF THE P3 USEHS COHMITTEE TIBABA

MAGERMAN, D.C:
QAO BEKV KLYSTRON BLOCXING-OSCILLATOR MODULATOR

940 BOEKV KLYSTRON BLOCKING-OSCILLATOR MODULATOR OAR521

2374 A BRIEF EVALUATION OF THE CONTROL ANO RF SYSTEMS IN THE CLINTON P. ANDERSON MESON OHYSITS 731 IBS

909 AEVICE FOR ABSOLUTE VELOCITY MEASUREMENT OF LINEAR ACCELERATOR BEAMS

DE VELOCITY MEASUREMENT OF LINEAR ACCELERATOR OE

23B AN OESGMC $101 / 40 M W$ AMPLIFIER FOR ACCELERATOR SERVICE

197 ANALOG DOLUTION OF THE PHABE MOTION PROBLEM

2373 DELTAGT D REFFRECE LINE EXPERIMENT: NOV. 15,1976

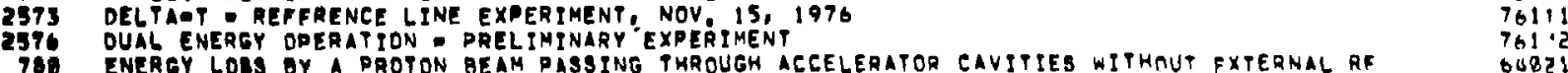

TEE EXITAYION OY A DROTON BEAM DASSINO THROUG

2359 PORE IOH TRAVEI TRIP REPORT (HAGERMAN)

MIGH DUTY FACTOR RF SOURCES AT BOB MHZ

2567 HIGHER PEAK CLIRAENT TESTS JULY 1 月, 197

25T8 INEREABED GRANIENTS?

752 MACHC COST MINIMIZATION

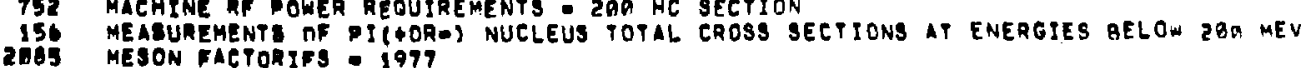

2ABI NEUTRON RADII OF CALCIUM ISOTOPES FROM PION TOTAL CROSS SECTION MEASUREMFNTS

346 OPERATINO PESIILTS ON PHE BOBWMEV PROTON LINAC AT THE LOS ALAMOS SEIENTIFTC LABORATORY

272 OPERATOR AND MAINTENANEE ACTION REPORTS

2379 PHASE BCAN OF PHE SIDE OCOUPLED LINAC

2575 PHABE SCAN OF THE 8 IDEWCOUPLED LINAE AS AN ENTITY

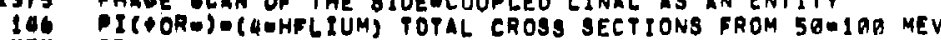

757 PRELIMINARY CHMPARIBON OF GOM ME TO 2 200 ME POWER AMPLIFIER TUBES

257T OUALITATIVE EXIERIMENT ON 21 IOMEV BEAM OUALITY

160 SOHE TOLERANCF REOUIREMENTS FOR A PROTON LINEAR ACCELERATOR

STAPUS OP LAMPF - 1976

263 THE LOS ALAMOH MESON PHYSICS FACILITY ACCELERATOR

1908 THE STATUS OF LAMPF

HEQ USE OF OPYMAINT REPORTS ANO WORKLIST

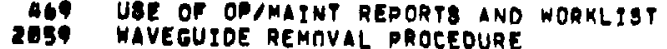

HAHA, A, 
HAMN, A. (CONTO.)

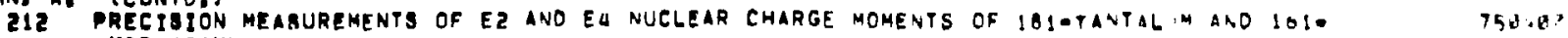
DYEPROSIUM

HAIMBON. 3

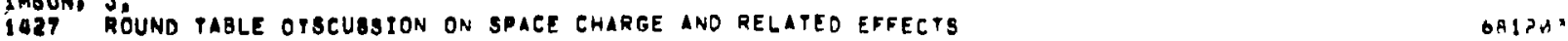

HAINES, E.L.

1645 THE FISSION TRACK RECORD OF APENNINE FRONT KREEP BASALTS T5O:D?

1467 THORIUMAURANGLM FISSION RADIOGRAPHY TRONT KREEP BASALTS TEM:R:

HALBACH, $K$,

159! A GENERAL ION-OPYICAL CORRECTION ELEMENT TOB?:

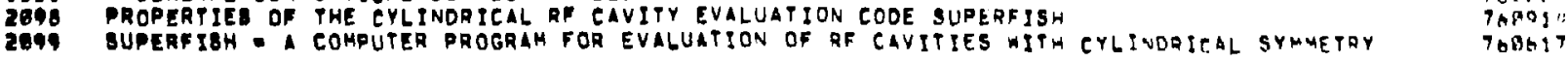

HALBIG. JGK. 20 I AND TR HARP PROGRAM

6) 201 GEAM SERADERS

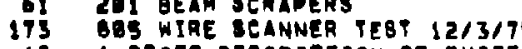

254 A MAENETgE DPFTION OF EMITTANCE OPEAATION

- MAGNETIC SPFCTROMETER SYSTEM FOR ON-LINE STUOIES OF GETA RAYS AND INTEANAL CONVERSION

263
i320 ABPDRER COLLFCTOR THICKNESSES
OEADPULL LOCAI CONTROL ELECTRONICS

172 BEAM EXPERIMENTS, PROPOSAL T6-

7 DEAM SCRAPERS

1949 CALIBRATION OF PHE 291 BEAM POSITION MONITORS - EXPERIMENT 7000

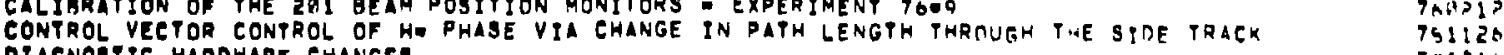

DIAGNOSTIC HARDWARE CHANGES

DIAGNOSTIC HARDWARE INTERLOCXS

DISK FILEd 32>0 ANO 389 (ACCELERATOR PARAMETERS ANO CALIBRATION OATA)

DISSIPATION POWER POR VIRIOUS OIAGNOSTIC DEVICES

EM EPECIFICATTONS

EM-11 MEABUREMENT

EMAz JAH STEERING EFFeTS

EmITTANER BIAS STUDIES

EMIPTANEE GEAR

EMITTANCE GEAR OPERATION REDORT TO ACCELERATOR ELECTRONICS COMMITTEE

EINAL TREATMENT OF PROHARP DATA

PUNCFION DESCRIFIION FOQ DEBUG BOX 1

GREAT BTARTU OHABEOSCAN ANOMALIES

INPERIM RESULTS OF ALIGNMENT SURYEY ON BO5 LINAC, PRANSTTION REGION ANT GNITCHYARO

235 INTERNALCCONYRSION COEFFICIENT DETERMINATION OF OOD PARITY COR PHE IHA, REXEV FIRST

2110 INVESTGAFION OF Th STEERING SOLUTION

74052 ?

751 हो

74446

740 A

751231

7 hinat

75613:
7 a

$75 ! 120$
74910

75 a

739 ?

7ुम?

7 703an

70035

7 Than?

Tul2e?

95025

750024

731192

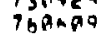

7580.40

Thite?

isar?

$75 \mathrm{~A} . \mathrm{A}$

$7+12^{3}$ 


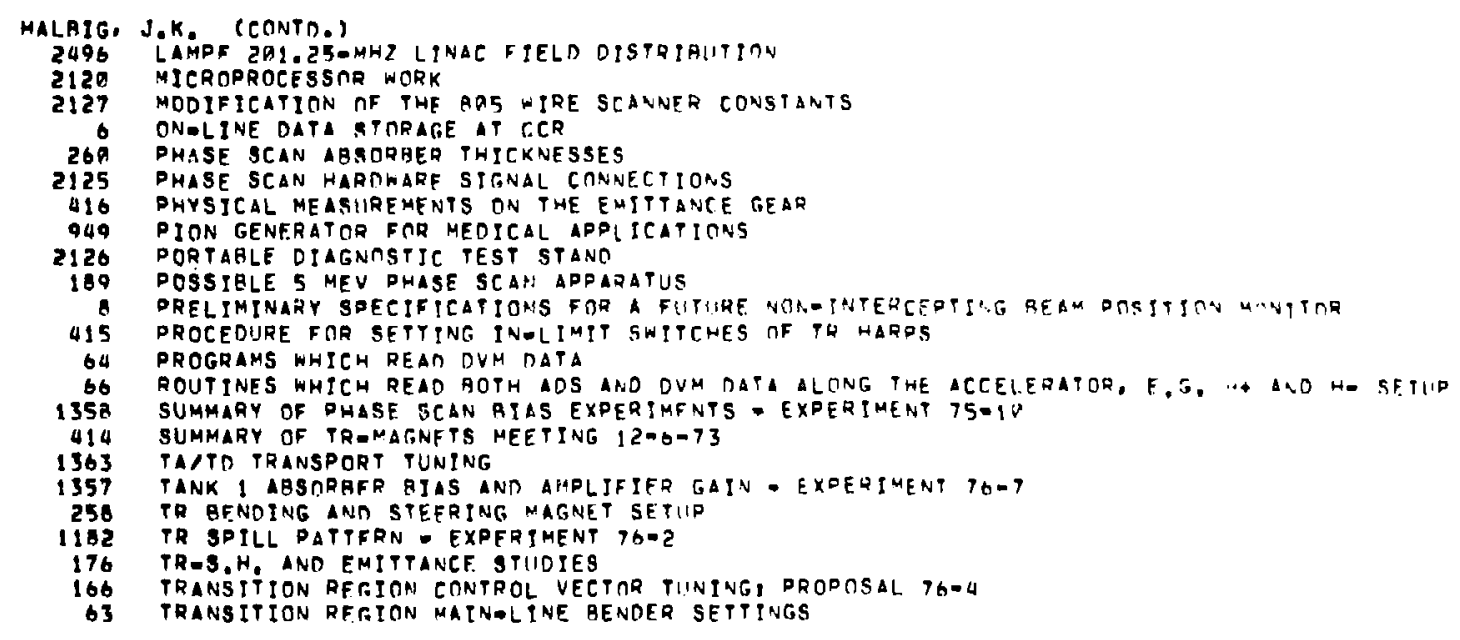

7 เงว2*

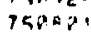

$77 \times 21$

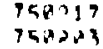

$711: 16$

Tarit

Tathe?

Than ?

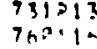

Thapes

75952

7 noint

751231

751231
$14+16$

MALE, G, M.

145 ELASTIC SCATTFRING OF 7-12 MEV TRITHAS BY ALPHA DARTICLFS

HALPERN, I

156 MEASIIREMENTS HF PIC+TR-) NUELEUS TMTAL CROSS SECTIONS AT ENERGIES AELO. SHA WFV

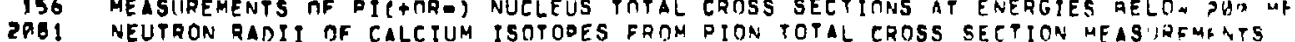

146 PI(COR-)OCQMHFLTUMS TOTAL CROSS SECTIONS FROM 5A-IMG MEV

1640 RECENT MEASURFMENTS OOFAL CROSS SECTYONS EROU SA-IAH MEV

HAMMEL, E,F.

TG4 CRYOGENICS ANA NUCLEAR PHYSICS. PART II

$750+2^{2}$

$75 \operatorname{sinas}$

7 thase

$\tan (4,1,52$

$\operatorname{laghas}$

HANNA, W.S.

1179 EXPERIMENTAL MAGNET POWER SUPPLY PRFLIMINARY TEST REPORT

1169 HARMONICS IN PONTROLLED-RFCTIFIER CIDCUITS

2355 MAGNET POWER SUPPLY STANTARDIZATION

1668 MODULATOR POWFR SUPPLY MODEL STUDY, QUARTERLY REPOHT, APRIL I - JUNE 3.3, 106

23UB PRANSIENT TESTS ON THE RFCTIFIFR MODEL

Topap.

athat

borica

HANBON, K.M.

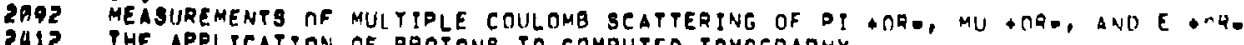

$\operatorname{logatac}$

111 
HARDEKOPF, R.A

145
220
$20 L A B T I C$ SCATTFRING OF $7-12$ MEV TRITONS BY ALPHA PARTICLES

HAROWICK, J.N.

24OS 2QBOAHZ DPERATING MANUAL

2366 FILAHENT TRANSFORMER - SPEC - 711119

2365 FILTER CHOKE SPEC - TI1199

HAROY, G.E.

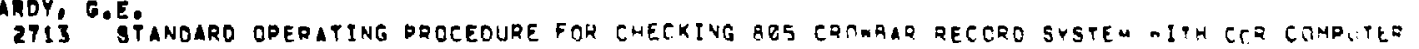

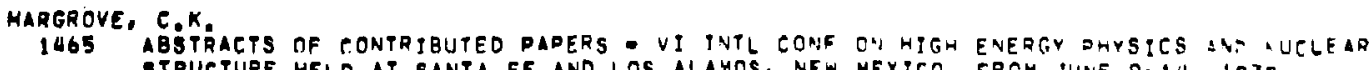

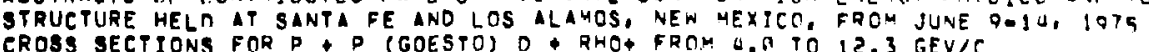

372 CROSS SECTIONS FOR P * P COESTOS D RHO FROM QA TO 12.3 GEVIC

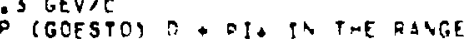

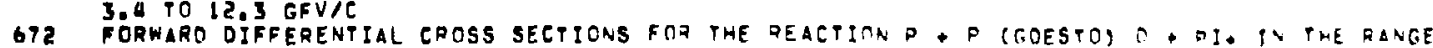

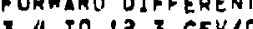

1463 DBSERVATION SF THE NUCLEAR RESONANCE EFFECT IN TME PIONIC IIP DALLAOIJU ATAM

ARP, 6.0.

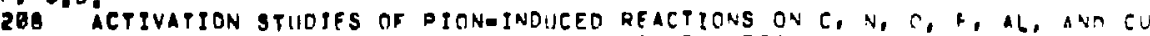

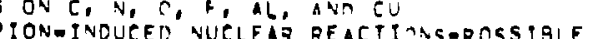

EFFETS OF THF FINITE LIFETIME OF PHE (3,3) ISOQIN

231 SPALLATION OF COPPER BY 190 MEV PICOORAS ANO 340 MEV PROTCAS

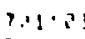

19110

APER, K, H.

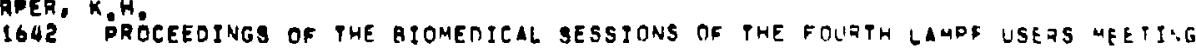

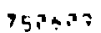

Paras

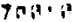

GAB 19 .

TALP 25

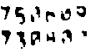

$75,1-20$

HARQ⿻B. R,E.

1746 MPQI PRINTED PIQCUTT CARTS IN USF

71020

$601 \cdot .48$

HARRISON, R,F,

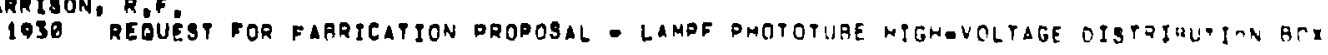

721.1.

HART, V.E.

1586 ALIGNMENY PHII OSOPHY, DESIGN ANO TECHNIQUES USED AT LAMDF

2035 BIOMED TARGET LOCATION

220. GENERAL SPECIFICATYONS BRIGHT ACID COPPER PLATING OF DIGLET IECFIERATOH PA.

2201 GENERIL SPECIFICATIONS FRR BIOMEOICAL BEAH LINE SUPOTRT STRUCTURE

INSTILLATION AND ALIGNMENT OF LAMPF 291.25-MHZ AND ABS=MMZ LINAC TANAS

7? LAMPE LLIGNMENT MONUMENTS 
HART, V.E, (CONTO:)

1986 PIGMI MECHANITAL FABRICATION
2264 TECHNICAL SPETIFICATIONS EPICS SPECTROMETER MIGNET INSTLLLATION A THS

HARTHAY, B.L:

2473 COMPACT PROTRAMMABLE CONTROL PANEL GOR COMPUTER CONTROL SYSTEMS

1609 A GUIDE TO THF LAMPF INTERPHONE COMMUNICATIONS SYSTE

AN OPERATORS CDNSOLE FOR THE LIMPF ACCELERATOR

1160 ENGINEERING STUDY FOR THE LAMPE COMMUNICATIONS SYSTEM

1409 MPOI BTANDARD NIM-AIN WIRING

1570 SECOND GENERATION CONSOLE INTERFACE UNIT (CIU) AND ASSOCIATED CONSOLE HAEDLAFE (DQAFT)

SPECIFICATION FD MIC HEADSET FOR LAMPF COMMUNICATION SYSTEM

E TWOWHAY PERSONAL FM RAOIO

HARYEY,

2016 A-G HARD AND TOROID CHANGE OUT

2012 CHANGES REOUTRED TO MAKE RAOIP OPERATIONAL

2919 DOSIMETRY AT THE RADIATION EFFECTS FACILITY, AO

1495 MINERAL-INSULATED CONOUCTORS FOR MAGNET COILS

1494 MINERALININGULATEO MAGNETS FOR HIGH-RAOIATION ENVIRONMENTS

1565 OPERATING EXPFRIENCE WITH LAMPF MAIN BEAM LINES INSTRUHENTATION AND CO TLOL SYSTEM

149 RADIATIONAHARTENED MAGNETS USING MINERAL-INSULATED CONDUCTORS

2918 RADIP DOSIMETRY IRRADIATIONS

HASSENŻAHL

705 AN EVALUATION OF WINOING TECHNIOUES AND NB-TI WIRES FOR A SUPERCONDUCTiNG, DUADRUPOLE

2053 COMPLETION OF G INCH ROOM TEMPERATURE BDRE SUPERCONOUCTING OUADRUPOLE WAGNET DOURLET CRYOGENICS AND NUCLEAR PHYSICS, PART I

673 QUADRUPOLE FOCUSING MAGNET

2839 SWITCHYARD BENDING MAGNETS

78. THE EFFECTS OF RADIATION ON SUPERCONOUCTING NB-TI WIRE

709 TWENTYOFIVE CFNTIMETER BORE SUPERCONDUCTING DUADRUPOLES FOR LAMPF

738004

711.20

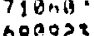

009023

0.5050
00105

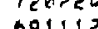

001119

73 pha?

HAETINGS, R, D:

I529 MANUAL HEAD ENNTROLLER (MHE) OPERATIONS MANUAL

$30,53,5$

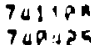

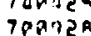

bOAHA:

75PAa:

Tรanus

74 anto

Triage:

papas:

Tepapa:

GAPADS

75031

T133\%?

tione

600915

$720 x$

$750+90$

$750.0 \%$

75868.

759129

HATCH, E.N.

1563 A6SOLUTE CROSA SECTIONS FOR PROOUETION OF PROMPT NUCLEAR GAMMA RAYS FY FAST DIONS

I391 FAOT PION INDIICEO PROCESSES IN COMPLEX NUCLER CROBS SECTION AND ALPHA RFMOVAL

1502 TAST PION INDIICEO PROCESSES IN COMPLEX NUCLEII PROSECTILE SYSTEUATICS

1505 PAST PION INDIICED PROCESSES IN COMPLEX NUCLEII SYSTEMATICS IN PRCDUCT .UCLE 
HAUSER, M.G.

664 AN EXPERIMENTAL STUOY OF OMEGA NEUTRAL OECAYS

$7 ! 1 \cdot 1=$

HAYANO, R,S.

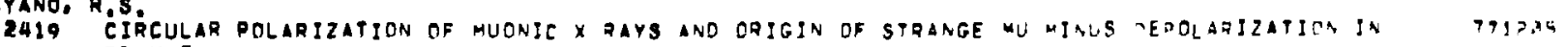
PO METAL

HAYWARO, T.D.

29 JOINT H- ION SOURCE DEVELOPMENT PROGRAM TLa"ith

HEATH, R,L.

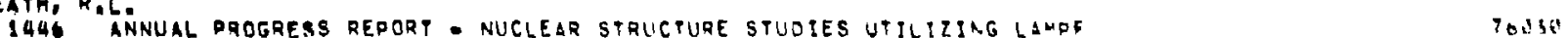

HEFFNER, R,HE

1625 OESTGN OF A PTON SPECTF.? YETER AT LAMPF

24I MASS DEPENDENCE OF PION DOUBLE CHARGE EXEHANGE

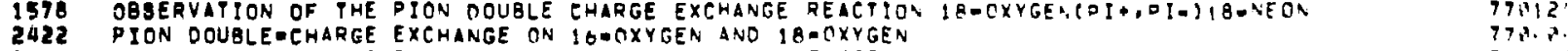

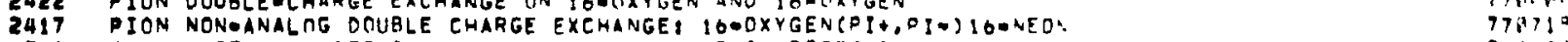

ISA THE ELASTIE BCATTERING OF LOW ENERGY PIONS ON BROTONS

HEINRICH, R,

162 NEUTRONS FROM A HIGH ENERGY PROTON BEAM STOP

HELLAND, J.A. BECONSTRUTTION OF SIMULATEO PRCTON RADTOGRAPHY OATA

JHO ABSORBED DOSE ANO RADIATION QUALIYY FOR BACKGROUND RADIATION FROM A TESATIVE PION REAM

240 ACCELERATOR PFRFRRMANCE TESTS " 711103

24. ACCELERATOA PFRFCRMANCE TESTS - 71111?

242 ACEELERATOA PFRFORMANCE TESTS - T11222

MCCELERATOR PFRFORMANCE TESTS F T11819

1PGI ANALYSTS OF STLICON ANO GERMANIUM RETECTOR OATA

IR64 BEAM REOUIREMFNTS FOR WEAPONS NEUTRON RESEARCH

1962 BIOMEDICAL BEAM VERTICAL SCANNING

900 MICRODOSIMETRY OF NEGATIVF PIONS AT LAMPF

1960 SPECIFICATIONS FOR HELIUM RAGS

998 TUNING OF PHE FIRST SECTION OF PHE BIOMEDICAL CHANAEL AT LAMOF

IA62 TWODIMENSIONAL VISUALIZITION OF STOPPING PION DISTRIBUTIONS

1306 WHOLE BODY DOSE FOR PATIENTS TREATEO WITH DIONS

$750 . \cdots$

711142

711119

711.19

?ing.

$\operatorname{tanns}$

$721 \cdot 140172111+1$

$75,3+19$

$749: 14$

$950 \mathrm{sat}$

Intand

Thing

HEL.MER, A, G.

2067 i34-AARIUM LEVEL SCHEME AS OBSERVEO IN THE OFCAY OF 13H-LAVTMAVIW

146 ANNUAL PROGRESS REPORP. NUCLEAR STRUCTIIRE STUMIES UTILIZING LAMDE 
HELMER, R,G, (CONTO.)

2065 GAMMA-RAY EMISSION FROM 134 -CERTIJM AND LEVELS IN 134 -LANTHANLIM

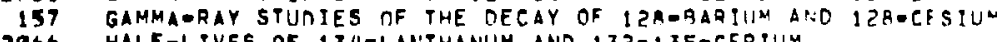

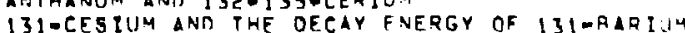

Thing

$741 \cdot 4$,

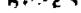

Thlid."

HENLEY, E,M.

$77112^{2}$

HENSLEY, W.K.

2964 MEASUREMENT OF TOTAL MUOM-CAPTUIRE RATES IV 232-TMORIUI, 235, ?3A-IRAVIUM, A4D 239-PLUTOVI.M

MEASUREMENT OF TRTAL MUON-CAPTURE RATES IV $235-T M O A$
MUON CAPTURE QATES ON ACTINIDE NUCLE!

WUON LIFETIME MEASUREMENTS IN THE ACTIVIDE ELEMENTS

$7+1 \cdot 5 n$

$7542^{\circ}$

HIERERT, J.C.

2A78 (P,N) ANO (N,D) MEASUREMENTS FROM TARGETS OF G-LIPHIIIM AND 7-LITHIUM AT AOR MEV

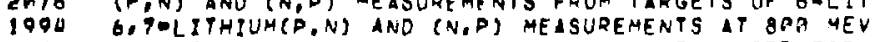

29A A MULTIWIRE PRDPRRTIONAL CMAMBER SPECTROMETER FOR NUCLEOVENLCLEO: EXOEOI 'ESTS

198 OATA ACOUISITTON SOFTWARE FOR A MWPC SPECTROMETER

2UIN EXTREME BACK ANGLE NO FLASTIC SCATTERING $1 T$ TO4 MEV

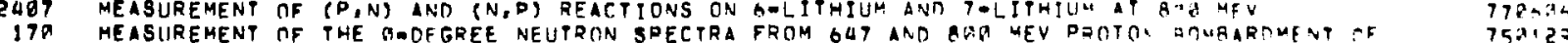

IGHT NUCLEI

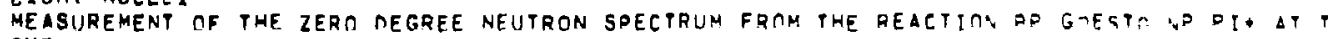

SUB P $=764$ MFV

NEUTRON SPECTRA AT ZERO DEGREE FROM (P,N) REACTIONS ON Q-BEEYYLLIHM, 1 z-CARUOH ANC 270

1633 ALUMINUM AT GUT AND BUA MEV NEUTRON SPEO

1960 NEUTRON SPECTRA AT ZERO DFGREE FROM PROTONOPROTON COLLISIONS BFTHEEN GLT AAS BIS MEV

NEUTRON SPECTRA FROM 5OA MEV DROTONS ON A=BERYLLIUM

NEUTRON SPECTOA FROM DROTON BOMBARDMENT OF DELITERIUM AT GAT AND RAU WEV

PRECISIION MEASUREMENT OF N-P CHARGE-EXCHANGE CROSS SECTION AT GUT WEV

PRECISION MEASUREMFNT OF NAP CHARGEAEXCHANGE CROSS SFETION AT GUH MEV

QUASIELASTIC C.HARGE EXCHANGE IN ND GOESTO PNN AT 704 YEV

$755^{2}+$

HIEBERT, R. O

2QBA PHOTRMULTIOLIFR TUAE GASE FOR HIGH OULSEO ANOOE CURRENTS

Thorpa

$7+n+45$

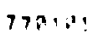

$700 \cdot 0 \cdot 0$

$75 \% \times 1$

$\operatorname{Ton} 2,4 \pi$

$720.60^{2}$

$77942 \pi$

Thin:

HIGHLAND, VIL.

1627 LIMITS ON THE DIRECT PRODUCTION OF POSITRONS AY 250 - AND BOGHMEY PROTONS

FOR DIRECT LEDTON PRODUCTION IN PD COLLISIONS $4 T$ ABA MEV AND 25 O "EV

1621 SEARCH FOR THF DIRECT PRODUCTIINN OF POSITRONS AY 256 . ANO ROR-MEY PROTIVY

HILL, J,C. 
HILL, J, C, (CONTO.)

Tas EFFECTIVE CROSS SECTIONS FOR THE (N,ZPN), (N, ZP), AND (N, ZPN) REACTIONS USING INTFRMEDIATEENERGY NEUTRONS

178 PRODUCTION OF NEUTRONARICH NUCLIDES USING HIGHEENERGY NEUTPONS AT LAMPF

2UI4 SEARCH FOR NEW NEUTRON-RICH NUCLEI PRODUCED OY FAST NEUTFONS IT LAMPF

179 STUDY OF THE DAFAPLATINUMO-200-GOLD DECAY CHAIN AT LAMPF

749 THE 209 PLATINUM - 2HA GOLD DECAY CHAIN

$7512^{0}+$

$741 \cdot 4:$

7 ho. 2 :

$741+0$.

$7 \operatorname{son} \theta 3$

HILL, R,E,

1490 "201.25 MHZ INTERLOCK

HINCKS. E.P.

572 CROSSS SECTIONS FOR P + P (GOESTOS D + RHO + FROM 4. T TO 12.3 GEVIC

574 FORWARD DIFFERENTIAL CROSS SECTIONS FOR THE REACTION \& P (GOESTO) * DI IN THE RANGE

672 FORWARD DIFFERENTIAL CROSS SECTIONS FOR PHE REACTION $P$ + D (GOESTO) D. DI+ IN THE RANGE 3.4 TO 12.3 GFV

HINDMARSH, A.C.

2734 DOUEIE DRIFT RUNCHER PART BI INCLUDING SPACE CHARGE

70970 !

Thesios

6ดควด:

606090

HINTZ, N.

2012 ELASTIC SCATTFRING ANALYZING POWER MEASUREMENTS AT BOG MEV

2012 ELASTIC SCATTFRING ANALYZING POWER MEASUREMENTS AT BOG MEVE-I
2013 ELASTIC SCATTFRING ANALYING POWER MEASUREMENTS AT BRG HEVH-II

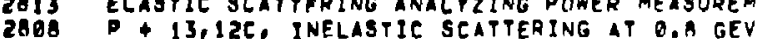

$77094:$

77090

HNATOHICH, D.S.

1633 MYOCARDIAL POSITRON SCINTIGRAPHY WITH SHORTOLIVET RURIDIUMOBZ

7 hasp.

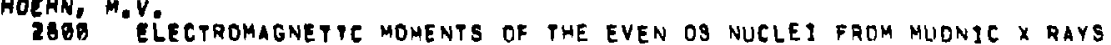

771121

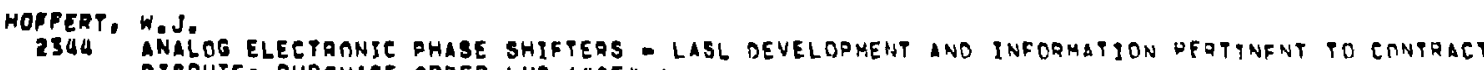
OISPUTE, PUREHABF ORDER LHD-14854-

236 ANALOG ELECTRANTE PHASE SHIFTERS - P.O, LHO-1485

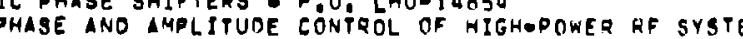

207 FAST RF CONTRAL WORK AT LASL

261 MICROWAVE INSTRUMENTATION FOR ICCEIERATDR RF SYSTEMS

23IS POWER SUPPLY GPECIFICATIONS

THE OF MASTER SOUPCE SYSTEM

MOFFMN, C.M. 
HOFFHAN, C:M. (CONTO,)

ABSTRACTS OF CONTRIBUTED PAPERS - VI INTL CONF ON HIGH ENERGY DHYSICS AND NUCLEAR

$750 \operatorname{son}$ STRUCTURE HELO IT SANTA FE AND LOS ALAMOS, NEW MEXICO. FRON JUNE 9-14. 1975

1542 LIMIT ON PARITY NONCONBERVATION IN PONUCLEUS SCATTERING AT O GEVIC

1628 LIMIT ON PARITY VIOLATION IN PONUCLEUS SCATTERING AT O GEY/C

1627 LIMITS ON THE DIRECF PRODUCTION OF POSITRONS BY 256- AND BAQEMEV PROTO.S

783 SEARCH FOR DIRECT LEPTON PRODUCTION IN PP COLLISIONS AT RQR MEV AND Z5G "EV

750505

968795

768715

760.20

HOFFMAN, E.W:

2541 CYCLE R TEST PLAN

2530 PROCEDURE FOR PHASE SPACE MEASUREMENTS IN LINE A-DIRECT

2530 REVISED CYCLE OPLAN

2671 TENTATIVE SPERIFICATION OF POLARIZATION DATA ACQUISITION SYSTEM

Tones:

7685365

$\operatorname{Tn} \ln 315$

HOPPMANN, G.

1148 "Dig:TAL TECHNIDUF FOR NEUTRON-GAMMA PULSE SHAPE DISCRgMination

1146 DIG. TAL TECHNYDUF FOR NEUTRON-GAMMA PULSE SHAPE DISERYMINATION

2612 ELAST C SEATFFING ANALYIING OOMER MEASUREMENTS AT BRO MEYMO

2813 TAPR: OCATTERING ANALYING POWER MEASUREMENTS AT OPO MEVEII

2008 - I2C. INELASTIC BCATTERING AT D. GEV

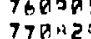

77979

77004.

ๆาด्म०

HOPTIEZER, J,H

2087 ANGULAR OISTRIBUTION FOR PO GOESTO 3HE PI ZERO REACTION AT TP 8 BAB MEV

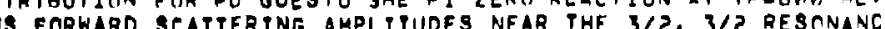

2073 IONONUCLEUS MMALL ANGLE ELASTIC CROSS SECTIONS NEAR THE 3/2, 3/2 RESONANEE

2074 PIONONUCLEUS POTAL CROSS GECTIONS NEAR THE J/2, 3/2 RESONANCE

Thua

$7 \mathrm{Th} 2$

771425

770425

HOGSTROH, $K, R_{\text {: }}$

1980 PION PRODUCTINN FROM THE REACTION DLP,D PISN AT 585 MEV

1982 PION PRODUCTIIN IN POP INTERACTIONS AT BAG MEY

761,50

HOLSINGER, R,F,

230: ACEELERATING ANO FOCUSING STRUCTURES FOR DIGMI

770719

77 1919.

796 bun

960214

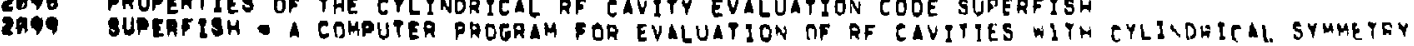

70 Q⿱一夕⿵冂⿱一口𧘇17

NOLP. J,A.G

1032 VHF CANCER THERAPY (JAN. 31, 1975)

1034 VHF CANCER THFRAPY (JAN, 3, 1975) 75A1901

HOLY,, , J. 
HOLT. R, S, TCONTO,

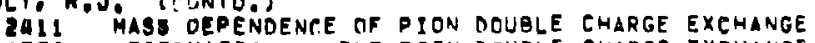

1978 OBSERVATION OF THE PION DOUBLE CHARGE EXCHANGE REACTION 180OXVGEN(DI+,DI-)18-NEON

2422 ION DOUBLEOCHARGE EXCHANGE ON IGEOXYGEN AND IBOOXYGEN

2417 PION NONGANALOG DOUQLE CWARGE EXCHANGEI 16-OXYGEN(PI+,PI-110*NEON

1653" "MYOCARDIAL POATTRON BEINTIGRAPHY WITH SHORT-LIVED RUBIOLUMERZ

วดคด.

HORNE, R.A.

260. A' SHORT-CUT RFMOTE HANOLING PROPOSAL FOR AREA A ANO REYOND

20A4 AOLUTION FOR REMOTE HANDLING IN ACCELERATOR INSTALLATIONS

2AI MERRIMAC STATIIS REPORT

796 MONITOR A VFRSATILE REMOTE HANDLING SYSTEM

2322 PROGRESS REPORT MERRIHAC IND MONITOR

75 and?

77 iाin

751121

ining

HORNSTRA, R,F, JR,

1184

$7 \times 39029$

HOAOLEY, R,F.

1697 CALCULATING JOSUA-B(X)

TION TIMES

1696 PAPER-TAPE COPY PROGRAM FOR TRE NOVA INO SUPERVOVA

1302 SUPERNOVA FLOATING-POINT EXECUTION TIMER

$\cos 250$

$\cos (0, a+1$,

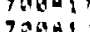

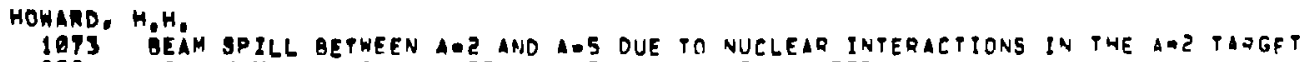

2324 FOLLOW UP OF ROME OUESTIONS ABOUT THE LINE X REDESIGN

1478 LAMPF USERS HANDBOOK

1627 UIMITS ON THE DIRECT PROOUCTION OF POSITRONS BY 250. ANA AGGAMEV DROTOLS

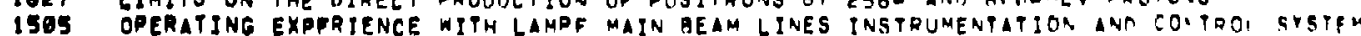

1 A76 PROCEEDTNGS OF THE FIFTH LAMPF USERS MEETING

$14 T 7$ PROCEEDINGS OF THE SIXPH LAMPF USEQS MEETING

2539 REVISEO CYCLE O PLAN

TA3 SEARCH FOR DIPECT LEPTON PROOUCTION IN DP COLLISIONS AT AGA MEV ANI ZSA WEV

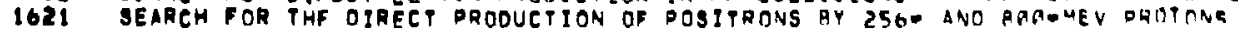

2674 SPECIFICATION OF STRIPDERS FOR LINE X. OHASE

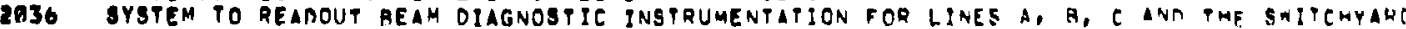

$75 \operatorname{14} 19$

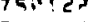

$7+110$,

$\operatorname{lnc} 715$

$75330^{\circ}$

73 a 85

inasy

$7 \rightarrow(9.029$

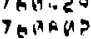

Japhe?

उउता2?

HOWARO. J.

Q53 Cell survival as a flinetion to depth for negative pions ant heavy iOMS

HOYT, H.C.

T93 DIMPLE METHOD FOR DETEQMINING DIMENSIONS DF AQS ME/SEC SHAPED LINAC TAVITISS

1 LOG COMPUTERADEBIGNED AMS MHZ PROTON LINAC GAVTTIES

05,11 
HOYT, H.C. (CONTO.)

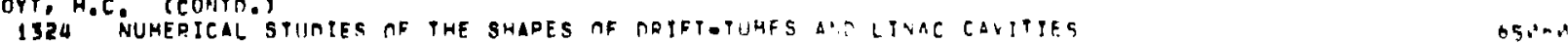

HUANG, C.Y.

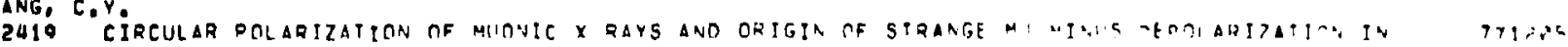
PD METAL.

HUO?S, J.

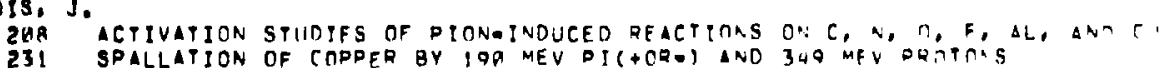

HUDOMAL JEGABITZSCH, J.

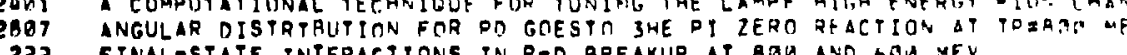

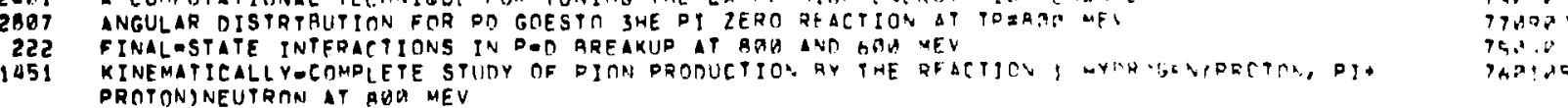

1981 PION PRODUCTION AY POD MFY PROTONS FROM G-LITHTUM

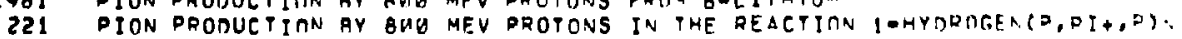

ION FROM THE REACT ION DCP,O DI)N AT 585 ME

D AT BAB MEV

PION PRODUCTION VIA A PQD GOESTO DHPI JUASTOFRE PROTONS IR DEUTERTUN

DION-NUCLEAR TOTAL CROSS SECTIONS NEAR THE $3 / 2,3 / 2$ RESONANCE

PIONONUCLEUS FORWARD SCATTFRING $\triangle M P L I T U D E S$ NEAR PHE $3 / 2,3 / 2$ RESCMARE

PION=NIICLEUS SMALL ANGLE ELASTIC COOSS SECTIONS NEAR THE 3/2, 3/2 OESOAME

PION-NUCLEUS TOTAL CROSS SECTIONS NEAR THE 3/?, 3/2 RESONA,CF

QUASTFFRE SCATTERING IN PAD RREAKMUP REACTIONS AT BIAG AND GRP MFV

QUASI-FREE SCATPFRING IN PROTON-INDUCED DFUTERON HRFAKUP AT 5 AS ANM RA. WE

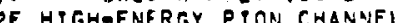

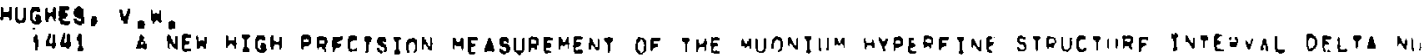

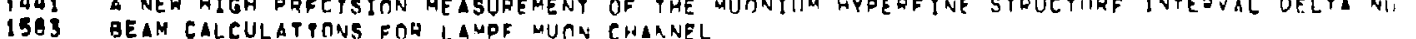

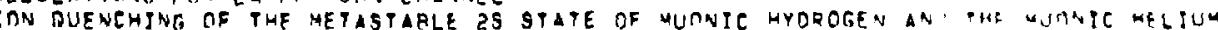

915 DEPOLARIZATION OF NEGATIVE MUONS IN HELIUM GAS, SEARCH FUR MUONIE MELI M ATOH

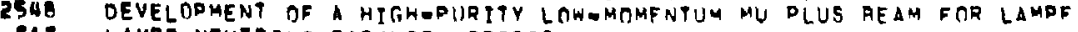

LAMPF NEUTRINA FICILITY PRDOOSAL MUTNIUM

MUONTUM CHEMISTRY: MUON CAPTURE.

MUNNYUM FORMATIOM IN NORLE GASES IND NOBLE GAS MIXTIJRES

DRECISION MEASUREMFNT DF MUONIUM HFS INTERVAL AND MUON MAGNETIC NOMEST

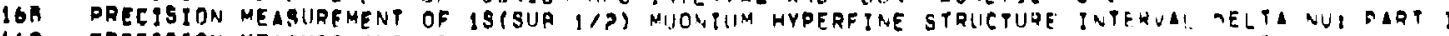
PREEISION MEAGUREMENT OF IS(SUB L/Z) MIJONIUM HYPEREINE STRUCTURE INTERVAL TELTA NII: DART II

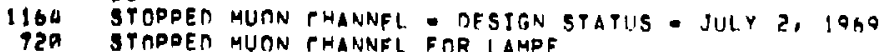

7500
750.0

Tuints

jilea

bogut

कh⿻1日)

Tyดog?

7T:

$750+20$

$750.2 \mathrm{a}$

bQAम? 
HUGHES, V.W. (CONTO.)

1963 SUMHARY OF STATUS DF DESTGN OF STOPPED MUON CHAVNEL

561 TESTS OF QUANTUM ELECTROOYNAMICS ANR PROPERTIES OF THE MUON

25G! THE STOPPED MIION CHANNEL AT LAMPF

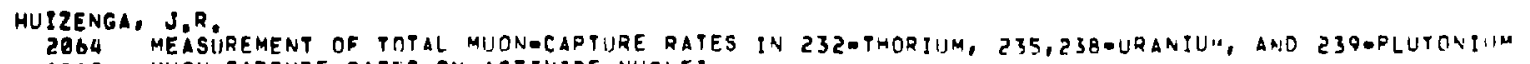

990 MUON CAPTURE RATES ON ACTINIDE NUCLE?

155 MUNN LIFETIME MEASUREMENTS IN THE ACTINIDE ELEMENTS 751.22

WUNGERFORD, E.V.
2BOY ANGULAR OISTRTAUTION FOR PO GOESTO 3ME DI ZERO REACTION AT TDEBOA MEV

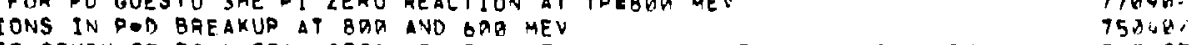

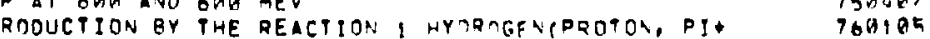

210 NUCLEAR - CDULOMB INTERFERENEE IN PI TOR. 16 OXYGEN SCATTERING

1981 PION PRODUCTION RY BAG MEV DROTONS FROY

221 PION PQODUCTION BY BAG MEV DROTONS FROM OOLITHIUH SCATTEQING

1980 PION PRODUCTION FROM THE REACTION D(P,O DI)N IT 585 MEV

19B2 PION PRODUCTION TN P-P INTERACTIONS AT OBO MEV

1096 PION PRODUCTION VIA A PQP GOESTO OOPI QUASI-FREE DROTONS IN DEUTERIUM

183 PION-NUCLEAR POTAL CROSS SECTIONS NEAR THE $3 / 2,3 / 2$ RESONANCE

1993 PION-NUCLEUS ORWARD SCATTERING AMPLITUDES NEAR THE 3 Z Z J Z RESONANCE

2933 PION-NUCLEUS

2974 PIONENUCLEUS TOTAL CROSS SECTIONS NEAR THE $3 / 2$ MT RESONANCE

223 QUASTOFRE SCATTERTNG IN PDO BREAKOUP REACTIONS AT BGB AND OAG MEV

2972 STUDY OF THE DEACTION OP GOESTO DELTACSUPER

$76018 \mathrm{~h}$

$7 \sin \theta$

$761+5$ i

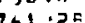

761.050
761.52

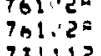

$331,1 ? 300$

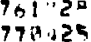

$779+25$

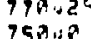

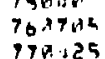

HUTCHEON, $190^{\circ}$
1645

HUTBON, R.L.

- METHOD FOR ACHIEVING CLINICALLY USEFUL PION DOSE OISTRIBUTIONS

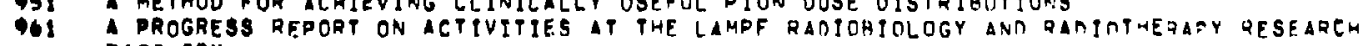

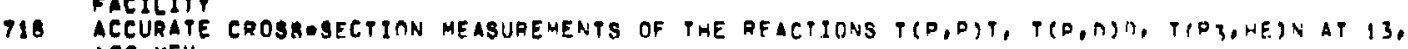
60月 MEV

1969 ANALYSTS OF MIIOMESIC X-RAY DATA FROM LIVER SAMPLES

397 GEAM SEPARATORSOSOME CONBIDERATIONS FOR LAMPF

1973 BEAM SPILL BETWEEN A=Z AND A-5 DUE TO NUCLEAR INTERACTIONS IN THE A-R TAHGE

1674 BEAM SPILL IN LINE A DUE TO NUCLEAR SCATTERING IN TARGETS

1983 BIOMEO CHANNEI PI- STOPPING DISTQPAUTION

1672 BIOMED LOW DENGITY TARGET

1139 BIOMEO BHIELO DDOR REQUIREMENTS

1985 BIOMED TUNING-SEOUFNCE OF TASKS

99. SIOMEDICAL JHTELOING REGUTREMENTS

683 CALCULATION OF RADTOFREQUENCY FIELDS IN TISJUE

$750: 00$.

71 a

$750 ? !$

Thancs

$75 \times 117$

730312

$70 \pi+15$

Tapiz

?5i?

748114

758011
7504 
MUTSON, R,L, CCONTO:S

917 CALCULATIONS FOR DOSIMETRY AND MICRODOSIMETRY OF NEGATIVE PIONS

CALCULATIONS OF TWO-DIMENSIONAL
ELECTRDOES IN CONDUCTING MEDIA

966 CAN NEGATIVE MUONS PROVIDE UNIDUE OR BETTER OIAGNOSTIE INFORMATION

1131 CHANGES TO BHIELD WALL CONFIGURATION FOR MAZE IN HIOMEO STUBOUT

960 CHARACTERISTITS OF ENERGY DEPOSITION BY PIONS

1225 DEPTH DOSE CAI CULATIONS

1 HOd EARLY RESULTS OF MIITMESIC XORAY EXPERIMENT

IA77 EXPERIMENT IOA. (IN VIVO CHEMICAL ANALYSIS WITH MUEMESIC X-RAYS), REVIEN TF WORK TO DATE.

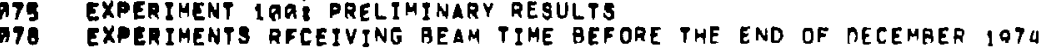

167

EEOMETR TCAL REOUIREMENTS FOR SMMPLES

GEOMETR ICAL RFOUIREMENTS FOR SAMPLES

IN REPORT ON TAMED CHANNEL

LA REPORT ON BIOMED CHANNEL
LETTER ABOUT SPECTRA DATA ANALYSIS

LEYTER AOOUT SPECTRA DATA ANALYSIS MU-MESIC XERAY MATERIALS ANALYSIS CENTER

MUDEESIC XERAY MATERIALS ANALYSIS CENTER

OBSERVAPION OF THE NUCLEAR RESONANCE EFFEC IN THE PIONIC IIT PALLAOIUIA ATMH

DERSONAEL SAFETY IN BDOMED TEEATMEN ROOM D 7 TRG

PLANS

PHYSICS FACILPTY

POYSICS TACTLYYY

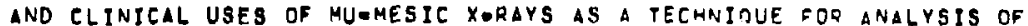

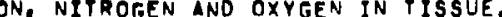

PROGRESS TOWARD USING MUGMESIC X-RAYS AS A DIAGNOSTIC TOOL

-ROJECTED MULTIPLE SCATTERING OISTRIBUTIONS

PROFONAPROTON ELASTIC SCATTERING FROM 9.6 TO 13.6 MEY

PROTONAPROTON SCATTERING AT $9.699,9,918$, AND 13.609 MEV

RADIATION THERAPY WITH NEGATIVE PI MESONS

RECENT EXPERIMENTS WITH MUOMESIC X-RAYS

RECENT PIF DEPTHODOBE DISTRIBUTION CALCULATIONS

SMALL SPOT TUNING OF BIOMEO CHANNE

SOME PROBLEMS OF TREATMENT PLANNING FOR PION BEAMS

SPATUS AND PLANS FE

PECHNIGUES FOR VISIIALIZING PION TREATMENT PORTS

TECHNIOUES FOR VISUALIZING PION TREATMENT PORTS AT LAMPE

PHE APPLICATION OF PROTONS TO COMPUTED TOMOGRAPHY

THE LAMPF BIOMEDICAL PION CHINNEL

THE USE OF MESONS FOR TISSUE CHEMICLL ANALYSIS

PISSUE CHEMICAL ANALYSIS WITH MUOMES IC XOPAYS

TUNING OF THE PIRST SECTION OF THE BIOMEDICAL CHANNEL AT LAMPF

TWO-DIMENSIONAL VISUALIZATION OF STOPPING DION DISTRIBUTIONS

XORAY DATA ANALYSIS

HWANG, CoF

1349 A MEASUREMENT TF PARITY VIOLATION IN PROTONEPROTON SCATTERING AT IS NEV

1345 A PROPOSEO A.

I5A2 LIHIT ON PARITY NONCONSEGVATION IN PENUCLEUS SCATTERING AT G GEVIC

2619 DOLARIZED TARGET CRYOSTAP TEST RESULTS

910707

730,75

$730730^{\circ}$

72952 ?

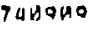

711119

750115

741.1 "

740827

7 taga.

740915

759зая

TaQj 5

701127

76 की

73 की 21
739540

75मर2व

73673 .

738100

71019

7ran:a

704105

74027
$71470 ?$

7uxpe

$73 \times 73$

Tรดमत०

75 मद

पiा

771.9

72452 ?

75411 ?

75113

Thbis.

759320 
HWANG, CAF, (CONTD.)

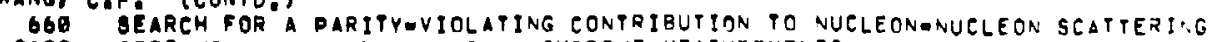

2622 SETIING OF MARNET FIELDS OY CURRENT MEASUREMENTS

222 SETTING OF MAFNET FIELDS BY CURREN MEASUREMENT

$160,6.3$.

2094 ELASTIC AND INELASTIC SCATTERING OF BOA MEV PROTONS ON 12,13-CARROA ANG ZBA-LEAD

2012 ELASTIC SCATTFRING ANALYZING POWER MEASUREMENTS AT BAD MEVU-I

2813 ELASTIC BCATTFRING ANALYZING POWER MEASUREMENTS AT BOQ MEY--II

2808 P $+13,12 C$. INELASTIC SCATTERING AT O.S GEV

770420

77490.

770009

IMAZATO, J.

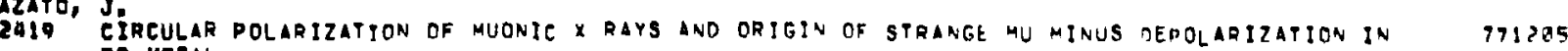
PO METAL

187 A PIOHUCLEUS RHEWMLOW EQUATION FOR FINITE NUCLEI

ISRAEL. H,I.

680 SHIELDING CONSIDERATIONS FOR THE DROPOSEO LOS ALAMOS MESON PHYSICS FACILPTY O5I!I5

687 THE RADIATION AND SHIELDING DESIGN FACTORS FOR THE LOS ALAMOS MESON DHYSTCS FACILITY OSI!!

IVERSEN, S.6.

2811 INELASTIE SCATTERING OF DI. LNO PI- FROM 18OXYGEN AT ILO ANO 230 MFV

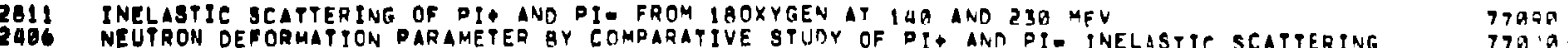

\section{JACKson, HeE}

1563 ABSOLUTE CROSA SECTIONS EOR PROOUCTION OF PROMPT NUCLEAR GAMMA RAYS HY FAST DIONS

1580 ALPHA AND MULTINUCLEON REMOVAL FROM SBONICKEL ANN GQENICKEL RY FAST DIONS

2416 ENERGETIC CHARGED PARTICLE YIELOS INOUCED AY PIONS ON COMPLEX NUCLE!

2561 PAST PION INDIICEO PROCESSES IN COMPLEX NIJCLEII CROSS SECTION ANO ALPMA RFMRVAL

Mast PION INDULCE PROCE

1585 PAST PION INDIIEED PROCESSES IN COMPLEX NUCLEI SYSTEMATICS IN PROOLICT QUTLFI

24I GAHMACRAY STUAY OF PIONEINDUCED REACTIONS ON

1993 INTERACTION OF FAST PIONS WITH G2, OUENICXEL

1562 GYSTEMATICS OF PION AND PROTON INTERACTIONS WITH NICKEL NUELIDES

JaCOS, N.P., JR.

206 ACTIVATION STIIDIES OF PIONOJNDUEED REACTIONS ON $C, N$, $O, F$, LL, LNO

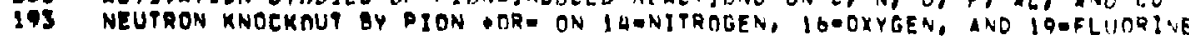

$75010 \times$

$75 \sin 2$

7794 A

750.10.

$75 \times 40$

75 Aind

int.i.

7 thi 50

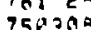

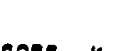

G22 Kinferaction of a gunemeo beam with tranguerse modes in pF cavities 


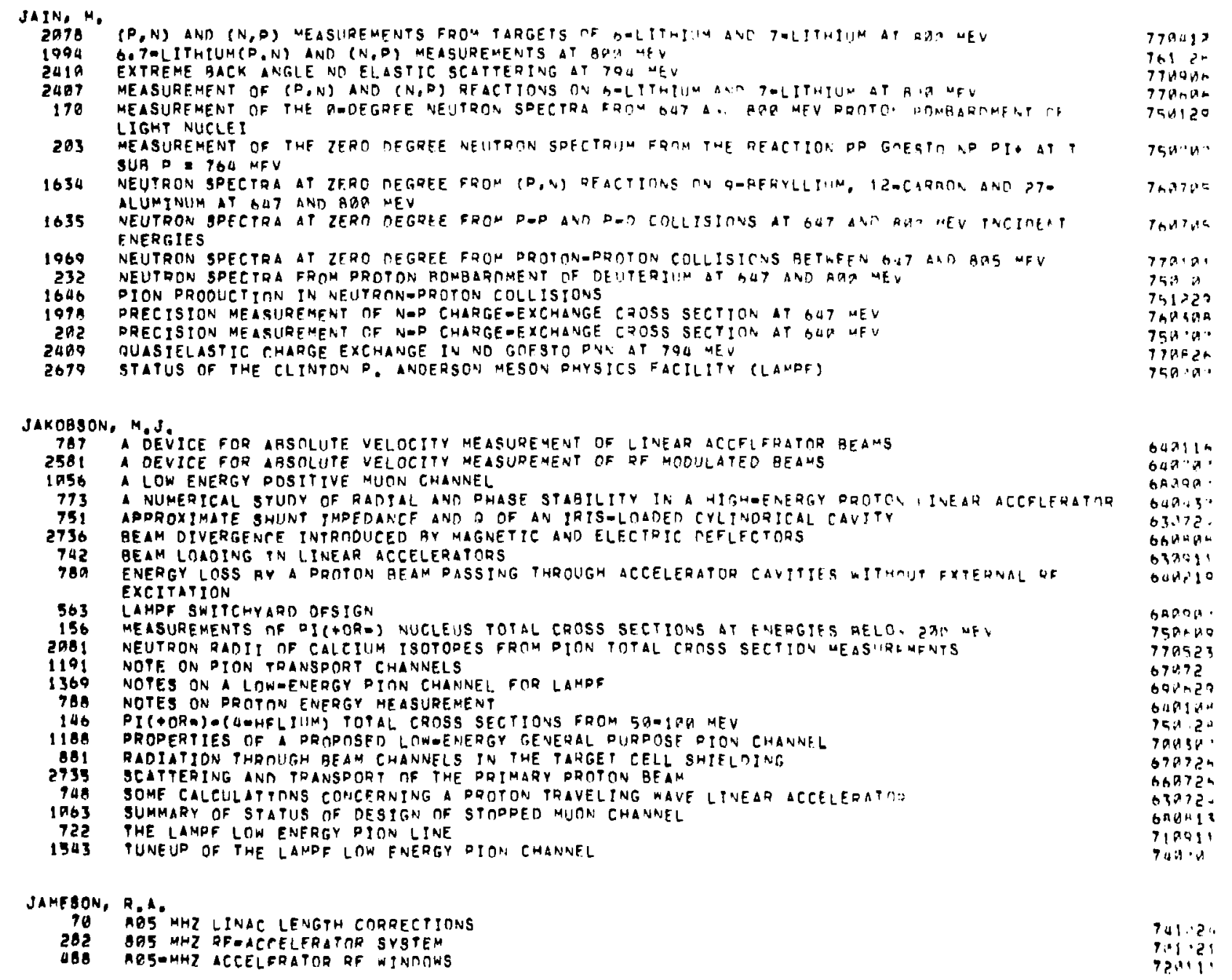


JAMESON, R.A. CCONTD.,

TIGNMENT

Bas-MHZ LINAC MODULE-TO-MODULE PHASING

2350 O05-MHZ PHASE OETECTOR SUBSYSTEM NO. 3, P. O. LHO-R6325 AND 2A1.25-MHZ DHESE OETECTOR

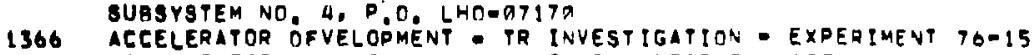

71 ACCELERATOR OFVELOPMENT THROUGH DECEMBER 29,1974

74 ACEELERATOR DFVELOPMENT JULY-NOV 1974

477 ACCELERATOR OFVELOPHENT SCHEOULE, $721113-7303$ O2
270 ACCELERATOR FIELO MEASUREMENTS AT HIGH PONER

2131 ACCELERATOR INSTRUMENTATION

2133 ACCELERATOR MODELING AT LAMPF

234 ACCELERATOR SYSTEMS DEVELOPMENT . LASL DEVELOPMENT ANR INFORMATTOA DERTPYENT TO CONTRACT ANALG ELECrR

AISPUTEI PURCHASE OROER LHO-14R5U-

PES OP, O, LHก-148S4

ACCELERATOQ QF SYSTEM ANO APPLICATY

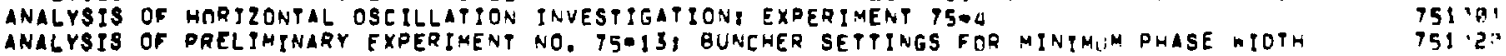
BUNEH

ANALYSIS OF PRELIMINARY EXPERIMENT VO $75-12$, TI-T A IOQ MEY SETTIVGS

AUTOMATIC CONTROL OF RF AMPLIFIER SYSTEMS

BABIC CONTROL SYSTEM THEORY

DEAD PULL MEASUREMENT TETHNIDUES AT HIGH DOWEO

BEAM OEVELOPMFNT WORK FOR HIGHOINTENS ITY

BEAM POSITION MONITOR EXPERIMENT ANALYSIS (EXF, :0, 76-3)

BEAM POSITION MONITORS

BEAM POSITION MONITOR EXPERIMENT, PROPOSAL $75=3$

BEAM DOSTTION MONITOR SYSTEM

BEAM POSITION MONITORS - 761195

AERKELEY PULSFDWWIRE ALIGNMENT METHOD

BRIEFING NOTES - MPOQ PROGRAM

AUNCHER OPERATION

CALIBRATION OF ANALOG OATA GHANNELS - JAMESON

CALIARATIONB

CAVITY TUNING FOR THE LAMPF BO5-MAZ LINAC

CHANGES IN DEI TANT ANSWERS WITH RA5 MHZ LINAC PESONANEE - EXPERIMENT 7500

CONBIDERATIONA ON PERSTNNEL IISE DURING PROPOSED ACCELERATOR SHITTHOW

OATA ACQUISITTON - SWYO USE FOR LINAC TUNSNG

OELTAET

OELTAET AND SOLIREE ROOM HAROWARE

DELTAOT TESTS - EXOERTMENT TSEI6

DELTIOT VS PEAK CURAENT - EXPERIMEAT NO, T6-17 - SUMMARY OE RESULTS TO DATE

OESIGN OF THE RF PHASE AND AMPLITUDE CONTROL BYSTEM FOQ A PROTON LINEAT ACCELLEATOE

DEVELOPMENT OF ANALYTICAL ANO NUMERICAL STUDIES

DYNAMIC MEASIJREMENT OF STOPBANO IN LAMPF BPS-MHZ ACCELEQATOR STRUICTULES ST WTGM ERWEO

USING HYBRID COMPUTER TECHNIOUES

I: ABSEMTIA

2132 EDITEO NOTES RROM CORRESPOHDENCE AND CONVERSATION MITH K. CRANALLL

2130 ELECTRONUCLEAR RAEFDER IRO PAEPARATION

EMITTANCE HEABUAING MARDWARE

7395मR

39411

$7605 \mathrm{~km}$

743020

$74 \sin ^{3}+1^{2}$

721131

770207

$77031 \mathrm{a}$
70.52

729714

1405

751.2

68129.

63393.

76 An 21

75091 ?

75 तो

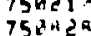

$75 a^{2}$
75105

761135

740 र?

73053

7 1)

720 in?

758547

7211 .

70012 :

$73 x^{3} \cdot 1$

720?"

Tha日en

$70112=$

7 ollth

Tarus.

T594.4.

751 ith

710 en.

774520

798195

7702 ? 
JAMESON, R.A. (CONTO,)

770 ENVELOPE RESPONBE OF A CHIIN OF COUPLED CIRCUITS

430 EXPERIHENTAL ICHEDULE THQU APRIL 1973

1364 FACILITY EOUIPMENT AVAILABILITY

ACILITY EDUIPMENT GVIILBILITY GRTGHT LINAC BEAMS

FAST LUTOMATIC PHASE AND AMPLITUDE CONTROL OF HIGHEPOHER RF 5 YSTEMS

FEEDOACK FROM SAFETY INSDECTIONS

FEEDFORWARD CONTROL OF ACCELERATOR RF FIELOS

- IRBT SUCCESSFUL DELTACT) RUNS

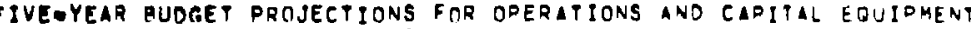

FOREIGN TRAVEI REPQRT (JAMESON)

PULL POWER OPFRATION OF THE LAMDF RO5 MHZ SYSTEM

GENERAL GRAPHTCAL PROGRAMS

GROUP ACTIVITTES

HIGH PULSE CUARENT TUNING - EXPERIMENT $96-16$

HORIZONTAL OSTILLATION STUOIES ANO MAROABPM OISCREDANEIES - EKPERIMEAT THEO

IMPERFECT DUANRUPOLE ANALYSIS

INJECTOR AND TSA KEV TRANSPORT PROCEDURES

INTERLACED PRAGRAMS W PROGRAM 734 REVISION

LAMPE COT ZSGHAZ LINAC FIELO DISTRIBUTION

LAMPF ACCELERATES THO

LAMPF PROTON I INAE PERFORMANCE

LAMP RF PHASF

LOSS MONITOR AND WIRE SCANNER INSTRUMEVTATION

MACHINE OEVEL TPMENT EXPERIMENTS - EXPERIMENT INDEX CONTINUEO

MACHINE DEVELTPMENT EXPERIMENTS

MAINTENANCE BACKGAOUND DATA BASE

MANAGEMENT INFOQMATION FOR BAMEF

MANAGEMENT INFORMATION FOR LAMPE

MANPOHER ESTIMATES FOR MEOICAL LINAC PROPOSAL

MEABURED DYNAMIC PERFORMANCE OF I.25 MW, MQT MHZ KLYSTRONS

MICROWAVE APPARATUS FOR BEADEPULL MEASUREMENTS

MICROWAVE INSTRUMENTATION FOR ACCELERATOR RF SYSTEMS

MP-O CONTRIBITTONS TO MAINTENANCE AND DEVELOPMENT SCHEDULE, FERRILOY 2R, 107

MP-9 INPUT TO DEYELOPMENT CYCLE, STIRTING FEB, 2 OR 311976

MPQO SUPPDRT ACTIVITIES

MP.DIVISION OATABASES

NOTE ON 4-ARM BRIDEE PHASE OETECTORS

NOTES FOR MEOTCAL LINAC PROPOSAL

NOPES ON PHASF AND AMPLITUDE PERMISSIVE SYSTFM

OPERATION/MAINTENANCE RECORDS DISCUSSION, SEPT, 11-12, 1972

OPERATIONG/MATNTENANCE DATA-RASE

OPTIMUM GENERATOR CHARACTERISTICS OF RF AMPLIFIERS FOR HEAVILY BFAMELOAOEO ACCELERATIRS

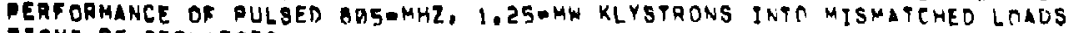

DIGMT RF ISOLATORS

PON GeneaATOR FoR HEOICAL APDLICMTIONg

HEDICAL APPLICATIONG

PREPARITION FOR HIGHOINTENSIPY EXPERIMENT.

640,25

730321

760511

$679 \cos$.

Ool?a

73 करा?

?

726317

731196

Tanne

परमवट

$7 \times 1: 14$

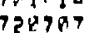

7מA34:

75 aी?

7 halle:

$75012=$

759425

$72079 ?$

7 Bम1日:

739697

Taitia

Thay

Tha?l?

73023

7ง

? 3 aरa

$75 x+a 1$

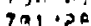

TI:

$603 n$ a

$36 x+15$
5

7 कराइ०

70 गुद 7

Poncitas

bi112.

75 pinte?

$60(x+5)$

TวसO:

73 a 323

G9Bसम

PaOA

$763^{3} 20$

15 and

75931 ? 
JAMESON, R,A. CCONTO,

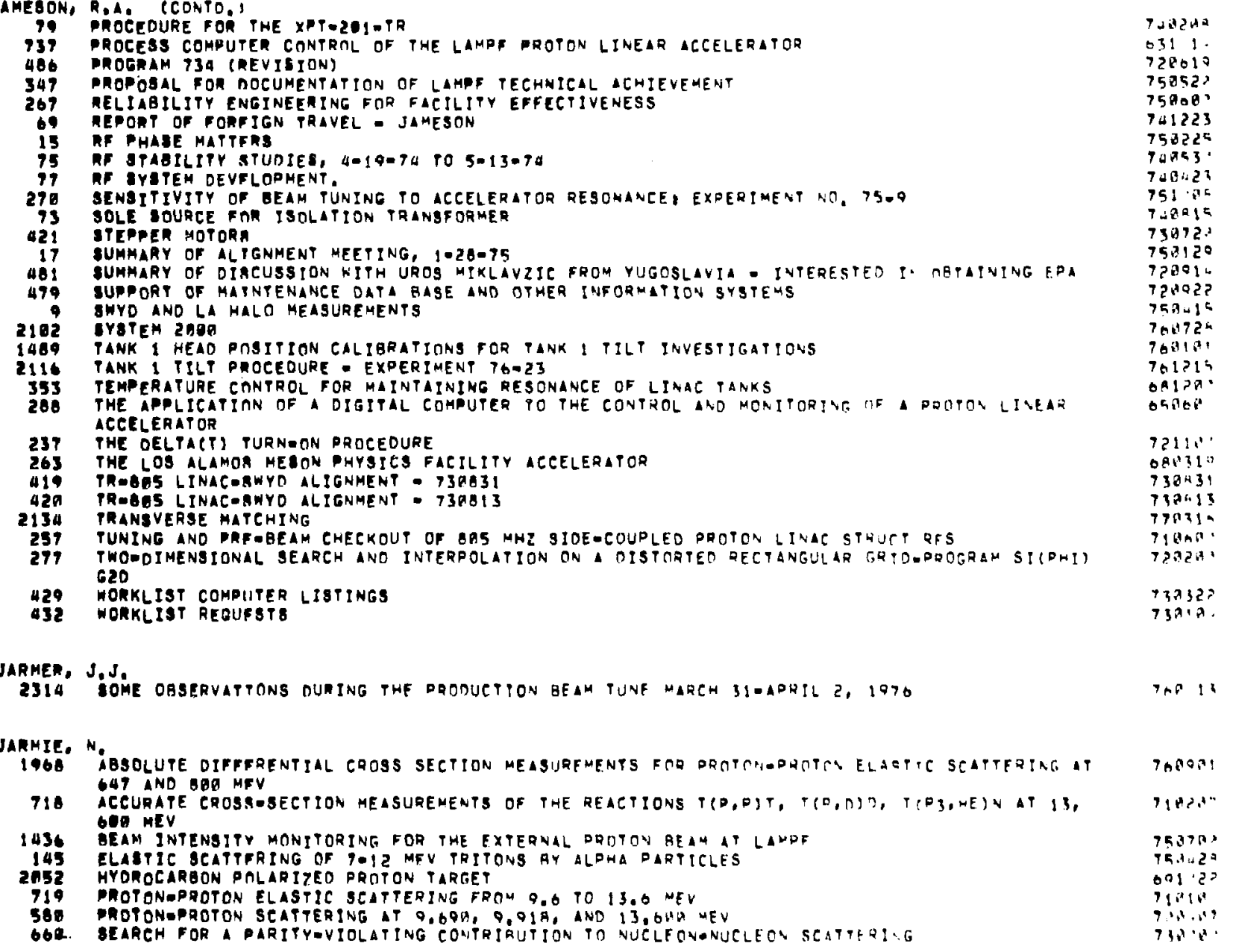

JENKINE, 0.1. 
JENKINS, D.A. CCONTO.

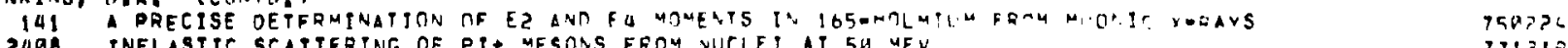

2UQS INELASTIT SCATTERING OF PI + MESONS FFOM VUCLE! AT $5 \mathrm{H}$ "EV

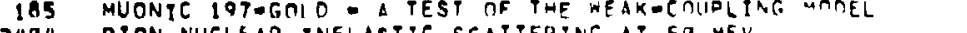

2484 PIONANUCLEAR TNELASTIC SCATTFRING AT SR UEV

JEPPESEN, R, H.

2585 COMPUTING IN SUPPORT OF EXPEATUEYTS AT LAMPE

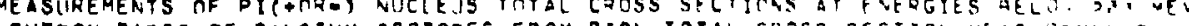

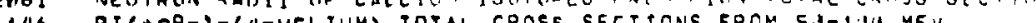


JOHNSON, H.

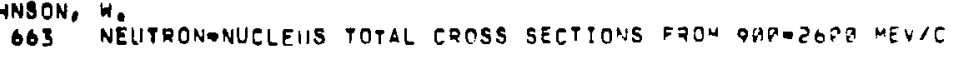

711115

JOHNSTON, M.O.

1 MANAGEMENT INFORMATION FOR LAMDF

80 OPERATIONSGMATTENACF DATA BASE MONTHLY REPORTS.

$3+x^{2}+\cdots$

$7 a^{3}:$

JONE8: O.F. EAST TOTAL TNF TRMATION REANOUT GYSTEY FOR MULTIMIRF DROPORTIOVAL CHAWEAS

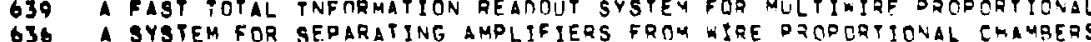

656 USING MULTIWIRE PRDPORTIONAL CHAMAER DECOOERS

656 USING MULTIWIRE PROPORTIONAL CHAMF

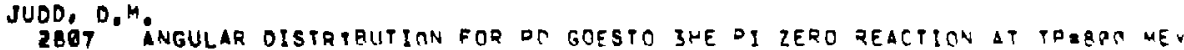

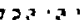

72110

JULE, $W, E$

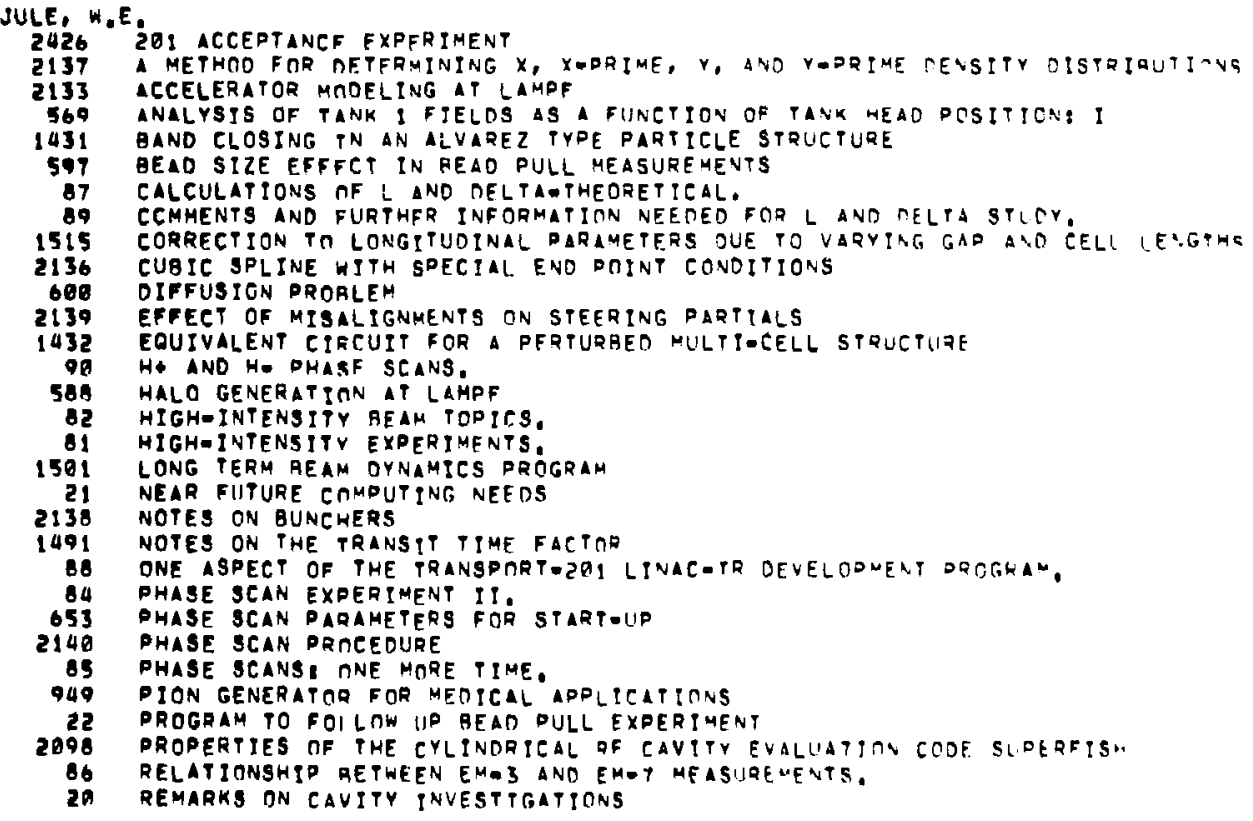

REMARKS ON CAVITY INVESTTGATIONS

$79+48$

$720,+58$

कel:1,

$79231 \mathrm{a}$

7anaj:

720015

34029

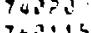

$7 n+115$
$7 n+1420$

$7 m+1420$
$75172=$

778 in

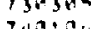

741.2
9

7.

$3+1110$

$365: 3$,

ini.

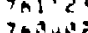

toivas

740531

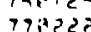

$7 \tan ^{2} 3$

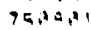

Tandis.

Thisis

Twa

$79.4 \times 1$ 


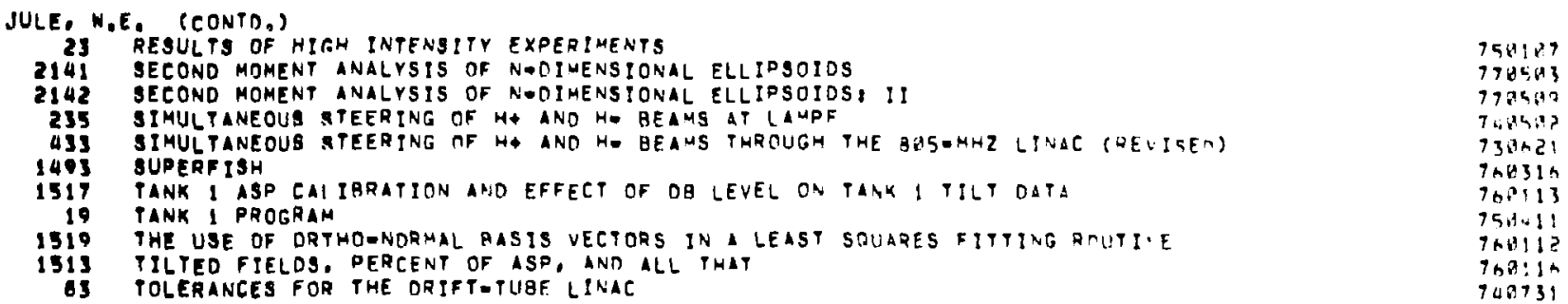

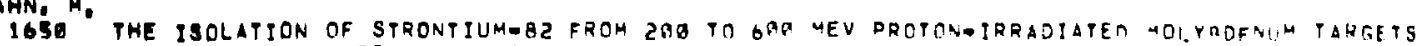
FOR BIOMEDICAI APPLICATIONS

$(5,4 \cdot 3)$

KALBACH, C.

155 MUON LIFETIME MEASUREMENTS IN THE AETINIDE ELEMENTS

$75 \%+2 \%$

KALLNE, S,

2025 SCINTILLATORS AND PHOTOMULTIPLIERS - SOME TESTS FOR EPICS TUNE-UP

1992 STUDY OF THE (PIF,N) AND (PI,PI PRIME P) REACTIONS IN LIGHT NUCLE

10,150

953 CELL SURVIVAL AS A FUNETION OF DEPTH FOR NEGATIVE PiONS ANR HEAVY IONS T5ATE.

KANEARIAN, R,

342 CAYITY PUNING FOR THE LAMPF 805.MHZ LINAC
2001 BHOEK WAVES IN LAMPF BEAM LINES

KAROL, M.J.

102 "MREPRATION OF POWDERED TARGETS

KABPAR, M,Y

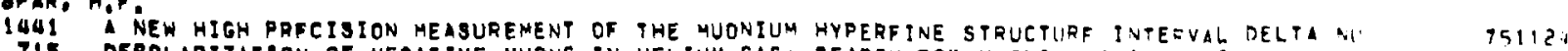

715 DEPOLARIZATION OF NEGATIVE MUONS IN HELIUM GASI SEARCN FOR MUONIC HELI:IM APON TUALI?

101 MUONIUM FORMATION IN NOBLE GASES AND NOBLE GAS MIXTURES

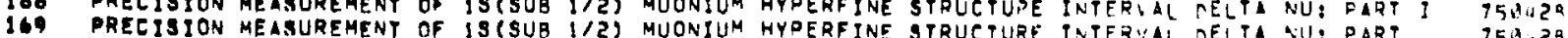

$753.2^{\mathrm{a}}$

KAUFMAN, $3, \theta$.

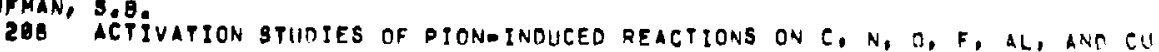


KAUFMAN, SOB COONTD I

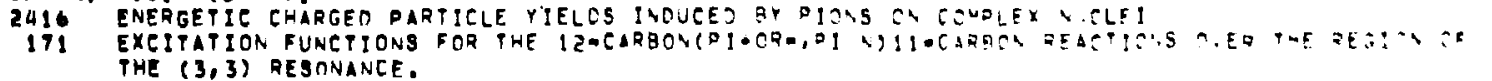

THE $(3,3)$ RESONANCE.

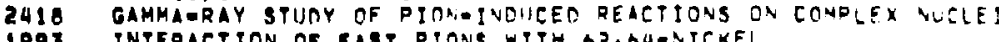

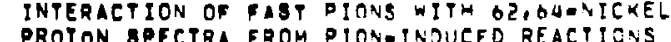

1199 REACTIONS OF FABT PIONS ITH IOT-GOLO

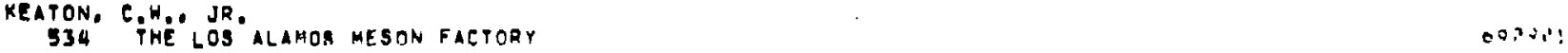

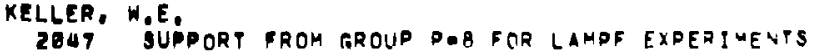

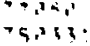

$+1,+35$

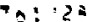

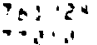

12,42

XELLOG, M,P.

2096 OATAGACOUISITION SOFTWARE

$\therefore i: i$

KELLY: L.M. SPEIFICATIONS FOA 291.25 MHZ DRIFT TIAE LINAC RF PELLEOS

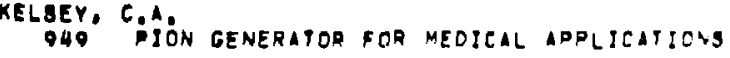

$\therefore ;$

$+6, \cdots$

KENEFICK, $R, A$.

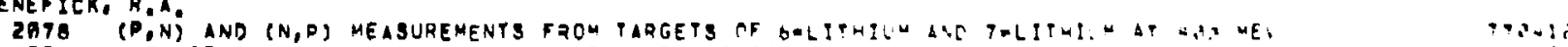

1994 6.70LITHIUM(D,N) AND (N,D) MEASUREMENTS AT BCO UEV

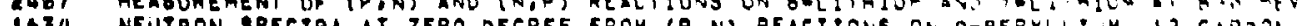

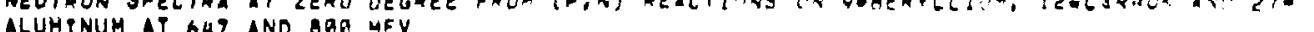

$3+\cdots$

inis

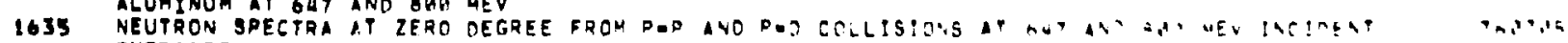

1970 PRECISION HEASUREMENT OF NOP EHARGE OEXCHANGE CROSS SECTIST AT AW WE

Dain

KERCHER, 0.0

159 BOS MHZ CONYROL BYSTEMS RACKS

32 BOS MHZ RACK AB MZ SPRID PARTS LIS

151005 MHZ RACK OUTLET

239 GOS MHZ RACK TERMINAL REOUIREMENTS

1032 BO5-MHZ MOOULF GROUND BAQ INSTALLATION

1004 DUPLEX INBTALI ATION IN MG5OMHZ MOOULES

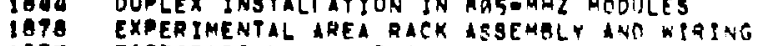

1037 FAGRTCATION SPECIFICATION FOR MUX TO TERMINAL STHEP CAHLE.

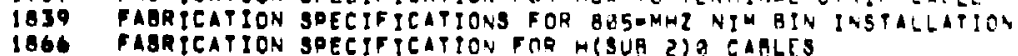


KERCHER, D.D. (CONTO,)

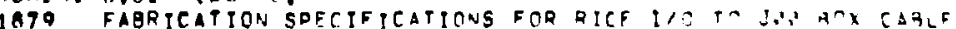

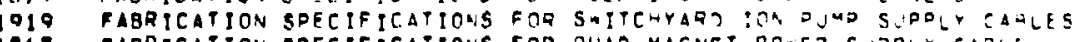

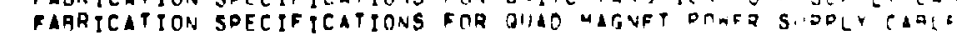

FABRICATION SPECTE PCATTONS FOR JU:AO UAGNET JUDER CARLF

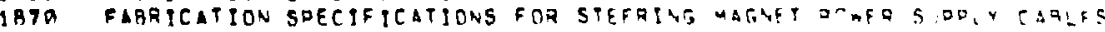

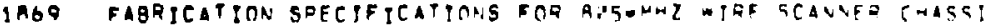

IA35 FABRPCATION SPECS FRR B.5OMHZ RACK W MAEDING

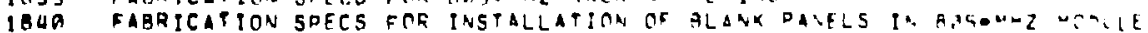

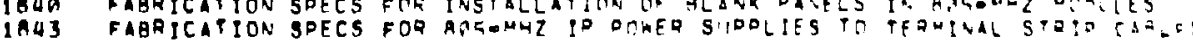

1A3B FAARICATION SPECS FOP FAST PROTECT TI TERUIVAL STPIF

1FU1 FARRICATICN SPECS FOP MASTER TIMIVG TO TERUIVAL STOIO

161 MODULE WATER SYSTEN STMLILATIO MONULE RACX B2

284 NIM REQUIREMENTS FOR THE RA5 HHZ SECTITY

1322 RICE I/O MUX FABLE DARTS LIST

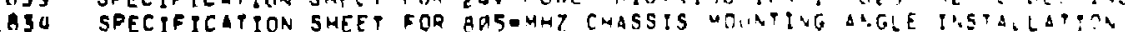

IOQU VACUIM CHASSIS TESTER CHECKOUT PROCETIRE

KERR, E, C.

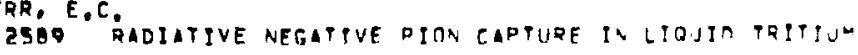

Pי⿻上丨

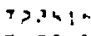

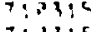

$\therefore \cdots$

?an

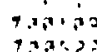

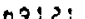

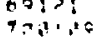

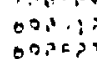

$\log 3$.

001210

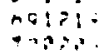

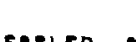

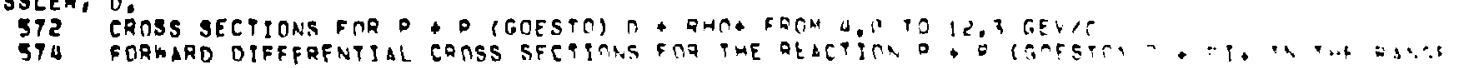

$\rightarrow,+, \cdots$

Y, , ?

3.4 TO 12,3 GFV/C

3.4 10 12.3 GFV

(n)

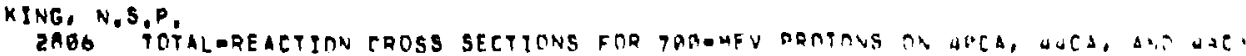

KING, T. A,

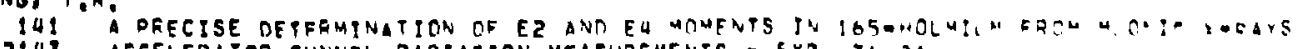

?143 ACCELERATOR TIINNEL RADTATITN MEASUREMEVTS - EXO THE?

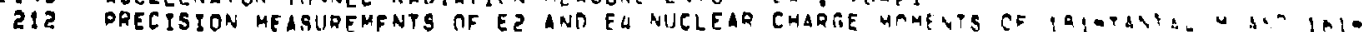
OYSPADSIUM

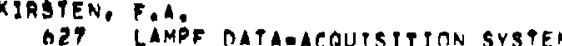

KISBLI INGER, LSS

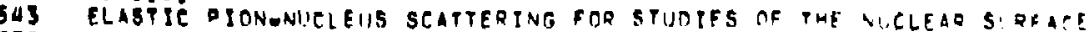

553 NUCLEAR WAVE FIINCTIONS FROM PARTICLE TRAVGFEO MATA

KLARE, K.A 


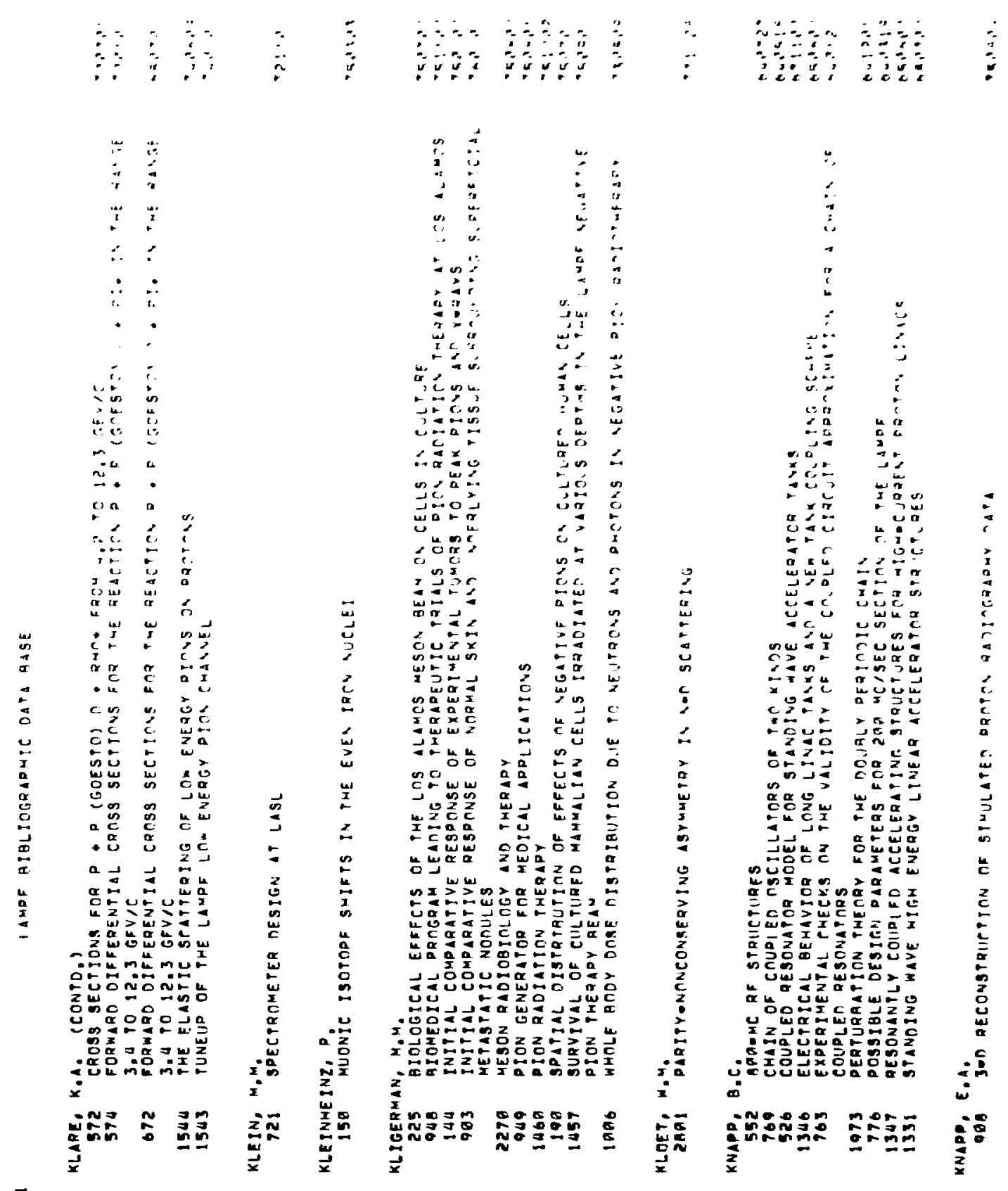


KNAPP, E.A. (CONTO,)

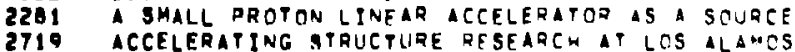

ACCELERATING STRUCTHAE RESEARCH TT LOS ALAMCS

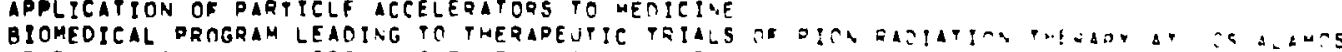

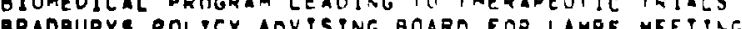

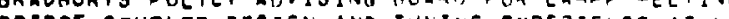

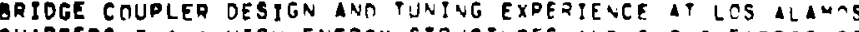

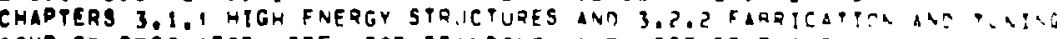

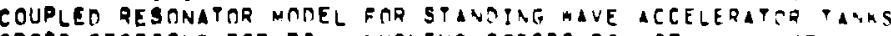

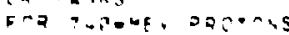

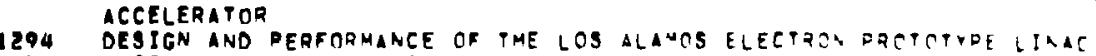

655 DESIGN OF HIGH INTENSITY HIGH EFFICIENEY LINEAR ACCELEQATORS

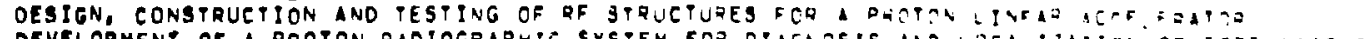

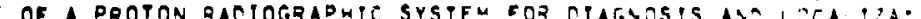
ABNORMALITIES

1986 DOSE RATES

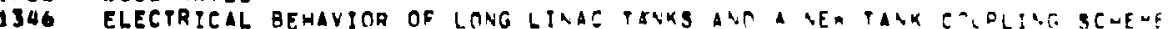

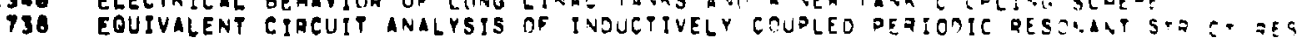

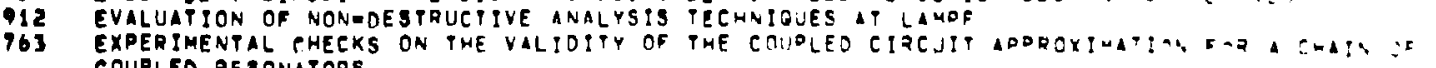
COUPLED RE3ONATORS

1209 PISCAL YEAR toba CONSTRUCTION OBLIGATIONS

1292 FOCLISING MAGNETS FOR SECONDARY BEAM LIVES

1292 FOCUSING MAGNFTS FOR SECONDARY BEAM LINES

HIGH ENERGY STRUETURES

HIGH POWER TERT ON A C

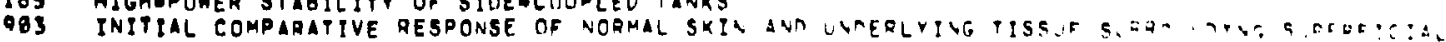
METASTATIC NORULES

1972 LAMPF ELECTRON PROTDTYOE ACCELERATOR - P.OTVISION TALK 101RAGA

909 LIGHT ION LINACS FOR MEDICAL AOPLICATIONS

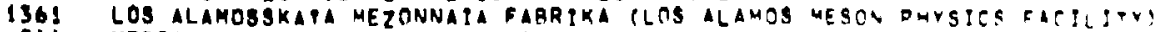

911 MEDICAL LINAC DESITN DOSBTGILITIES

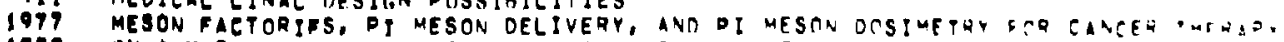

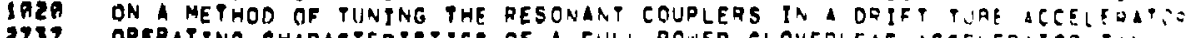

2737 OPERATING CHARACTERISTICS OF A FULL POWER CLOVERLEAF ACGFLEGATAR iA."

1974 ODIIHIZED CAVITY SHAPES FOR SIOE-COUPLED ACCELERATRRS

764 PARAMETERS FOR ELECTRON REAM EXPERTMENT

1073 PERTURGATION THEORY FOR THE DOJGLY PERIODIC CHAIN

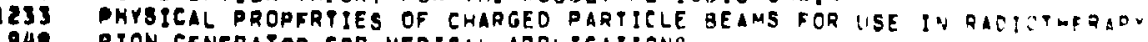

Q49 PION GENERATOR OMR MEOICAL APPLICATIONS

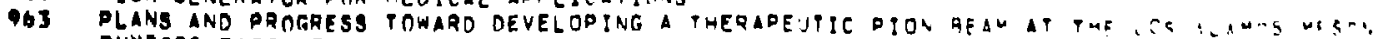
PHYSICS FACILITY

1362 POSSIBLE PION SOURCES FOR RADIMTHERAPY

1232 PRELIMINARY DFVELOPMENT OF THE AL PFANATIVG PWASE EOCLSER LINAC ST: G. EE

$\sin +2$

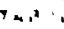

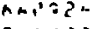

a : : :

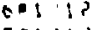

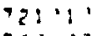

+1: $\cdots$

?⿻:1:?

$06: 3 !$

QAish

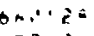

taing:

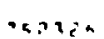

Bas, a.,

D3! $\because$

(a)

$0,12: 2$

ext:

"ד:

in

, an.

ini is

na. ? :

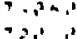

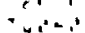

-11.20

mona

and : ?

די

t.: 271

1. 16.1 :

, 11,19

Aniz:

and 


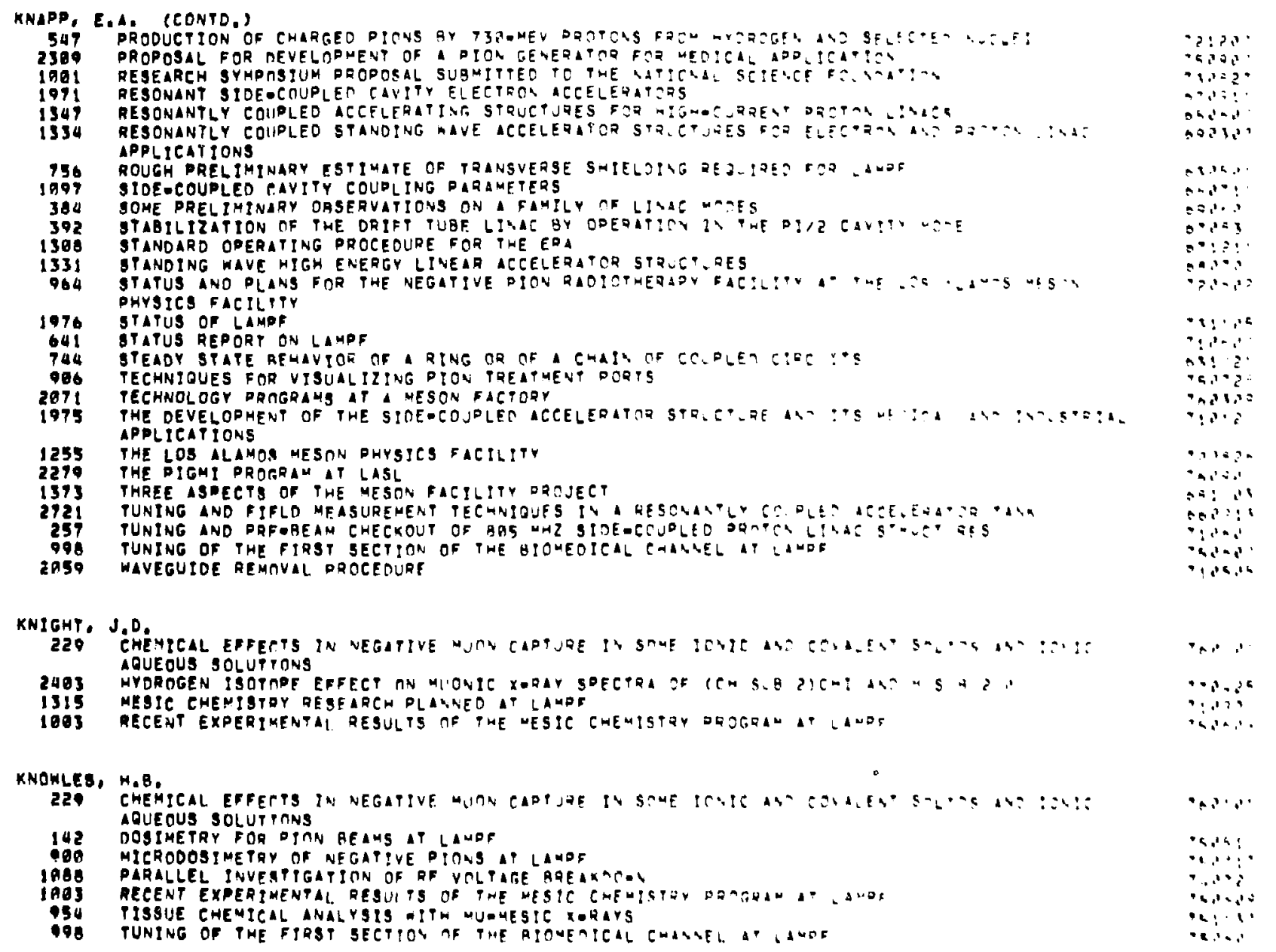

KNUTSON, L.D. 
KNUTSON, L.O. BEONTO.,

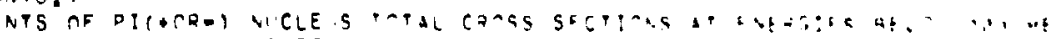

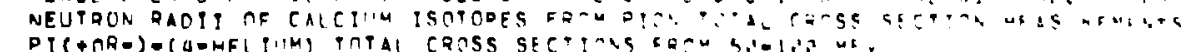

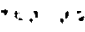

$\because \cdots i$

in, ia

KOCH, J.H.

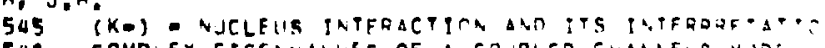

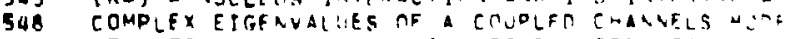

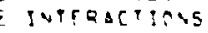

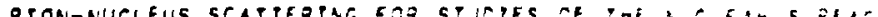

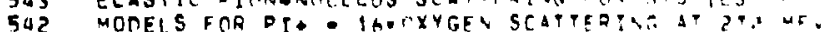

KOCZAN, S,P.

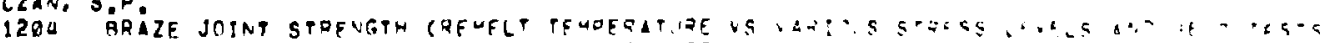

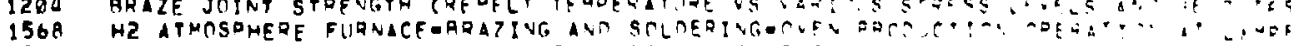

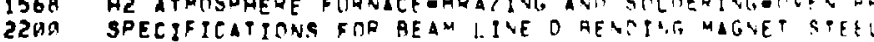

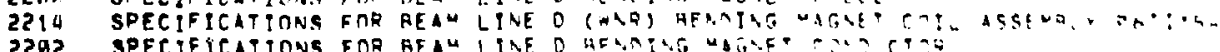

22 ดि

KOESTER, $K$

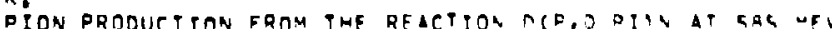

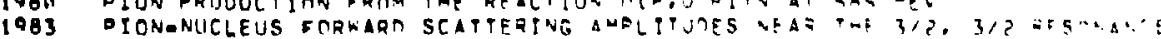

, : :

$\because:$

$\because \cdots$

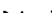

$\because \cdots$

$\because \cdots$

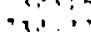

$\therefore$,

$\therefore ;$

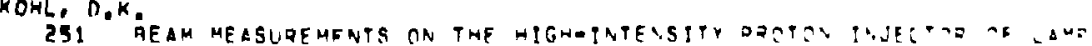

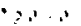

KastollLAs, I

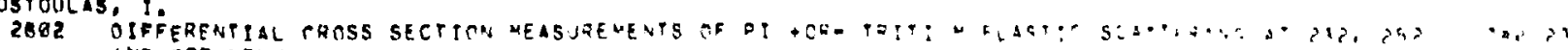

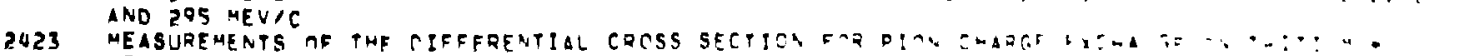

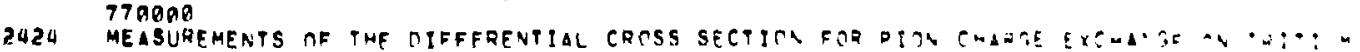
T61:3

2003

MEASUEEENTS

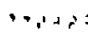

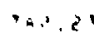

$\because \cdot$

KOVAR, 0.6.

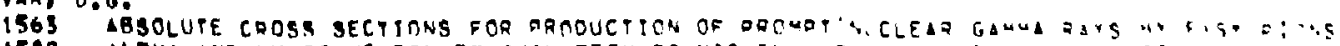

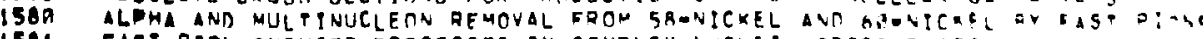

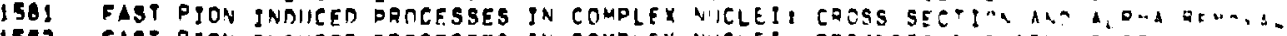

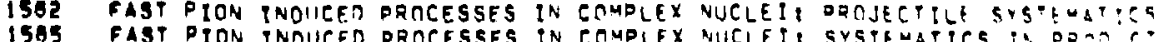

PIONDTNOCED REQCTIONS OV COMPLFX

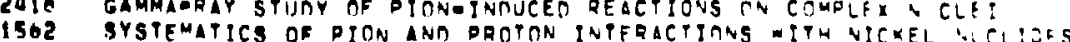

KOZIOL, H.

2a, in

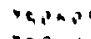

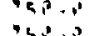

(is

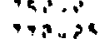

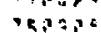




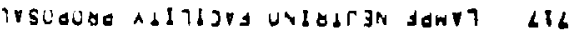

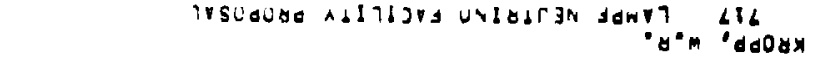

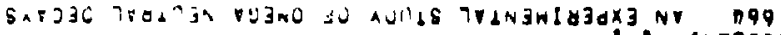

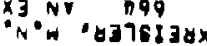

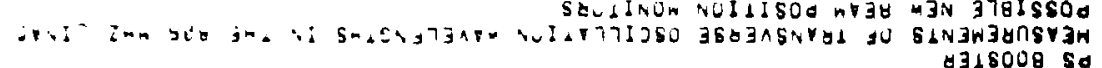

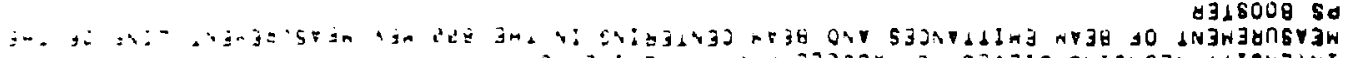
Stijn:

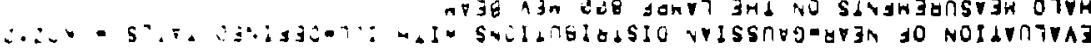
$\therefore i r$ $\therefore$

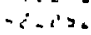

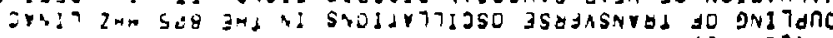




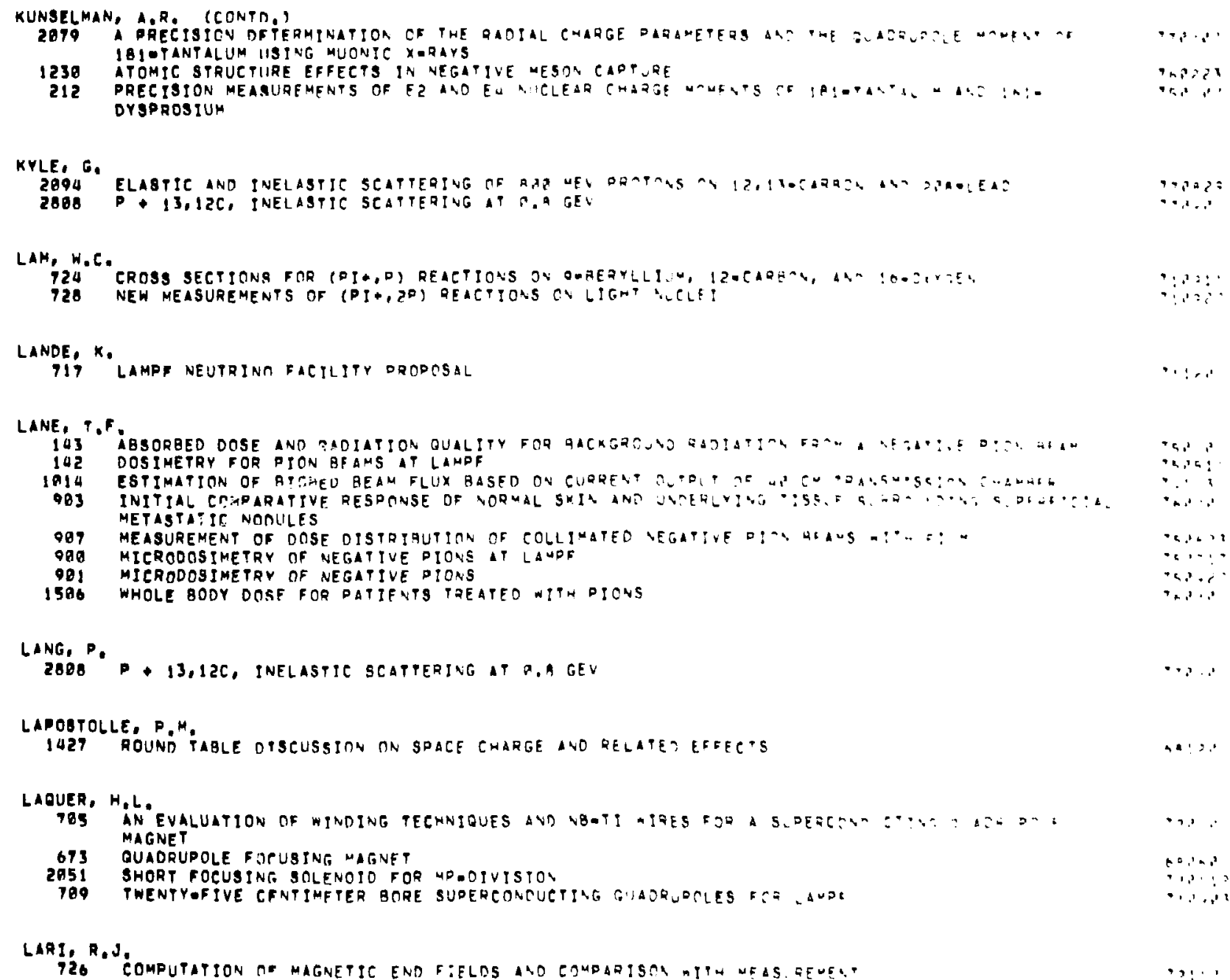


LAROTONDA, A.R.

LAMPE SWTTCHYARD

1165 MINIM JEXTUPOIE STRENGTH LOJUSTING DEJG2A

$30: \vdots:$

LARSON, D,A.

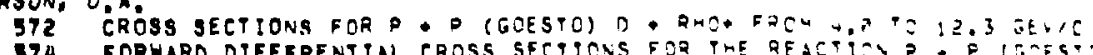

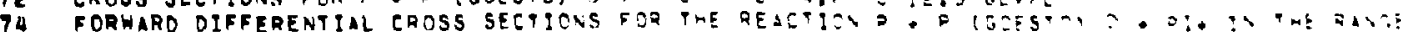
3.4 TO 12.3 GFVIC

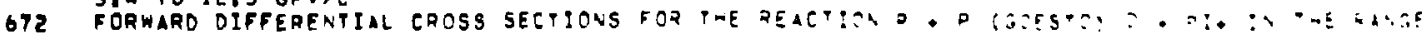
3.4 TO 12,3 GFV

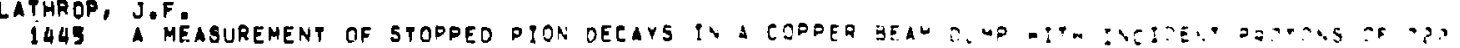
MEV (A CALIBRATION OF THE GAMF NEUTRIVO SOURGE IVEASITY

"A P :

2412 THE APPLICATINA OF PROTOMS TO COMPLTED IOMCGQAOAY

$\because:$

LAW, A.G.

2560 LINAC BEAH CORE MDOELING FROM WIPE-SEANTER CAT

2140 THE DECEMBE A-19. 1976 GLUEKSTERA VIS!T

an:

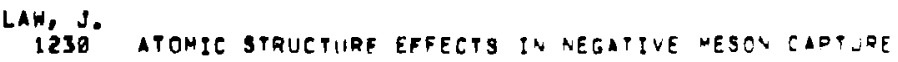

$\operatorname{na:2}$

LAWRENCE, G.P.

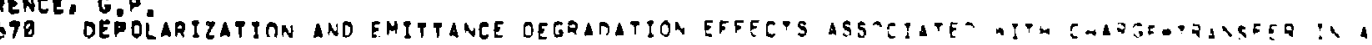

aง.?

JOINT H. ION SOURCE DEVELOQHENT DROGRAM

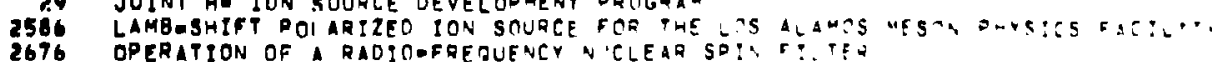

$\cos : 3$,

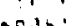

\section{LEAVITT, C.P.}

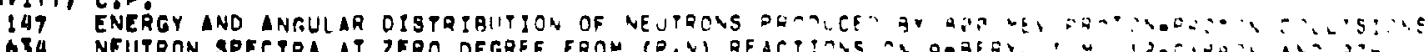

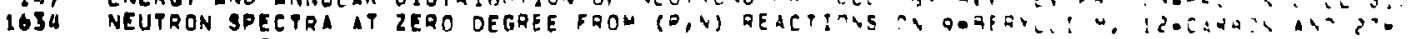

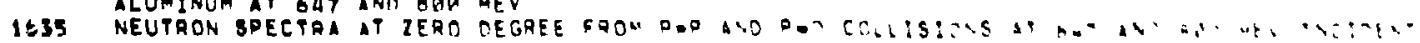

ENERGIES

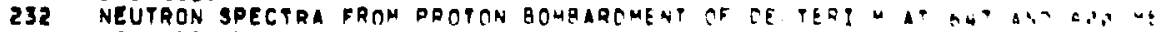

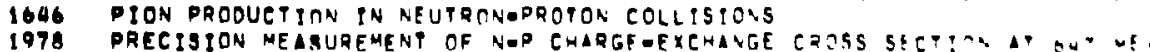

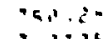

, a. ?

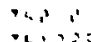

9,11

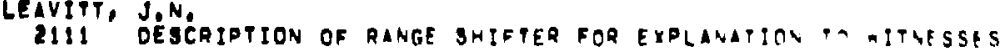


LEAVITT, J.N, CCONTD,

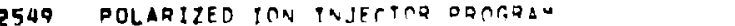

LEDERER, $\mathrm{H}_{2}, \mathrm{H}^{\circ}$.

528 ARE MODULATOR SIATIIS RFPMRT - AOPT?

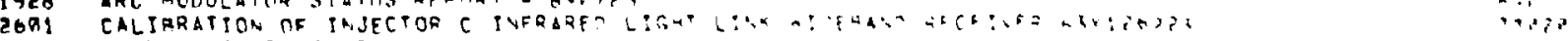

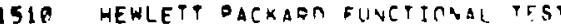

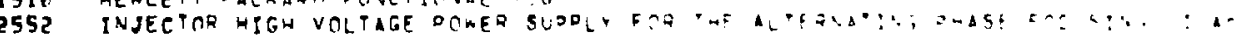

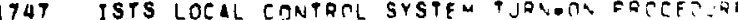

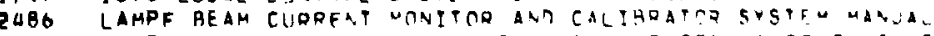

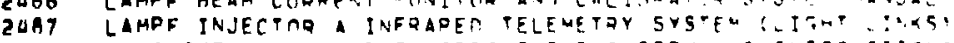

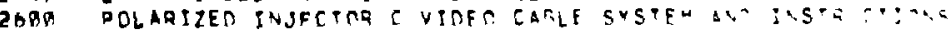

1512 PRELIMINARY TFST MEASURE UENTS

1673 SPECIFICATTON FOR DIPANITTYPE VACU: GAUGE

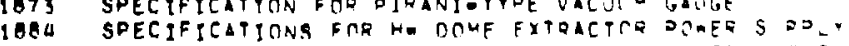

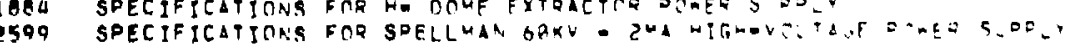

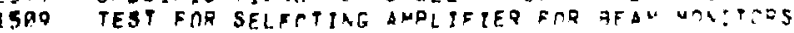

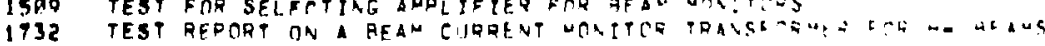

$15 ! 1$ TRYGON POWER SUPQLY TEST

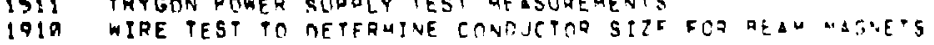

LEE, D.M,

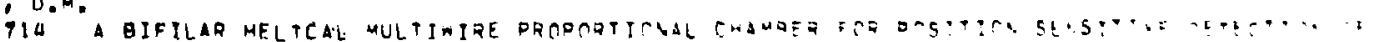

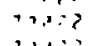

$\log 3$

$0,0,1,0$

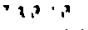

$+1,1-2$,

in:

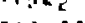

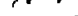

in?

$\because \because \vdots$ :

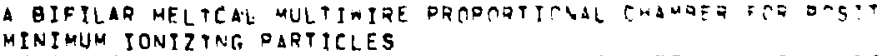

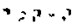

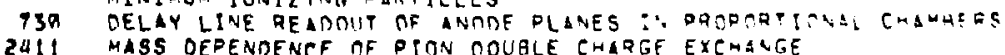

1578 OBSERYATION OE THE PION DOUBLE CHARGF EXEMAVGE

2U22 PION DOURLE-CHARGE EXCHANGE ON IG-OXYGEY AMG IR-OXYGEY

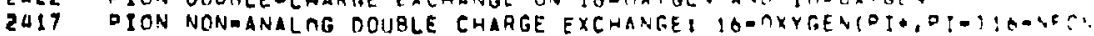

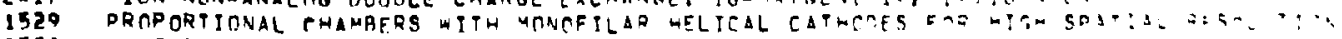

2539 REVISET CYCLE O PLAN

LEE, L.Y.

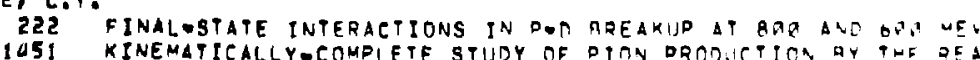

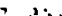

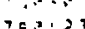

$\log 20$

$\therefore$,

$\rightarrow,+:$

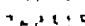
PROTONINEUTAOA AT LAMG ST

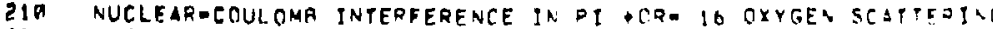

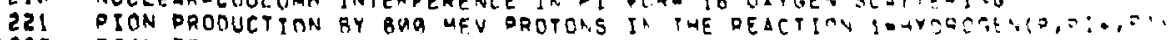

1980 PION PRODUCTION FROM THE REACTION $n(0$,$) DIIN AT 585$ LEV

1982 PION PRODUCTION IN DMP INTERACTIONS AT AMR MEV

IA3 PION-NUCLEL O TOTAL CROSS SECTIONS AEAR TUE 3/2, W/2 OESTNACE

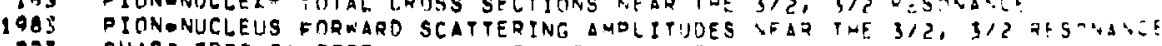

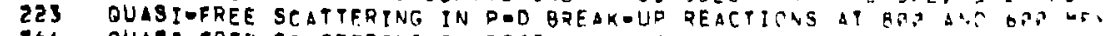

T66 DUASIOFREE SEATTERING IN PRDTONEINRICED DE TERCV GPEAKL A. 5A5 AN

2 UAR TUNEOUP OF THF LAHEF MIGHEENFRGY DTOU. CMANEL 
LEE, Y.K. (CONTD,)

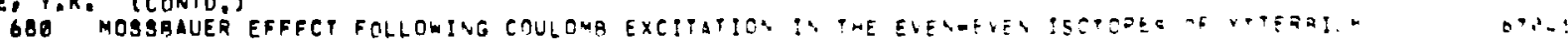

LEON, M.

$1107^{M} 189$ CON RIBUTION

9B1 ATOMIC CIPTURF OF NEGATIVE MESONS, THE FUZZY FEATETFLLEQ UESE

QBO ATOMIC CAPTURF OF NEGATIVE MUONE

999 ATOMIC CAPTURF OF NEGATIVE MESONS - 931020

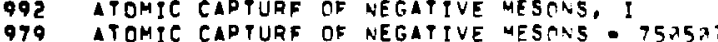

1230 ATOMIC STRLCTLIRE EFFECTS IN NEGATIVE MESON CADT JAE

1109 CASCADE CODES

Q97 OEPOLARIZATION OF NEGATIVE MUONS IN L IOUIO MELIJN

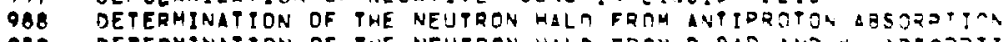

QBQ OETERMINATION OF THE NEUTRON HALO FRCU D ALR ANO CO ABSORPTIS

1101 DIPOLE TERM OPBCAROEO FFOM MESONONUCLEUS IMTERACTES

175 E2 NUCLEAR RESDNANCE EFFECT IN PIOYIC ATOMLS

180 HAORONIC ATOMS AND TICKLISH NUCLEI

987 KO ABSORPTION ANO THE NEUTRON HALO OF LEAD

1108 LETTER CONCERNING MESON CAPTURE YIELOS

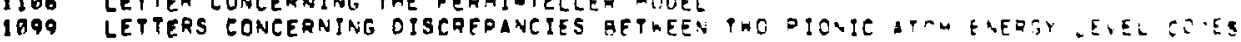

199

NUCLEAA MOTION CORPECTION TO EXOTIC

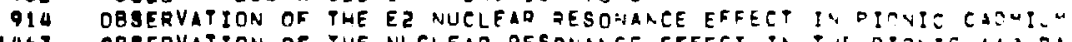

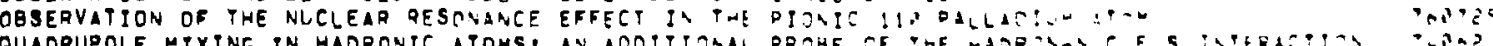

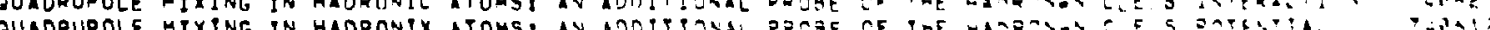

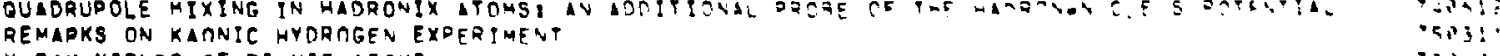

(3.,

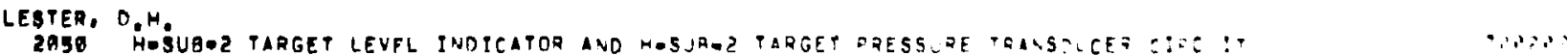

LEWIS, H.R.

GO1 COMPUTATION OF ELECTROSTATIC AND RAPIOLY PLLSEM .ACGET:C E!ELGE

LEYDIG, R,

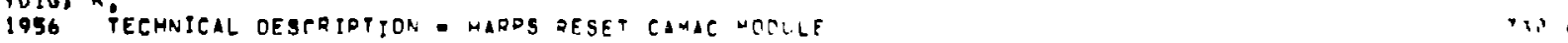

LILJESTRAND, R.

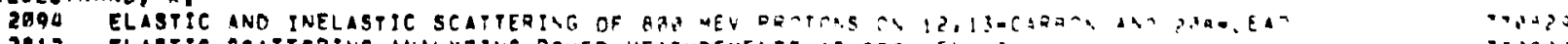

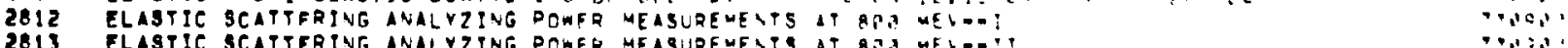

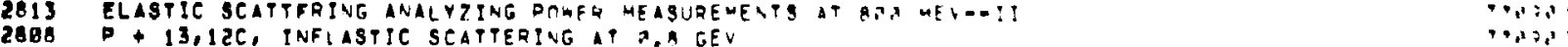

LINO, V.G. 
LIND, Y.G. (CONTD.)

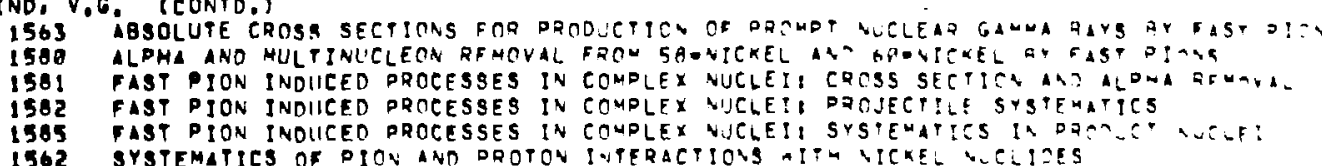

and

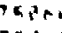

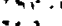

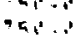

LINDOUTST, L.O.

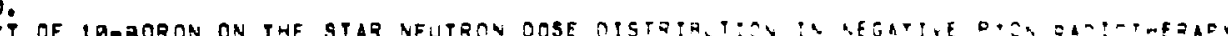

1 GQ8 EFPC

1348 LUW MOMENTUM PHOSPHORS, EXPERIMENT 75015

1223 OHOSPHOR STUPY - EXPERIMENT 76-1'1 (REF $75-15)$

2621 PIO THICK TARGET EXPERIMENT

2III RADIATIONORESTSTANT PHOSPHORS FOR AMA-YEV PROTOV gEAUS

1342 REQUEST FOR BFAM TIME, PROPOSAL 75-15

2001 SHOCK HAVES IN LAMPF BEAM LINES

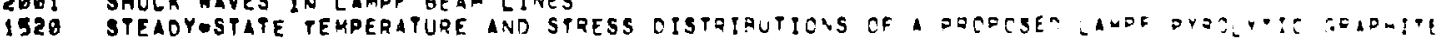
PION PRODUCTION TARGET

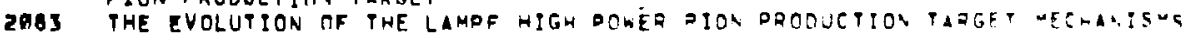

tis

iation

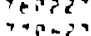

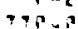

$7 x^{2}:=$

This

$\because \cdots \cdots$

799
LN EXPERIMENTAL STUOY ON OMASE AND AMPLITUDE CONTROL ADOLIEATIMIS E

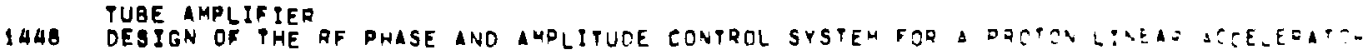

$02,1,0$

35,2,

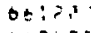

$\cos +3$,

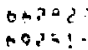

G78 LAMPF RF PHASF ANO AMPLITUOE CONTROL SYSTE REVISEOI

278 NOTES ON PMASF AND AHPLITUOE PERMISSIVE SYSTEM
2676 OPEAATION OF A RAOIO-FREQUENCY NUCLEAR SPIN FILTER

LISKA, 0.J.

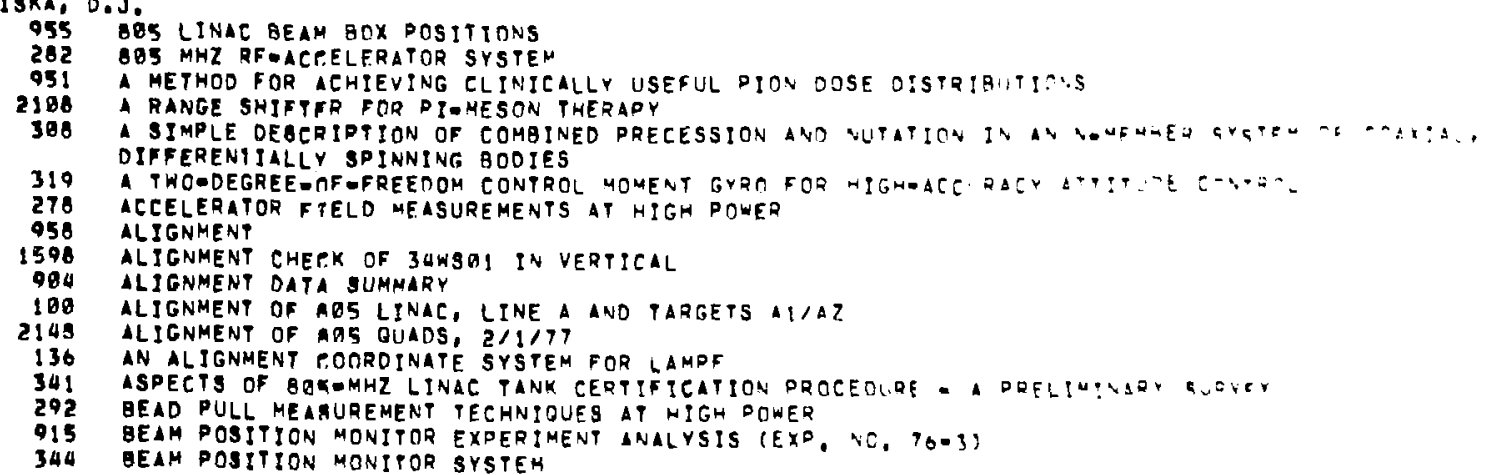

12,38

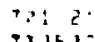

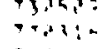

,

a $9: 2$ :

7 .

inision

acis?

\begin{tabular}{ll}
7 \\
\hdashline
\end{tabular}

$\rightarrow+1$

ine

$\because$

icas: 
LI8KA, 0.3. (CONTO,)

2114
443 BEAM POSITION MONITOPS - 761195
394

297 BEAM SEPARATOR PARAMETER RANGE FOAOPITS

369 BEAM SEPARATORS-BOME CONSIDERATIONS FOR LAMPE

GIOMEO PION ENERGY CONTROL RCIJATOES

2106 BPM GYSTEM OUALIFICATION - LATE SEPTE MGER SHUTSOM

CALIBRATION OF RANGE SHIFTER DURING DATIENT CYCLES

1549 CALIERATION OF THE 2AI BEAM POSITION MOMITCRS E EXPERIUENT TOEO

CALIBRATION OF THE BEAMESPILL CONTROL SYSTEM AT LAMPF

34 CAVITY TUNING FOR THE LAMPF BASAMHZ LIVAC

492 CONSTRUCTION INO TEST OF EPICS DARTICLE SEPAAATOR ELECTRONICS

2109 COUCH CONTROL SYSTEM OESIGN

314 CROBSED FIELO ELECTRON MOTION

DESCRIPTION OF EPICS PARTICLE SEPARATON

437 DESCRIPTION OF HYORAULIC SYSTEM REDUIRE MENTS

2111 DESCRIPTION OF RANGE SHIFTEA FOR EXPLANATION TO N!TNESSES

2110 DESCRIDTION OF THE SERVDOCONTROLLEO COLLIHATOR CONCEPY

ILT OESIGN REVIEW OF RANGE SHIFTER ANO SERVOCOLLIMITOR CORCEPTS

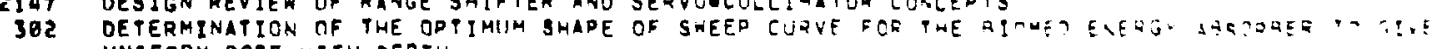
UNIFORM DOSE WITH DEPTH

2146 DEVELODMENT HORK ACCOMPLISHEO ON MAY 13, 1070

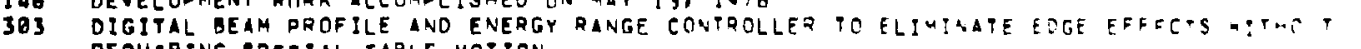
RE QUIRING BDETIAL TABLE NOTION

313 EDDY CURGENT SHIFLDING OF MAGNETIC FLUX IN ELECTROMAGVET CBRES ANO MIF

317 ELECTRIC FIELFi HEASUREMENTS TN KLYSTRON CAVITITS

1599 ELECTRONIC SYITEM REQUIREMENTS FOR THE EPICS BEAM SEPARATCO

2105 EPICS BEAM BEPARATOR (DROGRESS REPORT - 769731)

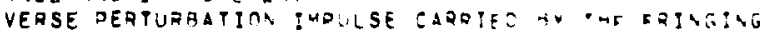

MIELO OF THE FDICS PARTICLE SEPARATO

FEAS

3 FEASIBTLITY STUDY OF HIGH DOWER BEAD DULL ON MCOULE 5

985 FINAL ALIGNMENT CURVES ON $201-$ LINAC

102 FINAL ALIGNGENT DATA FOR BRSEMHZ LINAC

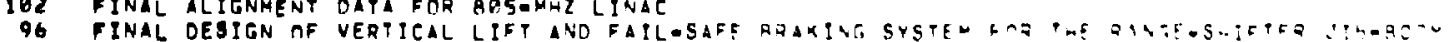

10

181

444

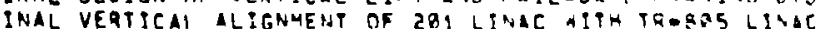

PULL OOWER OPFRATION OF THE LAMPF RQS YHZ SYSTEM

H- STRIPQER FAILURES UNDEQ HIGM DOHER PULSED CONCITIONS

HEAT EXCHANGE IND DOWER DISSTPATION IN THE BA5-UMZ MUVE DTE

HIGH VOLTAGE INSULATORS FOR EPICS PARTICLE SEPARATCH

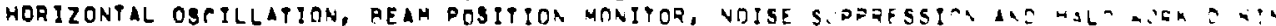

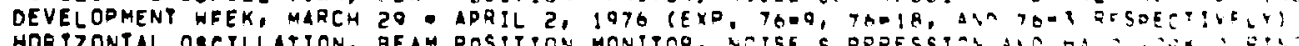

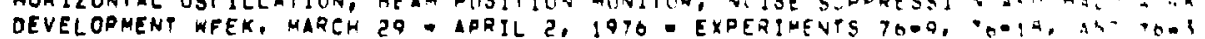

RESPECTIVELY

1400
2103

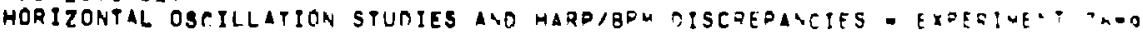

HYORLULTC BETUOARM (DROGRESS REPORT - T6R731)

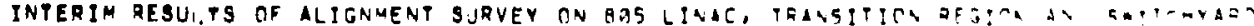

Th: : is

thent?

Tings

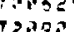

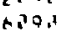

10,952

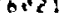

$75,3+s^{3}$

$72 ! \cdot 0$

$07 \mathrm{An}^{*}$

105010
75025

acsia

The.

72,551

(n)

anies

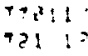

inica'

7al 2

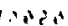

$7 x^{2}$

ratis

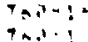

? ? 12 ?

4.?:

5.! $2=$

$\because 1 !$

acous -

and

-130

?a!?

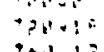

and =

9. : :

and

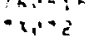


LISKA, U.J. (CONTD.)

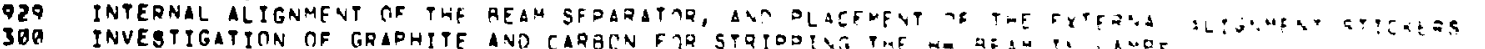

LAGER-CUT SLITS AND SURGHOLES IN GRADHITE

LOSS MONITOR ANO WIRE SLANNER INSTQU.NEVTATIOS

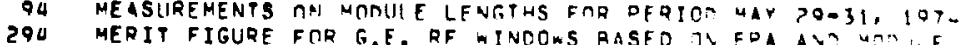

25 MOST RECFNT AI IGUMENT MEASUREMENTS OS MI LOCAC

435 MOUNTING OF THE QANGE SHIFTER QELATTVE TO WO

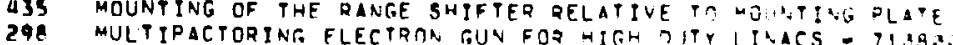

396 MULTIPACTORINT FLECTRON GUN FOQ HICH OJPY LIUACS a TPI"

TAL2 NOTES ON SINGIE PADTICLE THERQY IN CROSSEJ-FIEL MFVICS

2187 PERFORMANCE OF THE LAMDF PARTICLF SEFAQATCA

2077 DI-MESON RANGF SHIFTER FOR CLINICAL THFZAPY

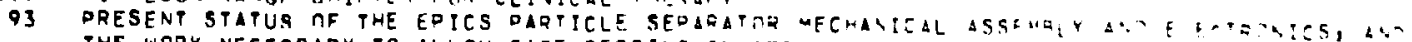
THE WORK NECESSARY TO ALIOW SAFE TESTIYG IS AREA A.

215R PROPOSAL FOR REDACKAGING QANGE SMIFTER ELECTRODICS

295 DUALITATIVF ANALYSTS SUMMAFY OF G.F QF H MDCH FAIL DES

445 OUESTIONS REGIRDTNG GRAPMITE FRIL SAUDLFS WITH PIOY WLLS

1524 RANGE SHIFTER STATUS ANT COLLIMATOR DROOTSAL

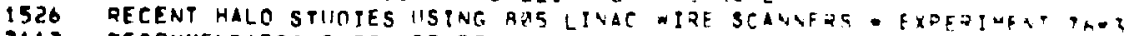

2112 RECONMENDATIONS FOR 3TARTING INSTALLATIOY OF ROM SYSTEW

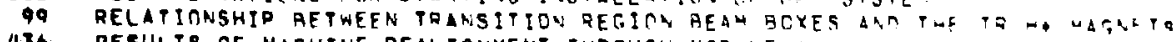

RESULTS OF MACHTNE REALTGNMENT TMRCURAH MODILF IU

SPROGRESS REPTION RANGF SHIF

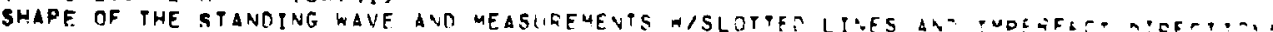

COUPLERS

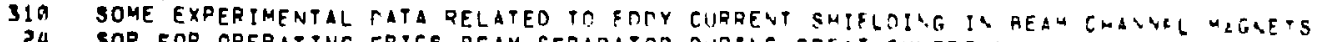

24 SOP FOR OPERATINF, EPICS AEAM SEPARATCQ DURINT, GREAT SHITACA

2363 SPECIFICATIONS FOR MOLLO TUBULAR CERAMIC INSILATORS

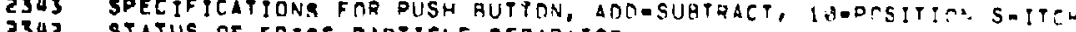

2342 STATUS OF EPITS PARTICLE SFPARATIRR

$152 !$ STATIIS OF FAST BPM SYSTEM

17 SUMALRY OF ALTGNMENT MEETING, $1028-75$

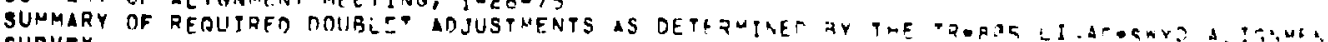

SURVEY

02

SUPTARY OF TANK

984 TANK 30,1974

TEST DO DECHNTAE QLJGNMENT aFTEQ OAISING T

493 THE EPICS HIGH VOLTAGE LINF

134 THE LAMPF COORDIVATE SYSTEM

THE PAPTICLE SEDATATOR AT LOS ALAMCS

257 THINGS TO WATCH POR IN OFIZER CARBON STRIPOER FOILS NITH TIWY HCIES

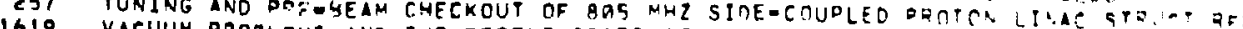

98 VENTICALOBLEHS. NNO THE RECENT TFSTS OF THE FPICS SEPARATCO

97 VERTICAL ALIGNAFHT OF FRONT END OF MACHINE

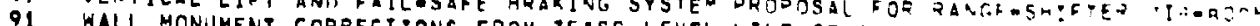

WALL MONUMENT CORRFCIIONS FROM ZEISS LEVEL LINEOAF-SIGHT IS Firi=LINA

पaर?

na, 0 ? :

LITTLE, J.O. 
LITTLE. S.D, (CONTO,

2440 120MHZ/201-HH7 TIMING SYSTEM

11 DOS MHZ TRUNK-LINE REOUIREMENTS

1280 ANALOG COMMANA INTERFACE CIRCUITRY

IA1S ANALOG DATA ATOUISITION SYSTEM REVIEN ANO PROPOSAL

770416

601125

INARY CDHMAND ANO ISSOC IATED BINARY INDICATION INTEREACES

688701

CURRENT SHITCHYARD INSTRUMENTATION AND CONTROLS STATUS

EPICS TARGET MECHANISH

1411 EXPERIMENTAL AND SWITCHYARD $H 2$ A SYSTEMS DESCRIPTION

$15 \Delta 0$ EXPERIMENTAL AREA MAGNET THERMAL ANO FLOW INOICITION WIRING

EXPERIMENTAL AREA HAGNET THERHAL AND FLOW

HRS ANO TARGET CONTROLS TO CAMAC PQOPOSAL

INOUIRYA-SPECYFICATIONS FOR FOCUS MAGNET PQWER SUPPLIES

PNQUTRY-ABPECIF ICATIONS FOR STEER ING MAGNE T POWER SUPPLY

NGTRUMENTATION OF THE EPA YICUUM SYSTEM SADDENDUM NO, I

ISTS ANALOG DATA SYSTEM FAILURE

LOW-LEVEL ANAI OG ACQUISITION SYSTEM SDECTFICATIONS

MISCELLANEOUS FABRICATION SPECIFICATIONS FOR COMPUTER COMPONENTS

PROPOSAL FDR HEATING ANO VENTILATINN GYSTEM FOR GEAM SWITCHYARD AND EXDEAIMENTAL AREA

RF PHASE DELAY INTERFACING BETWEEN RICE IJO, FAST PROTECT, AND TIMING SYGTEMS

RM-16 SYSTEM PROPOSED MODIFICATIONS

BPECIFICATION FOR HIGHABTAGILITY, LOWGCURRENT DC, PRINTEDACIRCUIT CARD-TYPE PUWER SUDPLIES

SPECIFICATION FOQ LAMPF ION PUMP POWER SUPPLIES

SPECIFICATION FOR LAMPF DATA TERMINAL POWER SUPPLY

SPECIFICATION POR LAMPF ION DUMP POWER SUPPLIES

SPECIFICATION FOR STEERING MAGNET POWER SUPPLY

GPEIFICATIONS FOR 24-V CONTROL POWER SUPDLIES - 719610

SPECIFICATIONA

SPECIFICATIONS TOR $240 V$

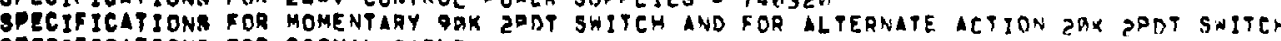

SPECIF ICATIONA FOR SIGNAL CABLE

OPECIFICATIONG FIR THE ANALOG DATA SYSTEM

BPELLMAN HV SIIPULY FOR SEPARATOR

MAGE AOJ BEAM PLUG CONTROLS DESCRIPTION

THIN TARGET EXPERIMENTAL CONTROLS DFSERIPTION

VIDEO AND ADS MEASUREMENTS

2449 YOUT REMOTE HLNOLING PROPOSAL

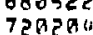

770323

779323
$7 \log 89$

$721>04$

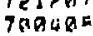

70 कीजि

$77922^{\circ}$

GRAQ2?

ba451h

b?

on! 1 and

opizita

671131

$\operatorname{Tin} \rightarrow \cos$

601402

IhaAz

OवसEदक

60001 ?

7 intulat

$001 \cdot 07$

710.54

$718 \mathrm{~A}$.

$121 \%$

74932

75938 ,

7241625

600327

7 on>11

73111 ?

731107

$710 \times 12$

7950

LIVINGITON H.

2689 HIGH INTENSITY MESON FACILITIES

Tan '9:"

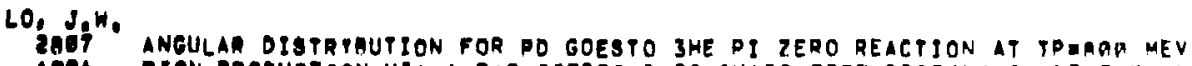

1996 DIOH PRODUCTION VIA PAP GOESTO OQPI OUASI-FREE PQOTONS IN DEUTERIUM

77000 .

$761 \cdot 2 a$

LOGAN, M.K. MONTE CARLO STUOTES OF PION PROUUCTION

$63691 \%$ 
LOMON, E.L.

2069 THE DEUTERON DESTATE AND THE PI+ D GOESTOP + REACTION 761.25

LOPEZ, T,A.

I3I TIEE POWER SUPPLY TEST GQRALR

Lovol, P.A.

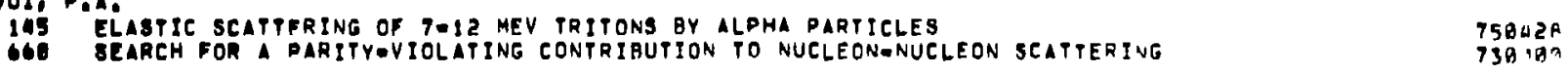

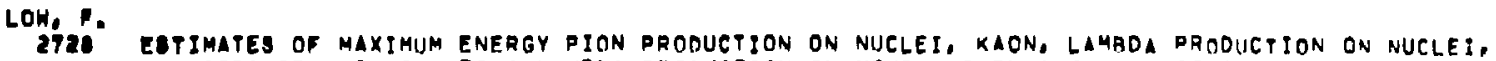
AND STRANGENESS VIOLATING LAMBDA PRODUCTION ON NEUTRONS AY GQB MEV PROTONS

GhOAN"

LUCAS, 6.3.

1346 ELECTRICAL BeHAVIOR OF LONG LINAC TANKS AND A NEW TANK COUPLING SCHEME

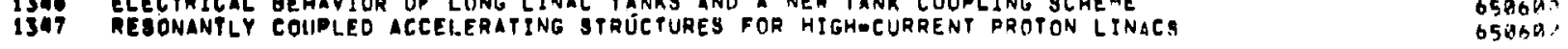

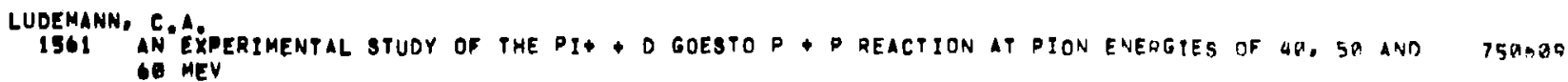

$773 \cdot 4 \cdot$

771219

2005 ELASTC SCATTERING OP POSITIVE PIONS ON 160OXYGEN AT UQ. ANO 49,7 ME.V

INELABPIC SCATIERING OF PI + MESONS FROM NUCLEI AT SE MEV

1991 MEASUREMENTS TF PIONENUCLEAR ELASPIC SCATTERIN
2404 PIONONUCLEAR TNELASTIC SEATTERING AT SQ MEV

2669 THE OEUTERON DASTATE IND THE PI+ + D GOESTO P + P REACTION

2421 THE PI+ D GOESTO \$P REACTION AT HO, 5B AND GO MEV

361425

$770 \times 10$

LUMDGAARD, $\mathrm{N}$

25S3 BEÁ CUREET REDUCTION, I PULSE IN IA

$76 \times 393$

LUNDY, A.8.

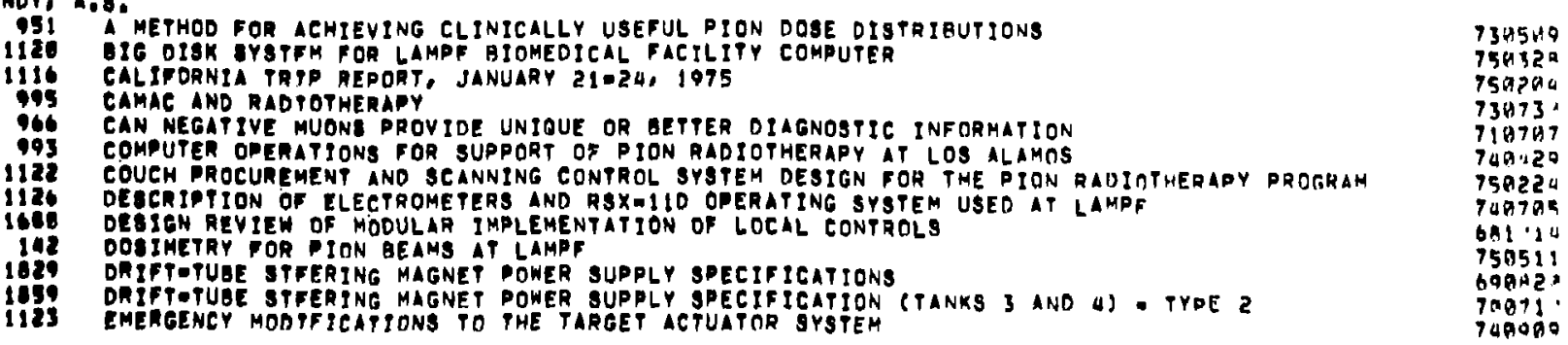


LUNOY, A.S, (CONTD,)

1096 EPA FORUS MAGNET CONTROL CHASSIS - OPEQATION NOTES OT1"10

1989 EPA STEERING MAGNET CONTROL CHASSIS SMZJ-SMGGMOPERATION NOTES OTI.IO

1906 EPA STEERING MAGNET CONTROL CHASSIS SMOI-SMOZOOPERATION NOTES

1819 FABRICATION SPFCIFICATION - LAMPF NIM LOCAL CONTROL MODULES OQR3ID

19IS FAERTCATION SPECIFICATION - LAMPF NIM MODULES, MECHANICAL GOAIZ-

1125 MAGNET POWEA SUPPLY PROBLEM AT BIOMED FACILITY

1790 HISCELLANEOUS FABRTCATION SPECIFICATIONS FOR COMPUTER COMPONENTS

1730 OPERATING INSTRUCTIONS - QUAO MAGNET PEMPERATURE MONITOR ANO ALARM TEST NIT, G3Y-14AOBI, GQIIZ"

963 PLANS ANO PRORRESS TOWARD DEVELOPING a THERAPEUTIC PION REAM AT THE LOS ALAMOS MESON

1393 POYSICS FACILPTY

1673 POWER CONNETTRS FOR EPA CHASSIS

T5O PROGRESS TOWARD USING HUMESIC X-RAYS AS A OIAGNOSTIC TOOL

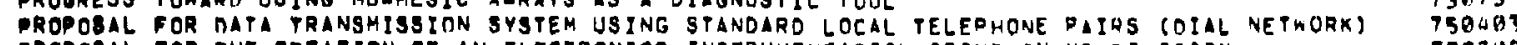

G6I PROPOSAL FOR THE CREATION OF AN ELEETRONICS INSTRUMENTATION GROUP IN MD MIVISION

III3 REPORT OF FORFIGN TRAVEL. LUNBY

1811 REQUEST FOR FABRICATION DROPOSAL. LAMPF NIM LOCAL CONTROL MODULES

SOME OESIGN FFATURES DF THE LAMPF GIOMEDICAL FACILITY

1121 SPECIFICATIONA FOR AQ MEGABYTE OR LARGER OISK STORIGE SYSTEM FOR THE LAMDF BIOMEOICAL FACILITY COHPIITER, PO QUS12

119 SPECIFICATIONA FOR A PORTABLE CAT TERMINAL PTTY COMPATIBLES WITH ACOUSTIC COUPLER

1 1月 BPECIFICATIONA FOR AN ISODOSE MEASURING INSTRUMENT

996 STATUS ANO PLANS FOR PION RADITTHERAPY AT LAMPE
964 STATUS AND PLANS FOR THE NEGATIVE PION RAOIOTHERAPY FACILITY AT THE LOS DLAMOS MESON

1909 TESTER FOR MOTOR BTEPPER BOARDS (O3Y-12579B AND G3Y-125815)

1727 TESTING PROCENURE POR ANALOG COMHAND MODULE I G3Y-125804 USING TFSTER O3Y-125823

1728 TESTING PROCERURE FOR ANALOG COMHANO MODULE II G3Y-125805 ISING TESTER GTY-175R22

II24 TREATMENT LOGRING CODE FOR USE OURING THE FIRST PATIENT EXPOSURES IRING ON EPA

LYKAN, S.T:

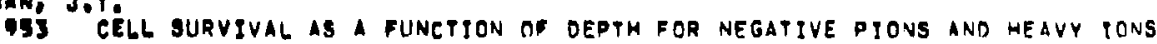

LYONB, K.M.

2797 IA IN LINE - VACUUM WINDOW AND HAROWARE ISSEMBLY

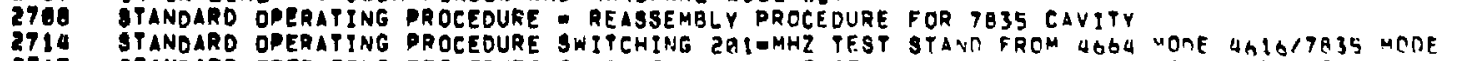

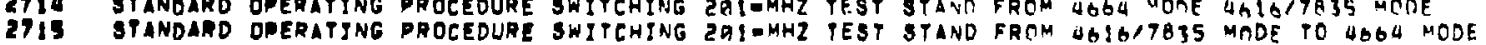

750346

60052

726015

69012

$75332^{\circ}$

749.00

730293

721117
75020

LVERKo, w,

1583 MEAM CALCULATYONS FOR LAMPF MUON CHANNEL

2129 EFFECF OF SPACE CHARGE AND OPHER FACTORS ON LINAC EMITTANCB GROMTH

2SGS EOUILIORIUM PHABE SPACE DISTRIBUTIONS AND SPACE CHARGE LIMITS IN LINACS 2156 HIGH ORIGHTNEAS LINAC BEAMS

क०त? द्र

Gognl?

60001 ?

740.09
$673011^{\circ}$

75 ana

$75071:$

751 .

7riando 
LYSENKO, W.P: CCONYD,1

2IS4 PRELIMINARY RFSULTS ON RZED PROBLEM

2151 REVIEW OF GLURKSTERN WEEK JULY 19 , 1976 (SPACE CHARGE EFFECTS)

2155 GPCE CHARGE FFECTS, PROGRESS REPORT NOV. 1970 . JAN 1077

2IS2 SPACE CHARGE BPFECTS STUOY

2153 3PACE CHARGE FFFETS

25. PHE STOPPED MUION EHANNEL AT LAMPF

761120

thasac

61115

70000

MAC ROBERTS, M.D.J.

IAGI LAMPF SPECIFICATION FOR DIGITAL CONTROL COMPUTER SYSTEM

IISS BPECIFICATION FOR DIGITAL CONTROL COMPUTER SYSTEM

690116

68123

MACEK, Ris.s.

240: A COMPUTATIONAL TECHNIOUE FOR TUNING THE LAMPF HIGH ENERGY PION CHANNEL

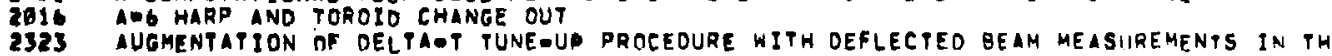
SWITCHYARD

124 CROSS SECTIONE FOR (PI+,P) REACTIONS ON OABERYLLIUM, I2-CARBCN, ANA 16-OYYTEA

159. CROSS GECTIONR FOR (PI+,P) REACTIONS IN GOLITHIUM, QUBERYLLIUM, 12ACARHON, AND 16-CXYGEN 2905 CURRENT MONITORS FOR LINE A IN AREA A AND LREA AEEAST

25U! CYCLE R TEST PLAN 2310 MARP AND SEANNER WJRE HEAT TRANSFER WIPH TIME STRUETURED REAM

2672 GIMITING APERTURES ON PHE SWITCHYARO

720 NEH MEASUREMENTS OF ( $P$ I, $2 P$ ) REACPIONS ON LIGHT NUCLFI

1500 PERFORMANCE OF THE HJGHEENERGY PION BEAM AT LAMPF

2673 PHASESPACE TAILORING FOR LX AND LE

711 PRECEPRON - PRINCIPLE FOR OBPAINING PIONGPION AND YUON-MUON COLLISIOUS

2613 PRELIMINARY MEASUREMENTS OF 6U7 MEV PROTON TIME-OF OFLIGHT SPECTAA (REVISEO)

1178 PRELIMINARY RFPORT ON SOME GENERAL PURPOSE TWO-BEND PION CHANNELS

2HIT PROTON BEAM DTAGNOSTIC INSTRLUMENTATION TASKS

2月IF AECOMHENDATIONS FOR SWITCHYARD IMPROVEMENTS

2339 REVISED CYCLE O PLAN

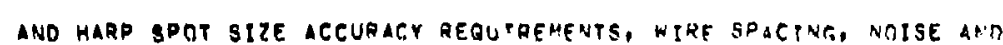
READ OUT ACCURACIES

2675 STATUS OF SWITCHYARO DECISIONS

2097 STATUS OF BWITCHYARO DECISIONS

2089 SWITCHYARD IMPROVEMENT PROGRAM

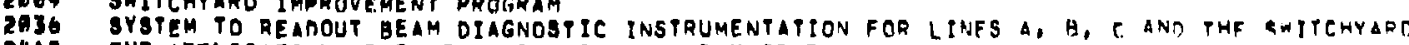

2U12 THE APPLICATION OF PROTONS PO COMPUTEO TOMOGRAPHY

2WEA TUNE $U$ UD OF PHF LAMPF HIGHAENEAGY PION CHANNEL

MACHEN, $0, R$

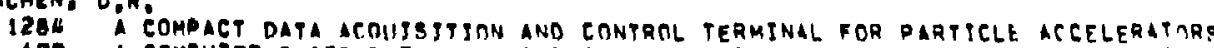

609 A COMPUTERTAARED OATA-ACAUISITION AND CONTROL SYSTEM FOR LAMDF BEAM HIAGYOSTITS

2482 LONG DIBTANCE CAMAC ARANCH VI DATAOLINK AND MICQOPROGRAMMED GHANCH TRTVER

1916 MICROPROGRAMMFD DIGITAL FILTER DESIGN FOR THE HP-?IGA CONPUTER

2481 BATELLITE CANTROL ANO DIAGNOSTICS COMPUTER SYSTEM FOR THF LAMPF INJECTURS

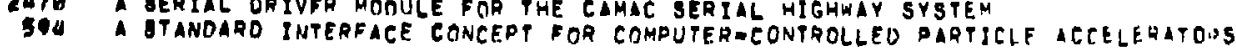

750.101

$74872 \pi$

$71001:$

74 ดीด

750317

76952.

76113

710020

75000.

7410

$60 B 02$

$741: 56$

pasia!

Tuम>2?

7मा1 मी

Th⿻一𠃋十 15

75म?

$150 ? 0 \mathrm{Am}$

750 Pan

74 A 2 h

73412 ?

77110

$75 a \cdot a$

6045 3h

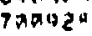

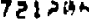

7 aाl? 5

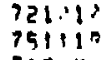

Pian 
MACHEN, D.R. (CONTO.)

393 AN OPERATORS CONBOLE FOR THE LAMPF ACCELERATOR TIBMB.

1214 BEAD PERTURBATJON MEASUREMENT

2478 CAMAC AT LAMPF

2467 CAMAC BASED COMPUTER-COMPUTER COMMUNICATIONS VIA MICROPROCESSOR DATA LINKS
686 CAMAC SYSTEMS AT LAMPF

DATAACOUISITION COMPUTER NETWORK

DESIGN NOTES. LAMPF CER

DIGITAL FILTER OESTGN FOR CAMAC MULTIWTAE PROPORTIONAL COUNTER

DART II THE OELERTOR DATA AND CONTROL SYSTEMS

OIGITAL FILTERS DART II THE OIGITAL PROCESSING

FABRICATION OF ADDITIONAL RICE MARK 118 LOGIC UNITS

HDTOHEX DEBUGRER FOR COMPUTER LOGIC MACHINE

INBTRUMENTATITN FOR REAM DIAGNUSTICS EXPERIMENTS

LAMPF SPECIFICATION FOR DIGITAL CONTROL COMPUTER SYSTEM

MINICOMPUTER APPLICATIONS AT LAMPF

OPERATING TESTS LAMPF PROTOTYPE DATA ACOUISITION ANO CONTROL TERMINAL

PERFORMANCE SPECIFICATIONS FOR A SERIES OF CAMAC CONTROL MODULES

PERFORMANCE SPECIFICATIOH FOR a CAMAC BTORAGE DISPLAY DRIVER/CQNTROLLER

DROPOSED LAMPF STANDARO LOGIC SYMBOLS ANO A OISCUSSION DF THE BASIS FOQ SELECTION

REFERENCE ANO MAINTENANCE MANUALI LAMPF OATA ACOUISITION AND CONTROL TERMINAL

REOUEST FOR FABRICATION PROPOSAL - LAMPF RICE MK IIB

REQUEST FOR FABRICATION PROPOSAL - LAMPF PHOTOMULTIPLIER TUBE BASES

REOUEST FOR FABRICATION PROPOSAL - LAMPF PHOTOTUBE HIGHEVOLTAGE DISTRIAUTION BOX

RICE MK II - MOD. L OPERATIONAL FELTURES AND RIUGRICEARIU INFORMATION FORMATS
RISE TIME AND EROSS TALK INVESTIGATIONS, CAMAC STANDARO DATAWAY

SIGNAL TEAM MNEMONICS - LASL RICE

GPECIFICATION FOR A CAMAC BINARY COMMANO MODULE WITH LATEH gACK INDUTS

GECCIFICATION FOR DIGITAL CONTROL COMPUTER SYSPEM

SPECIFICATION ROR LAMPF OATA TERMINAL POWER SUDPLY

SPECIFICATION FOR LH SUA 2 REFRIGERATOR AUPOMATIC CONTROL MARDWARE
SPECIFICATIONA FOR IS CHANNEL CAMAC STEPPING MOTOR CONTROL MODULF

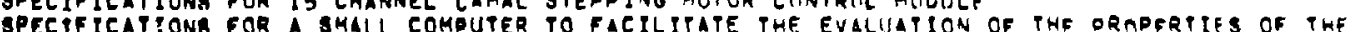
LAMPF PROTON AEAM

1920 GDECIFICATIONA FoR A MULTIOCHANNEL, 20 MHZ CAMAC SCALER MODULE

T2I SPECIFICATIONA FOR A MULTIOCHANNEL, GO MHZ CAMAC SCALER MOOUL

1920 SPECIF ICATIONR FRR REMOPE CONTROLLEO HIGH ACCURACY DFLAY

1026 SPEC PF ICATIONA FOR THE ANALOG DATA SYSTEM

1676 TECHNICAL DESTRTPTION OF THE LAMPF CAMAC DATAOLINK MOOULE

194 TECHNICAL OESTRTPTION - MAD TEST MOQULE . REFER TO PRINT B3Y-125RBO/1

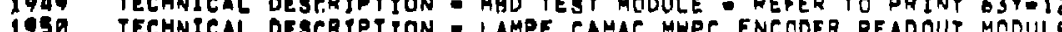

1933 TEEHNICAL DESCRIPTION - CAMAE PRIORITY ENCOOER MODULE - REFER TO PRIMT O3Y-149612

1962 TEEHNICAL DESCRTPYYON - LAMDF SERTAL DRIVER

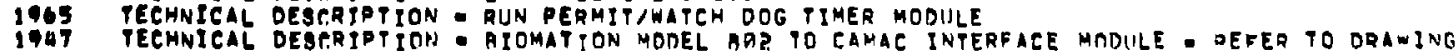
TECHNICAL DE

194B TECHNICAL OESTPIPTION - LAMPF ElGHT-CHANNEL EAMAE STEPDER MOTOR CONTHOL MUI.TTDLEXEO. DRAKING G3YGIDQS5E

9B3 THE CAMAC SERPAL SYSTEMS GESCRTPTION FOR LONG LINE, MULTICRATE APDIICATITN

$70118+$

$731: 27$

096519
$79190 ?$

730305

$711 ? 04$

71120

710406

7 ) 1203

600527

60011t:

73112.
609515

71123

72032

6月A! 2 ?

कीटा

72110.

วटा1ด

OBस72?

7 IAR?

botste

OA1 3 .

7 Jisne:

Tharas

740728

75ดวด

$75902:$

sha

b०ด3

7911 15

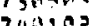

7409 त?

T5xदin?

700115

730.9

THE GATELLITE MINICOMPUTEA - A PRACTICAL SOLUTION TO ACCELERATOR CONTRAL

73091 
MACHEN, D.R, (CONTO,)

2471 THE USE OF MINICOMPUTFRS IN DARTICLE ACCELERATIRS

GBB USE OF a NOVA COMPUTER IN THE LOS ALAMOS MESON FHYSICS FACILITY

7319

MADOOCKS, H.C.

1766 PULSE-SIGNAI VIEWING SYSTEM FOR ACCELERATDRS

1762 AN OUTLINE OF A MATHF HATICAL ANALYSIS OF THE VIOEO SYSTEM FOR LAMPF

IOGI CALIRRATION AND TESTING PROCEOURE FOR THE WATER FLOW SWITEH LREVISFO

1993 CALIARATION AND TESTING PROCEDIJRE FDR THE WATFR FLON YUNTTOR SYSTEL

1763 PROGRAMS TO DFSIGN BRIDGEDAT FILTERS FIR THE VIDEO SYSTFM FCR LAMPF

1905 TABLE OF NOMINAL VOLTAGES FOR THE WATER FLON MONITIR CARD

1902 TROURLESHOOTING PROCEDURE FOR THE WATER FLON SWITCH

1984 TROUALESHOOTING PROCEDURE FOR THE WATER FLOM MONITOR SYSTEN

1761 VIDEO SYSTEM EOR LAMPF

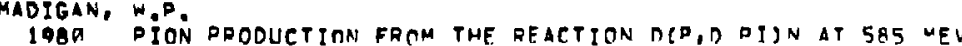

$60,41: 3$

$071+53$

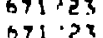

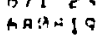

$67 ! \cdot 23$

BI:?

0.155

$7+125$

MADLANO, D.

2912 ELASTIC SCATTFRING MNALYIING POWER MEASIJREMENTS AT BAS MEVA-I

2013 ELASTIC SCATTFRING ANALYZING POWER MEASUREMENTS AT BOR MFVEI

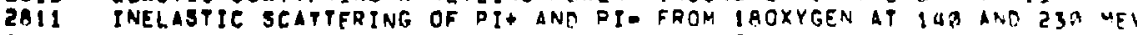

2BOB P + 13,12C, INELASTIC SCATTERING AT A. G GEV

7804

$\rightarrow 700$ in.

77 a日a

711 PRECETRON - A PRTNCIPLE FOR OBTAINING PIOVEPION ANR MUONAMIION COLLISITAS GOAMZU

MAH, R.
14QD GRAPHITEMTO-METAL BONDING TECHNIOUES

$7119 \cdot$

MALAROUGH, D.J.

ELASTIC SCATTERING OF POSITIVE PIONS ON 16-OXYGEN AT UA, ANS IOQ, T MEV

$17.4 \cdot 9$

INELABTIC SCATTERING OF PI + MESTA:S FROM NUCLEI AT SR MEV

MASS DEPENOENCE RF PION PDISBIE CHARGE EXCMANGE

MEASUREMENTS OF PIONGNUCLEAR ELASTIC SCATTERING AT LOW ENERGIES

- ION DOUBLE TCHARGE EXCHANGE ON 16-OXYGEN ANO IA-OXYGEN

PIDN NONOANALAG DOUBLE CHARGE EXCHANGE, 16-OXYGEN(PI+,PI-) I6-NEON

791210

780123

this 50

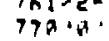

$\operatorname{lin} \rightarrow 10$

2UDU PIONANUCLEAR THELABTIC SCATTERING AT SH MEV

MANCA, J.J.

2306 ACCELERATING IND FOCUSING STRUCTURES FOR PIGMI

MANGER, C.E. 
MANGER, C.E. (CONTD.)

$1250^{\circ}$ NEW METHOD FOR MEABURING AND TUNING MAIN CELLS TABOBL

MANN, D. COMPUTATIONAL TEEHNIQUE FOR PUNTNG THE LAMPF HIGH ENERGY PION CHANAEL

(1)

$950.6 ?$

MUALESTATE INTERACTIONS IN POO BREAKUP AT BOB AND ODO MEV

PIONDNUELEAR TOTAL CROSB GECTIONS NEAR THE $3 / 2$, $3 / 2$ RESONANCE

183 PIONANUCLEAR TOTAL CROSS SECTIONS NEAR THE 3/2, 3/2 RESONANCE
223 OUASTOFREE SCATTERING IN POD BREAK=UP REACTIONS AT SDO ANO GPO MEV

35802

75 h $73 \sin ^{2}$

$7311 ! 2$

750.14.

MARLAM, P.G

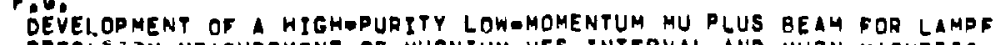

1891 PRECISZT: MEABUREMENT OF MUONIUM HFS INTERVAL AND MUON MAGNETIC MOMENT

790094

$790: 8:$

MARK, A,G
13Q9

$09020^{\circ}$

MAnKOWITZ, 3.s.

208 ACPIVATION STIIDIES OF PION-INDUEEO REACTIONS ON $C, N, O, F, A L$ ANO CU

$750 \operatorname{sen}$

75340.3

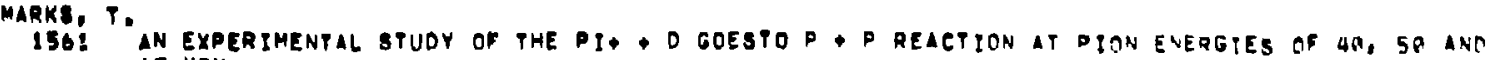

750 मी०

15O MEV

ELASTIC SCATTFRING OF POSITIVE PIONS ON 1600 OXYEN $1 T$ 4H, 9 AND 49,7 MEV

INELASTIC SCATIERING OF PI MESONS FROM NUCLFI AT 50 MEV

2411 MASS DEPENDENSE OF PION DOUALE CHARGE EXCHANGE

1991 MEABUREMENTB OF OIONGNUCLEAR ELASTIC SCATTERING AT LOW ENERGIES

1970 OBSERVATION OF THE PION DOUOLE CHARGE EXCHANGE REACTION 10-OXYGEN(PI+, DIO) 180NEON

2422 PION DOUBLE-CHARGE EXCHANGE ON 16-OXYGEN AND 18-OXYGEN

2417 PION NONGANALIG DOUBLE CHARGE EXCHANGE: 16-0XYGEN(PI+.PI-) 16-NFON

2ADa PIONANUCLEAR TNELASTIC SCATTERING AT 50 MEV

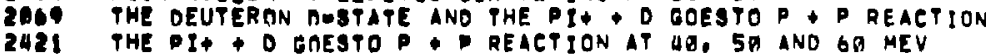

MARPS, R.E.ं.

156 MEABUREMENTS AF PI(+OR-) NUELEUS TOTAL CROSS SECTIONS AT ENERGIES AELOW 29 O MEV

2081 NEUTRON RADII OF CALCIUM ISOTODES FROM PION TOTAL CROSS SECTION MEASUREMFNTS

IA6 PI(TOR-) O(UEHFLTUMS TOTAL EROSS SECTIONS FROM 50-1OU MEY

770.19.

771219

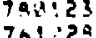

$701 \cdot 52$

778154

778716

773920

701.25

MARTIN, E,R.

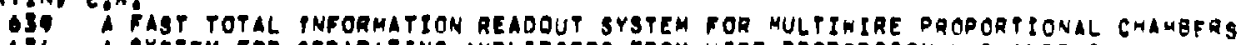

636 A SYSPEM FOR AEPARATING AMPLIFIERS YROM WIRE PROPORTIONAL CHAMBERS
1237 CAMAC ON TME PROTON COMDTON EXDERIMENT

$750 \sin$

750420

1237

$72 \pi \cdot$

7,052 
MARTIN. E.R. (CONTO.)

3 IL CAVITY PUNING FOR THE LAMPF BB5OMHZ LINAC

2427 CROSS SECTIONS FOR PI + NUCLEUS GOESTOPI +OR-... FOR TLOAMFV DROTOQS

549 PRODUCTION OF CHARGED PIONS RY 73 BAMEV PROTONS FROM HYDROGEN AND SFLECTER QUELFI

656 USING MULTINIRE PROPORTIONAL CHAMBER OECODERS

657 USING THE CA3A5U MWPC AMPLIFIER

MLRTIN, 3.T.

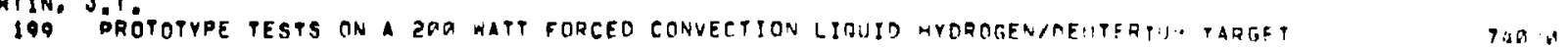

MARTIN, P,

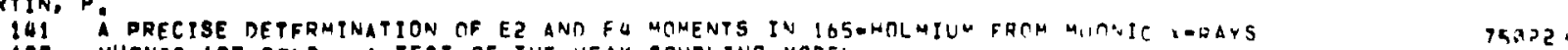

185 MUDNIC 197-GOIT. A TEST OF THE WEAK-COUPLING MONEL

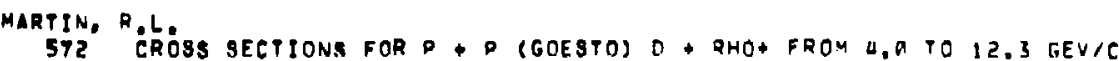

572 CROSS SECTIONS FOR P P (GOESTOSO P QHO+ FROM U,A TO 12.3 GEV/C

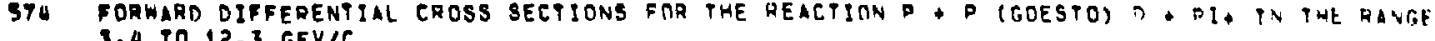

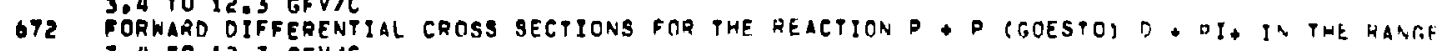
3.4 TO 12.3 GFVIC

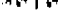

OR" 7 ?

MARTINS, B.I.

MATZ, N,

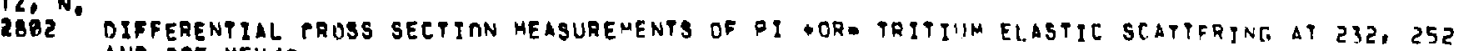

2423 MEASUREMENTS AF THE DIFFERENTIAL CROSS SECTION FOR PION CHARGE EXCHANGE ON TRITIUM.

2UZA MEASUREMENTS DF THE DIFEERENTIAL CROSS SECTION FOR PION CHARGE EXCHANGF ON TEITIUM.

2093 PI OOR. ELABTTC SCATTERING MEASUREMENTS ON H3 ANT HE3 AT 232, 252 ANI TOG "EVIC

75040

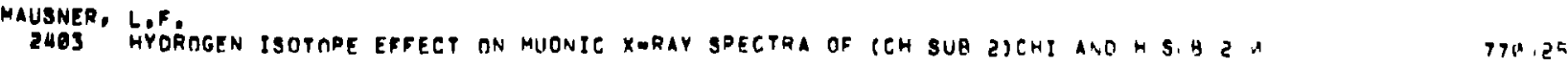

MAYER, E.G.

73G LOCALIZED HEATING AS IN AOJUNCT TO RADIATION THERAPY

926 THE POTENTIAL OE LOCALIZED HEATING AS AN AOJUNET OO RLOIATION THERAPY THIOB.

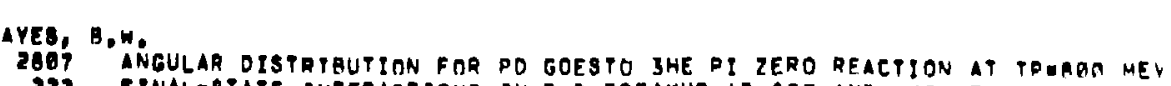

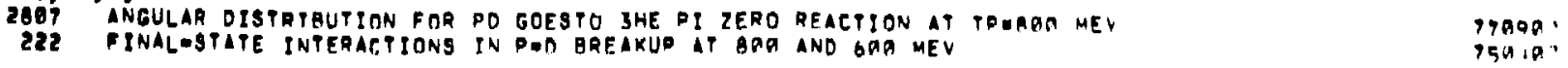


MAYES, B.W. (CONTO.)

KINEMATICALLY-COMPLETE STUDY OF DION PQOOUCPION AY THE REACTION, HYORAGENTDQOTOR, DI

$70 x+25$

IUS1 RINEMATICALLYOCOMPLETE STUD
PROTON) NEUTRON AT BBO MEV

210 NUELEAR-COULOMB INTERFERENCE IN PI ORD 16 OXYGEY SCATTERING

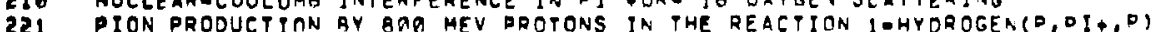

1990 PION PRODUETIINN FROM THE REACTION DCR,O PIIN AT 585 MEV

PION PROOUCTION IN P-O INTERACTIONS AT AQg MEV

PION PROOUCTION VIA A PQP GOESTO OPQI OUASITFREE PROTONS IN DEUTERPUL

PIONONUCLEAR POTAL CROSS SECTIONS NEAR TNE $3 / 2$, HORE RESONANCE

PIONENUCLEUS FORWARO SCATTER ING AMPLITUDES NEAQ THE J

PIONONUCLEUS GMLL NNGLE ELASTIC CROSS SECTIONS NEAR THE $3 / 2$ R

2974 PION-NUCLEUS TOTAL CROSS SECTIONS NEAR THE $3 / 2,3 / 2$ RESONANCE

223 QUASITFREE SCATTERING IN POD BREAK-UP REACTIONS AT BPG AND GPO MEV

OUAST-FREE SCATTERING IN PROTON INOUCEO OEUTERON BREAKUP AT 585 AN AOJ

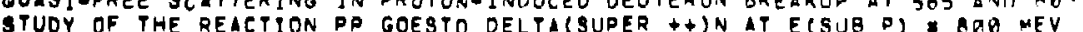

$45 \sin \theta$

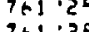

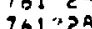

731115

301.52

198.25

$\rightarrow>P+20$

32000.000

$\tan 70$

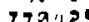

ME CABE, C.W,

2484 OOJMHZ CROWBAR LOGIC CHASSIS

2443 3 G-MHZ CROWRAR PRTGGER CHASS IS

932 A NEW ELECTROSURGICAL COAGIJLATING-CUTTING FORCEPS

929 TECHNIOUE FOR LOCALIZED HEATING IN TISSUE

CIRCUIY YIELOS LINEAR OULSE-WIDTH MOOULATION

936 LOCMLIZED CURRFN FIELD TUMOR THERAPY P PRELIMINARY RE

111250

$75042 \%$

721115

75310

$73630^{\circ}$

MC FARLANE, $W, K$.

25.5 COMPUTING IN SUPPORT OF EXPERIMENTS AT LAMPF

1627 LIMITS ON THE DIRECT PRODUCTION OF POSITRONS RY 256" AND BRBAMEV PROTONS

93 SEARGH FOR DIRECT LEPTON PROOUCTION IN PD COLLISIONS AT RPA MEV ANO $25 \mathrm{~S}$ WEV

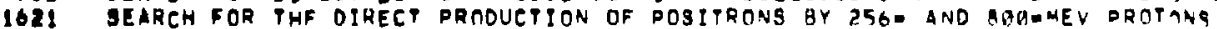

2310 STUOY OF DATA LOGGERS FOR LAMPF

Th1.6.

$10,3.150$

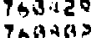

7of

MC GUIRE, A.O.

732 SOME" SECONOARY BEAMS FROM LAMPF

03122

MCADAMS, R.E.

1563 AGOLUTE CROSA SECTIONS FOR PRODUETION OF PROMPT NUCLEAR GAMMA RAYS RY FAST DIONS

1589 ALPMA AND MULTINUCLEON REMOVAL FROM SB-NICKEL ANO ORENICKEL BY SAST DIMNS

1501 PABT PION INOUCEO PROCESGES IN COMPLEX NUCLEII CROSS SECTION AND ALDHA RFMRVAL

ISA2 FAST PION INOIICEO OROCESSES IN COMPLEX NUELEII OROJECTILE SYSTEMATTCS

1385 PAST PION INOIICED PROCESSES IN COMPLEX NUCLEII SYSTEMIPICS IN DRCOULT NURLE?

ISG2 SYSTEMATICS OF PION AND PROTON INTERACTIONS WITH NICKEL NUTLIDES

$758 \times 2$

95 andia

$750+1$

750.04

750.906

MCEARTHY, J.

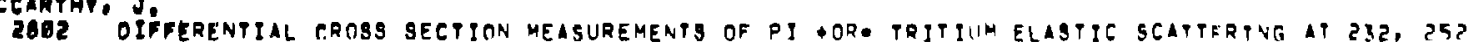

7 Aง12?

$2 A T S$ MEASUREMENT OF DIOD ELASTIC SCATTERING 
MCEARTHY, J. (CONTO.)

2803 PI' PORE ELASTTC BCATTERING MEAGUREMENTS ON HS ANO HES AT 232,252 AND 209 UEV/C

161.50

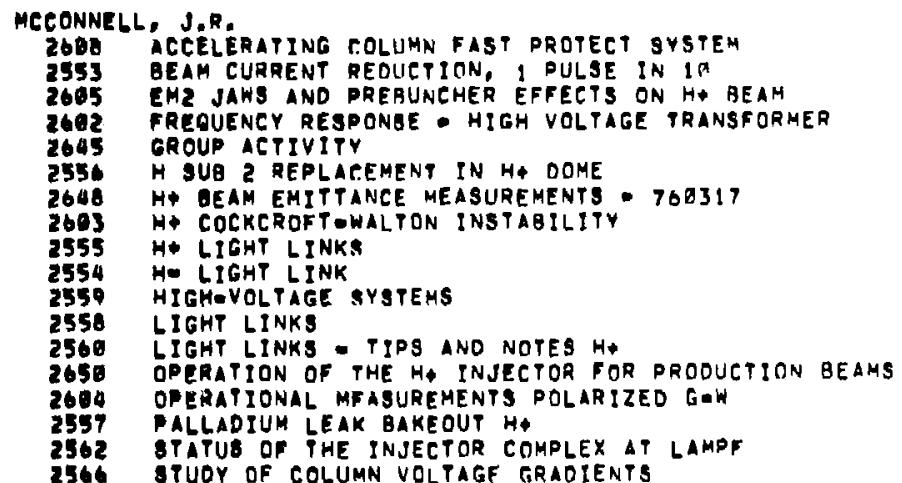

778713 760343

770205

77051,

769401
769028

36039

760319

7
7051900

761509

76, 90

$163 \times 14$.

$160: 8$.

Thases

77051 ?

To1>0?

$10,3,5$

MCOANIELS, D.K.

ELASTIE SCATTFRING ANALYZING POKER MEASUREMENTS AT BQD MEVE-I

ELASTIC SCATTFRING ANALYZING POWER MEASUREMENTS AT BAB MEVE-II

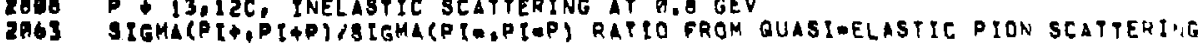

HeGILL, J,

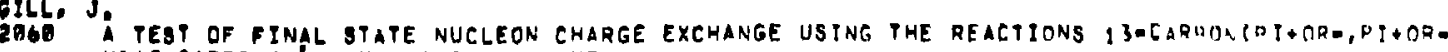
NII2-CARBON(4. $\triangle 4$ MEV AND 15.11 MEV

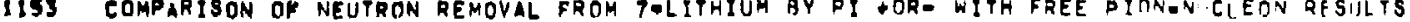

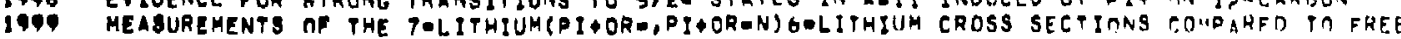

PION-NUELEON REBULTS

7รpapa.

$77 B \circ 9$

inlog.

MCKIBBEN, J,L

1549 A MEASUREMENT OF PARITY VIOLATION IN PROTON-PROTON SCATTERING AT 15 MEV

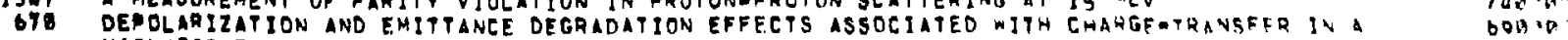

2542 LAMUOSHFY POIRTZED SOURCES AT LOS ALAMOS SEIENTIFIC LABORATOEY

2676 OPERATION OF A RADIO-FREOUENCY NULLEAR SPIN FILTER

228 DOLARIZED H. SOURCE FOR LAHPF

2549 POLARIZED ION INJEETOR DROGRAM

66 SEAREM OR AARITYGVIOLATING CONTRIBUTION TO NUCLEONUNUELEON SGATTERING

224 TEST OP PARITY CONGERVATION IN PAP SCATIERING

770.97

Thl 1 in

Thl 2 a

750 19.

bodith

$741 \times 2 ?$

$76 \sin$

73 . 
MEKIOBEN, J.L, (CONTD!)

2590 THE LAMPF POLARIZED ION FACILITYI STATUS REPORT 77MZIE

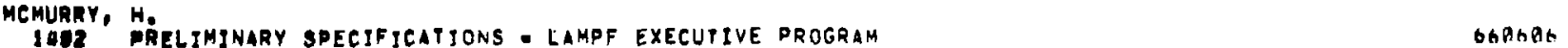

MENAUGHTON, MaW.

2585 COMPUTING IN SUPPORT OF EXPERIMENTS AT LAHPF

MEIER, K.L., TARGETS AND DRIVE MECHANISH PERFORMANCE

2317 HARP ANO SCANNER WGRE HEAT TAANGFER

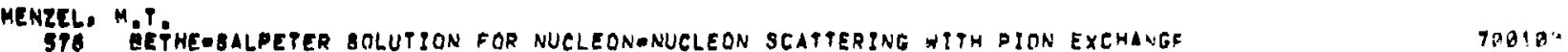

METHODOLS8. N.

b29 SIMULATION OF PHE SELGBga COMPUTER ON MANIAC II (SELMA) GTBzz?

HE9zGLA, $\mathrm{C}$.

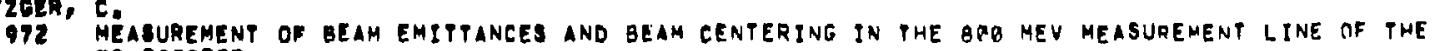
PE BOOSTER

601120

MEYER, E.A.

asei Co ÁCELERATING TOLUMN

251 QEAM MEASUREMFNTS ON PHE HIGH INTENSITY PROTON INJECTOR OF LAMDF

2305. CALIBRATION OF TON GAUGE

322 METALO TO AVOTO IN VACUUM SYSTEM DESIGN

325 OPERATION AND PERFORMANCE OF PHE HIGHEINTENSITY FROTON INJECTOR OF LAMPF

665 OPERATION OF THE LAMPF 7580KEV INJECTOR

2562 OTATUS OF THE INJECTOR COMPLEX AT LAMPF

230 STATUS OP THE LAMPF HE INJECTOQ

144 TEST OF LASL TON SOURCE WITM 75 OEKY ACEELERATING COLUMN

1443 TEET OP LABL TON SOURCE WIPH 2 GOOKV PIEREE ACCELERATIVG COLUMN

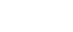

MEYER, M. O.

G35 A. OA IOENTIFYINT, MOMENTUMOSENSITIVE DETECTOR SYSTEM FOR NEGATIVE PIONS

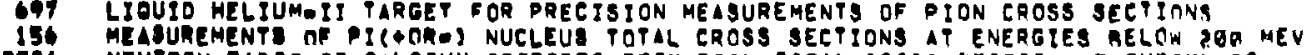

201 NEUTRON RADIT OF CALCIUM IBOTORES PROM PION POTAL EROSS SECTION MEASUREMENTS

70113:

72659

$791 ? 30$

$724 \times 31$

721191

Tionut

iat 5 is

7 hila 14

$720.10:$

c0902,

7 40790

Yuse?

750609
770523 
MEYER, H.O. (CONTO,

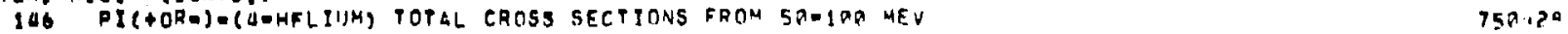

MEYER, R.E.

2ABd A SOLUTION FOR REMOTE HANOLING IN ACCELERATOR INSTALLATIONS 77OBIE

MEYER, S.L:

919 LAMPF NEUTRINA FACILITY PROPOSAL

BECTIONS IN CHLORINE-37 WITH MU OECAY NEUTRINOS

MEYER, T

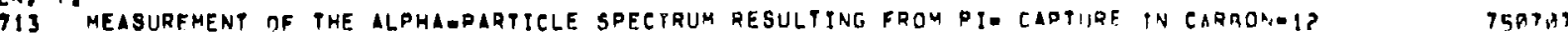

MEYER-BCHUTZME ISTER, L:

1563 ABSOLUTE CROSE SECTIONS FOR PRODUCTION OF PROMOT NUCLEAR GAMMA RAYS HY FAST DIOAS TSBVO

2416 ENERGETIC CHARGED PARTICLE YIELDS INOUCED RY PIONS ON COMPLEX NUTLEI

1581 FAST PION INDIICFD PROCESSES IN COMPLEX NUCLEI CAOSS SECTION AND ALPMA REMAYAL

158 FAST DION INDICEO PROCESSES IN COMPLEX NUCLEI! PROJECTILE SYSTEMATICS

15ES FAST PION INDIICEO PROCESSES IN COMPLEX NIJCLEII SYSTEMATICS IN PRODUCT * UCLEI

2418 GAMMAFRAY STURY OF PIONAINDUEEO REACTIONS ON COMPLFX NUULLEI

1993 INTERACTION OF FABT PIONS WITH GZ, GQENICKEL

1999 PROTON SPECTRA FRON PION-INDUCFD RFACTIONS

1562 SYSTEMATICS OF PION ANO PROTON INTFRACTIONS WITH NICKEL NUCLIDES

$75 \sin \theta$

7 7מAa

$754: A$

$750130:$

750 a

772125

$761 \cdot 28$

$761 \cdot 2 \pi$

75 ADSA

MILOER, $F$.

2UQB INELASTIC SCATTERING OF PI Q MESONS FROM NUCLEI IT 5 G MEV

2404 PIONONUCLEAR INFLASTIC SCATTERING AT 59 MEV

771719

MILIEH, C,P

749 ANALOG SOLUTIOTH OF PHE PHASE MATION PROBLEM

MILLER, B,F.

1496 PROCEEDINGS OF THF FIFTH LAMPF USERS MEETING 72A3H

MILLER, 6.A.

220 (PI,0) REACTIONN FOR SMALL INCIDENT PION ENERGIES

1979 SURVEY OF PION CHAAGE -EXCHANGE REACTIONS WITH NUCLE

167 JON CHARGE-FXCHANGE REACTIONS NITH NUCLEI - T4IZ?

215 DON CHARGE-FXCHANGE REACTIONS WITH NUCLET - 75GGPA

IT: DOSIPIVE PION PROOUCTION BY 185 MEV PROTONS

$2 ! 3$ ORDTONENUCLEISA SCLTIERING AT INTERMERIATE ENERGIES

1334 THE ROLE OF TWO NUCLEON SHORTORANGE CORRELATIONS IN PION CHARGE EXCHANGE RFACTIONS 
MILLER, G,H

141 A PRECISE DETFRMINATION OF EZ AND EU MOMENTS IN 165-HOLMIUM FROM MUONIC XORAYS

185 MUONIC 197-6OID. A TEST OF THE WEAK-COUPLING MODEL

$75 \times 32$.

MILLER, J.

2515 RADIATIVE NEGATIVE PION CAPTURE IN LIOUID NEON

2509 RAOITTYE NEGATIVE PION CAPTURE IN LIOUID TRITIUU

$\operatorname{Tin} 20$

773450

MILLER, J.H.

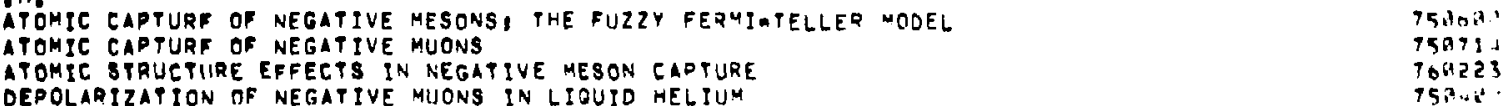

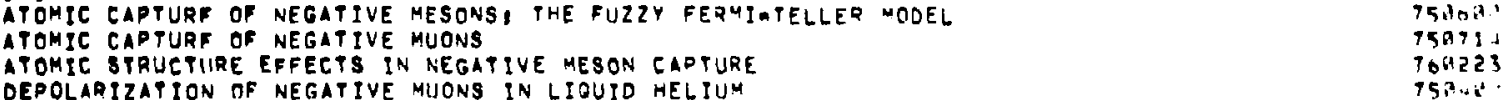

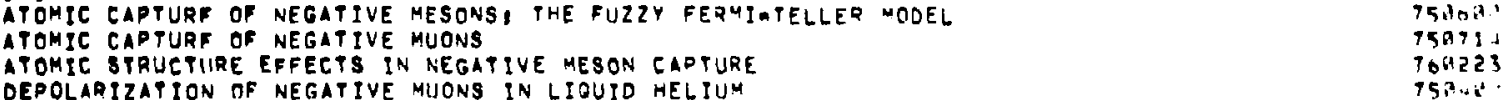

1230 ATOMIC STRUCTIRE EFFECTS IN NEGATIVE MESON CAPTURE

(5)

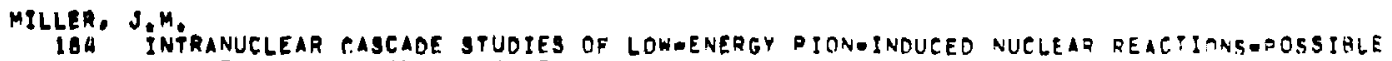

isan?

INTRANUCLEAR CASCADE STUDIES OF LOW-ENERGY PIONOINOUD
EFFETS OF THF FINITE LIFETIME OF THE $(3,3)$ ISOBAR

MILLER, A, C.

92. PHE POTENTIAL OF LOCALIZEO HEATING IS AN IOJUNCT TO RADIATION THERAPY

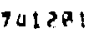

741.9.

MILL8, R.8:

1133 BACKUP of $32 \mathrm{~K}$ DATA BASES

15! GEAH MEASUREMFNTS ON THE HEGHEINTENSITY PROTON INJECTOR OF LAPF

2129 EFFEC OF SPACE CHARGE AND OTHER FACTORS ON LIVAC EMITTANEE GROWTH

ERE

2135 PACTORS AFFECTING HIGH-CURRENT, BRIGHT LINAC GELMS

26 INFORMATION RFTRIEVAL FROM THE OPERATIONGMMINTENANCE OATA BASE

MANAGEMENT INEORMATION FOR LAMPF

OPERATIONS/MAPNTENANCE AROS

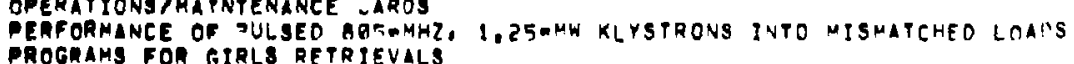

PURCHASE REQUFST FOR MRI TO MODIFY SYSTEM $2 R A G$

REVISIONS TO ACCFLERAPOR ODERATIONS OATARASE

REVISIONS TO ACCFLERAPOA OPERATIONS

TECHNICTAN TRAINING CLASS

TECNICIAN TRAINING CLASS

rwo

MINEHART, R.C.

2002 DITFERENTIAL C.RASS SECTION MEASUREMENTS OF PI OOR. TRITIUM ELASTIC STATTER:VG AT 232 , 252

IND 295 MEVIC

2075 MEASUREMENT OF PIOD ELASTIC SCAPTEPING

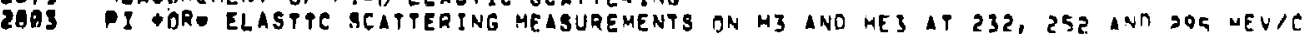

$7514 \cdot 3:$

1505 p.:

7035,4

778938

750.86

$773, \ldots+142$

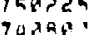

Tuis,

7 unas,

T)

Thane?

?nil

?

$758+10$

gुमp?

$791: 23$

774169

779.64 
HINEHART, R.C:, SCONTD!:

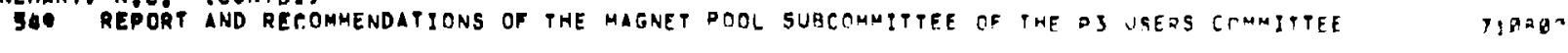

MINOR, MaM:

2460 A SOFTWARE SYSTEM GOR DATA ACOUISISTION IN NUELEAR PHYSICS EXPERiMENTS USTMG CAMAC

iIz? BATCH JOBS AT RITMED

ii16 CALIFORNIA TRTP REPDRT, JANUAAY 21-24, 1975

OA3 COMPUTER OPERATIONS FOR SUPPORT OF PIOV RAOTOTHERADY AT LOS ALAMCS

1122 COUCH PROCUREMENT AND SCANNING CONTROL SYSTEM DESIGN FOR THE PION RATITTHERADU PROCRAM

2AS6 DATA-ACQUIBITION SOFTWARE

13 PRACE

MIQCHKE, R.E.

19a7 a MEASUREMENT OF PARJTY VIOLATION IN PROTONePROTON SCATTERING AT IS UEV

1AGS ABSTRACTS OF CONTRIBUTED PAPERS - VI INTL CONF ON HIGH ENERGY PHYSICS ANM WUCLEAL

STRUETURE HELA AT SANTA FE AND LOS ALAMOS, NEW MEXICO, FROM JUNE 9-14, 1079

DGa AN EXPERIMENTAL stUDY GF OMEGa nEUTRAL DECAYS

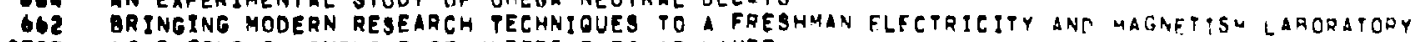
COMPUTING IN AUPPORT OP EXPERIMENTS -AT LAMPF

DIRECT LEPTON PRODUCTION

2843 EYALUATION OF EMIOBI3B PHOTOTUAE ANO OASE OESIGN

1542 LIMIT ON PARITY NONCONGERVATION IN PENUCLEUS SCATTERING AT G GEV/C

1620 LIMIT ON PARITY VIOLATION IN P-NUCLEUS SCATTERING AT B GEYJC

1627 LIMITS ON PHE DIRECT PROOUCTION OF POSITRONS BY 256- ANO BRPEMEV DQOTOSS

663 NEUTRONENUCLELIS TOTAL CROSS gECTIONS FROM QOR-2GGQ MEVIC

2UI3 PARITY VIOLATYON IN D.P SCATTERING

1623 PRODUCTION OF DIRECT POSIYRONG 250 AND 800 MEV

1920 REQUEBT FOR FABRICATION PROPOSAL - LAMPF PHOTOMULTIPLIER TURE EASES

1930 REQUEST POR FABRICATION PROPOSAL. LAMPF PHOTOTUBE NIGHOVOLTAGE OIBTRIQUTIOS BOX

6G BEARCH FOR PARITYOVIOLATING CONTRIBUTION TO NUCLEONENUCLEON SCATTERIVO

783 BEARCH FOD DIRETT LEPTON PRDDUCTION IN DP COLLISIONS AT BQA MEV AND 25 A EEV

1621 SEARCH FOR PHF OIRECP PROOUCTION OF POSTTRONS GY 256- ANO BQG- HEV DROTANE

224 TEST OF PAAITY CONBERVATION IN P.P SCATIERING

1344 THE ELASTIC SCATTERING OF LOW ENERGY OIONS ON PROTONS

1303 TUNEUP OF THE LAMPF LOW ENERGY PION CHANEL

$751+13$

Tabag

$10 \sin ^{2} 0$

15.0.

10805.

tyacs

MOINESTER, MAA.

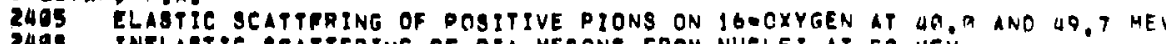

24 INELASTIC SCATTERING OF PI+ MESONS FROM NUELEI AT 5 S MEV

1991 MEABUREMENTS OF PIONONUCLEAR ELASTIC SCATTERING AT LOA ENERGIES

MEASUFEMENTS OF PHE (PION PLUS, PION ZERO) REACTION ON LIGHT ELFMENTS iN THE $(3,3)$ RESONANCE REGTON

2404 PIONENUCLEAR TNELASTIC SCATTERING AT 59 MEV

, in:

71112

$\log$

75111

$75 x+6$

75,450

Thatic

The?

Thi.1.

71112

$\rightarrow \rightarrow a, n$,

Tha?

igition

i⿱宀⿻三丨口

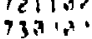

36,50

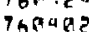

$74112 \mathrm{a}$

$\lim (\operatorname{lin} x \sin 0$

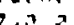

Magk, $D, C$

1627 LIMITS ON THE DIRECT PRODUCTION OF POSITRONS QY 256 - LNO BCH- MEV PROTOAS

TO3 OEAREH FCR DIREET LEPTON PRODUCTION IN PD COLLISIONS AT BOP MEV ANA $25 \mathrm{H}$ WEV

273.8

$771>10$

$761 \cdot 20$

$\rightarrow+2+20$ 
MOIR, D.C. (CONTO.)

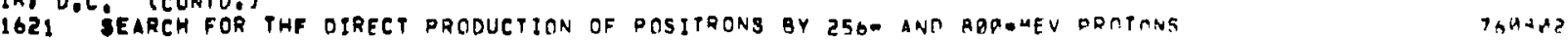

1544 THE ELASTIC SCATTERING OF LOW ENERGY PIONS ON DROTONS

MOORE, C.F.

1148 DIEITAL TECHNYQUE FOR NEUTRON-GAMMA PULSE BHAPF OISCRIMINATION

- TEST of Final gTATE nuCleon charge N) 12-CARBON (4. 44 MEV AND 15 . 11 MEV)

1133 COMPRIRON OF NEUTRON REMOVAL EROM Y LITHIUM OY PI OQ- WITH FOEF PIONGN CLEON RESULTS

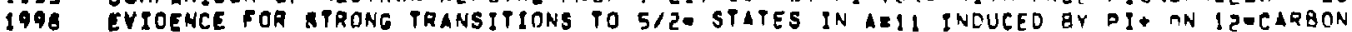

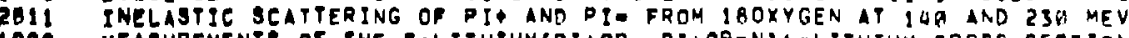

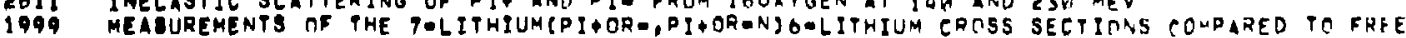
TONANUCLEON RESULTS

2406 NEUTRON OEFORMATION PARAMETER OY COMPARATIVE STUDY OF PI AND RIN INELASTIC SCATTERING

2091 PIAMESON INDUCED PROTON REMOVAL FROH OOBERYLLIUM TO THE D. QBI MEV ONE OLIS STATE OF RE

LITHIUM

2093 PION INDUCED NUCLEON REMOVAL FROM LIGHT NUCLEI

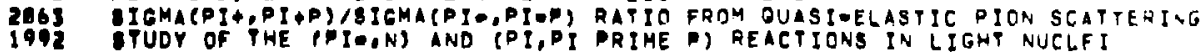

17ดรด9

Thl19:

$\operatorname{Tol} 2$ a

Tolisa

$978 \cdot 9$

97244.

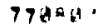

$76 ! 301$

Moone, R.H.

1653 MYOCAROIAL DORITRON SCINTIGRAPHY WITH SHORTELIVEO RURIOIUMEBZ THARA.

MORRIS, C

1140 DIGITAL TECHNIOUE POR MEUTRONGGAMMA PULSE SHAPE DISCRgMINATION TOHOA

$1: 52$ ATABLE LIGHT PULSER FOR GMIN STABILIZING PHOTOMULYIPL IER TUBES

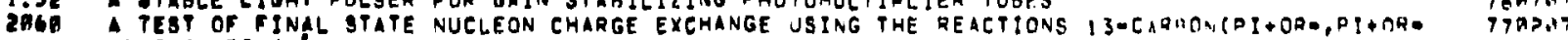

1133 COMPARTBON OF. NFUTRON REMOYIL MEV TOLITHSUM OY PI POR- WITH FREF OIONEN CIEON RESULTS

EYMARTON OF NFIITON REMOVIL MROM

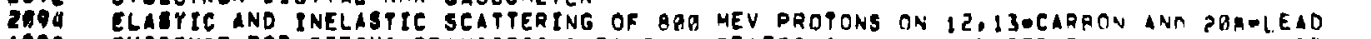

1090 EVIOENCE FOR STRONG TRANBITIONB TO 5/20 STATES IN AEII INOUCEO BY PI. ON 1?OEARBON

2811 INELABTIE BEATTERING OF PI+ AND PIE FROM 18OXYGEN $4 T$ 14O AND 238 MFV

2092 MEABUREMENTS OF MULTIPLE COULOMB SCATTERING OF DI OORM, HU OORE, ANDE + OR.

1999 MEALUAEMENTS TF PHE 7OLITHIUMCPI+ORM,PI+OR-NIG-LITHIUM CROSS SECTIONS C.OUPAREO TO FRFE

PION-NUELEON AESULTS

2106 NEUTRON DEFORMATION PARAMEPER BY COMPARATIVE STUDY OF PI+ ANO DI- INELASTIE SCATTERING

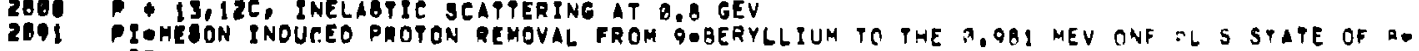

LITESTUK

2993 PON INOUEED NUCLEON REMOVAL FROM LIGHT NUCLEI

1992 STUDY OF PHE PPIO,N) ANO (PI,DI PRIME D) REACTIONS IN LIGHT NIJCLEI

216 THE JENIITIVITY OF PI. AND PIO INELASTIC SCATTERING TO DIFFERENCFS IV THF VEITRON AND PRÓTON AMPLITIIDE 


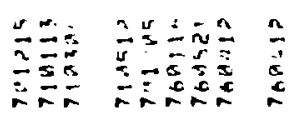

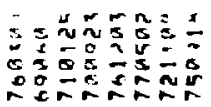

$\stackrel{n}{n}$

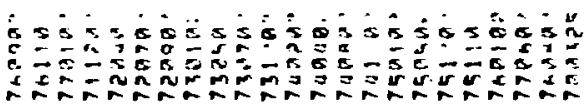

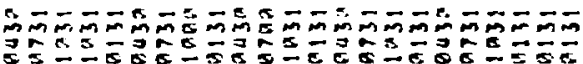

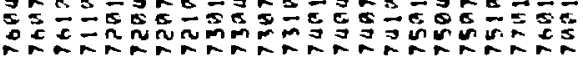

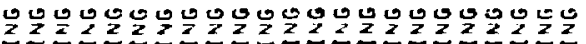

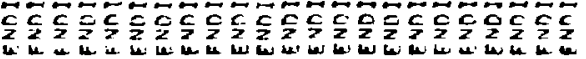

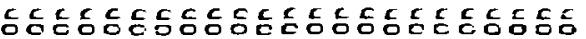

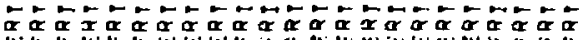

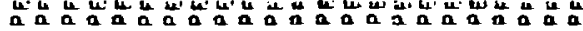

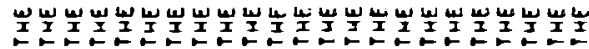

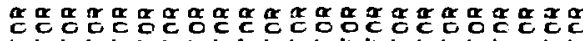

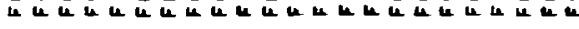

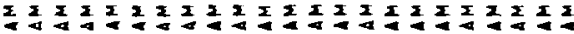

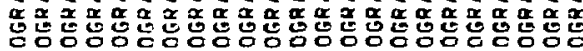

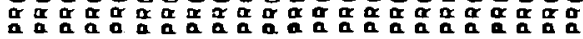

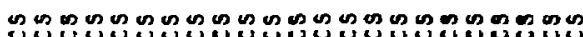

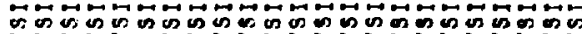

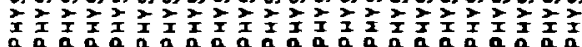

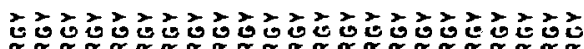

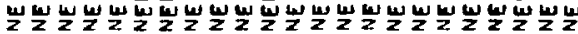

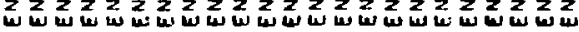

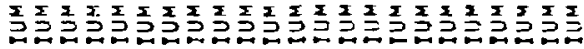

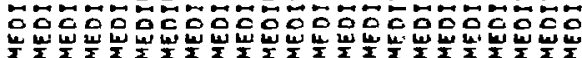

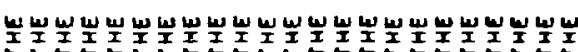

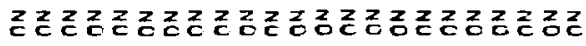

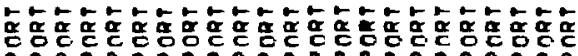

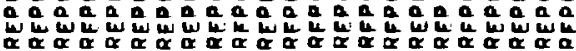

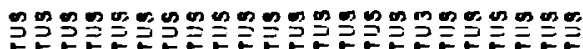

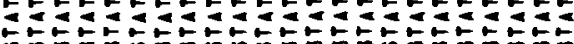

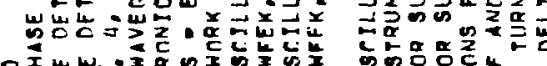

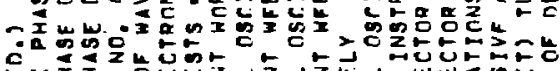

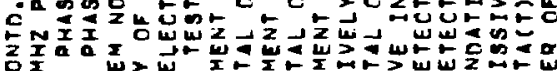

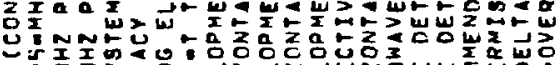

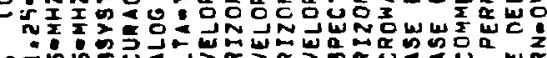

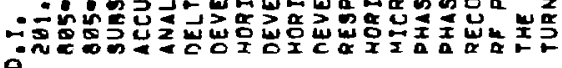

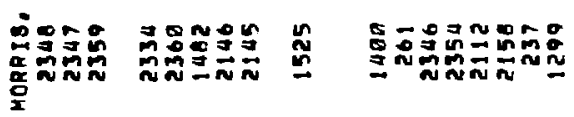

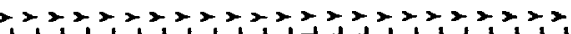

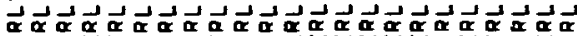

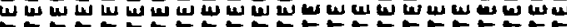

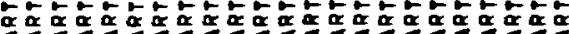
z 要 ง.ำ 
MP OIVISION (CONTDE)

62! QUARTERLY STATUS REPORT ON THE MEDIUM ENERGY PHYSICS PROGRIM FOR THE PERTON ENOING 7OIOJ! OUARTERLY STATUS REPORT ON THE MEDIUM ENERGY PHYSICS PROGRAM FRR TME PERTON FNDING T3UT3!

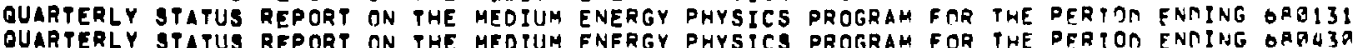
OUARTERLY STATUS REPORT ON THE MEDIUM ENERGY PHYSICS PROGRAM FOR THE OFRION ENITING OOAI31 QUARTERLY STATUS REPCRT ON THE MEDIUM ENERGY PHYSICS PROGRAM FOR THE DFRION ENDING THR 330 OUARTERLY STATUS REPORT ON THE MEDIUM ENERGY PHYSICS PROGRAM FOR THE PERION ENDING OTO43G OUARTERLY STATUS REPORT ON THE MEOIUM ENERGY PHYSICS PROGRAM FOR THE PERTON ENDING OTQU3A OUARTERLY STATUS REPORT ON THE MEDIUM ENERGY PHYSICS PROGRAM FOR THE PERTON ENOING OTP13 DUARTERLY STATUS REPORT ON THE MEDIUM ENERGY PHYSICS PRDGRAM FRR THE DERION ENDING G7973I QUARTERLY STATUS REPRRT ON THE MEDIUM ENERGY OHYSICS PROGRAM FRR THE PERTON ENDING OOGT3 QUARTERLY STATUS REPORT ON THE MEOTUM ENERGY PHYSICS PROGRAM FOR THE PERTOD ENDING TIO13I QUARTERLY STATUS REPORT ON THE MEDIUM ENERGY PHYSICS PROGRAH FOR THE DERPON ENOING TIQT3 QUARTERLY STATUS REPORT ON THE MEDIUM ENERGY PHYSICS PRDGRAH FOR THE PERYON ENDING TIOTSI QUARTERLY STATUS REPORT ON THE MEOIUM ENERGY PHYSICS PROGRAM FOR THE PERION ENOING GRIA3 OUARTERLY STATUS REPORT ON THE MEDIUM ENERGY PHYSICS PROGRAM FOR THE PERTON ENOING GQRIII TERLY STATUS REPORT ON THE MEDIUH ENERGY PHYSICS PROGRAM EOR THE PERTON ENDING 69 IIII QUARTERLY STATUS REPORT ON PHE MENIUH ENERGY PHYSICS PROGRAM FOR THE PERION ENDING GQR73 OUARTERLY STATUS REPORT ON THE MEOIUM ENERGY PHYSICS PROGRAM FOR TWE PERTON ENDITG OTIG3 OUARTERLY STATUS REPORT ON THE MEDIUM ENERGY PHYSICS PROGRAM FOR THE PFRTON ENOING 71 IDU3 QUARTERLY STATIIS REPORT ON THE MEDIUM ENERGY PHYSICS PROGRAM FOR THE PERTON ENDING GQDUJ OUARTERLY STATUS REPORT ON THE MEOIUM ENERGY PHYSICS PROGRAM FOR PHE PFRTOD FNDING OBIA3!

69121

$60866^{2}$

651104

600009

$071>015$

$7 !$. 45,

00 ith 20

00131

061132

\section{MPODIVISIO}

2305 201.25-MHZ PHASE DETECTOR SUASYSTEM - SPEC - 609015

2592 ACEEIERATOR ATSEARC

2358 ACCEPTANCE TEST DATA FOR MICROWAVE ASSOCIATES PHASE DETECTOR SUASYSTEM GIS.MHZ S/NI

2444 BIAS TRANBFORMER

2406 DATA-ACOUISITTON PROGRAM O

2303 ELECTRONIC PHASE SHIFTERS - O85-MHZ ANO 201.25-MHZ - SPEC - O91215

2353 EPAORF SYSTEM MINIMUH PEASONNEL OPERATION

2389 PSLAMENT PRANSFORMER - SPEC - GQP2QH

2398 PILAMENT TRANGFORMER : SPEC: 681022

2428 PILAMENT TRANGFORMER - SDEC - 6BIOAB

2434 FILAMENT TRANAFORMER - SPEC - TIRI

2043 PILAMENT TRANGFORMER - SPEC - 671127

2431 FILTER CHOKE - BPEC - 6APQOB

2AUZ PUSEDODISCONNFC SWITCH AND VACUUM CIRCUIT GREAKER

2430 HIGHOVOLPAGE CAPACTTOAS - 67900

2437 HIGH-VOLTAGE PAAACITORS - 6BIO39

2465 HISTOGRAMMING AND HISTOGRAM PLOTTING

2435 INDUCTORS (AEPORT NO, 13)

2436 INDUETORS (REPORT NO, 12)

2397 IOOLATION PALNGFORMER - SPEC - GALH2

2699 LAMPF PREPARTNG FON BECOND ASSAUIT

2389 LAMPP REPERENCE DRIVE LINE - SDEC - 690218

2A89 LOS ALAMOS. MANOY TIME FOR LAMPF

2379 MAGNET POWER MUMDLY PERERRMANCE SPECIFICATIONS

MOD OPERATIONG WITH MODSTO AND THE RBXOII HANOLEQ CC

099714

$75 \sin$

710.10.

$671: 24$

$768>31$

$601 ? 19$

7 aline

60024

OAI 5 द

Oning

T!Pla?

671129

bag.

ba119?

670.49

6月1 3

701110

671??

6า1

ontivis

9alap?

Q90210

7 blen

Thane! 
MPDDPVISION (CONTO,)

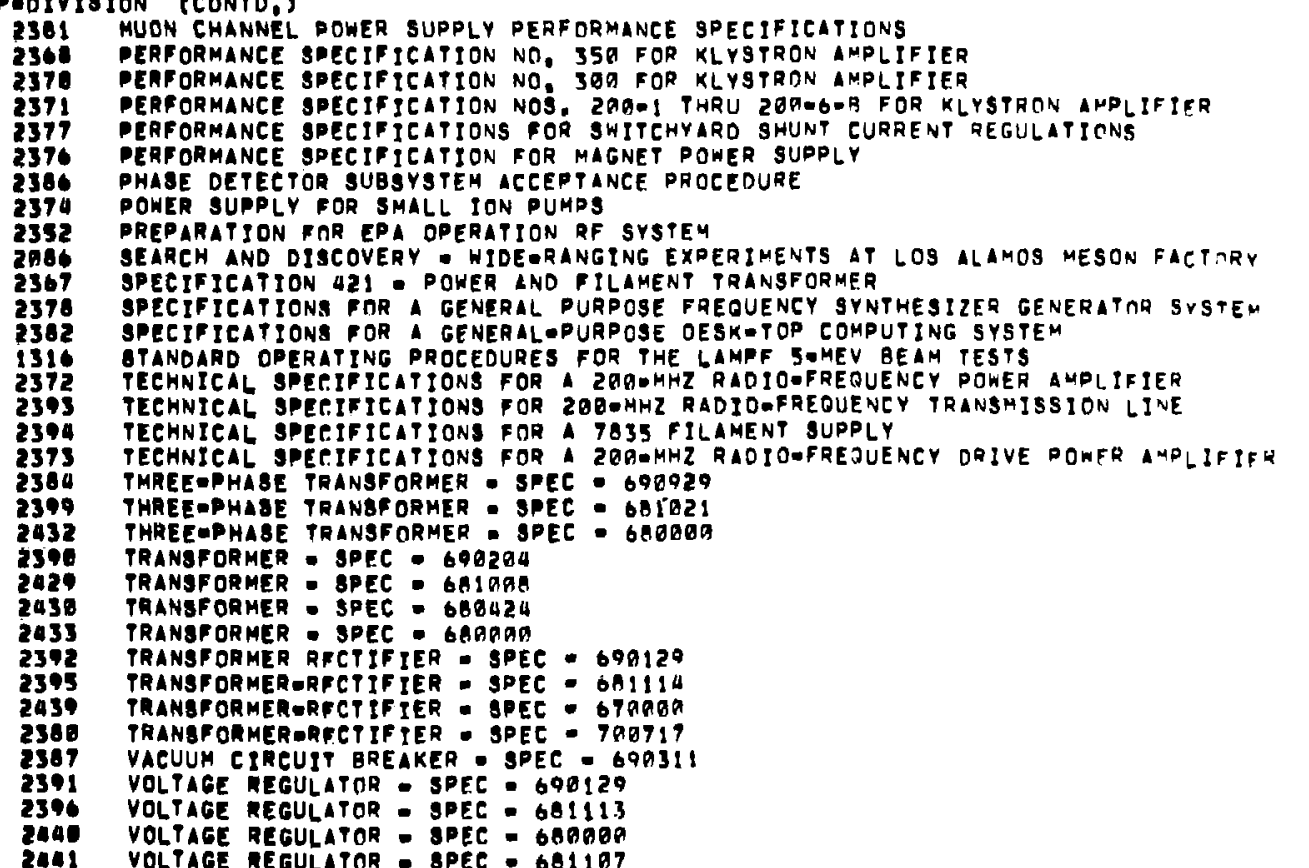

790513

600959

690129

671518,700611

7agis

791210

69953, 79324,

681194

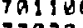

77020

GR1?1

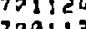

Torit?

tous 5 क

6ค11

(6)

onitis

6.

(6)

6892

6e日,

6.9.

GA1 625

$\cos 10$

$\cos 130$

691110

678.04

$\cos 71$ ?

$\cos x \sin$

600150

GR

GPIII?

691107

$750: 15$

APOTON LINEAR ACEELERATOR FOR USE IN A MOBPITALOBASEO PION THERAPY FACILITY

HUELLER, D,W.

231 BEAM MEABUREMFNTS ON THE HIGHOINTENGITY PROTON INJECTOR OF LAMDF

6 OEAM PROFILE MONITORING ANO ANALYSIS BY TELEVISION

DEVELOPHENT TN THE INJECTOR GYSTEMS

1AME CLECTRON ANALOG TESTS OF PROTON LINEAR ACCELERATOR STRUCTURES

2907 INJECTOR PROGRAMB

a96 INBTRUCTIONE TOR CHANGING ION BOURCEB (3RD REVISION)

1447 LAOL EXPERIENEE WITH A DUOPLABMATRON FEEDING A TSEOKV EXACT PIERCE COLIH.

aser MP DIVISTON SFMINAG

323 OASERVATIONB AND CALCULATIONS FOR A DUOPLABMATRON WITH EXACT PIERCE EXTRACTION
72054.

7100.

690518

65064.

$60052 \mathrm{~h}$

$72174 \%$

$67021 \%$

$67021 \%$ 
MUELLER, D.W. (CONTD,

325 OPERATION AND PERFORMANCE OF THE HIGHOINTENSITY PROTOY INJECTOZ CF LAMDF

439 OPERATION OF THE ION SOUREE

665 OPERATION OF PHE LAMPF T5A-KEV INJECTOR

BQI PART AI INJEETOR COMPLEX, PART BI ION SOUREE TEST STANO

227 PERFORMANCE OF THE LAMDF H- INJECTOR

324 RMS EMITTANCE ANO THE LAMPF BEAM

2506 SPELLLAN POWER SUPPLY FIRE

234 STATUS OF THE LAMPF HQ INJECTO2

1444 PEST OF LASL ION SOUREE WTTH $7580 K V$ ACCELERATING CTLUMN

1443 TEST OF LASL ION SOURCE WITH 2000 -KV PIERCE ACEELERATIVG COLUMN

1442 THE LASL HIGHAOUTY FACTOR ION SOUREE

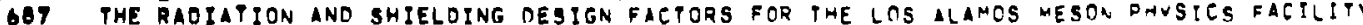

72118

$713 n+4$

$0 \rightarrow \sin 1$

$1,1.5$

7.

$759 \cdot 4$

730050

$698 \mathrm{ant}$.

OAR52:

MUELLER, R.O.

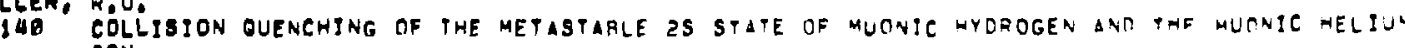

750.9 ION

2292 ON THE POSBIBILITY OF APPLIEATION OF PERMANENT MAGNET PUADRLOOLES IN TWE MIGH EVERGY PROTON LINEAR ACCELERATORS

MUTCHLER, 6.3.

2897 ANGULAR OISTRTBUTION FOR PO GOESTO 3HE PI ZERO REACTION AT TPEQAF HEV

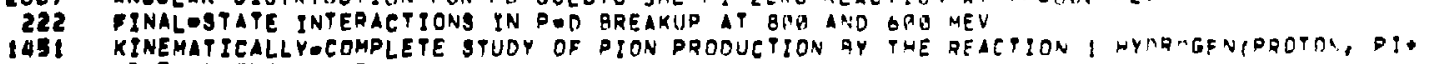
PROTONINEUTRON AT BGD MEV

210 NUCLEAR-COULOMB TNTERFERENCE IN PI *OR- 16 OXYGEN SCATTERING

1981 MION PRODUETINN BY BDO MEV PROTONS FROM GILITHIUN

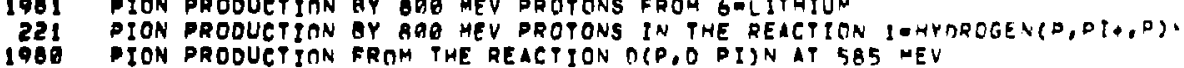

DERACYIONS AT BRA MEV

-iON PRODUETION VIA A PQD GOESTO DQPI OUASIOFREE PROTONS IN OEUTERTUM

IO3 PION-NUCLEAR POTAL CROSS SECTIONS NEAR THE 3/2, 3/2 RESONANCE

19.3 PONONUCLEUS CORWARO SCATTERING AMPLITUOES NEAR THE $3 / 2,3 / 2$ RESCNANCE

2673 PIONANUCLEUS SMALL ANGLE ELASTIC CROSS SECTIONS NEAR THE 3/2, 3/2 QFSCAMCF

2974 PIONANUCLEUS TOTAL CROSS SECTIONS NEAR THE 3/2, 3/2 RESONLNEE

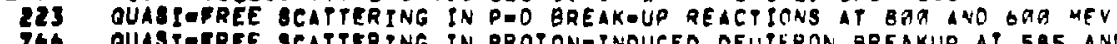

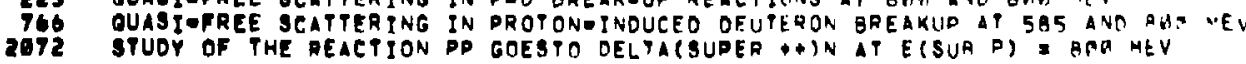

MAGAMINE, $K$

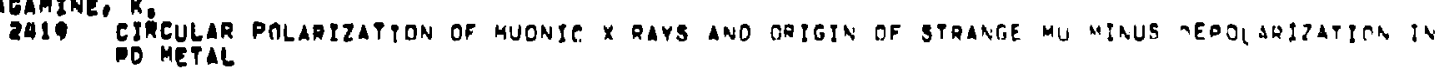

NAGLE, D.E.

ISHT A MEASUREMENT OF PARITY VIOLATION IN PQOTINAPROTON SCATTERING AT IS UE. 
NAGLE, D.E:, (CONPD,

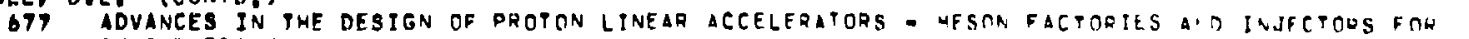
SYNCHRDTRONS

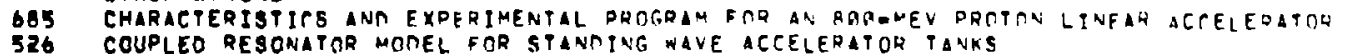

2427 CROSS SECTIONS FOR PI + NUCLEUS GOESTO PI +OR-... FOR TUA-MEV DEOTO.S

572 CROSS SECTIONA FRR P + D (GOESTO) D + RHO + FROM $4 . A$ TO 12.3 GEV/C

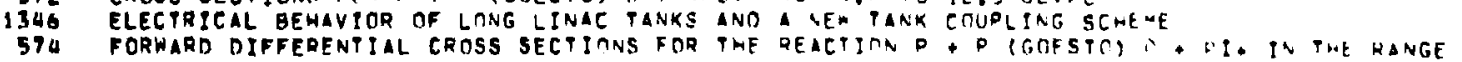

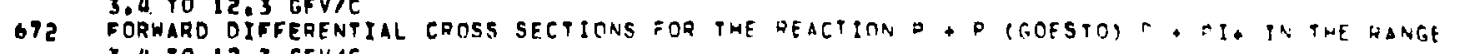

606 MIGH ENETGY PROTCN LINFAR ACCELERATOP

917 LAMPF NEUTAINO FAC TLITY PROPOSAL

909 LIGHT ION LINACS FOR MEOTCAL AOPLICATIONS

1542 LIMIT DN PARITY NONCONSERVATION IN PANUCLELS SEATERTNG

1620 LIMIP ON PARITY VIOLATION IN P=NUCLFIIS SCATTERING AT G GEV/C

1627 LIMITS ON PHE OIPECT PRODUCTION OF POSITRONS BY 256. ANO BUR-MFV PODTOS

573 LINEAR ACTELERATOR FOR PIONS

LINEAR ACCELERATOR FOR UNSTABLE RARTICLES

LINEAR ACCELERATORS FOR DROTDNSI NEW DEVELORMENTS

LINEAR ACCELERATORS FOR PROTONS

LONGITUDTNAL AND RAOIAL MOTION OF PROTONS IN THE LINAC

MACHINE RF DOWEQ REQUIREMENTS - 2 G MC SECTION

MESON FACTORIFS - 1967

MESON PRODUCTTINN

NOTE ON COUPLFO CIRCUIT PROALEM

PARITY NONCONAERVATION IN TWO-NUCLEON SYSTEMS

PARITY VIOLATTON IN PAP IND PGE REACTIONS

PERTURAATION TMEDRY FOR THE DOUALY PERIODIC CHAIN

PRDDUCTION OF CHARGEO PIONS AY T3PAMEV PROTONS FROM MYDROGEN ANO SELLCTE" ITLE!

DROOUESION OF PIONS AY 7LA MEV PROTONS ON VARIOUS TARGETS

-RDGRESS IN RIIILOING AN BgROMEV DROTON LINEAR ICCELERATOR

DROSPECTS FOR HIGH CURREMT ACCELERATORS

RESEARCH HIGHI IGHTS FOR THE FIRST HALF OF 1976

SEARCH FOR A PARTTYOVIOLATING CONTRTBUTION TO NUCLEON-NIIELEON SCATTERI:O

SEARCH FOR OIAECT LEPTON PRODUETION IN PP COLLISIONS AT GAG MEV ANO 25 A WEV

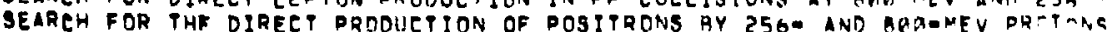

SEAREH FOR VIOLATION OF THE REFLECTION GYHMETRY IN PUD SCATTERINF,

SOHE DISCAETE TRANGFORMS AND THETR CONT INUNUS ANALDGISES

BTEADY STATE REHAVIOR DF A RING OR OF A CHAIN OF COUPLED CIRCUITS
TESP OF PARITY CONGERVATION IN PAD SCATTERING

PHE ELABTIC STAPTEOING OF LOW ENERGY DIONS ON DROTONS

575
1503 THE LABL MESON FACTORY

2425 UPPER LIMIT FOR A GMALL PARITY VIOLATING ASYMMETRY IN POP SCATTERING AT IS UFV

$65+1,1$.

67110

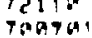

osians.

Yang.

onATA:

$65 a \cdot 4$.

$7112 \mathrm{OH}$

75oma

75 namh

Tharle

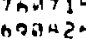

6al?b.

670910

o3tas:

6 bant?

bra ca.

Tapap

huil? ?

Yeana?

1CPAla

$64123:$

1? 120,

71ดวa?

ORI HO

thing

$73 x \cdot a$

7 ha. 25

7 bilni?

Thloz

651. Al

करांदा

701110

TaAnas

oup?

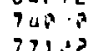

NAIVIR, F,J.

2467 CAHAC RASED CMMPUTERMCOMPUTER COMMUNICATIONS VIA MTCQODQNCESSOR DATA LINAS

2457 NEQRGA TO ORPFC OG7 INTERTACE 
NANN, H.

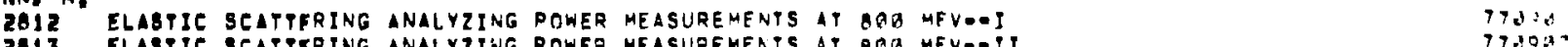

2013 ELASTIC SCATPFRING ANALYZING POWEA MEASUAEMERTS AT BRG MEV-OII

BQ8 P * 13,12C, INELABTIC BCATTERING AT P.8 GEV

NATOHITZ, J,B.

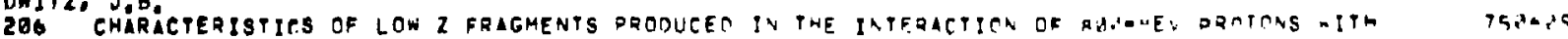
URANIUM

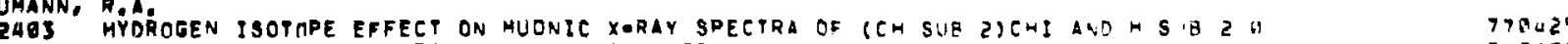

2335 PRDPOSAL FOR AN ONELINE MASS SPECTROMETER FACILITY IT LAMPF TO STUOY PQOAJETS OF

1903 GECENT EXPERIMENTAL DESULTS OF THE MESIC CHEMISTRY OQOGQAN AT LAMPF

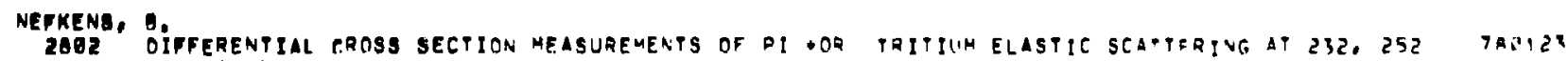

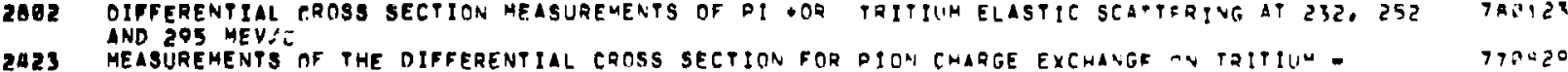

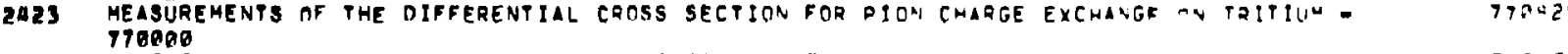

242A MEASUREMENTS OF THE DIFFERENTIAL CROSS SECTION FOR DION CHARGE EXCMAGF MV TRITIM - TARIZT

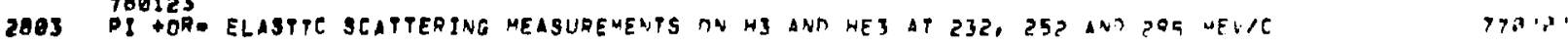

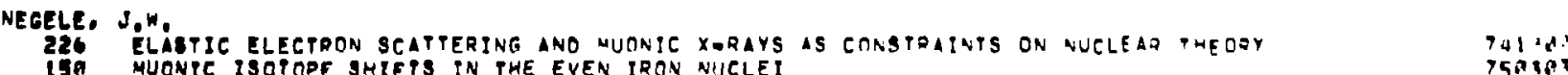

NEILOON, B.S:
IQU THE ISBOERBSUM GOESTO ISB-HOLMIUM DECAY SCHEME ANO OLSUB BETA) VALUE

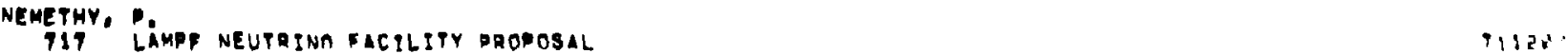

NEWCOM. Mo.
2Bu

NEHELL. R.H.

2364 CCCETANCE TESTING

2697 CALIBRATION PROCEOIIAE FOA BO5OMHZ SECPOR HIGM VOLTAGE

2706 HIGH VOLTAGE CABLE FAULT DEFECTOR SYSPEM CALIBAATICN PROCENURE

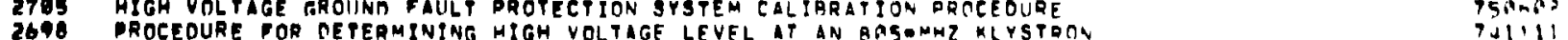


NEWELL, R, H. (CONTO.)

2902 PROCEDURE FOR HY METERING SIGNALS TO IVR ANO CCR TGB5RG

2901 PROCEDURE FOR OUAD MAGNET SUPPLY CALIBRATION

2917 SPECIFICATIONG FOR HIGH-VOLTAGE CADACITORS

TTANOARD OPERATPNG PROCEOURE G

224 STANDARD OPERATING PROCEDURE KLYSTRON FORMARO DOMER CRYSTAL CALIARATIOA

22 STANOARD OPERATING PROCEDURE IDE325B PROGRAM REMOTE OPERATING PROCEOURF TUEIZI

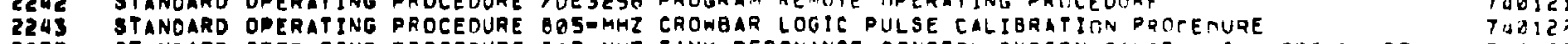

2253 STANDARD OPERATING PROCEDURE GOS MHZ TANK RESONANEE CONTROL SYSTEM GALIBAATIMA PROCE RURE

2250 STANOARD OPERATING PROCEDURE MAINTENANCE ANO REPAIR PROCEDURE NO. \& KGYSTRRN REPLACEMENT TARSIA

NEWMAN, R,D.

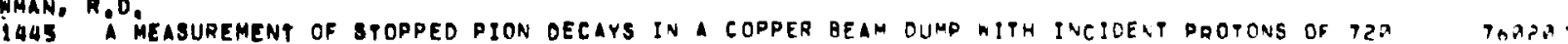
MEV (A CALIBRATION OF PHE LAMPF NEUTRINO SOUREE INTENSITY)

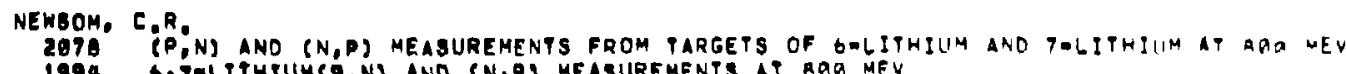

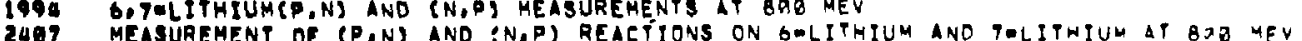

779612

$\operatorname{tingish}$

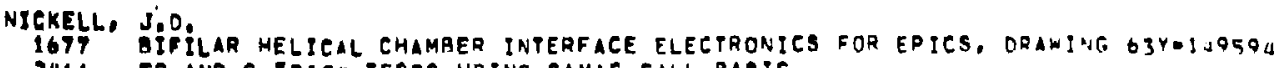
EG ANO G TOIGU TESTS USING CAMAC CALL BASIC TI 733 KSR I/O TERMINAL

1501 MODIFICATION OFF SUPER NOVA IPIN

2062 NOVA COMPUTER THERMAL

2463 RO-105 PDWER AUPPLY

1931 SPECIFICATIONA FOR IS CHANNEL CAMAC STEPPING MOTOR CONTAOL MODULE

1931 SPECIFICATIONA FOR IS CHANN
1575 TANKS WATER AI ARM SYSTEM

1737 TECHNICAL OESTRIPT ION 733ASR CASSETTE TERMTNAL OPERATION WITH CALL BASTC

1946 TECHNICAL DESTRIPTION - LAMPF BIFILAR HELICAL CHAMBER CAMAC DATA ENEODER. DOAMING OBYE 140505

1952 TECHNICAL DEBCRIPTION GOIN DATA MUS. ORAWING G3Y-190093

1957 PECHNICAL OESCRIPTION - LAMPF OUAL TWENTYOFOUR CHANNEL PULSE SOURCE. MRANING O3YM149507

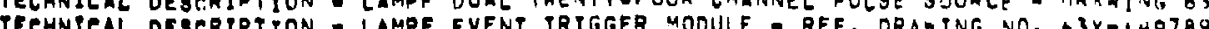

TECHNICAL OESCRTP

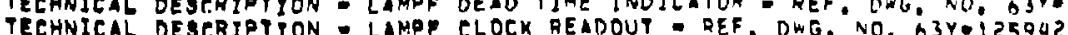

TECHNICAL DEERTPTON D LAMP CLOCX READOUT

TECHNICAL DEgCRTPYION DUAL AOC ITF CAMAC

TECHNICAL OEBCRIPTION - OUAL AOC I/F CAMAC - REF, DWG. NO. O3Y-149BRE

TECHNICAL DESTRIPTION - NIM POWER CONTROL CHASSIS . REFER TO ORANIMG O3Y-149G13

TEEHNICAL OESCRTPTION - STEPPING MOPOR BRAKE ACTUATOR

TECHNICAL OEGCRIPYION - CAMAC TEST CONTROLLER MODULE MELICAL CHAMBER - EDICS DEARIMG G BY 149596

1736 TEBT PROEEDURF STEPPING MOTOR BRAKE ACPUATOR O3Y-149608

IOI4 TEST PAOEEDURPS FOA LAMPF EVENT TRIGGER MODULE

1942 TEBT PROCEDURFS POR LECROY NIM UNITS

739500

76201

$74 x+16$

Thishon

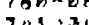

7497

7.

$105 \times 50$
$15015 n$

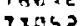

$70125 \mathrm{Sh}$

$7+4 \sin$

75an!

751.21

Tali:

inis?

the?
thu?

75i?

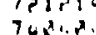

72023

72,420

$\cos 2$

NIEBCHMIOT, E.O. 
NRESCHMIOT. E.B. (CONTO',)

639 INTERLABORATORY COMPAAISON OF BPALLATION REACTION CAOSS SECTIONS FOR INO AN COPPER atTA THE B. 59ROHEV PROTONS

NIETHAMMER, A,C,

198 DATA ACQUISITTON SOFTWARE FOR A MWC SPECTROMETER

NIBHIKAHA, P.

1427 ROUNO TABLE OTSCUSSION ON SPACE CHARGE AND RELATED EFFECTS ORISP

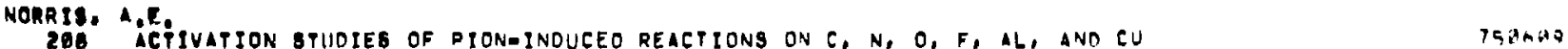

208 ACPIVATION BTIDIES OF PTON-INDUEEO REACTIONS ON C. N, O, FE ALA ANO CU

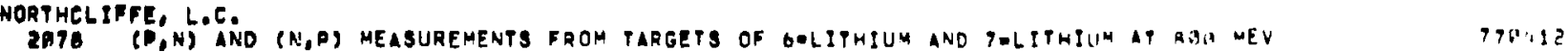

1994 6.7-LITHIUM(P.N) AND (N,P) MEASUREMENTS AT 8 QR MEV

SUREMENT OF (P,N) ANO (N,P) REACTIONS ON OOLITHIUM ANO TELITHIUM AT B.D MEV

MEASUAEMENT OF THE GODEGREE NEUTRON SPECTRA FROM G47 AND BGR MEV PROTO: BOMAARMEAT OF

750150

203 MEASUREMENT OF TME ZERO OEGREE NEUPRON SPEETRUM FROH THE REACTION OP GAESTO IP OI+ AT T

$750 .+1$

NEUTRON PHYSITS AT LAMPF

1634 NEUTRON SPECTRA AT ZERO DEGREE FROM (P,N) REACTIONS ON 9-BERYLLIUM, 12-CATAON ANO 5 TO

$70090 \mathrm{an}$

70909

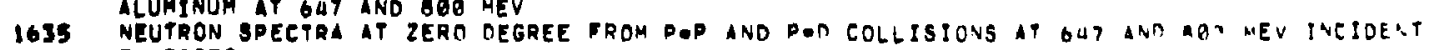

760705 ENERGIES

1969 NEUTRON SPECYRA AT ZFRO OEGREE FROM PROTONAPROTON COLLISIONS BETWEEN 647 190 8.15 HEV

200 NEUTRON SPECTRA FROM SAG MEV PROTONS ON QORERYLLTUM

FOR

1646 PION PAODUETION IN NEUTRONADROTON COLLIS:ONS

1970 DRECIBION MEABUREMENT OF NGP CMARGE-EXENANGE CROSS SECTgON AT G4T MEV

202 DRECIBION MEABUREMENT OF NO CHARGE WEXCNANGE CROSS SFCTION AT GUF MEV

2409 OUABELABTIC CHARGE EXEMANGE IN NO GOESTO PNN TT 794 MEV

1639 PHE NEUPTON OHYSICS PROGRAM AT LAMPF

NOYAK, J.K:

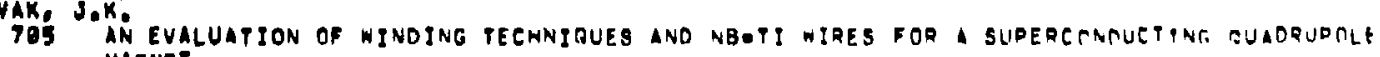

$176+0^{x}$

$74 x^{2}$

$750 \cdot 9 \cdot$

$7 a 1>20$

70 inise

124.14

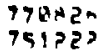

TOS MAGET

673 QUADRUMOLE FOCUSING MAGNET

25IS RADIATIVE NEGATIVE PION CAPTURE IN LIOUID NEON

298 RADSATIVE NEGATIVE PION CAPPURE IN LIOUID TRITIUM

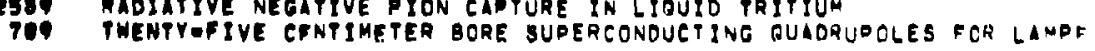

P.ANA

ofter.

77059
71050

774020

Piting

MUMANN, R.A. 
NUMANN, R,A. (CONTD.)

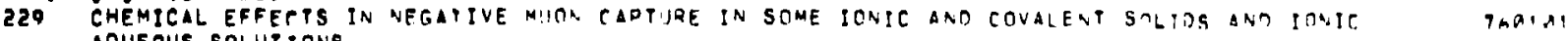
AQUEOUS SOLUTTONS

ORIEN, H.A., JR

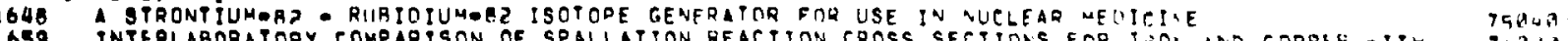

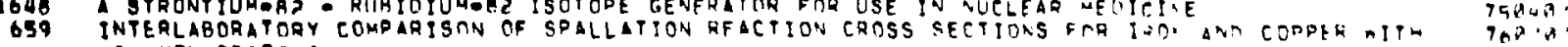
5900MEV OROTONS

1653 HYOCARDIAL POAITRON SCINTIGRAPHY WITH SHORT-LIVED RUATOIUIA-RC

IGII RADIOISOTDPE PRTDUCTION IT LAMDF

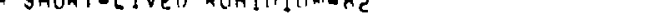

RADIOISOTOPE PRODUCTION AT THE LIS ALAYNB MESON PHYSICS FACILITY FOR MEDICAL ISE

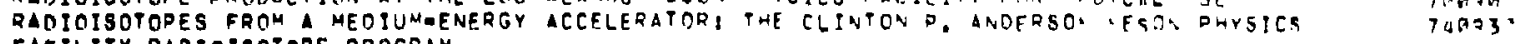

aACILITY RADINISOTOPF. PREGRA

1631 SPALLATION CROBS SECTIONS ANB THE LAMDF MEOICAL RACICISOTOPE PROCRAM

1654 STROMTIUMOAZ - RUBTOIUMOAL RAOIOTSNTOPE GENERATOR

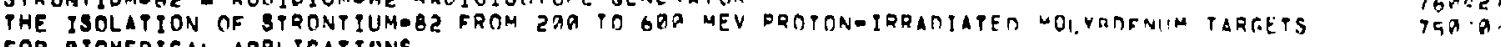

1647 THE RECOVERY OF SPALLOGENIC. CARRIER OFRE YITRIUM-RA FOR RAOIOISOTHPIC PWDTONEITRON SOURCES

OBST, A, W.

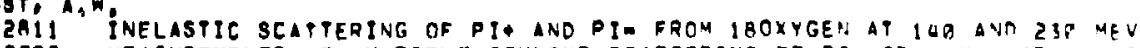

2992 MEASUREMENTS TF MULTIPLE COULOMB SCATTERING OF PI +OA-, MU +OR-, ANO E . P.

MULTI-COUNTER CHERFNKOU COUNTER FOR EPICS SPECTANMETER

EAO3 MULTICHANNEL AISC CERENKAN CONNTER FOP EPICS DION SPECTROMETER

NEUTRON DEFORMATION PARAMETER AY COMPARATIVE STUNY OF PI AND DI- INELASTIS ECATTERITG

DCINPILLATORS AND PHDPOMULTIPLIERS O SOME TESTS FOR EDICS TUNE-UO

OCONNELL, S,S.

554 NEUTRino OISINTEGRATION OF THE DEUTERON AT LAMPF ENERgIES

$75 \pi \sin$

ETIING, S.O.

596 BEAM SPILL HONITOR SYSTEM SOR AN BQU MEV PROTON ACCELERATOP

592 A BEAMESPILL MONITAR FOR LAMPF - 71969A

612 DEAMABDILL MONITOR FOR LAMPF - 7GMIII

77 map.

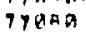

792131
790391

790.9 .

Ynllae

Ynd

DGARO, A.E.

1653 MYOGARDIAL POAITRON SCINTIGRAPHY WITH SHORTALIVEO RURIOIUMARE

1652 RADIOISOTOPES FROM A MEOIUMEENERGY ACCELERATORI THE CLINTONP, ANDERSON WESON DHYSICS

1651 SPALLATION CROSS SECTIONS AND THE LANPF MEOICAL RADIOISOTHDE PROTRAM

$\sin 30$

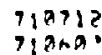

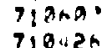

710.24
70511

OHLREN, G.6. 
OHLSEN, G,G, (CONTD.)

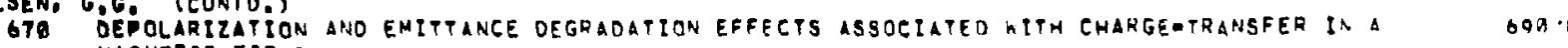

145 MAGNETIC FIELA

2676 OPERATION OF A RADIOAFREOUENCY NUCLEAR SPIN FILTER

SEARCH FOR A PARITYOVIOLATING CONTRIBUTION TO NUCLEONONUCLEON SCATTERIVG

2584 TRANSFER MAPRPX METHOD FOR CALCULATING SPIN ABERRATIONS IN THE TRANSPORT OF DOLARI7EN BEAMS

OHLSEN, S.D.

1481 LAMPF SPECIFICATION FOR BIGITAL CONTROL COMOUTEQ SYSTEM

IIS5 BPECIFICATION FOR DIGITAL CONTROL COMPUTER SYSTEM

150.20

$0901 \%$

730.6

$71+19$.

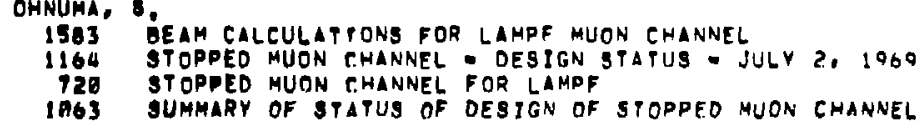

OOSFEN, J.

124 CROSS SECTIONA FOR (PIQ,P) REACTIONS ON Q-BERYLLIUM, IZ-CARBON, $\triangle N O$ 1 1 -OXYGEN

1558 CROBS SECTIONA FOR (PI+.P) REACTIONS IN O-LITHIUM, Q.BERYLLIUM, I COCARAO\%, AND IG-AXYGEN

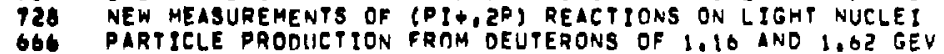

602119

60125.

OoTHOUDT, M.

29Q9 ELASTIC AND INELASTIC SCATTERING OF RGO MEV PROTONS ON 12,13 -CARRON ANM PQROLEAD

2012 ELASTIC SCATTFRING ANALYZING POWER MEASUREMENTS aT BOQ MEVE-I

WE MEASUREMENTS AT BQD MEVMII

2000 - 13,12C, INELABTIC BCATTERING AT G.B GEV
ORTH. C.J.
229 CHEMICAL EPFECTS IN NEGATIVE MUON CAPPURE IN SOME IONIC AND COVALENT S LLION AND IONIC 171 EXEIPATION FUNCPIONS FOR THE IZUCARBONEPIQOR-,DI NIIIUCARBON REACTTONS OVER THE REGITN OF
THE $(3,3)$ RESTNANCE.
EFFEC ON MUONIC $X$-8AY SPECTRA OF KCH SUP JJCHE AND H SIH Z
239 IDENFIFICATION OF PHORIUMO236
G59 INTERLABORAT ORY
1011 RADTOISOTOPE PRODUCTION AT LAMPE
TOE3 RECENT EXPERIMENTAL AESULTS OF THE MESIC CHEMISTRY PROGRAM AT LAUPF
231 SPALLATION OF EOPPER BY IOA MEY PI(+OR I) AND 349 MEY PQOTONS

OnTM, H. 
ORTH. H. (CONTD.)

GQAI A NEN HIGH PRFCISION MEASUREMENT OF THE MUONIUM RYPERFINE STRUCTURE INTERVIL DELTA NU

71 OEPOLARIZATION OP NEGATIVE MUONS IN HELIUM GASI SEARCH FOR MUONIC HELIIIM LTOM

181 MUDNTUM PORHATION IN NOBLE GASES AND NOBLE GAS MIXTURES

IBO! PAECIBJON MEASUREMENT OF MUONIUM HFS INTERVAL AND MUON MAGNETIC MOMENT

168 PRECISION MEARUREHENT OF 1S(SUB 1/2) MUONIUM HYPERFINE STRUCTURE INTERVAL DELTA NU: PART I

169 PRECISION MEABUREMENT OF ISESUB 1/2) MUONIUH GYPERFINE STRUCTURE INTERVLL DELTA NU: PART "11

OTTESON, D.H.

1563 ABSOLUTE CAOSA SECTIONS FOR PRODUCTION OF PROMPT NUCLEAR GAMMA RAYS AY FAST PIONS

1580 ALPHA ANO MULTINUCLEON REMOVAL PROM SBONICKEL AND GP-NICKEL BY FAST PIONG

1561 PAOT PION INDUEED PROCESSES IN COMPLEX NUCLEIS CROSS SECTION AND ALPHA RFMOVAL

1382 FAST PION INDUICED PROCESSES IN COMPLEX NUCLEII PROJECTILE SYSTEMATICS

15.S FAST PION INOHCEO PROCESSES IN COMPLEX NUCLEI I SYSTEMATICS IN BRODUCT NUCLEI

1562 SISTEMATICS OF PION ANO PROTON INTERACTIONS WITH NICKEL NUCLIDES

75abis

$150 \sin$

75940.

75949

$75(4,1)$

750008

PAcheco, C.

204 A MULTIWIRE PROPORTIONAL CHAMBER SPEETROMETER FOR NUCLEON=NUCLEON EXPERIMENTS

731802

aciorTI, $M_{0} A$.

371 IOE MEV GEAM AND ACCELERATOR TESTS

243 AGSORBEO DOSE AND RAOIATION OUALITY FOR GACKGROUNO RIOIATION FROM A NEGATIVE PION GEAM

10

710019

711183

711117 ACCELRATO PFRFORMANCE TESTS " THIGS

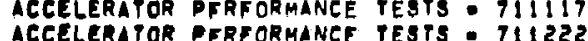

ACCELERATOR PFRFORMANCE TESTS: 711222
ACCELERATOR PFRFORMANCE TESTS - 711019

ACCELERATOR PFRFORMANCE TESTS T TIIRIQ

1061 ANALYSIS OF SPLICON AND GERMAN
1214 BEAO PERTUABATION MEASUREMENT

BEAO PERTUABATION MEASUREMENT

BEAM MEASUREMFNTS ON THE HIGHOINTENSITY PROTON INJECTOR OF LAMPF

BEAM MEASUREMFNTS ON THE FIRST TANK OF, LAMPF

BEAM SHAPING AND DEPTH DOSE ADJUSTMENT

BIOMED SHIELO DDOR REQUIREMENTS

BIOMEDICAL SHPELDING REQUIREMENTS

136 CALIGRATION OF ANALOG DATA CHANNELS PACIOTYI

$113 !$ CHANGES TO BHYELO HALL CONFIGURATION FaR MAZE IN BjOMEO STUBOUT

1139 DEVELOPMENT OF OPERATIONS INO REQUIRED SOFTWARE SUPPORT - PACIOTII

1139 OATVE LINE MEASUREMENTS ON TINK 1

2533 H+ BEAM CHOPDFR

1140 HIGH POWEA BEAM STOP

12S OPERATION AND PERFORMANCE OF THE HIGHOINTENSITY PROTON INJECTOR OF LAMAF

1130 PEASONNEL SAPEPY IN BIOMED TREATHENT ROOM - 72122

1112 Pensonnel aAFPY IN BIOMEO tRCATMENT ROOM - 73062

963 PON GENERATOR FOR MEOICAL APPLICATIONS

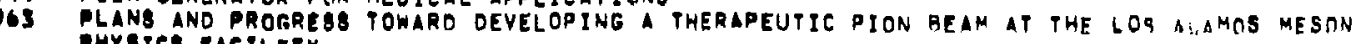
DHYICS PACILTIY

DOWER CAPABILTTY OF CERAMIL INGULATED CURRENT COLLECTOR

1130 SPILL MONITORA

BPILL MONBTORA IN AOEABT

11
11210

738516

79092

72050

$714 \sin$

76018

$7 ? 1 ? 10$

$75^{4}+1$

$7 \operatorname{sos} 30$

$73 \times 72:$

72821.

73111

70 मqe

$7 \because 120$

72119.

72152

$730 \mathrm{~B}$

$750 A B 1$

TJUSम0

71419

750.67

75452 ? 
PacioTrs, M.A., (CONTO!)

370 SUISTYSTEY REWORK SUGGESTEO BY 5 MEV OPERATION

2012 THE APPLICATINN OF PROTONS PO COMPUTEO TOMOGRAPHY

998 TUNING OF THE FIRST SECTION OF THE BIOMEDICAL CHANNEL AT LAMPF

150. WHOLE OODY OOSE FOR PATIENTS TREATEO WITH PIONS

Ta?d?

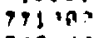

ios?

PALERMO, S.D.

251 BEAM MEASUREMFNTS ON THE HIGHAINTENSITY PROTON INJECTOR GF LAHPF T2A5G,

PALEVBKY, H,

2BES "P:" 13,12C, INELASTIC SCATTERING AT Q.8 GEY T7JOA

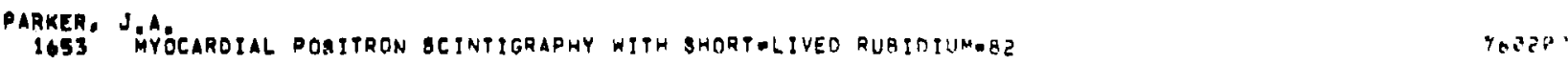

PARKER. J.R.

1614 BOSOHHZ MOOULE BEAMLESS RADIATION

590 A BEAM SPILL MONGTOR SYSTEH FOR AN BOB MEV PROTON ACCELERATOR

BEAM SPILLL MONITOR SYSTEM FOR AN BAD ME

AEAMESPILL MONITOR FCR LAMPF TIIOB

GEAM-BPILL MONITOR FOR LAMPF - TAG311

TALK TO THE AEC ADVIBORY PANEL - ACEELERATOR RADIATION SAFFTY (ADARS)

ACTIVATION CONTROL AT LAMPF

ACTIVATION CONTROL AT LAMPF

AN

ANALYBIS OF LAMPF INJECTOR OPERATION FROM MEASLREMENTS OF BREMSSTRAMLUAS DOORUCEO RY BACKO STREAMING ELEPTRONS

1617 STREAMING ELERTRONS

TTORING FOQ INTYISAL OPERATHON

1610 AREA CRADOSAEE GYSTEM - MANPOWER AND COST ESTIHATE

695 BEAM PROFILE MONITORING ANO ANALYSIS BY TELEVISION

1610 BIOMED RADIATON SAFETY SYSTEM

1887 CALIERATION OF NULL INOICATOR FOR RAYTHEON $250 \mathrm{KV}$ SUPPLY

1312 CALIBRATION OF THE BEAMGSPILL CONTROL SYSTEM AT LAMPF

1863 DESCRIPTIVE SPECIFICATION FOR BEAM SPILL MULTIPLIER-PHOTATUBF DOWED SUDDIY

DESCRIPTIVE SPECIFICATION FOR DOCUMENT READER IELEVISION CAMERA

ITTANCE OATA SYBTEM DROPOSAL

EMITTANCE OATA

OOF LOQUIO HYOROGEN IN OREMSSTRAHLUNG BEAH ERTM TUNESTEN

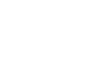

$310 \times 5$.

Tition?

tis

$71,0,5$

7.3.

73110

oson.

in

$\sin 2 !$

$\log 10$

?istat

(3)

$67+21 \%$

$350 \mathrm{ng}$

7

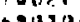

$691^{2} 196$

TARGET AT END OF

1603 EPL RADIATION SURVEY OUSE OF MODEL PNC-I (PORTABLE NEUTRON COUATER)

1635 EPL TELEVISION CABLE PLANT AND RACK SPACE REOUIREMENTS

1608 EXFENSION OF FPA RAO-BAFF SYSTEM TO EPA EXPERIMENTAL AREA

GENERAT ION OF RAD IOACTIVE HR AND NOXI

596 HIGH INTENBITY ELECTRON ACCELERATOR RIDIATION HAZAROS

1320 HIGH POHER PEATS ON A CLOVERLEAF CAVITY

1613 INJECTOR XORAY RAOTATION MEASUREMENTS

1202 LAMPF IXED RADABAE SYSTEM

185: LAMPF REMOTE AREAGMONITOR SPECIFICATION

6)

67,9710

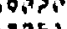

67.

$71,4=25$

65000.90

7opina

tingina

Bringige 
PARKER, J,R, (CONTO.)

1872 LAMPF SPECIFITATION EOR EXHAUSTOSTACK AIR VELOCITY MEPFQTNG SYSTEM TIODIZ

1657 LAMPF TELEVISTON SYSTEM

1530 CAMPF WIRING AND TERMINATION RECOROS OATGZ

789 PHASE DEVIATINN MEASUREMENTS QECOROS

1611 PREOTCTION OF ISODOSE GAMMA CURVES IN TARGET AREA AT EPA

602 PRELIMINARY MFASUREMENTS OF STRUCTURE BRE USSTRAMLUNG FROM DULSFO S MEV ACCELFRATR

1659 PRELIMINARY SOECIFICATIONS FOR CENTRAL CONTROL RTOU SCAN CONVERTFR TOAOIA

1605 PROPOSED ENVIQONMENT MONITORING RACXS GRA.H?

1595 RADABAFE DETERTION BABAL

1689 RADESAFE SYSTFM ORIDI?

1161 RADIATION DAMAGE IN THE LAMPF RAOIATION ENVIRONMENT

1749 RADIATION SUSFEPTIAILITY OF ECHO STOCK CPE

611 GAOIATIONDSAFFTY INGTRUMFNTAYION FCR AIGH INTENSITY, HIGN NUTY ACCELEAATAR

16 RE REMTE RADIATTON MONITORING FOQ MODEL L

1744 RF DRIVE LINE D RAOIATION EEFECTS

1863 SPECIFICATION FRR HV POWER SUPQLY FOR BUTT-LINE ZERO NEUTRON WUNITOR

1615 SUMMARY OF RATSAFE AND TV WORK IN PROGRESS

1250 TELEVISION BYITEM FOR LAMPF PHASE DESIGN AND SPECIEICATION ROIPIR

1650 TELEVISION SYMTEM FOR LAMPF, PHASE I DESIGN ANO SOECIFICATION GOI?10

1656 TELEVISION SYSTEMS FOR PRATOTYDE FACILITIES

614 TEST OF COMBUSTION PRODUCT DETFCTORS TN A RADIATION FNVIRONWENT

1660 TV MONITOR SCREENESIZE IN CENTAAL CONTROL RONM
2726 WIRING AND TERMINATION OESIGNATIONS

PARSONS, J.G

1694 AN ALTERNATE MAGNEF POWER SYSTEM FOR THE EXPERIMENTAL IREA

1967 EL RACK AE POWEA DISTRIBUTION

onithis

67129

Pantaloge, g.

1497 PROCEEDINGS OF THE S!XTH LAMPF USERS MEETING

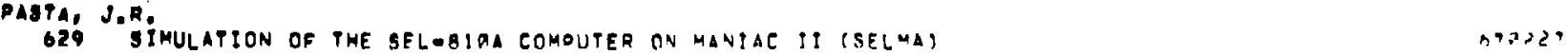

PATION, R.O.

27
320
320
BEAH POSITION MONITOR EXPERIMENT, PROPOSAL 75-3 JANUARY 1975

100 CALIBRATION OF THE GEAM OOSITION INO REAM CURRENT AMOLIFIER

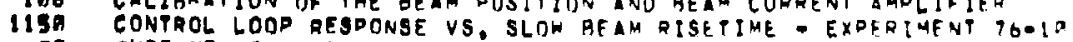

25 CURAENT MONITAR TAANGEORMER

2146 DEVELOPMENT WORK ACEOMPLISHEO ON MAY 13,1090

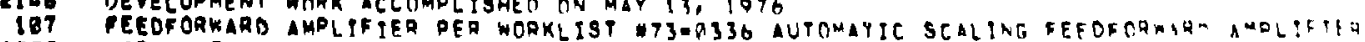

$\operatorname{tal} 1,19$

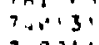

Top?:

inges.

tansin.

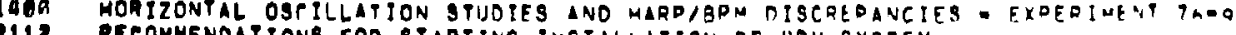

Tnl int

pauletta, g, 
PAULETTA, G, CONTD.

2012 ELABTIC SCATTFRING ANALYZING POWER MEASUREMENTS AT BMQ MEV--1

2813 ELABTIC SCATTFING ANALYZING POWER MEASUREMENTS AT BRG MEVEIII

PEEK, R.A

123, "AICRODOSIMETRY IN TISSUE EOUIVALENT FLUIO gaRAOIATEO BY DOT NEUTEONS TSP H

PEHL R.H. "UAE OF HIGHAPIRITY GERMANIUM OETECTORS FOR INTERMEDIATE ENERGY PHYSICS EYPERTMENTS TSAMTI

PENKROT, j.4.

158 ELASTIC SCATTFRING OF 58 MEV PI* AND 12OCARBON

163 STUDIES OF CHARGED PARTICLE EMISSION IN REACTIONS INOUCEO HY 220 AND SO "EV PLUSOPIOUS TSALE

164 STUPIES OF PARTICLE AND GAMMAOSPECTRA IN REACTIONS INOUCED BY BOAMMEV DRATONS TUB LAA

1632 USE DF AN INTRINSIC GERMINIUM CHARGED PARTICLE SPECTROMETER AT LAMDF
219 UIE OF HIGHAPUITITY GERMANIUM DETECTORS FOR INTERMEDIATE ENERGY PHYSICS EXPERIMENTS

DEREZ - MENDEZ, V.

106 DOUBLEGCHARGE-EXCHANGE AND INELASTIC SCATTERING IN PI- + 3-HELIUH

990 VISUALIZATION OF GTOPPING PION DISTRIBUTION

PERKINS, R',8:

2427 CROSS SECTIONS FOR PI + NUCLEUS GOESTO PI +OR. . . FOR T4QAMEV PROTONS T21100

190 MUONIC ISOTOPF SHIFTS IN THE EVEN IRON NUCLEI

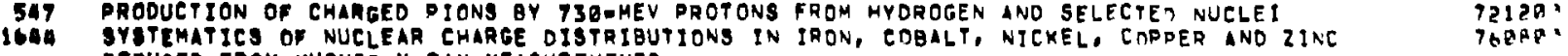

DEDUCED FROM MUONIE XORAY MEASUREMENTS

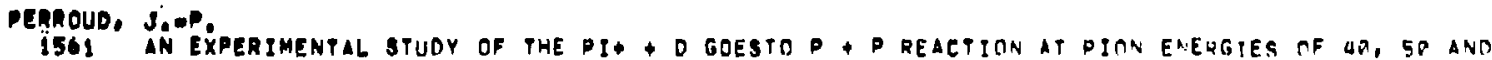

1633 PHOTON SPECTRIIM IN PION CAPTURE ON PRITIUM

2969 THE DEUTERON DASTATE AND THE PI+ D GOESTO D P REACTION

2421 THE P + D GAESTOP + PREATION AT 4D, 5H AND GB MEV

1393 TUNEUP OF PHE LAMPF LOW ENERGY PION CHANNEL

Tagnsta

700619

$\ln 125$

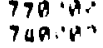

PERTY O.

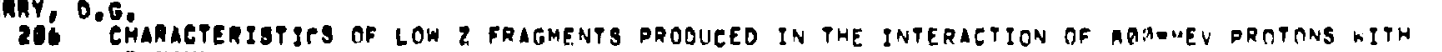

950402

ags compurgnt URANIUM

25AS COMPUTINE IN NUPPORT OF EXPERIMENTS

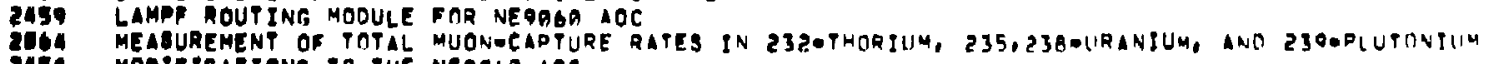

MODIFICATIONS TO THE NEQTGA AOC

701 is:

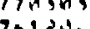

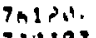

Thash

760.50
$7+1.54$ 
PERPY, D.G. (CONTD.)

2459 NEQGGQ TO ORTFC 46 ? INTERFACE

162 NEUTRONS EROM HIGH ENERGY PROION BEAN STRP

-

PETERSEN, D.F:

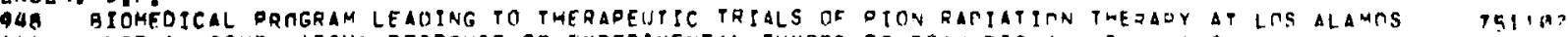

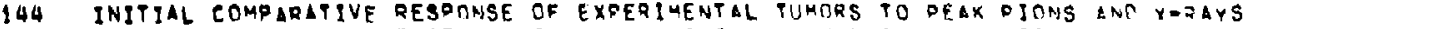

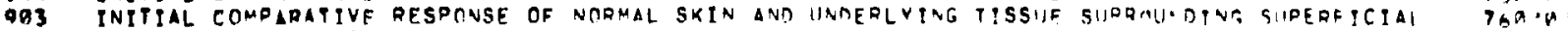
METASTATIC NONULES

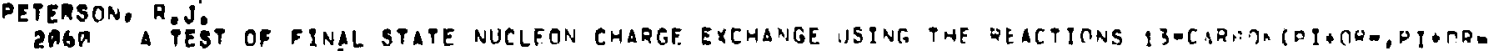
N) (2-CARBONCQI, MEV ANO 15,II MEV I

1533 A VERSATILE MILTTPOLE CORRECTION MAGNET OF RECTANGULAR APERTURE

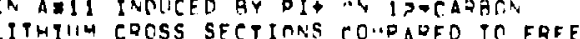

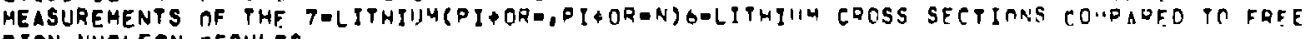

2A91 OIOMESON INDUTED PROTON REMOVAL FROM QABERYLLIUM TO THE M.QBI MEV ONE OL IS STATE CF E-

2093

2993 DION INDUCED NUICLEON REMOVAL FROM LIGHT NUCLEI
1995 SCATTERING OF 5 P MEV PI+ FROM LIGHT NUCLEI

$77 a>a>$

$759 \cdot 19$

76110

$7+24$
$7+1 \cdot 5 a$

77.4.

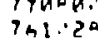

PETRY, R.

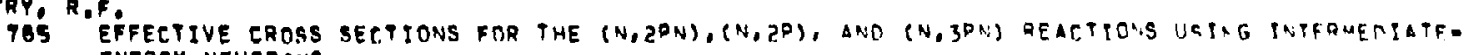
ENERGY NEUTRONS

198 PRODUCTION OF NEIJTRONERICH NIJCLIDES USING HIGHEENERGY NEUTRONS AT LAMPF

2414 SEAREM FOR NEW NEUTRONARICH NUCLEI PROOUCED AY FAST NEUTRONS IT LAMPF

179 STUDY OF THE PGQ-PLATINUME-ZGQGGOLO DECAY CMAIN AT LAMPF

7 THE 2 Ga PLATINUM - zMa GRLO RECAY CHAIN

$41 ? \mathrm{n}$

701.0

Thin

$7 \log \theta$

PFEUFER, G.W.

900 MICQODOSIMETRY OF NEGATIVE PIONS AT LAMPF

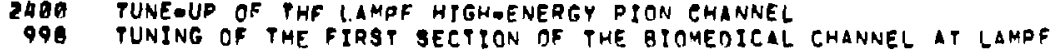

76417

$750 \cdot a$

75800

PHIL6?PS, 6.6.

2867 ANGULAA OISTRTBUTION FOR PD GOESTO 3HE PI ZERO REACTION AT TPEBAG MEV TYOUP:

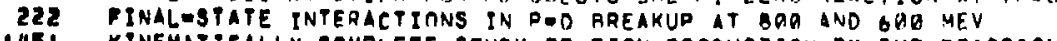

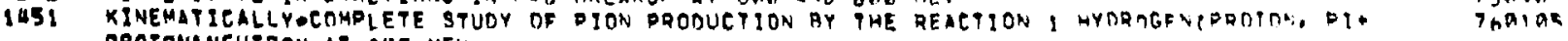

210 PROTONJNEUTRON AT AUA MEV

219. NUELEAR-COULOMA INTERFERENCE IN P! +OR= 16 OXYGEN SCATTERING

1981 DJON PRODUCTION RY BG MEV PROTONS FROM G-LITHIUM

221 PION PRODUETITN RY APO MFV PROPONS IN THE REACTION I-HYODOGEN(D,DI*,0).

1960 PION PRODUCTION FROM THE REACTION D(P,D OI)N AT SAS MEV

1982 PION PRODUETION IN PEP INTERACTIONS AT BOG MEV

$75 \tan$.

$761 \cdot 20$

That

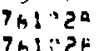


PHILLIPS, G.C. CCONTO:,

1996 PION PRODUCTION VIA A PAP GOESTO DQPI GUASIOFREE PROTONS IN DEUTERIUM

103 ION-NUCLEAR TOTAL CROSS SECTIONS NEAR TNE 3/2, 3/? RESONANEE

1963 PJON-NUCLEUS FORWARD SCATTERING AMPL ITUDES NEAT THE 3/2, 3/2 RESONANEE

2073 PONONLCLEUS SMALL ANGLE ELASTIC CROSS SECTIONS NEAR THE 3/2, 3/2 RESDVA.CE

2074 PIONONUCLEUS TOTAL CROSS SECTIONS NEAR THE $3 / 2,3 / 2$ RESONANCE

223 OUASI-FREE SCATTERING IN POD BREAKOUP REACTIONS IT BRG AND GAG MEV

966 OUASTOFREE SCATTERING IN PROTON-INDUCED DEUTERON BREAKUP IT 585 ANA AB A UEV

REA IFT AND RECOMMENDATIONS OF THE MAGNET POOL SUBCOMMITTEE OF THE DI UAFOS COMNITTE

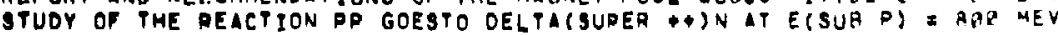

$24 B$ PUNE UUP OF PHF LAMPF HIGHOENERGY DION CHANNEL

PINSKY, L.

2609 ANGULAR DISTRTAUTION FOR PD GOESTO 3HE PI ZERO REACTION AT TPBBAT MEV

1961 PION PRODUCTION AY BUG MEY PROTONS FLOM GELITHIUM

1980 PION PROOUETITN FRUM TME REACTION D(P.D OI)N AT 585 NE

199. PIDN PRODJCTION VIA PQP GOESTO DQPI OUASIOFREE PROTONS IN DEUTERIUU

2073 PION-NUCLEUS AMALL ANGLE ELASTIC CROSS SECTIONS NEAR THE 3/2, 3/2 QESONA.C

2074 PIONANUCLEUS TOTAL CROSS SECTIONS NEAR THE 3/2, J/2 RESONANCE

$\operatorname{sins}$

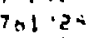

$10,1,50$

$101 \cdot 5=$

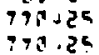

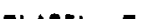

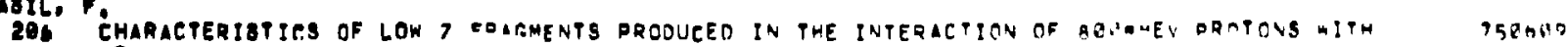
URANTUM

PLOPHER, CAM,

1481 LAMPF OPECIFITATION FOR DIGITAL CONTROL COMPUTER SYSTEM

liss SPECIFICATION FOR DIGITAL CONTROL COMPUTER BYSTEM
IBS

$00011 \%$

Thingi:

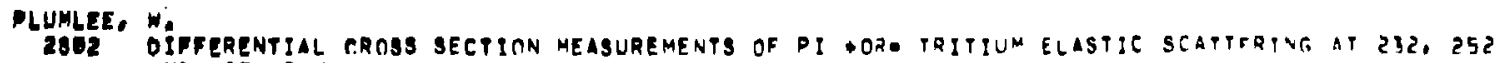

AND 295 MEVIC

2 A23 MEASUREMENTS OF THE OTFFERENTIAL CROSS SECTION FOR PION CHARGE EXEMAGE WV TEITIL"

2424 MEAGUREMETS GF PHE DIFFERENTILL CROSS SECTION FOR PION CHARGE EXEMATGE TA PAITIIM.

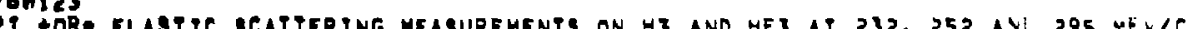

700128

$7 \rightarrow$ inc

$\operatorname{Tan}: 2$

$\operatorname{lom}$.

POORE, R.Y.

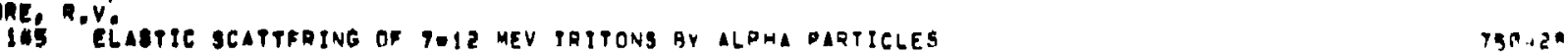

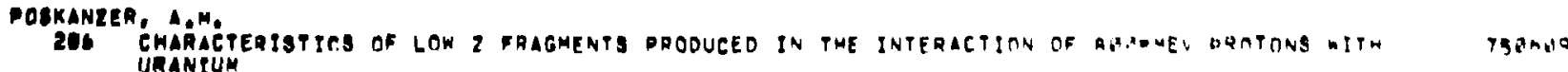

porten, D.C. 
POTTER, D.C. SCONTD.)

717 LAMPF NEUTPINO FACILITY PROPOSAL. 7I12A

POTTER, JAMES M.

552 BOR-MC RF STRIICTURES

1347 AEASUREMENT OF PARITY VIOLATION IN PROTONEPROTON SCATIERIAG AT IS MEV

A REAL-TIME SAMDLING BEAM DOSITION MONTTOR

2719 ACCELERATING STRUCTURE QFSEARCH AT LOS ALAMOS

795 AN ACCURATE METHEO FOR THE MEASUREMEAT OF HIGH P MICROAGVE RESOVATORS

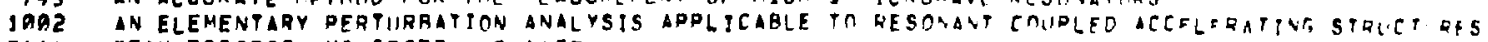

2114 GEAM POSITION NONITORS TOI11A5

143E BRIDGE COUPLER DESIGN ANO TUNING EXPERIENCF AT LOS LLATS

342 CAVITY TUNING FOR THF LAMPF BO5-MHZ LINAC

1346 ELECTRICAL BEHAVIOR DF LONG LINAC TANKS ANT A NEN TANM COIDLING GCWE."E

ISU2 LIMIT ON PARITY NONCRNSERVATION IN PONUCLEUS SCATTERING A O GEVIC

1620 LIMIT ON PARITY VIOLATION IN PANUCLELS SCATTERING AT O GEVIC

2737 OPERATING CHARACTERIBTICS OF FULL FOWER CLOVEQLEAF ACCELEQATOR TAYK

1169 DI/Z MODE PUNTNG EXPERIENCE WITH EDA

1119 PROPOSAL FOR THE CREATION OF AM ELECPRONICS INSTRUMENTATION GRAUA IN MO IVIGIRN

1071 RESONANT SIOE-COIIPLED CAVITY ELECTRON ACCELERATORS

1347 RESONANTLY COIIPLFD ACCELERATING STRUCTURES FOR HIGH-CURREAP DROTCA LINCS

G6A SEARCH FOR A PARITYOVIOLATING CONTRIRUTION TO NUCLFONONUCLFGA SCATEEI.

392 STABILIZATION OF THE ORIFT TURE LINAC QY ODERATIOA IY THF PIJZ CAVTY "OCE

1331 STANDING WAVE HIGH FNERGY LINEAR ACCELEQATOR STRUCTUQES

1563 SYSTEM DOCUMENTATION - ICR NOVA COMPUTER

224 TEST OF DARITY CONSERVATION IN POD SCATTERINE

1470 TESTS OF PARITY CONSERVATION IN P.P AND P.NUCLEIS SCATTERIVE

796 THE MODE SPECPRLIM OF AN INFINITELY LONG BIPERINOIC EHA

257

作

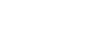

G4A72?

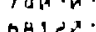

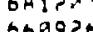

Q5! '?

67utpa

7?! 1

?? ?

acting

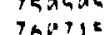

1001010

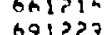

$15 \times 340$

1970

65 and

730.0

67,3531

67.453

7115

741,19

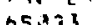

$2 \cos 20$

69023
71008

POTTER, JERRY $M$.

2482 LONG DISTANTE CAMAC BRANCH VIA DATA-LINK AND MICROPROGQANMEO GRANCW MQPVED

2489 A BATELLITE CONTROL AND TIAGNOSTTCS COMDUTER SYSTEM FOR THE LAMPF INJETT QS

2490 A SEAIAL ORTVFR MODULE FOR THE CAMAC SERIAL HIGHAYY SYSTEM

1719 ANALOG COMMANO TESTER INSTRUCTIONS CARO NO, B3Y $=125698$

IORA ANALOG DATA SYSTEM (AOS) TEST PROCFDURE AND RESULTS

1911 LNALOG DATA BVSTEM TESTER

1370 OIOAOI PCM RETEIVER

1729 BINARY COMMANA TESTER INGTRUCTIONS CARD NO, G3Y-125605

1729 BINARY COMMANO TESTER INSTRUCTIONS - FAST RINARY CORMAND CARO B3Y-125RZ!

1723 BINARY DATA TFSTER CARD NO, G3YOI25696

2469 CAMAC BASED EAMPIITFR-COMPUTER COMMIJNICATIONS VIA MICROOROCESSOR DATA LTVAS

1721 CHANNEL DECOOF TESTER INSTRUCTIONS CARD NO. G3Y-12572.

1350 DYM MUX AND INTERFACE

1735 ICA NOVA INTERFACE SYSTEM I/O WIRING

LAMPF CAMAC MWDC ENCODER REA DOUIT MADULL DETATLS

1922 ORALATE DATA CHANNELS COMMAND RUFFER TESTER INSTRUCTIONS EARO AO, HZY.125TUZ

$7>1,39$

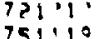

onisaz

6atson

770111

751100

की

boves;

ope??

करा11?

ORI ग19

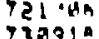

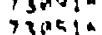

73 जद

QPa?!1 
POTTER, JERRY M. CCONTO.?

M. BCONOP

1676 TECHNGCAL OESPRIPT TON OF THE LAMPF CAMAC OATA-LINK MODULE 1676 TECHNICAL DESCRIPTION OF THE LAMPF CAMAC OATA-LINK MOOULE MOCLE
1950 TECHNICAL OESTRIPTION - LAMPF CAMAC MHPC ENCODER READOUT MOCLLE 1950 TECHNICAL OESCRIPTION. LAMPF CAMAC MWPC ENCODER READOUT MOCLLL
1962 TECHNICAL OESCRTPTION. LAMPF SERIAL ORIVER

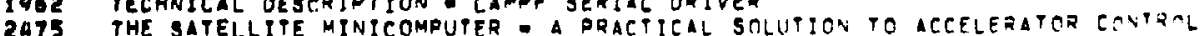

$700: 35$

7 3.

POWERS, R, J.

75,220

2979

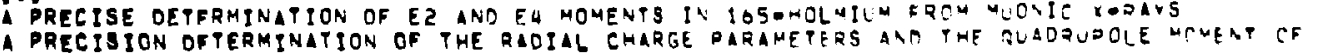

MUONIC IOT GOIO

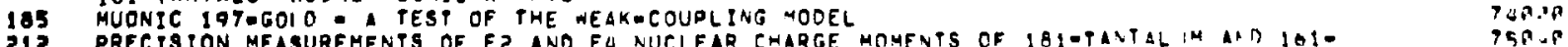
DYSPROSIUM

PRATT, J.C. COLLIOING BFAHS EXPERIHENT AT LAMPF, PHOTOOETACHMENT qESONANEES IN TWE h- ION

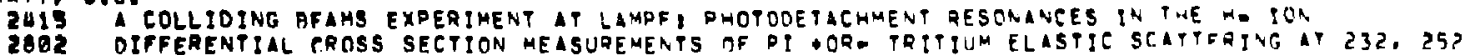
AND 295 MEVIC

2994 ELASTIC ANO INELASTIC SCATTERIMG OF AAM MEV PROTONS ON 12.13 OCARRON AW SARALEAD

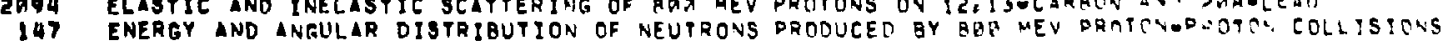

247 ENEGTENENTS OF MEASUREM THEBOB

2H24 MEASUREMENTS OF THE OIFFERENTIAL CROSS SEGTION FOR DION CHARGE EXCHANGE GN TRITIJM. 789123

1641 MULTICHARAEL, REMOTELY PROGRAMMABLF, LOGIC DELAY SYSTEW

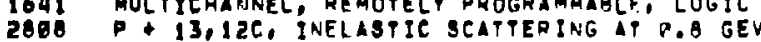

2803 PI I3,12C ORNELKSTIC SCATPERING

$77,3 \times 2=$

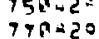

$783: 28$

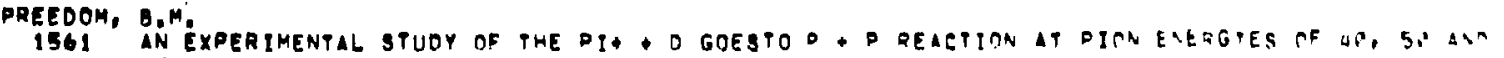

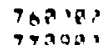

$7+4$

1561 AN EXPER

2585 COMPUTING IN RUPPORT OF EXPERIMENTS AT LAMPF

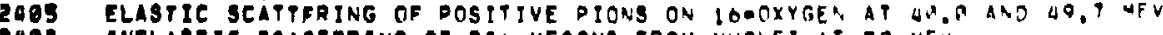

24QS INELASTIC SCAPPERING OF PI + MESONS FROM NUCLE: AT 5 M MEY

2411 MASA DEPENDENTE OF PION DOLIRLE CHARGF EXCHANGE

1991 MEABUREMENTS OF PIONONUCLEAR ELASTIC SCATTERING A I LOW ENERGIES

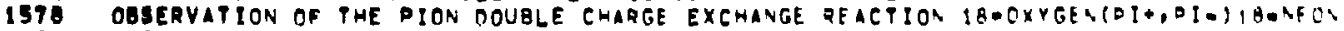

2422 PION DOUSLE OCHARGE EXCHINGE ON IOERXYGEN AWD IRNCXYGEY

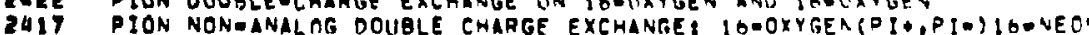

2HOU PIONONUCLEAR PNELASTIC GCATTERING IT 5 G MEV

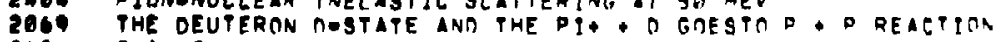

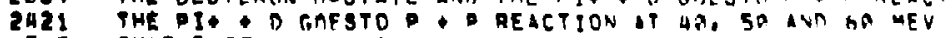

ISU3 TUNEUP OF THE LAMPF LOW ENERGY DION CHANNEL

$75,1+i c$

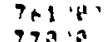

$\rightarrow 7 \operatorname{lig}^{2}$

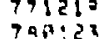

$3+1, s_{0}$

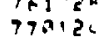

$773 \cdot 6$

$79,19.90$

$\rightarrow \rightarrow \cos 0$

$761 \cdot 25$

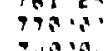

DUTNAM, T,M.

505 IN ODERATORS CONBOLE GOR THE LAMEF ACCELERATOR

1 IID COMPUPER CONTRO OF PHE LAMPF ACCELERATGR 
PUTNAM, T.M. SCONTO.?

1596 CONCEP PUAL OEAIGN OF PHE LAMPF COMMUNICATION SYSTEM PPLUS ADOENDIIM NA, 11 OA!:2A

IHOS DESIGN PERT PROGRAM INVESTIGATION

1535 INJECTOR CONTAOL ROOM CHANGES - LAMPF

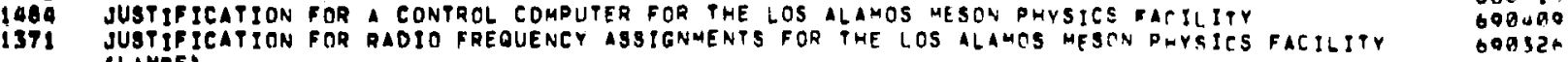

1426 LAMPF CONTROL BYSTEM DESIGN INO CONSTRUCTION

1437 LAMPF INSTRUMFNTATION ANO CONTROL SYSTEM

1314 LAMPF PERSONAFL SAFETY WARNING LIGHTS

1597 LAMPF RADIO COMMUNICATION EQUIOMENT

I313 MP OIVISION SAFETY PROCERURES FOR ACCELERATOR TURNOON AND BEAM TESTS

1892 MPO1 R AND D PROGRAM FOR FY=90

1889 MPOI R AND D PROGRAM FYOGQ

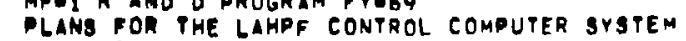

1669 POWER SOURCES

1401 PROCUREMENT OF A CONTROL COMPUTER FOR PHE LAMPF MOCKUP EXPERIMENT

630 PROCUREMENT OF A CONTROL COMPUTER FOR THE LAMPF MOCK-IJP EXPERIMENT

1381 REVIEW OF THE MPOI R AND O PROGRAM FOR 1069

1394 REVIBIONE TO RECTOR A PERSONAEL SAFETY SYSTEM (DSS), OPERATION MODES, AWA FNTRY PRCCEDURES

170 SECPOR DTATUS LIGHT ASSIGNMENT

614 TEST OF COMBUSTION PRDOUET DETECTORS IN A RADIATION ENVIRONMENT

$67001 \mathrm{a}$

600023

gopsza

60111 .

609720

671 '1!

6793 a?

068203

GQAD?

GOA口Q0

711.15

$670-23$

6500.9

RASALA, R,E.

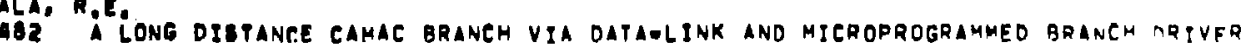

38. DESIGN AND OPFR TAM ORANE

620 RIgE TIME OPRATION OF A MICROPRDGRAMMEO BRANCH DRIVER FOR A PDPO

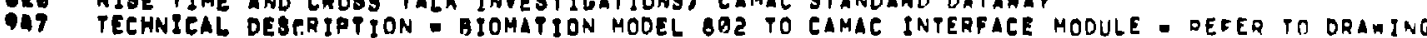
TECHNIEAL

1948 TECHNICAL OESTRIPTION - LAMPF EIGHT-CHANNEL CAMAC STEPPER MOTOR CONTROL UULTIPLEXER TECHNICAL DESTRIPTION

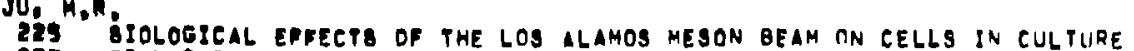

933 CELL BURVIVAL AB FUNCTION OF OEPTH FOR NEGATIVE PIONS INO HEAVY IONS

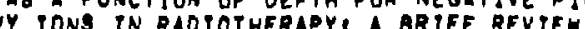

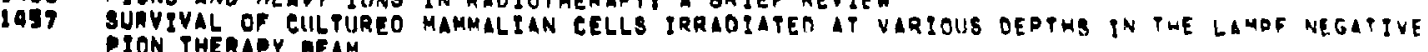

naY. L:

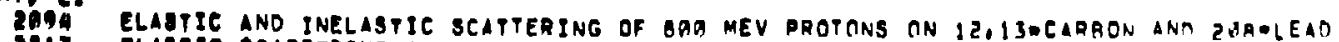

2813 CLABTIE SCATPFRING ANALYING POWER MEASUREMENTS IT AgO MEYOO II

$\lim 20$

- 13,12C, INELAGTIE SCATTERING AT G.B GEV

$73120 \mathrm{mon}$

13040.

7 thang

$73041 ?$

nEOKA, G.A, 
REQKA, G.A: (CONTD.)

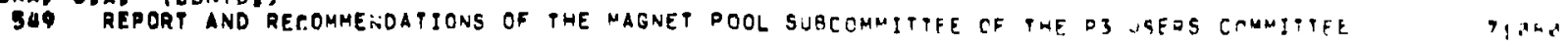

REDWINE, R,P

1563 ABSOLUTE CROSS SECTIONS FOR PQDDUCTION OF DROMPT NUCLEAR GAMNA RAYS AY FASP DIOVS

1561 AN EXPERIMENTAL STUDY OF THE PI+ D GOESTOP + P REACTION AT DIDN ENERGTES GF UN, 5P ANE

240560 MEV

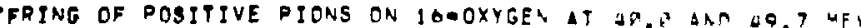

2418 GAHGETIC CHARGEO PARTICLE YIELDS INDUCED BY PIONS O.. COMDLEX NUELEI

2408 GAHMARAY STUNY OF PIONOINDUCED REACTIONS ON COMFLEX NUCLE

I\$A3 INTERACTION OF FAST PIONS WITH G2,64-NICKEL

2411 MASS DEPENDENCE OF PION DOUBLE CHARGE EXCHANGE

241

MEASUREMENTENEE OF PI

MEASUREMENTS NF PI (+ORE) NUCLEUS TOTAL CROSS SECTIONS AT ENERGIES RELO. TH: "EV

MEABUREMENTS TF PIONGNUCLEAR ELASTIC SCATTERING AT LOW ENERGIES

NEUTRON RADII OF CALCIUM

OBSERVATION OF THE PION DOUBLE CHARGE EXCHANGE REACTION 18-OXYGFAGPI+,DI-)18.NEDN

PION DOUBLE-CHARGE TOTAL CROSS SECTIONS FROM SB-IGg ME

PON NONAANALTG DOUBLE CHARGE EXCHANGE 16*OXYGENCPI+.DI-116-NEON

- ionanuclear tNelastic scatter jNG at

PIONONUCLEAR TOTAL CROSS SECTIONS

PRDTON SPECTRA FROM DION-INDUICEO REACTIONS

SYSTEMATICS OF PION ANO PROTON INTERACTIONS WITH NICKEL NUCLIDES

THE DEUTERON AMSTATE AND THE PI + D GOESTO D \& P REACTION

PUNEUP OF PHE LAMPF LOH ENEAGY PION CHANAEL

REICH, C.W.

2967 134-BAR IUM LEVEL SCHEME AS OBSEQVED IN THE DECAY OF 13 HELANTMANUM

14U6 ANNUAL PROGREAS REDORT - NUELEAR STRUCTURE STUNIFS UT ILITING LAMDF

1630 DECAY OF 1280PESTUM

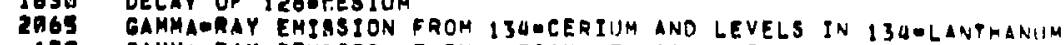

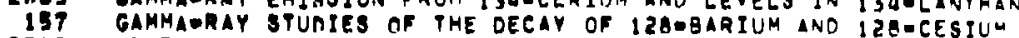

2968 LEVEL BTRUCTURE OF 131-CESIUM AND THE OECAY ENERGY OF I3IMBATIUM

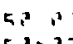

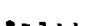

77 म 5 :

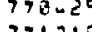

$717 !=$
$7812=$

72015

tamalis

7nt $2=$

770525
779120

750.2

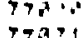

$773,1=$

Tophis

Th1.2

?n! ' 25

REIOY, J.s.

Pí eviluation OF NONODESTRUCTIVE ANALYSIS TECHNJOUES AT LAMPF

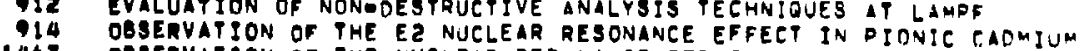

1463 OBSERVATION OF THE NUCLEAR RESONANCE EFFECT IN THE DIONIC IIP DLLLAOLU ATOM

996 THE USE OF MESONS FOR TISSUE CHEMICAL ANALYSIS

Q94 TISOUE CHEMICAL ANALYSS WITH MU MESIC XORAYS

1462 TWOOOIMENBIONAL VISUALIZATION OF STOPPING PION DISTRIBUTIONS

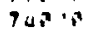

$3+1,6$

$3 \tan 30$

7nand

T41

$\ln 1 ! 0$

REINES, F

117 LAMPF NEUTRINA FACILITY PROPOSAL

$1450 \cdot 19$

$750 ? 1$.

76,725

Tद्131?

75113
76090.

RErst, H.W. 


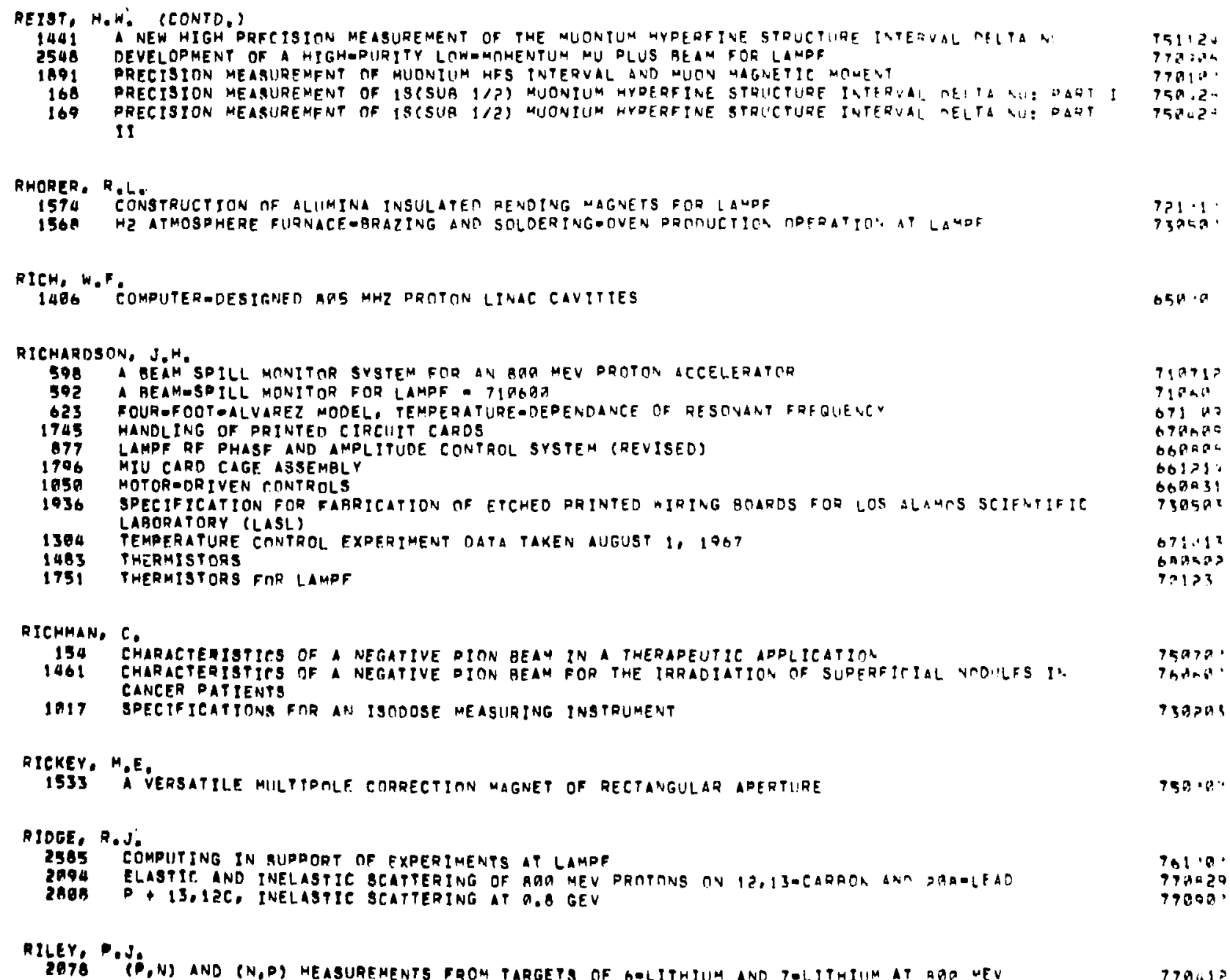


RILEY, P.S. (EONTO,)

1994 6.7-LIPHIUM(P,N) LNO (N,P) MEASUREMENTS AT BXA MEV TEI'ZC

EXTREME BACK INGLE NO ELASTIC SCATTEAING AT T9L VEV

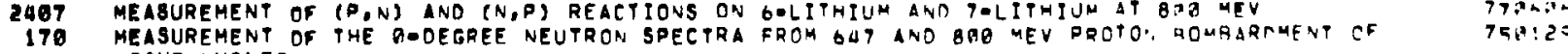
MEASUREMENT DF THE A-DEGREE NEUTRON SPECTRA FROM OUT ANO BGO MEV DROTO, ROUAGRTMENT CF

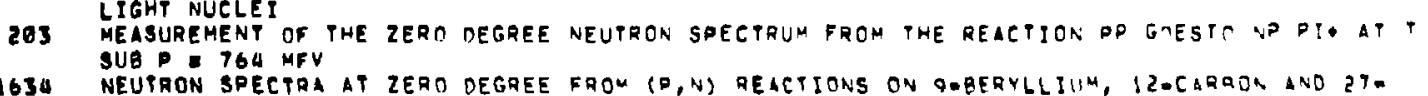

ALUMINUM AT GUT AND GQR MEV 1635 ALUMTNUM AT GU7 AND BQR MEV
ENERGIES

\section{$76,7^{3} \cdot 1$}

$\operatorname{lng}, 95$

1969 NEUTRON SPECTRA AT ZERO OEGREE FROM PROTONUPROTON COLLISIONS BETWEEN 0.17 4.0 845 NFV

200 NEUTRON SPECTRA FROM 59g HEV PROTONS ON QRBERYLLIUM

232 NEUTRON SPECTRA FROM PROTON BOMBAROMENT OF DEUTER IUM AT GUY AND BOA WEV

1978 PRECISION MEARUREMFNT OF NOP CHARGE-EXCHANGE CROSS SECTION IT GU7 MEV

202 OUISIELASTIC CHARGE EXCHINGE IN ND GOESTO PNN AT TQU UEV

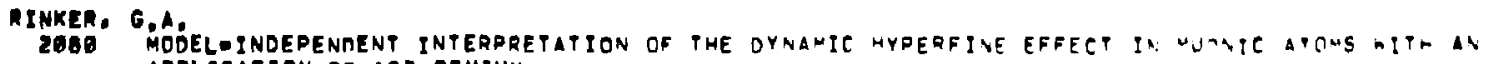
APPLICATION TO 19200SMIUM

150 MUONIC ISOTOPF SHIETS IN THE EVEN IRON NUCLEI

1644 SYSTEMATICS OF NUCLEAR CHARGE DISTRIBUTIONS IN IRON, COAALT, NICKEL, CMPDER ANO IINE SYSTEMATICS OF NUCLEAR CHARGE DISTRIBUT
DEDUCEO PROM MUONIC XMRAY MEASUREMENTS

PISLOE, S.E:

OTI 20. MHZ (SECPAR A) COOLING WATER CRITERIA

1 T70 DEBCRIPTION OF COOLING WATER SYSTEM FOR THE DRIFT-TUBE LINAC

302 DESCRIPTION OF THE COQLING WATER SYSTEM FOR THE SIOE-COUPLEO LINAS

390 DESCRIPTION OF THE COOLING WATER SYSTEM FOR THE TRANSITION REGIOR.

2314 SOME OSBERVATIONS OURING THE PROOUETION BEAM TUNE MARCH 3I-ADRIL 2, 1970

$\ln \log$

$77 n: \cdots$

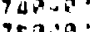

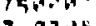

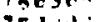

$770-2 a$

\section{MIBTINEN, R,A:}

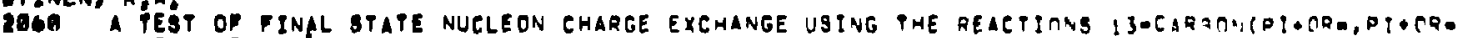
N) IZGCARBONIQ. DU MEV AND IS. 11 MEV

1533 A VERSATILE MIILTIOOLE CORRECTION MAGNET OF RECTANGULAR APERTURE

1153 COMPARISON OF NEUTRON GEMOYAL BROM TOLITHIUM GY PI OOR. WITH FREE OIINAN CLENN RES IL TS

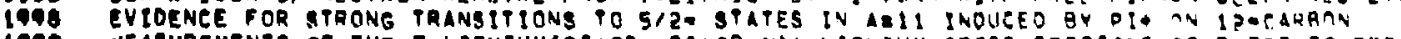

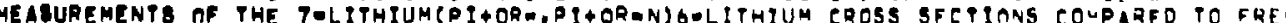
- IONANUCLEON EESULTS

20Q1 PIOMESON INOUTED PRDTON REMOVAL FROM OOBERYLLIUM TO THE R.PQI MEV ONF OL S STATE OF R. LITHTUM

2093 PION INDUCED NUCLEON REMOVAL FAOM LIGHT NUCLEI

TฺRup

$72, \pi 3 x$

GOA SAN EXPERIMENTAL SPUDY AF OMEGA NEUTRAL DECAYS

$063^{2} 5^{n}$

$00.11 \mathrm{in}$

0001.14

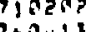

Prap."

15.9 .4

$7+1: \because !$

Th:

$\operatorname{ranap}$

ITaAas 
RiYTER, E.T.

150 MUONIC ISOTOPF SHIFTS IN PHE EVEN IRON NIJCLE

750383 DEDUEED FROM MUONIC XORAY MEASUREMENTS

RIVEA, O,M

998 TUNing OF THE FIRST SECTION OF THE BIOMEOICAL EHANNEL AT LAMPF

$75000^{2}$

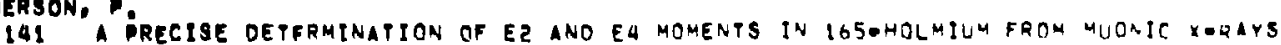

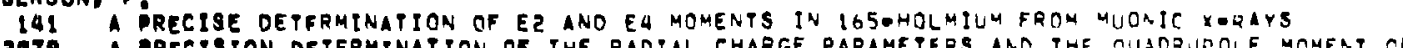

75022. 1810TANTALUM HSING MUONIC XORAYS

2408 INELASTIC SCATTERING OF DT MESONS FROM NUCLEI AT SP MEV

2004 PIONONUCLEAR PNELASTIC SCATTERING AT 5 M MEV

212 PRECISION MEABUREMENTS OF E2 ANO EA NUCLEAR CHARGE MOMENTS OF $181-T A N T A L$ M ANO 161. orsprosium

ROpBERG, L.

2728 EBTIMATES OF MAXIMUM ENERGY PION PRODUCTION ON NUCLEI, KAON, LAMADA PRND CTIAR OA WUCLE AND STAMTENEBS VIOLATING LAMBDA PROOUETION ON NEUTRONS BY BPB MEV PROTOCS

RODGER, E:

951 A METHOD FOR ACHIEVING CLINICALLY USEFUL DION DOSE OISTRIBUTIONS

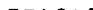

778620

1306 A SUGGESTED MFTHOD FOR OBTAINING A VERTICAL VELOCIPY OISTRIBUTION FOQ AV OSC ILLATING PIOA

achat

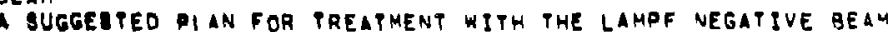

1398 ADDITIONAL COMMENTS ON VERTICAL SCANNING WITH DION BEAMS

THE GIOMEDICAI CHANNEL

IIAS UNIFORM DOBE DIBTRIBUTION WITH MOVING NEGATIVE PION REAMS

boinas

ROEDER, 0.6 .

143 ABSORBEO dOSE ANO RADIATION QUALIPY FOR GACKGROUNO RAOIATION FROM A MEGATIVE PION BEAM

1004 COMPUTER MODEI ING OF NEUPRON DOSE AT VARIOUS LOCATIONG WITHIN THE BOOY DIRIVG DION 2802 DIFFERENTIAL RROSS SECTION MEASUREMENTS OF PI TORE TRITIUM ELASTIC SCATTERING AT 232 , 252

laAs OOSE OTSTRIBUT

IAOs TISSUE

100 EFFEC OF IOAMORON ON THE STAR NEUTRON DOSE OISTRIRUTION IN NEGATIVE PIO. OATIOTMFLAOY

1505 OPERATING EXPFRIENCE WITH LAMPF MAIN BEAM LINES INSTRUMENTATION AND CO. TOOI SYSTEM

2803 I +OR- ELASTTC SCATTERING MEASUREMENTS ON H3 AND HE3 AT 232 , $25 ?$ PNT 295 WEV/C

262. SWTCHYARD BEAM TRANSFER. RESULTS OF PIELD VARIATION ANALYSIS

WHOLE BODY DOAE FOR PATIENTS ULTS OF FIELD VARI

RogacheV, V.l.

$73 \sin a$

721 is

7519118

731113
$730-1:$

$75 \pi \cdot 10$

74010

7 An: 23

T3ita.

$75 n^{n}+a^{n}$

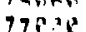

7 ival.

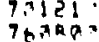


MOGACHEV, V.t: (CONTO')

2202 ON THE POSSTBILITY DF APQLICATION OF PERMANENT HAGNET QUADRUPOLES IN TME HIGW ENERGY PROTON LINEAR ACCELERATORS

ROCERA. D,T

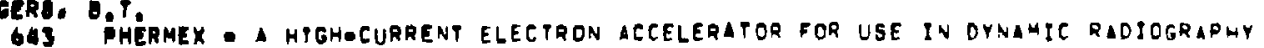

$051 \cdots$

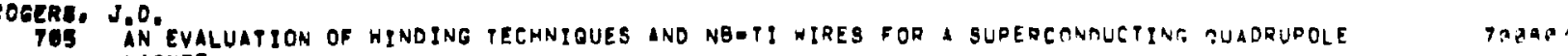

2053 MOMPLETION OF 6 INEH ROOM TEMPERATURE BORE SUPERCONDUCTING GUIORUPOLE "AGNET DOURLET COMPLETION OF 6 INCH ROOM TEMPERATURE BORE CRYOGENICS ANA NUCLEAR PHYS

QUADRUP OLE FORUSING MAGNE

706 RADIAL FLOW MFASUREMENTS OF HYDROGEN NEAR ITS CRITICAL POI
706 THE EFFECTS OF RADIATION ON SUPERCONOUETING NO-TI WIRE

799 TWENTYOFIVE CFNTIMETER BORE SUPERCONDUCTING OUAORUPOLES FOR LAWOF

$70,351 !$

7 ano:

$08000^{2}$

79019

79023

ROGERS, H.L.

1089 BINARY COMMANA MODULE - FABRICATION SPECIFICATION

1603 CURRENT SWITCHYARD INSTRUMENTATION AND CONTROLS STATUS

1802 ELECTRONIC TEMPERATURE CONTROLLER - FAARICATION SPECIFICATION

1920 FABRICATION BPECIFICATION LB/LE RACK ASSEMBLY AND WIRING

ISOA INSECTOR INTERLOCKS
1210 INOTRUMENTATION MODULAR CONCEPT

1790. MODULE NO. I COMPUTER CMANNEL ASSIGNMENT LIST

1706 SECTOR STATUS LIGHT ASSIGNMENTS
1003 PARGET WATEROPEMPERATURE, FLOW, DRESSURE MONITOR CHASSIS - FABRICATION SOECIFICATION

ROLFE, M.M.

7 I. LAMPF HIGH REROLIITION SPECTROMETER OATA ANALYSIS INELUOING HIGHER RRDEZ CORRECTIONS

723 MAGNETIC MEABUREMENTS ON THE LASL PROTOTYPE MAGUET

2600 13,12C, INELASTIC SCATTERING AT G, GEV

onacos

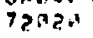

071100

$721 \cdot 29$

71,10

0711,9

67009

6709
67130.4

nosen, 6.

71: APPLICATION OF PARTICLE ACCELERATORS TO MEOICINE

567 COMPOUND ELABTIC SCATTERING IN NEUPAON-NUCLEUS INTERACTIONS

274 COMPUTERAASSIRTED ACCELERATOR MAINTENANCE

536 DESIGN AND CONBTRUCTION STATUS OF THE LOS ALAMOS MESON PHYSICS FACILITY

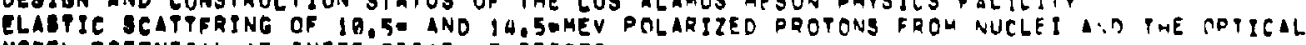

710029

751110

$77+00$

MOOEL POTENTIAL AT INTEAMEOIATE ENERGIES

70 EXPERIHENTS HTTH POLARIZEO PROTONS ANO THEIR INTFRPRETATION

INTERACTION OF MEOIUMOENERGY NUCLEONS WITH COMPLEX NUCLE?

INTROOUCTORY TALA FOR 1960 PROTON LINEAR ACCELERATOR CONFERENEE

LAMPF! MESON FACYOAY IN THE MAKING

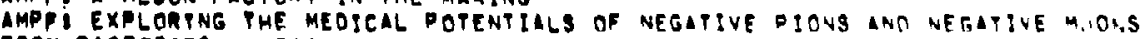

MESON FACTORIFB - 1966

Pa! 19

britdz

1931?

bop.a.

G5AMA.

05040,

$\log 4.5 ?$

03115

opt2."

Polat

$7 ? A+3$

onlen. 
ROSEN, L. (CONTD,)

S24 OUTLINE OF THF HROPOSAL FOR A MESON FACTLITY AT LOS ILAMOS

2601 PHYSICS WITH MESON FACTORIES
949 PION GENERATOR FOR MEDICAL APPLICATIONS

2684 POSSIBILITIES AND ADVANTAGES OF USING NEGATIVE PIONS IN RADIOTHERAPY

POSSIBLE USE OF NEGATIVE PIONS AND NEGATIVE MUONS IN THEPAPUETIC AND DIACVOSTIC MEOICINE

532 PROSPECTS FOR THE USE DF PIONS AND MUONS IN MEDICINE

IOII RAOIOISOTOPE DRODUCTION AT LAMPF

2689 RELEVANCE OF ACCELERATOR-RELATEO TECHNOLOGIES TO NATIONAL GOALS

53R RELEVANCE OF PARTIELE ACCELERATORS TO VATIONAL GOALS

1001 RESEARCH SYMPASTUM PROPOSAL SUBMITTEO TO THE NATIONAL SCIENGE FOUNDATITA

559 SCIENCE ANO SOCIETY

SOME MEDICAL APPLICATIONE OF ACCELERATORS

STATUS AND PLANG FOR THE NEGATIVE PION RADIOTHERAPY FACILITY AT THF LOG MLAMOS MESON oHYSiCs FACTLPTY

2602 STATUS OF CONGTRUCTION ANO EXPFRIMENTAL PROGRAM FOR THE LOS ALAMUS MESMN PHYSICS FACILITY STATUS OF LOS ALAMCS MESON PHYSICS FACILITY

STATUS OF THE CLINTON P. ANDERSON MESON PHYSICS FACILITY (LAMPF)

TECHNOLOGY PROGRAMS AT M MESON FACTORY

THE CLINTON P! ANDERSON MESON PHYSICS FACILITY (LAMPF)

PHE LOS ILAMOS MESON FACTORY

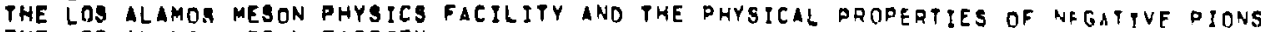

TME LOS ALAHOS MESON PACTORY

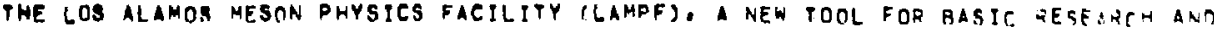

PRACTICAL APPI ICATIONS

$\sin 912$

$7 \cdot 11:$

67049.

15n.

ORlटa.

72 月द

ดा

7al?

$72 !$

76002

7 iine

7)

75isy

7)

73 3⿻

11तลत?

thpsos

7 7कP380

ด्व7द

70.4515

6997.4

60म7.

2515 RADTATYVE NEGATIVE PION CAPTURE IN LIDUTO NEON

2560 RADIATIVE NEGATIVE PION CAPTURE IN LIOUIO TRITIUA

$77 \operatorname{tin} 25$

ROSENTHAL A S.

2817 ANALYSIS OF THE INELASTIC SCATTERING OF 5O MEV PIONS GY $12 \mathrm{C}$

$771+99$

ROSENTHAL, H

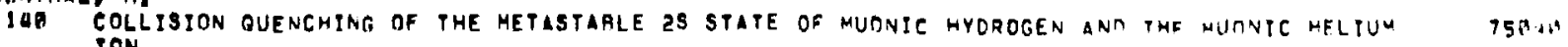
ION

nosT. E.

2019 ANALYSTS OF THE INELASTIC SCATTERING OF 59 MEV PIONS BY 120

2343 LOW ENERGY PION SCATYERING

Traph

ROUXOLOUGH, P,O,

1653 MYOCARÓIAL PQAITRON SCINTIGRAPHY WITH SHORT-LIVED RURIDIUMAOS

Thitsit.

nowe, P.C. 
ROWE, P.C. (CONTO.)

2515 RaOIATIVE NEgATIVE PION CAPTURE iN LIOUIO NEON TTAR27

550 PADIATIVE NEGATIVE PION CAPTURE IN LIOUID TRITIUM

RUHE, J,R,

2218 201 MHZ STRUCTURE VACUUM SYSTEM

305 DESCRIPTION OF THE HIGH VACUUM, SOFT VACUUM AND ROUGH VACULIM SYSTEMS FOR THE DRIFT TURE

1276 BESCRIPTION OF THE VACUUM SYSTEM FOR THE SIDE-COUPLER LINAC

1276 DESCRIPTION OF THE VACUUM SYSTEH FOP THE TRANSITION PESTON

GATION INTO POSSIBLE MECHANICAL REOESIGN OF TME RCA COAXITRON A:OVE

1254 SPECIFICATION FOR LAMPF ION PUMP POWEQ SUPPLIES

2213 SPECTFICATON FOR LOINCH VACUUME LINAC ION PUMP POWER SUPPLIES

1268 SPECIFICATIONS FOR 201 MHZ SIX INCH VACUUM VALVES

1268 SPECIFICATIONS FOR GOG LITER/SEC ION PUMPS

1260 SPECIFICATIONS FOR BBS MHZ SIOE-COUPLED LINAC VACUUM MANIFOLOS

1267 SPECIFICATIONS FOR B95 MHZ FOUR INCH VACUUM VALVES

2189 SPECIFICATION GOR LIMPF HRS ION DUMPS AND POHER SUPPLIES

2192 SPECIFICATIONG FOR ROUGH VACUUM PUHO PACKAGE FOR THE HIGH RESOLUTION SOECTROMETER AT LAMPF 72 TIZ

1266 SPECIFICATIONA FOR ROUGH VACUUM PUMO DACKAGE FOR ZOI MHZ ORIFPETUBE LINAT ORO

1233 SPECIFICATIONS FOR THE FOREPUMP FOR THE ROUGH VACUUM PUMP PACKAGES FOR THE 8 KM MHZ LINAC

2190 SPECIFICATIONS FOR VACUUM PUMP PACKAGE FOR THE LON ENERGY DION LINE OF LO YQF MAZ LINAE

G98 PWO CELL 201,25 MHZ ORIFT-TURE LINAC POWER MODEL OT,

RupP, i.

149 ENERGY AND ANGULAR DISTRIBUTION OF NEUTRONS PRODUCEO GY BEA KEV PROTONAPROTON COLLISIONS

RUSBELL, G.J.

162 NEUTRONS FROH A HIGH ENERGY PROTON BEAM STOP

2082 PARAMETRIC STIIOIES OF TARGET/MODERATOR CONFIGURATIONS FOR THF WEAPONS IE ITQON RESEARCH (WNR FACILITY

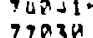

RUTLEDGE, L.L.

2416 ENERGETIC CHARGED PARTICLE YIELOS INDUCED AY PIONS ON COMPLEX NUCLEI

2910 GAMMARAY STUNY OF PION-INDUCEO REACTIONS ON COMPLEX NUELEI

1993 INTERACTION OF FASY PIONS WIYM 62.64-NICKEL
IQ99 PROTON BPECTRA FROM PIONOINDUCED REACTIONS

77446.

$7 \rightarrow 01.26$

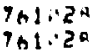

RYAN, V.A.

162 "NEUTRONS FARM A HIGH ENERGY PROTON BEAM STOP

SAITO, N. 
DAITO, N. (CONTO,)

23日0 ACCELERATINR AND FOCUSING STRUCTURES FOR PIGMI TTQTI"

BALOMON, $\mathrm{M}$.

T28 NEH MEASUREMENTS OF (PI +,2P) REACTIONS ON LIGHT NUCLEI T1BOZZ

1561 AN EXPERIMENTAL STUDY OF THE PI\$ + D GOESTOP + P REACTION AT DION ENERGIES TF 4R, 5H ANT T5BOAO

1991 MEASUREMENTS OF PIONONUCLEAR ELASTIC SCATTERING AT LOW ENERGIES

2069 THE DEUTERON DOSTATE AND THE PI* + D GOESTO P + P REACTION

2421 PHE PI+ + D GRESTOP + PEACTION AT 40, 50 AND 60 MEV

1543 TUNEUP OF THE LAMPF LON ENERGY PI ON CHANNEL

$\ln 12^{2}$

$7+1 \cdot 25$

770.9 .9

$769+6 \cdot$

SANDER, O,R.

2160 CABLE INSTALLATION DURING THE MAY 16,1977 MAINTENANCE PERIOD

2802 OIPFERENTIAL CROSS SECTION MEASUREMENTS OF PI OOR. TRITIUM ELASTIS SCATTFATNT, AT 232 , 252 ANO 295 MEVIC

2159 INITIAL RESULTS OF OO5 WIRE SCAN STUDIES MAOE ON $3 / 19177$

2M23 MEASUREMENTS OF THE DIFFERENTIAL CROSS SECTION FOR PION CHARGE EXCHANGF TN TRITIUM.

$\operatorname{TAP} 1$ P $^{\circ}$

TARI23

$777+17$

MEASUREMENTS TF THE DIFFERENTIAL CROBS SECTION FOR PION CHARGE EXCHAVGF IN TRITIIM.

7 คB:2?

2803 PI +OR- ELABTTC SCATTEAING MEASUREMENTS ON H3 ANO HE3 AT 232, 252 AND zQG MEV/C

SATCHLER, 6.9.

21I NEW GIANT RESHNANCES IN NUCLEI, aN INTERIM REVIEW

770

$7411 \%$

SCARBMOUGH, E.C.

1520 STEAYYSTATE TEMPERATURE AND STRESS DISTRIRUTIONS OF A PROPOSEO LAMPF DYROLYTIC GMIPHITE Ton product ton tapget

BCHALLER, LEÁ. TESTING AT IOG MEV
IIOG TARGET TESTA

77110

11429

BCHALLER, B.C.

1760 UEING THE LAHPF VIOEO SYSTEM (THE Y YOEO PATEH FILE ANO FRIENDS)

sehamaun, R,G:

1305 A8SEMBLY INO C.HECK-OUT PROCEOURE FOR INTER-TANK SPACER RING, TANKS I ANOZ

2196 SPECIFICATIONG FOR BIOMEDICAL BEAM LINE MAGNET SIEEL

2200 SPECIFICATIONA OR LINE 
SCHEFER, J,P.

1563 ABSOLUTE CROSA SECTIONS FOR PROOUETION OF PROMPT NUCLEAR GAMHA RAYS OY FAST OIONS

1586 ALPHA AND MUL PINUCLEON REMOVAL FROM 58-NICKEL ANO GO-NICKEL BY FAST PIONS

2a16 ENERGETIC CHARGED PARTICLE YIELOS INOUCEO BY DIONS ON COMPLEX NUCLEI

1301 FAST PICN INOIICED PROCESSES IN COMPLEX NUCLEI! CROSS SECTION AND ALPMA REMAVAL

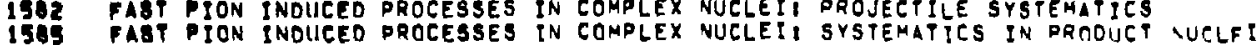

2418 GAMMATRAY GTUNY OF PION-INDUCED REACTIONS ON COMPLEX NUCLEI

2418 GAMMAERAY STUNY OF PIONEINDUCED REACTIONS ON
1993 INTERACTION OF FAST PIONS WITH G2, GU-NIEKEL

193 INTERACT ON OF PAST P ONS WNOHER G

15.2 OYBTEMATICS OF DION AND PROTON INTERACTIONS WITH NICKEL NUCL gDES

$750>0$ ?

758 Bat

770 aी:

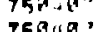

75 की

790.75

773425

701
761.52

custhact

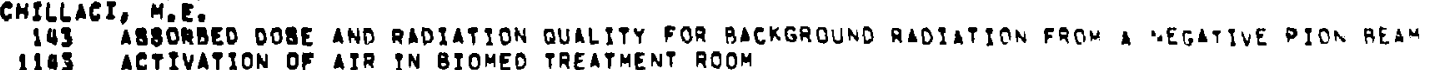

7509 कू

1143 ACTIVATION OF AIR PN BTOMEO TREATMENT ROOM
1097 AEYMMETRY IN PI PRODUCTION BY POP COLLISSONS

220 CHEMICAL EFPETTS IN NEGATIVE MUON CAPPURE IN SOME IONIC AND COVALENT S.LIOS ANO IONIC AOUEOUS

IOI2 COMPARISON OF LAMPF ANS RLIP FOR PRODUEING RADIOISOTOPES

1142 COMPARIBON OF LIS MOOEL TO ARMSTRONGS MONTE CARLO CALCULATIONS

IOES COMPUTER MODEI ING OF NEUTRON DOSE AT VARIOUS LOCATIONS WITHIN THE RONY D RIVIS DINN

1 DOS DOSE OIETRIBUTION DUE TO NEUTRONS IND PHOTONS RESULTING FRON NEGATIVE PITN CAPTURE IN

IMos Tose org

1 HOS EFFECT OF IOAMORON ON TME STAR NEUTRON DOBE DISTRIBUTION IN NEGATIVE PPOL QATIOTHERAPV

lial FY 76-77 109 TNFORMATION

2003 HYOROGEN ISOTAPE EPFECT ON MUONIC XERAY SPECTRA OF (CH SUB 2) CHE ANO H S 2

659 INTERLABQRATORY COMPARISON OF SPALLATION REACTION CROSS SECTIONS FOR IHO AVR COPPER HITH SOFEMEV PROTONS

1013 IOOTOPIC NEUPRON SOURCES FROM THE LOS ALAMOS MESON PHYSICS FACILITY (LAMJF)

1315 MESIC CWEMIITRY RESEARCH PLANHED AT LAMP

2805 NEGATIVE MUON CAPTURE IN BINARY SYSTEMS

1009 NEUTRON STREAMING IN BIO-MED CHANNEL

ON THE OIBCREPANCY BE TWEEN SOFT PION PQEOSETIONS

IIUA PRELIMINARY RFBULIS FROM LAST RUN, EXPERTMENT GO

MADIOLSOTOPE RRODUCTION AT LAMPF

57 RADIOI8OTOPE PRODUCTION AT THE LOS

IAG 3 MAOIOLSOTOPE PRODUCTION AT LAMDF

2331 RUTHENTUMAQ9 PROOUETION AT LAMDF

1006 WHOLE BODY DORE DIBTRIBUTION DUE TO NEUTAONS ANO PHOTONS IN NEGATIVE PIMI RAMIOTHERAPY

1596 WHOLE BOOY OOAE FOR DAPIENTS PAEATEO WIPH PJONS

$758 \times 8$

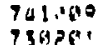

Topin

71001

750115

700910

758702

75878.

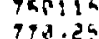

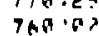

11010

Tinam.

77 a

771 Ha

70,725

$7.3+1$

$721 \%$

Thing,

$\tan 20$.

$75 \sin \theta$

Tintio

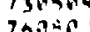

DCHIMMEAL INE, W.

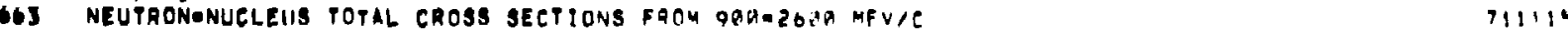

SEHLEUETZ, Fi:

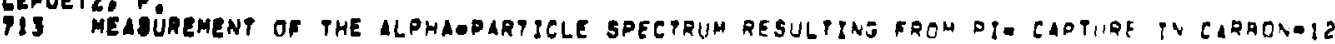




\section{SCHNEIDER, E.J.}

2604 BES QUAO MACNFT DOWER SUPPLY CONYRML

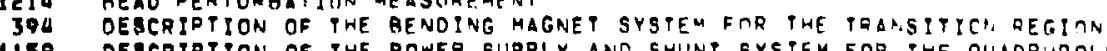

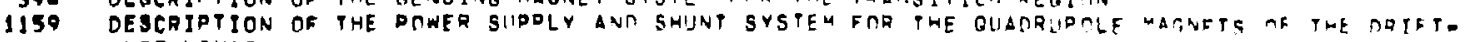
TUAE LINAC

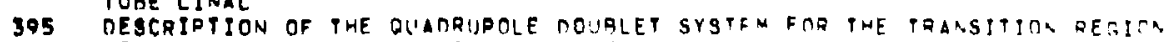

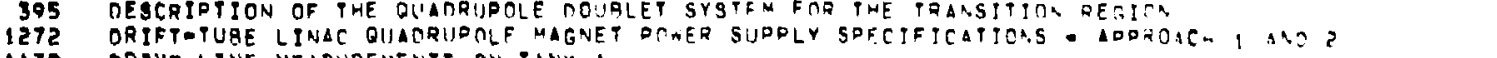

1139 DRIVE LINE MFASUREMENTS ON TANK I

1249 EXCITATION OF TANK I' 1206 TIELD PERTURBATIDNS IN THE ODST COUPLES DRIFT-TUAE LINAC

1251 POWER SUPPLY SPECIFICATIONS FOR TRANSITIONA REGION HENOINT, MAT,AFTS

RIPPLE CURAENT AND FLUX IN MINERAL INSULATEO MAGAFTS

2703 SPECIFICATIONS FOR BIPOLAR POWER SUPPLIES FRE TRANSITION REGION VERTICAL STEFRIVE MAGNFTS

2716 SPECIFICATIONA FOR MAGNET POWER SUQPLY IAI2OC2SG (प5PV)

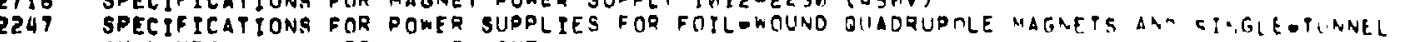
OUADRUPOLE MAFNETS - HAR LINE

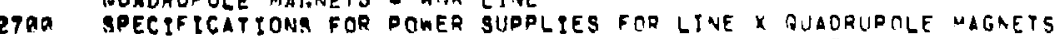

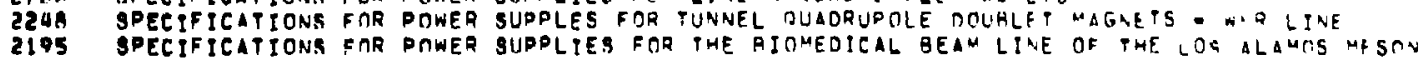

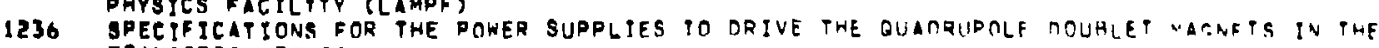
TRANSITION RERION

THE DRIFT TUBE LINAC BY OOERATION IN THE PI/Z CAVITY IOOME

10. TWO CELL $2 B 1,25$ MHZ ORIFTETUAE LINAC POWE MONEL

SCHNEIDER, F,

69 STEPPING MOTOR DPIVER WITH INTERPOLATEO STEPS DESIGNEO FOR REAM SCAAPEOS IN THE ISO

$\operatorname{tanses}$

710503

710529

कम 1125

7.1. in

$7 \cdot 1 \cdot 24$

brist

$319: 13$

$751 \cdot 5$

$75 \times 516$

70 is 5

Thatis

TaAs?

$720: 50$

$74025:$

$7>040$

$710 \times 14$

$\log 3$ a

SCHROOER, W.U.

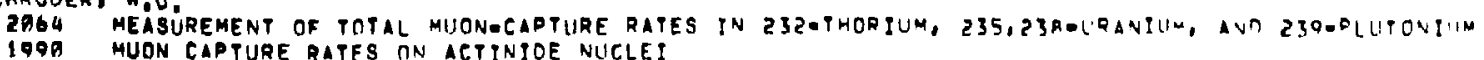

$761>a$.

int 2

SCHULPHE TS, P.M.
ITOP

1496 ACCELERATOR TIIRN-ON SERUENCE

ORAWING SYMAOI S STANTARDS

1739 GENERAL DRAWING RULES AND GRAPHIC SYMBOLS

1590 LAMPF COMMUNICATION REDUTREMENTS

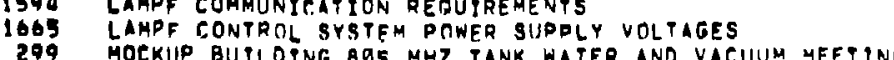

1492 MODULATOQ TURNOON SEOUENCE

MODULATOQ FURNAON SEQUENCE

DATA ACQUISITIINN AHO EANTACL SYSTEV

THE APPLICATION OF DIGITAL COMQUTER TO THE CONTROL ANO MONITOEINE OE A DOOTCL LiVAO ACCELERATOR

06,4519

651 in

bation.

65113

6hat

bhares

5110?

Q51110

65)

scort, L.D. 
SCOTT, L.D. (CONTD.)

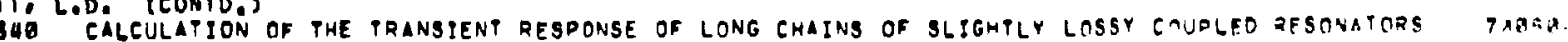

IOTI PRANGIENT ANALYSIS OF PHE SIOE -COUPLED LINAC ANO ORIFTEPIJEE LINAC

BEEGER, P,A.

162 NEUTRONS FROM A HJGH ENERGY PROTON BEAM STOO (WNR) FACILITY

BEGLL R.E.

1363 ABSOLUTE CROSA SECTIONS FOR PRODUCTION OF PROMDP NUCLEAR GAMMA RATS BY FASP DIONS

1580 ALPHA AND MULTINUELEON REMOVAL BROM 5A-NIEKEL ANO GQOVICKEL BY FAST PIONS

2416 ENERGETIC CHARGEO PARTICLE YIELDS INOUEEO GY DIONS ON COMPLEX NUCLE

1501 PAST PION INDUICEO PROCESSES IN COMDLEX NUCLE II CROSS SECTION AND ALPMA QEMTVAL

1582 PAST PION INDUICEO PROCESSES IN COMPLEX NUCLEII PROJECTILE SYSTEMATICS

1585 PAST PION INDIICEO PROCESSES IN COMPLEX NUCLEII SYSTEMATICS IN PRODUCT YUCLE:

2416 GAMMA-RAY gTUAY OF P:ONEINDUCEO REACTIONG ON COMPLEX NUCLEI

1993 TNTERACTION OF FAST DIONS WITH 62.64-NICKEL

1562 SYSTEMATICS OF PION AND PROTON INTFACTIONS WITH NIEKEL NUELIDES

SEKI, $R_{\text {. }}$

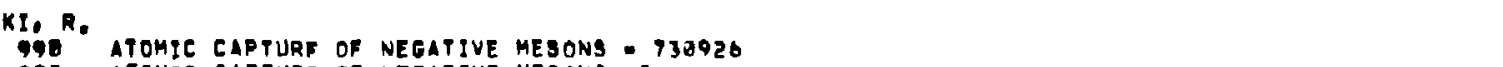

998 ATOMIC CAPTURF OF NEGATIVE MESONS - 730926

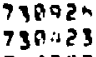

$75 \times 5019$

APOMIC CAPPURF OF NEGATIVE MESONS - 95A597,

DETERMINATION OF THE NEUTRON RALD FROM ANTIPROTON ABSORDTION

OETEAMINATION OF THE NEUTRON HALO FROM D BAR

NUELEAR MDTION CORREETION TO EXOTBC ATOM LEVEL NIOTHS

$93125 ?$

740130

730 aga

EeLYTOEE, $h$,

9at MEASUREMENY OF DOBE DISTRIBUTION OF COLLIMATEO NEGATIVE PION BEAMS MITA EILM

$750 \times 93$

DeREVILLE, . OE

G66 PARTIELE PROOUETION FROM OEUTERONS OF 1.16 AND 1.02 GEV

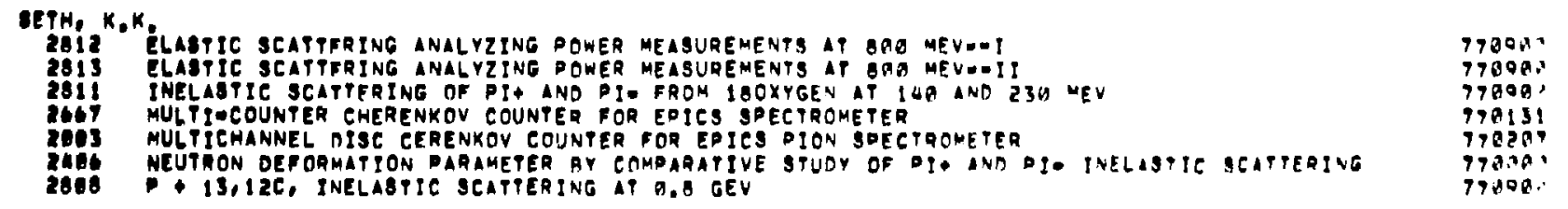

SHamal, $Y$, 
ghaMAT, Y, (CONTO.)

704 MEAGURMENTS OF THE (PION PLUS, PION ZERO) REACTION ON LIGNT ELEMENTS IN THE $(3,3)$

760118

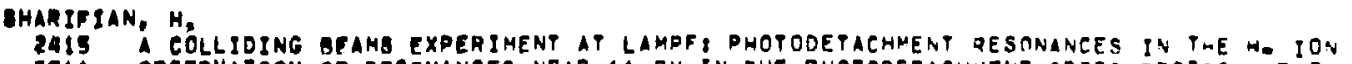

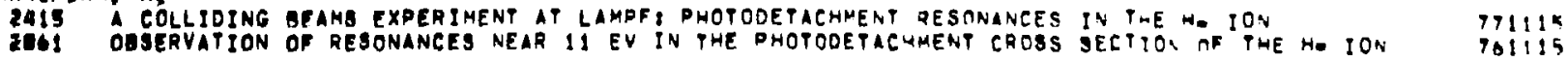

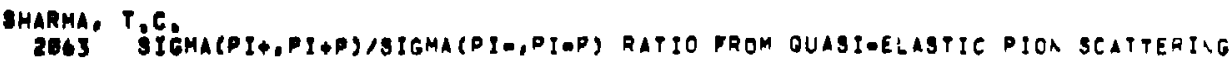

701291

SHARP, J.B.

282 Bés MHZ AF-ACeELERATOR SYBTEH

767 BQ5 MHZ TEMPERATURE CONTROL SYSTEM - WHAT, WHERE AND WHY

346 A DIGITAL RESTNANCE CONTROL SYSTEM FOR THE ORIFT-TUBE LINAC

270 ACCELERATOR FTELO MEAGUREMENTS AT HIGH POWEA

1537 CONBOLE INTERFACE UNIY - IIO INFORMATION

3 OESCRIPTION OF THE TEMPERATURE CONTROL SYSTEM FOR THE SIOEOCOUPLED LINIC

397 OECERTPTION OF THE TEMPERATURE CONTROL SYSTEMS

293 PUAT DOWER OPFRATION OF THE LAHPFE. AND WHY

GO4 RESONANCE CONTAOL GYSTEM FOR LAMPF SIOE-COUPLED LINAC

1926 BPECIFICATION FOR A OUAL OUTPUT, UNREGULAPEO, DC DOWER SUPDLY

1682 THE BIOE-COUPIEO LINAC PEMPERATURE CONTROL SYSTEM

SHELINE, R,K,

20BG MOOEL -INDEPENBENT INTERPRETATION OF THE OYNAMIC HYPEREINE EFFECT IN MUNNIC ATONS WITW AN

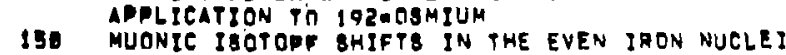

SHEAh, E.B.

250 ' COMPUTING IN GUPPOAT OF EXPERIMENTS AT LAMPF

200 ELETROMAGNETIE MOMENTS OF THE EVEN OS NUCLE! FROM MUONIC $X$ RAYS

2000 MOOEL INEEPENRENT INTERPRETATION OF THE OYNAMIC HYPERFINE EFFECT IN MUNNTE ATOUS ITM AN APPLTCATION TO IQzeOSMZUM

150 MUONIC ISOTOPY GHIFTS IN THE EVEN IRON NUCLE:

140 MUONIC XERAYS FROM THE TRANGITION NUCLE! $194,195,1960$ DLATINUM

I6A4 SYBFEMATICS OF NUCLEAR CHARGE DISTRIGUTIONS IN IRON, COBRLT, NICKEL, COPAER ANA ZINE DEDUCEO FROM MUONTE XORAY MEASUREMENTS

GHERTROOK, M.V.

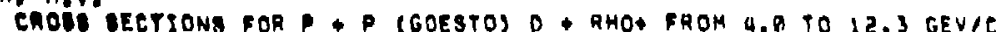

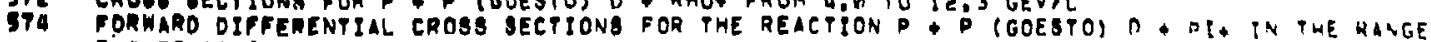

6723.4 TO 12,3 GFY/C

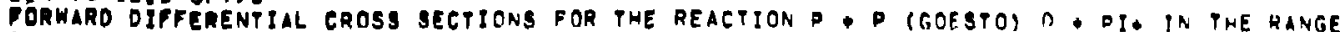
3.0 TO 12,3 GEV 10

$7 \rightarrow \operatorname{lag} 25$

70429

oor

Tana

TaA

bon.tis

$940 ? 2$

70025

$71+34$

714341

oble

779.2

750304

761.94

$7+1+5$

77240

$7503 a x$

$75 a$
$750+2 a$

TOAAC."

$72072 !$

6097 
BKEAMAN, J.D.

1995 BCATTERING OF 5B MEV PI+ FROM LIGHT NUCLE

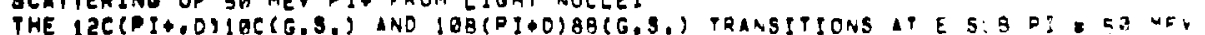

2810 THE ILCCPI,PJIIC REACTION ATE SUBPI SB MEY TPBO

BHERMAN, R. H

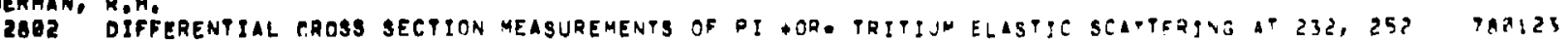

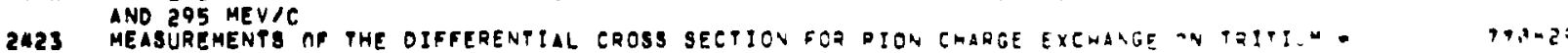

2424 MEABUREMENTS AF THE OIFFERENTIAL CROS3 SECTION FOZ PIOU CHARGE EXEHAGE VY TZIT! W.

1633 PHOTON SPECTRIIM IN PION CAPTURE ON TRITIUM

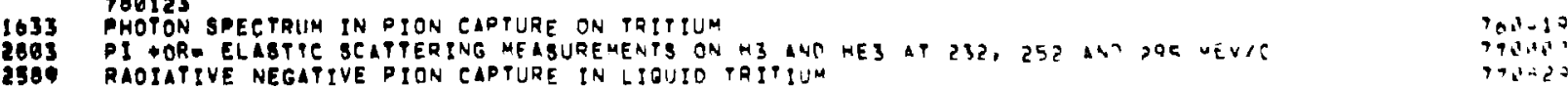

BHIRK, 0.6 .

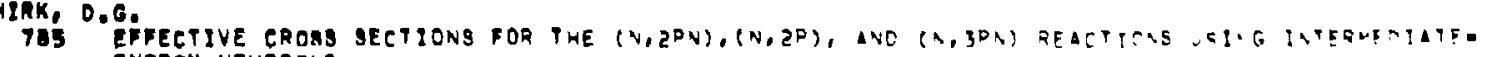

2914 ENERGY NEUTRONS

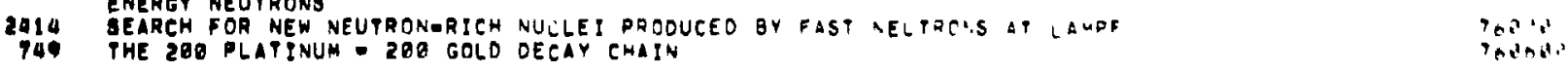

BHIVELF. F.T.

2030 BEAM LINES A ANO X MAGNET ELEGTRIC POWER SYSTEN

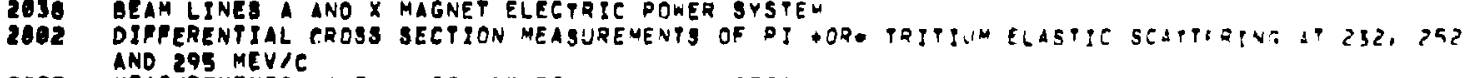

2423

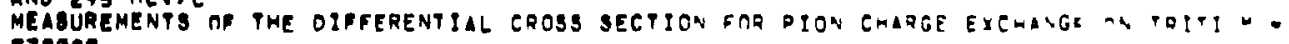
770000

2924 MEAGUREHENTS OF THE OIFFERENTIAL CROSS SECTICN FDR DION CMARTE EXCHAVGE A TOITI: .

2316 TOG123 ON TWOAFEASY MODE OF SWITCHYARO OPERATION

1633 PHOTON SOECTMIM IN PION CAPTURE ON TRITIUM

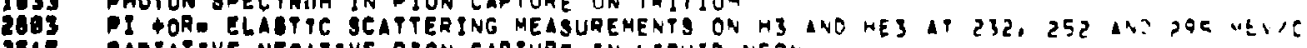

2515 AADIATIVE NEGATIVE PION GAPPURE IN LIOUIO NEOA

2509 RAOTARIVE NEGATIVE PION CAPTURE IN LIGUIO TRITIUM

SORPARE AEDUTREMENT FOR INITI IN BEAM TRA

D

DOPOLES TO CORPECT FCR BIELO VIRIATIAVS

2620 SWITEHYARD BEAM PRANGFER. REgULTS OF PIELO VARIATION ANILSIS

GW ITEMYRD BEAM TRANBFER

2040 SYSTEM OP SWITCMYARD BELMOMON!TORING DEVICES

SHLAER, 1,0 .

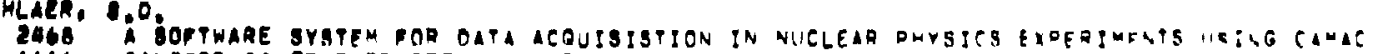

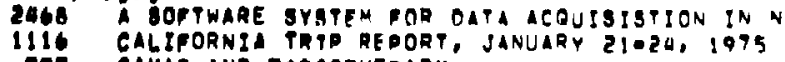

ITS CAMAC AND TRDPTHERARY

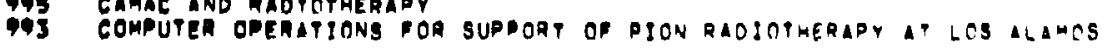

$72,332^{\circ}$

$\rightarrow a^{*} \cdot 2 x$

19,102

प0त!

72019

ㄷ․ㄴ.

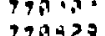

$190+29$

$170 \div 5$

$720: 1$
79025

710225
$71+319$

71m3!

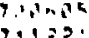

i.

79117

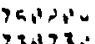

34730
$740-20$ 
SHLAER, S,D (CONTD.)

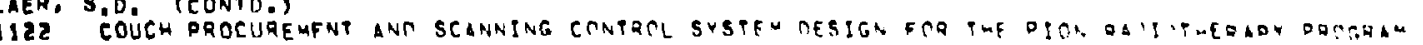

2A56 DATA-ACQUISITION STFTWIRE

6I3 INTERACTIVE GQAPHIES FOR ICCFLERATMR CTNTOCL

1694 PROGRAMMING THE CALENDAR CLOCK

PROGRAMMING THF LAMDF HASTER II UF Q

1695 PROGRIMHING THE PIU AND ALL ITS FPTELDS

17GG DROGRLMMING THE STIRAGE SCOPE

1791 PROGRAMMING TWE TRACKBALL
1782 TENTATIVE PLANB FOR GITEMEO CQUPUTER SYSTEM SCFTHAEE

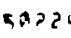

$3 ., 105$

001,17

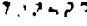

738210

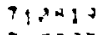

$\operatorname{tin} 5 \cos$

BHLAER, M.J. J

S52 BQBOMC RF STRIICTIIRES

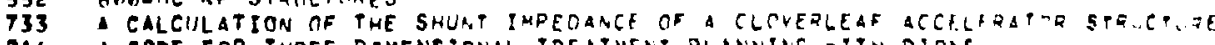

296 CLEANING OF G.E. WINOONS

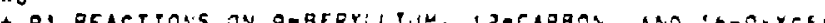

CROSS SECTIONS FOR

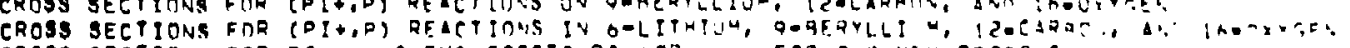

CRORA SECTIONS FnP DI

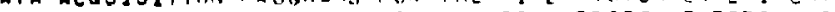

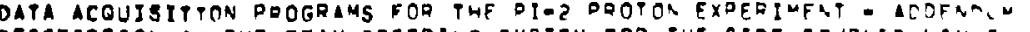

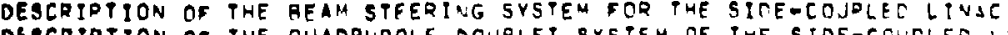

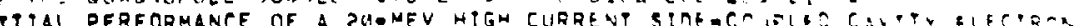
ACCELERATOR

1320 MTCLERATOR

NEW MEASUREMENTS OF (PI TOP) REACT IOAS ON LIGWT PUCLEI

NEW MEASUREMENTS OF (PI+, $2^{\circ}$ ) RELCTIONS ON LIGHT NUCLE

GQ0,

RESONANT STOE =COUPLEO CAVITY ELECTROA ACCELERATORS

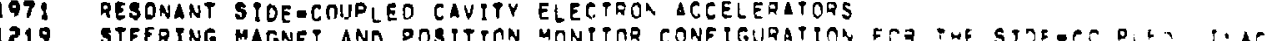

2920 SUPPLEMENT TO SKOMP $=3=\Delta 10$

797 THE ONE DIMENSIONAL APPROXIMATION TO THE COAXITRTI

$0,0.50$

o 31 is:

3,3

anditis

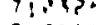

$1+\frac{100}{10}$

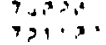

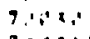

$\log (0,5)$

00155

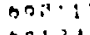

SHONK, C.R.

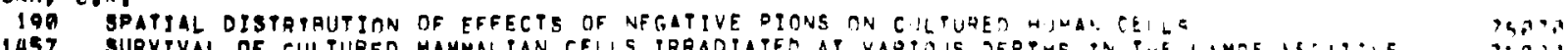

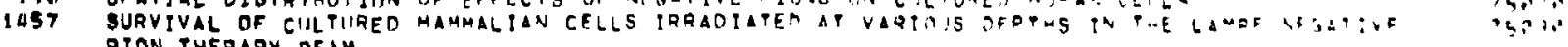

SILBAR, R,R.

1OS1 GEAM TRANSPORT FROM LAST TARGET TO BEAM STOP

COMAENTS ON GYMAETRIT PRTPLETS

FIT NUCLFAR STRUCTURE EFFECTS in (PI,PI N) PEACTITNS

546 MODEL DEDENDENCE OF FIONANLICLEUS TOTAL CROSS SFETIONG

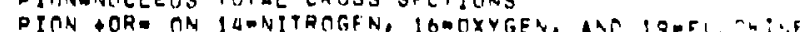

581 ON THE OISCAEPANCY REPWEFN SOFT PION PREOICTIOSS ANA EXSER

IASS PHIMARY BEAM TRANSOORT! FIRBT TO SF CONA TARGET

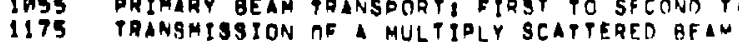

$\operatorname{sothan}$

$\log : 5$

in: $2,5,0$

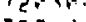

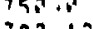

$\log 3 \cdot 1$

कondin.

$\therefore \rightarrow$ 
SILBAR, R,R, (CONTO.)

1177 WAIST-TOMLIST TRANSPORT WITH ONE THIN LENS

SIMMONDS, D,D.

2467 CAMAC BASED COMPUTER-COMPUTER COMMINICATIONS VIA MICROPROCESSTR AATA LYKS

$51: 17$

1 COG6 COMPUTER-DESTGNEO OQ5 MHZ PROTON LINAC CAVITIES

582 DATAOACOUISITTON COMDUTER NETWOQK

1081 LAMPF SPECIFITATION FOR OIGITAL CONTROL COMPIITER SYSTEN

1153 SPECIFICATION FOR DIGITAL CONTROL COMQUTER SYSTEM

$58 \cdot 13$.

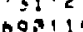

790,5,

$0 \times 1>3$

IIMMONO, J.E.

2978 (P,N) AND (N,P) MEASUREMENTS FROY TARGETS OF G-LITMIUM AND TOLITHY AO A. UEV

1994 G,70LITHIUM(O.N) AND (N,D) MEASUREMEATS AT BGM MEY

204 A MULTIWIRE PROPORTIONAL CHAMBER SPECTROMETER FOR NUCLEONENUCLEOA EXPEPIVEMTS

201 EARLY EXPERIMFNTS WITH NEUTRON BEAHS AT LAMPE

24I0 EXTREME BACK ANGLE ND ELASTIIC SCATTERING AT $794 \mathrm{MEV}$

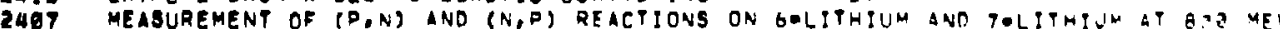

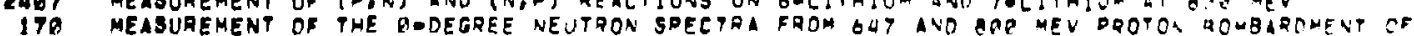

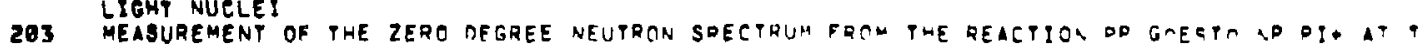
SUB D T6U MFY

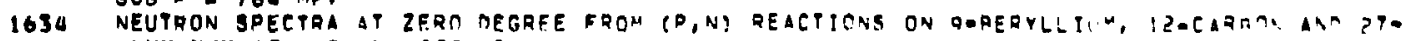
ALUHINUH AT 649 AND BPA MEV

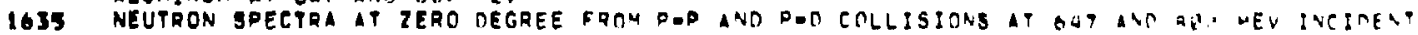
ENERGIES

1969 NEUTRON

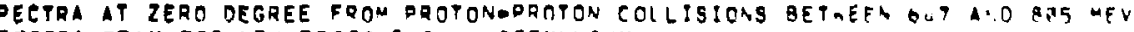

NEUTRON SPEETPA FRTM PROTON ROMBARDMENT OF DEUT

DDUCTION TN NEUTRONAPROTON COLLISIONS

202 PRECISION MEAMUREMENT OF NAP CHARGEREXCNANGE CROSS SECTION A? Di H

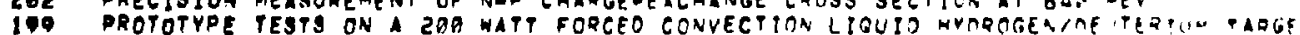

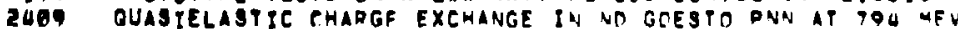

SIMMONS, M.L.

162 NEUTRONB FROM A HIGH EMERGY PROTON BHAM STOR

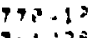

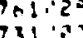

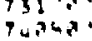

$7 \rightarrow 00$ a

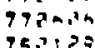

72,3

$7 n+3 \times a$

TAP 7,16

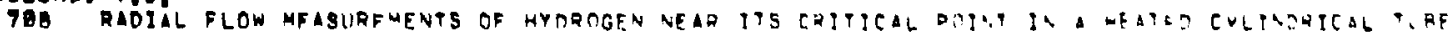

$779+\cdots$

$740+345$

$726 \cdots$

751253

70,7110

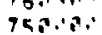

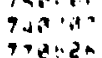

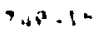

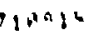

SMITH, E.

2811 INELASTIC SCATTERING OF PI. ANA OIN FROM IAOTYGES AT IAM ABE 231 MEV

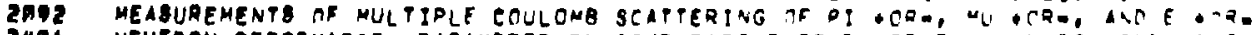

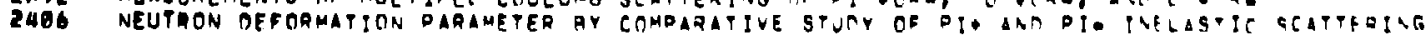

8HIYH, G. 
OMITH, 6. CCONTD,

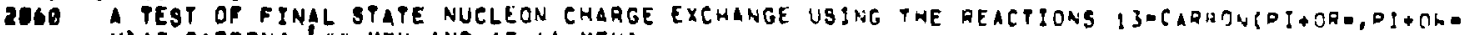
NJIE-CARBONCL. LA MEV ANO 15.11 MEV

1153 COMPARISON OF NEUTRON REMOVAL EROM TOLITHIUM BV PI OOR- WITH FREE DITAEA CLEAN RESULTS

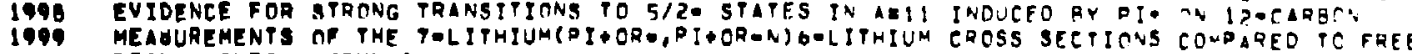
PIONONUCLEON RESULTS

2001 PIMHESON INDUREO PROTON REMOVAL FROM Q.BERYLLIUM TO THE A.QBI MEV ONE OL S STATE OF A.

2893 PION INDUCED NUCLEON REMOVAL FROM LIGHT NUCLE!

770247

761191 $7+1 \cdot 20$

$761 \cdot 28$

$77 \mathrm{H}+9$.

77040.1

SMITH, J.J.

ITAS CABLE TERHINATOR/ORIVER CHASSIS - FAQRICATION SPECIFTCATION

32 CLARIFICATION OF LAMPF COHPUTER BPECIFICATION NO, MPGITOA3

IAIS COMPUTER CONTROL OF THE LAHPF ACCELERATOR

1779 COMPUTER INTERFACE UNIT SPECIF
BTS DATA ACQUISITION AND CONTROL

1900 INTEGRATED CIRCUIT MOOULE ENCAPSULATION SPECIFICATIONS

1785 INPEGRATED CIRCUIT MODULE CONNECTOR ROARD ANO IRING SPECIFICATIOA

7 LAMPF DATA ACOUISITION AND CONTRDL SYSTEM

1424 LAMPF I ANO C SYSTEM MOOIFICATIONS

1776 LAMPF LEVEL ADAPTER CHASSIS SPECIFICATION

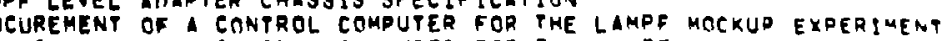

TOQ THE LAMPF HOCKMUP EXPERTMEAT

BPEIFICATION FOR ONELINE DIGITAL COMPUTER SYSTEM

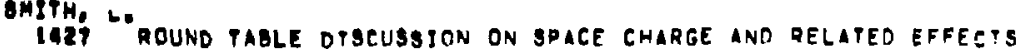

061110

bh: in:

$6 n 3505$

05490 ?

obopia

Obe?3:

$600 \%$

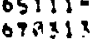

$6 n+25$

60000 .

600099

05132 .

on $1>4^{n}$

SMITH, L.E.

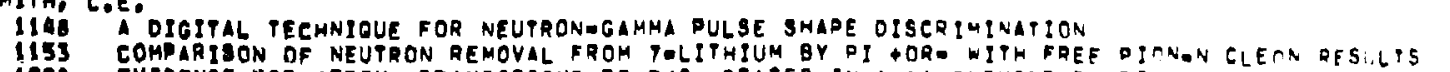

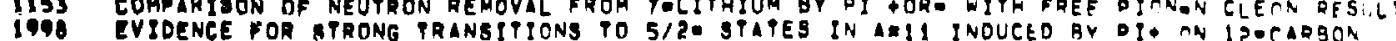

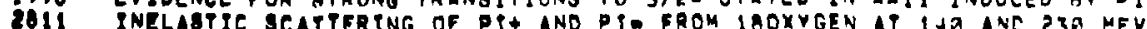

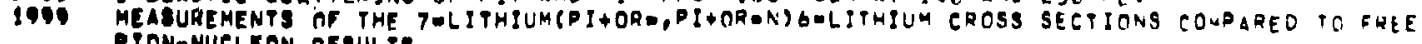

Then:

$701: 52$

$101 \cdot 2 \cdot 2$

ont PH, T.W.

IUS3 MYOCARDIAL POAITRON SCINTIGRAPHY WITH SHORT-LIVED RUBIDIUMOBZ

Tan.a.

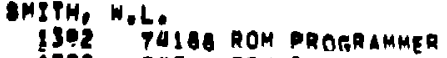

1993 DHE HARDWARE MOOTSTRAP READ ONLY MFMORY

560 40 MO PRIORTYY INTERRUPT STATUS OISPLAY

ISOA DHO MP PROGRAM SENBE REGISTER INO PRIORITY INTERRUDT ENCODER 
8MPTH, WL TCONTO

I592 SUA REMOTE RESTART

ANALOG DATA SYSTEM (AOS) TEST DROCEOURE AND RESULTS

DOVDE HEAO CONTROL UNIT

POVA EMITTANEE DIAGNOSTIC DAC?

1320 LAMPF RICE I/O MODIFICATION FOR FAS

1731 MOBILE REX

2454 POPAI OOWNL INE LOAO/REMOTE RESTART

1341 PORTABLE ADS FXERCISER

1509 DORTABLE HANDHELO COUNTEO

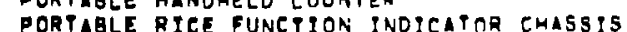

1335 PRESENT STATUS OF RICE, RICE IO RICE POWEQ SUPDLIES ARO AMALOC OA A SYSTEUS

1983 REFERENCE ANO MAINTENANCE MANUAL: LAMPF OATA IGQUISITICN ARO CONTROL TFR I*AL

RICE CHECKOUT US ING PHE REX (WITH NO 10 OR CONTROL MOOJLES)

1310 RICE CHECKOUT USING THE REX (WITH RICE I10 CONVECTOE J3 TESTER)

1350 RICE I/O BINARY DATA UNIT (BOU

1372 SAMPLE AND HOID EXERCISER

SOS RICE BINARY COMPARATOR EARD TESTE

1340 SEQUENCE OF EVENTS FOR RICE I/O TATA PAKES ANO GCMMAVOS

15.20

30,18

7ane

75032.

$3 \sin 2$

ำ

Tratis

Tisats

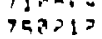

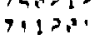

710.3

7509

00 a

$737=51$

$70,1,12$

73250.

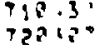

BNYDER, J,N

627 LAMPF DATAGACAUISIYION SYSTEM

$7 \because \cdots$

BOBOTTKA, B.E.

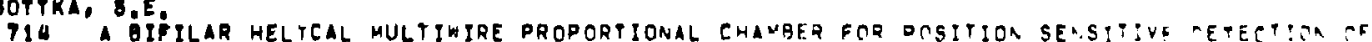

$\sin$

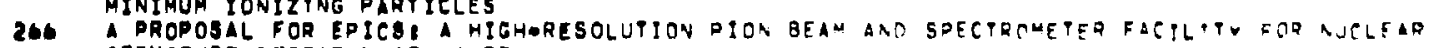
TrROPSAL

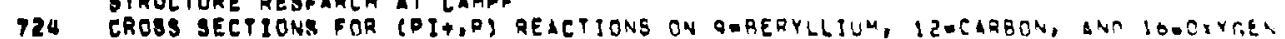

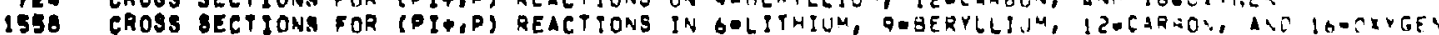
DELAY LINE REAOTUT OF ANODE PLANES IN PROPORTIONAL CMAMAERS

NEW MEASUREMENTS OF (PI\$, 2P) REACTIONS ON LIGHT VUCLEI

PARASITE PION CHANNELS

PROPORTIONAL P.HAMBERS WITH MONOF ILAR HELICAL CATMOOES FOR MIGH SPATIAL RESA THO

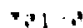

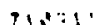

$1+4,3,4$

Thing

lings:

$73,2 \cdots$

BORENBEN, L,B,

1347 A MEASUREMENT OF PARTTY VTOLATION IN PROTONEPRTTON SCATTEHING AT IF UE

15 L LIMIT ON PARITY NONCONSERVATION IN PONUELEUS SCATTERIVG AT O GEVIE

1620 LIMIT ON PARIYY VIOLATION IN P.NIIELELIS SCATTERING AT O GFVIE

740.06

in?

SOUDER, P A

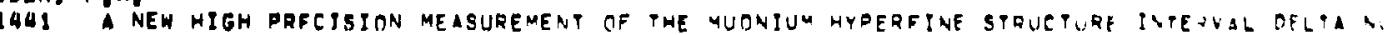

DEPOLARIZATION OF NEGATIVE MUONS IN HELIUM GAS, SEARCH FOR MIONIC HELT MATOU

23HE DEVELOPMENT OF A HIGHAPURITY LOHAMCMENTUA MU PLUS PEAM FOR LAMPF

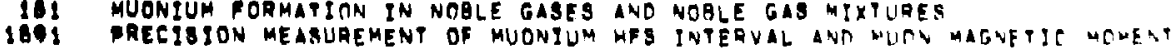




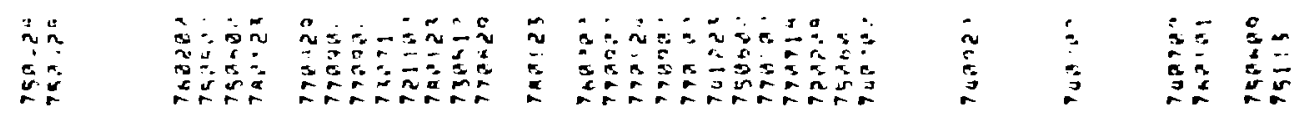
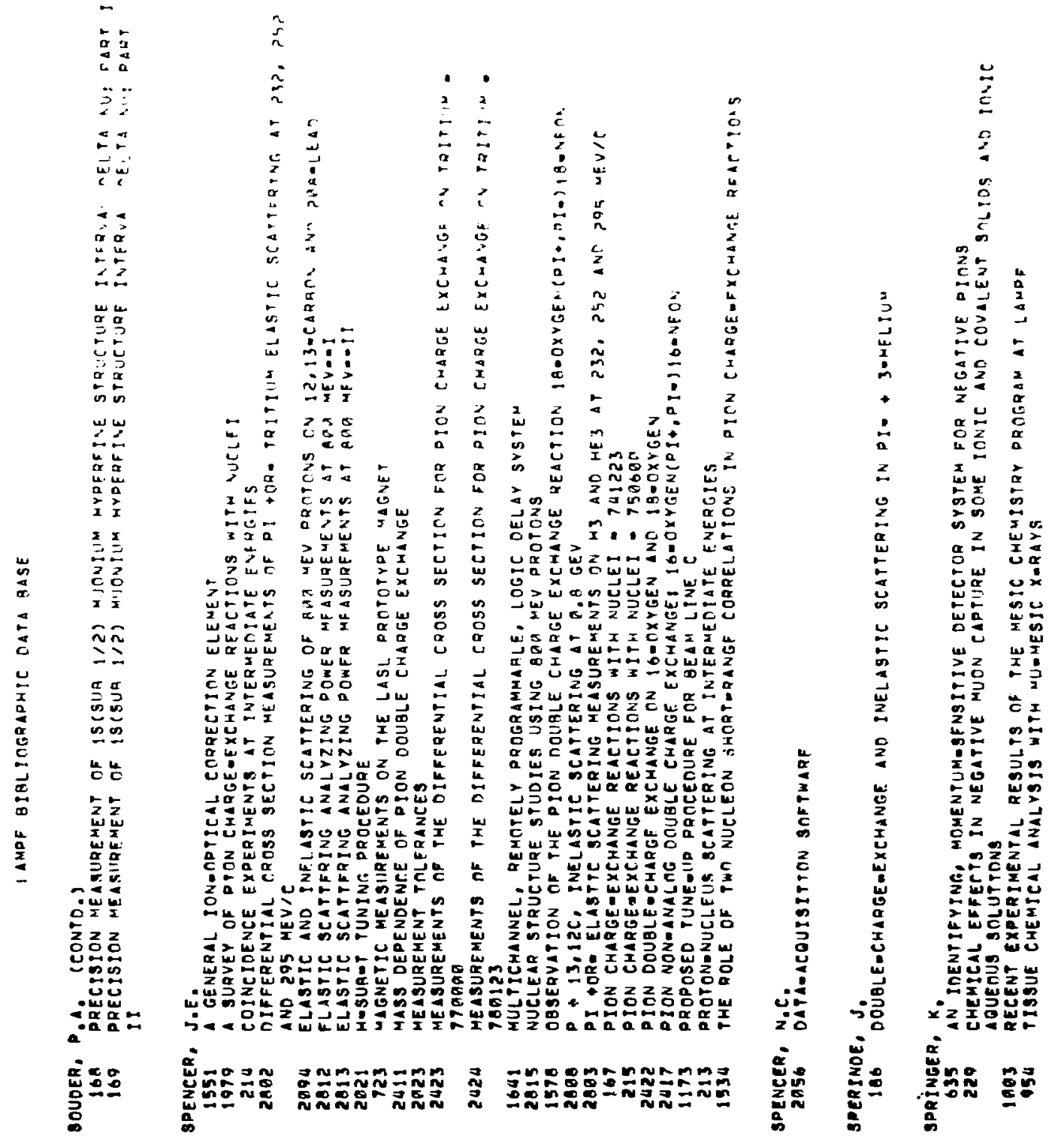
STAMBAUGH, R.D.

14AI NEW HIGH PRECISION MEASUREMENT OF THE MUONIUM HYPERFINE SPRUCTIIRE INTELVAL DFLTA N.

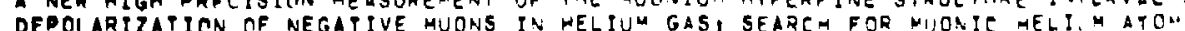

101 MUONGUM FORMATION IN NOBLE GASES AND NOBLE GAS MIXTURES

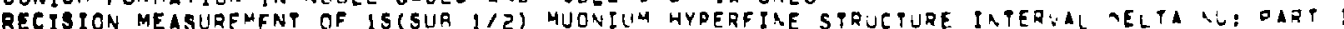
II

STIRNER, J,W.

194 THE 158-ERGIUM GRESTO 15A-HOLMIUM DECAY SCHEME ASD QCSIB BETAS VALUE

$7 a 1 \cdot 3$

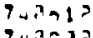

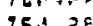
75,30 II

STEARNS, R.

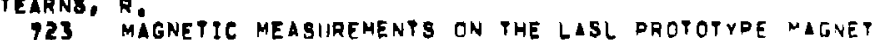

$14 a \cdot .9$

72113.

BTEFFEN, R. M.

ROBQ ELECTROMAGNETIC MOMENTS OF THE EVEN OS NUCLEI FROM MUONIE $X$ DAYS

150 MUONIC ISOTOPF SHIFTS IN THE EVEN IRON NUCLEI

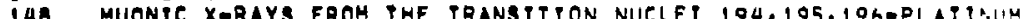
IGUA SYSTEMATICS DF NUCLEAR CHARGE OISTRIBUTIONS IN IRON, COBALT, VICKEL, CMDOER AMA ZIMC

STEIN, N.

2078 (P,N) ANO (N,P) MEASUREMENTS FROM TARGETS OF G-LITHEUM AND 7OLITMIIM AP Q,3 "EEV

1994 6,7०LITHIUH(D,N) AND $(N, D)$ MEASUREMENTS AT BAG MEY

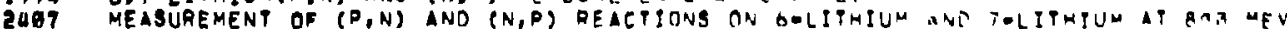

STEINOERG, E,P

I199 REACTIONS OF FAST PIONS WITH 197 MOLO

BTEDHENSON, G.J,

2016 AN OPTICAL MONEL STLIDY OF LOW ENERGY PION BCATTERISG FROM IOOXYGEY

2BGi PARITYONONCONBERVING ASYMMETRY IN NOO SCATTERING

Mi:

STERNHAGEN, C.J.

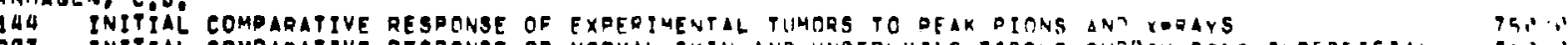

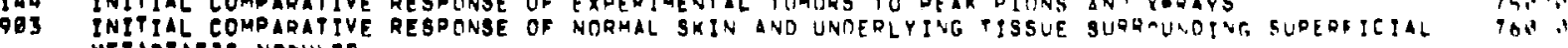
METAGTATIC NONULE

\section{BTERNAEIM, M, M.}

SHS (KA): NUCLEUS INTERACTION AND ITS INTERDRETATION

SHB COMPLEX EIGENVALUES OF A CNUPLED EHANNELS MONFL

2962 DRAMATIC NUCLFAR STRUCTURE EFFECTS IN (PI,DI V) REACTIONS

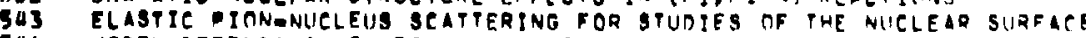

SHG MODEL DEDENOENCE OF DION-NICLEUS PATAL COOSS SPCTIONS 
CTERNHEIH, M.M. (CONTR,)

- ItOOXYGEN SCATTERING AT 27 A MEV

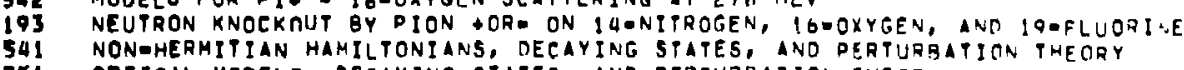

OPTICAL MODELS, OECAYING STATES, AND PERTURBATION THEORY

1370 PI PLUSOMINUS ABSOAPTION CROSS SECTIONS AND NEIITRON DISTRIEUTION PARAMTTRS

1195 PION ABSORPTINN CROSS SECTIONS AND NEUTRON DISTRIAUTION PARAMETERS

THEORY OF INTFRACTION OF MESONS ANO NUCLEONS WITH NUCLEI

STEVENS, R,R.. JR,

2614 1:10̈ PULSING SYSTEM

2630 IN 10 CHOPPTNG MODE

2583 AROTATING WHFEL BFAM SCANNER FOR THE ELECTRON PROTOTYPE ACCFLERATAR

2600 ACCELERATING ROLUMM FAST DROTECT SYSTEM

110 ACCELERATOR DEVELOPMENT STUDIES FROM NOVEMBER 20 TO NOVEMBER 20

2640 ACCELERATOR DFUELOPMENT FOR PROOUCTION BEAMS

2503 ARC CONTROLLER CUTPUP TRANSFCRMER SPECIFICATION

2606 ARC PULSE MODILLATOR PROTECTION CIRCUISS

458 ARE-OONN PRDBIEN ON THE H* INJECYOR

SO4 ARC-DOWN PROBI EM WITH COCKCAOFYOWALTON GENERATOR

458 AREDOWN PROBLFM IN THE HIGH INTENSITY INJECTOR

2612 BEAM BRIGHTNESS CONSIDERATIONS

2632 BEAM DEVELOPMFNT PLAN

4S1 BEAM OEVELOPMFNT TESTS

431 BEAM EXPERIMENTERS MEETING

2SI BEAM MEASUREMFNTS ON THE HIGH-INTENSITY PROTON INSECTOR OF LAMPF

BEAM PROFILE MONIIORING ANO ANALYSIS BY TELEVISION

BEAM PROPERTgFO

TEN OF OUAORUPOLE MAGNETS

914

GEAM TRANSPORT DESIGN FOR THE HIGHEINTENSITY PROTON REAM LINE IN THE LAMOE INJECTOR

326

2508

OEAM TRANBPORT SYSTEM IN THE INJECTOR COMPLEX OF LAMPF

CAW PROBLEMS AFTEA OYERVOLTAGE EVENT

CHANGES IN CONTROLS TO H+ ION SOURCE

CIRCUITRY FDR SENSING HIGH VOLTAGE FAULTS

COMHENTS AND RECOMMENDATIONS PERTAINING TO THE ACCFLFRATOR DAO BEANS

COUPLING BE TWEEN TRANSUERBE OSEILLATIONS IN LINACS

COUPLING BETWFEN TRANSVERBE DSEILLATIONS IN LINACS MATNETIC FYELT

1110

MAGNETIC FIELL

DESTON OF AN LCHROMATIC INFLECTOR FOR THE DUAL BEAM TRANSPORT SYSTEM IA PHF LAMPF IMJECTAM DESTGN OF SOLFNOID LENS INOR PIGMI

He

ACTE ION SOURCE FOR LAMPF

2615 DEVELOPMENT OF A HIGH CURAENT HE ION SOUR

IIS DOME AMPLIFIER SYSTEM

1330 DOME VOL TAE FAST PROTECT SYSTEM

II4 ELECTRON TRAP ELECTRONIES

2624 EMITTANEE DAMPING EXPERIMENT -761217
2633 EMITANCE DAMPING EXPERIMENT: 761912

761.5

621364

71713

7 ist? 30 ?

Thith

$73 ! 2$.

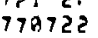

73.912

$72353 !$

75152,

77 Anin

$7 n 115$

Thones

730717

$72,450$.

Tinnd.

718 al

073324

$721 \div, 4$

ग1?

730427

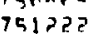

$75 A^{4}+11$.

750 .

670.4

bo.1 4

ande?

7 hagh?

741?1?

7unat?

$99.1:$

$74002 x$

75 andith

$744+3$ ?

7nle! ? 
SPEVNS, R.R., JR, (CONTD.)

2618 EMPTPANCE OAMPING STUDTES

2643 EMITTANCE JAW GROUNDING

$265 !$ EMITTANCE JAHS REVISITED

TEVTSNTS ON THE H* BEAM

PAsT PROTECT NUMERING ON THE HQ INJECTOR

PAST PROTECT QROTECTION FOR PHE ACCELERATING COLUMN

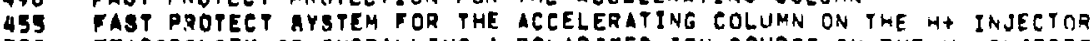

329 PEASIBILITY OR INSTALLING A POLARIZED ION SOUREE IN THE HO INJECTOR

2634 H\$ QEAM EMITPANCE MEASUREMENTS - 761001

2648 HQ BEAM EMI TANCE MEASUREMENTS: 76031

2661 H. INJECTOR PARAMETERS POR CYCLE 15

2662 H* INJECTOR DAFAMETERS POR CYYLLE

2520 H RECTIFIER TESTS

2649 HE BEMM CHOPPER

2625 HE BEAM GHOPPFR DEVELOPMENT - 761218

2631 Ho DEAM CHOPPER DEVELOPHENT - 761015

2626 HE BEAM EHOPPER IMPROVEMENTS

2639 HO GEAM TEETS

2636 HE CHODPER OPFRATION

565 H. INJECTOR CONSTRUCTION PROGRAM

2316 HE INJECTOR CANTROL SYSTEMS

2659 HE INJECYOR PARAMETERS FOR CYCLE 15

2660 HE INJECTOR PARAMETERS POR EYCLE :

HSU HE INJECTOR TURNGONITUANGOFF PROCEOURES

11: HE BOURCE TUNING

2644 HIGH PEAK CURRENT BEAM TESTS

113 HIGH POWER OPFAATION OF THE H+ INJECTOR - 741198

499 HIGH PONER DPFRATION OF THE H* INJECTOR = 72120

I10 HIGH VOLTAGE MEASURING PROBLEMS IN THE INJECTOR COMPLEX

2619 HIGM-CURRENT HE INJECTOR FOR LAMDF

148 INITIAL OPERATION AF THE BEAM TRANSPORT SYSTEM IN THE LAMPF INJECTOR COMPLFX INJECTDR BEAM LINE DEVICE TOLEAANCES FOR CCR

INJECTOR ENERAY VARIATION EXPERIMENT

REVISITED

INJECTOR HIGH VOLTAGE POWER SUPPLY GDR THE LLTERNATING PMASE FOCLISTNG LINAE

116
2649
INJECTOR MAINTENANCE

2649 TNJECYOR MOOIFICATIONS FOR 1 MA

2635 INJECPOR STAPUS MESBAGES CU CCE CONTROL SCTPE INJEE

INJECTOR BYSTFM

TUNTNG EXPERIMENT

INSTRLLATION GF CURAENT MONITOR AT TANK 1 L

INON SOUREE ANA INJECTOR INFOAMATI ION

ION SOUREE ANA INJECTOR INFORMATION

SOENF HO GON ROUNCE DEVELOPHENT OROGQAM

1447 LASL EXPERIENRE WIPH A DUOPLASMATRON FEEOING A 75B-KV EXACT PIFHCE COL M.

436 LIMITING BEAM CURRENT TO EXPERIMENTAL AREAS

760520

7600902

$751.3 ?$

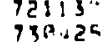

75 की 40

761391

76017

$770+1.0$

778050

75050.

7 म०315

70121.

$701 \cdot 15$

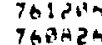

7 thatem

$76092 \mathrm{x}$

724203

75111.

Trant:

$779+20^{\circ}$

730503 ; 7315401

741110

76120

$760+26$

711190

921305

740319

7 110

71 74a?

751 ilp

751 3\%

70174 ?

70 10

700233

160 ?

70 करन

$741>11$

7 aral?

7 HAt $2^{\circ}$

71119
43935

71079 
STEVENS, R,R..JR, TENNTD,!

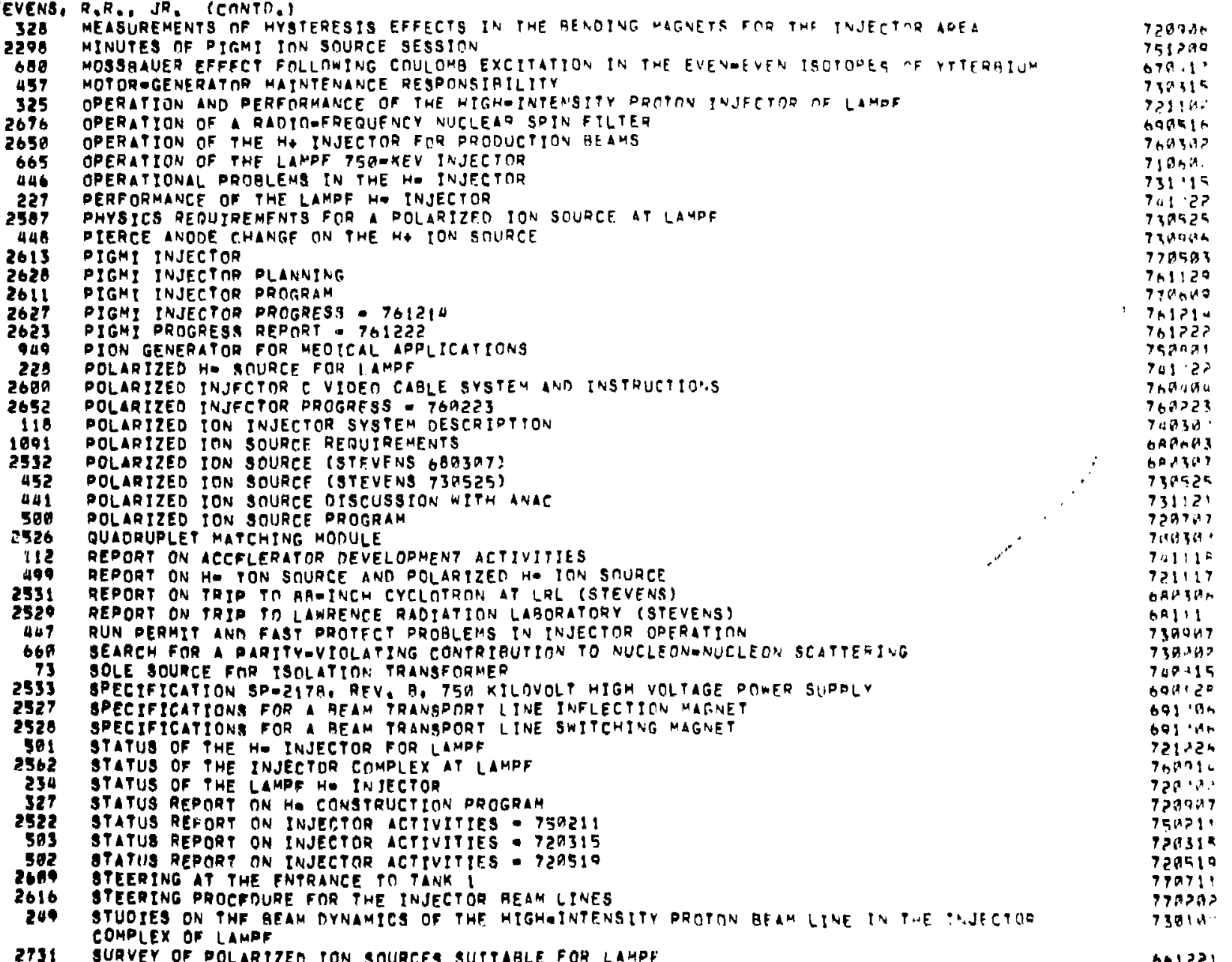

2731 SURYEY OF DOLARIZEO ION SOURCES SUTYABLE FOR LAHPH 


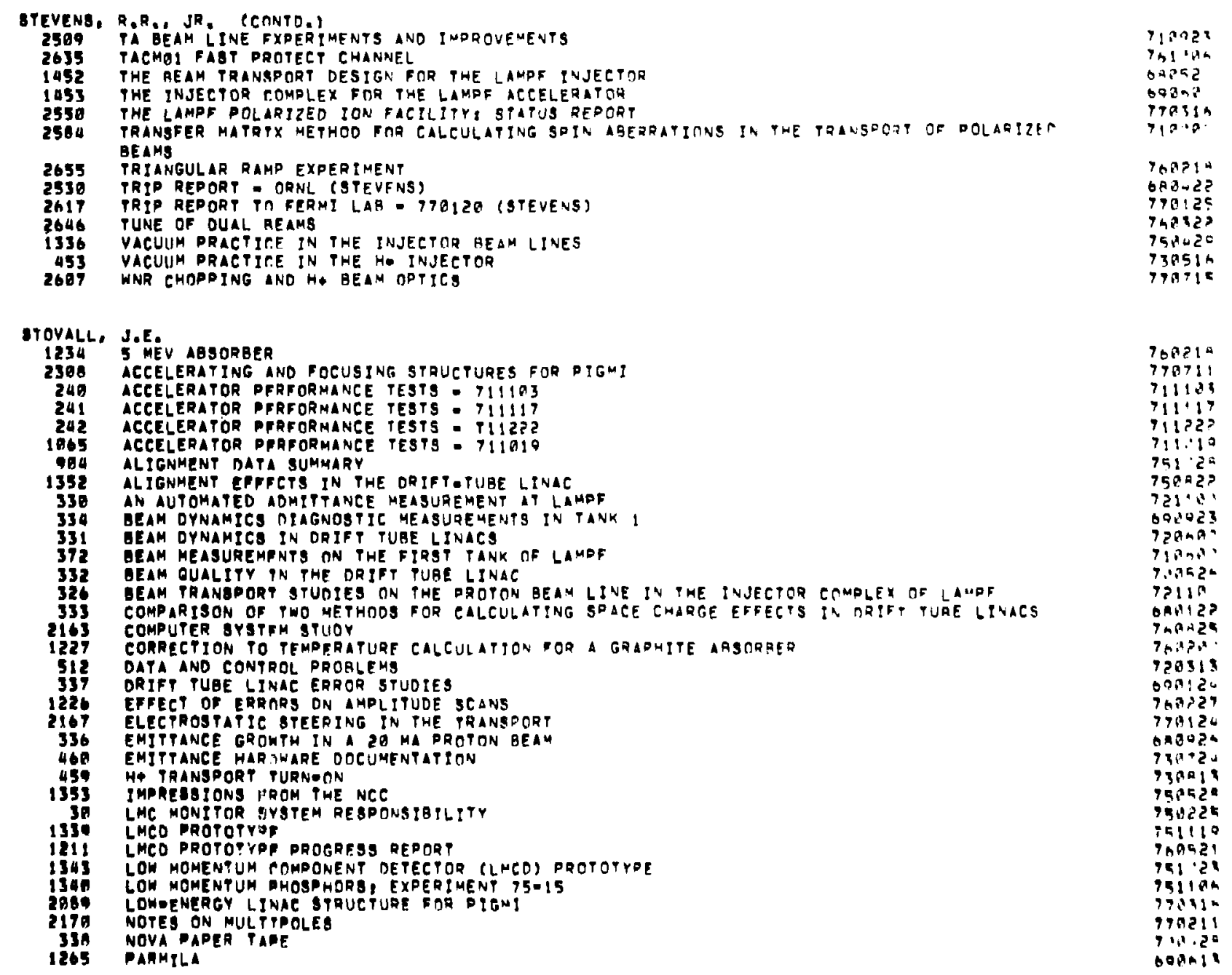


STOVALL, J.E. ICONTD,

H13 PHASE SCANS ON MODULES I THROUGH I

1223 PHOSPHOR STUDY - EXPERIMENT 76-14 (REF 75-15)

30119

POSPHOR- STUDY - EXPERIMENT T6-1 d (REF 75-15)

this
thes

1246 PIGM CONTROL SYSTEM MEETING: JANUARY 23.9970

2165 PIGM? CONTROL SYSTEM MEETING JANUARY 6,1979

2166 PIGMI CONTROL SYSTEM MEETINGS JANUARY 12 AND 14, 1977

2169 PIGMI CONTROL SYSTEM HEETING SANUARY 26.1979

PIGMI DRIFT TIIBE LiNAC PARAMETEAS

- ION GENERATOR FOR MEDICAL APPLICATIONS

POST ALIGNMENT ADMITTANCE STUDY

PRELIMINARY SERAPER STUDY

RAOIATIONGRESTSTANT PHOSPHORS FOR BAPAMEV PROTON BEAMS

REQUEST FOR BFAM TIME, PROPOSAL 75-15

RESULTS OF PARTICLE DYNAMICS CALCULATIONS

SCRAPER STUDIFS - EXPERIMENT $76-5$

SELECTION OF DUAORUPOLE STRENGTHS FOR ORIFT TUAE LINAC

SOME FACTORS PNFLUENCING THE SELECTION OF THE BUNCMER

SPECIFICATION SPMU316

2162 SUECRFISH STUOY OF DIGM ORIFT TUBE LINAC

2161 PECHNICAL SPECIFICATION FOR A COMPUTER SYSTEM FOR THE COVTROL ANT MIAGAORTICS CF A PROPOTYPE ACCFLERATOR

STRABSNER, 6.

2315 RADIATIVE NEGATIVE PION CAPTURE IN LIBUID NEON

2380 RADIATIVE NEGATIVE PION CAPTURE IN LIOUID PRITIUM

$\operatorname{Tin} 20$

Torist:

$\rightarrow \rightarrow 19$

770129

77a??

750201
$75200 \mathrm{~A}$

Thalu?

77040.5
751.20

Inie?

76031 ?

6 ดी 73

693325

Thl?

Thinch?

ITROIK, P.J.
BQO TWO CELL 201,25 MHZ DRIFTOTUBE LINAC POWER MODEL

$072+28$

STROVINK,

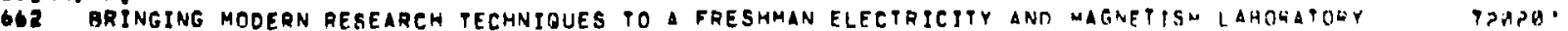

STUDEGAKER, J,K.

19A4 AFAST VERSATILE PREAMPLIFIER FOH IISE IN PARTICLE PHYSICS

1990 AUTOMATIC 24 VOLT CROWBARECAMAC SUPDLY

1959 CAMACOPOWER SIIPPLY FAULT DETECTOR

1670 DANA 533A DIGPTAL VOLT-METER TO CAMAC INTERFACE

MIGHESENSTYIVTTY BEAM CURRENT MONTTOR SYSTEM

1689 LAMPF DUAL FAST WIRE SCAMNER AMPLIFIER

1606 LAMPF GENERAL PUAPOSE THERMOCOUPLE AMPLIFIER ANI OVERPEMPERATURE TRID

1943 LAMPFERCA 4525 PHOTOTURE BASEIAMPLIFIER

1915 RESPONSE OETERMINATION IP SINGLE DOMIVANT ORLE DPEHAPIONAL AMPLIFIFRS

1699 SIXPEEN HIT MADIFICATION MWPC ENCOOER

DTUPIN, D Ma

IAS ELASTIC SCAPTERING TF T-12 MEV PRITOUS BY LLPHA PARTTCLES 
SUAZO, G.

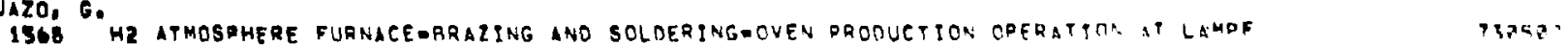

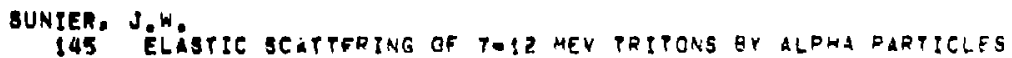

$750 \cdot 2 m$

sUTYER, R.J.

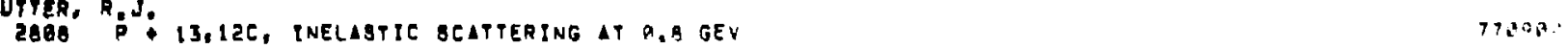

SAIN: G.R:
1430
282
2805 RESONANCE CONTROLLER

OOSOMHZ ACCELPRATOR INSTALLATION CHECKLIST, STEOS AETER FINAL MODULE T INIVE

BOS-MHZ LINAC REAM STEERING . EXPERTMENT NO THOS

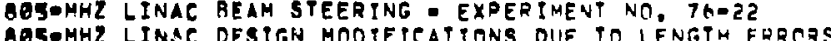

DOS-HHC LINAC DFSIGH MDOIFICATIONS OUE TD LENGTH FLRCZS

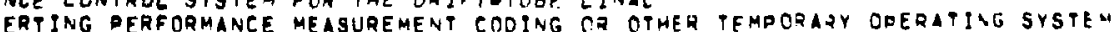
MOTHCO FR FiBERT ING QERFORMANCE

MOOTFICATIONS ON THE ALA COMPUTER

ANAYYIS OF MULTSPLY COUPLED RESONATORS US ING GY CONSOLE AT CCR

ANALYSIS OF MILLTIPLY YCOUPLED RESONATORS USING A MATRIX FORMULATION

A8PECTS OF BAF-MHZ LYNAC TANK CERTIFICATION PROCEDURE - A PRELIMINARY QULVFY

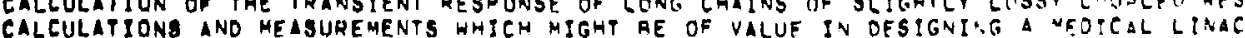

CALCULATIONS SIMULATING DELTAOT O REPEREVEE LINE EXPERIMENT O PRELIMINARY OESULTS

CALCULATIONS SIMULATING DEL TOT O REPERE

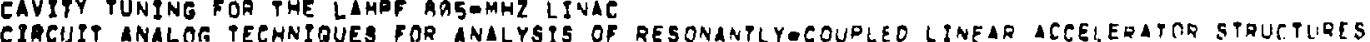

COMPUTER CONTROL SYSTEM EFFICLENCY STUDIES

CONTROL PHYLOSOPHY FOR PIGM!, AN INFORMAL NOTE

DEADSTARY PRDCEDURES FOR OELTAST DISK FILES

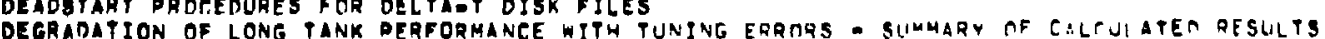

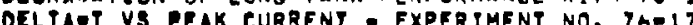

OELTAT Y PEAK CURRENT: EXPER MENT NO: T6-17. SUMMARY OE RESUL TS TO OATE

DELTAOT Y PEAK CURRENT EXPERIMENT ND, YGOI PART

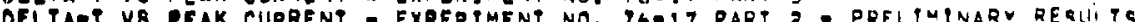

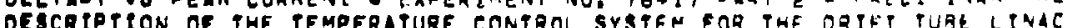

ORIFT TUBE LINAC RESONANCE CONTROL SYSTEN DESIGN REVIEN

FARMOA COMPUTFR COOE FOA ACEELERATOR RAOTAL MOTION STUDIES

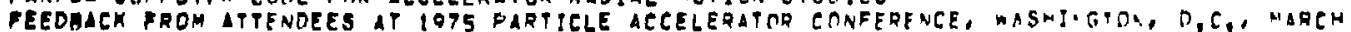

I2-14. 1975. ON POSTER SESSION PAPER HE5, INCREASING THE HELEYANEE OF ATA DRESENTEO TO

THE APERTTORS IN AN ACCELERATOR CONTROL SYSTEM

FREE FIELD COMPUTER CARD READ ROUTINE

283 PULL POWER DOFRATION OF TME LAMPF OQS YHZ SYSTEM

BI HIGHOPNTENSTTY EXPERIMENTS.

330 ILLUSY:ATEO LPST OF LINAC PRANSTENT ANALYSIS MOVIES AASER OA COUPLEO RESAMATOQ MADELS

1434 INEREASING THF RELEVANCE OF DATA PRESENTEO TO TME OPERATORS IN AN ACCELELATOR COATROL

SYBTE

MEETING TO CONSIOER COAE MEMARY SITUITION

$751 \cdot 5$,

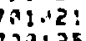

?

Tueri:

$693 \mathrm{na}$

$74 \mathrm{Pl}$.

$74+1 ! 2$

67071:

7 and 15

7590.15

75 tow

Th! 2 ?

कराश,

6क? 5.

75)

7 1120

on 2 a

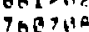

itgatats

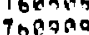

7 Thu 20

$7 \mathrm{hav}$

00,3 be

0.1

041217

750

40.325

7 पित्व

701112

o0日य?

461

$7 x, 192$ 


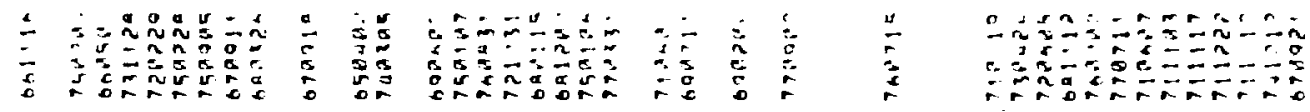

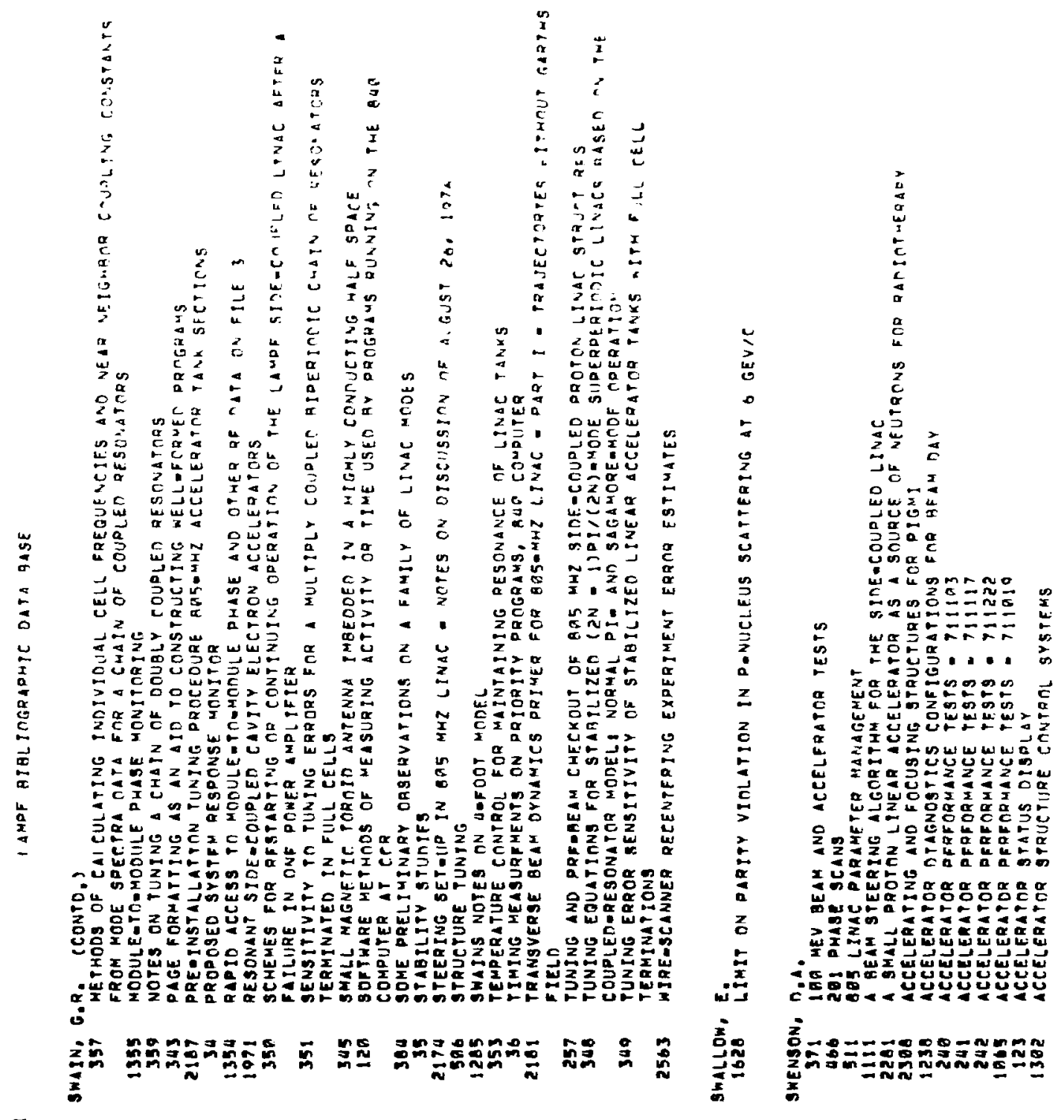


SWENLON, D.A, ECONTD,'

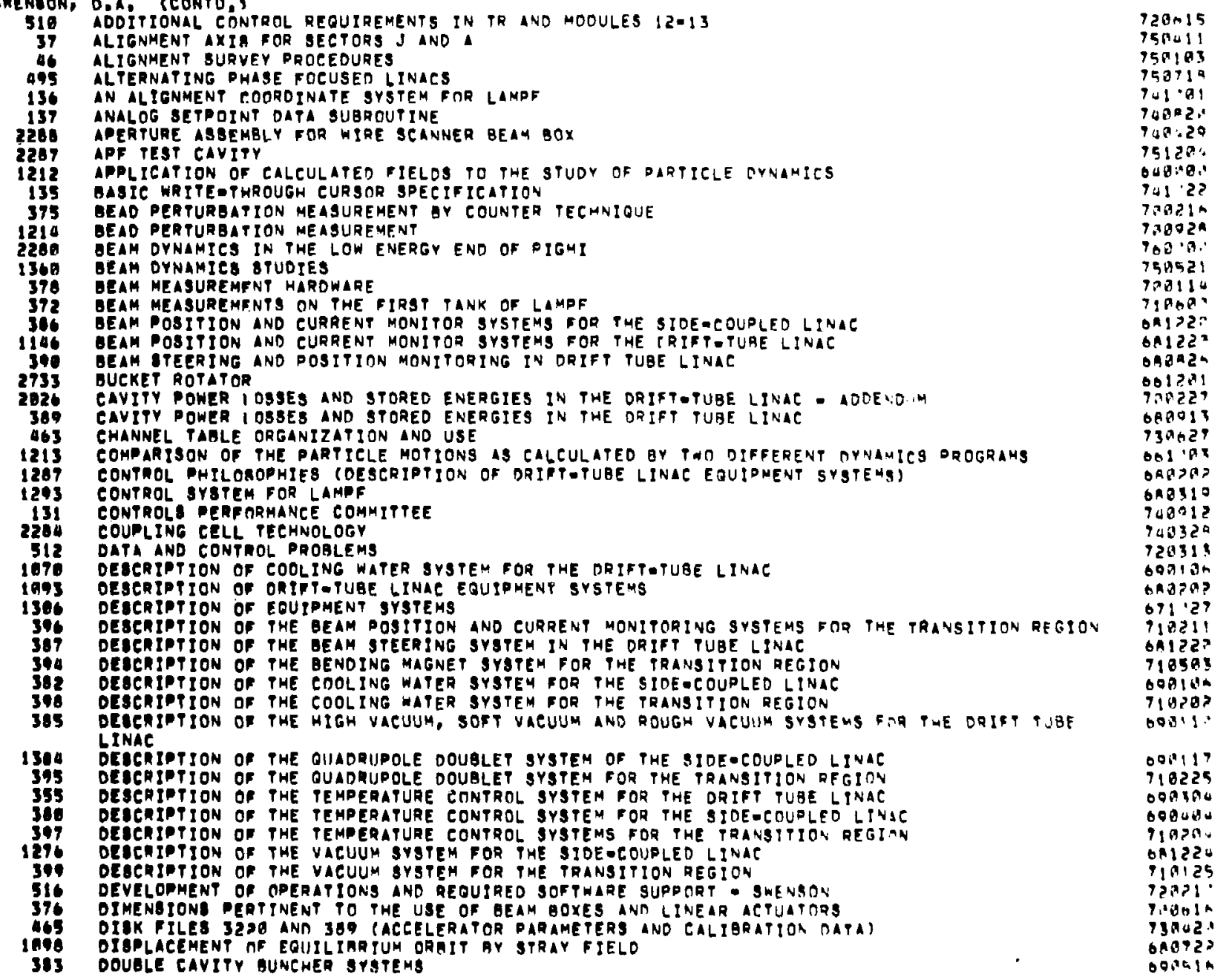


BHENBON, D.A. CCONTO, I

I30S DRAFY OF A CONTROL PHILOSOPHY FOR ACCELERATOR STRUCTURES

1350 DRAFY OF PRELIMINARY DESIGN STUDY

391 ORIFTOPUBE LINAE DIMENSIONS

1130 DRIVE LINE MEASUREMENTS ON TANK

1301 ORIVE LOOP LOCATIONS IN DRIFTETUBE LINAC TANKS

1240 EXCIYAYION OF TANK I

1206 FIELO PERTURAATIONS IN THE POST COUPLEO DRIFTUTUBE LINAC

27. FIRSP SUECESSFUL DELTACT) RUNS

132 FIXTURES FOR HORIZONTAL MEASUREMENTS

243 FORTRAN SUPPORT ROUTINES

245 GENERAL GRAPHTCAL PROGRAMS

368 GENERAL ISOMETRIC OISPLAY PROGRAM

393 GENERATION OF GEOMETRICAL OIMENSTONS FOR OR:FT PURE LINACS

2090 HIGH ENERGY ARCELERATING STRUCTURES FOR HIGH GRADIENT PROTON LINAT ADPLITATICAS IMMEDIATE AL IGNMENT ACTIVITIES

INSTRUMENTATION FOR BEAM OIAGNOSTICS EXPERIMENTS

INTERPRETATION OF FIELO DISTRIAUTIONS IN VARIAGLE CELL LENGTW MONEL

INTERPREYATION OF LALA OUTPUT

KNOB TEST ROUTINE (3191)

LAMPF ALIGNMENT MARKERS-

LOW MOMENTUM COMPONENT DETECTOR

LOW-ENERGY LINAC STRUCTURE FOR PIGMI

HAINTENANCE UND OIAGNOSTIC PROCEDURES

MEASUREMENTS OF TRANSVERSE OSTILLATION WAVELENGTHS IN THE ARS MHZ LIVAE

HINUTES - CONTROL SYSTEM DEVELOPMENT COMHITTEE - 75R328

MINUTES - CONTROL SYSTEM DEVELOPMENT COMMITTEE - 759327

MINUTES - CONTROL SYSTEM OEVELOPMENT COMMITTEE - 758310

MINUTES - CONTROL SYSTEM OEVELOPMENT COMMITTEE " 750219

MINUTES - CONTROL SYSTEM WORKING GAOUP - 759290

MINUTES - CONTROL SYSTEM WORKING GROUP : 950116

MINUTES - CONTROL SYSTEM WORKING GROUP - 759116

MINUTES - CONTROL SYSTEM WORKING GROUP - 741295

MINUTES - CONTAOL SYSTEM HORKING GROUP - 741204

MINUTES - CONTROL SYSTEM WORKING GROUP : 741184

MTNUTES - CONTROL SYSTEM HORKING GROUP - 74IAZI

MINUTES - CONTROL SYSTEM WORKING GROUP - 7419OL

MINUTES - CONTROL SYSTEM HORKING GROUP - 940925

MINUTES OF FAAT PROTECT DIAGNOSTICS MEETING

MINUTES: CONTROL SYSTEMS DEVELOPMENT WORKING GROUP - IUMO18

MINUTESI REVIFW OF APF COLD PROBE MDOEL

MPA3 CONTROL PHILOSOPHY

NOVA EMITTANEF MEASUREMENT

OPERATICN OF THE FIRST TANK OF LAMDF

OPTIMUM GEOMETRIES AND FIELD STRENGTHS FOR ALVAREI LINACS

parameter spate

marmgla

PhaBe caliaration pRocedure

PHABE SCAN EXPERIMENT ON PHE DRIFT TUBE LINAC

DHASE SMIFTER DAIVE SYSTEM

PIGLET DARAMETER.

PIGMI ORIFT PHBE GEOMETRY

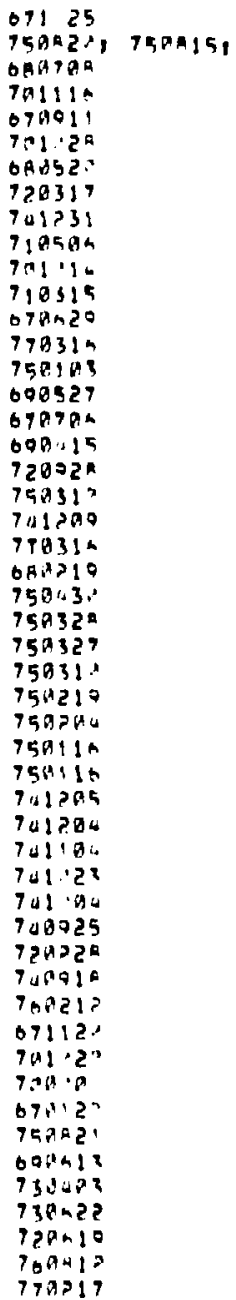


SWENSON, D,A, (CONTD,)

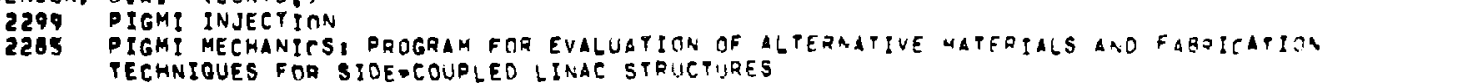

306 PIGMI MICROPQNCESSOR APPLICATIONS

2286 PIGMI MINUTES. OCTARER 31 1 1975

2291 PIGMII PRELIMTNARY SCL CONFIGURATION

Q49 PION GENERATOR FOR MEDICAL APQLICATIONS

363 POST COUPLERS FOR TRIFT TUBE LINAE

1232 PAELIMINARY DFVELOPMENT OF THE ALTERNATING PMASE FQCUSED LINAE STR:TC T.7E

PAOPERTIES OF ATMPLE PERIODIC BEAM TRANSPORT SYSTEM

PROPERTIES OF THE CYLINDRICAL RF CAVIIY EVALUATION CODF SUDERFISH

PROPOSAL FOR NEVELOPMENT OF ADION GFNERATOR FOR MEDICAL APPLICATIOA

DUANTITATIVE SYSTEM SERVICE MONITOR

REOUNDANT MONTTORING

RESONANT FREOIENCY VERSUS TEMPERATURE

REVIEH OF PROPOSED DESIGN

SAMPLING OF ANALOG DATA
SCAN CONVERTER FOR CCR

JEMIANNUAL PROGRESS REPORT FOR THE OIGUI PROGRAM AT LASL FCR THE OERION ENMIAG DTCEMGER

240 3IDE 1976 COUPLED I INAC TURNOON PRORLEM

2301 SINGLE CELL Q TEST

247 SOME FACTORS TNFLUENCING THE SELECTION OF THE BUNCMEP

69 SOME FEATURES OF THE RESONANTLY COUPLED ALVAREZ LINAC

30. SOME PRELIMINARY OBSERVATIONS ON A FAMILY OF LIVAC MOOES

SPECIFICAT AMPF DROTON REAM

392

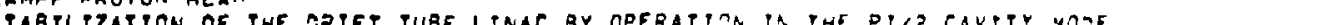

751217

76119

$751: 29$

$7 n x^{2}+2$

$75 a+a$

07,129,

$00 \times 2 !$

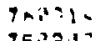

$12,3,4$

75,512

$750+24$

$071>1$.



क्र 121

790131

$\operatorname{tangt} 0$

$796+1=$

$\cos 32=$

oritab.

oogace

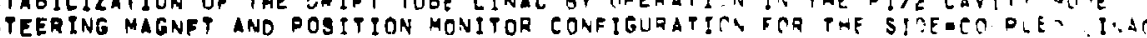

TEM PERTUREATI ONS IN ALVAREZ LINACS

TEPPING-HOTOR PUISE RITE

TTRAGE OSCILI OSCOPES IN CCA

TORAGE SCOPE CURSOR USAGE

BY 5 MEV OPEATTION

STLSTSTEM STATUS AND CONTROL

MED BY MPDO ON MP-1 5 YSTEMS

SUPERTISH EVAL UATION OF SLEEVE PERTURBATION

SUPERFISH STURY OF AOF TETMETRIES

SUPERFISH STURY OF ADF DIAMETER

TEMPERATURE CONTROL EXOEQTMETET

TEMPERATURE CANTRO EXPERIMEN OATA TAXEN AUGUST 1,1907

THE QUCXEY QOTATOQ

THE BUCKET ROTATOA

THE DELTACT) TURNATN

THE GEOMEPRY TF APF

THE GEOMETRY AF THE OIGMI PROTATYPE

THE LAMPF COORDYNATE SYSTEM

THE PIOMI PROTRAM AT LASL

THE PRANSITION REGION

TRANGPORT CALCIJLATIONG

$69 i+3$

og1 23

$\cos (3, \cos x$

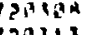

19.0.

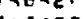

this?

7 7)

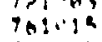

9791,4

7ol. 9 .

071 i

bople.

(3),

$7 \rightarrow 12$

741.31

7opan

inal,

$720: 14$ 
SWENSON, D.W,

WENSON, DEWEL GATE FQOM MASTER TIMEQ

SWENBON, W.

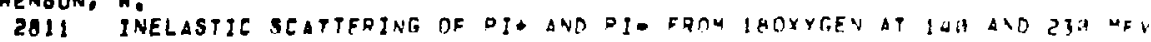

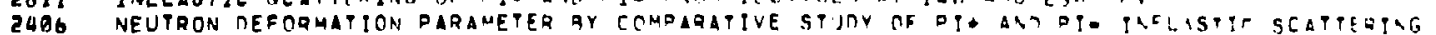

72B NEW MEASUREMENTS OF (PIQ, ?D ? REACTIOSS ON HIGMT NUELET

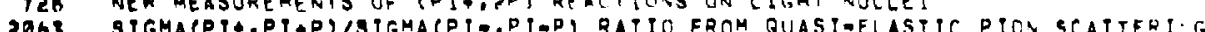

$77, a \cup a \cdot$

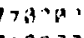

this

TABOR, S.L.

2416 ENERGETIC CHARGED PARTICLE YIELOS INOUCEO BY PIONS CN COMPLEY DJCLF

24IG GAMMARAY STUNY OF PION-INNUCED REACTIONS ON COMPLEX VIICLEI

1997 INTERACPION OF FAST PIONB WITH OZ, B.MENICKE

$77 a+\infty$

7704525

$\ln 1,24$

TALAGA, R,

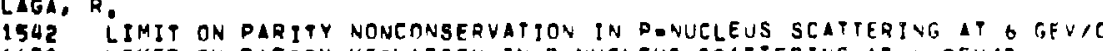

1542 LIMIT ON PARITY NONCONSERVATION IN DENUCLEUS SCATIERIVG AT 6

750595

TALBERT, W.LEA JR,

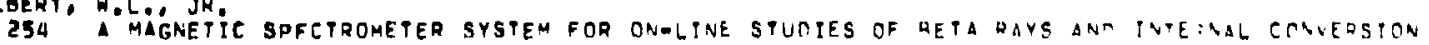

ELECTRONS TF RHORT-LIVED MASSESEPARATED ACTIVITIFS

255 INTERNAL-CONYFRSION COEFFICIENT DETERMINATION OF ODD DARITY FRR THE IHA, QOKEV FIRST-

$76 a^{2}+x^{2}$

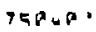

2335 EXCITED STATE OF DIARURTDTUM

作

$731 \cdot$

TALLERICO, P.J

637 AN EXPERTMENTAL KLYSTRON FOR LAMPF

223. AN EXPERTMENTAL KLYSTRON FOR LAMI

SB2 DETA DIAGNOSTPC DREUMENTATION

633 DESIGN CONBIDFRATIONS FOR THE HIGHAPOWER MULTICAVITY KLYSTRON

2339 FORETGN TRAVEI TRTP REPORT TIALTEQICOS

647 HIGH PERFORMANCE KI YSTRONG FOR ACCFLERATOR ADPLICATIONS

2108 KLYSTRON PLRTS HANDL. INE PROEFDIIRES

ZIOI KLYSTRON TURNAON PROCEOURE

GUL LARGE-STGNAL FFFECPS IN THE MUITICAVITY XLYSTRON

1162 LASL CONFINED-FLOW KLYSTRON COOE ODEQATION

2000 LOW FREQUENCY KLYSTRON FOR ACCELERATOR IPDLLICATICNS

2237 DERFORMANCE BPECIFICATION FOR KLYSTRON AMPLIFIEQ

2369 PERFORMANCE SPECIFTCATION NO ZGM-GOL FOR KLYSTRON AMPLIFIER

2255 PERFORMANCE GPECIF TEATTION NO

2340 PROCEDURE FOR REACTIVATING OXITE CATHOOES ON VA-BGEA KL STREAS

1985 RELIABILITY IND RPFRATING EXPERIENCE OF THE LAHF RPFE UHZ OF SYSTE

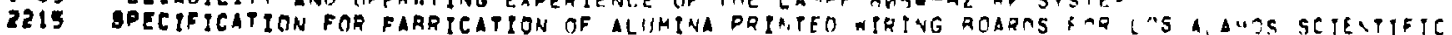

2A3U PHE CURRENT MANTIOR READOUT GYGTEM

$7111:$

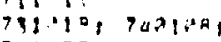

$731,5\}$

Thinat

tiban?

$75 x^{2}, 75,7512001$

150.521 7505501

73.5

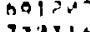

750125

15025
715025

1
7 (a)

T)म?

Tैand

Tुकरण

7 sand. 
TALLERICO, P.j. (CONTH.)

Q31 THE DESIGN AND PERFORMANCE OF THE LAMPF 101/4 MN KLYSTRON MCOULATOR T3OQID

1497 THE EFFECTS OF LOAD MISHATCH ON THE HIGH POWER MIJLTICAVITY KLYSTROA

AQB THE LAMPF EXPFRIMENTAL AREA BEAM CURPENT MONITORS

$14 B 6$ THE LAMPF KLYRTRON REPAIR FACILITY
1180 PAANGVESE EFFECTS IN THE HIGHOPOWER MULTICAVITY KLYSTRON

75110.

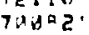

TANABE, $K$,

1964 GTOPAED MUON CHANNEL OESIGN STATUS - JULY 2, 1960

TANAKA. No

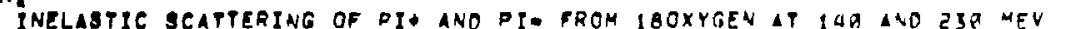

2466 NEUTRON DEFORMATION PARAMETER BY COMPARATIVE STUOY OF OI AND DI. IVELISTIC SCATTERIVG

P + 13,12C, jNELASTIC SCATTERING AT B.8 GEV

REPORT ON HRS MEETING

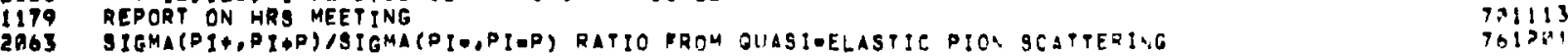

2412 FHE ADPLICATION OF PROTONS TO COMPUTEO TOMOGRAPHY

$71 \cdot 2 \cdot$

TERRY, F.D.

G16 AVERSATILE TFCHNGOUE FOR INTERFACING A CONTROL COMPUTER HITH REMOTE DATA ACTUISIPION AN?

60.7605

CONTROL STATINNS RLONG PARTICLE ACEELERATORS

laTS COMPARYSON OF TIMED DATA SCAN SYSTEMS FOR LAMDE

1229 COMPUTER INTERFACE UNIT (CIU) INTRODUCTION

1527 MANUAL HEAD ENNTROLLER (MHE) OPERATIONS MANUAL

1536 MANUAL HEAD EANTROLS

1102 PROTOTYPE COMPUTER INTERFACE UNIT (CIU) OPERATIONS MANIIAL

1428 RECOMMENDEO LAGIC TYPE FOR LAMPF COMPUTER CONTROL SYSTEM

RICE, DEVICE, ANO CHANNEL DESIGNATION SCHEME

124B RICE -COMPUTER INTERFAEE UNIT (RIU) DESIGN CONCEPTS ANO TWO SUPRLEMENTS

1533 SOME DESIGN CONEEPTS PERTAINING TO THE INJECTOR CONTROL QOOM

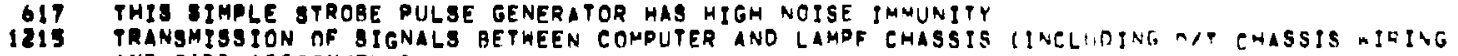
IND CARD ASIITNMENT?

TESCHE. F $R$

696 ENGineERING AMPECTS OF THE LOS ALAMOS MESON DHYSICS FACILITY

643 PHERMEX - A HTGHICURRENT ELECTRON ACCELERATOR FOR USE IN OVNAMIC RAOIOFRADHY

6909 !:

$\operatorname{coshos}$

$071 \cdot 25$

60,019

OA, $25 ?$

OAdOI:

071.23

OAB? 14

GII?

oring.

oritai

THERIOT, E.D.

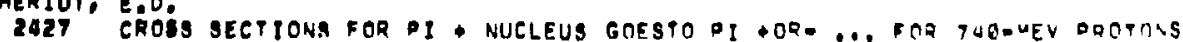




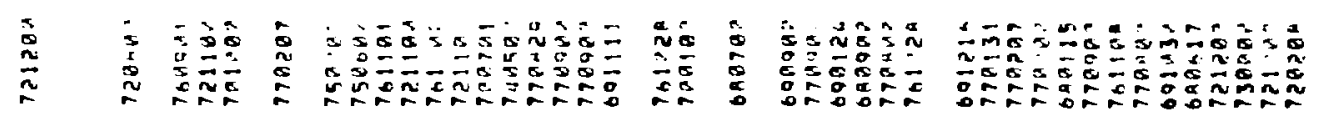

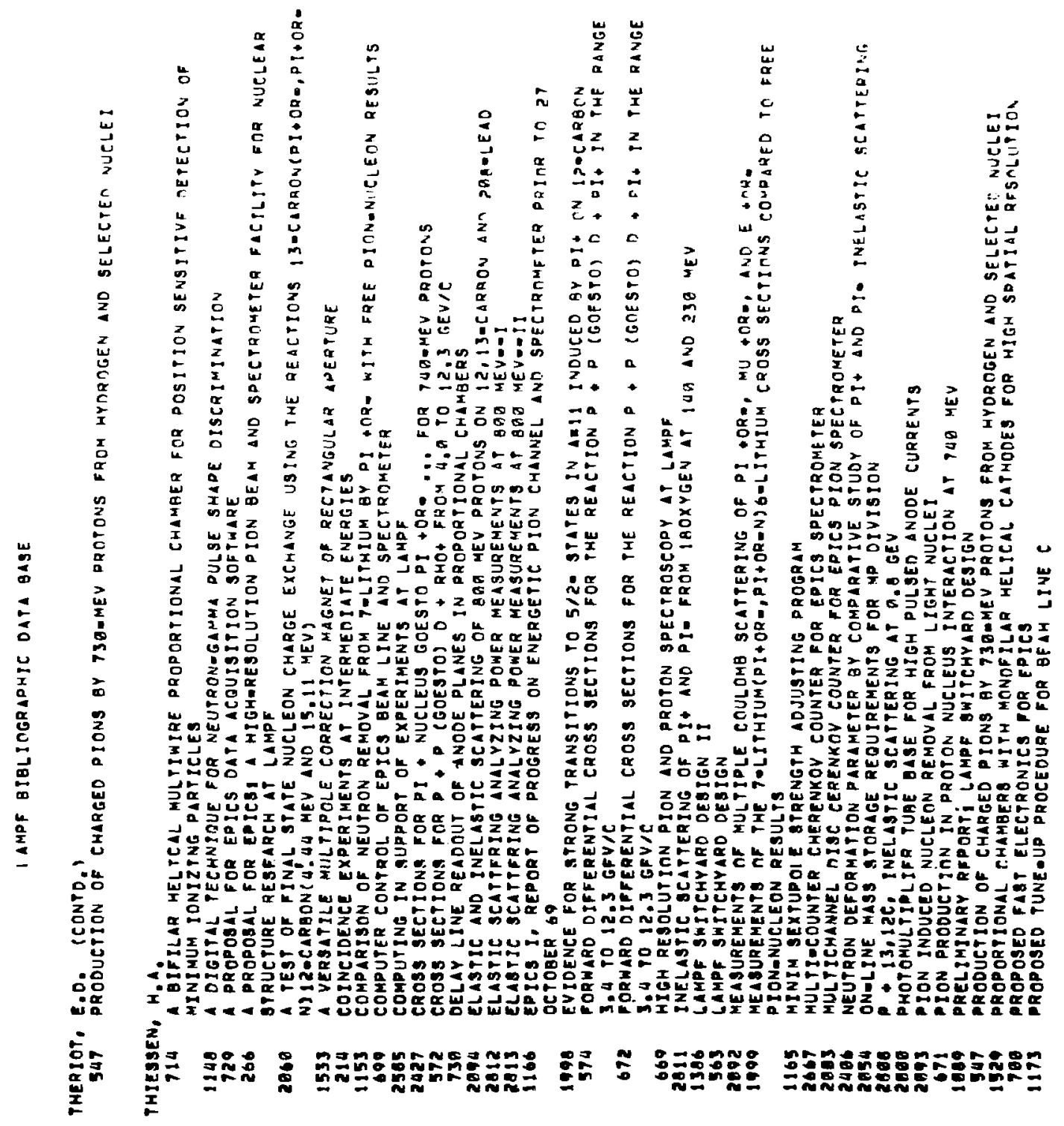


THIESSEN, H.A. (CONTO!),

1995 SCATTERING OF 58 MEV PI \& FROM LIGHT NUELE]

2023 SCINTILLATORS ANO PHOTAMULTIPLIERS - SOME TESTS FOR EPICS TUNE OS

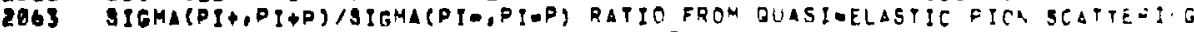

131 3LIT SCATTER ING IN THE EPICS BEAM LINE

121 BPECTROMETER DESIGN AT LASL

STUOY OF PME PPIE,N) AND (PI,PI PRIME OI REACTIONS IA LIGMT RUCLFI

1063 SUMMARY OF STATUS OF DESIGN OF STOPPED MUON CMANTEI

2536 PUNEUP OF HRS

$701 \cdot 2{ }^{2}$

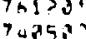

131,3

$7+1.5=$

$080 \times 13$

$7+9,75$

$750 \cdot \because+$

144 INITIAL COMPARATIVE RESPONSE OF EXPERIMENTAL TUMOES TO DEAK PICNS AVI) XOGAYS

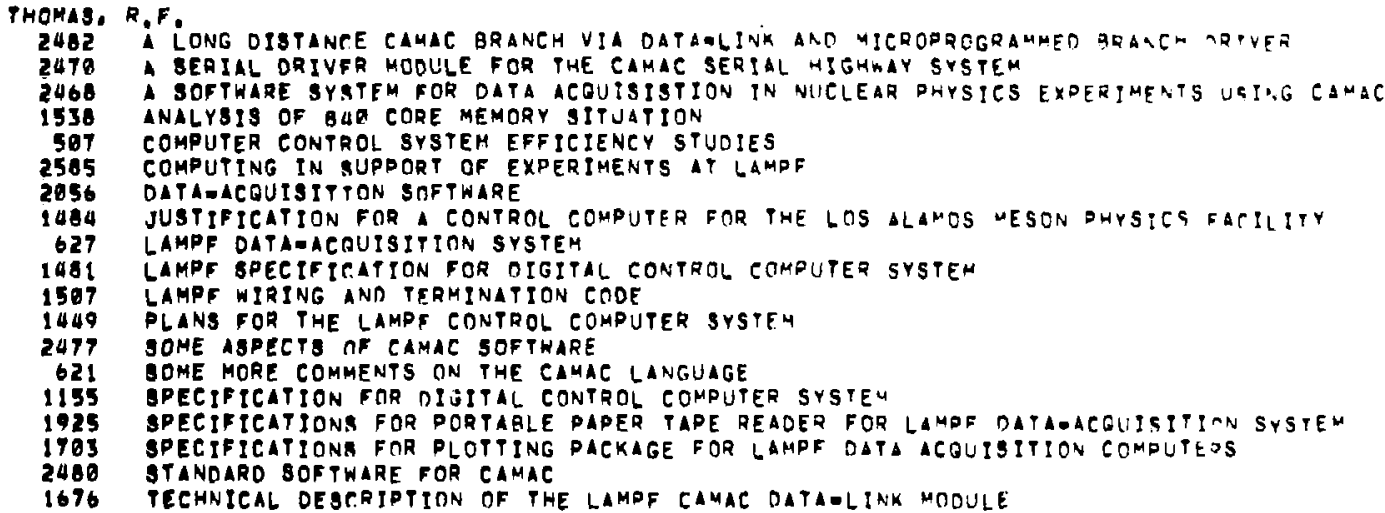

1676 TECHNICAL DEBCRTPTION OF THE LAMPF CAMAC DATA-LINK MOOULE

THOMAS, w,

1646 PION PROOUCTION IN NEUPAONAPROTON COLLTSIONS

$7213 \ln$

751110

$720-3$

$721 \cdot 10$

Thl ia:

$7002:$

600409

Topan.

ood114

bat?

671.13

7 3a?

$71,40.0$

OA. 33

$721 \cdot$ Aक

7pमpब

$72120 \mathrm{~m}$

THOMASON,

2AMS SOFT. RADIO MAPPER STEP SIZE

HOMASSON, 9.0 .

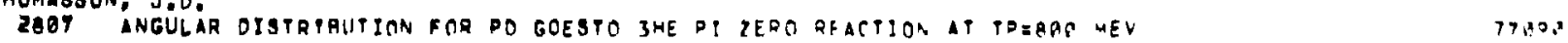

THOMPBON, AC

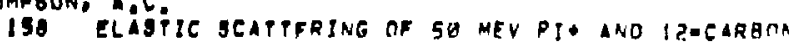

74,296 
THOMPSON. A.C. CCONTO:?

163 STUDIES OF CHARGED PARTICLE EMISSION IN QEACTIONS INDUCED FY 22.4 AND 51 WEV PLI15-PIONS 750.20

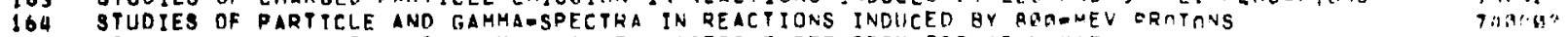

1632 USE OF AN INTRTNSIC GERMANIUM CHARGEO PARTICLE SPECTROMETER AT LATPF

219 USE OF HIGHOPIRITY GERMANIIMM DETECTORS FOR INTERMEDIATE ENERGY DHYSITS EXPFRTMFNTS,

THOMPSON, P.A.

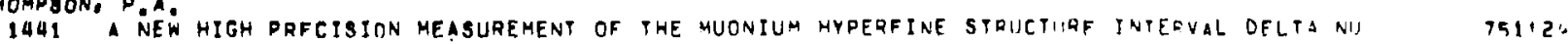

1583 BEAM CALCULATTONS POR LAMPF MUON CHANNEL

915 DEPOLARIZAFION TF NEGAFIVE MUONS IN HELIUM GASI SEARCH FOR MUONIC WELI IM TOM
2540 DEVELOPMENT OF A HIGHOPURITY LOW-MOMENTUM MU OLUS REAM FOR LAYOF

2914 HEATING OF TARGFT CELL COMPONENTS AY SECONDARY PARTICLES

181 MUONIUM FORALTITN IN NOBLE GISES AND NOBLE GAS MIXTIIRES

2015 OBSERVED INTENBITIES AT MUON CHANNEL OURIHG ENFRGY CHANGES SEPTEMBER Ih, 10YU

109! PRECISION MEASUREMENT OF MUONIUM HFS INTERVAL ANO MUON MAGUETIT MOMENT

I6O PRECISION MEASUREMENT OF IS(SUB 1/Z) MUONIUM HYPERFINE STRUCTOPE TNTERVAL TELTA NU: PALT

160 PRECISION MEARUREMENT OF IS ISUA I/2) MUONIUM HYPERFINE STRUCTURE INTERVAL RELTA NU: PART II SETTING OF MARNET FIELDS RY CURRENT MEASUREMENTS

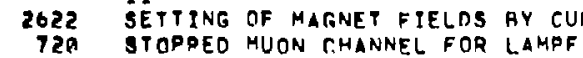

2561 THE STOPPED MIION CHANNEL AT LAMPF

THORN, L.L.

1952 ROLLER TESTS - JUNF 1968

$29 B 3$ THE EVDLUTION OF THE LAMDE HIGH POWEQ PION PRODUCTION TARGF MECHAMISMS

75 int.p.

Tatang,

77 urath

$73+301$

7ם90月?

7 andन.

7क्या:

55 12

750420

739415

71, तर ?

$77 x a n$

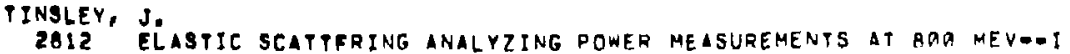

2BI3 ELASTIC SCATTFRING ANALYZING POWER MEASUREMENTS AT PRG MEVAII

OADYAE

T7isis

POBIAS, E,A.

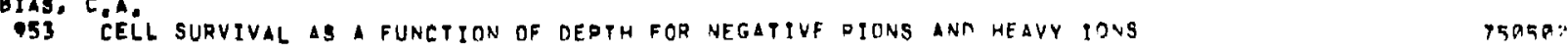

TOOD, P,W.

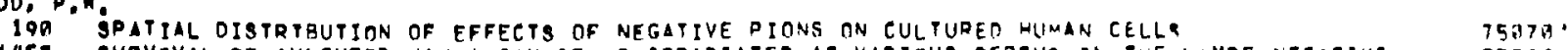

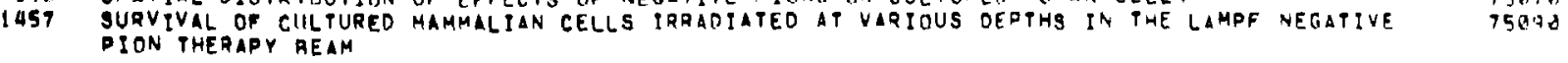

TOOTOONCHI. H.

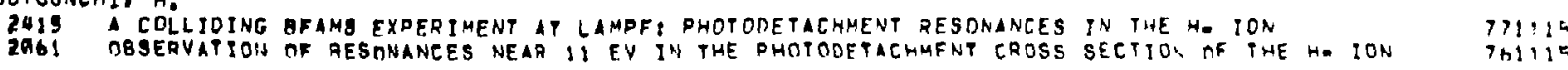

TAEGELLAS, R.T.

2695 BEAM DIAGNOSTTCS DEVICES IN THE ICCELERATOH AREA T5B HA?

UQD CONCEPTUAL DESTGN OF EONTROLS FOR MPA 3 EOUIPMENT SYSTEMS BOAMT 
TREGELLAA, R.T. SCONTE,S

COOL ING WATER SYSTEH FOR THE DRIFT-TUBE LINAC

OECRTP

DESCRIPTION OF THE BEAM POSITION AND CUROENT MONITORING SYSTEMS FOR THE TRANSITIOS REGIOA

DESCRIPTION OF THE GENDING MAGNET SYSTEM FOR THE TRANSITION REGION

OESCRIPT ION OF THE BEAM POSIT ION ANO CURQENT MONTTORING SYSTEMS EOQ

DESCRPTON OF THE EOOLING WATER SYSTEM FOR THE TRANSITION REGION

OESRIPTION OF THE FREOUENCY TUNER AND FIELD TILT TUNEA SYSTEMS FOQ THE MRIFT TUDE LINAC

DESCRIPTION OF THE MONITOR LOOQ SYGTEM FOD THE DRIFT-TUBE LINAC

DESCRIPTION OF THE QUADRUPOLE DOUBLET SYSTEM FOR THE TRANSTTION REGION

DESCRIPTON OF THE TEMPERATURE CONTQOL SYSTEM FOQ THE DRIFT TUBE LINAC

DECRIPTION OF THE TEMPERATURE CONTROL SYSTEM FOR THE GIOE COUPLED LINAC

OESCRIPTION OF THE TEMPERATURE CONTROL SYSTEMS FOR THE TEANSITION REGION

OEOCRIPTION OF THE VACUUA SYSTEM FOR THE SIOE-GOUPLED LINAC

OESERTPTON OF PHE YACUUM GYSTEM FOP THE TRANSITION REGION

EPICS PARTICLF SEPARATOR SUPPLY

MAINTENANEE OF BEAM OIAGNOSTICS SYSTEMS

GTEPPING MOTOR DRIVEN OEVICE DIAGNOSTIC EQUIPMENT

GUMMARY OF PHASE SCAN BIAS EXPERIMENTS - EXPERIMENT 75-1B

1357 TANK IBSORBFR AIAS ANO AMPLIFIER GAIN - EXPERIMENT TH

PRUJILLO, T.T

225 BIOLOGICAL EFFECTS OF THE LOS ALAMOS MESON BEAM ON CELLS IN CULTURE

Q53 CELL SURVIVAL AS A FUNCTION OF OEPTH FOR NEGATIVE PIONS ANO HEAVY IONS

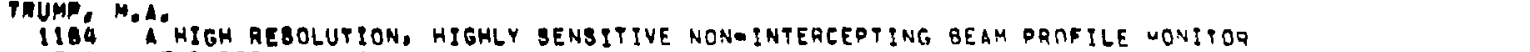

1214 BEAD PERTURBATION MEASUREMENT

386 BEAM POSITION AND CURRENT MONITOR SYSTEMS FOR THE SIDE-COUINLED LiNAE

作

346 OESCRIPTION OF THE BEAM POSITION AND CURRENT MONITORING SYSTEMS FOR THF TRANSITIINN REGION

1243
QQHO LAMSF ACEELERATOR AEAMOLINE MONIPORING SYSTEM

70005 B

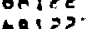

71051

$71125 \times$

711221

inuol,

1633 "HOTON IPECTRIIM IN PION CAPTURE ON TRITIUM

QSG. RAOTATIVE NEGATIVE PION CAPTURE IN LIDUIO TRITIUM

7001.10

$778 \times 52$

77,1920

TUeB, G,E.

2465 2OHOMHZ OPERATING MANUAL

MUON CHANNEL POWER SUPPLY PROTOTYPE TESTS

2212 PERFORTANCE SPECIFICATION FOR MAGNET POWER SUPPLY

GPECIFICATION FOR MATNET POWER SUPPLIES

PECIFICATJONA FDR TO35 FILAMENT SUPPL

214 OPECIFICATIONA FOR MAGNET POWER SUPPLIES: 72069

701193

701117

73 คa.'A

131119

740,13

72Ana! 
TURNER, R.O

2598 GL RUADRUPOIE STEEL PERMEABILITY TESTS

ANO X MAGNET ELECTRIC POWER SYSTEM

2039
709 THE LATIPF SWITCHYARD MAGNETS

7117.43

120315

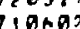

TURNER, T.F.

DUA Bo. I KLYSTRON BLOEKINGAOSCILLATOR HODULATOR

676 HIGH VO'. IGE DESIGN PRACTICE

2725 INTERIF KLYSTRON MODILLATOR DESIGN

PULSE CURRENT TRANSFORMEAS

PULSE TRANSFOAMERS

PULSEO RF DOWFA SYSTEM FOR LAMPF ACCELEQATOR

760 RELTAOILITY. AVATLABILITY, ANO MACHINE OESIGN

$650 \sin$

60032 ?

60032 ?

$061>29$

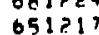

051217

000910

UBERALL,

TIT LAMPF NEUTRINA FACILITY PROPOSAL

71137

VAN OYCK, 0,8 .

2326 MEASUREMENTS TF HE EMITTANCE TAILS

1505 OPERATING EXPFRIENCE WITH LAMPF MAIN GEAM LINES INSTRUMENTATION ANA COMTSOL SYSTEM

233. REVISED CYCLE O PLAN

2325 SWITCHYARD FRTNT END REDESIGN

2319 SWITEHYARD MATNEY CURRENT MEASUREMENTS

2666 SWTTEYARD MEASUREMENTS OF HE PRANBVERE OSCILLATION $(4 / 4 / 77)$

thas

74 arरs

$751,10 \mathrm{hn}$

VAN OYKE, W.J.

2HO1 SHOCK WAVES in LAMPF BEAM LINES

UH ISOLATION valves

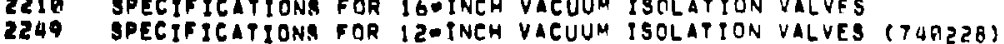

2238 SPECIFICATIONA FOR 2NINCH VACUUM ISOLATION VALVES (VAN OYKE 731212 )

2296 SPECIFICATIONA FOR GIINCH VACUUM ISOLATION VALVES

2262 SPECIFICATIONS OOR GOINCH VACUUM ISOLATION VALVES

SPECIFICATIONA GOR ROUGH VACUUH PUUP PACKAGE FDR THE EXPERIMENTA AFEA OE GUDF

1242 SPECIFICATIONS FOR SIX INCH RADIATION HARDENED VACIUM ISOLATION VALVE OD: THF IAMPE EXPERIMENTAL AREA

2259 SPECTFICATIONG FOR TIIRADMOLECULAR VACUIJM PUMP DACKAGSS FOR USE IN TME GMTTCHYARD AND OY GINE IN THE LAMPF EXPERTMENTAL AREAS

2269 SPECIFICATIONA FOR THRFEOINCH VACUUM ISOLATION VALVES

1259 SPECIFICATIONB FOR VACUUM ISOLATION VALVES

2263 SPECIFICATIONA FOR VACUUM DUMP PACKAGE FOR THE STODPEO MUOA CHANAFL IFF LiMDF

75155

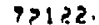

748520

131515

$753 \% 5$.

$751: 01$

75iग

71 iा

7504 a t

trinas

$\cos 319$

VANBUREN, D,

GIS A PAST PQOTECTION SYSTEM FOR LINEAR ACCELERATORS 


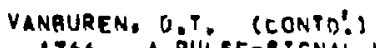

1766 A PULSE-SIGNAI VIEWING SYSTEM FOR ACCELERATORS OOPGMS

1220 AMPLITRON ANALOOG ANO VIOEO EHANNELS

1786 ANALOG-TCODIGTTAL CONVERTER SPECIFICATION

1269 BINARY COMMANA CONTROL CIRCUITRY

1794 CAMAC SUBRTIUTINES FOR NOVA SINGLF USER BASIC

1706 CHANNEL IDENTTFICATION - 608787

1707 CHANNEL IDENTTFICATION - 600713

1705 CHANNEL IDENPFFICAYION - 669023

624 COMPUTER CONTROL OF THE LOS ALAMOS LINEAQ ACCELERATOR

1171 DEFECTIVE LOGIC MODULES

1741 DEVIEE SYHBOLA AND HAROWARE DRAWINGS

1235 DFL LOGIC STINDARDS

1672 EPA MOOULE CONTROL POMER

1216 EPA RICE LAMP AND CONNECTOR WIRE SCHEOIJE

1993 FABRICATION RFOUEST NO. ZSI LOGIC SIMULATOR

1671 INJE 1567 COR CONTROL ROOM RACK AND DISPLAY LAYOUT

1567 INJECPOR CONTROL ROAM RACK IND OISPLAY LAYOUT

725 LAMPF CONTROL SYSTEM YIMYNG
1303 LAMPF FAST PROTECT SYSTEN

1303 LAMPF FAST PRATECT
1151 LAMPF VIOEO SYSTEM

1151 LAMPF VIOEO SYST
1198 LOGIC MODULES

1198 LOGIC MODULES

290 MOCKUP

BQD MPOI CONTROL SYSTEM MEETING FOR DISCUSSION OF LAMPE OATA ACOUTSITITN A.O EMVTROL SYSTEN

1889 MPOI R AND D DROCRAM FY=AQ

1688 PROGRAM DIRECTS

1714 PROPASEDSIHUI ATOR
1783 RICE CHASSIS SPECIFICATION

1763 RICE CYASSIS SPECIFICATION

1702 RICE IVO CHASAIS SDECIFICATION
I573 SECTOR CONTROI

I775 SECPOR CONTROI PANEL SPECIFICATION

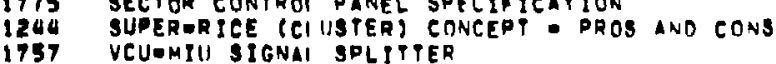

67111

$\operatorname{Bns}+2^{2}$

OAH1'10

75120,5

000727

008713

660423

62 मी

601225

69110

67119

67वसla

671 knda

ongiz:

05119 ?

$05122^{\circ}$

00611

6al 2 a

67 win?

6711,1

670514

(5)

वरा

ofith?

651128

$\cos \cos ^{2}$

Do! 11:?

6aras

oxhly

कराम?

671304

VANVESSEM, T, G.

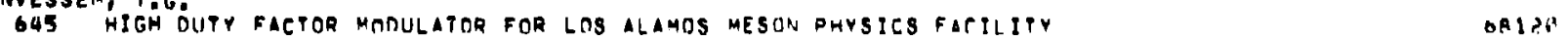

VARGHESE, P.

281? ELABPIC SCATYFRING ANALYZING POWER MEASUREMENTS AT BOR MEVE-T

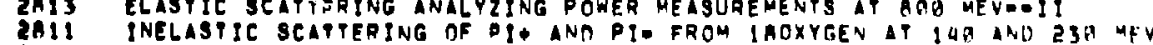

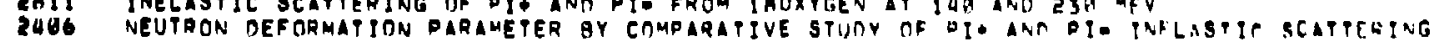

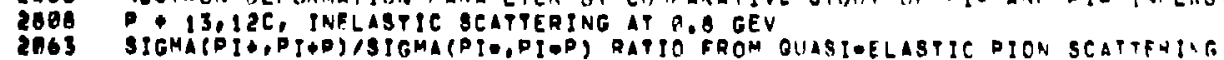

VARGO, P.M.

T7AOA.

17 म09

770

7 ?日ing

79ang 
YARGO, D.M. (CONTD,)

1715 DIGITAL SYSTEM SYNTHESI2ER OOBTZC

1716 AUTOMATIC MODILE TESTEP OKADSZ

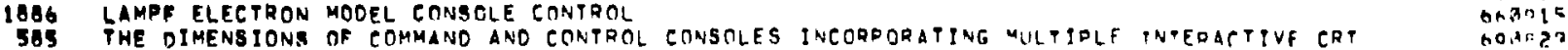
DISPLAY SYGTEMS

2203 FOUR POLE MAGNET RINGS FIR FUEUSTNG FEAYS OF CHARGED OARTICLES

556.8

VEGORS, 5 .

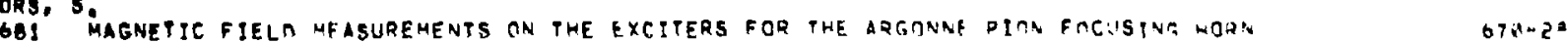

VENAELE, D

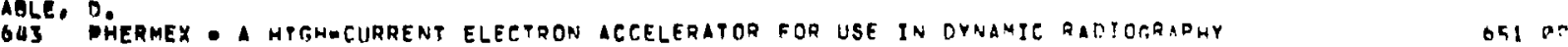

VERBECK,

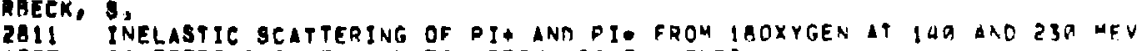

1995 SCATTERING OF SQMEV PI FROH LIGHT NUCLEI
1902 BTUDY OF THE PPIE,N) ANO (PI,PI PRIME P) REACTIONS IN LIGHT NUCLEI

$79000^{2}$

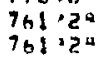

VETTER, J

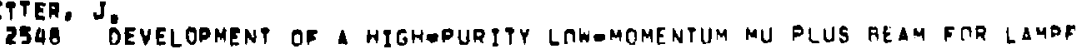

17040.

$\checkmark 1600 R, 8$.

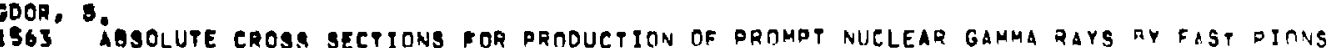

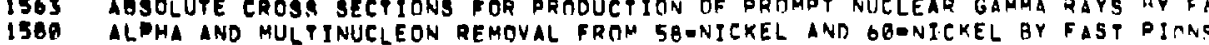

1 ONO

1502 RAST PION INDICEO PROCESSES IN COMDLEX NUCLET, CQOSS SECT ION AND ALOMA

1505 FAST PION INDIICEO PROCESSES IN COMOLEX NUCLEI SYSPEMATICS IN PRODIJCT. UTLEI

2418 GAMHARAY BTURY OF PION=INOUCEO REACYIONS ON EOMPLEX NUCLEI

1993 INTERACTION OF FAST PIONS WITH GZ, GA-NICXEL

1562 SYSTEMATICS OF PION AND PROTON INTERACPIONS WITH NICKEL NUCLIOES

VISSCHEN, W,M.

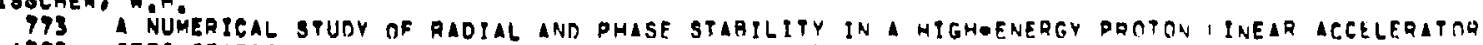

1323 OPTIMIZATION OF MAGEPIC LENGES PORE STABTLITY IN MTGHEENERGY DROTON

Vookt, Hof.

127 3D MAGNETIC FTELO COMPUTATIONB ANO COMPARISON WITH MEASUREMENTS

1363 OEAM CALCULATTONS FOR LAMDE MUON CMANNEL

2394 COMPUTATION OF MAGNET-END FIELOS

$750 \cdot 3$.

$750 \operatorname{so}$

77200

75a

75p iA

758 in

7०1

7คI ?

$7+1 \cdot 250$

64243

canp

72110

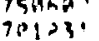


VOGEL, H.F. (CONTD,

726 COMPUTATION OF MAGNETIC ENO FIELDS ANO COMPARISON HITH MEASUREMEAT

1103 CROSSEOTIELO VELOCITY SEOARATOR FOR PROOUCING TRANSVERSEDOLARIZEN UUANG

$1: 93$

SThe

FFFETS OF AN INTENSE FLUX OF FAST AND SLOW NEUTRONS ON THE MAGNETIE PRODERTIES OF STEFL

$60,721,13$

761 ELECTROAMECHAN

1360 ELLIPSE MATCHPNG WITM DOUBLET THIN QUADRUPOLE LENSES

1100 FIELO BETHEEN FINITE PARALLEL DOLES AND ITS OPTICAL IVTERPRETATIOA

880 GRAPHICAL REPRESENTATIONS OF THE PION, MUON DECAY KINEMATICS

265 INOUCEO OLARTTY IN PWD OIMENSIONAL MAGNETIC FIELDS WITH FINITE IRON

G61 MAGNETIC FIELO MEASUREMENTS ON THE EXCITERS FOR THE ARGONNE PION FOC

1184 MAENEPIC PIONEMUON SEPARATION IN THE ANALYZER OF THE STOPPED MU CHANAE

1105 MUON ORIGINB AND TIME STRUCTURE IN THE STOPPED MUON CMANNEL

1 is9 ON PHE OUESTION AIR. MELIUM, OR VACUUM FOR THE STOPPEO MUION CHANNEL

66. DERPURBATIONG FROM STEEL ENVIRONHENT CLOSE TO A C MAGNET

1 G54 PROPOSAL FOR A BTOPPEO MUON CMANNEL

2920 RADIAL EXTMACTION OF SECONDARY BEAMS IN AN AXIAL MAGNETIC FIELD

1187 SOME REMARKS MELRING ON QUAORUPOLE FIELO QUALITY

1164 STOPPEO MUON EHANNEL - DESIGN STATUS - JULY 2,1060

720 STOPPED MUON C.MANNEL FOR LAMPF

1863 SUMMARY OF STATUS OF DESIGN OF STOPPED MUDN CHANNEL

2361 THE STOPDEO HIION CHANNEL IT LAMPE

VDGEL, D.

IAI ARECISE DETERMINATION OF EZ AND E4 MOMENTS IN 105 -HOLMTUM FROM MUONIC YERAYS

212 PRECISION MEARUREMENTS OF E2 AND E4 NUELEAR CHARGE MOMENTS OF 181 FANTALIM ANO 101 . DYSPROS IUM

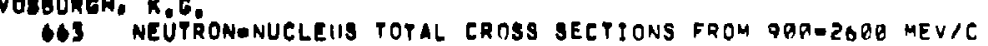

$\cos 32 n$

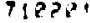

070910

$001 \frac{17}{20}$

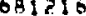

GA1

$71332 n$

690.100

$\cos \theta 005$

136725

19645

$00079=$

190201

$\angle A O=13$

Tिक्ष

OLINGER, E.A.

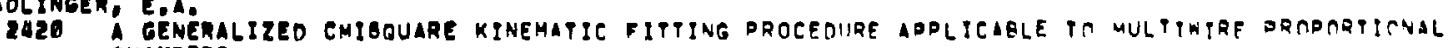
CHAMBEA

2602 DIFFERENTIAL RROSS SECTION MEASUREMENTS OF PI OOR. TRITIUM ELISTIC STATTLING AT 232 , ZSE

2075 MEAQUREMENT OF PIOD ELASTIC SCATTERING

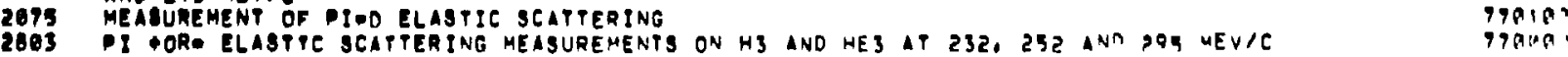

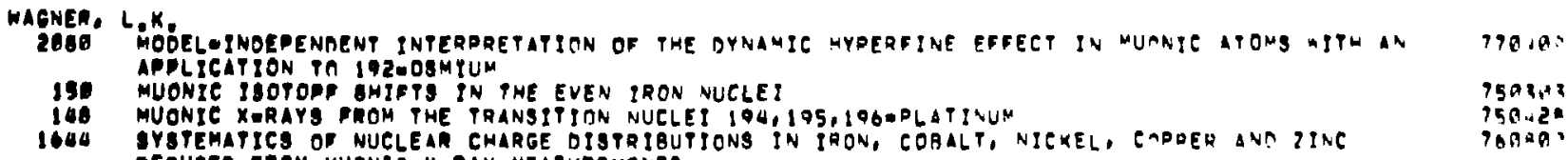

WALKER, 0. 
WALKER, D. (CONTO,)

179. DIOACRO DUNCH AND DIE SPECIFICATIONS

195 CIBRTCATION BPECIFICATIONS FOR WARNING SIGNS - 7 PBZDE

1917 FABRICATION SPECIFICATIONS FOR WARNING SIGNS - T2P3I4

1203 INJECTOR PERSANNEL SAFETY SYSTEM

NEPESONNFL BARRIER IN SECTOR G

1934 PERSONNEL BAFPTY KEY BANK SYSTEM

20

WALKER, J,F.

948 Complex eigenvalues of a coupled chanNels mode

MOSBEAUER EFFFE FOLLOWIAG COULOMB EXCITATION IN THE EVENGEVEN ISOTOUES OF YTTERAIIHM

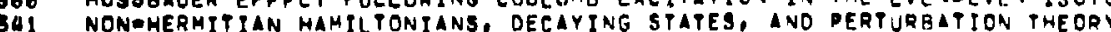

551 OPTICAL HODELA. DECAYING STATES, ANO PERTURAATION THEORY

$71 ! \cdot 9$

070.1

72039

WALKER, S,A

1404 MINERAL-INSULATED MAGNETS FOR HIGHARADIATION ENVIRONMENTS

$698 \rightarrow 40$

HALLACE, J.O. WHZ RFOACRELERATOR SYGTEM

ACHIEVING ELINICALL USEFUL DION DOSE DISTRIBUTIONS

27日 ACCELERATOR FTELD MEASUREI

510 ACCELERATOR SYSTEM STUDIES

1391 APPLICATION INFORMATION ON THE EAI380 MYRATO COMPUTER BIOMED BEAM SHAPING

B

M OHEO PION ENERGY CONTROL ACTUAT

322 BIOMEDICAL CHANNEL BEAM CONTROL

TIMPF BASOMMZ ACCELERATCR STRHICTURES AT HIGH POWEO

US ING HYBRID COMPUTER TECHNIOUES

TEEDFORHARD C.OMPUTER TECHNITUES OD RE FIELDS

279 CULL POWER OPFATION OF THE LAMPF AD5 YHZ SYSTEH

521 MASNTENANCE -729622

521. MAINTENANCE O 729622

2696 PRELIMINARY SOECIF ICATIONS PHAGE HONITORING SYSTEM

$24 B 9$ OUASIELASTIC PHARGE EXCHANGE IN ND GTESTO PNN AT TO4 MEV

203 REPORT ON TRgD PO NAL

2月3日 SLAC ORGANIZATION

237 TUNING AND PREOBEAM CHECKOUT OF BOS MHZ SIDEOCNUPLEO DROTON LINAE STHUPTIAFS

$7 \log ^{2}$

100010

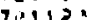

$75975 n$

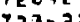

$\operatorname{Tin} 2020$

$71060 ?$

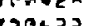

trangs

$758+11$

158163

reang

Tugh

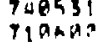

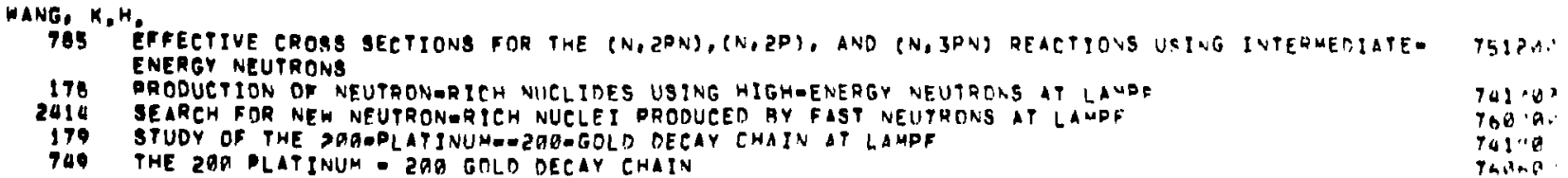

WANGLER, T.P. 
WANGLER, T.D. CCONTD,

1563 ABSOLUTE CROSR SECTIONS GOR PRODULTITN OF PROMPT NLCLEAR GAMUA RAYG RY FAST DIONS

1500 ALHA ANO MULTINUCLEON REMOVAL FROM SB-NICKEL ANO GPENICKEL OY FAST DITNS

1581 FAST PION INDUICED PROCESSES IN COMPLEX NUELEI CROSS SECTION AND LLPA REMCYAL

1582 FAST PION INDULED PROCESSES IN COMPLEX NUCLEIL DROJETTILE SYSPEMATCS

1585 FAST PION INOUEED PROCESSES IN COMPLEX NUCLEII SYSTEMATICS IN PRODICT WCLE

2410 GAMMA-RAY STUNY OF PIONEINOUEED FEACTIONS ON COMDLEX NUCLEI

1562 SYSTEMATICS OF PION AND PROTON INTFRACTIONS -ITH AICKEL NUELIOES

$75 x \cdot 6$

75 inm?

750.20

$75,-2$

$77,10.25$

$75,4=4,8$

WARNEKE, $M$

222 FINALESTATE INTERACTIONS IN PAD BREAKUP AT BOD AND OHO MEY

$753: 2 A^{2}$

IA51 KINEMATICALLYOCOMPLETE STUDY OF PION PRODUCTION BY THE REACTIOA I HYARAGNGDROTOA, DI*

$7+9105$ PROTDN) NEUTAON AT

1981 PION PRODUCTION BY ORQ MEY DROTONS FROM G-LITHIUL

221 PION PROOUETION RY BRE MEY PROTONS IN THE REACTION IEMYDQCGENTD,PI,DO

1982 PION PRODUCTION IN PAD INTERACTIONS AT BAP MEV

1996 PION PRDDUETION VIA A PQP GOESTO O+PI BUASIOFREE PROPONS IS AEUTERPUW

1983 PIONONUCLEUS FORWARD SCATTERING AMRLITUDES NEAE THE $3 / 2,3 / 2$ RESONANCE

223 QUASTOFREE SCATTERING IN P.D BREAKOUP REACTIONS AT 83 TINO G3O MEV

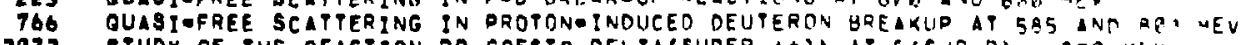

2072 STUDY OF THE REACTION PO GOESTO DELPA(SUPER +\$)A $\triangle T F(S U R D)=$ RTP MEY

2400 TUNE-UP OF THF LAMPF NIGHOENERGY PION CHANNEL

WEBER, D.W.

1204 A COMPACT OATA ACOUISITION AND CONTROL PERMINAL FOR PARTICLE ACCFLFRATRRS

1269 BINARY COMMANO CONTROL CIRCUITRY

1417 BRINGING OUT STAPUS INDICATIONS AND PHE COMPUTER CLOCK FAOM THE DOPAIIh POUP.,TEQ

1263 CHANGING THE RICE OATA FORMAT TO ENABLE MORE ICCURATE DATA MFASUREMENTS HFRE OE ?UIRFO

1800 COAXIAL RELAY MATRIX, 10 INDUTS, 2 OLTPUTS

1217 COMMENTS ON AMPLITRON TEST STAND NOISE

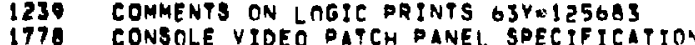

1900 ELECTRON MODEI CONSOLE UNTT REVISED SPECTFICATION

IOQS ELECTRON MOOEI HEAO CONTROL UNIT

1994 FABRICATION RFOLEST NO, 261 HEAD CONTROL CHASSIS

1250 ION SOURCE TEST STIND

1297 TON TEST GTANA RICE I/O CHASSIS

IAIA LAMPF RICE IOA WIRING HARNES SPECIFICATIONS

1942 LOGIC ELEMENTA

179 MISEELLANEOUS FABRICATION SPECIFICATIONS FOR COMPUTER COMPONENTS

1693 NPUT POST PRORESBOR

1956 OPERATION AND CHARACTERISTICS OF THE VIDEO SYSTEM

1273 PROPOSAL FOR THE RIEF 110

127 RICE $1 / 0$ CONVFRTER

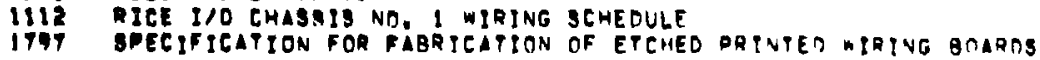

$701+24$

int 20

Tol 124

inl 2 政

$753 \ldots 3$.

$779+2 a$

$757 \times 0^{3}$

Inl Phy

oopsas

कीA $1=$

orituta

oxal:

ort 3 :

brang.

6711 the

ool.il

ont.?

601.1?

कAplz?

680515

conila

G.alin

67!!

ogs5i"

al. 2

GAMBI

GAOPE?

on 67 aी 
WEBER, O.W. (CONTD:)

FOR LAMPE RICE IJO FABRICATION

181. SPECIFICATIONS FOQ PQINTED CROUIT BOAQDS

1016 TENPATIVE SPEPIFICATION FOR PRIATED CIRCUIT CARD SIZES

1 149 THE OS MHZ RTCE IOO CHASSTS

232 THE HANOYMANS GUIDE TO THE RICE I/O CHASSIS

I960 THE HANDYMANS GUTDE TO THE MCDULE VEU

1298 THE HANDYMANS GUIDE TO THE LAMDF RICE I/O

1755 VCU LOGIC SIGNAL CONVENTIONS

1759 VIDEO BUFFER AMP IFIERS

I7T3 VIDEO CONTRDL UNIT SPECIFICATION

WEEMSLER, P.J.

2167 ELECTROSTATIC STEFRING IN THE TRANSPRRT

RIAJ PHE SENBITIVITY OF WILLAROS FINGERS

60011

$\operatorname{san} 130$

69167

6R1

Gain

orgses

608435

6)aps

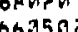

WEINGECK, $n, 3$.

235 INTERNALGCONYTRSION COEFFICIENT DETERMINATION OF OND DARITY FOE THE IBR, AOKEV FIOST-

$\operatorname{lint2}$

CIS8. 6.K.

GQZ LOCALIZED CURRENT FIELO TUMOR THERIPY. PRELIMINARY RESULYS IITH SPONTANEOUS ANIHAL TUMORS

36 NOYSEOREE ECR DATA DUR ING ELECTROSURGICAL PROCEDURES

WEISS, J.R.

1645 PHE FISSION TRACK RECORD OP APENNINE CRONT KREEP BASALTS

$750 \cdot 10^{2}$

inata:

WELSH, R.E

141 PRECISE DETERMINATION OF EZ AHO EL MOMENTS IN 1650HOLMTUM FROM MIIONIT YORAYS

LES MUONGE IOT

$50>24$

WREEK, R.O.

1308 PERPORMANCE OF THE HIGHEENERGY PION BEAM AT LAMDF

2QI3 PAELIMINARY MEASUREMENTS OF GUT MEV DROTON IIMEOOF -FLIGHT SPECTAA (REVISFOI

2ATO TUNEGUP OF THF LAMPF HSGHEENERGY PION CHANNEL

$7 \sin x$

?at.?. 20

WERREN, $D, W$,

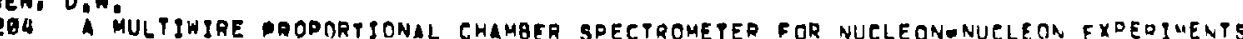

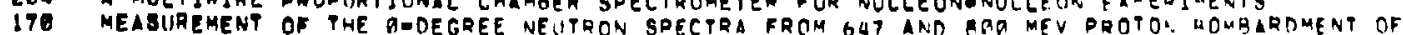

$131 \cdot 0 x$

203 LIGHT NUELEI THE PEDEGRE NEUTRON SPECTRA FROM GUT AND RGA MEV PROTOL. WOMBLRDMENT OF

SUE P . $904 \mathrm{MFV}$

1634 NEUTRON SPECTRA AT 2ERO DEGREE FROM SP, NI REACTIONS ON GEBERYLLILI, 1Z-CARROH: ANO 27-

$794 \cdot 9$. ALUMINUM AT GA 9 AND BOA MEV

Taptak 


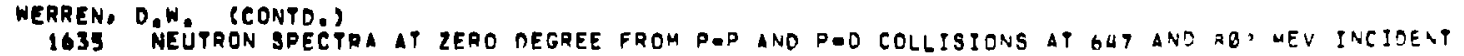

762785

ENERGIES

200 NEUTRON SPECTRA FROM 5OG MEV PROTONS ON OARERYLLIUH
232 NEUTRON SPECTRA FROM PROTON BOMARDMENT OF DEUTERIUM AT OUT AND ROA YEV

1978 PRECISION MEASUREMENT OF NAP CHARGE-EXCHANGE CROSS SECTION AT GU MEV
202 PRECISION MEASUREMENT OF NAP CHARGE OECHANGE CROSS SECTION AT GU MEV

WEAT, 6.8.

2490 COULOMB-NUCLEAR INTERFERFNCE WITH PIONS

770.49

190 PPATIAL OISTR

190 SPATL OISTRTBUTION OF EFFECTS OF NEGATIVE PIONS ON CULPUFEO MUMAN CELLS PION THEAAPY AEAM

WHARTON, W.R.

WARTON, WCRP SCATERING OF SO MEV PI P FROM LIGHT NUELEI
1995

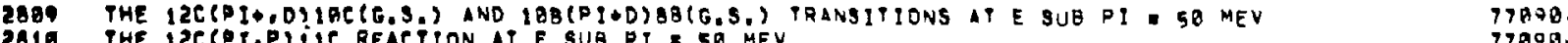

WHETBTONE, S.L.

330 STATUS OF THE LASL MONTE CARLO INTRANUCLEAR CASCAOE CaLCULATION (1964) OUI?!

WHITENTON, 3.

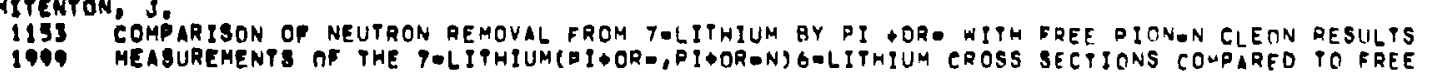
PIONGNUELEON RESULTS

WHITTEN, CoA.

2090 ELAOTIC AND INELABTIC SCATTERING OF

2812 CLABTIC SCATTFRING ANALYZING DONER MEASUREMENTS AT SPO MEVE-I

2013 ELASTIC BCATYPRING ANALYZING DONER MEASUREMENT

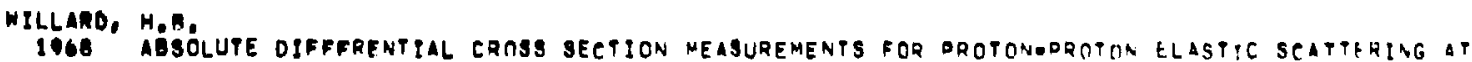

$701+301$

1436 6A7 AND OEO MFV

200. POTAL -REACTION CROSS SECTIONS THE EXTERNIL PRCTON AEAY AT LAMPF

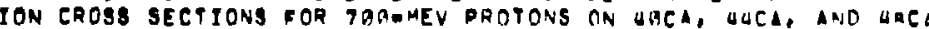

7501700

WILLZAMB, H.E.

1933 APRLICATION OF A NEW LIMIT SWITCH FOR IH-TURN DOTENTIOMETERS

25UD PLARIZED ION INJECTOR PROGRIM

TECMNICAL DESTRIPIION - BECTO 
WILLIAMS, R,A.

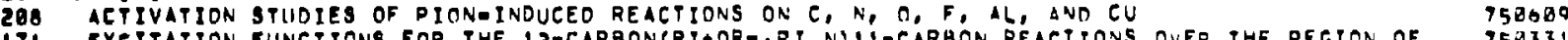

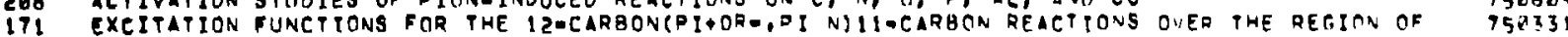

231 THE (3,3) RES ONANCE, BY 1 OQ MEV PI( +OR-) AND $3 \triangle Q$ MEV PROTONS

195 BTATUS REPORT ON THE INITIAL NUCLEAR CHEMISTRY EXPERIMENTS AT THE CLINTO. P. ANOERSON MESON DHYSICS FACILITY (LAMPF)

WILLIAMS, T.M.

222 FINALETATE INTERACTIONS IN POD BREAKUP AT BQR ANO ORO MEV

1H5! KINEMATIEALLYOCOMPLETE STUOY OF PION PRODUCTION RY THE REACTION I HYOROGFNOROTON, PI+ PROTON) NEUTRON AT BAQ MEV

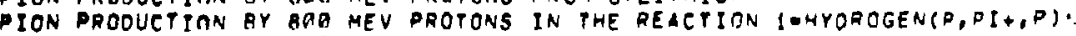

PION PRDDUETIION FRDM THE REACTION DCD,O DI)N AT 585 MEV

PION PRODUCTITN VIA A P+D GOESTO DQPI TUASIOFREE DROTONS IN OEIITERIUUA

PION-NUCLEUS SMALL ANGLE ELASTIC CROSS SECTIONS NEAR THE $3 / 2,3 / 2$ QESONA.CF

IONONUELEUS TOTAL CROSS SECTIONS NEAR THE $3 / 2$, 3/2 RESONANCE

UASIOFREE SEATTERING IN PUD BREAKOUP REACTIDNS AT RAD AND WAV MEV

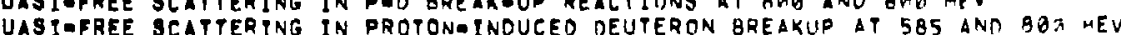

766
2072 OUASI-FREE SCATTERTNG IN PROTONAINOUCEO DEUTERON BREAKUP AT 585 AND

WLLSAMSON, K.D. JR.

S45 A PROPÓSED B. 4 POLARIZEO PROTON TARGET CAYOSTAT FOR LAMPF

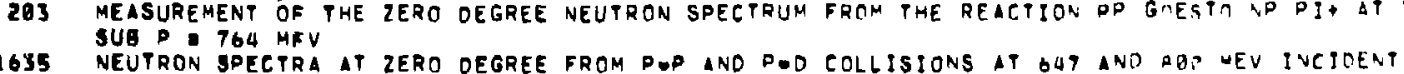
ENERGIES

232 NEUTRON SPECTRA FROM PRDTON BOMBAROMENT OF DEUTERIUM AT OUT ANO BQA MEV

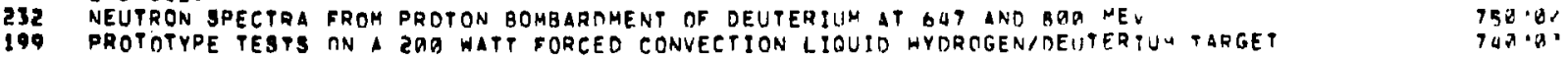

WILLMEQ, H.

1968 ABSOLUTE DIFFFRENTIAL CROSS SECTION MEASUREMENTS FOR PROTONOPROTHN ELASTIC STATTERIVG, AT 649 AND 8 GQ MEV

WILMARTH, J.E.

397. FABRIEATION SDECIFICATIONS FOQ BEAM POSTTION CABLE

1879 FABRICATION BDEEIFICATIONB FOR IOV EXEITATION TO ADS CABLES

$254+4$.

की

70129

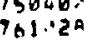

$76 !$ द

$7+1 \cdot 20$

$761 \cdot 52$

$\tan 052$

$750 \times 0$

700705

WILSON, $K, B$

2月OU KOLUTION FOR AEMOTE HANOLING IN ACCELERATOR INSTALLATIONS

$75,12$.

7 क⿻ 795

Thค9:4:

WILSON, METEA A

$71110:$
$71111:$

79014

72010 
WLSON. M.T. COONTO.)

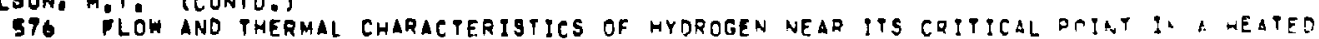
MUN

1522 KILOTON SHIELD DODRS AT LAMPF

2 GA2 MERRIMAC BOX DIMENSIONS

TON PQRT AT LAMPF

RADIAL FLOW MFASUREMENTS OF HYOROGEN NEAR ITS ERITICAL OOINT IS A WEATFO CYLIAORTCAL TI AE RADIOACTIVE ATP E

RADIOACT TVE TATER GYSTEM

RAOIOACTIVITY. SHIELOING. RAOIATION TAMAGE, AND QEMOTE MANOLING

REMOTE HANOL ING PHFLOSOPHY ANO EQUIDMEVT FOR LAMDF

REMOTE MAINTENANCE CONCEPTS FOR TME LAYPF

REMOTE MANOLTNG PHTLOSOPHY AND EQUTPMEUT FOR

ROLLER TESTS - JUNE 1968

THE EVOLUTION OF THE LAMPF

THE LAMPF HOT CELL COMPLEX

$00,141^{2}$

75119

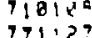

$711: 2$ ?

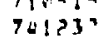

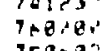

OB:Th:

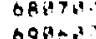

opesicis

करका

$77031 \mathrm{~A}$

bog?

WING, J.D.

IIIS OEHINERALIZEO WATER DISTRIBUTION SYSTEM IN BIOMED FACILITY

750.01

HITEN, T.R.

222 GINALOSTATE INTERACTIONS IN POD BREAKUP AT BGB AND OBO MEV

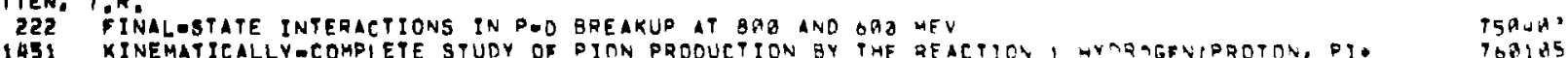

160125

PROTON INEUTRON AT BOR MEY

$15 n^{4}+a^{2}$

221 ION PRODUCTION GY OGg MEY PROTONS IN TME REACTION 10HYDROGEN(D,DIDO)

1980 PION PROOUCTION FROH THE REACTION D(D,O PI)N AT 585 MEV

183 PIONANUCLEAR TOTAL CROSS SECTIONS NEAR THE 3/2, 3/2 RESONANCE

223 OUASI-FREE SCATTERTNG IN OTD BREAK-UP REACTIONS AT BQB AND OQG NEV

966 OUASIOFREE BCATTERING IN PROTONGINOUGEO DEUTERDN BREAKUP AT SBS ANR RP WE

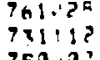

$\operatorname{son}+0,5$

WOHLFAHRT, H.O.

1SB MUONIC ISOTOPF SHIFTS IN THE EVEN TRON NUCLEI

1644 SYSTEHATICS OF NUCLEAR CHARGE TISTRIBUTIONS IN IRON, COHALT: NICKEL, CAPOEP AVT 2INE

$\operatorname{Tan} 3013$ OEDUCED FROM MUDNIE XORAY MEASUREMENTS

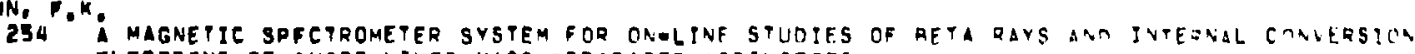
ELECTRONS OF SHORT-L IVEO HASS-SEPARATED ACTIVITIES

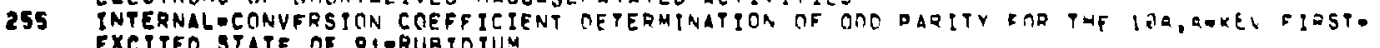

Tunart 75. :

WOLFE, D.M.

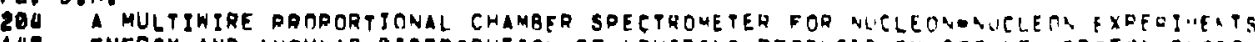

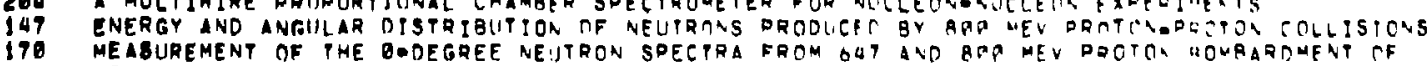
LIGHT NUCLEI 
WOLFE, O.M. (CONTD,)

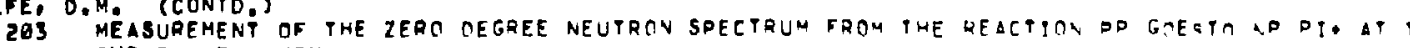

$790,0.9$ SUAD 764 MFV

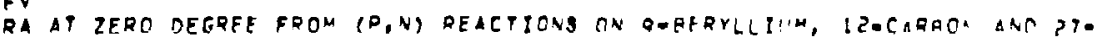
ALUMTNUM AT GUT ANA BAT ME

AT TERO OEGREE FROM PAP AND P.O COLLISIONS AT GU, DNS QR. WEV INCINET ENERGIES

200 NEUTRON SPECTRA FROM SAG MEV PQOTONS ON Q-BERYLLIUM

232 NEUTRON SPECTRA FROM PROTON HOMBAROMENT OF OEUTERIUM AT OUT AMO AHG UE

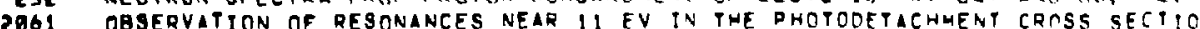

1646 PION PRODUCTION IN NEUTRONGPROTON COLLISIONS

PREC PSTON MEEAUREHENT OF NOP CHARGE-EXCHANGE CROSS SECTION AT O4 7 WE

202 PRECISION MEASIREMENT OF NOP CHARGE EXCHANGE CQOSS SECTION AT GW WE

ratares

$\ln x>\operatorname{sen}$

Tथर००म

75 . HA

7 in: 1 's

$74 \cos 30$.

$75, x+x$

WOOOARO, C.E.

2235 GTANDARD OPERATTNG PROCERURE TURN-ON PROCEDURE - 291.25 4Hz

$731>07$

woobr, C.L.

LOU I GUIOE TO THF USE OF PRECESS

405 ABSOLUTE ENEPCY CALIBRATION OF THE H. COCKCROFTOWALTON IUJECTOQ

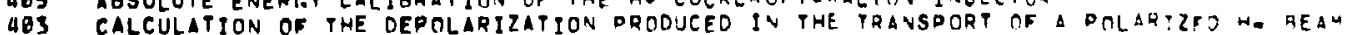

UGB CRELIHINARY OF THE DEPOLARIZATIONDROOCECHANGE CELL AND FILLER ASSEMIY ERR THE M DOLLRIZED TON SOURCE

$73 a .5 .54$

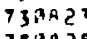

73025
73025

WOOLUM, D, S

1069 THOR IUH-URANIUM FISSION RADIOGQAPHY

$960+0$.

WORSTELL, H, G

197 BQG MC CLOVERI EAF FABRTCATION

2336 BOG-ME CLOVERIEAF RABRTCATION SYSTEM

DESTGN, DEVELOPMENT. AND FABRICATION OF MULTICAVITY ABS MHZ ACCELERATOR TAMKS

651 FABRICATION OF IIMPF IINAC STRUCTURES

15 HE HZ ATMOSPHERE FURNACE ARRAIING ANO SOLOERING OVEN PROOUCTION TPERATIOA AT LAMPF

HYDROGEN FURNACE BRAZING OF LAMPF ACEELERATOR STRUCTURES

1040 LAMP. 2 I HHZ YACUUM SYSTEH

LOCAL COOLING SYSTEM FOA BOA-MC CLOVERLEAF PROTOTYPE

652 MECHANICAL DESIGN OF LASL LINAC STRUCTURES - (LAMPF)

MECHANICAL ENGINEERING BQS HHZ STRUCTURES

METHOO FOR OETERMINING SOS MHZ STRUCTURES MOOULE NOMENCL ATURE OUTGABSING OF ETST KICXER FERRITE RLOCKS

2275 OUTGASSING OF FAST KICXER FERRITE RLOCKS

TANE HUGHES ION PIIMPS

B93 PRELIMINARY PARAMETERS OF 291.25 TANKS TO DETERMINE CLAD PLATE THICKOES5 AND SIZES PRELIMINARY PARAMETERS OF 2 LI.

O23 SAFE OPERYTION HF ION PUMPS

SPECTPICATION FOA PION DECAY VACUUM CHAMBER

SPECIFICATIONA FOR EGI MHZ DRIFTETUBE LINAC ION PUMPS

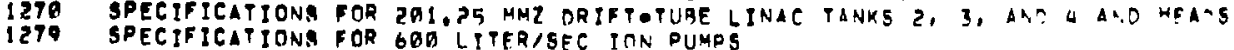


WORBTELL, H.G. (CONTS:

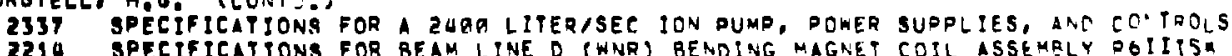

2245 SPECIFICATIONS FOR BEAM LINE D (WNR) 3O OEGREE BENDING MAGNET IRCN CORES CAIVOI

2244 BPECIFICATIONS FOR BEAM LINE D (WNR) 3Q OEGREE GENOING MAGNET COILS CB? VOB

1300 SPECIFICATIONS FOR BELL PYPE TYDROGEN BRAZING EURNACES

2225 SPECIFICATIONS FOR BEAM LINE O JUNCTION MAGNET CONOUCTOR

2226 SPECIFICATIONA FOR BEAM LINE O SWITCHYARD QUADRUPOLE MAGNETS

1297 SPECIFICATIONS FOR COPPER CLAO STEEL PLATES

2231 SPECIFICATIONS FOR LINE D MATN OUADRUPOLE MAGNETS

2229 SPECIFICATIONA FOR LINE D JUNCTION BENDING MAGNET TYPE P71168/

2189 SPECIFICATIONS FOR LAMPF HRS ION PUMPS ANO POWER SUPPLIES

2221 SPECIFICATIONS FOR TUNAEL QUADRUPOLE MAGNETS FOR LINE D

2227 SPECIFICATIONS FOR WNR Ja OEGREE RENOING MAGNET STEEL CORE FORGING RLANKS (CAIVOZ)

2233 SPECIFICATIONS FOR WNR 3P DEGREE BENOING MAGNETS - TYPE C BIV O

2250 SPECIFICATIONA FOR WNR QUADRUPOLE MAGNETS (406)

2265 VARIAN GOE LITERISEC (SGR LITER/SEC) ION PUMP RERUILOING PROCEDURE

140,3

0770,5

$73001=$
731 , 3 ?

orentis

$731: 13$

711,05

$7 \rightarrow-5$.

730019

$731: 12$

73115

748153

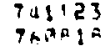

HORTHINGTON, J.N.

2416 ENERGETIC CHARTED PARTICLE YIELDS INOUCEO BY PIONS ON COMDLEX VUCLEI

INTERACTION OF FAST PIONS WITH G2, GUANICKEL

Thaneas

$\tan 11.52$

nORTHAN,

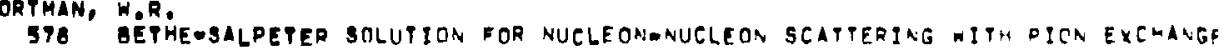

wU, $c, 8$

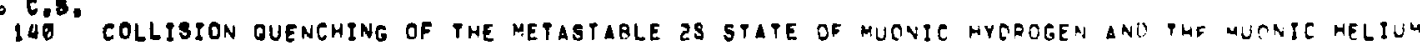
ION

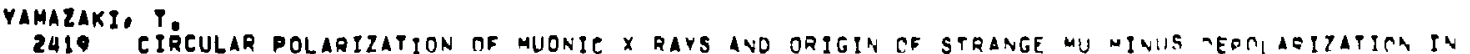
D METAL

Yamazaki. Y.

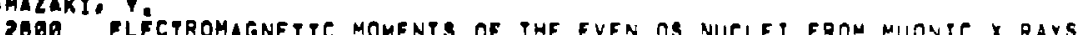

$171 \cdot 2$

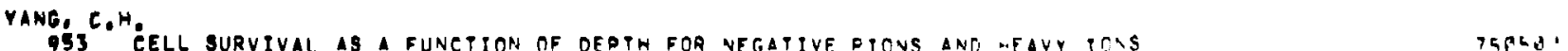

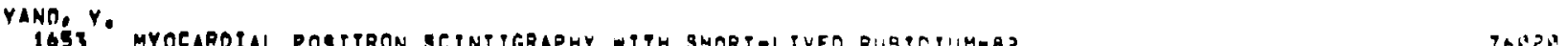

YAPEOEHILLIAMB, M.A. 
YATEBUWLLIAMS, M,A. (CONTO.)

2415 A COLLIDING BFAMS EXPERIMENT AT LAMPF PHOTODETACHMENT RESONANCES IN THE H. ION

ACTIVATION STIIDIES OF PION-INDUCED REACTIONS ON C, N, $D$, F, AL, ANA CU

ENERGETIC CHARGEO PARTICLE YIELDS INOUCED BY PIONS ON COUPLEX RUCLEI

171 EXCITATION FUNCTIONS FOR THE IR-CARBON(PI+ORE,PI VIII-CARBON REACTIONS OVER THE REGION OF THE $(3,3)$ RESOHANCE

2418 GAMMARAY STUAY OF PIONOINDUCED REACTIONS ON COMPLEX NUCLE!

I993 INTEAACTION OF FAST PIONS WITH O2. GU-NICKEL

$241 !$ MASS DEPENDENCE OF PION DOUBLE CHARGE EXCHANGE

IO97 PROTON SPECTRA FROM PION-INOUCED REACTIONS

YAVIN, 4.1.

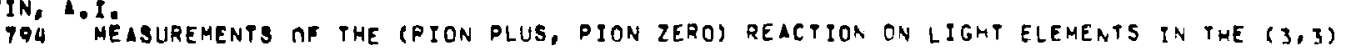

$70011 ?$

YOUNG, D.E.

1213 COMPARISON OF THE PARTICLE MOTICNS AS CALCULATED BY TWO DIFFEREAT DYNAWICS PROGRAMS

$601 \cdot 63$

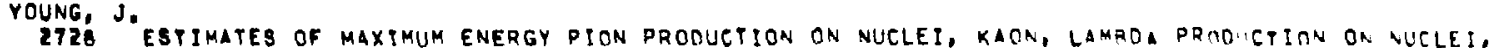

boBis?

ZENNDER, A.

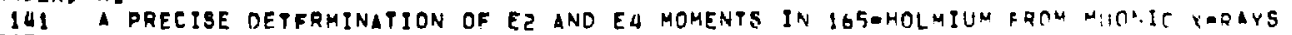

2979 PRECISION OFTERMINATION OF THE RADIAL CHARGE PARAMETERS AND THE OUADQUDOLE MOMENT OF 8I-TANTALUM USTENG MUONIE XMRAYS

212 PRECISTON MEARUREMENTS OF E2 AND EA NUCLEAR CHARGF MOMFNTS OF 18I-TARTALIM AND 101-

$770: 0$. OYBPROSIUM

759.2.

ZEIDMAN, B.S.

2811 INELASTIC SCATTERING OF PI + AND PI- FROM 18OXYGEN AT IHA ANO 239 MEV

24I MASS DEPENDENTE OF PION DOUBLE CHARGE EXCHANGE

1578 OBSERVITION OF THE PION DDUBLE CMARGE EXCHANGE REACTION 18-DXYGEN(DI+1,DI-)18-VEOR

2422 PION DOUBLE-CHARGE EXCHANGE ON $16-0 X Y G E N$ AND 180 OXYGEN

2417 PION NONAINALRG DOUALE CHARGE EXEHANGEI 16-OXYGEN(PI+, PI-) 16-NEON
364 PHE HRS SPECTROMETER SYSTEM

ZIOCK, K.O.H.

113 MEASUREMENT OF THE ALPHAOPARTICLE SPECTRUM RESULTING FROM PIO CAPTIIRE IN CARRON-IL

1543 TUNEUP OF THE LAMPF LOW ENERGY PSON CHANNEL

ZU PUYLIYZ. G.

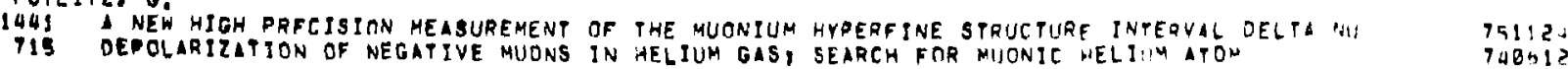


ZU OUTLITZ, G. (CONTO!)

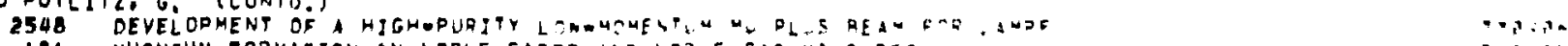

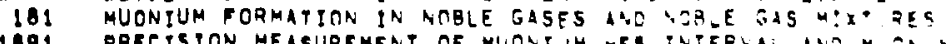

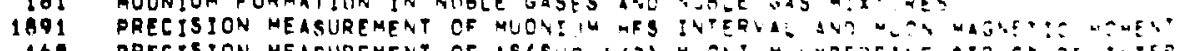

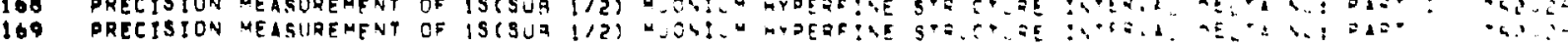
II 

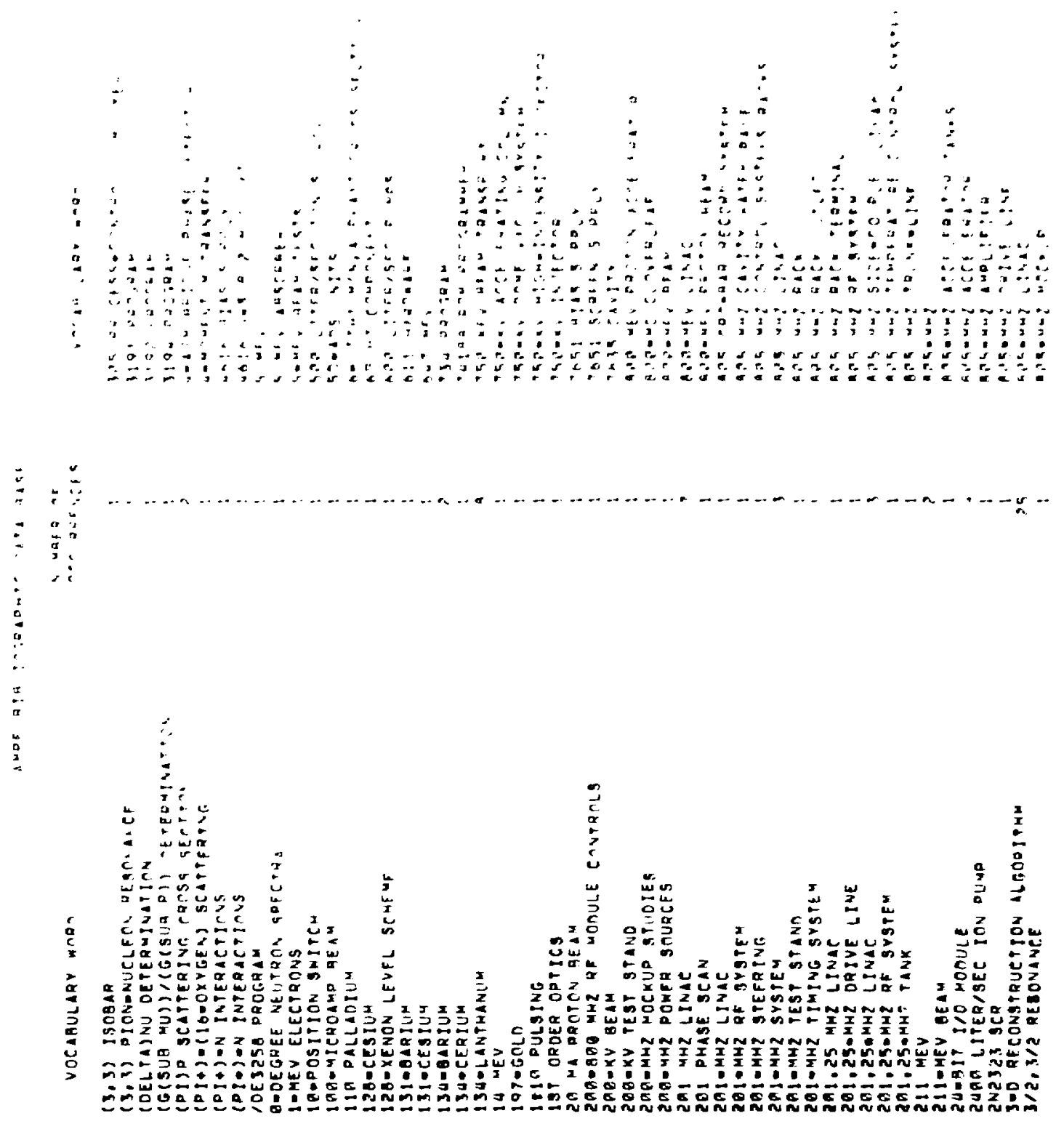

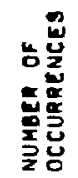


NUMBER OF
DCCURRENCE

voCaRULari moRn

105-MHZ

695-MHZ MODEL STUDIES

BOS= MHZ MODULE

G5- MHZ POHER SOUACES

BAS- MNZ RF SYSTE

E5-MNZ SIDE - COUPLE: LIOAC

BQS-MHZ STEERING

BASOMHZ SUBSYSTEM DATA

BOS-MHZ SYSTEM

040

DU CCNTROL SYSTEN

Bug CORE MEMDQT

QUD HARDWARE BCCPSTEAO OEAD CHLY UEUTAV

BUA MP PRIORITY IATFRQUSP STIPLS I ISOLA

BUP PROGRAM SENGE REGTSTEQ

GLO REMOTE RESTIRT

BAP SYSTEM

BSO-KV POAER SUDULY

O IN IA CMOPP ING MOOE

obog camac adC

AOS TARGET

$A \rightarrow E$

A.E CONTRACT

AOE PQEPARATIONS

A/O CONGERE?

ABERRATIOAS

ABSOLUTE CROSS-SEETJOYS

ABSOLUTE ENERGY CALIBRATIOY

ABSOLUPE SHAF DOS:TINY ESCAJFA

ABSOLUTE VELOEgTY GAJGE

ARSOLUTE YIELO

ABSORBED DOSE

ARBOABED DOSE MEASJPEUE.

ABSORBE SPRAGGLING

ABSORRER PHTCKNESS

ABSORHER/COLLECTOR

ARSORBERS

ABSORBING WEOGE

ABSORBTION CROSS SECTIO.S

ABsorption

ABsoRptION IN SOLIDS

ABSORPTIVE REGIOA EIGPNGTATES

AC INPUT

AC MOTOR DRIVERB

C POHER CONNECTOO

AC SRSTEM ADVANTAGES

AC STSTEM OISAOVANTAGE

ACCELERATING GAND

ACCELPRATING CAVITIE
TEE 20

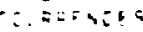

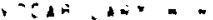

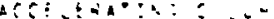

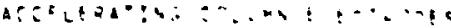

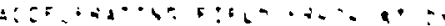

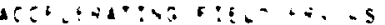

ter

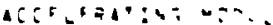

tore

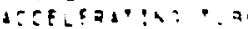

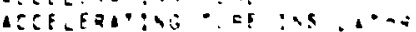

ices rasio

acticteatia bi:-mat

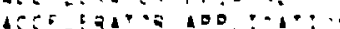

acc

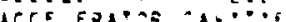

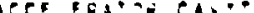

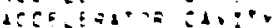

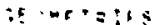

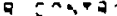

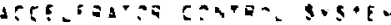

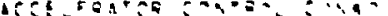

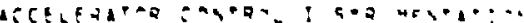

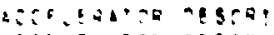

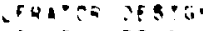

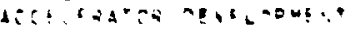

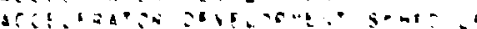

ACद.

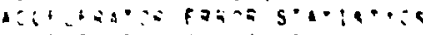

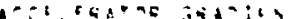

Aerich

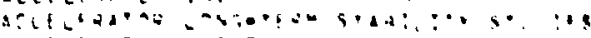

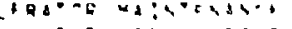

1.?.

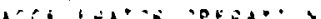

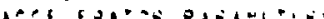

वृC

वृद

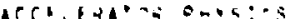

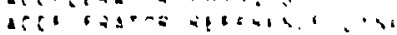

arce

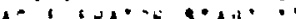

ac

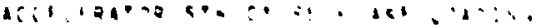

atritinias

$1,10, \cdots 0$

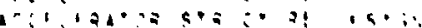

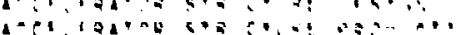

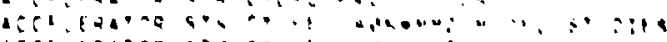

$1,1,1+\cdots=$ 
NUMGER OF
OCCURRENCES

1
1
1
1
1
2
2
1
1
2
1
1
1
1
1
1
1
1
1
1
1
1
1
1
1
1
5
1
1
1
1
1
1
1
1
1
1
VOCABULARY war

ACCFLERATHR SYSTEN ST D:ES

ACCELERATOR TANK

ACCELERATOR TANK CO.J. T.

ACCELERATOQ TANKS

ACCELERATOR TECANAL OO

ACCELERATOR TUVIAG

ICCFPTANEE

ACCEPTANCE TESTS

ACCFOTANCE THEORFUS

ACCFSS SUAVEILLANCE

ACCUMULATOR

accuracr

aCCURACY IN OOSimetar

ACHROMATIC INFLECTOR

ACMS

ACRYLIC JACKET

ACRYLLIT JACKE?

ACS

ACTINIOF ELFMENTS

actividoe NUCLE

ACTION PROGRAM

aCPiVATION ANALYSTS

ACTIVATION ANALYSISIRESTDLAL ACTIVITV

activation or pions

activation CONTEOL SVGTF

activation hazaRos

aCTivarion METHOD

acriverson

ACTIVE HANDLING SYSTE"

ACTLAiOR

AC PUATOR ORIVER MOOULE

ADE VOCARCINAMA

SOXIMLTInN

ADMTTTANCE

AOMPTTANCE RUCKET

ADMITTANCE IN GDORENSITNAL OHASF SPLCF

105

LOS SYSTEM
LEC INSTRUMENTS

$A E C L$

AECL PHETATRON COBALT MICWINE

AEROBIE CONDITIONS

AGAsTaf TIME OELAY RElaV

AGCHAN PROGRAM

agRICULTURAL ANALYSIS

IfP

Ais activity

Are netivity nonitons

ATR EXHAUST SYBTE

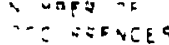

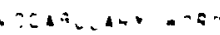

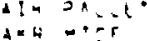

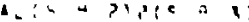

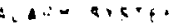

$4-A-45$

$\sin 20$

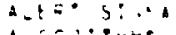

$\because \because+2, y$

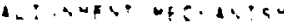

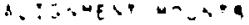

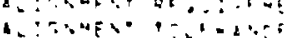

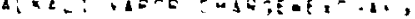

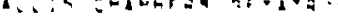

C..

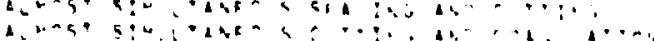

$A, 0-1 \geq \therefore=0$

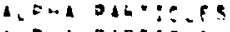

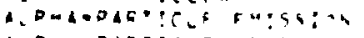

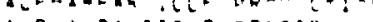

A.

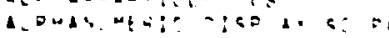

16

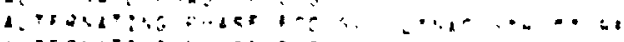

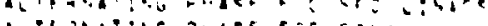

$A+\infty ! y$

aisit,

A. Wat? a

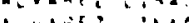

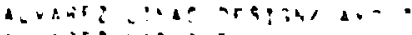

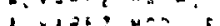

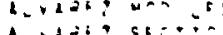

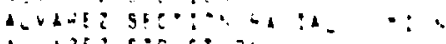

Andatios

AWE It: it:

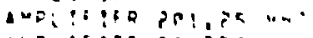

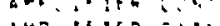

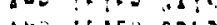

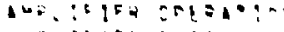

a

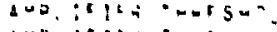

ano riva 
NUMAER OF
OCCURRE VCES
VOCABULAAY WORO

AMPLITRON

ciacuition

AMPLITRON PROGRAM

AMPLITSOON

AMPLITUOE AOJUSTMENT

AMPL ITUOE CONTROL

IMPLI ITUDE CONTROLLER

AMPLITUOE FUNCTION

AMPLITUDE MODULATIOS gPOJE PURE

AMPLITUDE MOOULAT?OS

AMPLITUDE SCAN

AMPL ITUDE GETPOINT

AMPL I PUDE -PMASE CONTROLLEQS

AMPLITUDE TPWASE

ANALOE

ANALOG ACOUISITION SYSTEM

ANALOG CMANNEL

ANALOG CMALNELS

ANALOG CIRCUI PY

ANALOE CLOSEO LOOD

ANALOG COMMAND

ANALOG COMMAND TNTERF ACE CIOS IITEV

ANALOG COMMANE MONULE

ANALOG COMMANA MONULE II

ANALOG COMMAND PESTIY:

ANALOG COMPUPI ING COMDNFTS

ANALOG CONTRDL

ANALOG CONYOOL VALVES

ANALOG DAPA

ANALOG DATA CHAMAEL

ANALOT DATA CHANAFLS

AMALOG OATA MIU

ANAL OG DATA SUASYGIE

ANAL OG OATA BYSTEM

ANALOG OLTA SYSTEM OH INS

AvAl OF, EXPEDIMEN?

ANALOG MODULES

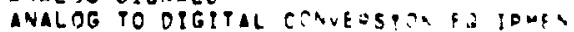

ANALOGOTOOOIGITAL C"NAEPTFQ

AYALYTE OESIGN

ANALYTIC POOLS

ANALYTICAL ACCURAE

ANALYTICAL TECWNIDUES

ANALYZER

ANALYZER DUR

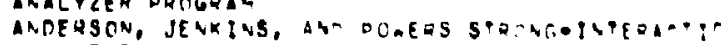
ATAMETERS

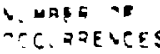

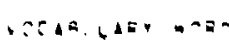

4.,

ATis onta

The.

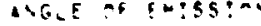

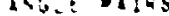

ATGLE PDAS

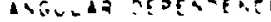

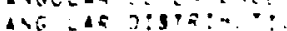

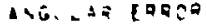

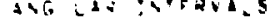

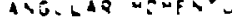

1)

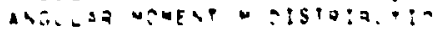

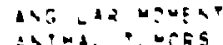

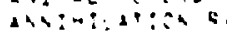

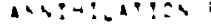

MNEF C, TRE:

AUE OLITS

DSEE Dinfo

ASTE Pith SoD.,

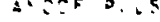

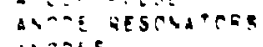

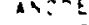

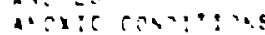

an ienevoris is

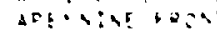

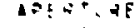

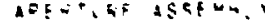

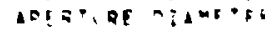

AOE $=1$ in

Aor

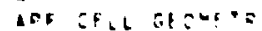

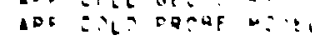

ab Liver

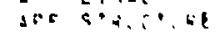

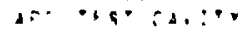

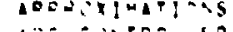

tit:

19:5

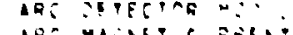

10: vacist conat

atc

10600
106

$16, \ldots$

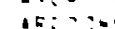

$1=2,1=2$.

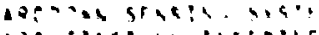

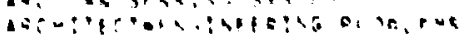


NUMBER OF OCCURRENCES
ARC ING

AREA ATE A S

ARE A $A$

AREA C

ARE MORITCRS

ARGON

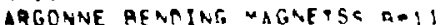

ARGONNE LINAC

ARITHMETIC UNI

ARRESTOR

ARTEQIAL TRANSPLAMT PQOCEOUDE

ARTIFICIAL FEVER THFHAPV

ASYMMETRY

ASYMMEPRY in Pis DRCO CPIMA

ASYMPTOTIS FOPMULAE

MTOM LEVEL WIOTHS

ATOMIC RFAM SOURE

ATOMIC CAPTURE

NEGATIIE MESMSB

ATOMIC CADTURE OF MFSOVS

ATOMIC CASCADE CORE

ATOMIC GEEEXCITATIO

APIMIC HYERFINE STHUCTIQR

ATOMIC TOENTITES

ATOMIC NUMBER

ATOMIC POTENTIAL

ATOMIC STRUETURE

ATOMIC TRANSITIONS
ATOMIC WETGHT (A) DEDENDECE

ATIENULTION EMARACTENISTICS

aTTENUATIONGPHASE CONREOTS

ATTENUATOR

AUGER OE-EXCITATION RATES

ALGER MOCESS

LUGER TRANSITIONS

aitocollimating TOOL IVG LaSFe

atomateo collimató

automatic CONTROL SYSTE."

autonatic Data acsurstition systeu tFste

autovatic Data actolistition

auromatic oeltát paderidoe

auromartic Light COMPENSATION CIRCUI

automatic LOGGING OF DATIENT COMTOUO

AUTOMATIC SCALING

AUTOMATIC SEF POINT

AUPOHATIC SPEERING DRACFDIIRF

AUTOMATIE BHITCHING

MUTOMATIC TUNEUP DROCEDARS

AUXILIARY OPERATINALL SYSTEUS
AUYILIARY BYSTEMS

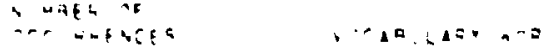

A,

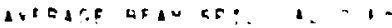

and

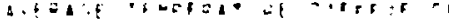

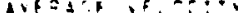

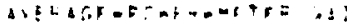

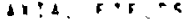

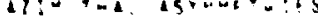

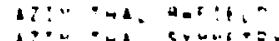

$1: a^{2}-4$,

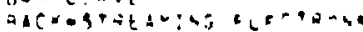

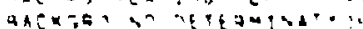

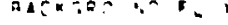

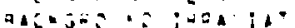

axco-as

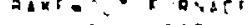

4a, sit?

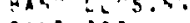

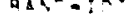

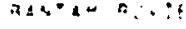

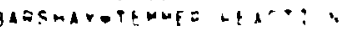

Hasl: -?

heter poitses

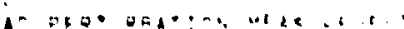

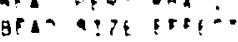

arsinas

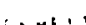

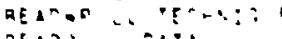

af

........

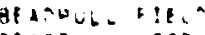

a 1 a $\cdots$.

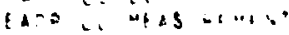

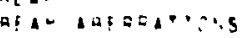

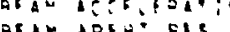

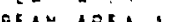

पiv $10+1$

aric acta

aी An Anticis:

911 active

uf a $a a_{n} a^{\circ}$

पर

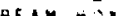

QP

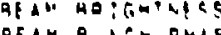

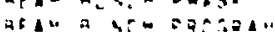


NUMBER OF
OECURAENCES

VOCABULARY NORO

BEAM BUNCHING

BEAM CAVITY INTERACTIJNG

BEAM CENTERTYG

BEAM CENTEROIO STEEO

BEAM CENTROIO SHIFT

BFAM CHANAEL

OEAM EHARAETEQISTICS

GEAM CHOPPER

DEAM CHOPPEQS

BEAM CONTROL

BEAM CURRENT

BEAM CURRENT INTERLITES

GeAM CURREAT LEVEL

GEAM CURRENT MONLO

BEAM CURRENT MONPTOR AMOLIFIED

BEAM CUFAENT QRETUETIO

BEAM CUARENT TESTS

BEAM CURRENT TAANSFI:R.

BEAM OESTGN

GEAM OEVELOPMFN

BEAM DIAGNOSTIC IASTR UEVTAYITA

GEAM DIAGNOSTICS

BEAM DISSIPLTION

ofaM nose Disirioution

DEAM DUMP

DEAM DYNAMICS

BEAM ELLIPSOIOS

DEAM EMITITANC

GEAT ENERGY 3DECTRUN

GEAM ENVELOPE

BEAM ExCITATION

BFAM Ex ERTEN

BEAM EXERIMENTAL IREA

BeAM COCUSING

BEAM Gate

oeAm malo

beam halos

BEAM MANOLIAG

BEAM MARP

BEAM HEATING

BFAN IMP INGEUE:

BEAN INHTBIT grSTE

BEAM INTEASITY

BEAN INTERRUIPTICN

BEAN JANS

BEAM LINE

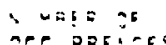

2
3
$\vdots$
$\vdots$
$\vdots$
$\vdots$
$\vdots$
$\vdots$
$\vdots$
$\vdots$
$\vdots$
$?$
$\vdots$
$\vdots$
$\vdots$
$\vdots$
$\vdots$
$\vdots$
$\vdots$
$\vdots$
$\vdots$
$\vdots$
$\vdots$
$\vdots$
$\vdots$
$\vdots$
$\vdots$
$\vdots$
$\vdots$
$\vdots$
$\vdots$
$\vdots$
$\vdots$
$\vdots$
$\vdots$
$\vdots$
$\vdots$
$\vdots$
$\vdots$
$\vdots$

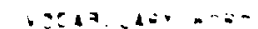

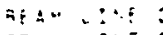

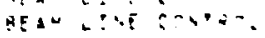

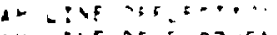

aga

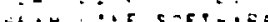

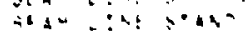

ats

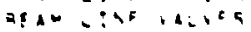

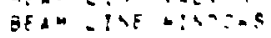

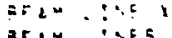

area

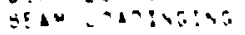

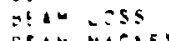

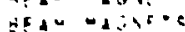

acat

afix vatio

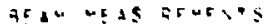

न

ar a w w in

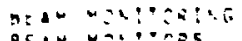

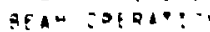

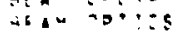

as an $-15,10$

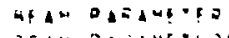

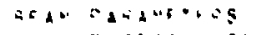

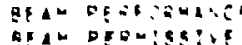

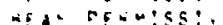

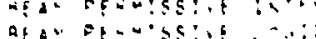

hat E! $=0$ ind : $\cdots$

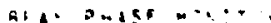

aris ain

ar a.. :

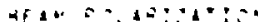

स.

ค!

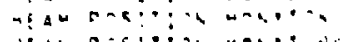

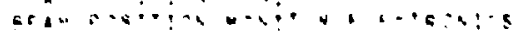

atis

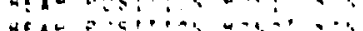

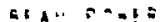

asio os is

nith nas?

$4 a$
an

an $1,2, ?, \ldots$ 


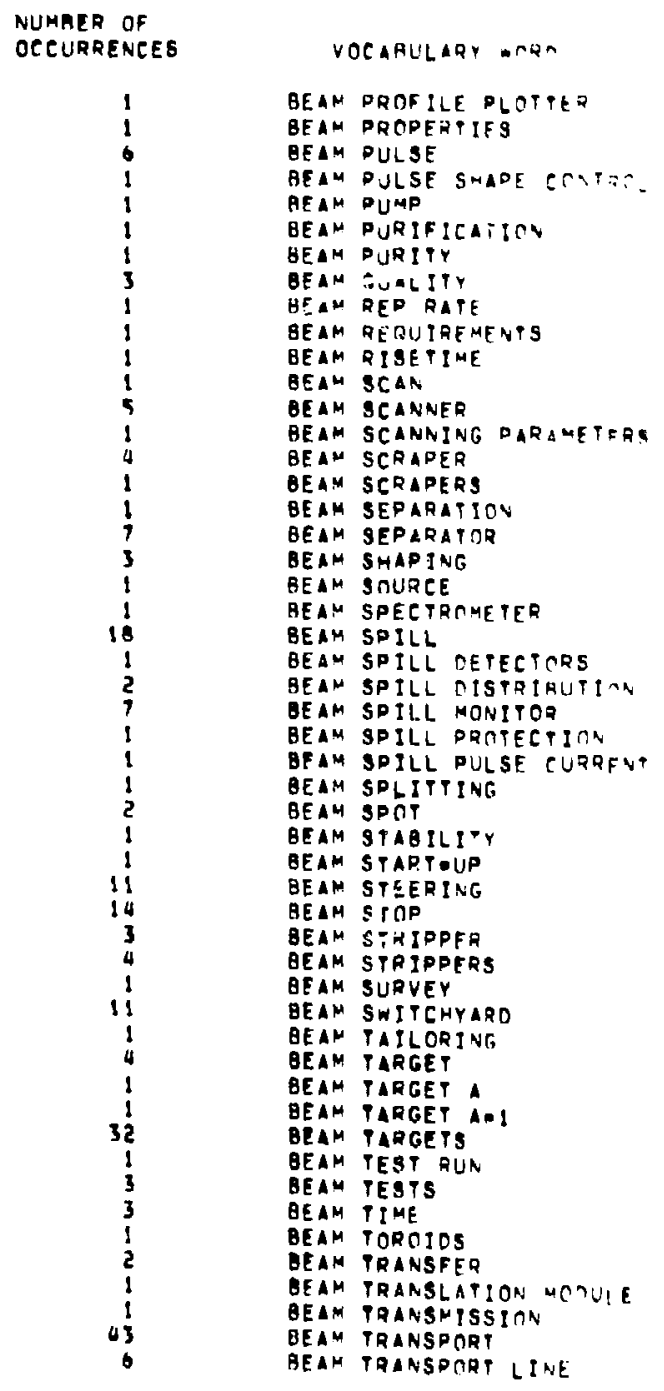

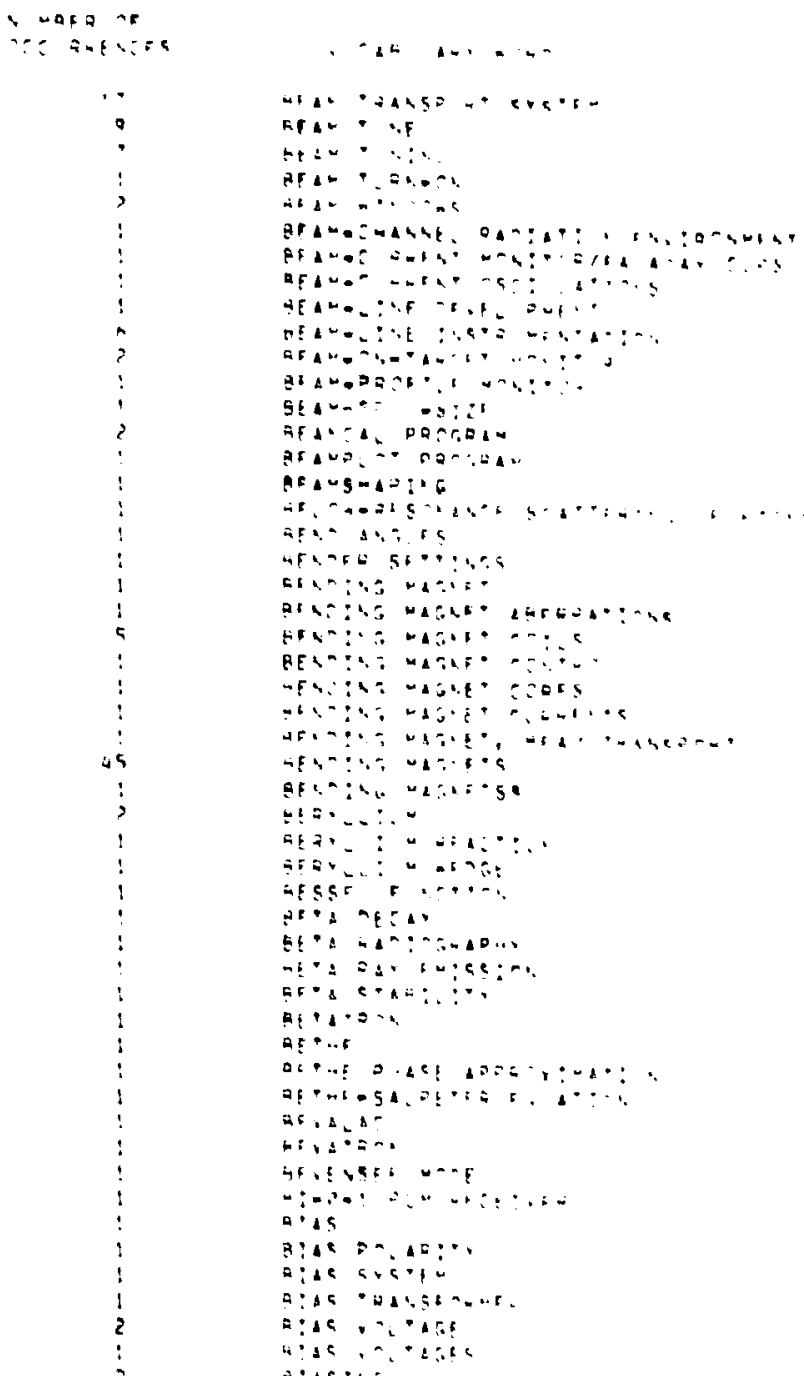




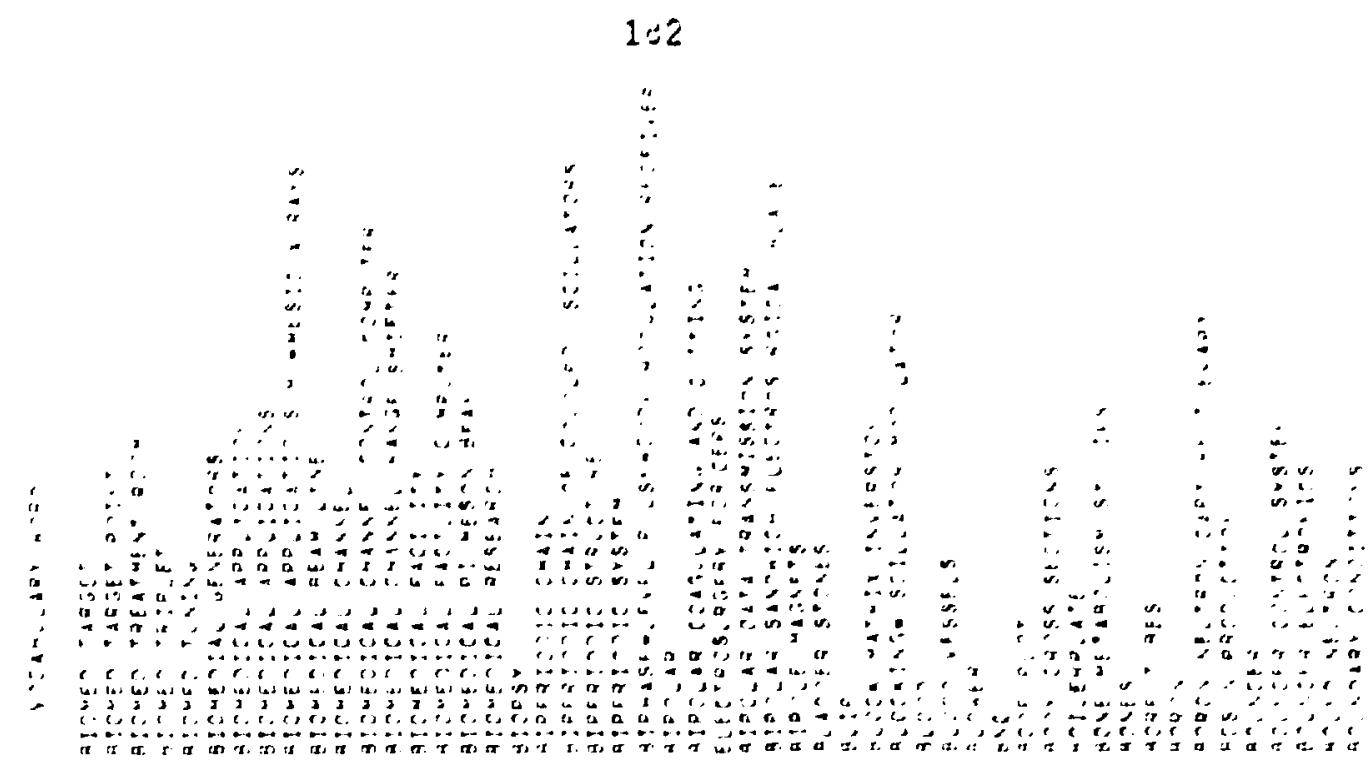

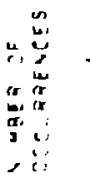
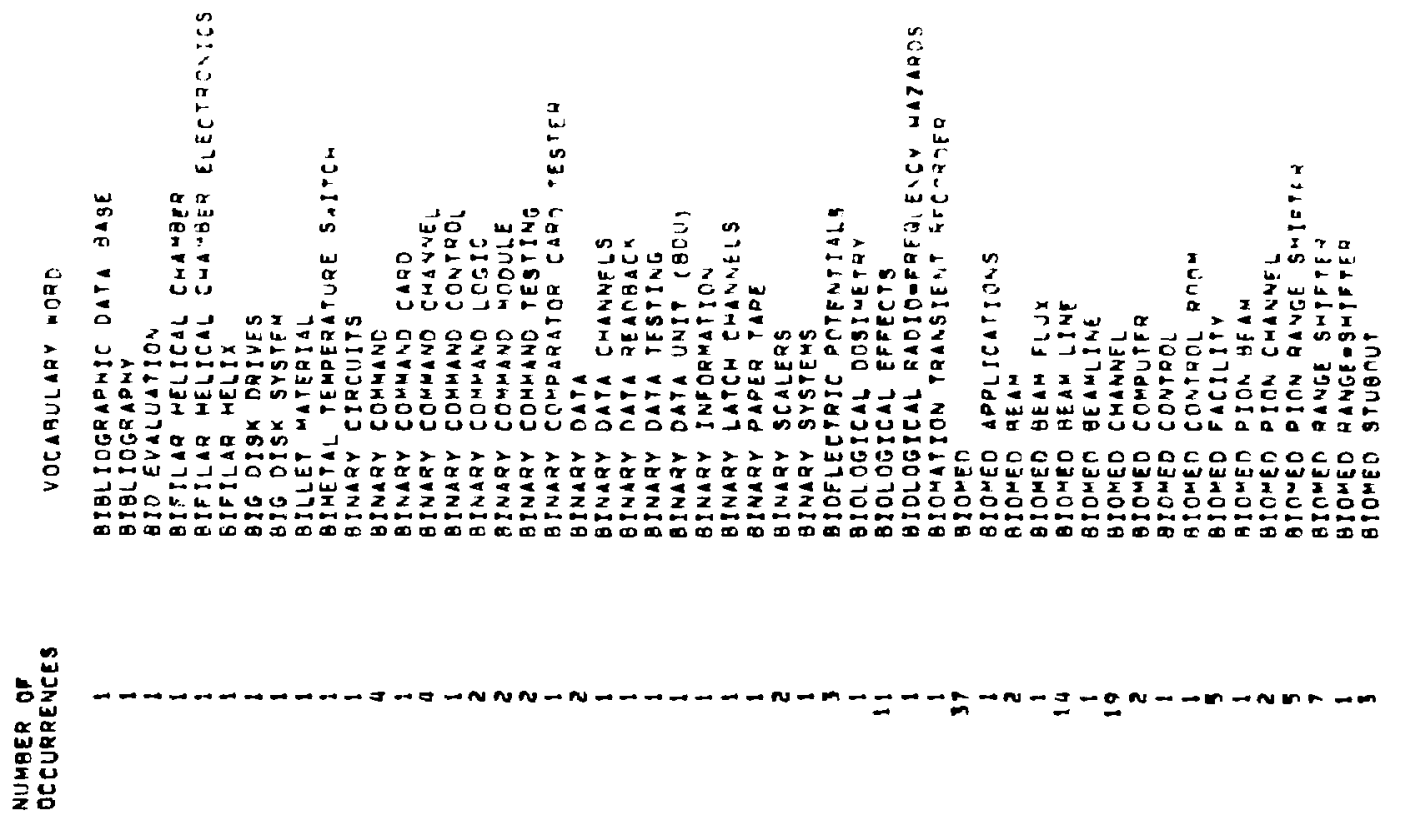
NUMBER OF

OCCURRENCES

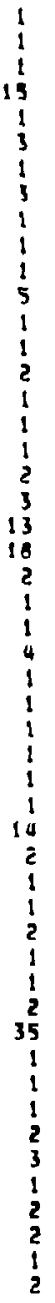

VOCABULARY ATR?

BOU.DARY DERTIRAATIRYS

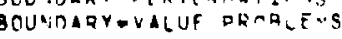

BDM

GRAGG PEAK

GRANCH ARTERIES

GRANCH CRIVEQ

BQINCHES

GRABSIERE

BRALE ALLOY

BRATE JOINTS

QRAZEO COLL

BRAZING

BRAZING FILLER METALS

BRAZING TFCHNIDUES

BREAK-UP PROCESSES

BREAST

BREAST CANCER

BREAST CARCINOMA

BREAST MALIENANE

BREAST SKIN TEMPERATUAE

BREMSSTAAMLUNG

RRINGE COUPLERS

BRINGE RECTIFTER

BRJOGE RECTIFIER CIRC. IT

BRIDGE WITH THEDMISTOZ GEISCR

BRIDGEOW STIER

BRIGWTAESS RATIOS

BROAD RANGE SPECTRONETE:

BROOKHAVEN FACILITY

BROOKHAVEN LINAC

BTC CABLES

BTC DEVICE

BUCKET

BUDGET

BUFFER

RUFFER AMPLIFIER

BUFFER AMPLIFIERS

BIIILOING MATERIALS

BUNC SHIELOING

BUNCHED

BUNCHER CAVITY

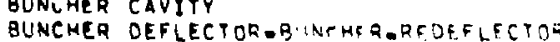

BUNCHEA OEFLECTOREA.
BUNEHER DOURLE WATS

BUNEYING

Busy

GYPASSES

CACIR LINE ORIVER

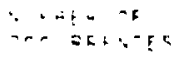

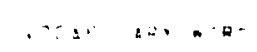

$\because \cdots$

玹!

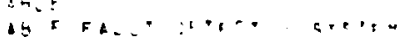

祭,

$\therefore 0,100$

A. : : a

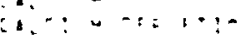

in

$169 a+1,36$

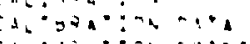

$\because \leqslant \cdots$

सL:

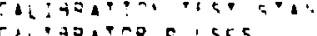

cisoming

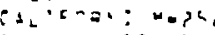

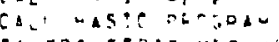

ci, $-2,0,2=2 \pi$

isus:

isus

cava:

cas: $=02+1$

cans

cant

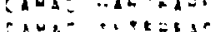

Canic ine

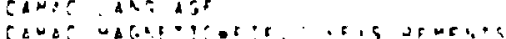

cavas and

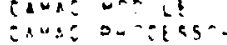

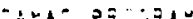

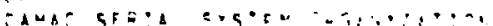

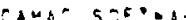

Canat saur tores

caicis

calce Har

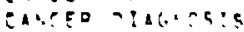

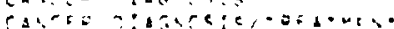

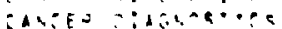

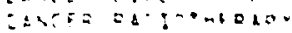

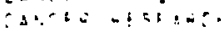

ares - ation.

idere isfacer.

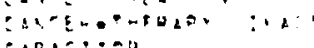


NUMBER OF

OCCURRENCES

VOCARILLARY WORD

CAPACITOR BANK

CAPACITOR ROOYS

CAPACITY

CAPE PROGRAN

CAPTIJEE CROSS SECTICA

CAPTURE EFFICIENCY

CAPTURE OF NEGLTIVE M.10:S

CAPTURE RATIOS

CARBON

CARBON CONCENTRATIO.

CARBON FILATENTS

CARBON STRIPPER FOILS

CARBON XORAY GACKCRROJ.O

CARCINOMA OF THF RREAGT

CARD ASSIGNME:

CARD FORMATS

CARD REAOER

CARTRIDGES

CASCAOE

CASTINGS

CASTS

CATEGORIES

CATEGORY NUMBERS

CATHODE

CATHODE CURRENT

CATHOOE FAILURE

CATHODE POLLOHER

carnoDe gar TuBe

CATHODE STRUCTURE

Cais

CAUCHY INTEGRALS

cavities

caviry

CAvitr amDlifieg ASSE Bi-

caviry chala

cavitr cleanitug

cavitr end closures

cavitr exeitation

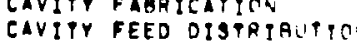

cavipi fielo gignal

Cavitr FREGUENCY

Cavitr LENGTh

CAVitr MODEs

cavitr pmase

Cavity POWER LOS

cavitr oRODIJTIION

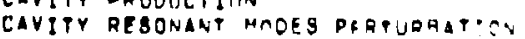

cayity suppont sioucti

CAVity TUNING

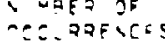

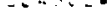

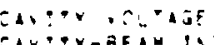

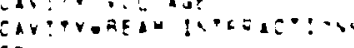

5

ace

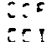

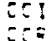

cer :avset

$c c$

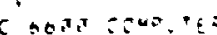

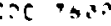

erc galves

chat

erti inger

$\cdot, \cdot$

CFL TES:a

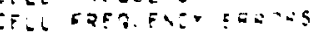

CFLL LENGTH

cros and

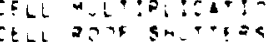

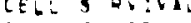

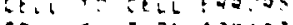

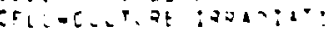

SEL 1,5

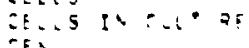

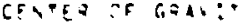

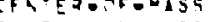

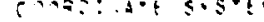

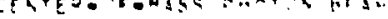

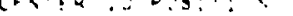

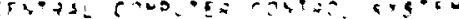

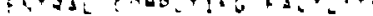

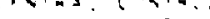

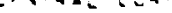

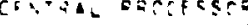

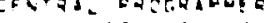

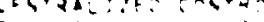

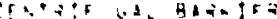

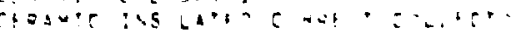

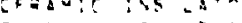

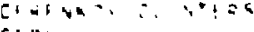

ctas

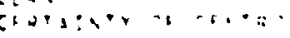

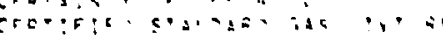

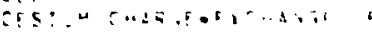


CHAIN OF COUPLEO CELLS

CHAIV OF NOI CEILLS

CHANVEL

CHANNEL CALIBRATION DaOCEMJRES

CHANNEL CALIHZATI IT

CHANNEL CALIARATIINNS

CHANNEL CHECKOUT

CHANNEL DECCOE TESTIV:

CHANNEL DESIGN

CHANNEL ENGINEEQINGG
CHANNEL INDF X PQITRAM

CHANNEL LENGTH

CHANNEL LIST

CHANNEL NUMAFR

CHANNEL OPTICS

CHANVEL SELECTION

CHANNEL SIMULATION

CHANNFL TOLERANCES

CHANNEL THPF

CHARGE DENSITY

CHARGE NISPLACEMENT
CHARGE DISTRIBUTIONN

CHARGE EXEHANGE

CHARGE EXCHANGE CANAL

CRARGE EXCHANGE REGIO.

CHARGE FXCHANGE SYSTEN

CHADGE RADII

CHARGE SYMMETRY

CHARGE WEXCHANGE

CHARGE-EXCHANGE CROSS.SFCTINAS

CHARGE-EXCHANGE REACTIO.S

CHARGE-TRANSFFR

CHARGED PARTICLE

CHARGED PARTICLLF DETECTITRS

CHARGED PARTICLE EMISIIAN

CHARGED PARTICLE FNERCY

CHARGED PARTICLE RADINGRADHY

CHARGED PARTICLF RADIMGRADHIC SYSTEUS

CHARGED PARTICLE SPECTRAMETER

CHARGED PARTICLE YIELIIS

CHARGEDAPARTICLE REAM TRAMSPOHT

CHARGING INDUCTANCE.

CHASSIS

CHASSIS MOUNTING

CHASSIS NO, I

CHECKDUT PANEL

CHEMICAL BONDINO

CHEMICAL EFFECTS

CHEMICAL ENVIGONMENT
CHEMICAL SEPARATIINN

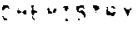

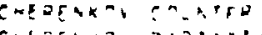

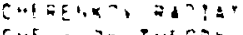

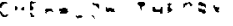

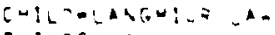

OhIs

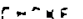

$\operatorname{cin} \cos 20$

zancos ans

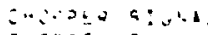

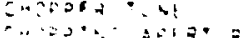

CWE:COS DA-

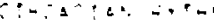

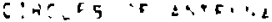

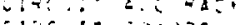

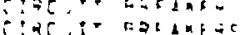

$\cos 30$

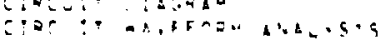

300

$\underset{\mathrm{E}}{\mathrm{E}}$

ciar cion on

ciocinotion

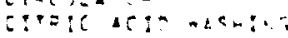

chezice inas

$c: i=t$

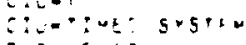

condic is on

eivice

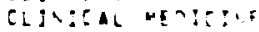

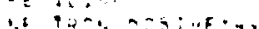

CbIVICAL Ta!A.S

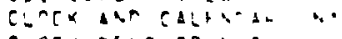

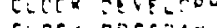

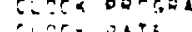

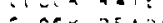

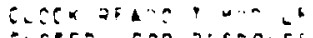

Casenocion $a$ an

Clcsen-ces

chasen-i ins Buck

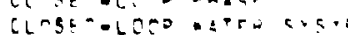

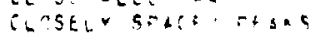

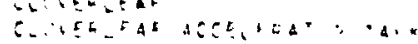


NUMAER OF
DCCURRENCES

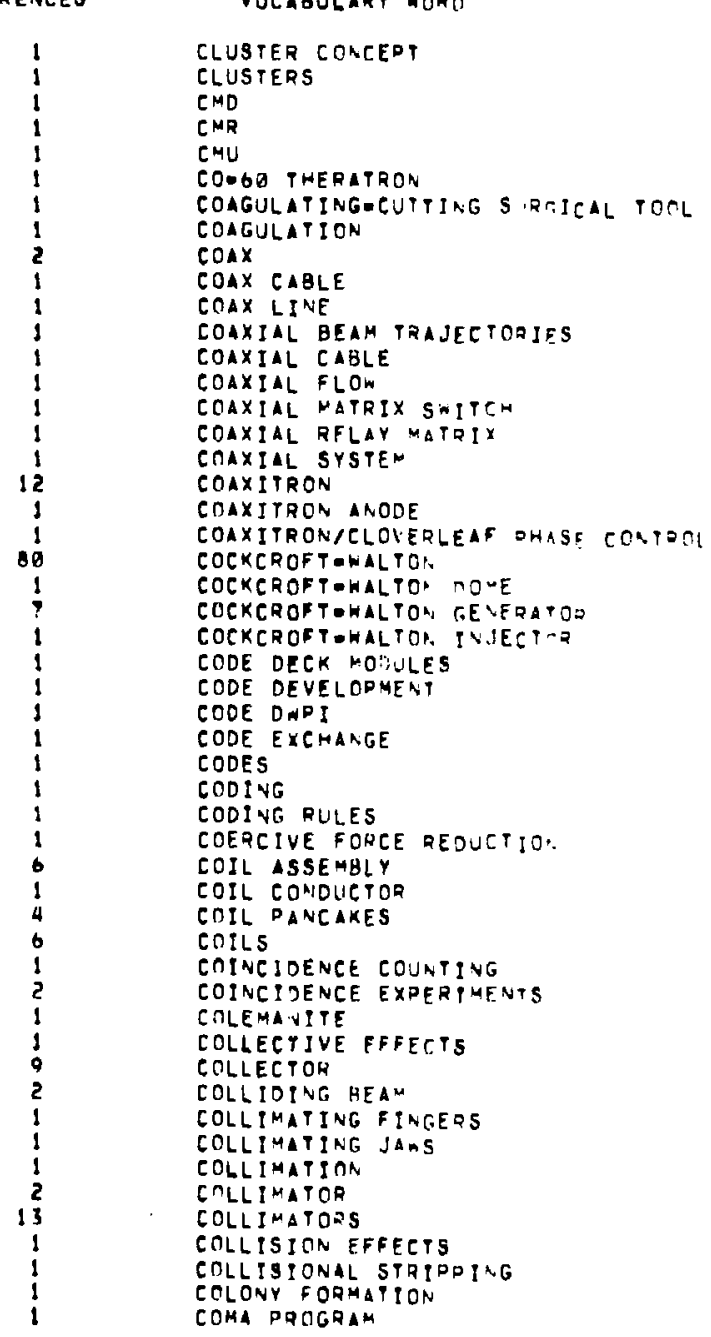

V.T.25 T5 CCC $27 E V Z S 5$

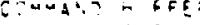

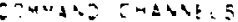

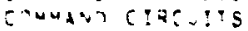

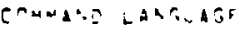

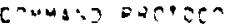

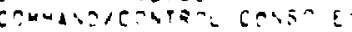

cruvisos

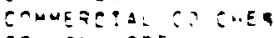

conuge ang

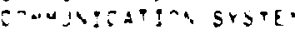

cind vications

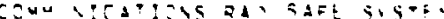

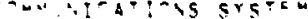

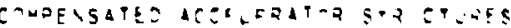

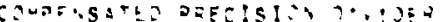

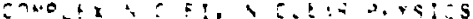

anp ㄴ.

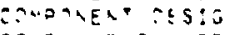

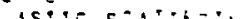

govom

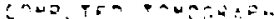

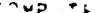

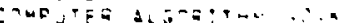

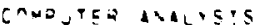

CSUE tha

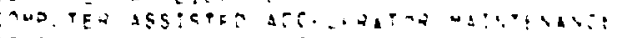

Gup tga jac at

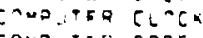

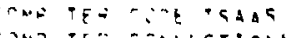

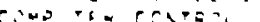

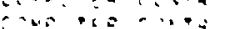

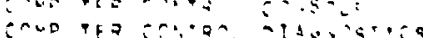

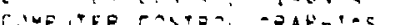

chus

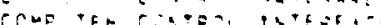

cravo

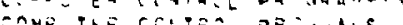

Chu

cono iev cratsat gartan.

cono iso

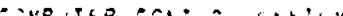

, , , , ,

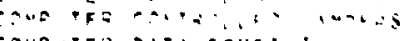

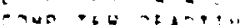

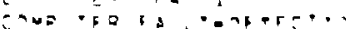




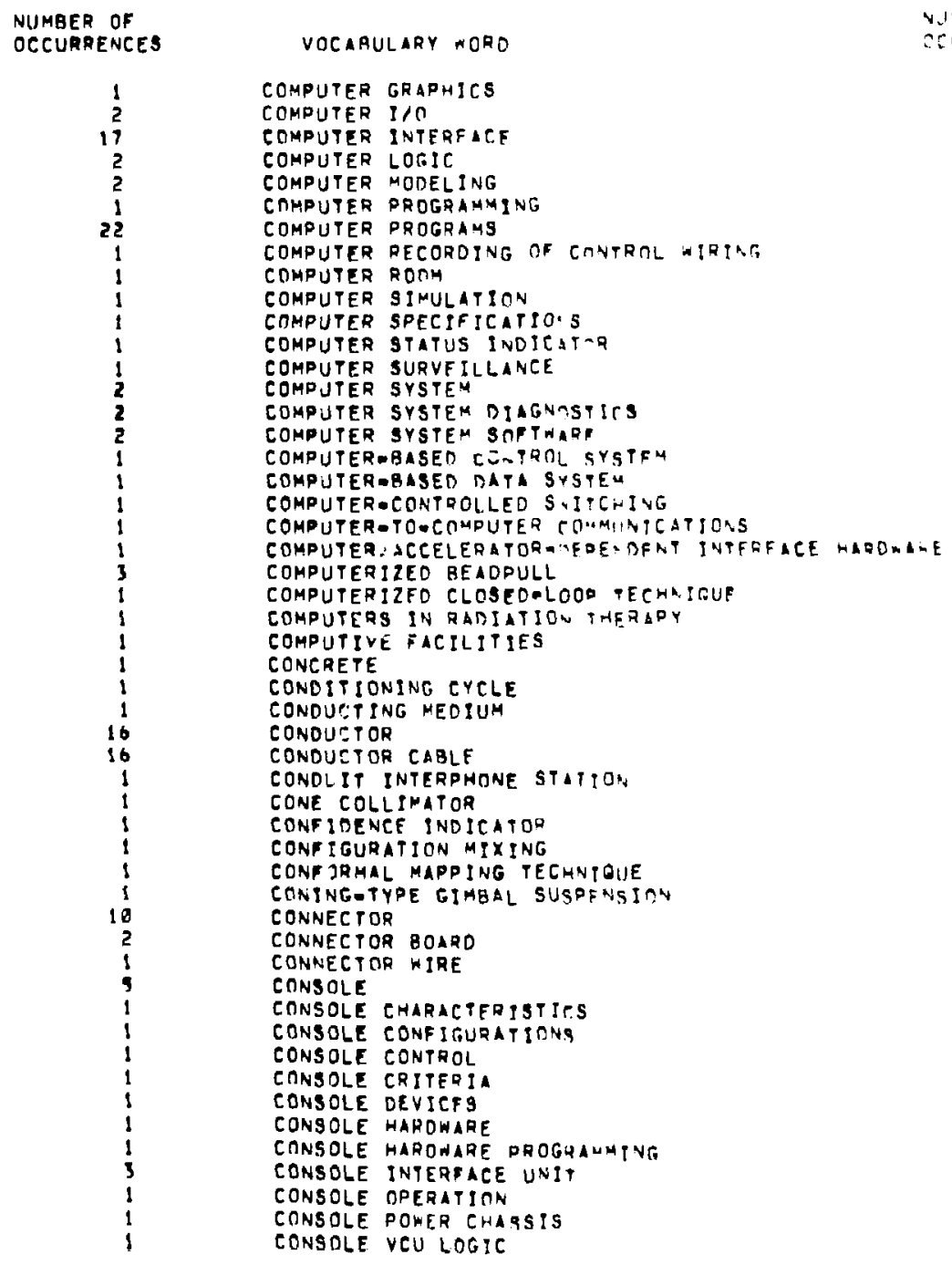

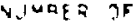

CC:ATENCFs

GCAHULA2t AMan

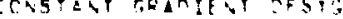

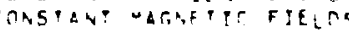

EAsti Ct?

crataction

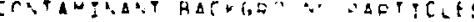

congugeste

contaugaten ataro syatio

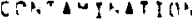

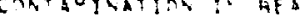

con? 2 ? inguart?

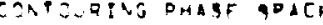

chistas

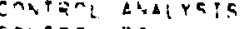

CNiscl Bax

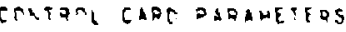

crotanis carns

CMTrCL CHADACTES

crivath chassis

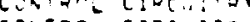

cervari cioc. its

certzol col on

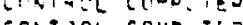

625 25

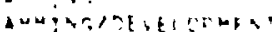

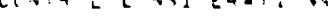

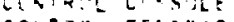

ccrogatorach $L$ : D

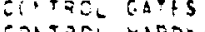

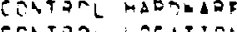

critasl lacatis

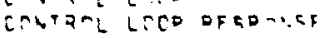

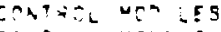

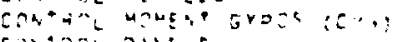

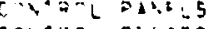

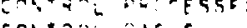

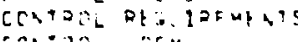

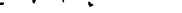

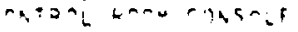

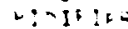

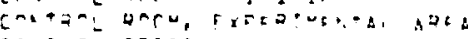

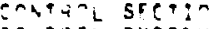

carpac 5 retso

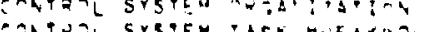

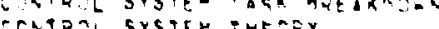

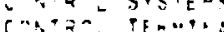

$m_{x}=-i$ in 
NUMBER OF OCCURRENCES
VOCARULARY WORE

CONTROL VECTOR

CONTRDL VOLTAGES

CONTROLLABLE DELAY LI HES

CONTROLLEDARECTIFIER PI?CIIIT

CONPROLLEOI-THERMONUCLEAF-FUSIOA EXPFAIMEVT

CONTROLLERS

CONTROLS

CONVENTIONAL TIMEAOISTAA.CF

CONVEATENT BEAM

CONVERSIOHS

CONVOLUTION THEOREH

COOLANT FLOW AATE

COOLING CHAMBER

COOLII'G CIRCUITS

COOLING JACKET

COOLING SYSTEM

COOLING SYSTEM OISPLAY

COOL ING TUBE SOLDERING

COOLING PUPES
COOLING WATER INTEPI.OCK

COOLING WATER SUPOLY

COOLING WATER SYBTEY

COOL ING WATER SYSTEMS

COORDINATE SYSTEM

COOROINATE SYSTEM ROTAT:ON

COOROINATE SYSTE ROTATION

COORnina

COPPEA

COPPER BEAM OUMP

COPPER CLAO STEEL PLATES

COPPER TEMPERATURE

CORE ASSEMOLY

CORE CONTROL

CORE FOAGINGS

CORE MEMORY

CORONA EFFECT

CORONARYTTRANSPI ANT DOOEERURES

CCRRECPEO $I$ LAW

CORBELATION

COSMIC WRAY TESTS

COST ESTIMATES

cosis

COUEH PROCUREMENT

COUL DMB AMPL ITUDE

COULOMG OEPENDEMT TFaus

COULOME DISTORTION

COULOMB EXEITATION

COULOMB INTFACTION

COULDMB NUCLEAR INTEREERECCE

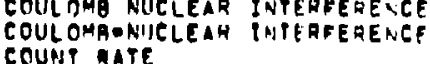

NIMRFO TF

CEC: FEE YCES

1
1
3
1
1
2
2
1
5
5
1
1
2
1
1
2
1
1
1
1
1
1
1
2
1
2
1
1
1
1
1
1
2
1
3
1
1
1
1
1
1
1
1
1
1
1

SCAPLiar a:A.

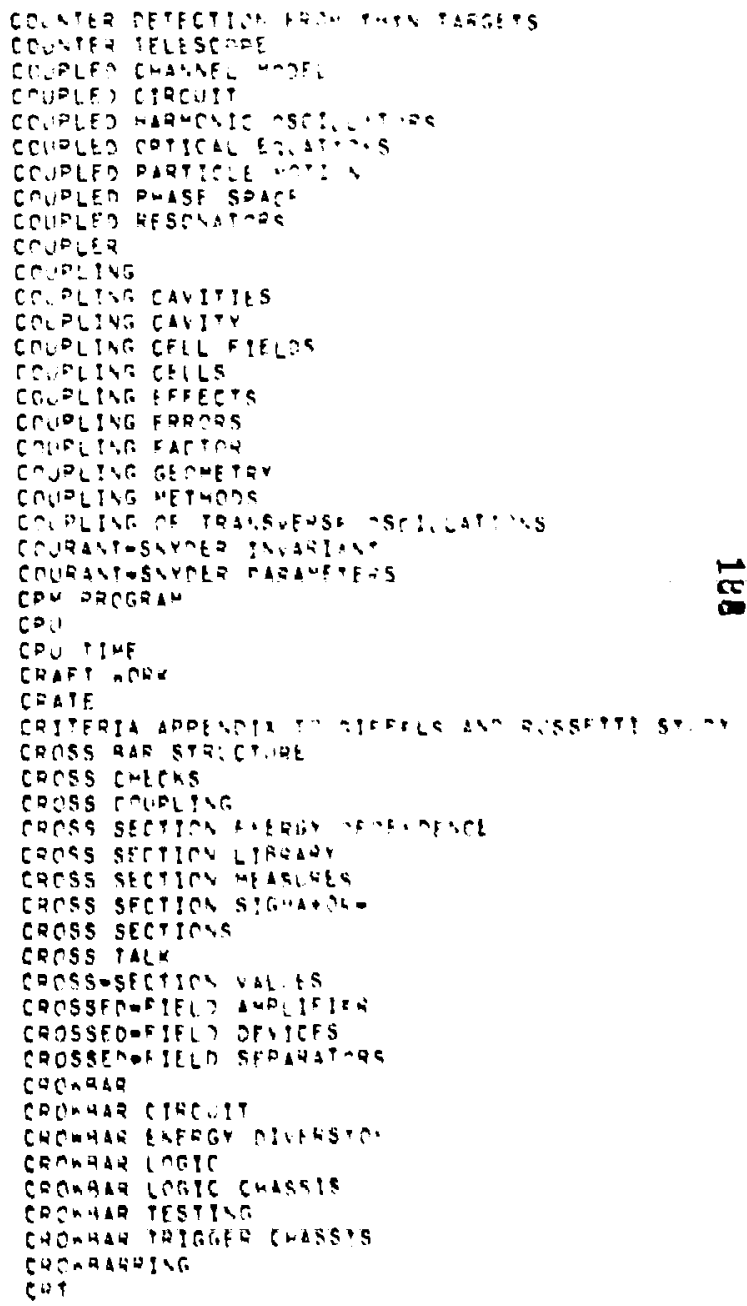


NUMAER OF OCCURRENCES

VOCABULARY WORO

CRT DISPLAY

CRT TERMINAL

CRYOGENIC DEWAR SYSTE

CRYOGENIC LINAC

CRYOGENIC MUON CHAN'EL

CAYOGENIC PUMP ING

CPYOGENIC SYSTEM

CRYOGENIC TARGETS

CRYOGENICS

CRYOMAGNETISS

CRYOMAGNETS

CRYOSTAT

CRYSTAL OSCILLATOO

CTR

CUBIE SPLINES

CULT TUREO HUMAN CELLS

CURRENT

CURAENT OENSITY

CURRENT DISCRININATIR CIRCUIT

CURRENT DIVIOER

CURRENT FORH OF WEAK INTERACTION THE ORY

CURRENT IMPEDANC

CURFENT LIMITATION

CURRENT LIMITING DEVITES

CURRENT LOOP

CURRENT MEASUAING SYSTEY

CURRENT MONITOR

CURRENT MONITORS

CURRENT OUTPUT

CURRENT POSITION

CURRENT REGULATSR

CURRENT BERVOS

CURRENT SHUNT

CURRENT SOURCE

CURSOR IISAGE

CURVE FITTING

CUT OFF VALVE

CVC VACUUM

CYCLE COUNTING CLOCK

CYCLE :

CrClE R

CYCLOTRON

CYCLOTRON SOURCE

CYLINDEAS

CYLINORICAL STOPPING DEGINN

CYLINORICAL TARGE

DELENG

O/A CONVERTER

o/T CHABSIS

DACS
NUAED JF

SCC REEYCE

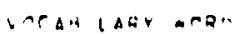

oulier alpon:

Dassr+11

DANAGIVA ARES

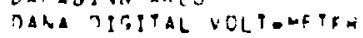

DLPLTVGTOC CIRCLIT

oita

Data acougision

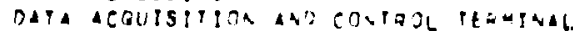

sut aceussige cr.

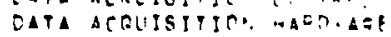

Cata aealisgion a

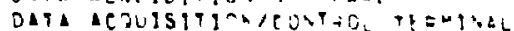

OATA anabrsis

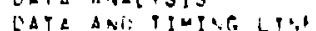

CATA HASE

DATA Tit

DATA QREAK Ta IOUES

OATA MISHE

OATA RIFFETH.

TATA CHEMEL

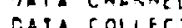

cata chitfct

Jata îsplat

Jatz oisplar iatLax

bata ficrega

DATA FILE EXCBA.GE

DATA FILE TRALWISSIO

Data olinat

DATA MACMLISE FLFTTMO.ITS

CATA MADELANG SYSTE

Cata lisk

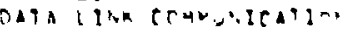

Data logeino

chita bencessing

Cata juF TYG DaORLE

Data SeAa

DATA SINKS

DATA STIEAGE

OATA SYSTE

OATA TARE

DATA TRANSEEA RATH

DATA TRANSNISSION

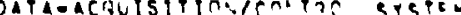

Data-gas

DATAELINK MCOULF

DATAELINK ITRUTHA

DATA-LCGGER

MATANAY

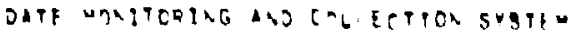

OAY C.OCK

OC AMBLIFIFH NOIFT 


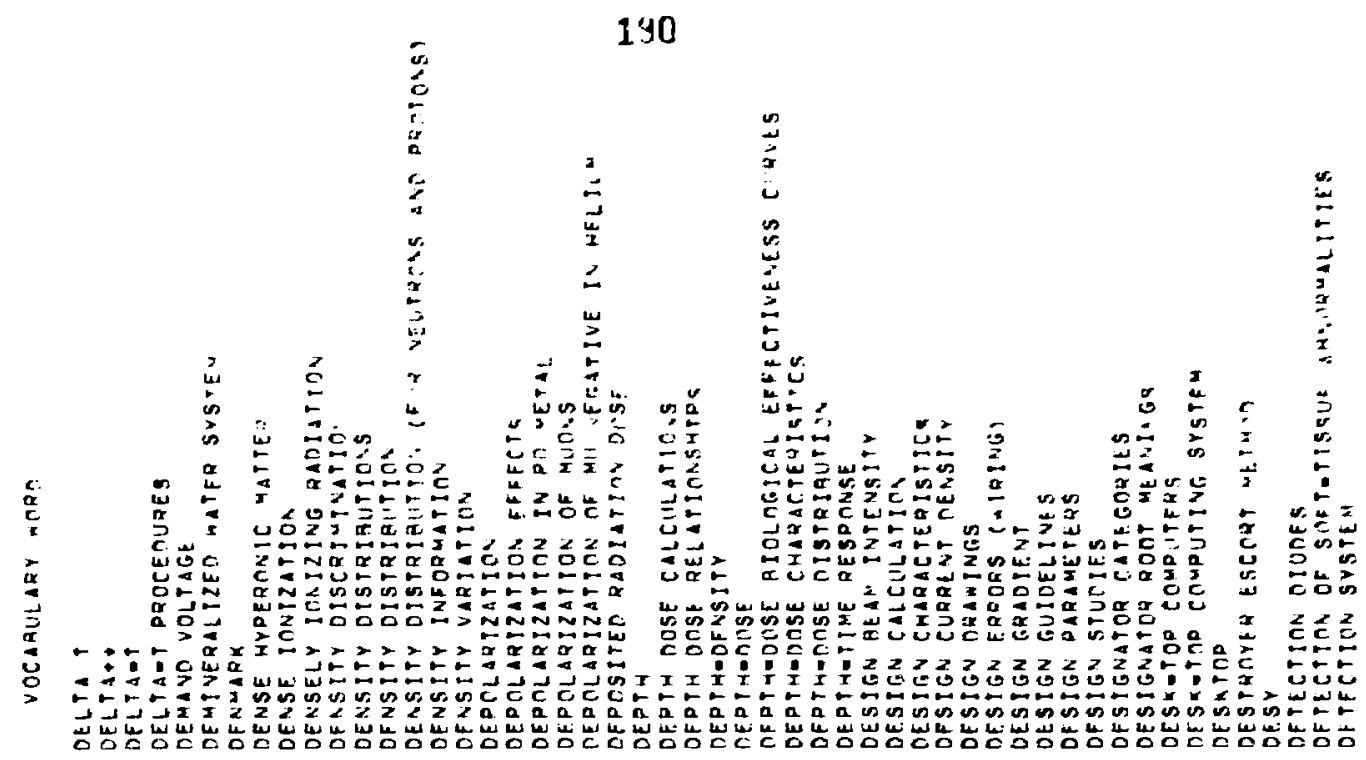

空

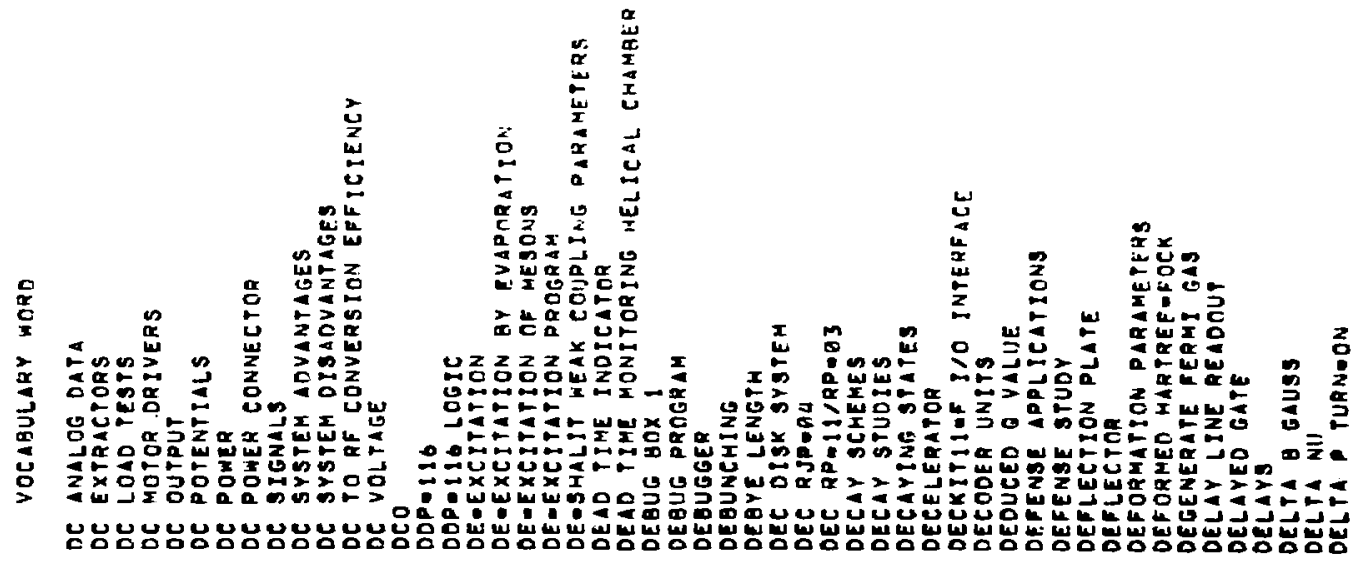


NUMBEA OF OCCURRENCES

VOEAAULARY WORA

DETECTOR

DETECTORS

DETUNING

DEUTERIUM

DEUTERON

DEUTERON DESTATE

DEUTERON MISSING MASS SOERTTOMETER

DEUTERON MOMENTUM

DEUTERON MOMENTIUM OISTRTBUITIONS

DEUTERON PROOUCTION

DEUTERON SPECTRA

DEUTERON WAVE FIINETIONS

DEUTER ON WA

DEUTERONS TRTIONS

DEVICF CONES

DEVICF CONES

DEVICE DECLARATION
DEVICE DESIGNATION

DEVICE DESIGN

OEVICE SIMULATION BOX

DEVICE SYMBOLS

DEVICE-SELECTION

DHF POTENTIAL

OTONEUTRON WAVE FUNCTIO

DIAGNOSTIC EQUIPMENT DEVELODMENT

OIAGNOSTIC EOUIPMENT

DIAGNOSTIC EQUIPMENT

DIAGNOST IC INSTRUMENT

DIAGNOSTIC PROCEDIJAFS

DIAGNDSTIC DROCEDIJRF

DIACNASTIC SOFTWAE

DIAGNDSTIC TOQL

DIAGNASTIC

DIAL SYSTEM

OIELECPRICIINSULATIDN TEST UNIT

DIFFERENCE IN TEMPERATUUE

DIFFERENCE MESHES

DIFFERENT IAL AMRL IFIER

DIFFERENTIAL GREAST TPMDEAATURE RECBRDING

DIFFERENTIAL CROSS SECTTONS

OIFFEAENTIAL CROSS SECT TON

OIFFERTIAL CROSS SECTION DEFLCTION

OIFFERT

DIFFERENTIAL PRESSURE TAANSTUCE

DIFFERENTIAL SCE

OIFFERENTRATOR

DEFFUSION

Digital computer

DIEITAL COMPUTER CONTRDI.

DIETTAL CRT

DIGITAL DATA SERVO SYGTEM

DigiTal DATA TRANGMIOSION
NUARER AF

OCCURPENCFS

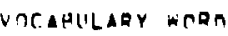

DIGITAL DAIVE.

DIGTIAL HLECTEONITS

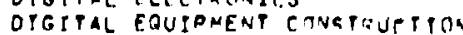

DIGITAL FILTEG NESIGN

DIGITAL FILPERS

DIGITAL GAUSSMFTFW

OIGITAL PROCESSING

DIGITAL DEADTIT

DIGITAL SERVO

DEgTtAL SYMTHESTZFE

DIGITAL SYSTEM

DIGITTZED IHECRMATIDA

OIMESSITNAL SEASITIVITY

OIMENSIONS

Dincris alvo

Dincinia

DIOne

DIOCE nETECTING CDECUTtS

DIORE DETECTOR

DIUDE RFTECTORS

DIODE PROTECTIVF DEVIRES

DIONE SCUACE

DIODES

DIPOLE ANTEYNA

DIPRLE ELECTAODES

OIONLE FIELO

DIPOLE INIERACTION

DIPOLE MAGNETS

OTDOLE STEEDING COILS

DIPCLES

DIAAC-HBATAEEFFCK URAL

DIRACEMARTREE -FOCK DDPF. TTAIS

DIRECT

OIRECT COUPL:A.G

DIRECT EXTRACTION

DIRECT LEPTON PRONUCIIO.

DYRECT LEDTANS

DiRect DOSTTRANE

DIRFETIONAL COIFLER

DIRECTIVITY

DISC ANO CAT DISPIAY

DisC COUNTE TEFHNIRUES

DISC COUNTERS

DISC COUNTER
DISC MRIVER

DIST RRIVER

DISC 3YSTEM

DISK ANO WASHFR GPRAC

DISK IVD WASHFL STRIICPULE

DISK CAULCITY

OISK MEMORY

DISK STOH AGE CEVICE 
OISPERSION/MAGNIFICATION OISPLAY

OISPLAY CONTROL CHASSTS

DISPLAY gYSTEMS

OISPLAY SYSTEM

OISPLAY UNIT

DIBSOCIATOR

DISTORTEO EVENAEVEN NUCLEI

OISTORTEO RECTANGULAR GRID

DISTRIBUTEO OPEAATIONAL SYSTEMS

DISTRIBUTEO OPERATIONAL SYSTEM

DISTRIBUTED SYSTEMS

DISTRTBUTION AMPLIFIERS

DistrinuTION FUNCTIONS

Ortarg

OISTRIBUTOON OF PRAJECTORIES

ONA DF MALISNANT CELLS

DNA OF MALI

DO FACTOR

DO VALUE

DOCUMENTATION

DOG

DOG BLOOD

DOGE

DOLDRUMS PAPER

DOME IMPLOSION

DOME LIGHTS

DOHE RADIATION

DDHE VOLTAGE

DOME VOLTAGE SIGNAL SYSTEM

DOMES

Dones

DOOR INTERLOCKS

DOSAGE

DDSAGE DSSTRIBUTION

DOSE

DOSE CALCULATION

DOSE Cal Culations

DOSE DisTRIBUTION

DOSE EOUTVALENT

DOSE RATES

DOSI ME TERS

dosimetric calculations

DOS IMETRY

DOSTMETRY MEASUERERENTS

DOSTMETRY TECHNOLOGY

DOUALF SLIT

ONUBLET ARM SPECTROMETE"

COUALE-CHARGE EXEHASG

ONLBLEODFIFT BUUNCHER

DOUALFT

DOURLET DEWAR

ROUBLET MAGAFTS

DOURLY COHPLED PESOBATO.

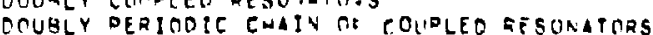
OCURLY DERICOIC CHAIN

ORAWING RULES

ORAWING RULES
ORATIVG SYWAOLS

ORIF

DRIFT LENGTH

ORIFT PROBLEMS

DRIFT SDACE

DAIFT TIJAE

ORIFT TUHF OIMENSTOSS

DRIFT TUAE LINAC

DRIFT TUBE LINAC URITTOR LOP/TANER CONTECL

DR:FT TUBES

DRIFT TUAES OUADAIIPRLF

DRIFT-TUAF

ORIFT-TUBE ACCELERATO

DRIFT-TIARE ACCFIERATIIG STRICPIIDE

ORIFT-TIIRE ANO STFM ISSEMRLY

ORIF POTIBF COOLINA SYSTEM

ORIFT-THEE INSTALLATION TUNTNG SLIIG

DRIFP-TIRE LINAC

DQIFT=TUBE PLATE ASSEMAIY

DRIFT-TUBE PLATE PF CONTATT

DQIFT-TILPE RESONANEE CO. TOOL

DRIF T-TUBE STFEQING MSG.ET

DQIKT-TUAE STFMS

DRIFYTTISE STRUETIIR

BRIFT-TIJAE TANES

DRIVE

ORIVE APEATIRE

DRIVE CONTROL

DRIVE LINE

DQIVE LIAE AYOLIFIED GYETE HE

DRIVE LINE UEASUHEMENTS

ORIVE LINE FHASE

DRIVE LOOD

DQIVE MECWANISM

DRIVE MAT RAS

DRIVE PONER

DRIVE, PALFO AMPLIFIER

DRIVE SHAFY

ORIVE-LINF ANDLIF 
EFFECTIVE OOSE CISTUIAUTIOA EFFFCTIVE OPTICAL POITETIAL EFFECTIVE RANGE THENRY EFFECTIVE SEATTFRING HOTHS EFFICIE:CY

EIGENFUNCTIONS

EISEVAERG AND KFSSLFR CODE

ELASTIC $(01+)$-NUCIEl'S SCATTERTNG

ELASTIE CWARGE-EXCKAMGE REAETIOAS

ELASTIC DIEFEREATIAL COASE SECTIOUS

ELASTIC PION CHARGE-EXCHAMGF QEACTIIONS

ELASTIC PIOAS

ELASTIC DRODUETTON

ELASTIC SCATPFRTVG

FLASTIC SCATTERING FXPE'IIMENT

ELASTIC SCATTEATVG OF LOA E. EHGY PIONS ELASTIC SCATTEATVG OF LAA EDEHGY PIONS
ELASTIC SCATTFRING, PALBRTLED NICLEONS ELECTOIC REHAVIOK

ELECTRIC TEFLECTOR

ELFCTRIC DIPOLE YOMFN

ELECTRIC FIELT

ELECTRIC FIELO AREAKDOA'S

ELECPGIC FIELO DISTRIAUIIION

ELECTRIC FIFLT FRROLS

ELECTHIC FIELD INTEVSITY

EIECTRIC FIFLD RESCLUTIEN

ELFCIRIC REMOTEDATF COETHOL

ELECTRIC SUPPLY

ELECTRICEFIFL D GAMOIEAT

ELECTHICAL CONTROIS

FLECTRICAL FIRE

ELECTRICAL INSTALLATIINAE

ELECTRICAL SHOCM

ELECTAO-METHAA ICAL "AvIOUIATUR

ELECTHOAMCHANICAL POIEDANCE

EIECTROCHEMICAL CELL

FLECTRODE CONFIGURATITAS

ELECTRODE DICKLING

ELETTOOOE SHAOE

ELECTLOONS

ELECTROMAGNET

FLECTQOMACNETTC INTEQACTIINNS

ELECTDJMAGNETIC ISOTONE SEPAPATION

ELECTROMAGNFTIC MAMENTS

ELECTRTMUECHANICAL MANTO LATOR

ELECTROMETED AMPLTFIEA SYGIFM

ELECTROMETERS

ELECIRON

ELECTRON BEAM

ELECTRON EXPERTMEDIS 
ELECTRON GUN

ELECTRON GUN FII LMENT

ELECTRON INJECTION

ELECTRON LINAE

ELECTRON MICROSCOPY

ELECTRON MICROSC

ELECTRON MOOEL

ELECTRON MOOEL CONSOLE
ELECTRON MODEL EXOERINENT

ELECTRON MOTION

ELECTRON NEUTRINO

ELECTRON OPTICS

ELECTRON PROOUCTION

ELECTRON PROTOTYPE ACCE! ERATOR

ELECTRON SCATTERING

ELECTRON SHIELOING

ELECTRON SOURCE

ELECTRON SPECTRIIM

ELECTRON SPIN

ELECTRON SUPPRESSOR

ELECTRON TRAP

ELECTRON TRAP EFFECTIVENESS

ELECTRON TUBE

ELCTRON TUBE

NTFACTIONS

ELECTRONIE CONTROL

LECTRONT OEvices

ELECTRONIC DRIVE

ELECrRONAC ENGINEFING

ELECTRONIC LOGIC CHASOIS

ELECTRONIC PHASE SHIFTE

ELECTRONIC RACKS

ELCTRONIC SHUTTERS

ELECTRONIC TEMPERIPURE CONTROLLES

ELECTRONIC TIME DELAY

ELECTRONIC TAINSFORMEQS

ELECTRONICS

ELECTRONICS INSTRUMENTATION GROUP

ELECTRONICS READOIIT SYSTEM

ELECTRONS

ELECTRONUCLEAR BREEDER

ELECTRONUCLEAR Fuel DPOMIJETION

ELECTROPOTENTIALS

ELECTRONUCLEAR FUEL BoOTICTION

ELECTROSTATIC EFFETS

ELETROSTATIC FIELOS

Eecroostarte piekolup

ELECTROATATIC ORINTER/PI OTIFR

ELECTROSTATIC SEPARAT

ELECTROSTATIC SHIELC

ELECPROSURGERY-INDUCEA AOISF
NIMAER OF

OCCUARENCES

VRCABULARY HCRT

ELECTROSURGFAY-INDUCE" FEA UNISE

ELECTROSURGICAL BLATE

ELECTROSLRGICAL CTAGULATIAGECIITTIAG FCOCHOS

ELECTROSURGICAL CUTTITE BIALE

ELECTROSURGICAL DEVICES

ELECTROSURGICAL PQOCEDUAE

ELECTROSURGSCAL THCDNTOUE

ELFYENTARY PATT TCIES INA FIELOS

ELTVTED IE MPERATURES

ELLTOSE EGLATIOI.S

ELLIPSE HALF EVVFLOEE

ELLTPSE MATCHINE

ELLIPSF PARAUETERS

ELLIPTICAL REGION

EM⿻? JAW STFFRING

EMTEEAR JAW 5

EN? Jin

FMEGENCY MODIFICATIOT.S

EMF

EMISSIOR LEVEL

EMITTANEE

EMITTANEF DAMPING

EMTTYANCE OEGRATATITA EFFECTS

EMITTANCF FUERGIES

EUTTTANCE FIGUNEQAF- YFRIT

EMITTANCE GFAR

EMITTNCE GRTMT

EMPTTINUEE JALS

EMIT TANCE ME ASUREUE:T

ENiTTANCE MFASUPFUEVTS

EMITTANCF NFASUE? I:

ENITANCE MFASUEDAG DEVTCES

ENTTPANCF DATPEEN

EMTTTANCF SCAHATE

ENITTANCF SPFCTFA

E"ITTANCE SPREATING

EMITIANCE STATITNS

EMITTANCE SYSTEM

EMITTANCE TAILS

ENPHESYML

EMPIHIEAL CONSS SECTION FORWILA

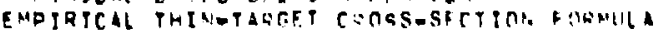

EMULSION TEEHNIOULS

ENULSIDY TRACK

ENCTDER QFADOUT MODULF

ENC NOE 45

EACrelopaEOIC FMTEY

END STTBFEAER HiNGS

ENERGETIL PION CHAN'EL AND SPFCTROME TER

ENFRGFYIC DION CWANAEL ANA SPFCTRCMETEQS

EAERGEIC PIOA THANE 
ENERGFTIC SECONCARY NEUTHONS

ENERGY

ENERGY 3.5 MEV

ENERGY ABSORAER

ENERGY CONFIGUHATIONS

ENERGY CONTROL SUASYSTEI"

ENERGY DEGR ADEP

ENERGY DEGRADING MATERIALS

ENERGY DEPOSITFD

ENERGY DEPOSITI INN

ENERGY DISCRIMINATION

ENERGY EIGENVALUES

ENERGY GAIN

ENERGY GENERATION

ENERGY GRADIENT

ENERGY LOSS

ENERGY PROPAGATION

ENERGY RESOLUTION

ENERGY SHIFTS

ENERGY SPECTRA

ENERGY SPECTRIJM

ENERGY SPREAD

ENERGY VARIATIONS

ENERGY OOEPENDENT EFFECTIVF NUCLEAR RADIUS

ENERGY DEPENDENT DHASFMBMTFY ANALYSES

ENERGY I INTENSITY DE UUIREMFNTS

ENG INEERING

ENGINEERING DESIGN CALCHLATIONS

ENGINEERING OIFFERENCES

ENGLANO

ENI AMPLIFIER (MONEL 24"'L)

ENVELOPE RESPONSE

ENV IRONHENT

ENVIAONMENTAL STUDIES

EPA

EPA BEAM POSITION MONTTMRS

EPA CONTROL CAMPUTER

EPA CONTROL SYSTEM

EPA EXPERIMENTAL AREA

EPA INTERLOCK SYSTEM

EPA MECHANICAL ENGINEFRINT

EPA PROTOTYPE

EPA STATUS HONITOR

EPA STRUCTURES

EPA SYSTEM ALIGNMFNT

EPB

EPICS BEAN CHANNEL

EPICS BEAM L.INE

EPICS BEAM SEPARAYOR

EPICS BRNOING MAGNETS
- Hater OF

NCCURREVCFS

VDCARULARY moRT

EDICS CWANAEL

EPICS HIGH VDLTLGE LIQE

EPICS PARTICLF ACTELE:ATOR

EPICS PARTICLE SEDAHATOA

EPICS DION SPFCTRAMETFR

EDICS SEPAFATOR

EPICS SPECTROMETE

EDL

EPL RAOIATION SI:EVEY

EPOXY IMDREGNATION

EPOXY OATTING

ERUATION OF STATE

EOUIDENSITYEELLIPSE

EQUILIBRIUM DISTRTB:TID. FUNCTITNS

EOUILIHRIHIO OISTRTRITIOAS

EQUIPAFNT

EDUIPNENT AVAILABILITY

EDUIDUENT DOME

EQUIDMFNT KXPERIMENTS

EDUIPMEAT FIILURE MODES

EOUIPRENT IT.VENTORY

ERUIPUEAT MATUTFNANCE

EDUTPMENT PODL

EOUIPMERT STABII ITY UEVFLOPMENT

ERUTDMENT STACK

SHIELO FLTCTRODE

EQUIPOTENTI IL SIIRFACF

EQUIVILENT CIRCUIT MONEL

EOUIVALENT EIRCUTTS

ERARA ANALYSIS

EARTR CWE EKIAG

ERROA CIINTROL

ERROL FUNCTIONS

ERROAS

ERRORS FUO DIONS

ERYTHFMA

FSONE

E\$1

ETL AHILOIAG

ETL RUILOINT, KLYSTRCN

EUR-AI BAE

EVACUATION SYSTEM

EVAPORATION CALCULATI INE

FVAPORATION MONEL

EVAPORATION THINNING

EVE: OS NUELE:

EVENT SPECTRA

EVEAT IRIGGER MOUULF

EXCITATION ENERGY SOEPTDA

ExCITATION FUNETITING

ExETtation OF OTHEA mODES 
NUMBER OF OECURRENCES

VOCABULARY WORD

EXEC ODERATING SYSTEM

\section{EXEC SEAN \\ EXECUTIVE PROGRAM \\ EXECUPIVE TERMINAL}

EXHAUST SYSTEM

EXIT PAATICLE DISPLACEMENT

Exotic apoms

EXP, 1 ag

Exp, 182

ExP 103

Exp: 104

Exp 105

Exp: 185

Exp: 111

Exp: 118

ExP, 121

EXP: 123

Exp: 120

ExP, 125

ExP. 120

Exp: 129

Exp. 131

Exp. 132

Exp: $132 / 160$

ExP, 132/160/42

EXP. 137

EXP: 137A

EXP: 142

EXP. 147

ExP. 149

EXP. 150

EXP. 153

ExP. 160

EXP, 161

Exp. 162/179

ExP. 163

Ex: 160

Exp: 167

ExP: 179

Exe. 190

Exp: 180

Ex०. 181

ExP. 189

ExP. 191

Exp. 192

ExP. 195

Exp. 196

Exp, 2

ExP: 214

EXP, 26

Ex: 27

Exp: 31
NISMAER OF

OCCUIRRENCFS

VOCARULARY WORA

EXP, 31/160

EXP, 32

EXP, 34

EXP. 35

Ex० 5

EXP: 5

Exp: 53

EXP:56/125

EXP: 561

EXP: $62 / 12$

EXP. 67

Exp: 7

EXP, AD

EXP, AI

EXP, Bu

EXP: 86

EXP: 98

EXP: 96

EXP:99

EXPANSTON SECTION

EXPANSION

EXPF

EXPEVSES 184

EXPERT MENT 137

EXPERTMENT 179

EXPERIMENT 195

EXPERI MENT 32

EXPERTMENT 5

EXPER? पENT 6

EXPERIMENT QH

EXPEHIMENT NO,

EXPERIMENT NO IZ

EXPFDIMFNT ND, IZG

EXPERI MENT NO, IIZ

EXPERIMENT NO, I3a

EXPEQ I MENT NO, I37

EXPERIMFNF MO: IHI

EXPERTMFN NO: IA

EXPER MEN NO"

EXPERTMENT NO,

EXPERIMEAT NO, 32

EXOFQTMENT NO, 37

EXDERIMENT NO, 47

EXPERIMENT NO, 90

EXPFR!MFNT NO: 7

EXPERTMENT NOO O6

EXPFRIMENT ND, TQ

FXPELIMENTAI AREA

EXPFRTMENTAL IRFA

EXPFRTMENTAL HHEA COMDUTEOS

EXPFRIMFNTAL AREA TIRGETS 
NUMBER OF OCCUARENCES

VOCABULARY WORA

EXPERIMENTAL AREAS

EXPERIMENTAL BEAM ERNTRAL

EXPEAIHENTAL CONTAOL HOOM

EXPERIMENTAL DATA

EXPERIMENTAL OEAD TIME

EXPER IMENTAL DOSIMETRY DROGRAM

EXPERIMENTAL MDNITORING

EXPERIMENTAL PROGRAM

EXPERIMENTAL PROPOSALS

EXPERIMENTAL RESULTS

EXPERIMENTAL SCHEOULE

EXPERIMENTAL SCHEDULE DATA AASE

EXPERIMENTAL SHIELOING

EXPLOSIVELY DRIVEN METAL CONFIGURATIONS

EXPLOSIVES PROPERTIES

EXPS, 56, 125, 129, 295

EXTERNAL CONTROL CIHCJIT

EXTERNAL FIELOS

EXTERNAL INPUT SIGNALS

EXTERNAL PROTON REA:A

EXTRACORPOREAL GLOOD

EXTRACTION

EXTRACTION ALGNET

EXTRACTOR CURRENT SIGNAL

EXTMACYOR VOLYAGE

F-LENS

FABRICATION PROCENURE

FaILOSAFE

\section{FalluRE RATES}

FAILURE REPORTIHE

FAILURE SYMOTOMS

FAILURES

MIRCHILO LOGIE

FALL TIME

FANO CORRECTION

FARADAY

Fahaday cage

Faraday cup

FARM PNOGRAM

fast analog command

Fast BINARY COMMAND

FAST CHDPPER

Fagt deFlection PLate

FAST FAULTAPROTECTION LOGTC

Fat Fil

mast regating electróse

Fas INJECTOK SHUTDOWA

FAsT ION CHAMBERS

FAST KICKER MATNE

Fast LogIC

FAST MODULAPOR PROTECTION
AUIMPER OF

OCCURAENCES

VOCABULARY WORA

FAST NEITHON BEAM

FAST NEUTAONS

FAST PIONS

FAST PRTTECT

FAST PROTECT CHANNEL

PAST PROTECT CHASSIS

FAST PROIECT EIPCINITS

FAST PROTECT DIAGNOSTICE

FAST PROTECT MESBAGES

FAST PROTECT PROTECTION

FAST PROTECT SYSTEM

FAST DAD-SAFE SYSTEM

FAST SHUTDOWN

FAST WIRE SCANHER

FAST-INTERLOCK

FAST-KICKER POWFO

FALILT ANALYSIS

FAULT CONOITIO
FAULT CURRENT

FaULT JE.TECTION EIRCUPT

FAUIT DETECTOR

a AULT PROIECTTIN

FAULT RATES

FAULT RESE

FAULTS

FEATIVES OF THF SPM

FEED FORWARD CONTROI

FEENAFOQWARD

FEFDTHROUGH

FFRMI DISTHIAUTION

FERNI FNFRGY

FERMI SHADE

FERMI-TELLER MOOFL

FERHE-TELLER MONEL MESOR CADTURE

FERMT-TELLEE T I H

FERMILAH

FERRELLS FORMULA

FFRRITE BLOCKS

FERP ITE TORES

FERAITE ISOLATOA

FFRAITE MAGPE

FERRITES

FET

PEEARS

FIHER OPTICS

FIEID

FIELD AMPLJTUNE

FiELO BFHAVINO

FIELD changes

- IEl.o clamp

Fiela conerence 


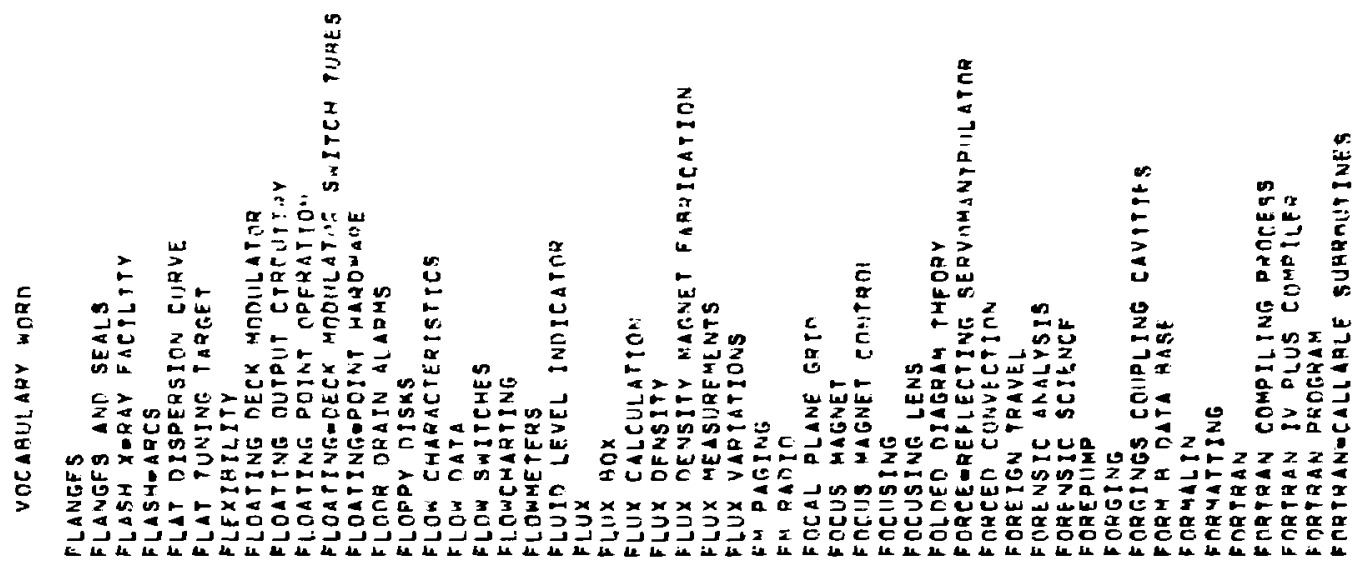

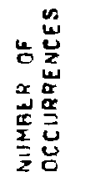

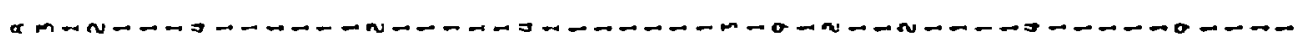

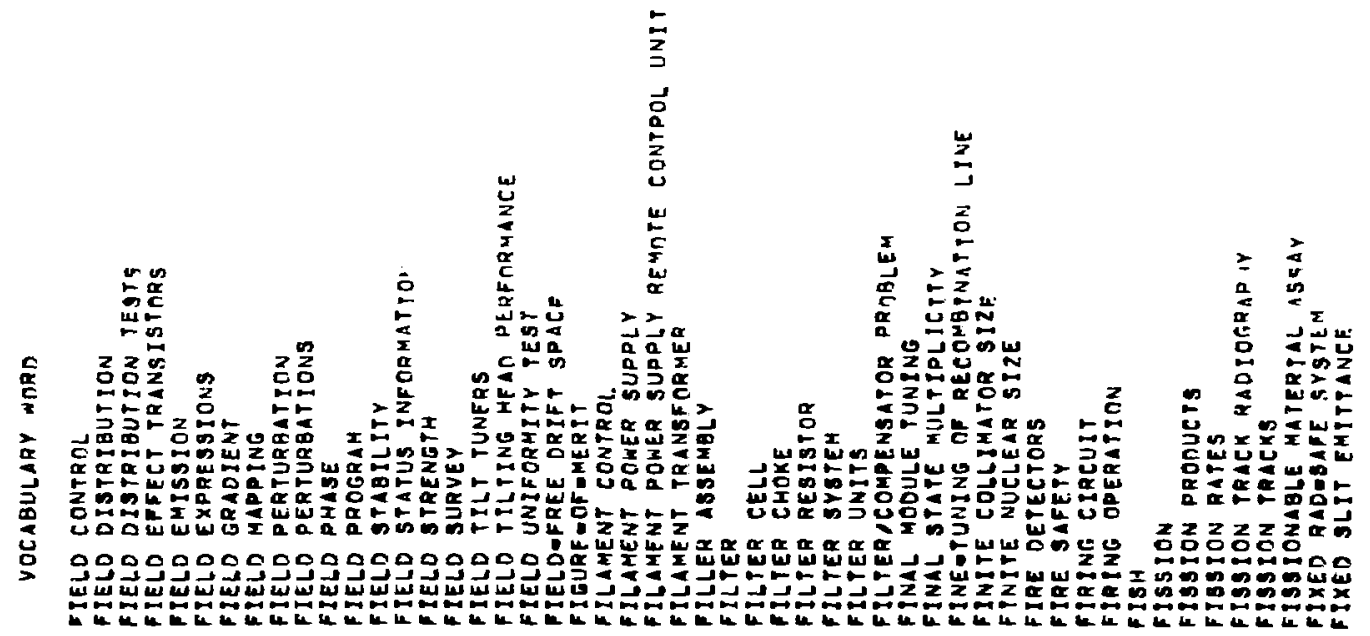

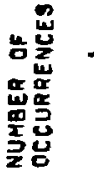


FORWARD ELASTIC SCATTFRING

FORWARO SCATTEREO ELECTRONS

FDRWARD SEATTERING, ELASTIC SCATTERTNG AMPLTTHIOE FOUNOATION

FOUR-FOOT DRIFT TUBF MOLEL

FOURDJAW APERTURE

FOUROJAW EMITTANCE

FOURIER ANALYZE

FOURTER TRANSFORM

FOURTER TRANSFORM TEENBACK SYSTEM

PPB/ACCELERATOR INTEREATE

FRACTION OF TREATMENT DOSF

FRAGMENTATION

FREE +MUON PROPERTIES

FREE ELECTRON DENGITY

FREE FIELD COMPUTER CARM READ ROUTIME

FREE MUTN

FREE PIONONUCLEON RESULTS

FREE - NUCLEON RESONANCE

FREDUENEY

FREDUENCY CHANGE

FREOUENCY DISTRIBUTIO.

FREQUENCY ERRORS

FREOUENCY GEMERATOR

FREQUENCY MODULATION STOUCIURE

PAEQUENCY HUL TIPL IER JEVELOPMENT

PREDUENCY RESPONSE

PREOUENCY RESPONSE

FREQUENCY RESPONSE

FREQUENCY SOURCF

FREQUENCY TPHASE REFERENCE

PREOUENCY PHABE STUDIES

FRIEDOMARTIN CORRECTIINN

FR INGE FIELO CORRECTION CONSTANT, FLUX PLOTS PRINGING FIELO

FRONY

PRONT PORCH/BACX PORCH APFRATION

FULL-USAGE TIME

PUNCTION

FUNCTION GENERATOR

FUNCTIONAL REQUTREMENTS

FUNDAMENTAL PARTICLE STIDY

FUNDIMENTAL PASSBANO

FUNDTNG/CONSTRUCTION STATIIS

FURNACE BRAZING

FUSEDODISCONNECT SWITCH

FUSION REACTIONB

FUSION REACTORS-OFSIG

FUZZY FEHMIOIELLER YONEL

G.F, MAXITRON

G, E, WINDOWS

GAIN CURVE

GAIN SEL

GALILFAN-IAVAFIANT ABSUOPTIINN

GAMAVAL

GAMMA

GAMMA DECAY

GAMMA EMITTFRS

GAMMA MON

gamma RaY calculatione

GAMAA RAYS

GAMMA SCINTILLATIONS

GAMMA SENSORS

GAMMA SPFCTRUM

GAMMA TRANSTTIOHS

GAMMA-NETFCTOR MONULES

GAMMA-F IITING DR ODICTS

GAMMA-GAMMA COINC IOLNCE

GHMMA-GAMMA MEASUDEMENTS

GAMMA-NFUTRON ACT TVATTO.

GAMHA-PARTICLE REMTVAI.

GAMHA-HAY EMISSION

GAMMA-RAY ENFRETES ANA INTENSTIIES

GAMMAORAY SPECTRA

GAMMA-RAY SPECTROMETE

GAMHAORAY SPECTPOMETRY

GANTQY

GANTRY STATUS RFVIEW

GAP CHANGFS

GAP CHANGF

GAP LFNGTHS

GAP LITNGS

GARAAGE COLIECT

GARTENHALIS

GAS CELL

GAS FLINW SYSTEMS

GAS PAOPORTIORAI COINTERS

GASEOUS HYDRDTE

GATED SPFCTRA

GATES

GALISSIAN OISTRIRUT TON

GAUSS IAN IHTEGHAT TO

GAUSSIAN MULTIPLF SCATERTI

GAUSSI GAN

GE (LI) DETECTORS 
GE(LI) GAHMAORAY TECHNIOUES

GE (LI) 3 PE

GE(LI) SPECTROMETER ME AGUREUENTS

GELATIN

GENERAL DESIGN REVIEW

GENERAL ELECTRIE (T2UA) IGNITIONS

GENERAL GEOMETRY PAOGRA

GENERATOR CHARACTERISTICS

GEOMETAICAL EFFICIENEY

GEOMETRICAL REQUIREMENT

GEOMETRY

GERMANIUM

GERMANIUM SPECTROMETEQ

GIANT MULTIPOLE RESONANCES IN NUELEI

GIRLs

GIRLS OATA BASE

Gro OROCRAM

GL

GLOUDER APQROXIN

GOALS STHARG

GOALs

GODFREY CODE

COLD

GORK PROGRAM

GRADIENTS

GRAFT PROCEDURES

GRAM-SCHMIDT ORTHCGONALIZATION IECHNIOUE

GRAPHIC ARTS

GRAPHIC CONPROL PANFLS

GRAPHIC DISPLAYS

GRAPHIC BYMEOLS

GRAPHIC OTIME - HIST ARY

GRAPHICS DISPLAY SCOPR.

GAAPHICS OISPLAY SEOPES

GAAPHICS PROGRAM

GRAPHICS SOFTWARE

GRAPHICS TERMINAL

GAAPHITE

GRAPHITE FINGEAS

GAAPHITE FOIL

GRAPHITE-TOOMETAL BONIIAS

GRAY COOE OUTPUT

GREASE PIT

GREASE PIT

GREATEST-UPPER-ROUNO (G.4H)

GREENS FUNCPION

GRID AOMBING

GRIO CHARACPERISTICS

GAIO EMISBION

GRID-CLAMP CIRCUIT

GAIDDED AMPLIFIER TUHE

GROUNO GAR beanclatar ana

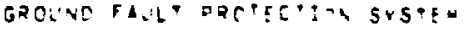

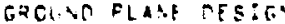

GROLIVIDISCLATIOC

GRCNTA DELAT

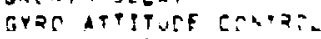

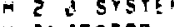

H INJECTOR

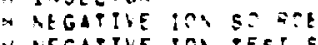

a NFGaTIVE Iro test so

hoostrite se tegative inectio

H. AVO HO REAUS

H. PEAH

H. PEA CHCODER

Hi BEAMS

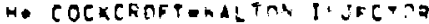

H. CoCxchesteandition

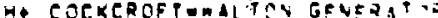

nQ INJECTCR

H. INJECTORS

H ION SOULCE

mo DOLIRIZES Rit

H* SWIN

m. PRAYSPCRT T:OLOS

HAINA INJECTORS

Ho ACCELFATISC Cne wa

H. ACCELERATIAS CW W

H. HEAT

H. REAL (REAN LITF B)

H. REAM CMROPEA

H. DEAU STPTODEAS

H* QEAपS

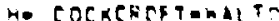

HE DJME SYSTE Th

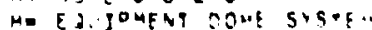

H. IYJECTOR

mo ivictca naseria

m. i iv aEA

m. In schece

N. I. ITS

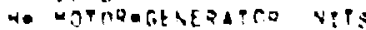

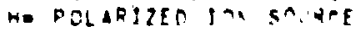

H. DRTAES

mon $S \pi, \operatorname{tit}$

wo STilace

H. STR IPDFE

M. STQJPPEA FAlIS

M. STPाण्ING

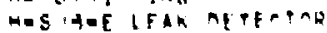

mestia e p ivise

mes 4 et a ing ines

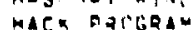

N 
NUMBER OF OCCURRENCES
VOCAGULARY IORR

HADRON-NUCLEUS INTEMACTIO HADRONONUCLEUS POTENTIAL

HADRONIC ATOMS

HADRONIC FORLE

HAORONIC XORAY LIMES

HAEFELY CO

HALF MEGAWATT OLTPUT

HALF - LIFE

HALF-LIVES OF 134-LA ANT 132-135-CE

HALL CHSP

HALL PROBE MEASIIRFHEVTS

HALO

HALO FACTOR

HALO FACTOR

HALO MEASUJREMENT

HALO MEASIIRE

HALO SIZE

HALO-GENERATING MECHANISM

HAL OADRODUCING MECHANISM

HAMILTONIAN

HAMIL TOVIAN FORMAI ISM

HANDLER

HANOSAOFF OPERATINN

HANOS ON OPERATION

HANF ORD MEPHOS

HARO PUBE MOOULATOR

HAROHARE

HAROWAGE COST

HARONARE COST
HAROWARE DOCUMENTATION

HIRDNARE DRAWINGS

HARDWARE DRAWINGS

MARONARE FLUCP ION GENERAT OR

MARONARE FLUCY ITN
HARDWARE STRUETURE

HAROWARF TEST PROGRAM

HAROWARE TEST PROGRAMS

HARMONIC

MARMONIC BLNCHER

HARMONTC CONTEN

HARMONIC CONTENT MEASHRE HENTS

HARMONIC CURRENTS

HARHONIC CURRENTS

HARMONIC FLTEA

HARMONC FUNGTION

HARMONIE MEASUREMENTS

HARMONIC POLLNGMIAL EXPANGION

HARP AGSEHBL

MARD GOAROS

HARP DISPLAY

HARP HPOI

HARP MEASUREMENTS

HARP MEISURING OEVITE

HARP PROBLEMS

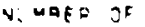

occihafices

SOCAALLAT NEET

HAD SPTE SIZL

$\triangle A D=5 \times 5 T+4$

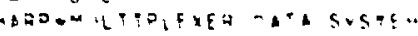

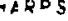

HARPS W. LTIOLF AFE

MAOPS PUCGLA

MAPTDECBCC

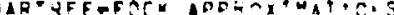

Mrit

MEAT ASSI UHL:I

MEAE COHPCL CHESTIS

we to conpori ing

uninge

upation Darsice

然

MEALPO SAFEPY

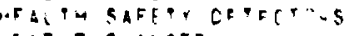

AF $A$ EXCHARGE

HWA EL

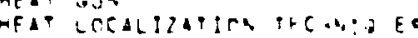

WFAT Magass in Win

HELT PRTEES

MEAT SMIELCG

WE TFO

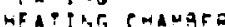

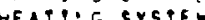

matr pon accelosation

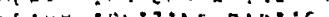

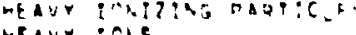

Wavr iolic

WBAt TCLTCE

ELA CELLS

-ELICAL CADETES

MELICAL COAMES

HELICAL OELAV LIOTS

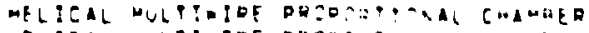

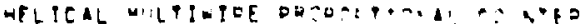

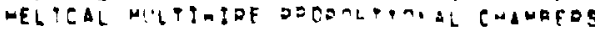

MELICAL MOPE AFUEI CDHER

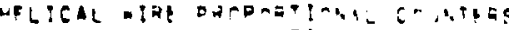

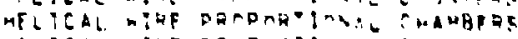

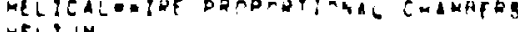

HLidj

HLi, L OS

WEL? RaGS

AFLI, CCATATCER

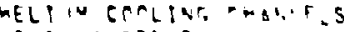

uftix cioglit 
NUMBER OF OCCURRENCES

VOCABULARY WRD

HEL IX PITCH

HEMOCHROMATOSIS

HEP

HERHANASKILLMAN POTENTIAL

HETERODYNE DETECTORS

HEWLET P PACKARD

HEXADECAPOLE MOMENT

HF 3

HFS OF 30 MUONIC STHTES

HFB TRANBITION IN HUONI IL (MEASLREMENT CF) HIPOT TINO

HIGH BEAM CUARENTS

HIGM BEAM BFILLS

HYGH BDIGHTNESS LNAC OEAHS

HIGH CURRENT

HIGH CURRENT EFFECTS

HIGH DENSITY IIOUTD

HIGH DUTY FACPOR

HIGH DUTY FACTOR ONE-SHOT

HIGH DUTY OPERATION

HIGH ENERGY ELECTRON DOSINETAY

HIGH ENERGY NEUTRON REACIIONS

HIGH ENERGY PION CHAMHEI

HIGH FREQUENCY OPERATTOL

HIGH FREOUENCY SIGNALE

HIGH INTENSITY

HIGH INTENSITY H ION SOURCE

Hign INTENSITY INJECYOR

HIGH INTENSITY PRITAN ATCFLEAATCA

WIGH INIENSITY PROTON AFAM

HIGH LET RIOTITIOA

MIGH MOMENTUH PRANSER

HIGH DEAK OEAMS

HIGH DOWEA

HIGH POWER OPERATIJA

HIGH RULSE CUARENT

HIGH OULBED ANONE CHRQE.TS

HIGH D

o eavitr

HIGH OUALTYY OUADRUPDI,E

HIGH AESOLUITON HEAM SCANAER

HIGH RESOLUPTON POON GPFETAOMETE

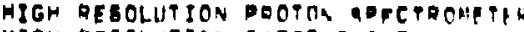

MIGH REBOLUTION SPECTAORCAPY

HIGH RESOLUTIIIN SOECTOONETER

HIGH RESOLVING OOREP SPLCTHOME IF

HIGH SENGITIVITY RACIAGEAOHIC SUSTE"

HIGH SPATIAL RESOLUTION

HIGH TEMPERATURE PEASILS GPQECGT

High VACUUH

HIGH VACUUH THERMOCOUELE
VIVAFR AB

TCL DSEYCE

Meablater mas

mlom VWF FLEC

Higm volitace carblo

Mig unitiog capacis

nigh VGLTLGE LiaE

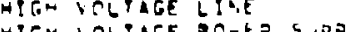

High vol iage sristom

MIGM VRLPAGE SYSTEM

HIGM 2 ELEMFUTS

MIGHOCVIFENT HERM

-igmac jagas: me ind s unes

MIGHOEJPREAT TESTINW

MIGWEEVEQGY LIVAT

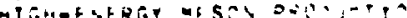

Mlomer

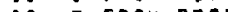

HJGH. EAFQGY PQJTES

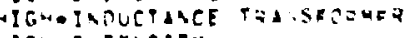

Migmeis?

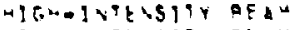

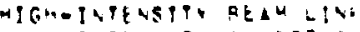

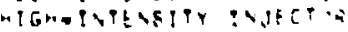

HGR-INTENS!T, WIOU HEA.

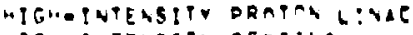

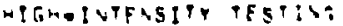

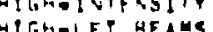

Ming

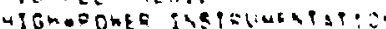

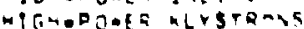

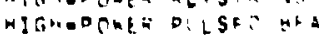

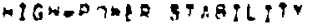

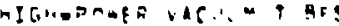

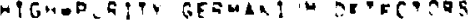

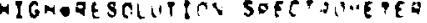

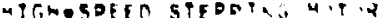

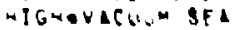

HIGMOVITTHGE

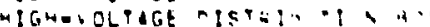

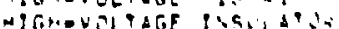

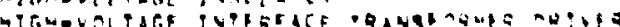

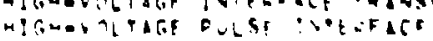

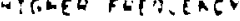

"ITHEQ DASSHALES

-IGHE SUACE RESITT

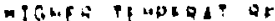

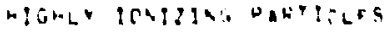

i $10 \mathrm{~m} \rightarrow 00$

$\operatorname{lin} 15$

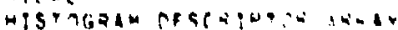

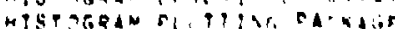

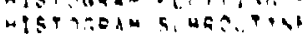


NIIMAER OF

OCCURRENCFS

VOCARULARY WMRE

Hist OGRAM

HISTORY

HODOSCOPE MAGNETS

HOLLERIYH FORMATS

HOLL OW CONOUCT!

HOME PEAMINALS

HDNEYCOMB CONVFATFRS

HORIZONTAL MOTICN

HORTZONTAL OSCILLATIOA POWTOOL

HDRIZONTAL OSCILLATIO

HORIZONTAL SCAN

HOR IONTAL SC ANTIS:G

HORIZONTAL STEERING MAG.ET

HOA $20 \mathrm{~N}$

MORSESHDE MAGNFTS

HOSFITAL ACCELERATOR

HOSPITAL-QASED LINAC

hot

HOT CELL COMPLEX

HOT CELLS

HOT HIRE SENSOA

HOT-CELL OPERATIONS

HOUSTON $X$ G RECORDF

HPOZIOA COMPUTER

HRS EXPERIMENTAL PRIIGRAU

HRS MELSUREMENTS

HAS TESEARCH PROPDSALS

HAS SPECTROMETER

HRS SPECTROMETER BYSTEM

\section{HSABY}

HT WINDINGS

HTM EVOLUT ION

HUMAN CLINICAL TRIAL

HUAAN KIONEY CELLS

HUMAN LIVER

HIJMAN RADIOBIOLOGICAL EXPERTMENIS

HISMAN RADIOGZOL RGY

HUMIOIFY?NG

HV CONTROL

HV SFCTION

HY SEPARATOR SUPPLY

HV SWITCH

HVDC SUPPLY

HVP MONIFORING CHASS IS

HYBR ID COMPUTER TFCUNIOUES

HYBRTO INTERFACE COMP TAENTS

HYDRAULIC ACCUMULATOR

HYORAULIC CIRCUITS
3. whith of

OCC:PUE NCE

octan hat ane

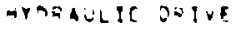

MYOEA Lis OQF SE $=$

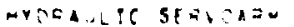

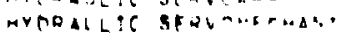

wrata lit suster

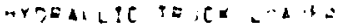

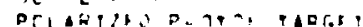

artontis.

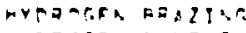

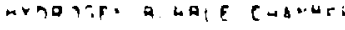

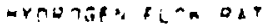

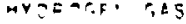

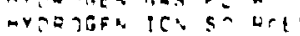

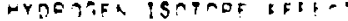

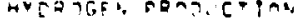

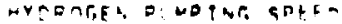

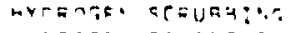

Areages TAQDE C

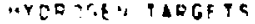

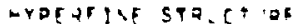

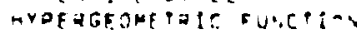

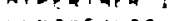

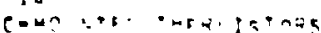

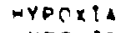

aroryit contipa

mYSTETFSIs

HYSTEOFSTC TERET

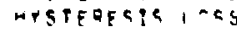

I AND C SHSTEL

iel conglisio

ils caule una. bs

$1 / 2$

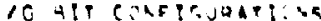

ic baude umb. i.

i,o caritits

ic cmassis

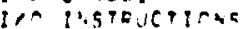

ire gratenas

in tiani:

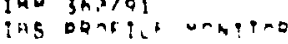

icics

ic

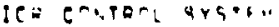

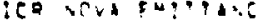

inealize anar ol wetules

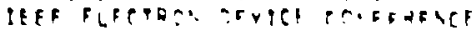

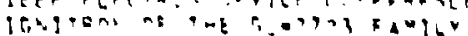

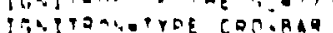

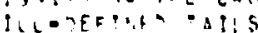

N: 
INTERACTIVE GRADHTCS

INTERACTIVE MODE

INTERICTIVE TREATMENT DI ANNING

NTERCOM SYSTEM

INTERCONNECTING BFAM LINE

INTERFACE

INTERFACE AMPLIFIER

INTERFACE CONCEPT

INTERFACE CROSSING

INTERFACE ELECTRONICS

INTERFACE ELECTRONICS

INTER

INTERPACE HARDWARE

INTERFACE MODULF

INPERFACE MOOULE

INTERFACE SYSTEM
INTERFACE PECHNIOUE

INTERFACE TECHNIOUE
INTERFACE TRANSFOQMFR

INTERFACE TRANSF

INTERFACING DECOOER UNITS

INT ERLOCK CHAIN

INTERLOCK CIRCUIT

INTERLOCK PROBLEMS

INTERLOCK SYSTEM

INTERLOCKS

INTERMEOIATE ELFCTRUDE

INTERMEDIATE ELLCTROD

INTERMEOIATE ION SOURCE

INTERMEOIATE ION SOURCE
INTERMEDIATE PONER AMDLIFIER

INTERMEOIATE POWER TMDLIFIER

INTERPHINE COMHUNICATIO:S

INTERPOLATION

TIC ARnGRAM

INTERRUPT PROCESSOR

INTERRUPTS

INTERSECTING STORAGE RING

INTRACRANIAL MALIGENANT OL TOMATA

INTRANUCLEAR CABCABE CALCULATIOHS

INTRANUCLEAR CABCADE INH FVAPORATION MONEL

INPR INSIC GERMANIUM DETFCTOR

INVIRIANT CROSS SECPIONS

INVASIVE METHOD

INVAS IVE.
INVENTORY

INVEST MENT CASTING

ION CHAMBEA

ION CHAMBER PROAE

ION CHAMAERS

ION EXCHANGE

ION GAUGES

ION INJECTOR

ION INJECTORS

ION OPTICS SYSTEM

OCC

1
2
1
1
1
1
01
1
1
1
1
1
1
1
1
1
1
1
1
1
1
1
11
1
1
1
2
1
2
1
1
1
1
1
2
1
1
1
1
1
1
1
1
1
1
1
1
1
1

VOCABULART mert

IC. DAlES

ICN Dhup

ICA DUMF CUPRE

IC' DUAP REPUILC

ICA OUND FEAUILA FACI. ITY

ICN DUMP REPUILOITG

ICS PLIMP SUPPIY

ICA SCURCE

ICA SCURCE ACCELERATITG CALIMA,

ind SO RCE COSTOCI

ITA SCJRCE CEVELOUEAT

Soutice injectiog

ION SOURCE CPFRATION

ICA SCIRCF OISER

jor sculce test 5 TANO

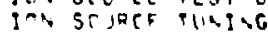

ind $2070 \mathrm{cos}$

IOS SPECIFS

ICA TF MPERATIRE

ICAIZATICN PFAM SCANAG

ITAIZATICV PHAMRE

IONIZATIOA CURRTAT

ICNIZATION CURVE

ICNIZATICN HETWADS

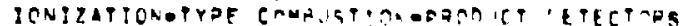
ns IF DORER SLPDLIES

IFA

IPA AMOCE SEREEN SUDPIY

IPA EILANEAT DOAER SUDDLY

IRIS IDFETUAE

IRIS LOADED CAYITY

IRIS LOACED STZI.CTURES

IRIS LOADEO WAYEGIITE

TRIS LOACYNC.

IOCN COYCEAPATIOAS

IRCN COFE OF SIOA.

IOON WOIISE

IDON 61140

inON a IV

IARAIIATJP

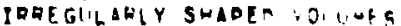

ISAM

ISOBAR CADYHIO

ISORAR WOAEL

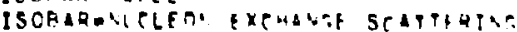

is nemanveus cycintory

iscousf conin:as

ISODOSF CURES

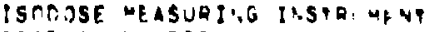

ISCNOSE PLCPTER 
NUMAER OF

OCCURRENCES

VOCABULARY WORO

ISOLATED COLLECTOQ

ISOLATION

ISOLATION TRANSFORMER

ISOLATION VALVES

ISULATORS

is $O P$

I3ORAD

ISOTOPE PRODUCTION

ISOTOPE PRODUCTION

ISOTOPE DRODUCTION AREA

ISOTOPE SEPARATION

ISOTOPE SEPARAT
ISOTOPE SHIFTS

ISOTOPE

ISOYOPES

ISOTOPIC NEUTRON SOLHCE

ISTS

ITEP ACCELERATOD

IVR CONTROL SYSTEU

J3 TESTER

JANUS PROGRAM

JOINT DESIGN

JOINT STRENGTH

JOBEF STEFAN INSTIIUTF

jovstick

JUMPER CABLES

JUMETION BENDING MAGVER

JUNE TION BENDING MAGNETS

JUNE TION MAGNEY CONOUETRR

K- ABSOLUTE YIELDS

Ka ABSORPTION

$K$ - BAP CAPTURE

Ko XoRAY DATA
KoRAY EXPERjHEATS

$k=x \cdot \operatorname{xars}$

Kamesic ATOMIC CASCADES

$K D M E S I C$ ATOMS

$K \in N$ SCATtERTN

KOSHELL

KOSHELL MHONIE XERAYS

$K=S H E L L$ XORAYS

$K=V$ DISTRIOUTION

$x \in E$ LASER

KAE LASER

KAHILS SPORAGE MOOULE CTINTROLLEO

KAON PRQUUCTION

KAONGNUELEUS ODTICAL DUTENTIAL MONEL

KAONIC ATOMS

KAONTC HYORTGEN

KAONIC MOLYBOENIIM
KAONIC XORAY YIFLOS

KAONIE XERAYS

KAONS

KAPEHINSKIJOVLAOMERTKIJ EOUATIOK
QHALEF 25

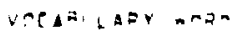

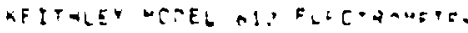

KFWI CELl

xHT SATCCMA

MICKFA UAGAES

KIETEY TAI CELIS

XILPATZT6 CRITEZTO

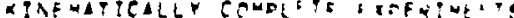

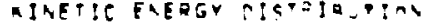

DJAETIC SOSTEU

Tiet siset

XidT RER $S+S T E$

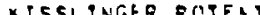

KLetrogranga es aita

KLYSTRO

KLYSTETS AMDLITIE

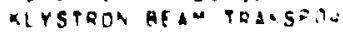

KLYSTOAN CAVITIOS

kitriach ablog

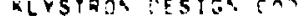

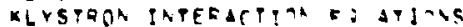

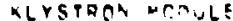

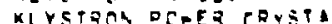

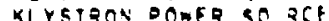

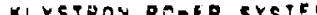

Klystoor aERJil:

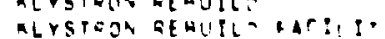

ablition IEs stian

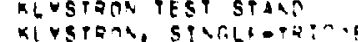

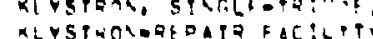

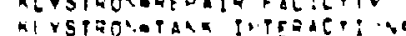

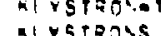

al.

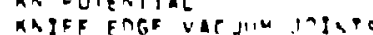

MAOA QESPATSE

MREEO HASALIS

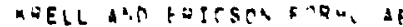

ketrez a 2 at

b tho on bia

Lot Filita

L- FiliEAS

Lanstritavitos

LASFueit

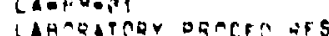

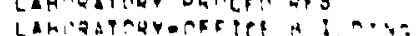

LC:

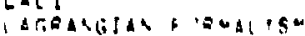

LGEANGIAN IATEEORLAT

LALA

LLA Cith

LaLa Dacgan

n

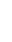


NUMBER OF OCCURRENCES

VOCABULARY WORO

6
1
1
1
1
2
1
2
4
1
1
1
2
1
2
1
1
1
1
1
1
1
1
1
3
2
1

LAMA SHIFT SOURCE

LAMRESHIFT

LAMP

LAMPF

LAMPF BOO HEV BEAM

LAMPF ACCELERATOR

LAMPF FACILITY

LAMPF LINAC

LAMPF PERT

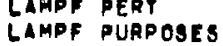

LAMPF gTATUS

LAMPF STATUS

LAMPF USERS GROUP

LAMPF VIOEO SYBTEM

LANGUAGE STRUEPURE

LAPLACE PROGRAM

LAPLACE TAANSFOAMS

LAPLACES EOUATION

LAPLA

LARGE AMPLITUDE

LARGE SAMPLES

LARGEOSIGNAL THEORY

LASER GEAM

LABER CUTTING
LABER HEATING

LASER SHOT

LASEREEUT SLITS

LABERS

LAURENT SERIES

LCF

LCR CIRCUIT

LDDL PRTGRAM

LDMD PROGRAM

LEAO

LEADING EDGE TIMING

LEAK DETECTOR
LAME SHIFT POLARIZED ION SOURCE

LAMPF CONSTRUETION STATUS

LAMPF ELECTRONICS AND EOUPPMENT POOL

LAMPF ELETIRONICS EQUIPMENT POOL

LAMPF ELECTRONBCS PNOL

LampF Facility descoiption

LAMPF NUCLEON PHYSICS LAB

LAMPF PELEVISTON SYSTEM

LAMPF USERS EOUIPMENT AD ELECTRONICS POOL

LAMPF USERS GROUP, INC.

LAMPF/LOL COMPUPEQ LINK

LARGE SIGNAL DISK-MOOEL KLYSTRON CONE

LARGE -SIGNAL EFFECTS

LARGE OPPCCTROMETER SYGTFMS

lol aia spectrometer

LEAO SHIELO
NIUAFO OF

OCCIIRRE VEES

VOCARULAOY WRP

LEAK TESTING

LEAFAGE

LFAK $\rightarrow G E$ IOMI CTA.CF

LFAnS

LEASP SOLAEES

LEAST SOUAEFS OLCH, FM

LEEDON NI ANITS

LEO

LFE

LENGTH

LEAGPN FERCE

LFAS COVTROL

LEAS FILIE SUSTF

LEP

LFP CHANAEL

LEPTON CONSERVATIOA LAN

LEDTO PROCUCTIAT

LFDTONS

LESIOA

LET

LET CHIMEEO

LET OISTRIRUTIO

LET RAOIATIOO

LEY SPECTRA

LFINETII

LELEETA MATUPENANCE

LEVFL IDAPTER CHASSIS

LEVELING CAPARILITY

LN SJR 2 REFRIGFDATAÖ

LIAISTN OFFICE

LICHA LISA RECEIVER

Lifint Livk SOSTEN

LIGA LINAS

LIGNP VIICLE

Lign ory

LIGNT PIOE CADETE OATM

LEGMP DULSED

LIGHT-LIAK CATEAL

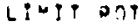

LIMIP SAITCHES

Livitg?

LgMTHU ADEOTJOES

LINAC

LINAC IPDLICITIANS

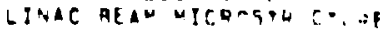

LINAC AELU TESTS

LIVAC MUNCMER

Linac cavity relsigas

LINAC CHARICIERISPI'S

LINAC Comonate te

Lina Covion 
NUMAER OF OCCURRENCES

VOCARULARY WARA

LINAC OATA

LINAC DESIGN

LINAC DESTON DARAMETEAS

LINAC FIELO DISTRTRITIO

LINAC HOUSING

LINAC MOOES

LINAC MOOULES

LINAC PARAMETER MANAGEMENT

LINAC PROGRAM

LINAC READY LIGH

LINAC STARILITY

INAC STEERING

LINAC STRUCTURE PARAMETIR

LINAC TANK OESIGN

LINAC TUNTNE

LINDA OROGRAM

LINDY OROGRAM

LINE A

LINE A-DIRECT

LINE $B$

LINE CURRENT HARMONICS

LINE

LINE D

LINE ORIVER

LINE-EXPENDING TECHNIOUF

LINEAL ENERGY

LINEAR ACEFLEAATOA

LINEAR ACEELERATOR TEEMVOLOEY

LINFAR ACTUATORS

LINFAR AMPLIFIER

LINEAR BEAM PROF ILES

LINEAR CONTROL GYSTEM TIIETRV

LINEAR LENSES

LINEAR MASS FLOWMETER

LINEAR MOMENTUM TRAHSFER

LINEAR MOTIONS

LINEAR NEGATIVE RAMP

LINEAR PULSEOWIDTH MOMUI ATION

LINEAR OENERGY TRANSFE

LINEARITY

LIQUIO DEUTERIUM

LIQUID DEUTERIUM

LIOUTO HELIUM

LOU IO MYOROGEN

LIOUTD HYSAOGEN/OEUTEQ?'M TARGE?

LJOUJD NEON

LIOUIO PNANTOM MEASUREMENTS

LIOUID RANGE SHETPER

LIOUID TRITIUM

LIOUTO WABTE OISPOSAL
NH URER TF

OCC RAENCES

vacambilativ mat

LOU IO-COCLEC IARAE

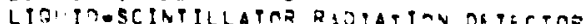

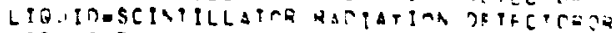
Lithing

LITH!UN

CeCSS SFCit-ve

LITNIJU FSUORIOE

LITHILHETEIFTEO SILICAN METECTOES

L"

Lu novipos

Laco

Lus

LV TENDERATISES

LOAC OF WBDLLATTR

LOAO PANK

bCARE HAVFGUTDE

LOAOING COIL MOCULF

LOBE IENGTH

LOCAL CARTRAL SYSTF"S

LOCAL CMATROLLEE ALTENATTSE

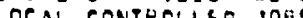

LOCAL CONTROLS

LOCAL HEATING

LOCAL WEAT LO

LCCAL nOT TCAL UTCEL

LCCAL POTENTIAL PARAWL TIITATION

LOEAL-DENSTTV AOPOOXI-ATIAS

LOCALISATION

LOCALIZFO BRFAST TANCER

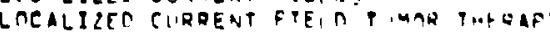

LOCALIZEN HEATITH

LOCALIZEO RF CLIRRENT FIELAS

LOCALIZING BEAM

incitin.

Logir

LOGIC CiRCuits

LOGIC COMPONENTS

LOGis ENATGOL

LOGIC ELEMENTS

LOGIC HAROHARF

LOEIC HAROWARF

LOCIC INPER

LCGSC LIAES

Lrege MOCULES

LCGic SINULATOR

LCGTC STANBARES

LPGIC SYMROLS

LOEIC/PAOTEETIVE CIAC ITAY

LONG AlPERI ODIC CHA

LANG livie Tatys 
LONE PENULTIMATE TAIFY LENGTH

LING TANK FEASIAILITY

LONG-TERM STARILITY

LONGAC

LONGITUDINAL

LONGITUDINAL ACCEPTANCE

LONGITUDINAL CAVITY YTDE

LONGITUDINAL OYNAMICS

LONGITUNINAL EMITTANCE

LONGIYUDTNAL FOCUSIVG

LONGITUDINAL MOTION

LONGITURINAL MUON POLARIZATION

LONGITUDINAL OSCILLITIONS

LONGITUDINAL SPACEOCHARTE EFFECTS

LONGITUDINAL SPACEECHARTE EFFECTS

LONGITUDINAL TUNING

LONGI IUDINAL TUNING

LOOP/TUNER CONTROLS

LOOPS

LORENTZ TRANSFORM

LOS ANGELFS

LOSS MONITORS

LOSS OF GUADRUPOLE MAGNET SECTION

LOST BEAM TIME

LOW AND HIGH Z MATER IALA

LOW CURRENT LEVEL MONTTIRTINO

LOW OENSITY

LOW OENSITY

LOW OENGIL

LON ENEAGY BEAM

LOW ENERGY CHANHEL

LOW ENERGY LINAC STRUCTURES

LOW ENERGY MUON CHANNEL

LOW ENERGY PIDN

LOW ENERGY PION CHANNEL

LOW ENERGY PION SCATTERINE

LOW FREQUENEY OSE PLLAF ION

LOW MARMONJCS OTAGNOS IS

LOW INTENSITY PION BEAMB

LOW INTENBITY RF CURRPNYS

LOW LEY COMPONFNT

LOW MOMENTUM COMPANFNT OETECT OR

LOW PATIENT DOSE

LOW PRESBURE COB

LOW PQESSURE GAS

COOLING SYSTEN

LOW TEMPERATU

LOW VOLTAGE

LOW $Z$ TANK MATEPIAL

LOW $Z$ TARGET MATERTALS

LOW-COST ELECTROMETER

LOW-ENERGY CYCLOTRONS
VOCARULLARY WTRA

LANOENFRGY NEGATIVE DIOAS

LON-ENERGY NEGATIVE MIIT. S

LOW-ENERGY REGITV ACGELFAATOAS

LOHE IMTENSTTY HELN

LOW-INTEASITY DILSEN AEAM

LONEINTENSITY PILSES

LON-LFT BEAMS

LOW-LFYEL MUL TIOLEXF

LOW-L VEL OF SYSIEM

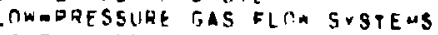

LOWFA CURREN HODF HOJWNARY

LS2 DFDUCTYAN FOR WAI ISM

LUMINESCET RESOCWSE

LUMINCSITY

LUMOEO CONSTANT MADEL

LIMPEO DARAMFTED THEBHY

LUNAR SOIL

$L X \cap A M a P !$

LVMAN INTENSITIFS

MECHINE (LINAC) PHVSIES

MACMINE AUTRMATIDV

MACWINE CYCLE

MICHINE DFVELODHEN:

MACWIAE EXPRIMFNTS

MACMISF PARAMETFAS

MACMINE PROTECTION

MACMINF RISONATCE

MAEMI'E SAFFTY

MICHIQE STARILITY

MECHIVE STATUS CMASSTS

MACNIVF STURIES

MACWIVE VERIEICATIO.

MAEMLETT MLATZLA TETRODE

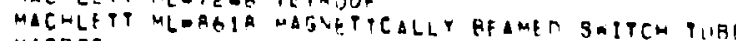

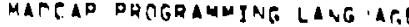

HIGIC GAS MIXTUDE

MIGNESIUM

MAGAESIIM OXIDF ONWEL

MAGNET

MLGNE (C-TYDE)

MAGNFT COMDITIONING C-DES

MAGNET CONTROL

MAGAET COOLIAE

MAGNET CUPRENT

MIONeT OLSIro.

MAGNET FIELD MAOPIVT

MACNET PNTELOEKS

UAGPET TMUETTORY

MGNET IRAA ABSFMALY

MAGNET "ADPTNG 
NUMAER OF

OCCURRENCES

VOCABULARY WRRM

MAGNET MATERIAL

MAGNET MOUNTING PLATE

HAGNET POOL SUBCOMMITTEE

MAGNET POHER

MAGNET PQHER DARTICLE GEPARATOR

MAGNET PONER SUPPLIES

MAGNET POWER SUPPLY

MAGNE 7 TUAORUPOLE LENS

MAGNET REPRIEVAL OPERATION

MAGNET SMX

MAGNET SUPPORT STRLCTILF

MAGNET GYSTEM REQUIREMENTS

MAGNET SYSTEMS

MAGNET TESTING

MAGNET TESTING AND YETROLOEY LAB

MAGNET WARNING LIGHTS

MAGNET, 3D CALCULATIO:S

MAGNET, 3D CALCULAT

MAGNET-TESTING ANO METROLOEY LAB

MAGNET-TESTING ANO
MAGNETIC ALIGNMENT

MAGNETIC ALIGNAEN

MAGNETIC ANALYSTS
MAGNETIC ANALYZER

MAGNETIC BEAM PRANSPORT SYSTEM

MAGNETIC CMARACTERISTICS

MAGNETIC COIL

MAGNETIC COLLECTION CHAVNFL

MAGNETIC CORE

MAGNEPIS OEFLECTOR

MAGNETIC FIELD

MAGNETIC PELD

MAGNET TC IELD DIBTH IA

MAGNETIC FIELD MEASUREHENTS

MAGNETIC FIELD SETTING

MAGNETIC FIELO VARIATIONS

MAGNETIC FIELOS

MAGNETIC FIFLOS (RAPIDLY PULSFD)

MAGNETIC HYSTERESIS

MAGNETIC LENSE

MAGNETIC MEASUREMENTS

MAGNETIC POLES

MAGNETIC SEPARATION

MAGNETIC SEPARATION

MAGNETIC STRIPPING

MAGNETIC TAPE

MAGNETIC TECHNIOUES

MAGNETIC TOROTO ANTENMA

MAGNEPIT VS, MECMANICAL AXIS

MAGNETRON

MAGNETS

MAGNETS POWER SUPPLY
NUMAER OF OCCURRENCES

VOCABULARY WORO

MAGNETSIE FIELOS

MAGUIFIEO OATA

HAGNIFIED RAOSOGRAPRS

MAGSET

MAIN MEMURY

MAIATEVARCE

HAINTENANCE GICKGRTLNO "ATAOBASE

MAINTENANCE COSTS

MAINTENANEE FACILITY

MAINTENANCE MANULLS

MATNTENANCE PQOCEDURE

MAINTENANCE RFDORTI.G ETRNS

MAL IGNANT CELLS

MALIGNANT CELLS

MALIGNANT PUMORS

MALIGNANT PUM

MALPQACTICE

MAMMALTIAN CELLS

MAMARY ARENDCARCTNOMA

MAFMAFY CARCINOMA

MANOMACHINE COMNUNICATIONS CONSOLES

MANEMACHIAF INTERACTION

MAN- MACHINE INTERFACE

MANAGEMENT IVFRQMATZM.

MANAGFMENT INFORMATTO. SYSTFN

MANDFLSTAM RESTRANCF “D?FL

\section{MANIAC}

MANIAC II MACHITE LANGII.GE

MANIFOLI

MANNED OKHITISO TELFSCONE

MANPAIER

MANPOHER ESTINITES

MANUAL FORMAT

MANIIAL MEAC CONTERLLEG

MANIALL MASTER PIILSE

HARIJAL OPERATION

MANY AODY DRORLEM

AANYAROMY (GFT) ADPLOACM

MARK IIR LOGIC UNITS

MASS EFFECTS

MASS FLONMETERS

MASS SCREF INE

MASS SCREPN?NG

MASS O NUELEAR SYSTHM

MASSTVE RREAST CANCFR

MASSIVE SPILL CANTHOL

MASS IVE SPILL.

MASTEO CONTROL CHASSIS

MASTEQ CONTROL CHASSIS

MASTER COATROL FRAGEA

MASTER FIISF

MASTED TIMF

MASTELOSLAVE SWITCM 
MAXIMUM PION DOSE

MAX IMUM YRANSPORT EFFICIENCY TUNE

MAXWELLIAN OISTDPRUTIITN

$\operatorname{MAX}$

MAD

MAD CAPABILITIES

MAO OPERATION

MAD PROCESSOR

MBDSTD

MBDSTD PROGRAM

MCQETH DENSITOMETER

MCD

MCS

MEASUREO MUON-OISAPDEARANCE RATES

MEASUREO MUONOOISA

MEASUREMENT LINE

MEASUREMENT OF EXCITATINN FIMNCTIONS

MEASUREMENT OF NELITRON BPFCTRA

MEASUREMENT TOLERANCES

MEABUREMENTS

MECHANICAL PUMP

MEDICAL ACCELERATORS

MEDICAL APPLICATION OF YUONIC $X$ RAYS

MEOICAL APPLICATIONS

MEDICAL DIAGNOSPIC APPLICATIONS

MEOICAL LINAC

MEOICAL PHYSTES

MEDICAL PION GENERATOR

MEDICAL PLANS

MEDICAL PLANS
MEDICAL RADIATION EOUTPHENT

MEDICAL RECORD KEEPTNG

MEDICAL USE OF MUONJE $X$ RAYS

MEDIUM ENERGY

HFOIUM ENFRGY COLLISIONS

MEDIUM ENERGY NEUTRON SDECTROMETER

MEDIUM ENERGY PHYSITS

MEOIUM-ENERGY NULLEONS

MEOIUMOZ PARGET MATEHTALS

MELANOMA CFLLS

MELTTEM

MEMOAY AND HIGH SDEEO DATA PRANSFER CONTROL

MEROAY BOX

MEMORY PARITY DROREAM

MERRIMAC AOX

MFREIMAC GARTRY

MFRRIMAC TV

MESHATYPE CALCULATIUN

MF SHATYPF

MESIC ATHM

MESIC CHFMISTRY

MESIC XORAT SPECTRA

MESTN CAPTUEE A.D MIION ABCLICATIOAS

MFSON CAPTURF YTELTS

MESON CAAWREL

MESON CHF MISTR

MESOV FACILITY

MESCN FACTORTS

MFSON FACTORY

MFSTN FACT TRY
MESON DHYSICS

MESON PHYSICS FACILITY

MESTV PROEES

MFSUN PAORUCTIOA TAHGETS

MESONENUCLEUS IATRRACIITN

MESOVIC ATOMS

MESTVIC X-LAY TDIPENSITILS

MFSONS

MFSSYMESH

MESSYMESH CALCULATTINAS

MESSYMESH DATA OROCFSTI. I

MESSY ESH DROCRA

METAL DIATING,

METALIZING EEQANIC

METALI OGAAPHY

METALLUUGY

METASTAALE AEAM

METASTAALE DOLADIZLU DANTIV SOUACFS

METASTATIC AONULES

METER

METERING CHASSIS

METERTNG SYSTE

METRDLOGY LABCRATIRY

MAC

MHL UnCXUPS

MICF.

MICPOCCNMP:JTEA

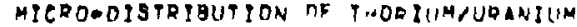

MICRODOSIMETRIC GALCUI TITANS

MTCR: IDSIMETRIC TEEWNIOIES

MICRODOSIMETRY

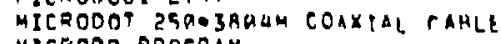

MICEOPB DQOTEAM

MICROPHORES 
NUMBER OP

OCCURRENCES
YOCABULARY WORD

\section{MPCROPROCESS OR}

MICROPROCESSOR CROSS- ASSEMBLER

MICROPROCESSORS

MICROPROGAAMMED BRANCH RQIVER (MBD)

MICROPROGRAMMED BRANCM DRIVER

MICROPULAE

MICROSWTTCHES

MICROTRON ACCELERATOR

MICROWAVE CAVITIES

MICROWAVE IRRAOTATIONS

MICROWAVE MAGNETIC RESONANCE METHRD

MICROWAVE POHER SYSTEM

MICROWAVE REBONATOR

MICROWAVE TECHNIOUES

MICROWAVE TUBES

MrOAS PROGRAM

MINERAL - INSULATEO CABLE

MINERAL INSULATED CONDURTORS

MINERAL-INSULATED MAGUET OREPARATION

- INBULATEO MAGNeTs

MINERAL-INOULATEO SOUARE-SECTION COPPER CABLE

MINICOMPUTER

HINICOMPUTER OISK SYSTEMS

MINICOMPUTERS

MINIMUM PHASE WIOTH BIJNCH

MINIM

MIP

MIRROR

MI8

MISALIGNMENT

MIBALIGNMENT ERROR

MISMATCH FACTOR

MISMATCHED LOA

MISSING MASS GPFCTROMETFR

MIU

MIU GINARY COMMANO

MIU CARD CAGE ASSFHALY

MIU CONMECTORS

MIUARICE 110

MIXEO LET

MEXEO NEUTRON-GAMMA IEIO

MIXEO RADIATION FIELO

MIXING TOLERANCE

MK II B BOARD

MK II BOARD

MOQILE REX

MOCK UP FACILIT

Mock

mockum

MOEN COMMINICATIONS SYSTEM
NUMAER OF OCCURAENCES

VOCAULARY WTRD

HOCKIJP CONTROL SYSTEM MCEKUP EXPERIMENT

MOCKUP FACILITY

$M O D=A N O D F$

MODE SPECTRA

MODEL GA SURVEY METER

MODEL 73-115 TEMPERAT,RE PONTROLLEA

HODEL DEPFNAENCF

MODFL DESIGN

MDDEL $F$

MODEL F ACCELERATOR

MODEL $K$

MODEL $K$ ACCELERAT OR

MODEL $K$ PRIDGE COUPLEQ

MODEL L

HOOEL L ACEELERATOR

MODEL L ACCELERATAR TANL

HODEL L EXPERIMENT

MODEL $M$

MODEL

MODL M ACCELERATOS

MOOEL MAGNET

MODEL MAKIN

MODIFIED ROSSI COUNTES

MODULAR OIGITAL SYSTE.

MODULAR FORMAT

MOOUL TR LOCAL CONTRCL

MONULAR SYSTEM

MBDULATING ANODE

MOOULATING ANMNE WOLULATOR ROEKV

MOOULLTOR

MODULTTIR 2A1,23 MHZ

MODULATOR DEVELOPME:T

MODULATOR GRIDTCLAMP

MODULLTOR POHFR SUPPLY

MCOUL POR PROTECTIOA

MODULATOR SYSTEM

MEDIILATIR TAAK

MOOULATCR TESTING

HOOULATOR TIIBF

MODIILATIHA TUBE VOLTAGF "OL DECFF

MONULATOH TUQRen

MODULATORS

MDDULE 1

MODULE

MCOULF CONTROL

MODULE EQUTPMFNT

MODILLE I

MONULE INTERFAC

MOOULF LDGIC 
NUMBER OF OECURAENCES

VOCABULARY WOAD

MODULE TESTER

MODULE TESTING

MODULE VCU

MODULE VCU LOGIC

MODULE WATER SYSTEM SIMILATOR

MODULE $X$ CDNTROL POWER

MODULE TOMMOULE COMMINTCATION

MODULE-TOMMODULE FIFLD ADJUSTMENT

MODULE TOAMODULE PHASE

MDOULES

MODULES $201.25 \mathrm{MHZ}$

MODIULES BOS-MHZ

MOEA CDNFERENCE

MOLDS

MOLECULAR SYRUCTURE

MOLYBOENUM

MOLYBDENUM TAROETS

MOLYBDENUM

MOMENTARY 2 POT

MOMEVTUH

MOML IUM (P) DEPENOENCE

MOMENTUM ACCEPTANCE

MOMENTUM AITE

MOMENTUM COMPONFNTS

MOMENTUM DETERMINATIOOI

MOMENTUM ME SSUREMENT

MOHE YTUA OF CHARGEO PION

MOMENTUM OF PROTON

MOMENTUM RESOLUTION

MOMENSUM RESOLUTION

MOMENTUM SPREAD

MOMENTUM SPREAD

MOMENPUM-ANALYZFO GEAM

MOMENTUM

MONITOR CARD

MONITOR DETECTOR CRITERIA

MONTTOR LOOPS

MONITIRA SCREENASIZE

MONITOA SIGNALS

MONITOA SOFTHARE

MONITOA SYSTEM

MONI TORING

MONI TORING SYSTEMS

MONI TORS

MONOENERGETIC BEAM

MONOF : LAR HELICAL CATHONE

MONTE CARLO

MONTE CARLO CALCULATIONS

MONTE CARLO CASCANE

MONTE CARLO CASCADE CODE

MONTE CARLO CODE
NUMBER OF

OCCIIRRENCES

OCABULARY WORD

MONTE CARLO PROTRAM

MONTE CARLO TECHNTGIJES

MONTE CARLO TRANSPORT CODE

MONTE CARLO TRANSPORT PAOGRAM

MONTE CARI DOTYPE CALCILATIONS

MONUMFNT

MONIIMENT STATSOHS

MOSSAAUER EFFECT

MOTLR DROGRAM

MATOR

MOTOR BAAKE ACTUATOL

MOTOR CONTLCL

MOTOR PROGRAM

MOTOR STEPPER BOAROS

MDTOR ODRIVEN CONTEIILS

MOTOR-GENERLTOR

MOT MR-GENERATOR SET

MOTOHIZEO CONTROL OHIVE

MATOROLA GOAO MICROPRACLSSOR MOTOROLA LOGIC

MOTOAS

MOUNTAIN STATES TFLEPHOHE CO,

MOUNTING PLATE

MOUSE TIIMORS

MOVING PION BEAMS

$M P-13$

MP-I FOUIPMENT SYSTFME

MP - 3 PRE AMPL IF IFR

MP-Q TO MP-B TRANSIIIIN

MP 3 AMPLIFIER

MPU

MRA PROGRAK

MU MESIC LIFET THE

MUAMESIC $X \in R A Y$

MU. MESIC XORAY MEASURFMENTS

MUAMESIC XORIY SPECTRA

MUSOMFSIC $X=R A Y S$

MUOMESON FLEMENTAL ANALYSIS

MIJPPROCESSOR

MUINTSLE PROGRAM

MULLARA SL-75 LINAC

MUL T - ENERGYOGR

MULT IOEAIN

MUL I ? INPUTOOUTPUP FEFDHATK CONTKOL SYSPEM

MUL IIOPROGRAMMING MCOS

MULT I-SECTOR TRANEFER

MULTI-TASKIAG OPERAPING SYSTEM

MUI TIOWIRE PQODORTIONAL COUISER

MULT I $\triangle P E R T H Q E$ GRIDDED SNUUCF

MULTIBRANCH CONTROLLER

MULTICAPILLARY COLLIMST 
MULTICAVITY XLYSTROO

MULTICHANNEL CHERENLOV COHNTER

MULTIEHANNEL OISC CERENKOV COUNTER

MULTICHANNEL LOGIC OELAY SYSTE

MUL TICAATE APPLICATIOUS

MULTICRATE SYSTEM

MULTIPACTOR

MULTIPACTOR ELECTRON GU: (MEG)

MULTIPACTOR ELECTRON CUN

MIJLTIPARTICLE EVENTS

MULTTPLE APERTURE SOURE

MULTIPLE COULOMA SCATIER ING

MULT IPLE DRIVE

MULT POL TNFERACT IVE CRT OISPLAY SYSTEMS

MULT TOLE TNTEAACTIVE CAT DIS

MULTIPLE KEYBOARD TTERFACE

MULTIPLE SCATTERING

MULT TPLE SCATYERING

MULTIPLE SCATPERING ANGIES

MULTIPLE SCATTERING TWERRY

MULTIPLE SCATTERING OISTRTEUTIONS

MULTIPLE STORAGE SCOPES

MULT IPLEXER

MULTIPLEXING

MULTIPLIER ENVELOPE BRONNTNS

MULTIPLIER WPHOTOTLBE

MULTIPLIERS

MULTIPOLE FIELOS

MULTTODLE HAGNET

MULTIOLLE SCATTFRING

MUL PIDRDEESSOR CONFIGHATION

MULT TSTEM

MULTIVARIATE ARALYSIS

MULTIWIRE CHAMBFQ

MULTIWIRE PROPORTIONAL CHAMRERS

MULTIWIPE PROOORTIOUAL CMAMHER SPFCTROMETER

MULTIWITE PROPORTIONAL COLINTERS

MULTIWIRE PROPORTIORAL CHAMAERS DEVELTOUES

MULTIWITE PPOPORTIONAL CHAMBER

MULTIWTRE PROPORTIONAL OEVELOPHEN

MUMPS

\section{MUON GEAM}

MIION CAPTURE

MUON CAPTURE PROBABILITV

HUON CHANNEL

MUON CHANNEL, DION ACCETTANE

MUON ELOUD

MIION DECAY ELECTRONS

MUON DEPOLARIZATION IA. LITUTO HELIUL

MUON ELECTRODYNAMICS

MUON EXPERIMENTS
VOCABLLAE MRD

Mach Fux

HIOA. GA? EYOEPINEAT

HUOV INTEASITY

MIJON MAGNETIC MUMEST

MUOC MOMEAT

MIJOS WULIIPLE SCATIEPINE

HUC, ORIGINS

HUO: DQDPERTIES

MUOS TELSSCHOE

MUOR XQRAYS

MUON-CAPTLRE SACCESSES

MUCHOLLTCTROA SYMUETR

MUCVAnURA CCLIISICVS

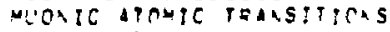

Nisito atoms

MIONIC MYMRAGEY

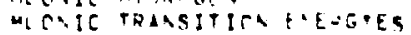

MUNIC X RaYs

mionic xorat lises

mucaic regar miasiagments

muchic ropar sofetra

MUORIC $x=0 A Y S$

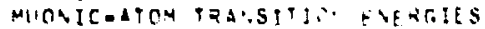

MIST.IUM

mIONIUA T10\%

MUCYI.1W CrE HISTAY

MUONIUM FORUATIOA

MUD? IUA HFS

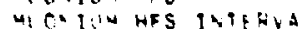

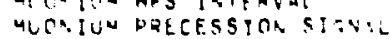

Mi!ONS

MLOASIJM GESORACE CUABS

Mा104

MUTLI-TASKING MORE

atix

mux caAle

Mix PLJ?

$H_{n} D C$

HaDE AMRLIFIER

unde ristoingatha

WADC ENCONER

Mmot ENCONERS

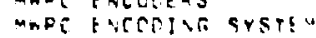

MHDC STFTTETMETER

WYOCAROIAL IUACINO

MYOCARBIAL STABAINO

$\checkmark$ RFA. Dll sis

NiD afatTiOM

VACE PLFE CAVITIES

vo inisacitios

A. D SCATERTAG 
NOP SCATTERTAR, EXOENIMEATS

NAT DETECTCES

NAL

NAND GATES

NANOS

NATIINAL COMPUTER CONFEDENCE

NO ELABTIC SCATTERING

NEAR OEGENERACY OF VUCLEAR EXCITATITA

NEAR-GAUSSIAN DISTRIBUTTOUS

NEGATIVE HADRON

NEGATIVE ION INJECTIDR

NEGATIVE MESON CAFTURE IN MATTEO

NEGATIVF MESON CADTURE

NEGATIVE MESONS

NEGATIVE MU MESON

NEGATIVE MUON

NEGATIVE MUON BEAM

NEGATIVE MUON CAPTULE

NEGATIVE MUONS

NEGATIVE PI MESONS

NEGATIVE PI MESONS FRO CANCFR TUFHAOY

NEGATIVE PI D GOES TO NGAMHA RFACTIOA

NEGATIVE PION

NEGATIVE PION

NEGATIVE PION CAPTURE D. TRITIUN

NEGATIVE PION CAPTURE

NEGATIVE PION CAPTURE

NEGATIVE PION RAOTOTHFRAP

NEGATIVE PION RAOIDTHFR

NEGATIVE PION

NEON IONS

NEOPLASTIC CELL POPULATION

NEUTRAL BRANCHING RATIOS

NEUTRALIZATION

NEUTRINO

NEUTRINO AREA

NEUTRINO CAPTURE

NEUTRINO DIEI NTEGRATION

NEUTRINO FACILITY

NEUTRIND FLIIX

NEUTRINO FUX SFCONDAOY AFA US

NEUTRINC PLUX

NEUTRINO HOUSE
NEUTRINO SHTELOING

NEUTRTNO SATELOTNA

NEUTRINO SHUTTER

NEUTRINO BOURE

NEUTRINOANUCLFUS REACTIAN

NEUTRON AYERIGING METHOH

VE TARD AEAN

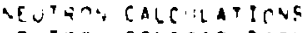

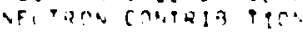

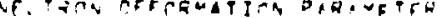

A.

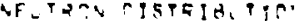

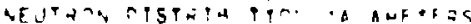

a.Fitiona cese

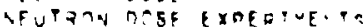

ifuizon bosimtior

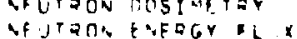

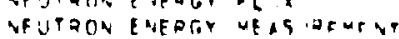

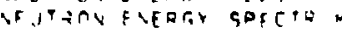

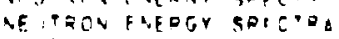

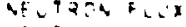

Neutiry flux stavinat

WFiT2ON FLIX G TOFATU?

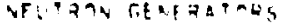

AF

AFC TEC HALC CF LOA

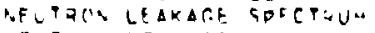

arolach ucatiog

QEvinas oursics

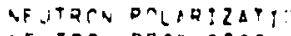

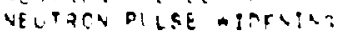

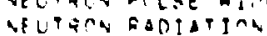

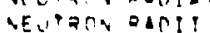

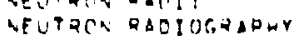

DF.TRON RBUTVAL

AELTOCL NESFAEC.

AEUTRON SCATTEIT,

NFUTOON SCINTILATIMAC

NEUTION SETSTRS

NEUTRON SWUTER

NEGTHAN SOLIRCF

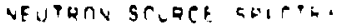

NEUTSCN SOECTRA

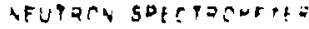

TEUTरण 501610.

DEUTCOSTLR

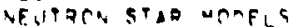

WFUTRJA STRFAC:0

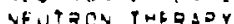

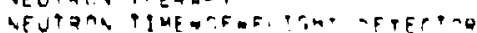

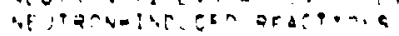

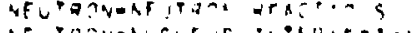

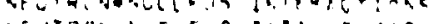

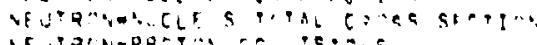

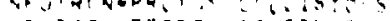

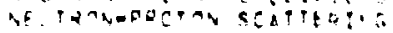


NEUTRON/PROTON AMPLITUOO

NEUTRONICS

NEUTRON

NEVIS

NICHOLS CHART

NICKEL ISOTOPES

NIDRUS PROGRAM

NIM

NTM OTN

NIM CONTROL MOOULES

NIM CONTROL MOOULE

HIM ELECTROHETER
NIM ELECTRONIC MONULE

NIM MOOULE

NIM PROGRIM

NIM-BIN WIRING

NI MR OD

NIOBIUM GERMABIOE

NITROGEN

NITROUS OXIOE

NMTE PRDGRAH

NOALE GAS AOHIXTURES

NOBLE GASES

Noise

NOISE ACCURACY

NOISE ANALYZER

NOISE IMHUNITY

NOISE SOURCF

NOISE SOURCES

NOISE SUPPRESSION

NOISEOFREF ECG

NOISE-FREF ECG

NON-DESTRUCTIVE ANALYSI

NONDDESTRUCTIVE TESTIVG

NONOGALILEAN INVARIANT OITN PRORUETION RDERATIR

NONEHERMITIAN HAMILTONIAN

NONOLINEAR SIFERING FIELO

NONEMDNDTONIC ZOVARIATINNS

NON-PERIODIE STRUETURES

NONGAESONAN

NONG THERMAL EFFECTS

NONDESTRUCTIVE TESTINE

NONL INEAR GADIO SEAN

NONLINEAR RADIO

NORTM ORT ARF

NORTN FORT BEAM

NORTMPOR P RUNS

NOSF OSPRE TCWER

NOTCH AREA

NOVA

MON COMPUTEQ

NOVA INTERFACF

NCVA PANGEA

VEVA SISGLE USEOS ALS:C

Mracavac conizolits

VCXITIS GAS GEWER

verinks Gosts

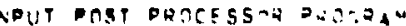

NAz CEDF

NuCl 5 AR ABSORET

VucleAR caDivat

NHCLEAT CBNPE NIOJE MUMENT

villear CHARG Distolajeita

NUCLEAR CHARGE WODE

NUCIEAR CHEUISTIZY

NUCLELR CMEMISTRY HLILOTNG

NUCLEAR CHEHISTRY EXOER MENTS

NLCLEAR CMEUISTAY LAO

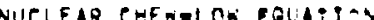

NUCLEAR EFFECTS

NULLEAR ELECTAIC OULDIUNTL WAUTAT

NUCLEAR ENERG JSES

NUCLEAR EXCITATIOS NERTV

NUCLEAR FORCES STUR

NUCLELR INELCSCIC INTGAICTIA

NUCLEAQ INPQRACIITN

NUCLEAR LEVEL SPQ,ICTUIE

NUCLEAT MATTEF

AUCLEAR NEOIEIS

NiELELG MOATEOCARL: THA.SOCEI COAI

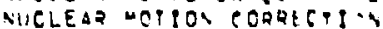

vicifia ontsics

HCIEAz OATEICS RFSEATC

W.CLEAP PICA. CAOP

NUCLEAR REACTITAS

NUCLEAR FESTNINCF

WCLEAR OESCAMACE ERFE

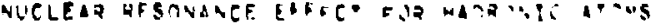

YUCLFAH SCATTEQTSE.

TUCLEAQ 5PECTYOSCOD

WUCLEAR 5 TH CTURE

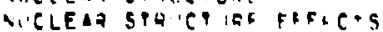

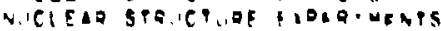

$\checkmark$ Cleat gin et aE Hisia:c.

A. Clecta girletiof SiJay

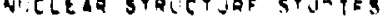

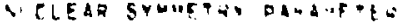

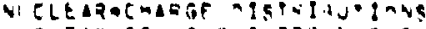

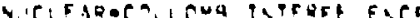

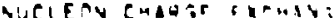

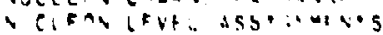



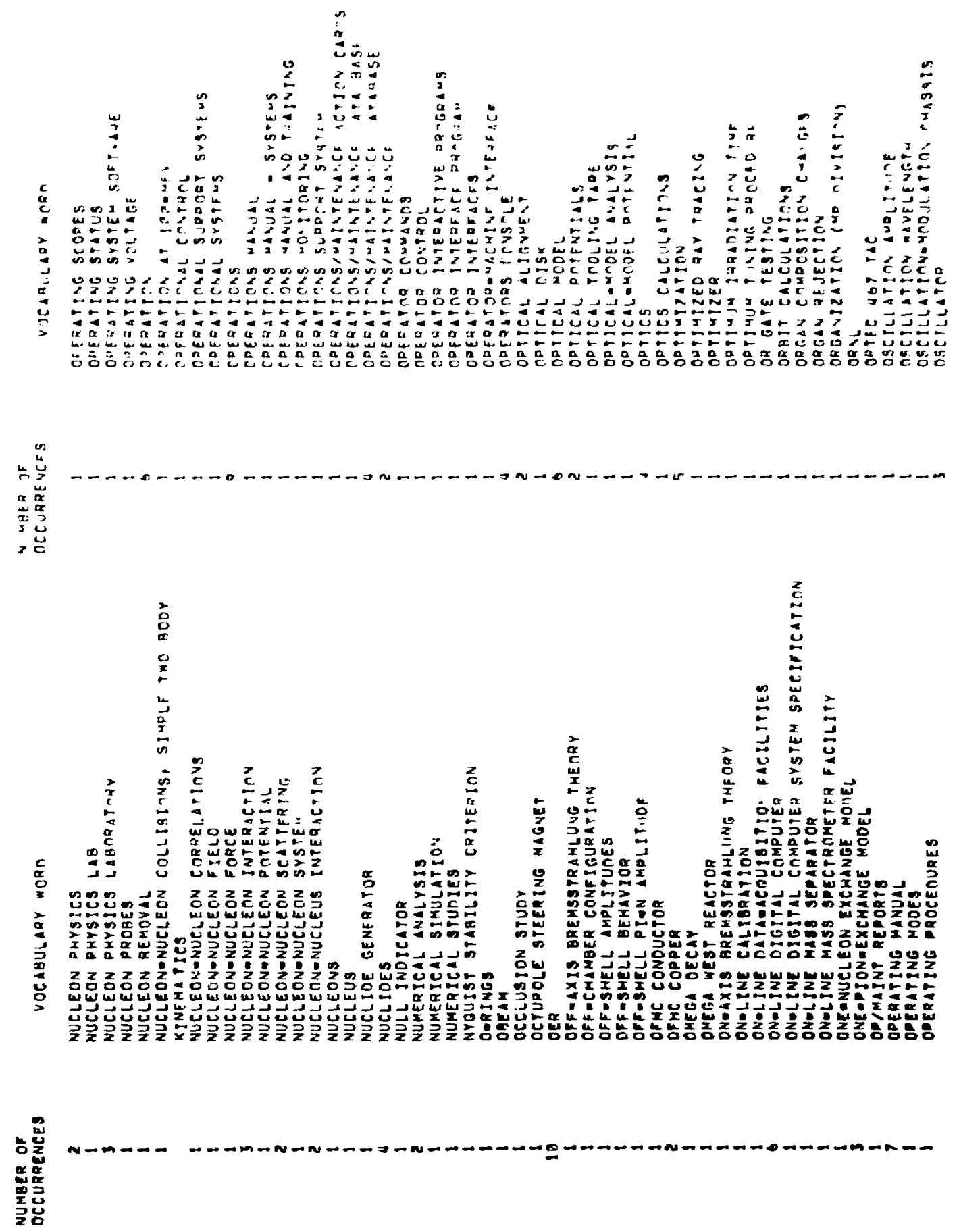
OSEILLOSCOPE CONNFCTINN TEST BOX OSMIUM

QSTEOGENIE SAREOM

OUTGAS

OUTGAS MATERIALS

OUPGASSING

OUPBY

OUPPUT EIRCUITRY

OUPOUT CURRENT

OUPPUT GATES

OUTPUT PULSE

OUTSIDE CONTRACTS DATA RASE

OUTSIDE CONTROL POINTS

OVERLOLO PROTECTION CIRCUITS

OVERRELAXATION COAES

OVERRIDES

OVERSHOO

OXYGEN

OXYGEN CAPTURE

OXYGEN EFFEC?

OXYGEN ENHANCEMENT RATIM

OXYGEN ION BEAH

OXYGENATED CONDITIONS

OZONE

OZONE GENERATION

P BAR ABSORDTION

P BAR XTRAY EXPERIMENTS

PQP DATA

P,N REACTIONS

- OP COLLISTONG

Pop INTERACT IONS

Pop scatTERING

-ap tRIPLE scatTERING

pomater scaptfoing

Pohave

Dave PIONUCLEUS interaction

PuWAVE PIONANUCLEON INTERAGTION

Pawaye plon-nueleus

P3

PJ REAM

-3 ChanNel

Pac

pace paogay

PACIFIC ELECTRIC MOTOR CO.

packagine

paer formatting

piging system

paig spectogmeter

Pallabiua

pallagija hripoger lesk

PALLADTUW LEAK

PALC ALTO

DANEL

ANEL ANE RACK LAY:

$P \triangle D E P A T A P E$ CODY PROGPAM

PARA HYDROGEN

PARAIIELEPLATF CADACI OE

DAFAHETER WANAGFMEAT

Paqa veTEL OPIIMIzaTio.

PARAMFTER SEADCH

PAR AMETERS

DARAMFTRIC STUNR

PARASITE BEL

Darevit icas

paritr rufFer

paritr consezvation

olaifir hixing

paritr voneconsepving comalageve

paigit no.conseguato.

oaritir srmaftar

papjty violativg asYMMETAY

olritr violatina

PARITYUVONCONSERVING ASYMAETAY

PAFITYOVIOLATING ASYMIETAR

DARITYOVIOLATIAG FFHECTS

PRMILA

DAfimilia coDof

PARMILA HQOGRAM

PARTIAL OFEIUATIVES

DARTIALS

-articlel acCalfratols

OARTICLF ACCELFPATOLS

dapticle accelehator

Darticle deAH

DAFTICLE MFCAYS

onericL DE VSt

oavticle distriaugina $5 \cdot t+120$

Daticle ornamits

Dapilel interatigo.5

ohiticle ution

Darticif phrsics

dapticle physics atsengen

pIRTIELE DHCDUCTI!"

DARTICLF SCMEME

olaticla stpatatoo

DAHTICLE SPECTAOUFTF

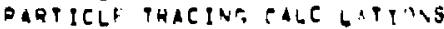

oarticle pajectoger

onepicle yeloc Itr

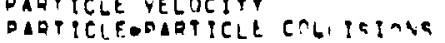




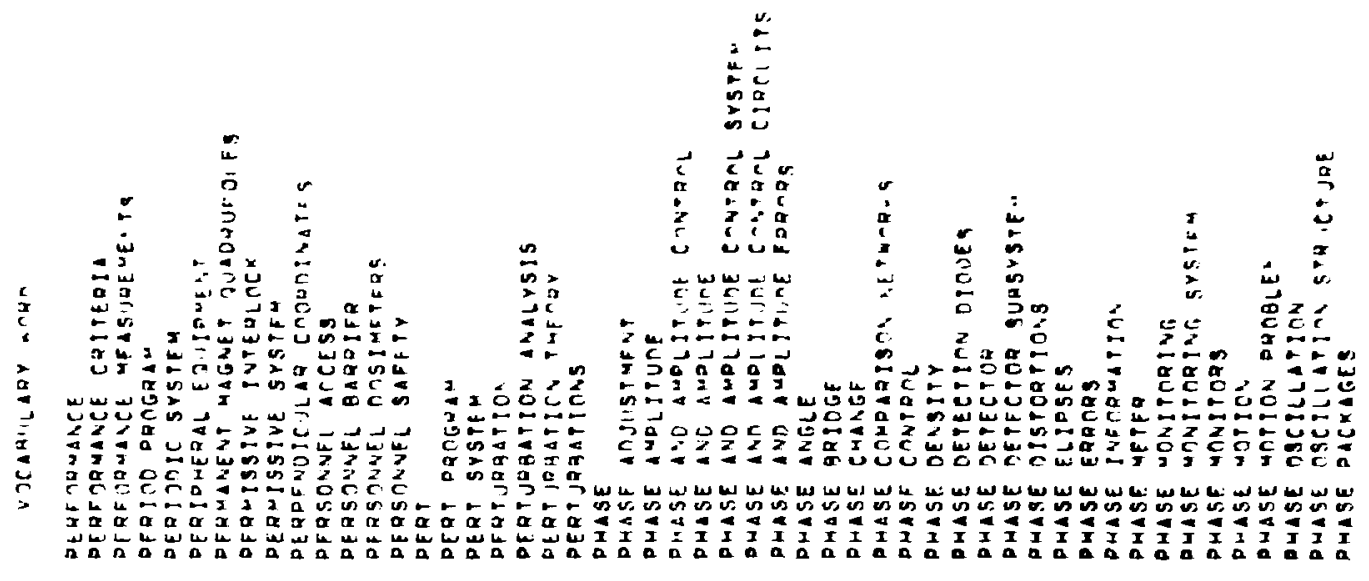

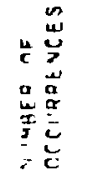
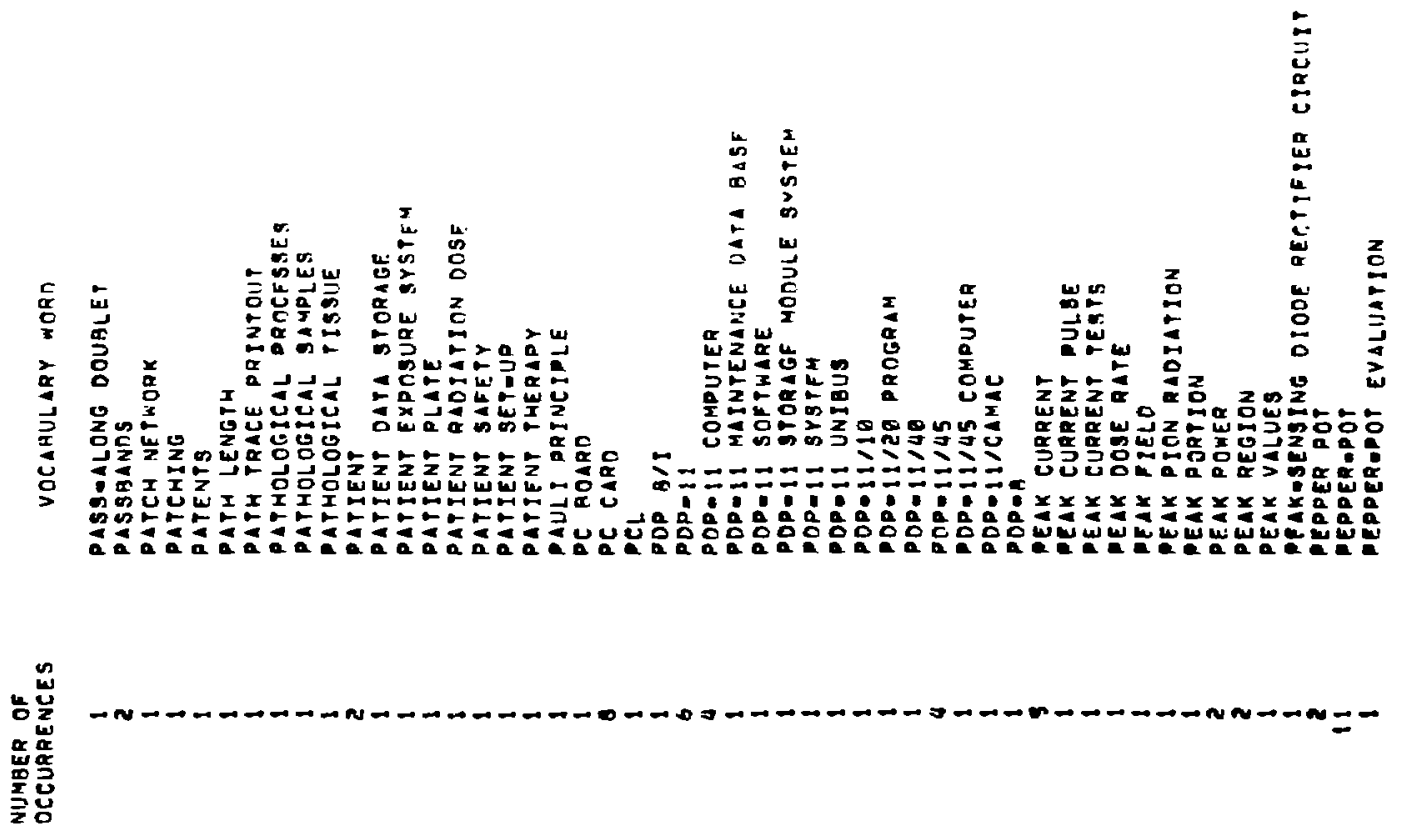
phase RegPonse

PHASE SCAN

PHASE SCAN bIAS

PHASE SCAN GEAR

PHASE SCAN PROGRAM (317?)

PHASE SENSITIVE DETECIION

PHASE SHIFP

PHASE SHIFT ANALYSIS

phase shifiea

PHASE gHIFTER CALIBRATINN

phase SHIFTER DRIVE SYSTEM

phase shitier positions

PHASE SHIFIERS

PHASE SHIFTING

phase soace

phase spice acceptane

PHASE SPACE BOUNDARIES

PHASE SPACE DISTRIBUT IO:S

phase space mapehing

PHASE gPaCe PAPTERN

PHASE SPACE TAILORING

PHASE SPACE

PHASE STABILIYY

PHASE STAOILIFY

PHASE SUBSYSTEM

PHASE POLERANCF

PHASE VELOCTYY

phasemalpi ys andoe subply voltage

PHASEOSPACE

phaseramolipuoe conirol system

PHENOMENOLOGJCAL OPPICAL DOTENTIAL

PHERMEX

PHOSPHOR LIFETIME

phOSPHOR LUMSNOStTY

PHOSPHOR MONITORS

PHOSPHOR SAHPLES

PHOSPHORE

PHOSPHORS

PHOPODEPAEMMENT CROSS SECTION OF HO

PHOPODETACHMENT RESONANCES IN THE Ho 10 .

PHOTOQETAEMMENT STUDLES

PHOTOOISINTEGRATION

notolecthic codsosecitans

PHOTOGRAPH PLATE OETEPTION

-hotomulPiPlien light COLLeTOA

PHOTOMUL PIOLIER SEINTILL ATOR

DHOTOMULTIOLZER PUEE AAGE

PMDTOMULTPOLIEAS

D.TOTOEUTROA

PAOTONEUTAOA 50HRCES

DHOTOUS

DHOTOTJAE BASE

OHOTOTUBE CNECK FAG STI.TMLGTSES

PHOTITJAES

PHOTOTJAES RASE

DHYSTCAL BTRUET RE

PHYSICS EXPEAI MENTS

- I CMARGE RIOIUS

- I CJRED CHASAEL

- I NESON DOSTHETRY

DI UODE

DI NOOE ExETtitia

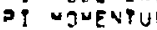

DI DI INTERACTIONS

DIt, REACTIOAS

$P I+C F A$ IKELASTIC $5 C A T+E=T A$

DI. AEAM SOILL

- i. SeAmg

of. mesor a ojopheraer

PI. STAQ

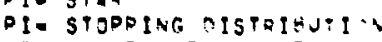

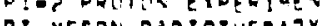

Di-nESON Rarictuegad

Din"esos inERADY

DiomesONS

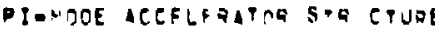

- IovORE ORIVE

DI-MOCE SYSTEMS

F-AJCLEAR CRCSE SFCT.O.

D indelFus SEATTERIVG

DIIN STRTROERS

Dis noDe

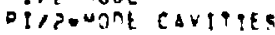

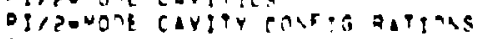

Disounat fatiugner

- Ia 1

diccaudaf Daver

pioria phogolu

Dieve

- peqce innof

- ieder crlana

- geve nistognupina

Ditoer electamors

Disoce fitoactirs

Diface fiel?

- ieqeF Gecuetar

DIflu

oge ix ognegan 


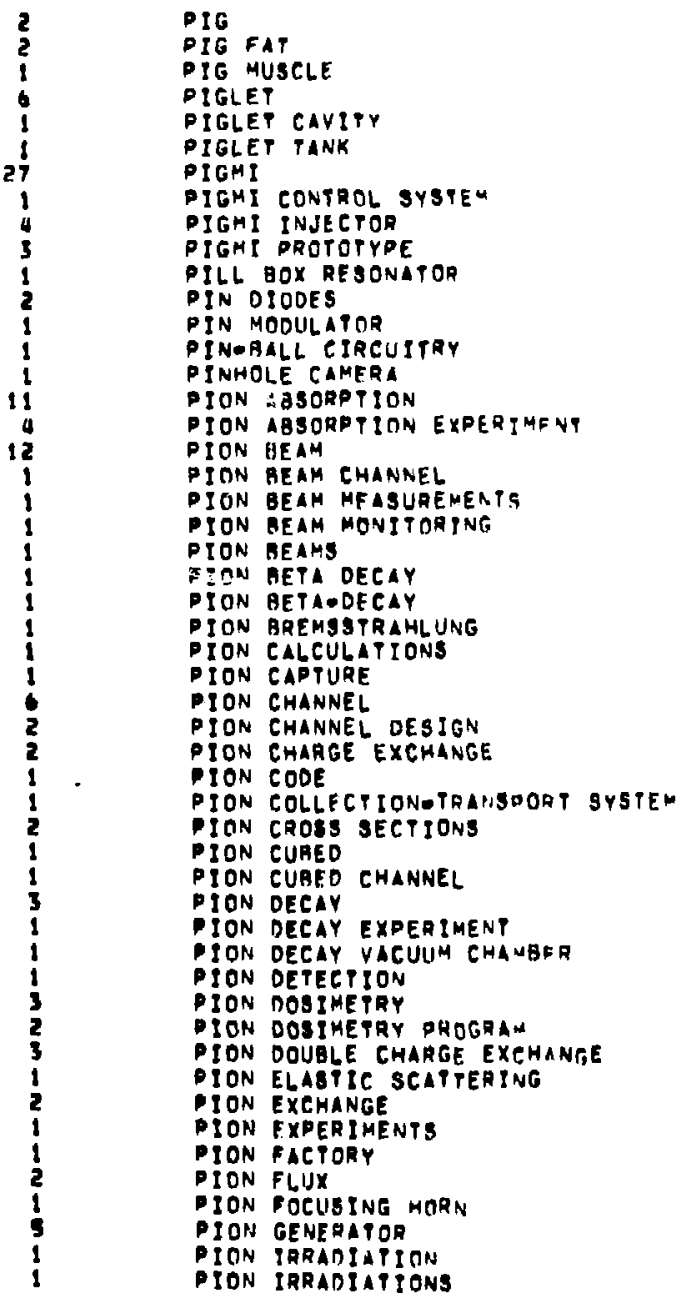

10.

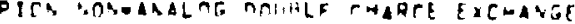

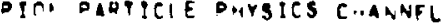

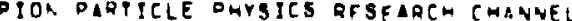

pich pamolictitan

pion ponfuetion higke

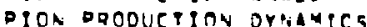

pion oqnojetion expepias

pion Dannifetion iage?

pior ponnuepion rapoets

ogn. DQRouetiay intal

pion poCgRay

PIOA PADTation

Pop. FaOTCBIOLOG

DIO. RADIOTWFADY

Dics QANGF SHIFter

DIn!, RATF

Dicis scattFatato

DIOV SELF EVEFGY

- iri. snilace

Dion SPECTRA

DPC: SDECTOCLETEA

- ionsot size

DIOV STIRS

Dion STCPDIQ

oing pHerip

- goa imehapy Facllity

PION TOTAL CanSE SECPiO.

D TON TRFATMENT CONEO

PION, MUOCN

DION-CHARGE EXCHASGE

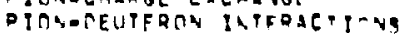

DgOr-nOSIMEPRY

- ION-INDUEEO NUCLFAR DEACTIONS

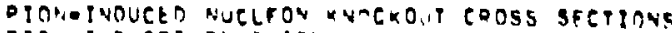

DICAAINOUCER a ACTICVA

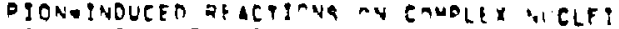

DIONGMUON SFPLATION

- TOVODUCLECS

PIOM-NJELF OS AMDLY TOF

PIOSENUCLE ON INTERIETYO. S

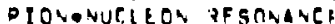

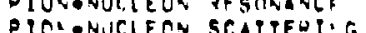

Pionanjelfus

PIOA-NUCLEUS TNTERACTiO.

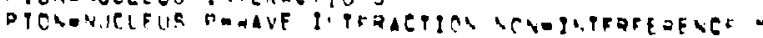
ODS

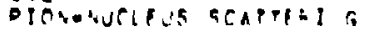

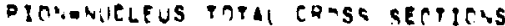

ION IRRADIATION

DIOA-DION COLLISITAM 


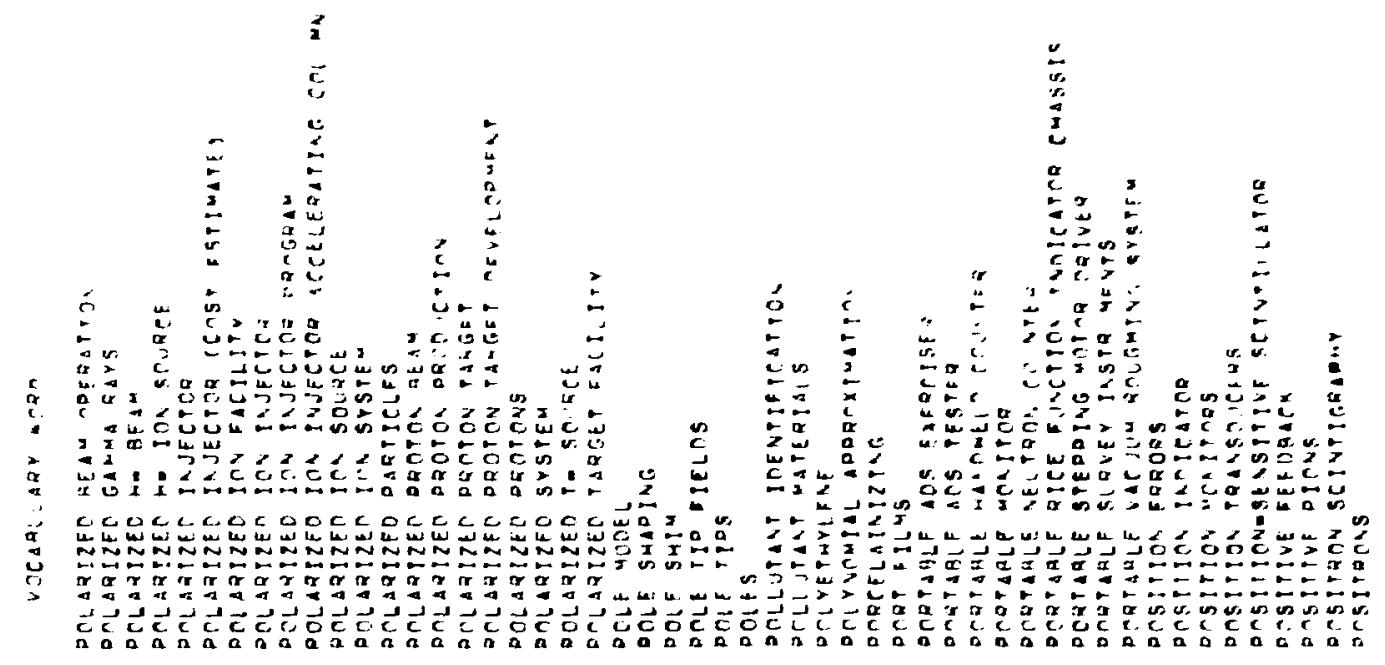

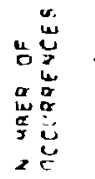
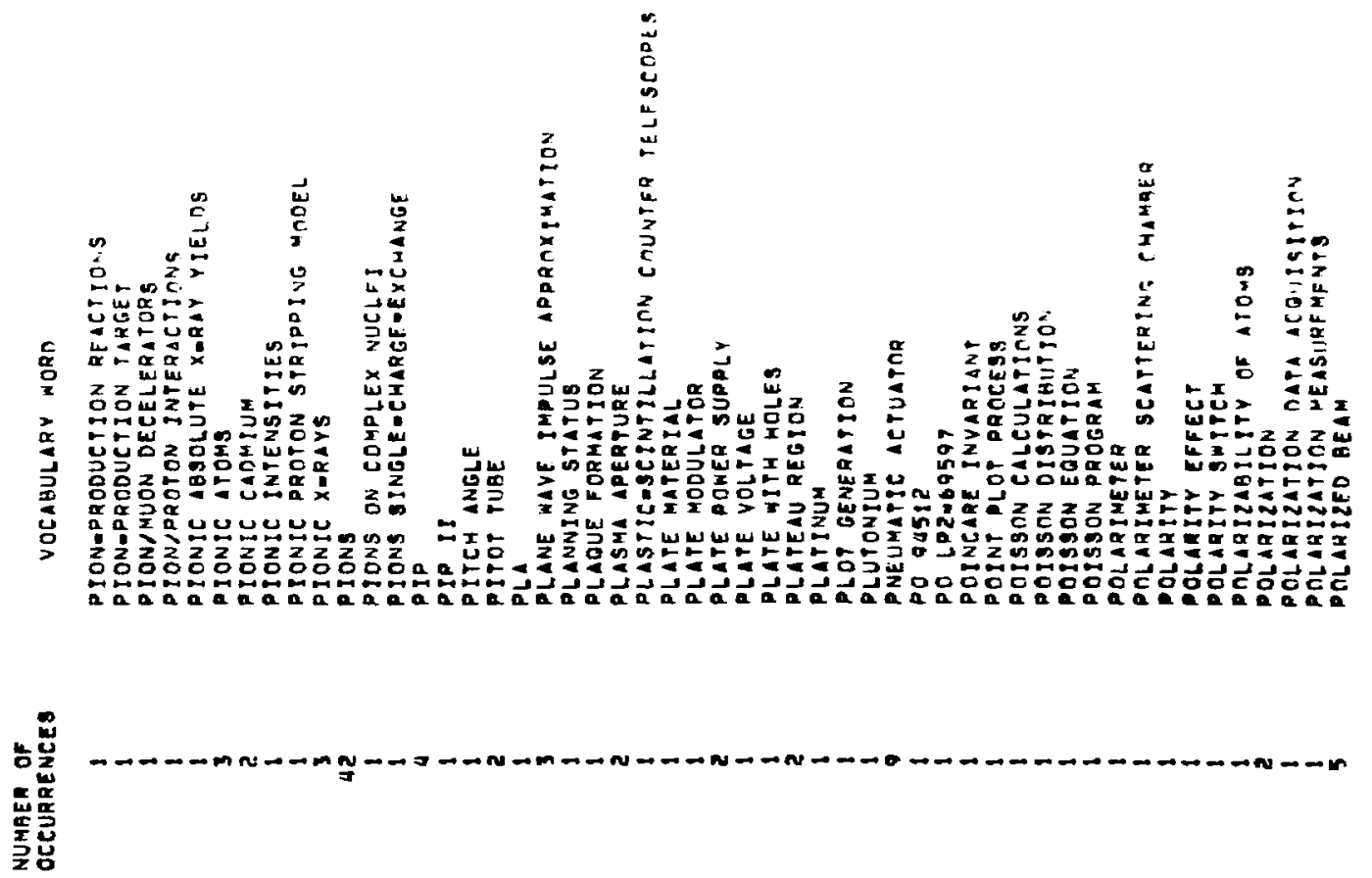


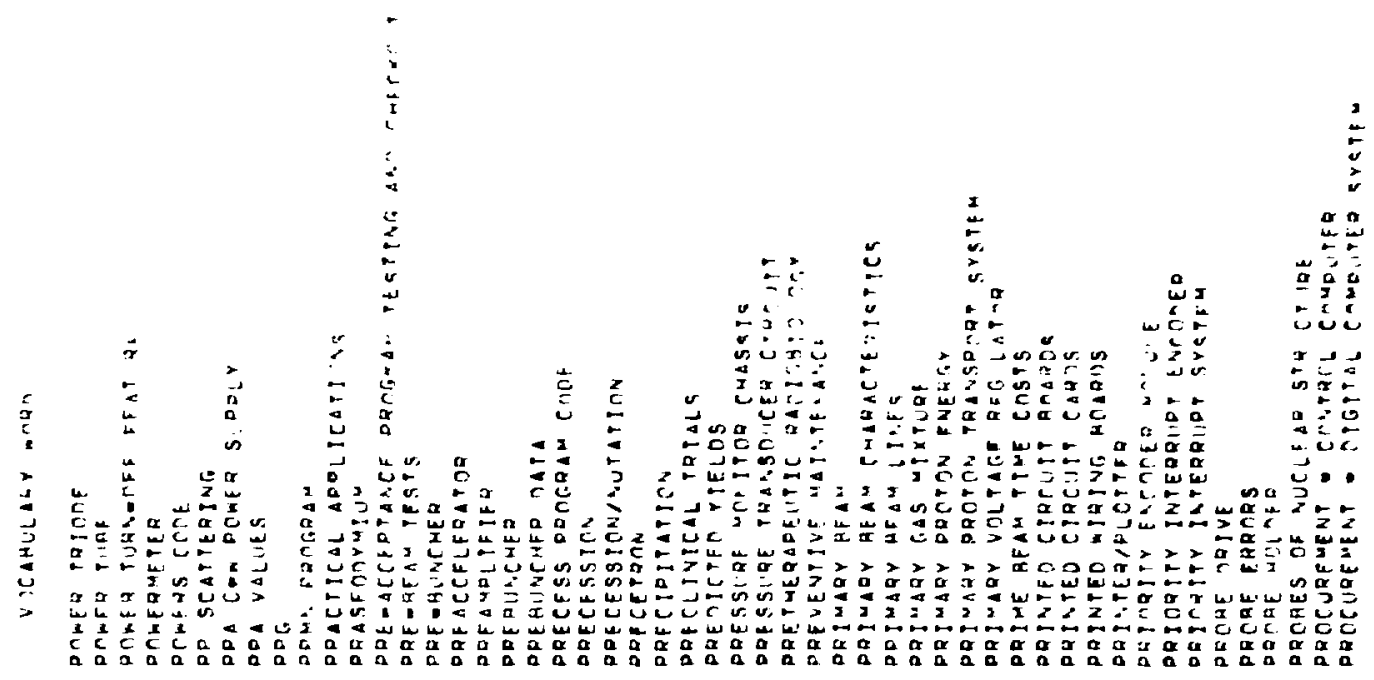

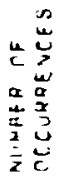

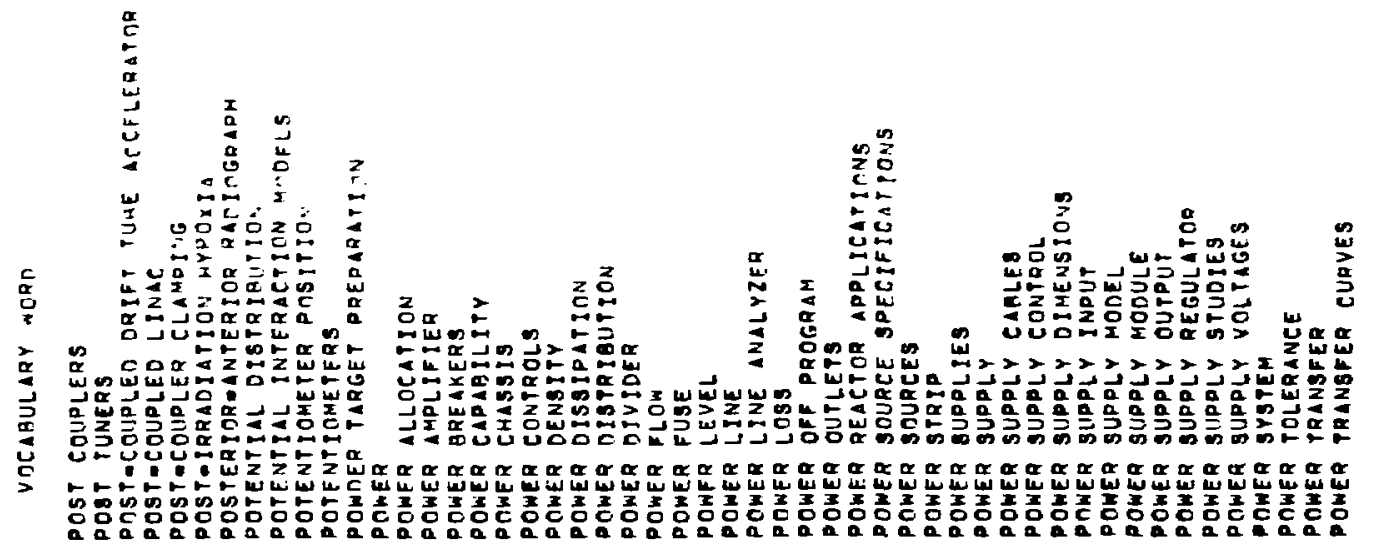

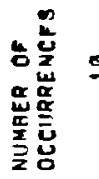


PRODUCTION RUN

PRODUCTION RUNS

PRODUCTION PARGETS

PRODUCTION YIELOS

PROFESSIONAL WOMEN

PROFILE MEASUAEMENT

PROFILE MONITOR

PROFILE MONITORS

PROGRAM 3:7A

PROBRaM CoUnter

PROERAM EXP

PROGRAM MODULES

PROGRAM O

PROGRaMmLBLE CLOCK

PROGRAMMAQLE COMTROL DANEI

PAOGRAMMERS CONBOLE

PROGRAMMING

PROGRAMMING MaNUALS

PROGRESS REPORT

PROJECTIONGOPERATOR TECWNTQUES

PRomet gavia RaYs

propagation

PROPAGATION
PROPERTY CONTROL

PROPORT IONAL CHAMBERS

PROPDRTIONAL COUNPER

PROPOSAL

PROPOSAL NO, 114

PROPOSAL NO. 32

PROPOSAL NO: 59

PAOPOSAL NO: 96

PROTECTION EITCUIT

- Rotection logle

PROTECTIVE CIRCUIPQY

-Rofective oEvice

PROPECPIVE GUAGE INDUETAR

PROTEIN OF MALIGNINT CELLS

DROTON

PROTON ACCELERATOR

PROTON ANALYZINE PONEH

PROTON ASYMMETRIES

PROTON BEAM

L

PROTON BEAM EMITTANEE

PROTON BOMBAROMENT

PROTON BOMBAROMEN ON Q. BFAYLLIUM

PROTON CHANNELS

DROTON COLLISIONS

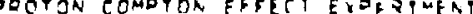

DaOTH AISTRIBUTJAL

PQCTTOR EMISSION

PDOTOQ EAFRGY

PROTON GENERATORS

DRTTON LINAC

PEOTON LINAC DESIGN

DOOTOR LIME

PAOTON LENEAR ACCELFGATAR

PRTIOS OEAK

DRCPOA RAOIOGRAPHY SYSTEM

DECTOD. RANGE

DQOTOS REACTIOAS

Donion genovil

Dooroy simulation

ontion souree.

- DOTON SPULLATION

PROTON SPILLATION

PROTON SOECTRA
POCTON SPECTOCMETF

PROTON STOEAGF DINO

DROTIN SYNCHROTDRA

DKOTOS. TARGET

DROTOA THERADV FACIIITY

DHOTOn COLPTON

DEOTON-COMDTOA EXDEUIVE. T

PROTONEINOUCEO OEHTERON GREAKLIP

DROTONAUULLFUS SEATEEI.G

PROTONGRROTON COLGISTRVE

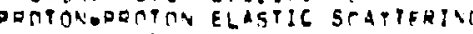

- potonadarton scatifatni.

PROTONARECTIL SEECPSOUETEOS

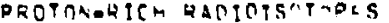

DROTINS

DROTONSFERTINAS A. EIE

DROPOTYEE

DoTOTYOE CONSOLE.

DROTOTYPE FLECTECU SCEEIEUATUD

DEOTHYTE WIGNET

DDOTDIYDE TESTI'G

os ajosté

DEEIITUVECTUR

$D$ SP

DTOTOAPARALIEL

piag pocGray

PIRLIC DORRFSS SYSTEM

- Diplications

DULSATING DE FIFLT

DULSE

- ULSE CHANRELS

DIISE COAF uOMULATIOA 
PULSE CURRENT

PULSE CURRENT TRAASFOQMFRE

pulse delar ungt

PULSE GATE

PULSE GENERATOR

PULSE HETGHT ANALYZER

PULSE NEIGHT OISTRIBUTITNS

PULSE HEIGHT EVENTS

PULSE HEIGNT RESOLUTIAN

PULSE HEIGNT RESOLUTIA

PULSE HEIGAT

PULSE LENGTH

PULSE MOOULATION gYSTEM

PULSE MOOULATOR

PULSE MOTOR

PULSE MLATE

PULSE PLOT

PULSE RATE

-ULSE RESOLUTION

PULSE SHAPE

ULSE SOURCE

ULSE SOURCE

DUL SPECTRA

PULE STAETCHER

PULSE STRUCTUR

PULSE TRANSFORMFR

PULSE VIEWING

PULSE-TOAULSE

PULSED AMPL IFIERS

PULSED GEAM DEFLERTIO

PULSED SIGNALS

PULSED-WIRE METHON

PULSER

PULSER TESTS

PULSERS

PULSES OF VARIABLF WIATH

PUMP

PUMP BOX GRILL

PUMP MAGNET

PUAP POWER SUPPLIES

PUMPING BOX

PUMPING ELEMENTS

PUIAPING GRILL

PUNCH AND DIE

PURITY VIOLATIONS

PUSHOBUTTON CIRCUIT

PYROCARBON TARGE

PYROLYTIE CARRON

OYROLYTIE GRAPHITF

c me asuREMEnTS

ADD SDECIRTMETER

QUIAT ALIGAHENT FORTE ST IDV

GIIAT CURRFATS

BIAD GRAMIER.TS

JHAO LENGTHS

quan magnet coil conliar. sugith

DIIAO MAGNET SLPFL

OUAD MAGNET TFSTIAG

QUIAD MAGNETS

JUAC MATCHPAG

OUAN REVERSAL

GUAD STREAGTHS

QUAREATIE PERTUERATIO.

JUACAUPLET LER,S SYSIE."

DUAMRIIPELAR IRON CORIG HATIOU

DIAORLIPCLE

JHACRIIOCLE APERHITINAS

JIARRIPOLE DOUHIFT FITITATIM.

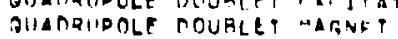

JUANRHOOLF NOLRLETS

JUADRUPCLE FNEQGY SPLITTIMGS

IIADRLIDCLF ERPORS

JUADRUPCLE FACTOR

DU $\triangle M A U P O L F$ FIFLR

OUADLUOCLE FOCUSIDG MaGIETS

DHATHIDOIF FOEISING

WUADRUOOLF LENS SYSTH"

QUATRLIPCLE LEVSES

OUADRUPCLE MACONFT

DUACQUDOLF MATERT SYSTE

JUAC210OIF WAGMETS

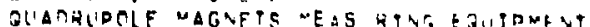

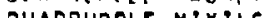

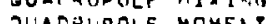

Juratiode umear

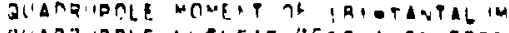

JUANRIPOLE NUCLFAO AFGC A.CE FEFET

JULRTIDCLE STREVGTHS

DLACRIDOLE TQTPIFT

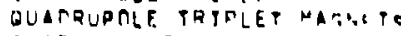

3I AMRIDOIES

JuAOS

J'ANS AR ELECTROSTATJC DRTICLE SEPAHITOR

JIALITY CHATROL DRICERUIEE

j. ARTIZATIC. FRODOS

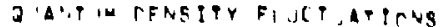




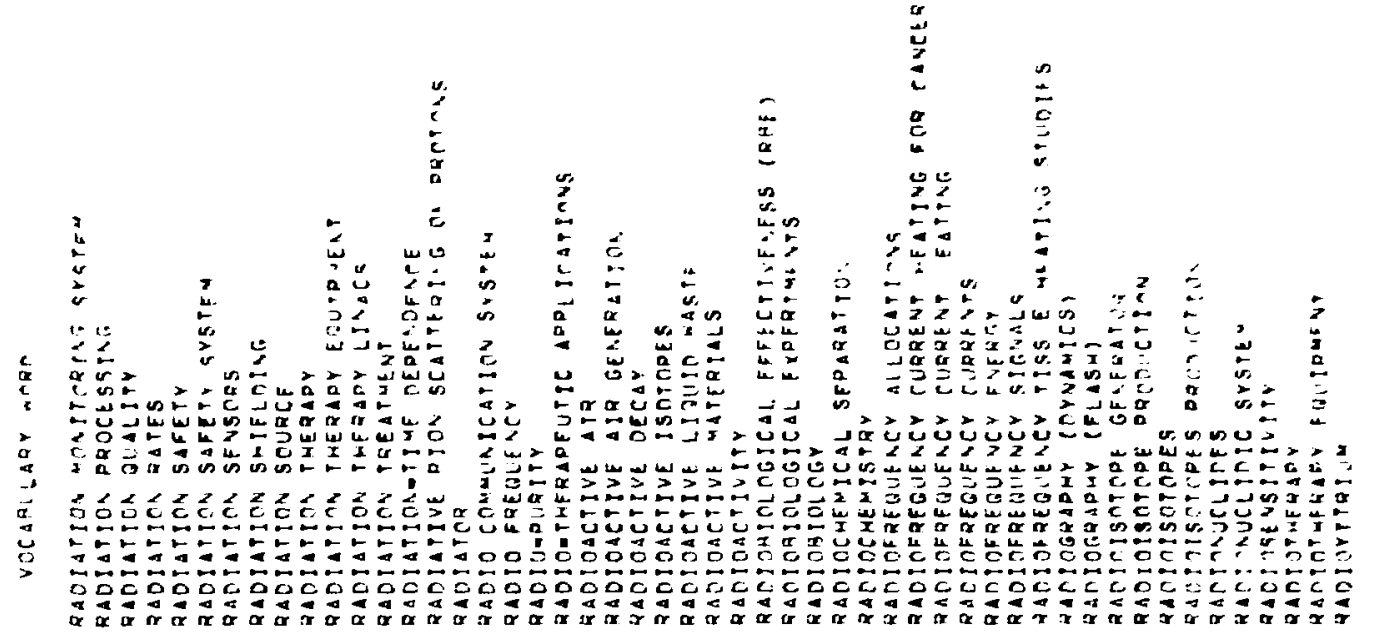

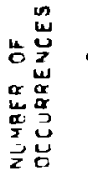

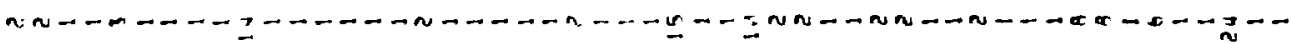

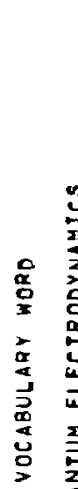

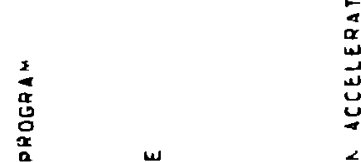

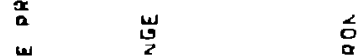

紧

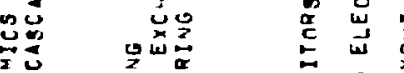

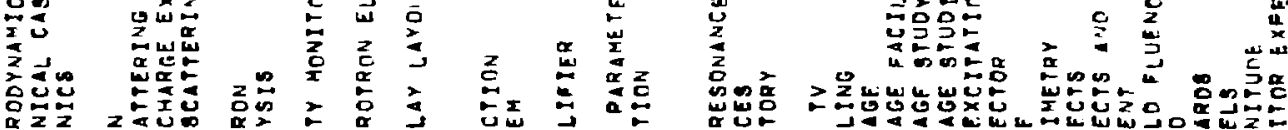

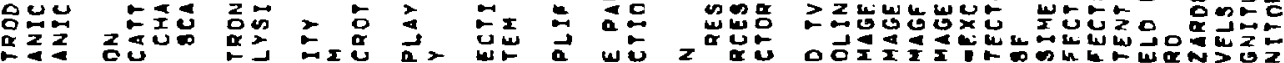

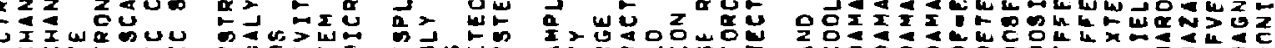

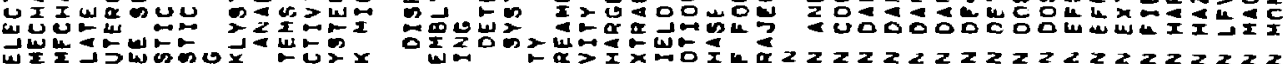

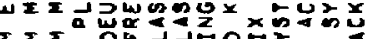
O 우애

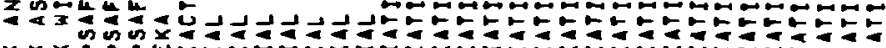

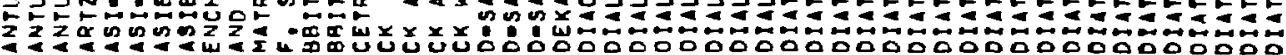

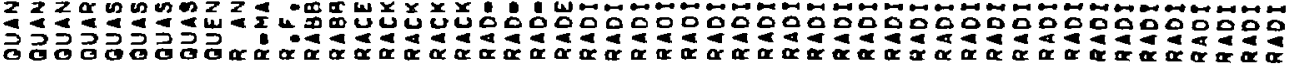




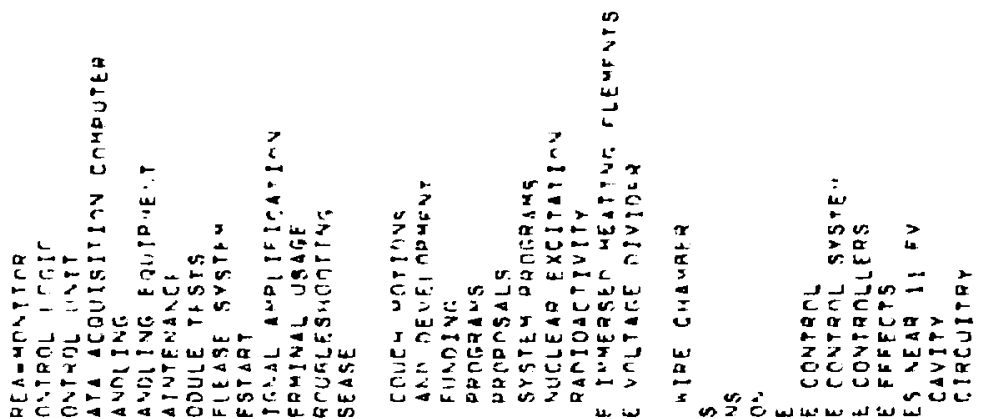

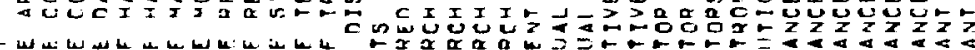

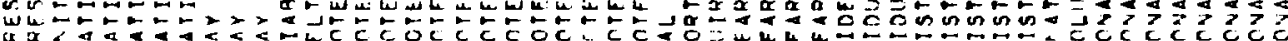

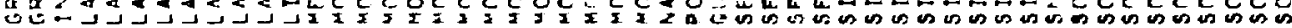

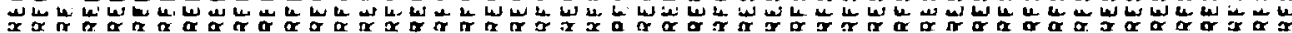

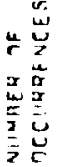
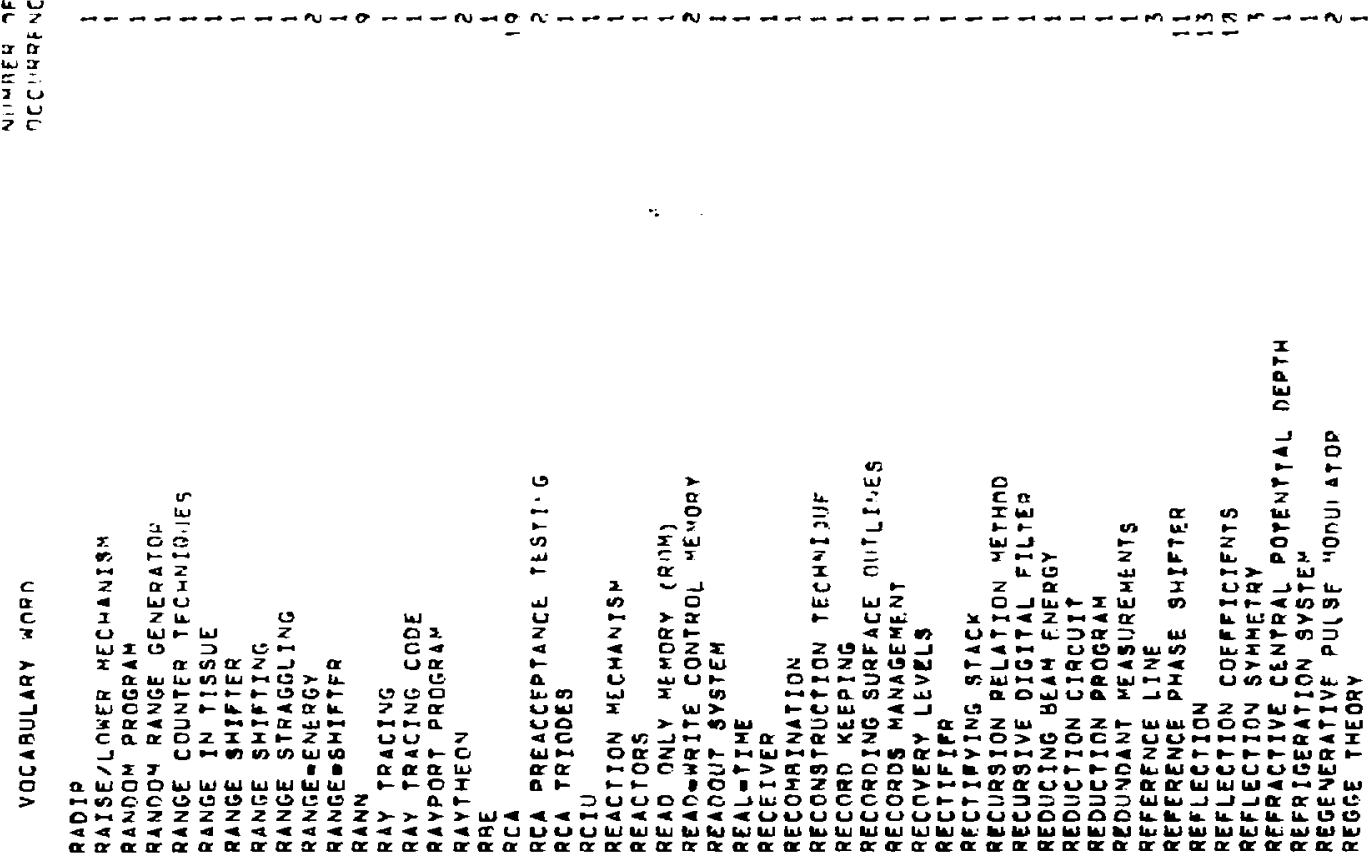

L

$0--\infty-0-n-m-n-m$ 
NUMBER OF

OECURRENCES

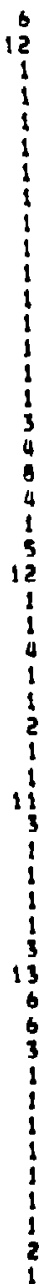

VOCABULARY WORT

RESDNANT COUPLING

RESONANT FREQUENCY

RESONANT POST TIJNFRS

RESTNANT POSTS

RESONANTLY COLPLEO STANIING WAVE ACCELERATORS

RESONANTLY COUPLED STRUETURES

RESONANTLYOCOUPLED LITAT

RESPONSE PREOICTION

RESPONBE RATE

RESTORATION PROCENURE

RESTRICTEO USAGF

RESTRIETING COLLIMATORS

RESULTS

RESULPS

REVERSED OIODE

REVERSED OTODE

REX

REX

RF ACCELERATOR SYSTEH

RF AMPLIFIER

RF AMPLIFIER CAVITIES

RF AMPLIFIERS

RF AMPLITUDE

QF BELLOWS

RF BURNS

RF CAVITIES

RF CAVITY

RF CHOPPER

RF EMOPPING

RF CIRCUIT

RF COMPONENTS/BIOLOGICAL FFFECTS

RF CONTROL

OF CURREN

RF ORIVE

RF ORIVE
RF DRTVE LINE

RF DRTVE LINE

RF DRIVE LOOPS

RF DRIVE LOOPS

RF IELL REHAVITH

AF FIELO CON

RF FREQUENCY

RF FREOUEN

RF GATE

RF GENERATOR

RF HTATINO

RF INPERFACE UNTTS

AF INT FRF ERENCE

RF IBOLATION TRANSFORMER

RF LEVEL SFRVO CONTQOL

RF LONPS

RF MANIFOLO

RF MASTER SOURCF SYSTEM
Ni) MHER OF

OCCHRRENEES

VDEARILADY aORA

RF MODULATION

SF MITULE

RF MOOULE CONTZOLI

Q5 NULTIPLIFAIOWASE REE RFNRF STUNIFS

QF OUTOUT

RF DERMISSIVF GiPCi!t

QF PEQWISSIVE SYSTE"S

RF PHASE DELAY INTEPFACIVE

aF PHASE SGAA

RF PHASE SMIFT

RF PHASE/AMPLITIIDF CORTSO

RF PAWER

QF DOHER AMPLTFIENS

RF FONER I DSS

QF P तMEQ METEP

QF ORAE UEVER

QF POWER SOlRCE

RF POWER SPLITTE

RF PONER SUDPY

RF PONER SYSTFMS

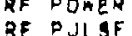

SF PULSF

RF DULSING OUT

RF SERVO CONTROL DECAVEZY

RF STABILITY

RF STANDS

RF STRUETIJRE

RF SYSTEM

QF SYSTEMS

RF TANK

RF TESTINT

QF TIMEOCFOELTGMT

HF TRANSITION

RF THANSITIOAS

RF TRANSMISSION

QF TRANGMISSION LIVL

RF VOLTAGE

RF VILTAGE HAEAKDONN

RF MAVE LENGTH

RF HAVE SPRING FALRICATION

QR MAVES

RF WINDOW

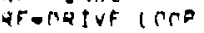

ZF-F DHEL חUTPIT

QF-BTEERIVG

QFFT

DFPS

RFT

RFT

AICE 
RICE DARITY ERROR ALIFEE.

RICF POWER SUOPLY

RICE TEST MODULE

RICENICR

RICEOMIU CHAIN

RICE-RICE I/OAMIU CCMaI.ATION

RICE RIU

RING DYNAMICS

AING OF $2 N$ CELLS

RING -COUPLEO CAVITY SYSTFM

RIPPLE CURRENT

RIPDLE FLUX

RISETIME

RIU

RME 16 SYSTEH

RMS

QMS BEAM

AMS DEVIITION

RMS EMITTANCE

RMS PHASE

RHS RADII

RMS VALUES

RHS - WIOTH

RO.105 POWER SUPPL

ROLLER ASSEMBL IES

ROLIER TRUEKS

ROM

ROOT LOCUS PLOT DESIGN

RISS I-TYPE COUNTER

ROTATE MECHANISH

ROTATING MAGNETIC FIELD DEFLECTOR

ROTATING SCINNING WHEEL

HOTATING WHEEL BEAM SCAMNFR

ROUGH PUMA DACKAGES

ROUGH VACUUA

ROUGH VACIJUM SYSTFM

ROUGHING PUMP

ROIJGHNG PLIMP PACKATE

ROUGHING SYSTEM

ROUPING MODIUE

RPEA

RSX 11 HANDL $E$ I: CC

ASX-IID OPERATING SYSTEN

RUOSTAM THINETARGE

RUDSTAMS EMPIOICAL FORMILLA

RUDSTAMS EMPIDIC
RIIN DFRMISSIVE

RUN DFRUISSTVF SYSTEM

R.'A OERTIT "ORUI.E

Dun DFRMit STATIS

RUN PFR TY SYSTEM

RIIN SUPDI IES

QUNEDERUIT SYSTFM

ri,naatir oscillaticas

PIINAIVG ON TOQA

RUNAING HAVES

PII THE VIUM

RUTHENIUMAOT

RIITHERFARN I $\triangle A B D A T O A Y$

$S=$ HATRIX

S-WAVE PNTERACTION

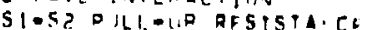

25

SACIAY

SAFFTY

SAFF TY INTERLRCKS

SAFFTY SYSTFM

SAFETY SYSTEMS

SATAMARE $M$ MUE

SALIVF-FII.LFO RLETTOUNE VESSELS

SAMPLE AND HOID

SAMPLE TITE

SAWDLE GATE GEVEHATME

SAMPLEORTATA CO.TPOL SYCTEU

SAMPLIVE MOA ITOO

SAMPLING SCOPE FETHVI JaS

SANDAICHECOUNTER DETFE TIDR SYSTEN

SATELLite COMPIJTE STSTH

SATELLife CnM

SCALITG LAn

SCALING LAW

SCAVVER WIRE

SCAnivint cont

Scat TECHNTHUS

scatieling

SCATIFRING P MAUREOS

SCATTFETNG NATA

SCATPRTNG IBTFEATIS

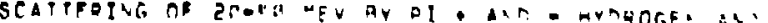

DEUTERIUA

SCHEO.LL IA

SCHE QULING

SCMECF

SCLFFF I

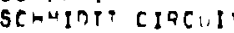

Stivillation cruate

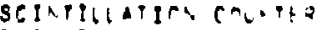

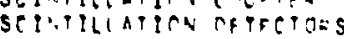


SEINTILLATOR-PMOTODIONE COMRINATION SCINTILLATOR-RADI TION NAMAGE CINTILLATORS

\section{SCL PARAMETERS}

ROGRAM

SCOPE EXECUTIVEIDISPLAY LANGUAGE

SCOPE INTERFACE

SCOPE/HYDRA INTERFACE

SCR

SCR POWER SUPPLIES

SLR POWER SUPPLY

SCR UNITS

SCRAM SWITCH

SCRAPER STUDY

SCAMPEAS

OCRAP ING PROCEDURE

SCRATCH PAD MFHORY (SPM)

SCRIPTOAGRAPHICS DATA TABLETIDIGITTIER

SERUB (NS) ROUTINE

SEULPTEN FIELDS

SOS RICE

SEALS

SECOND HARMONIC CAVITY

SECONDARY GEAM CHARACPEOISTICS

SECONDARY BEAM DIAGNOSTIE

SECONDARY GEAM HANDLING SYSTEM

SECONDARY BEAM LINES

SECONBARY BEAM LINES

SECONDARY EMISSION

SECTNDARY EMISSION MO:IITOR

SECONDARY PARTICLES

SECONOAAY PROTON CONTRINUTION

SECONDARY REACTIONS

SECTION TWIST

BECTOR

SECTOR A AND TRANSITITN REGION

SECTIR A PERSONAEL SAFETY SYSTEM

SECTOR CONTROL I TEIC (SCL)

SECTOR CONTROL OANEL

SECTOR WATER OISPLAY PA:EL

SECTORS BAN

SEL

SEL ASSEMBLER

SEL COMDUPER

SEL $=$ BIAA

SELABAM

SELF -ABSORPTION CORRECT IOAS

SELMA SIMULATRR

SENS ING WEAOS

SENSITIVITY

SFNSORS
SEFT IA

SERTAL CRAIF TONTDOLLERS

SERIAL DRIVER

SEMTAL HIGHTAY

SFR IAL SYSTEH

SERIES APBODXIYATIO

GERIES HAFDETUBE MOMULATSE

SERIES MONULATCa

SERVICE CELL

SERVO HYDRAULIE PIIHE

SERVD SYSTE

SFRVOARM

SFRVOMANIPULATOR

SERVOAATIPULATORS

SERVOMECHANISM

SERVOME CHANTS MS

SERVOMOTHRS

SETOPOINT TRANSFER

SETPOINT

SEXTUOOLE COIIS

SEXTIPOLE FIEID

SFXTIPOLE MAGAET

SWATOW SHIEID

SHAF $P$ ENCODER

sHELl STRUCTURE

SWIELO MOOR

SMIFLD PLIIG

SHIELTINO

SHIFLING

TING SEINTILLATOHO

SHIFLDING SDECIFICATIANC

FiFQ CiRCUT

SHIVANTISHIM

SUIDOTNG AND INSTALLATIN

SHU I MFY ACCFLFNATIR

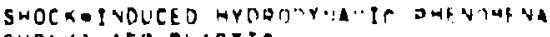

SHOSKA 15R DLASTIP

SHONKA CHAMAER

SHORTAEANGE CEATAML REP LSITY

SHIVNT IUPEOANCE

SHUD.T RESISTANCE

SHUD? VAITARES

5 साיis

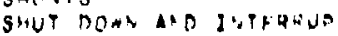

SwUTOAnय SCHF rul I.

SHUPOOWNOINTERRIFP

SHUPTFA DOORS

Si(l) J) TFETRES

SIUF PLATES

SIDF YOKES 
SIDE - COUPLEN LINAC

SIDE - CAVITYOKLYSTRDN

SIOE-COUPLED ACCELERATOU

STOEACOUPLEO ACCELEHATOO TANKS

STOE-COUPLEO CAVITY CUATVS

IOE-COUPLED CAVITY

SIOF-COUPLED GUTDE

SIDF-COUPLEN IIMAC

SIDE-CDUPLEA GTRUCTURES

SIDE-COUPLED TANKS

SIOF-COUPLEO WAVECGUARM ACCFLERATOD

SIOF-COUPLEO KIVEGUIDF STQUCTIIRF

SIDFTRACK

SIEVE

STGNAL AMPL ITUOF

SIGNAL CHARACTERIBTICE

SIGNAL DETECTION

SIGNAL PROCFSSOR

STGNAL PULSE

SIGNAL SOURCE BOX

IIGNAL SPLITTER

TIGNAL STRENGTH

SIGAAL TRANSMISBION

SIGNALICONTROL CABLF

SIGNALS

SILAAR-STERNHETM POTENTIAI

SILICON ANO GFRMARIUM OF PFCTURS

SILICAN CONTROLLEO RECTIFIEQ

SILICON DETECTOR

SILICON SURFACE BARITE SEMTEONDJETOR OETFCTOO

SILUER-SILVER-CHLORIDE FLFCTROPLATING TECHNIPHE

Truar

SIMALAC

AC PROGRAK

SIMILAC

IMIILATEO INPUT DATA

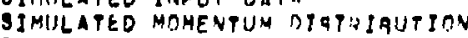

SIMLILATED HEDSFS

SIMULATION TF THE MAGDETS POHEA

STMULATOR

SIMULTAHFOUS ACEELEQATION

SIMULTANE OUS REAMS

SIMIJLT ANE OUS BUNCHING

SIMULTANEDUS STEERING OF WH AEAMS

$\sin$

SIN CYCIOPROA

SINGLF APERTURE EXTRACTION GYSTFM

SINGLE APFAPUDE SOUACE

SI WGLE CAVITY HUNCHEA

INNCLE CELL TEST PROGAA.

INTLF COLLIMATOR JAN

SINGLE CRATP GYSTFM

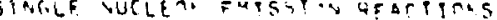

STNELE PARTICIF

SINTLE PARTTLLE EFHFTS

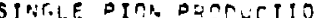

SINTELE SCATTEATA.G

Sincine chLL Cavitr

SinToval control caAle

Sitif Fatilitifs

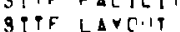

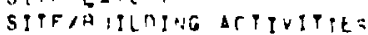

SKE* JJADRUPOLE CAMUNE.T

SKIS EFFELT

SKIOTER DRCGLAN

SI A A

SLATERS FORUULA

SLIVE ARM

SI EFP

SIFW RATE LIMITATIOSS

SLERIVG CAR.TRCL

SLINIVG TRAASTIIOAS

SLIT ASSENELY

SLIT ASSE MELY STEFEINR. AGNET

SIIT SCATTEDING

SLCTTEO LIAE

SLOTTEN LIAF MF ASHRE ME WTS

SLOTTEOADLATF

SI TH F LL

SLOW FREQUEACY COAPII

SLCK INTELLCE

SLOH MESOR.

SLRn SiARILIZE

SLOH STABILILEQ SYSTE.

SMALL ANGLE DIFEFUE TI PRESS StTTION

SMALL ANGLF ELASTIC SCATIERINT.

SUALL NNELE ELASTTC CUOSS SFCTITANE

SMALL BIGNAL PRANSFER F NETPOAS

SNALL SPOP IUSING

3HALGOSIGUAL KLYSTREA CNDF

SMALLOSIGNAL THE RDY

Sue

BMOCTH MOSF NISTOTH TIO.

SCOIUM CMAQGE-FXCHALGE +AVAl

SONIUW IONIILE DETFCTOLS

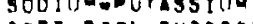

SCFT DION ENISSIOS TA.C LATTONS

SCET DIOA PDFEITTIAAS

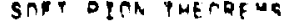

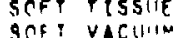


SOLENOIO

SOLENOIO CHANNEL

SOLENOIO DRIVER CARD

SOLENOIO LENS

SOLENOIO LENS

SOLENOID MAGNET

SOLENOIO VAL

SOLIO ANGLE

SOL IO ANGLE SCALE

OLLID CORE MAGNE

OLIO STAPE APRLICATINN

30LIo state OETECTOAS

SOLID sTATE OIOOE

SOLIO STATE SOURCES

SOL IO TARGET

SOL IO-ANGLE-DEFINING SLTT

SOLVENT EXTRICTTOON

SONALERT MLARM

$s 0 P$

SOURCE GEOMETQY

SOURCE PROCUREMEN

SOUREE ROOM

SPACE CHARGF

BPACE RAOIATION FFFECTS LABORATORY

SPACE CHARGE

GPACE-CHARGE EFFECTS

Pallation

SPALLATION CROSS SECP10.9

mPALLATION NEUPRONS

opallation of copoer, vegas

SPALLATION DROCESSES

SPALLATION PRODUCTS

SPALLATION REACTIONS

GPARE UNITS

OPRE UNITS

SPARK LIMIT

SPARK DROTECTIOH

SPARKOOWA

BDARKING THRESHOLO

90ATIL OISTAIBUPFo

SPATIAL RESOLUTION

SPEC IF ICATINAS

SPECIFIED PROTON ENEREY

OCCUPEEVCES

SPECTRA

SOECPRAL DENSIPY FOUNALISM

SOECTRAL DATTIOAS

SPECTROMICAFTIC POEER S POLV SPECTAONETE

SPECTRCUEPER LIAE

SPECTROYETER UIGYET CMNATOL

SPECTROMETERS

SPECTROSCORIC FACTURS

SPECTRUM

SPECTRU TU OF TICI IOES

gPEctaun or viclities

JDMERE-COUNTIUG EFFIC IESCIES

SPHERICAL LENSER

SDMFRICAL PROPORTIOAAI OD:IVTER

SPRERICAL STODPIAG AETI TV

SPMERICAL TISSUF MATEL

SPNERICAL TREATUENT YLL YE

SPILL MONITOR JNSERTIAY

ODILL MANITORS

SDIN AGAERATION

SPIT CONPAOL

SPIV OEPENDEVEE

SPIN TRTVE TRAT

SOIN FILTER

SPSN CAIENTATION

Soiv opeCESTES

SPIA REVERSAL

SOIA-CORRELATIOA

SPINAFIL TER

SPINOORAIP POTENTIAL

SPIN-ZOIN TNTERACTIST

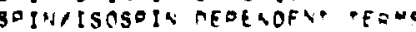

gDLIT DAASE CODF

SPLIT SHASE CADING

SPLIT DUASE SPACE OEAYS

SOLITAOMASE OECOCER

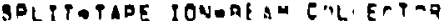

goONTANEOUS TUMAOS

SPUV REAL LIVES

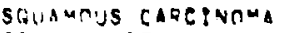

SLKLY Cone

stantilit

gratilit stUDIFs

STAILIZEO STRUET JRES

STAPLF OSCILLATICA, OAM"ISO

MARLF. OMISF AVGLF

STABLE TEMOERAT AT CO T.: TI

STACX DIISTE

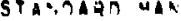

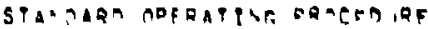

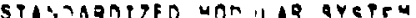


STANOSNG WAYE MODE

GTANDING WAVE RF SEPIRATORS

GTAR

TTAR DOSE

STAR EVENTS

STAR NEUTRON

GTAR NEUTRON DOSE OISTRTBUTTON

STAR NEUTRONS

STAR PARTICLES

STAR PHOTONS

STAR REGION

STARK AOMIXTURE

STARK MIXING

START SUPPLIES

START UUP CODE

START-UP PROEEOURES

STATE EQUATION

STATE OF AGGREGATION

STATIC ELECPRiC F PELO

STATIC ELECTRIC GUAORIIPILE AND MEXADECAPOLE MCMEN

STATIE FIELO DC SEPARATARS

STATISTICAL APPROYIMATITN

STATISTICS

STATUS PANEL

STEAOY STAPE CONTROL

STEADYOSTATE

STEADYOSTATE FIELT

STEEL

STEEL BACKING

STERL BILLETS

STEEL FORGING

STEFL PESTINE

STEEL PHICKNESS

STEFL WALL LINEAS

STEERING

STEERING COILS

STEERING CURRENT

STEERING OIFFICULTIES

STEERING DOUBLEPS

DTEERING MAGNET

STEERING MAGNET COIL

STEERING MAGNEY CONPRL

STEFRING MAGNF

STEFFFN PROGRAM

STELLAR EVOLUTION

STEN RELLOHS

STE" DERTJPA4 I AVS

STE BRBIINC

STEO SITE NEASURE "WE

STEPOF PUAS

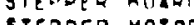

STEDSTUG WOTOR

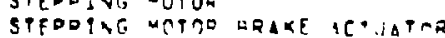

STERILITY

STCPAAND

STROAAND CLOS QE CHECK

STCODEO ATIPROT TS AWI l1 ATIOA.

STODEF H DO CHANATL

STOPDFO HICVS CHAVNEL

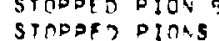

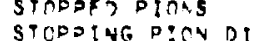

STCDOTHG PECN DISTRIH TUA.

STOPDIVT REGIONS

SPOHAGF RAV

STURAGE DISOLAR MTDIL

BTORATE OSEILLOSCAPES

STOEAGE SCOPES

STOEE ENERTY

SPRAGGLING

STRAVTENESS VIMIATITIT,

SPASBOHSG PAAAUETESS

STRAY FIELOS

STREAMER IMAURER

STRESS REDUCTION

STRTNGESS

BTRIDPEA

STETDOING

STRIDPIVE COAF

STRTODIVG COOLIVG ATIR SYSTEM

SPROBF DLLSE GEV.FDATOS

STRCNG FIELO 10:IIZE

STROVG IATEQREPT

STARVG STEFRING

STACNGEINIFRACTIOU, ASOONOPION AATSS

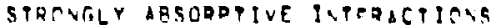

STRONTI JU ADEAKTMDOSOA.

STRUCT JPE LENGTM FRAOST

STR CIJPE TUVTR

STUHUIT

S'lR5YGTFM CODIVG

SUA5YSTEM SONTQOL

SLBSYSPEM FIELD

SUASYSTEU INTEACTIN

SUBSYSTEMS SCMFUATITS 
SUNNYVALE

SUPER NOVA

SUPER•NOVA

SUPER-RICE

SUPERCONDUCTING COILS

SUPERCONDUCTING LINAC

SUPERCONDUCTING MIGNETS

SUPERCONDUCTING PJON EHAVNEL

SUPERCONDUCTING QUADOAJPALF MAGNETS

SUPERCONDUCTING OUARRIIPNLF MAGNET

SUPERCONDUCTING OUADRIPALF NOURLET

SUPERCONDUCTING SOLENAIISS

SLPERCONDUCTING STRUCTUIES

SUPERCONDUETING SYSTE.4

SUPERCONDUCYING WTRES

SUDERCONDUCTOR

SUPERCONDUCYOR TRTON SYNEMPOTRON

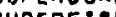

SUPFRFLUID UEHELIUM TARAET

SUPERFLUID PHASE HELIJM

SUPFRHULTIPLEY SYMMETRY EXPERIHENTS

SUPERNOVA

SUPERNOVA PLOATINGEDOPNT EXECUTION TIMER SUPEAPERIOOIC LINACS

OUPPLY PROBLEMS

SUPPORT ACTIVITES

SUPPORT CONE

SUPPORT CONES

GUPPORT FRAME

SUPPOAT RODS

SUPPORT STRUCTURES

SURFACE TMPEOANCE /O MEASUREMENTS

SURFACE PREPAQATION

BURGICAL INTERVENTIIIN

SWEDEN

SWITEH BOUNCE SUPPRESSINA

SWITCH TUBE

SWIPEH WIRING

SWITCHOEAG

SWITCHING DEVICE

SWITCHING MAGHET

SW ITCMYARD

SWITCHYARO AEAM TRANGEE

SWITCHYARD DESIGN

SWITCHYARD HEAT EXCWAIGF UTA ITOAS

SW I PEHYARD MAGNET

SWIPZERLAND

SWYT

SYMPOSIUM

VOTHULARY aran

SYMEHRONOL'S ENERGY

SYACWRONOUS PARTICLE

SYNC MROTROA

SYNCWROTREN SITIRTF

SYAFAGISTIC EFFFETS

SYNERGISTIC LETHAL EFFEET

SYSTEW

SYSTEN 5 MAB

SYSTE GARON1OTH

SYSTEM OESIG.

SVSPEM DEVFLOPUEVT

SYSTEM DOCUMESTATIES

SYSIEM FIFI

SYSTEN NAROWARE

SYSTEN LOGIS

SYSTEN NCISE

SYSIEN OPERATION

SYSTE PARALETEAS

SYSTEM ORCGRAN

SYSTEM REARRAVGL MEAT

SYSTE PFLIABILITY

SYSTEM SEQVICF WONITOP

BYSTEM SOFTIAAE

SYSTE STAHILITY CO.SIDE LATIOAS

SYSTEUATIC EQTOAS

SYSTEMS DESCRIPTICNS

i $S, A=2 F R C$

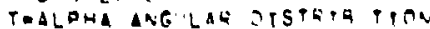

TOBAL CQUPLER

TOE HUMAN DHASTOO

TOMATRTEL

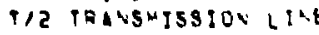

TA AEAM GIAE

TACODNA

TAII

isin:

tatse 5

TIAK

PANE

iAdK Cavity

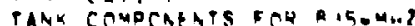

TANK COUPLING

TANK ERLE AMOLIT IMF

TANa FIELCS

TANK FLATUES

TANK GEAMTPE

TANE WEADS

TANK JAEKET COOLTLO

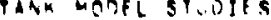

TAAK WOVENE II

rat. OESOMARE enditis 
TANK SHELL

TANK TILT

TANK TUNING

TANK TUNING SLUGS

TANK WILL CONTROLLER

TANK WATER SYSTEM

TANK-WALL-COOLING SYSTE"

TANKS

TANKS 2, 3, AND a

TANTALUM

TAP

TAP CTECHNICAL aDVISOQY PANEL?

TAPE EONTROL

TAPE TAANSPORT

TARGET

TARGET AI

TARGET AZ

TARGE ABSORDTION

TARGET ACCURACY

TARGET ACTUATOR MOTOR

TARGET ACTUATOR SYSTEM

TARGET AREA

TARGET CELL

TARGET CELL A-5

TARGET CONTROLLER

PARGET CAYOSTAT

PARGET OESIGN

TARGET OEVELOPMENT

TARGET DEVELOPMENT HO BFAM STRIPPERS

TARGET LEVEL INDICATOD

TARGET LEVEL INDICA
TARGET MECHANISM

PARGET MECHANISMS

PARGET MECHANISHS
TARGET MONUMENTS

TARGET PLANE

TARGET STATIONS

TARGET WHEEL

TARGET-TOETARGET TRANGOTRT

TARGET / MODERATOR

TARGETS

TAUT-WIPE DOSITION INAICATOR CHECK

rocmas

rocs

TDI

TE WALL

PECHAICAL DRAWINGS

TECHNICAL REOUIAEMENTS

TECHNICIAN ATTITUAES

PECHNGOUE OF OPTICAL DOTENTIALS

PECHNYOUE
TELEARMS

TELFVISIOA

TEMPERATURE

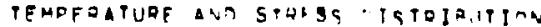

TEMOEOAPULE RFMAVIOT

TENDEQATLOE AQAZITE

TFMPERATURE CMaTAOL SUSPE

TF MFEFATUE CUNTRAL CiRTJTIOT

TFUFFATIOE OIFFFOF. TIA.

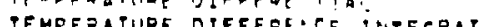

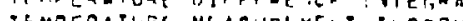

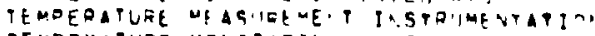

TEUPERAT

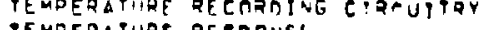

TEMTERATUR QFSDONSL

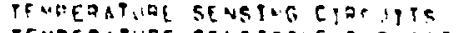

TFUDERTIIDE SENGITIVE QI STSTOAS

TE HDERATUAE SENSOR

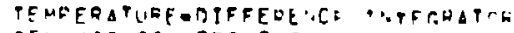

IEN HIT CONVEASATIOS

TERUIAAL UMGEH ING

TERUI'AL COFAATION

TEREINAL STRIO CARLE.

TERPTNATS

TFZMINATING RESISTMOS

TFRUINATIOA

TFRMINATION COOE

TEST HENCW

TFST CONFIGI RETITA.

TFS CANFTG

TFST MODULE

TEST STANO

TEST STAND

TESTIVG

TESTIVE IS MEETRLCTIO.

TFTR DOE

PHE TQFTICAL K/GETA. HATITE

THE RRETICAL PRECITTIO.S

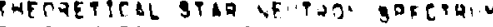

THEOAPEUTIC ADOLITATITS

THEOAFEITIC FAD BEA

THERAPY

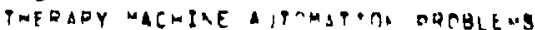

TNFRMAI DEFINING

PNEPUAL EFFPCTS

THERMAL HEAT CUSMION

THFOUAL OHOALFU

THFOMAL POOTFETO

THEOULL STRESS

THEOULL STRESS
THFOUAL SAITCUES 
THERMAL TREATMENT OF LOCALIZEO CANCER

THERMAL PUMOR THERAPY

THERMAL VELOCITY DISTHIAUTION

THERMIONIE EMISSION

THERMISTORS

THERMOCOUPLE

THERMOLUMINESCENT OETECTORS

THER MOLUMINES CENT ONSTMETERS

THER MOLUMINESEEN T- TYDE GENS CRS

THER MOLUHI NES

PHERMOPILE

THICK LENB MODEL

THICK TARGET

PHICKOTARGE?

PHICKNESS GAZING, ELEMENTAL CONCENTRATION

PHIMBLEOTYPE IONIZATION CHAMBER

THIN LENS MODEL

THIN SAMPLES

THIN SAMPLES
THIN TARGET

THIN TARGET

THIN TARGET CONTROL SYSTEN

THIN WALLEO AFPERLOADINT. CELLS

THINGTARGET

THOHASEFERMI POTENTIAL

THOMASAFERMI STATISTIEAI MOOEL

\section{THOR IUM}

THORIUM 236

THREE-GENO PION CHANNEL

THREE GODY EREAKOU

THREE-OIMENSIONAL DENGITY DISTRIBUTION

PHRE EOOIMENS IONAL TPEATUENT PLANNING

THREEOPHASE GRIDEF CIRCUIT

PHREE OPHASE TRINSFORHFR

PIEKLISH NUCLEI

PILT INUESTIGATION

TILT INVEST

PILTED DOUELET STEELITC

TILTEO PIELO DISTAIBUTION

TIME DELAY

TIME DEPENDENCE

PIMF DEPENOENT PARAMETERS

TIME RESOLUTION

TIME RESPOASE

PIME STRUE TURE

PIME -DEPENDENT OUANTU" . ECWANTCS

TIME - INDEPENDENT PERPIRRAY IUN PHEOAY

TIME OFFFLIGWT

IIMFO DATA

TIMING

TIMING GATE

DCCURREVCES

VOCARULARY ACLO

IMING, GATES

IIMING GYNCS

TTMIYG, SYSTEM

TINTANIUM FOI!

TISSUE

TISSUF ABSORDTICMETUV

IISSUE CHEMICAL ANAITEIS

IISSJE EMF

TISSUE EQIIIVALENT LIO IT

TISSUE EQUIVALENT PLAFTIC

TISSUE in Vivo

TISEUF SAMPLES

TISSUE TYPES

TISS'JE-EQUIVALENT CHLWHE

IISS JE-ERUIVALEAT CAJP TFR

IISSUE-EQUIVALENT MEDIU.

TITANIUM

TLO

TLO MEASURE UEVT

TLO SYSTEMS

TLDS

TM RAND

TM(SUR BIP) MODF

TOGELE SUITCHES

TOCGLF-TYPF CIRCUI

POLERANCE ON CENTFRINT.

TOLFANHCE REQUIRE UF NTS

TOLFANCFS

TONGUE MALIGNANCY

TOROIO

TORNNACO MACHIAFS

TORTJE OUTPUT

TOTAL CAOSS SFCTIANS

TATAL LAAD

TOTAL MIGCAECAOT IRF HATES

TOTAL NEUTRON INTENSITY

TOTALEAASCRDTICL CC VEE:

TOTALAREACPIOL CRASS SEPTIOAS

TOUCHODANEL

rCWFa wath activiti yo. tTog

$\log$

PSL.'3

toLs

TR H TitanCE

TQ HDFLATION

TRARAS ALIGNHENT

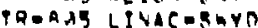

TOACE FLE UFA AAMIYSIG

PHACE OHUGDa 
TRAINING

TRAJECTORIES

TRANSDUEER

TRANSDUETOR

TRANBDUCTORS

TRANSFER COOES

TRANSFER FUNCTION

TRANSFER MATRTX

TRANSFORMATION MATRIX

TRANSFORMER

TRENSF ORMER SFNSITIVITY

TRANSF ORMER-RECT IF IFR

TRANSTENT BEHAVIOR

TRANSIENT CURRENT

TRANSIENT EFFECTS

TRANSIENT FIELOS

PRANSIENT MISMATCHES

PRANSIENT RESPONSE

TRANSIENT VOLTAGE

TAANSIENT WAYE FOAMS

TRANSIENTS

TRANSISTOR SWITCH

TAANSISTORIZEO ARC PULSE MONULATOR

TRANSIT TIME FACTOR

TRANSITION

TRANSITION ENEREY

TRANSITION NUCLEI

PRANBITION RATES

TAANSITION REGION

TAANSITIONS

PRANSMISSION

TRANGMISSION CHAMBER

TRANSMISSION DATA

TAANSMISSION EXPERIME TT

TRANSMISSION LINE

PRANSMISSION LINE (FPXL)

PRANSHISSION LINES

TRANSMISSION MONITORIVG

TRANSMISSION SYSTFM

TRANSMITTER

TRANSPAAENT TANK MATERIAL

TRANSPLANTED MOUSE TUMORS

TRANSPLANTES TUMOPS PO MICE

TRANSPL LNTED TUMORS

TRANSPORT

TRANSPART (AEAM OPTICS PODEI

TRANSPORT AREA

PRANSPORT CALCULATIONA

TRANSPQRT COOE
TRAMSPOAT MAGAET

TOA.TPOHT MAGUETS

TRANSPORT MATRICES

TA $A$ TSOOPT DF D

TRA.SPOAT DOOERAM

TRANSPORT STEEOING

TRANSPOLT SYSTEM

TRANSPOAT THFORY CALC LATTOAS

TRANGDORT TUNING

TRATSDTJ

TRANSVERSE

TRAP.SVEOSE AGEEOTANCE

TEA.SVERSE ADMITTANTF

TRAASVERSE AMPLITIIDF VAETATION

TRAESVERSE HEAM BLONOP

TRANSVFRSF DEFLECTIN'

PRAMSVFRSF TIYAMICS

TRANSVEASF AYMAUICS DEF" WTITONS - COMFALATIVE

TRAASVEDSE GMTTANCE

TRANSVERSE EMITTANCE UATCHINE

TRARSVERSE EMITTAHCF VALIATION WITM HUNCHFE AMPI. ITUOE

TRANSVERE FOCISTNG

TRAPISVFRSE MATCHING

TRANSVEOSE NODFS

TRA'SVERSE NEEDLE MEA BUEENENTS

TRARSVERSE TSCILLATIONS

TRANSVERS OSEILLATIOD

TRA'ISVERSE PERTURAATION INDILSE

TRANSVERSE DUALITY AF HA AM

TRANSVERSF SPACF

TRANSVERSE TUYING

TRALSVEASF VELOCITY

TRAASUERSEAPOLARIZEO WITVE

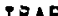

TRAVELING WAVE ACCELEAATJH

TAQVELING WAVE RESOMACF DING

TRAVELING WAVES

TREATMENT COUTH

TRE ATMEAT IA DEDTH

TREATMENT LINES

TREATHENT LCGTING CEDE.

TRE ATMENT DLARATNE.

TREATHENT PROTAL FILN

TREAPMENT DQRCENULE

TREATHENT AODH

THEATUEVT TAPLE CAORIAGF ASSE WHL

TREATHENT TIME

PQFATHEU VERTFICATIOU EII.

TQEATMEWT VOLINE

PREATMEAT VCLIIME VISUSLIZATION 
TRIGGER MDOULE

TAIGGER PULSE AMPLIFIFR

TRIGGFRED SPARK GAP CROAHARS

TRIGGERING CIRCUITS

TRIM PROGRAM

TRIODE

TRIOOE FINAL AMPLIFIERS

TRIDOE MOOULATOR

TRIDOE TUOE

TRIODE, AHPLITRON, RLYSTAON

TRIODES

TRIPLET

TRISTAN

TAITIUM

TRITON PRODUETION

TRITON SPECTRA

TR IUMF

TRUMP CURRENT KONITOR

TRYGON POWER SUPPLY

TTL LOGIC

TUAE CALCULATIONS

TUBF FOREST GEAM STIOP

TUEE PAHAMETER STUOTES

TUBF PROOUCTION

TUBE SLEEVES

TUMOR OATA STORAGE

TUMAR DETECTION

PUMOR MEAT THERAPY

TUMOR LOCALIZATION

TUMOR SENSITIVITY

TUMOR THERAPY

TUMOR VOLIIME

TUMORS

TUNE-UP PROCEDURE

TUNERS

TUNGSTEN

TUNGSTEN TARGET

TUNTNB

TUNING

TUNING OF CHINNEL

TUNING PROBLEM

TUNING PROCEDURF

TUNING PROCES

PUNIVG SLUGS

PUNING TECHNIQUFS

TUNELING

TIRAJUOLECLLAE TIUDS

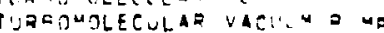

TLA+-CFF PFCCEE.RF

TURP.OON

TLRVOON ALGOR!TH

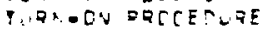

IIRT-CQ DROCRANS

II QR. ON 5 EQUE?CE

iv ANALYSIS

th uJuttudis

iv Dateh

iv seat

iv SYITEM

iv tai UK LIAF

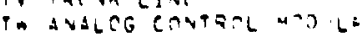

Priadx allar

thisteo bita

the acor Diol auplitine

THS IMENSICVAI. REA A P T

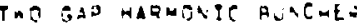

THC Y CLEON SYSTEN

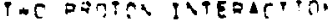

TWOAEAW SPERATION

Twrodeam scivt toy

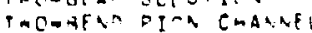

ing-anna RFACTinas

inO-300r $5 T$ T TS

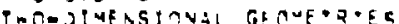

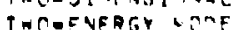

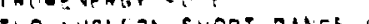

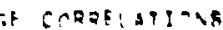

incoonte analng fíp

inCOAAY RAOIn SYSTE

ULTFC

UIBUS

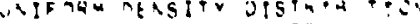

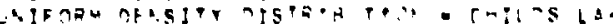

Juibjan tese

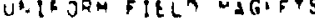

Net CFLI, OPIF i at

BDT I/C CMANBE

UNIT NJMAER SYSTEM

UAlT WHAESS

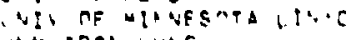

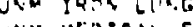

Jom iasicat semect

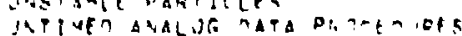

invi

USER S DOCLT 


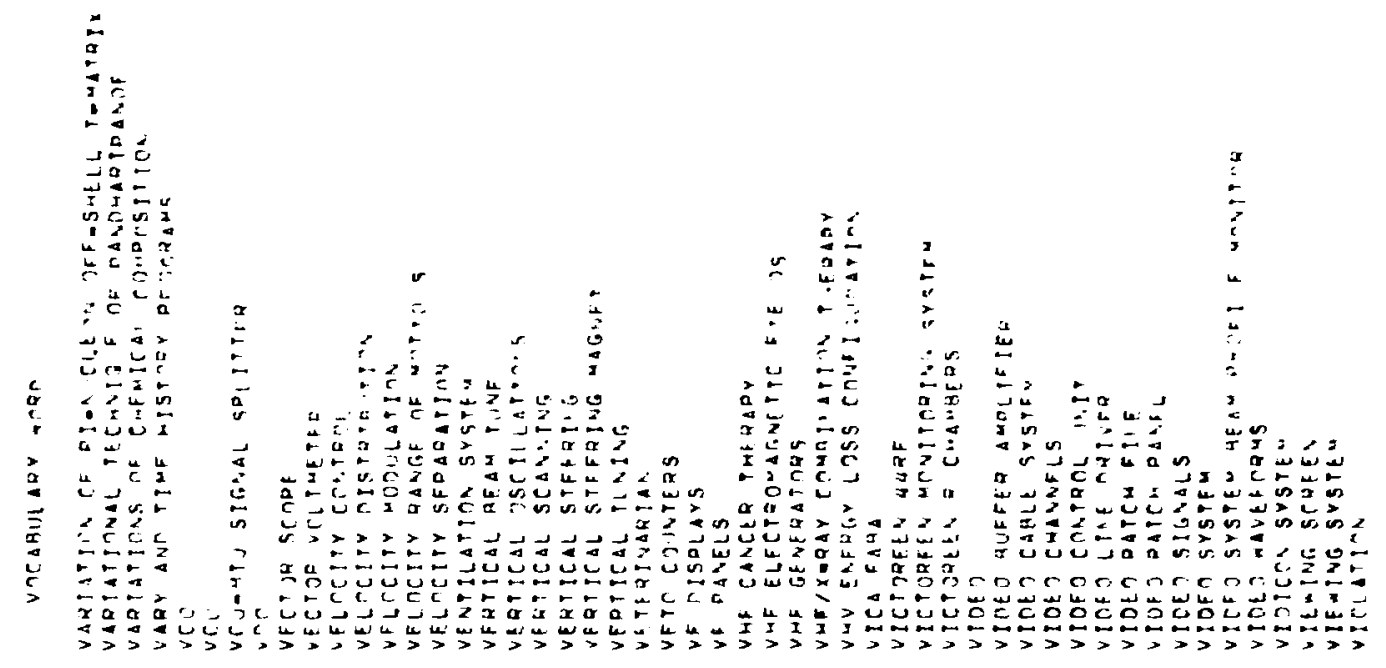

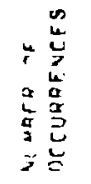
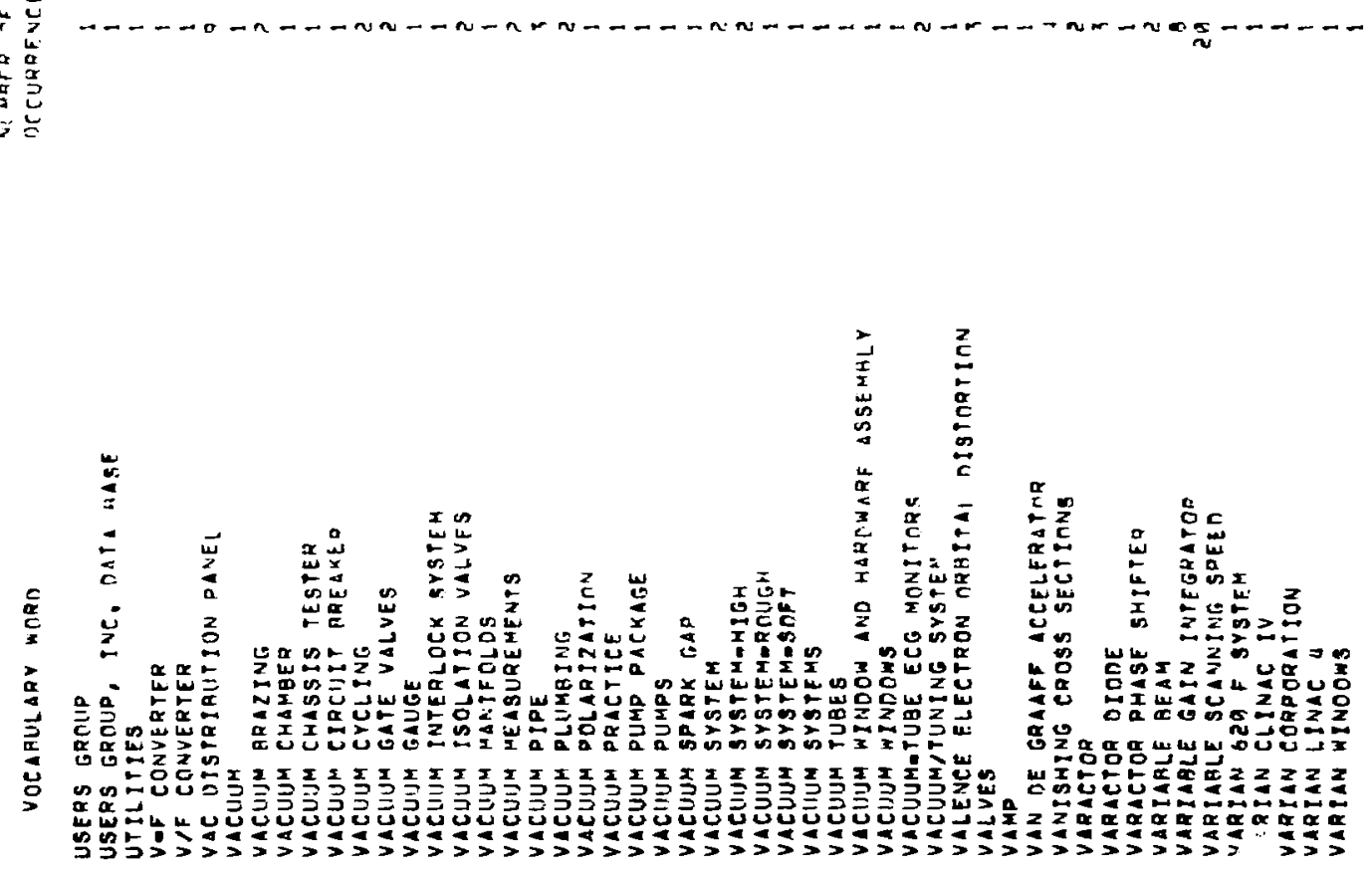

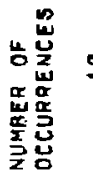


NIJAER OF OCCURRENCES

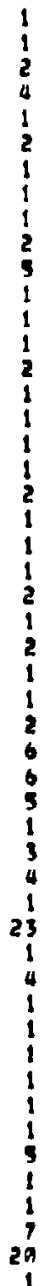

VOCABULARY WORE

VIOLATIONS OF UNITARITY

VISITORS CENTER

VISITORS CENTER DATA TASE

VLASOV EOUATION

VOLTAGE OISTRIBUTIOS SYETFM

VOLTAGE DIVIOFR

VOLTAGE GRADIENT

VOLTAGE HOLOEOFF OEGR $10 A T I O N$

VOLTAGE INSULATION

YOLTAGE LOOP

VOLTAGE REGULATOR

VOLTAGE STANDOFF

VOLTAGE WAVEFORMS

VOLLIHE SHAPING

VSWR VARIATION

WAIST-TOOWAIST TRANSFOR.ATITN

WALL CUPRENT DETECTRA

WALL STICKERS

WARNING LIGHTS

HARNING SIGNS

WASTE DISPOSAL CELL

WASTE OISPOSAL

WASTEO TIME

WATCHDOG TIMER DAOGOA

WATER ALARM SYSTEM

WATER BATH

WATER CABINETS

WATER CABLES

WATER EART

WATER CIRCUIT

WATER COOLINT, SYSTE"

WATER FLOW

WATER LEAK

WATER PASSAGE

WATER PHANTOH

WATER PRISH

WATER SYSTEM

WATER BYSTEMS

WATER TEMPEA ATURE

WATERICOOLED APEATULES

WATER-CBOLING SYTTEMS

WATERDCOOLING SYSTEM

WATERCART

WATUSI PROGRAM

WAVE FILTERS

WAVE FunCtions

WAVE TUNCTIONS

WUE NUMBER
WAVE SHIFT

WAVEFORMS

WAVEGUIDE

WAVEGUIDE CNUPLFRS
Qindite If

CCCIIDF YCES

HOCAR:ULAFY -720

MVFGUITE IINAC

maVEGUIDE MCOJLES

MVEG! ITF REMCVAL

aVAGJinE SPAZX

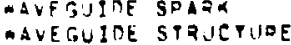

MAVEGUITE TASK

MAVELFBGTH

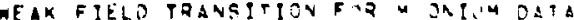

- EAK iniERLETION

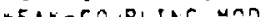

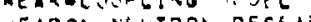

C- EAcitiv

WEADONS NEUTRON QFBE

AEADOUS VEUTRON RFBFAEC. FaCIIITY AEA LISE

MEDTE ALSCQFE:

WEOGE DEGRAOER

WEOGE ENERGY CFCOADOER

nEL NOEVAL JATICS

MELPING PECHVIJIIES

DELI. LOCGING

-FIL -F DRMEL DRDTRAMS

-HEEL TIRGETS

MOLE hOOY OOSE

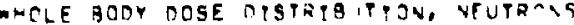

aCLE GOCY HYOEATHEMMTA

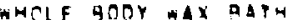

* arlfancer nose

aroleanon hestgut.

*holegior tmencal tarathest

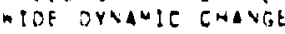

- INCIVE

Wist uESH

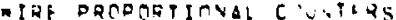

- ipr savile

WiRE SCAN

- iá SCANYEa

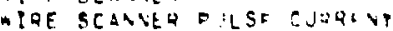

- ira scanicos

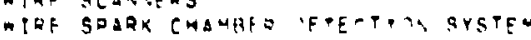

- THE TARLF

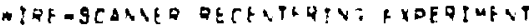

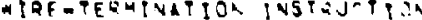

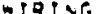

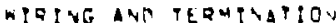

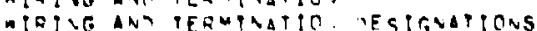

- IP TVe hoARos

mivise cont

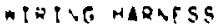

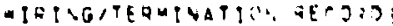

nivilo opegam 
NUMBER OF

OC CURAE NEES

WKE APPROXIMATION

WNR

WNR BEAM CHOPPER

WNR BEAM LINE

WNR DEVELOPMENT

WNR FACILITY

WORKLIST

WOUNO CONDUETOR

WOUND REPAIR AIOLOGY

WRITE = THROUGH MOOE

WY YLE

WYLE LOGIE

$x=A x$ is COLLIMATOR

$X$ RAY DATA ANALYSIS

XORAY DOSTMETRY

$X$ XRAY EXPERIMENTS

$X$-RAY INTENSITIES

$X$ RAY INTENSITY RATIOS

XORAY LINES

X-RAY PRODUETION

$X O A A Y$ SELFOABSORFTION

$X$ RAY SHIELOING

$X=R A Y$ JINGLFS OATA

$X$ XRAY SPECTRA

$X=A A Y$ PHERAPY

$X$ OAAY PRANS ITIDNS

$X=R A Y Y I E L D S$

$X \in R A Y S$

$X=Y$ COUPLING

$X E N O N$ GAS

$X X$-PRIME RECONSTRUCTIMN

XY RECONSTALICTION

Y SOECTRA

YELLOW SPRINGS HYOERDERUIC THERUISTOH

YELLOW SPRINGS TELETHER YOMEYER

YTTEABIUA

2 LAW

2 MATERIAL

$Z$-VARIATION

2ENFR DIODE BIAS CIRCIIT

ZERO ANGULAR MOMENTUM

ZERO CURRENT BEAM

ZERO FMITTANCE

ZERD PDTENTTAL CONE AYG

ZERO STEERING CURRENTS

CERO-CROSSING DETECTOR

ZEROACURRENT ADHITTANPE

ZIA CRAFT COOROINATOH 

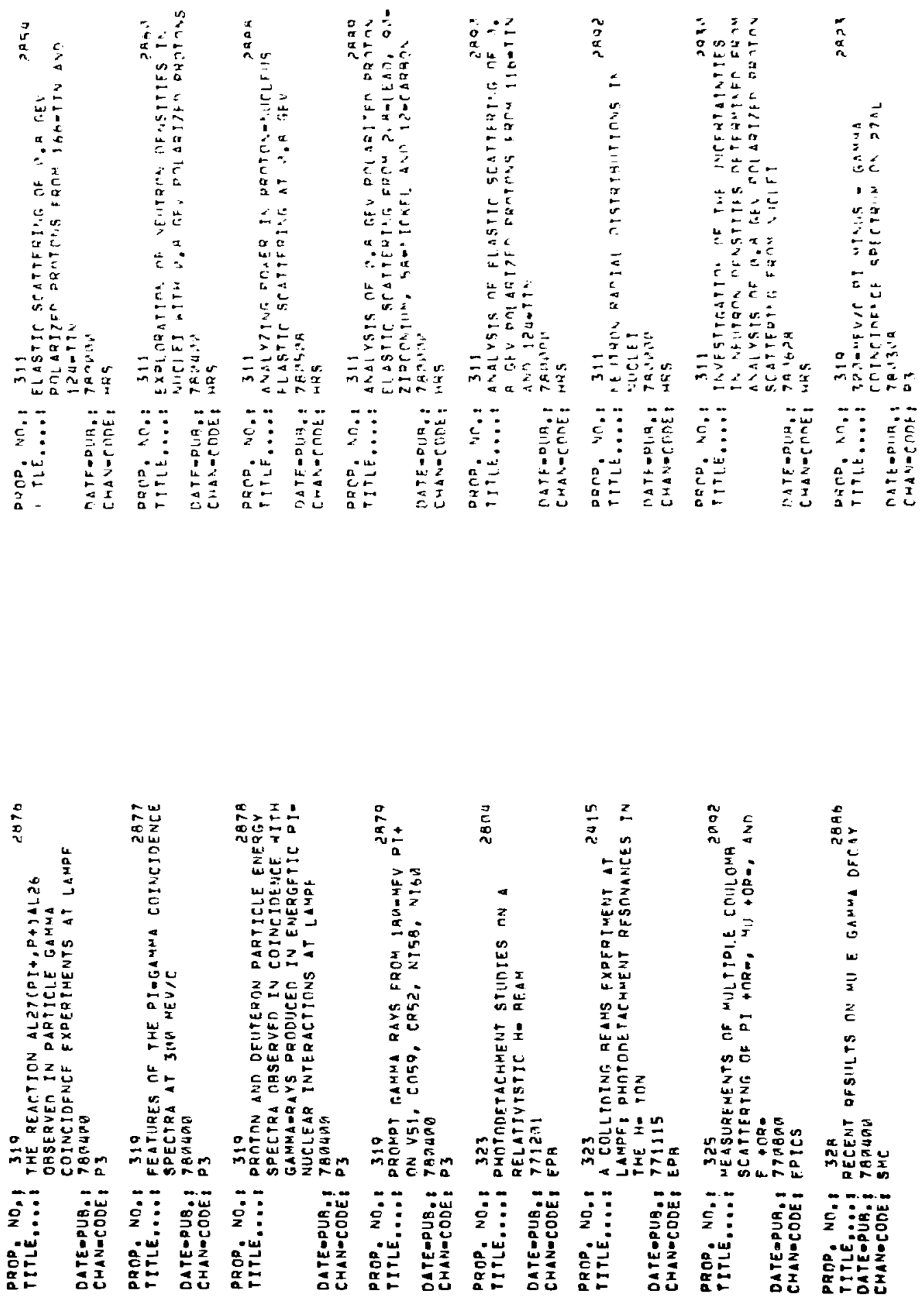

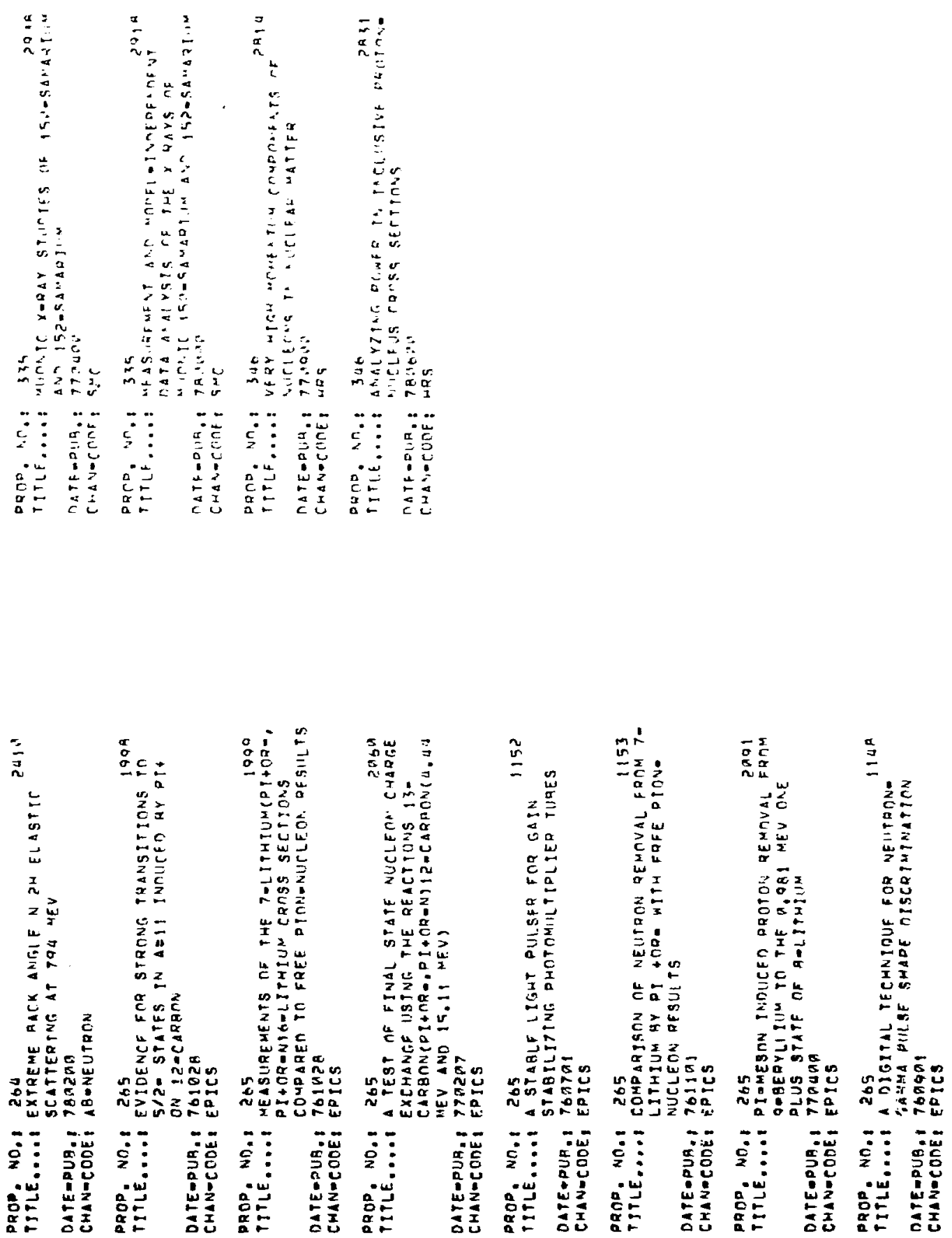


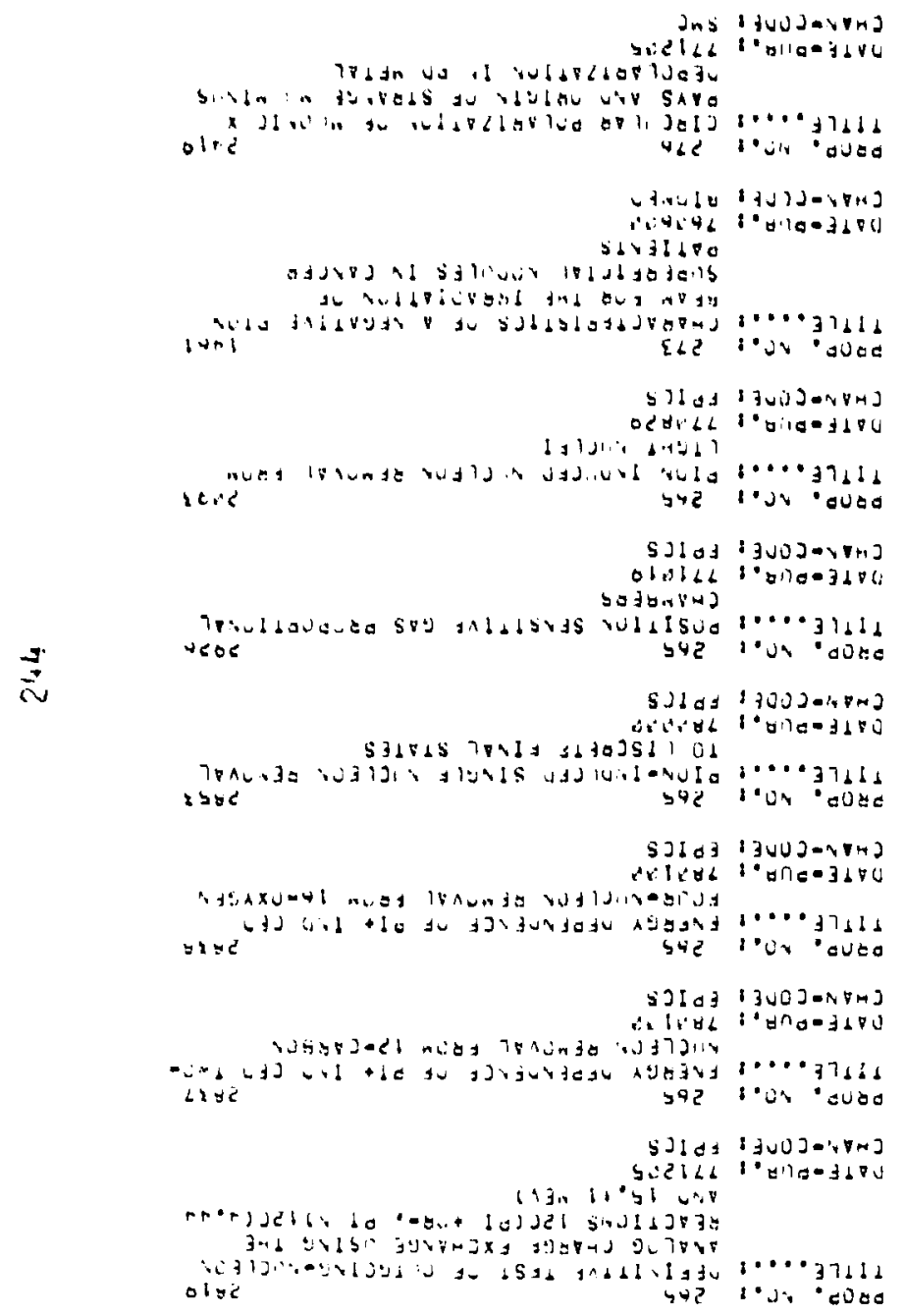

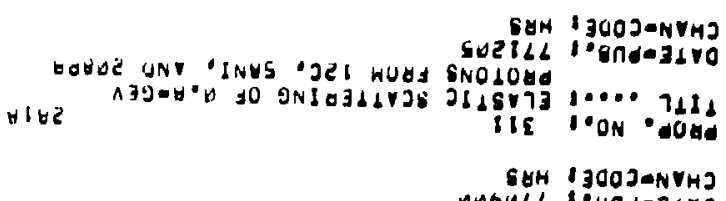

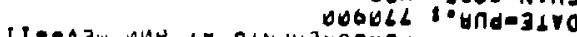

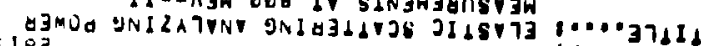
हliz

Sah I zO0Jomihz

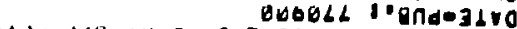

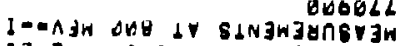

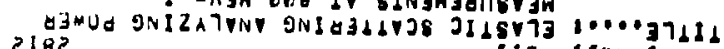
ite ron dodd

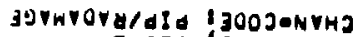

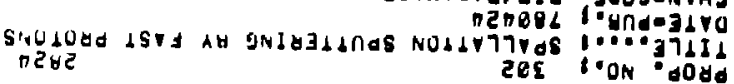

JWS I J00JenVHT

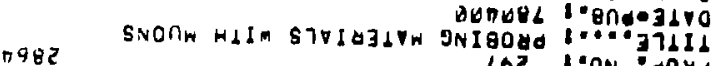
LOS I.ON JOAd

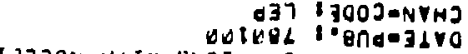

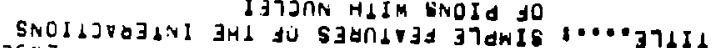

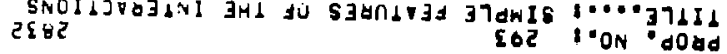
Ed :300J-nYho

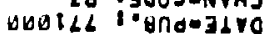
AHAVOOUWU⿺ UJindwOJ

$2 \ln 2$

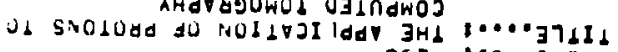
985 l.ON GOAO

JWE JjOOJONVHJ 028046108 Ond-JAYO

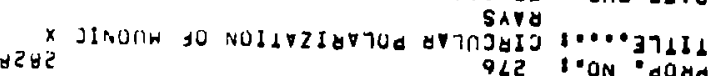

JWS $13003-n V H$ J 658046 ignd=3r.

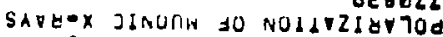
$42=2$

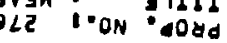


PROP. NO.1 234

3400 5 MEV

DATEOPUB, 779820

CHAN-CODE, LED

PROP. NO.1 234

TITLE....I INELASTTC SCATTERING OF OI MESONS DATE-PUB, FRDM NIITLFI AT 59 MEV

CHAN-COOE! LEO

PROP. NO.1 2UA

TITLE OOO CHARGE OISTRIBUTTON PARAMETERS $2 B 7$

IF (SIJB TIS) SHELL NUCLEI DEDUICFO
FROM MIJNNIC XORAY MEASIJREMFNTS

DATE-PUB, TBMUGA

CHANOCOOEI SME

PROP. NO, 24 M

TITLE...I MUNNIC TSOTOOF SMIFTS IN THE STAGLF

DATEOPUA, TBMAGG

CHANOCODE S SMC

PROP. NO,1 ZUM IF(SUAB 7/?) SHFLL NUCLET

DATE-DUA I TBABa

CHAN-CONE; SMC

DROP. ND.: 241

TITLE...I SEARCH FOR THF DIRECT PRODUCTION OF DOSJTRONS RY ZKG- AND BAMGMEV PROTONS

DATE-PUR, T6HBAR

CHAN-CODE, EPB

PROP. NO.1 241

TITLE....I PRODHCTION OF DIRECT DOSTTRONS 1 T

DATE-PUB 256 ANT AHA MEV

CHANOCOOE: FOR

PROP. NO, 54

TITLE...I LIMITS ON THF NIRECT PRODUCTION DF

DATEDPUA, T6ATI5 OATEDPUA 76A715

CHANECOOE, EPR
PHCO WO 201

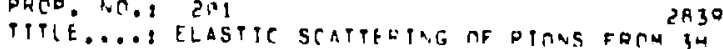

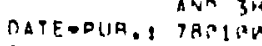

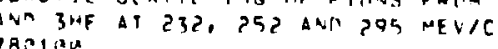

CMAS-CONE, PB

PRCD. MC.1 525

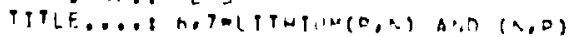

1004

CATF-PUH, : TOIMZR

CMANGCOTE, ABONFBTIOAN

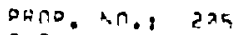

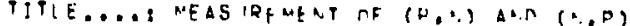

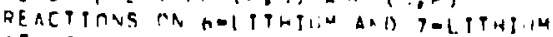
at Ben ufV

CATE-PUA, T7MBA

CHAMECNAE: ARENEUTROA

DROP AOA POS

TITLF..... TP TOS

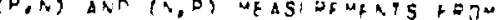

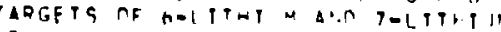
AT DaA UFV

DATF-DIIA.: 77:3412

CHARECOOE, AREREITAOA

PQDD. nn, zon

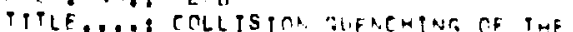

CHANOCOAE: 5 MCOA

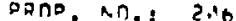

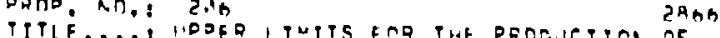

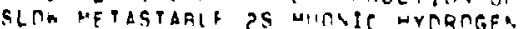
In

DATE-DHA TR, Ta,

CHAHOCOAF: SME

DQRD. NM. : 5?

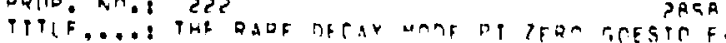

DATE=DUA. TR, Th,

CHAC-CDTE; D 3

PRND, M. : : ?25

TITIF...08

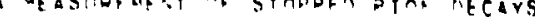

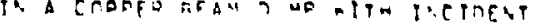

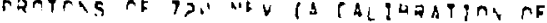

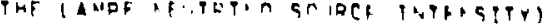
DATFEDUA.: ThD?:P

CHAAECCDE: "B ITATARA 
PRON. NO.1 163

TITLE...I SYSTEMATICS OF DIFFERENCES OF

NUCLEAR CHARTE FAHAMETERS IN THE FE NUCLEAR CHARTE EAMAMETEQS IN THE FE, NC. ZN RFGION FROM ELECTRTA SCATPERTNG ANO MUTAIC XORAY MEASUREMENTS

DATE-PUB. TGAGAR

CHANOCODE, SMC

PROP, NO.1 163

TITLE.... SYSTEMATICS OF NUCLEAR CHARGE

2006 DISTRIAIITIONS IN THE REGIDN FE TO ZV DEDUCED FRAM MUONIC $X-R A Y$ MEASUREMENTS

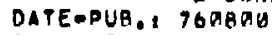

CHAN-CODE: SHC

PROP. NO.L $1 \mathrm{~h}$

20,10

TITLE.... SYSTEMATICS DF NUCLEAR CHARGE

DISTRIAIITTON TM THE CA TO YO REGION NEOUCED FROM ELASTIC ELECTRON SCATTERTNG AND MUONIC $X=Q A Y$

MEASURFMENTS

OATEAPURA, 77 OSZO

CHAN-CODE: SME

PROP. NO.8 163

$29 ! 1$

TITLE,.... SYSTEMATICS OF NIICLEAG CHARGF

DISTRIAIITINNS IN THE RFGION UH .LT. - LT. iga orducen fRom flastic

ELECTREN SCATTERING ANO MUOMIC XARAY MEASUREMENTS

DATE-PUA, T7MQRA

CHANDCODE: SMC

PROP. NO.1 163

2917

TITLE.... ELECTRON SCATTERTNG ANO MUTNIC RESULTS IN THE MASSOGP REGION! COMBINEA ANALIYSIS

DATE-DUB \& TBAMAR

CHAN-CONE, SME

PROP. NOII 165

TITLE...II MUONIJM FOHMATTOM PV NOALE GASFS IVI NOALF GAS MIXTURES

DATEOPUA, TUAQU?
PRRD, MOE: SAj

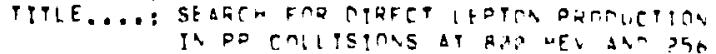
IN $\mathrm{V}$

DATENDUA.: THA420

CAAT-CODE: FDA

PQOD, AOE: JU:

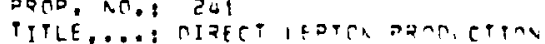

TAS

TITEODUR: T5!:111

CHAVECME: EPQ

DQCD. AR.: 205

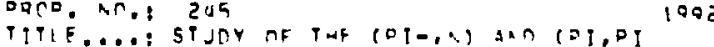

TATE-DUR: TRIMEDE

CHAAOCOME: EPTCS

DRCD, ACE: ZuS

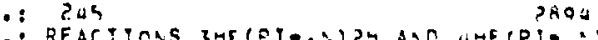
ba at of $50, \mathrm{TEEV}$

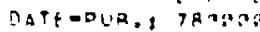

CHANGCOE: FDICS

BQNO, AN.: 24b 1005

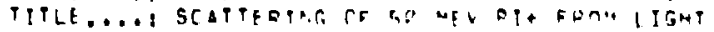

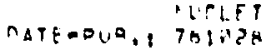

CHANECOMF; EDTES

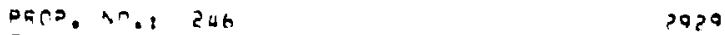

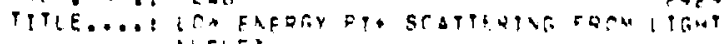

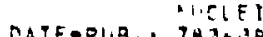
MATFOPLA.: THMARE ChanceChi, GDich

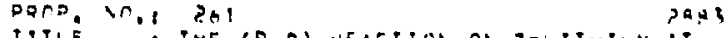

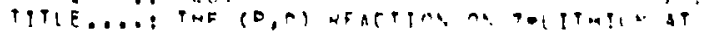
G)

chatecrobi: has

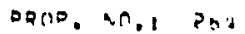

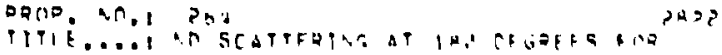

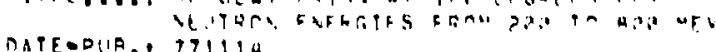



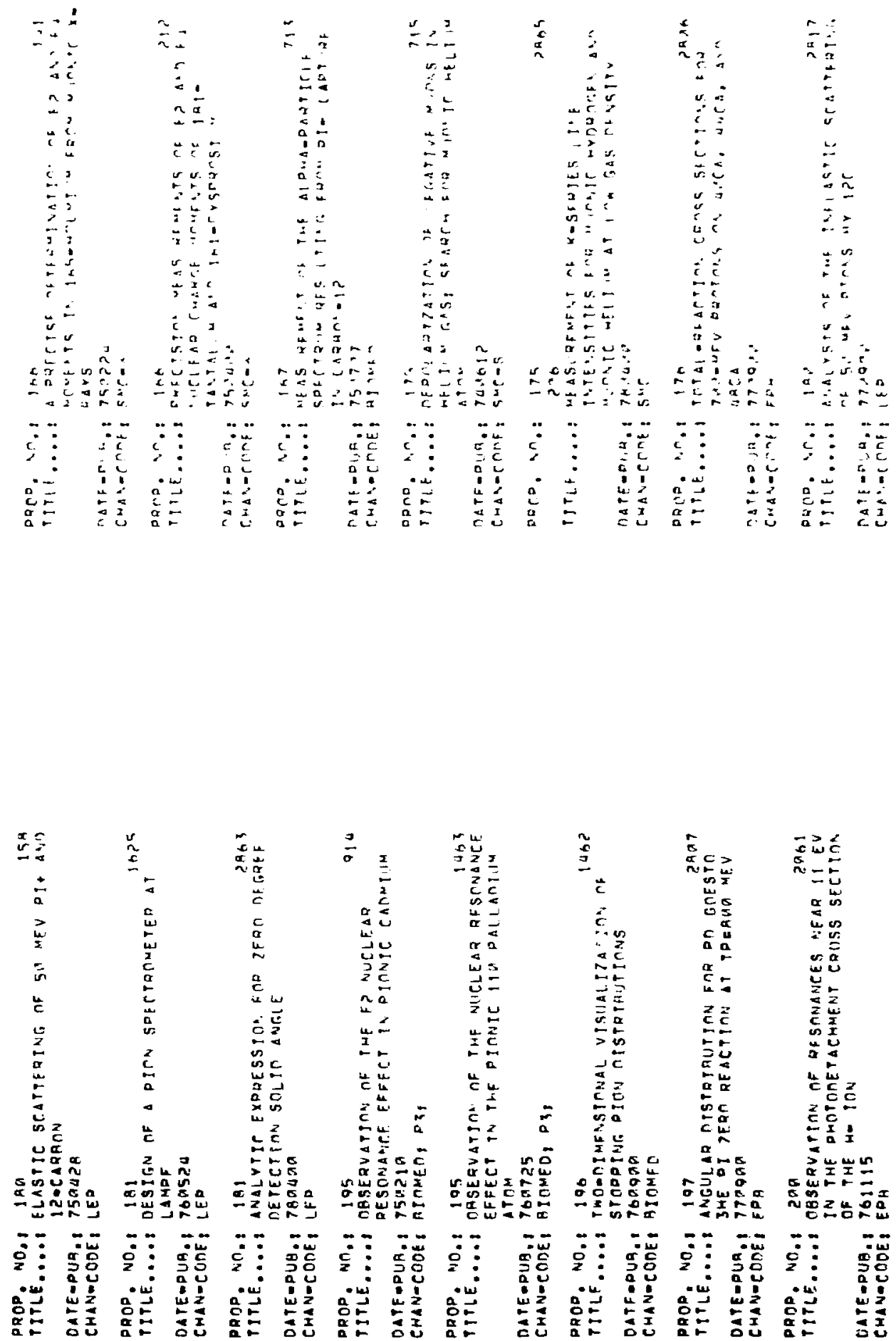

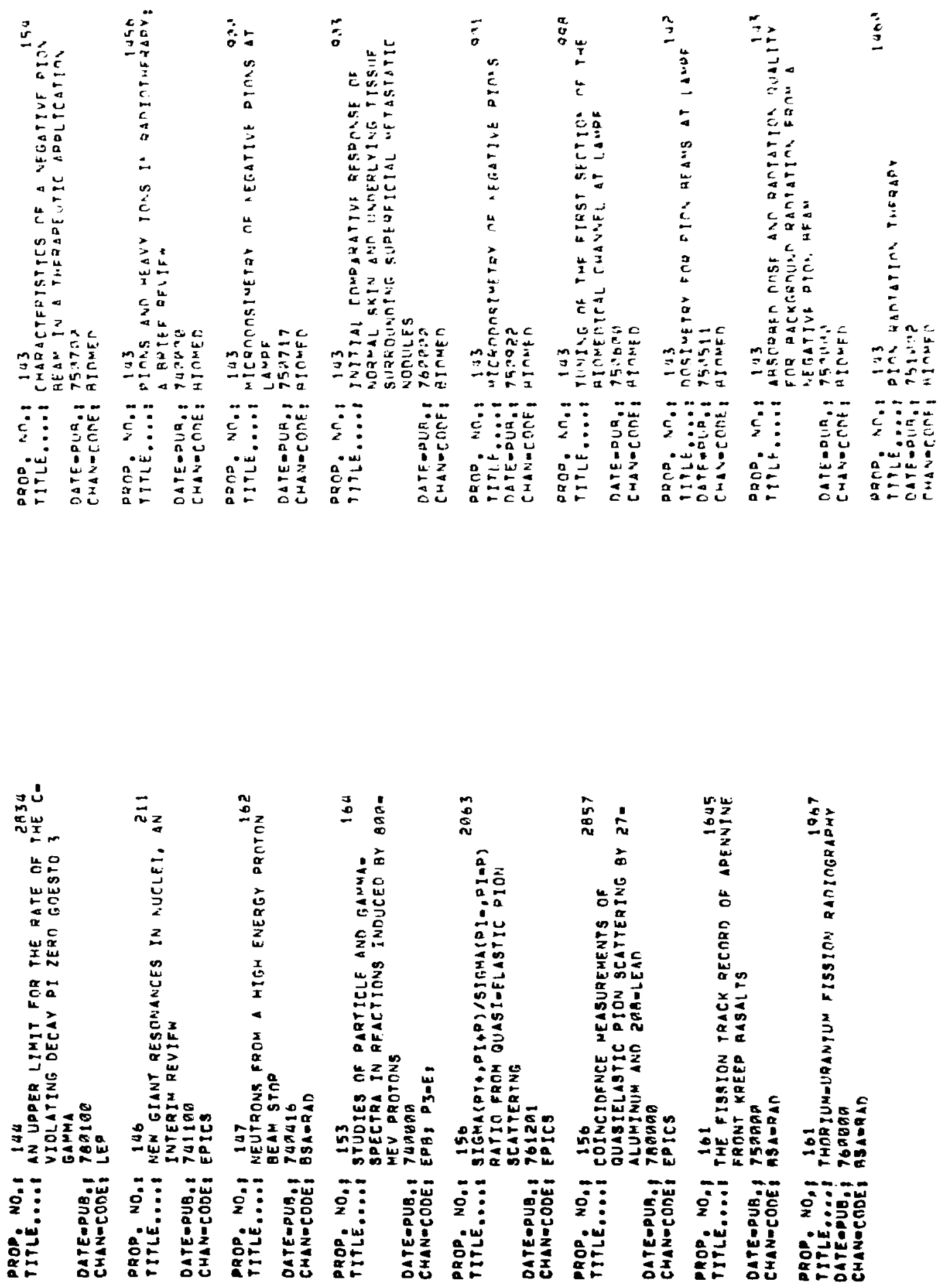
PROP. NO.1 130

TITLE OOO ANALYSTS OF PROTON INDUCEO TNELASTIC REACTIINNS AT H.8 GFV

DATEAPUB, TAGLAB

CHANOCOOE, HRS

PROP. NO, 139

TITLE.... ANALYSIS OF PROTONANICLEIIS ELASTIC

TSTE.... SCATTERTNG AT B.A GEV

B.! 78P4E

CHANECODE, HRS

PQOP. NO. 130

ELASTIC SEATTERING ANALYTING POREQ

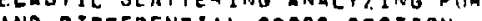
AND DIFFERENTIAL CROSS SECTION MEASUREMENTS ON CALCIUM ISOTOPES AT ด. $G 5$

DATEOPUB, 78 BQ

CHANGCODE, HAS

PROP. NO.1 139

TTLE 01 COUPLEDECHANAELS ANALYSIS OF INFLASTTC PROTON SCATTERTNG FROM ITC CARBON AT G, G TEE

DATE-PUB. TBHOLI2

CHANOCODE, HRS

PROP. NO., 130

TITLE. D. PROTON FLASTTC AND INELASTIC

2891 SCATTERTHG AT M,A T,EV FROM I2.13 CARBON AND 2OB-LEAD

DATE-PUB, TERMAO

CHANOCODE, HRS

PROP. NO.8 130

TITLE,...: (P,P) SPATTERING OH: $12,13-C A R B O N$ AND

DATE-PUA 2HB-LEAT AT MGA MFV

CHaNeCODE Thana

opopo noug iso 20,3

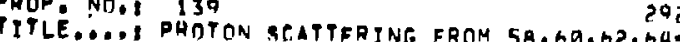
NICKFL AT AMg MEV

OATEOPUR, TTOOOA

CHANDCCDE: HRS

PAOP. NO.S 130 PIPLE... THF HITH RESOLIITIOA, SPECTROMETFR 295

DATE-PUR FACILITY AT LAMPF

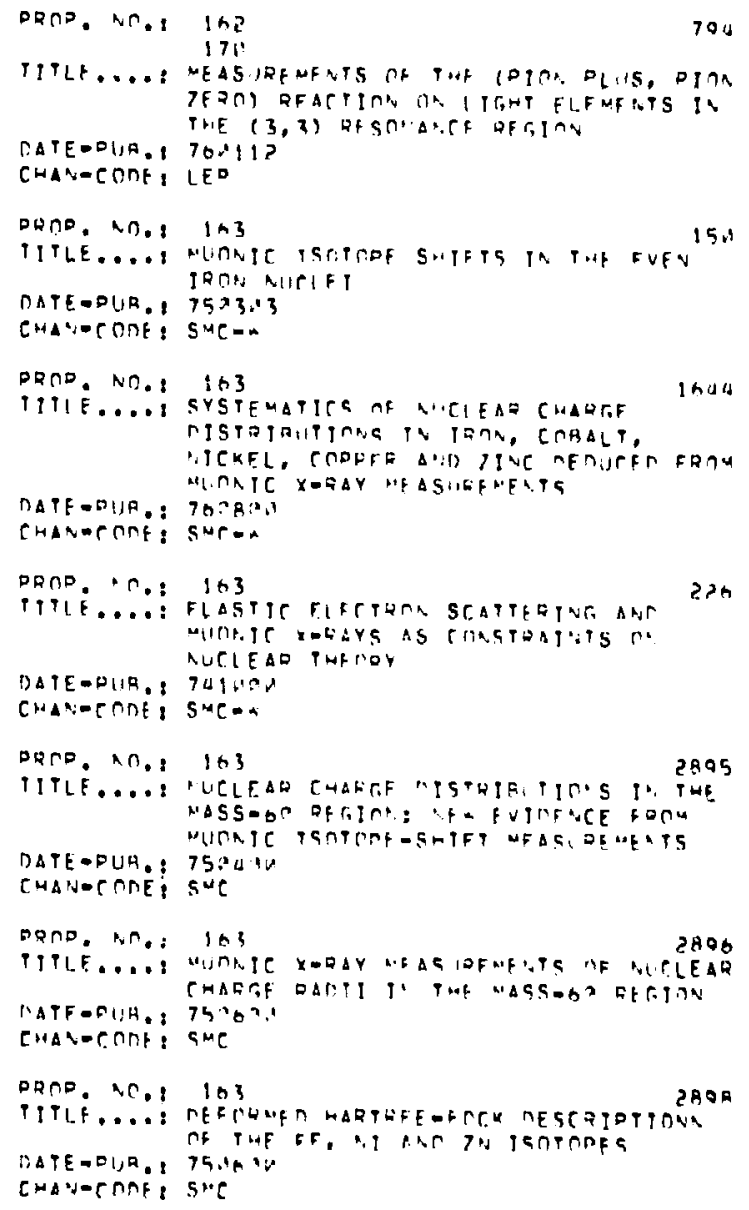



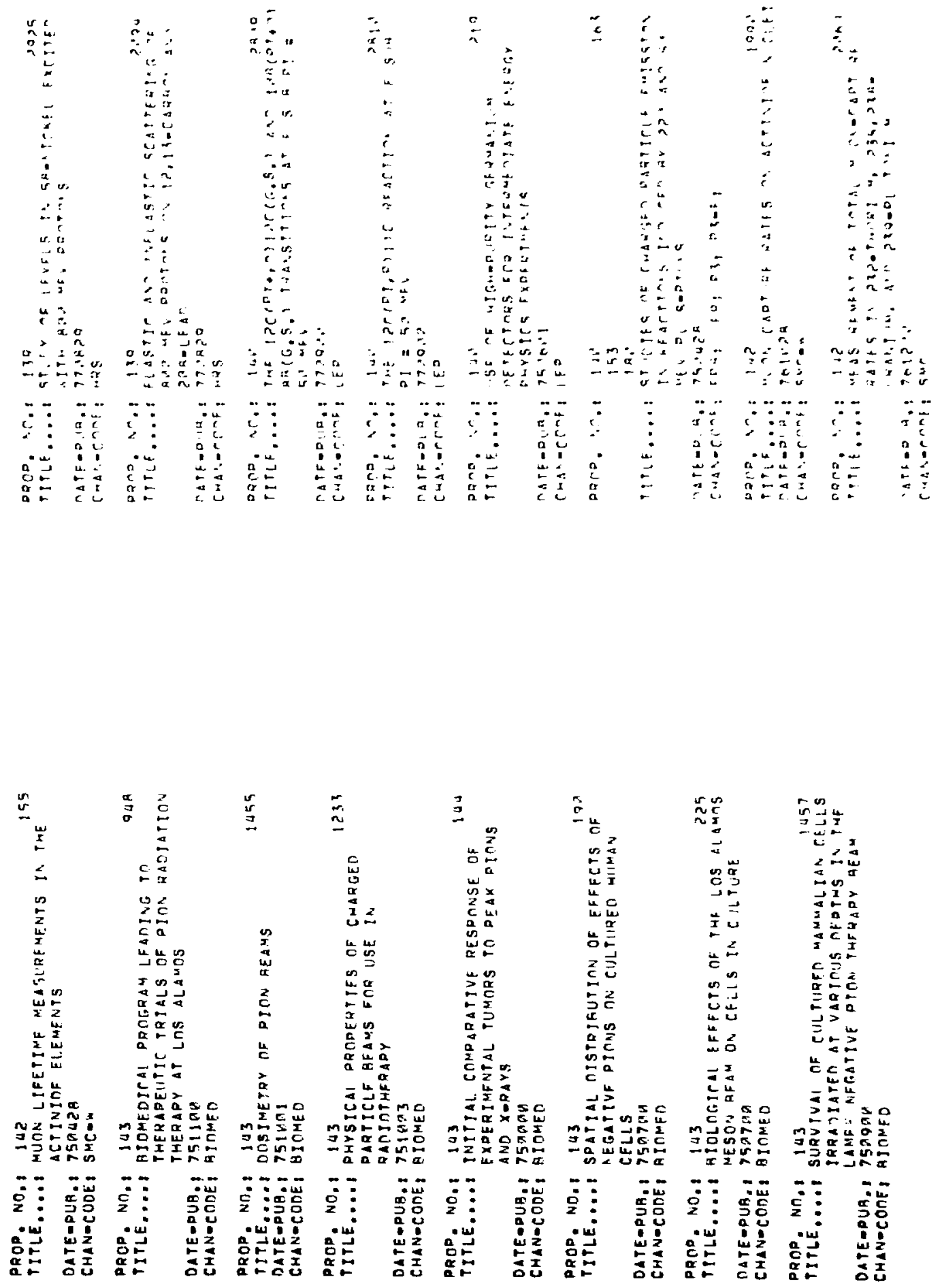
PROP. NO. 125

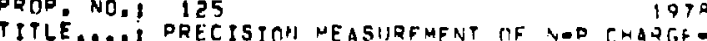

DXChANGF ConSS SECTICV AT hUT MEV

CHANGCONE: ABONEUTROA

DROP. NO.1 129

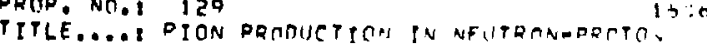
COLLISIOAS

DATE-PUB, I 751220

CHAN-CODE, AA-NELTORA

PROP. NOE: 13a

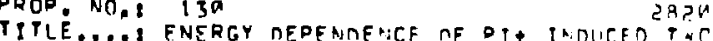

AND FOUIA-NUCLENN QFHOVAL

CATEOPUA, 771210

PROP. NO.I $13 H$

PROP. NO.: IJH INELASTPC DINN SCATTERING ON THF (3,

DATE-PUR, TODIGA

3) DION-NUCLEOIN QESONANCF

CHANACONE: EPICS

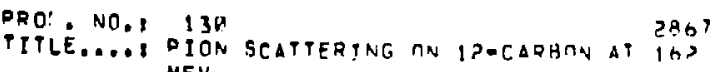

DATE-PU⿴囗十 TEV

CHAN-CONE: EPICS

PPDP. Nח.1 136

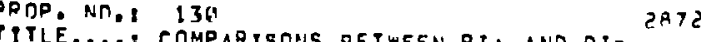

COMPATISTHS RETWEEHPT+ AND DI-

JNELASTTE SCATTEPIHG IN A STHBY JF PALL B BI RCKING EFFFCTS IA: COI LECTIVE ThARSTTTONS WITHIN SINGLE-CLNSE DSHELL BIIICIFI AEAR CALCTUN

DATE-PUR OS TR CHANGCODE: EPICS

DROP. NOB 130

2993

TITLE.... INITIAL RFSULTS WITH EPICS DITH,

SPECTROMFTER SYSTE.

DATE-PUA I 77 A

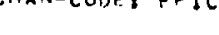

PANP. NO, 131

TILE.... THE RFIITFRON DESTATE AWR THE PIQ +

DATE-PUR GOESTO + D REACTIOTM

CHANOCODE: LED

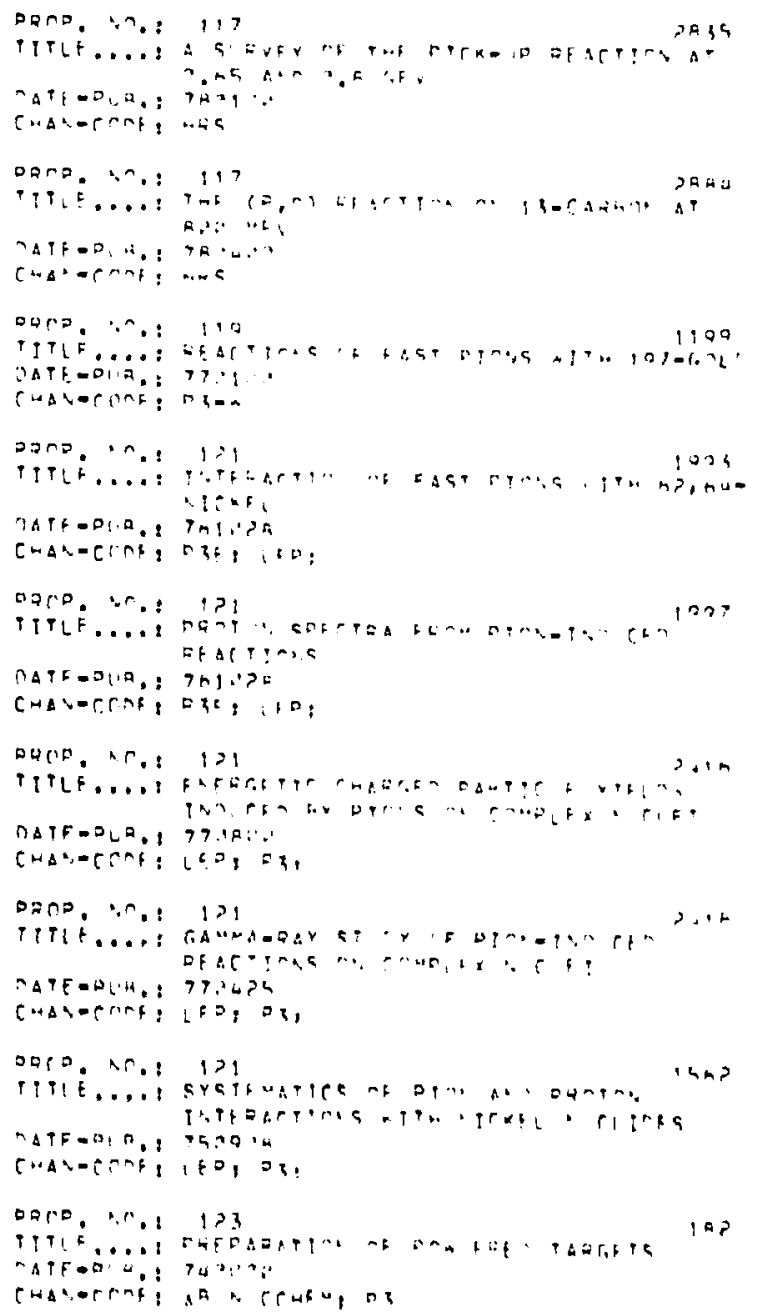


PROP, NO.I ITA
TITLE.... IN VIVG TISSUE ANALVSIS ISING MU. IAR
MESIC X RAYS

DATE-PUA, 76 ABOS

CHANOCODE: SMCOW

PROP. NO.I laz

TITLE.... NEUTRON KNOCKOHT BY PION DOR. ON 19NITROGEN, 16-OXYGEN, AND 19-FLUOPINE DATE-PUB,: 75A4A

CHANOCODE: P 3 OE, LFO,

PROD. NO.I IR3

ORE) ANR 340 MEV OFOTONS

TTEDPUA, 759830

CHANOCODE: O3: $A B=N U C C H E M$

PROP. NO, 195

TITLE...? GAMMA-RAY EMISSION FROM 13U-CERIIIM AND LEVFLS IN 1340 LANTHANUM

DATE-PUB, 769610

CHAN-CODE, AB-NUCCHEM, SWY-LABS,

PROP. NO,I 195

TITLE...I! HALFELIVES OF 134-LANTHANUM ANM 1320 $135=$ EERTIJH

DATE P PUB I 760223

CHANDCODE, AR=NIICEHFM, SWY-LASS

PROP. NO.1 1 A5

TITLE...1 130-AARTUH LEVEL SCHEME AS OASERVED

DATEOPUE IN THE RECAY OF 134ELANTHANIJN

CHANOCODE, AB-NUCCHEM, SWYOLAPS

PROP. NO.: laS

DATE THE DECAY FNERGY OF 131 - HARIIIM

DATEAPUA, 76119 AR

CHANOCODE: AB-NIJCCHFM, SWYOLARS,

PROP. NO, 105

TITLE.... TAMMAGRAY STUDIES DF THF DECAY OF 159 I2B-BARTIIM AND 158-CESTUM

DATE-PUB. 741 TOAH

CHAN-CODE, ABONUCCHEM, SWY-LARS

DROP. NO.1 195

TITLE...i d DECAY OF 1 ?R-CESIUM

DATE-PUB, 77 TUAG

1639

CHANOCODE, AB-NUCCHFM, SWYOLAAS,

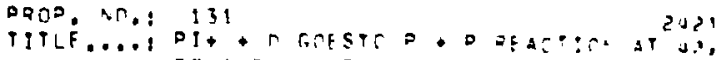

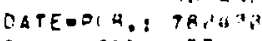

CHAvecti: ifo

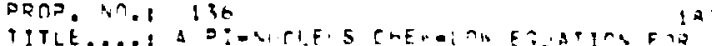

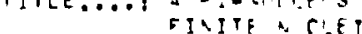

DATE-DLA. 19.2.53

CHAOCOCE: EFICS

DROP. An.: 137

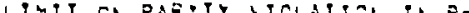

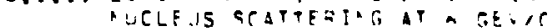

NATE-OUR: 7Q:7I5

CHAV-CCOE: FD

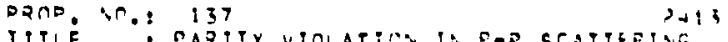

TTTLE,....

CATEOPLAR: TTIO?

CHANOCOAE: FFi

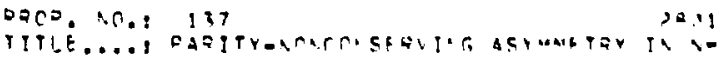
C SCATtFAD"

CHAT-CCAE F 771138

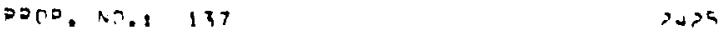

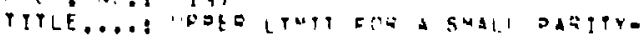

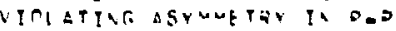

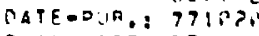

CATTEETAO AT IS MT

CWASCCHE: EPA

DQCO. IT: 137

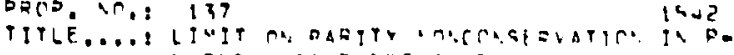

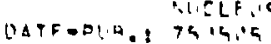

Chavechatis to:

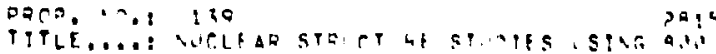

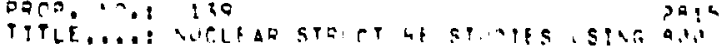

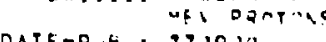

Chatecchis bes

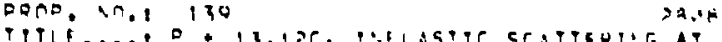

Tht

chatecmil: mes 

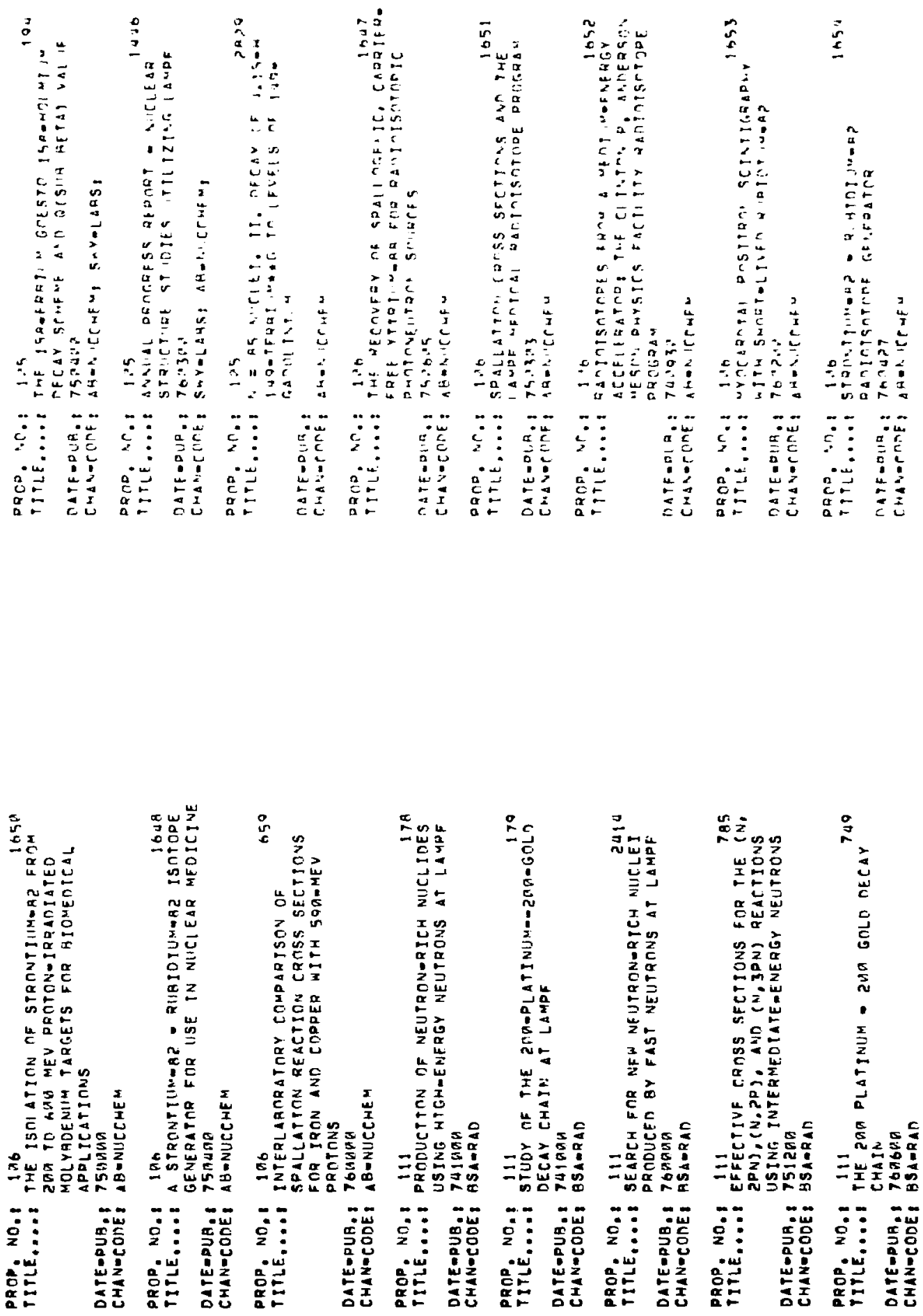

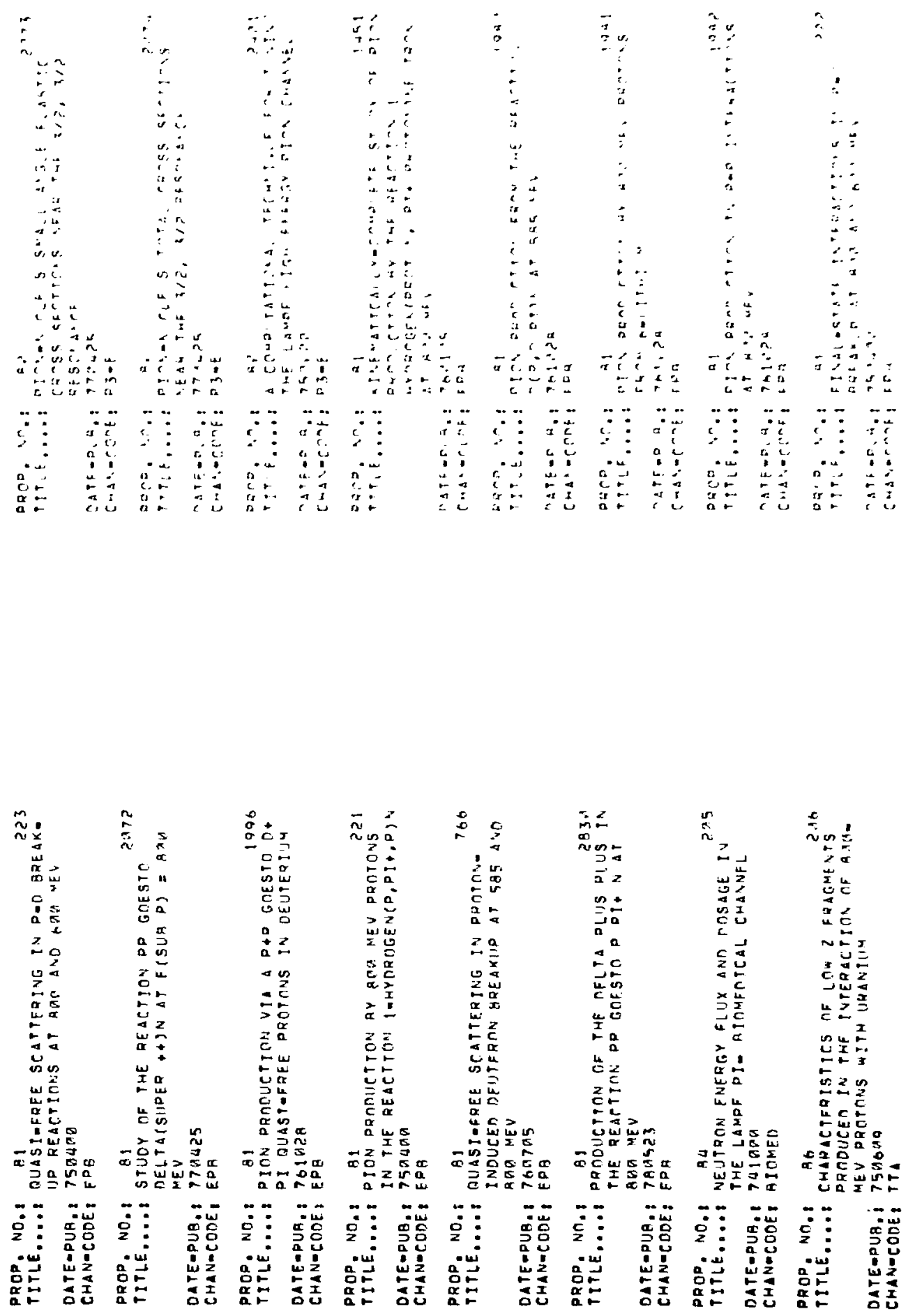


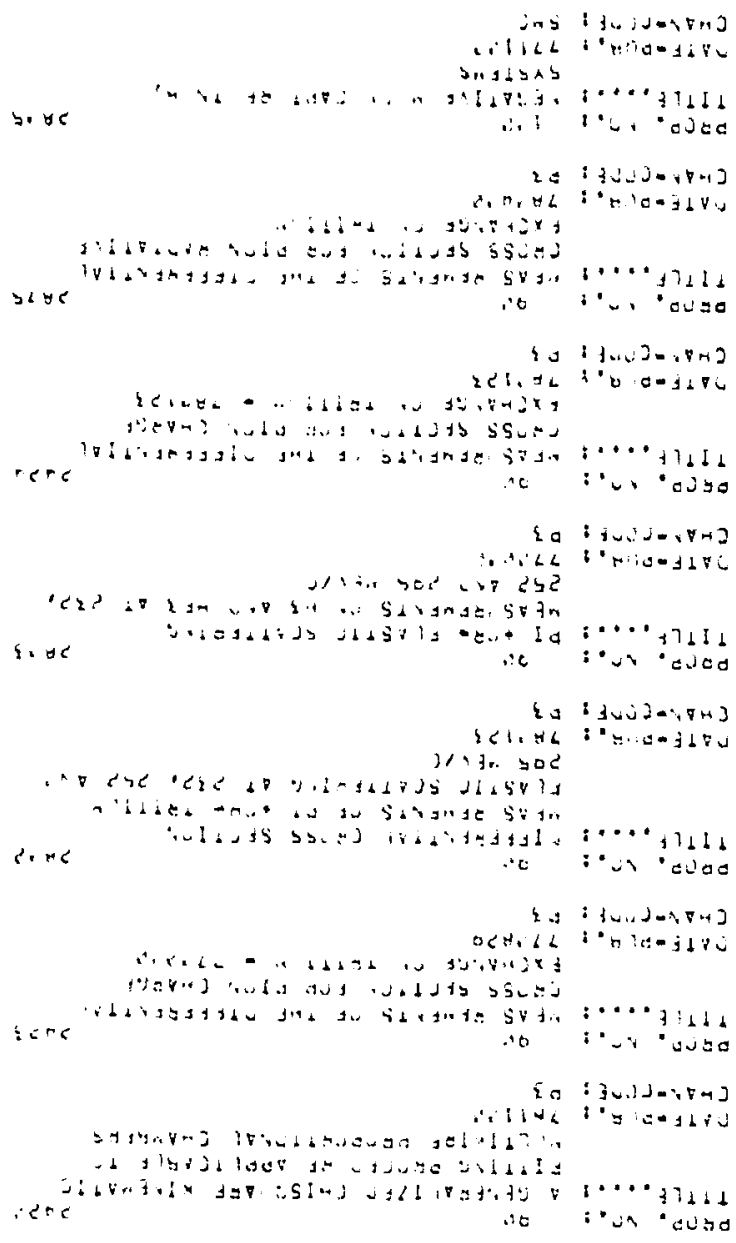

NUOLOBNER IJOOJONVHJ

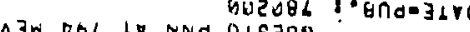
T3isrne $\cdots \cdot \cdots 371$

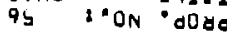

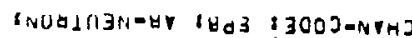

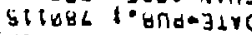

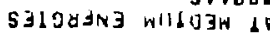

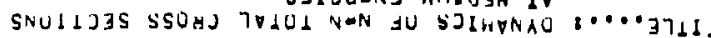
458 is is in aho

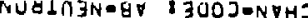

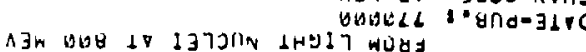

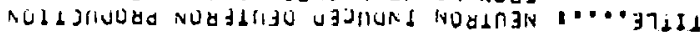
$4<\forall<$

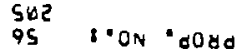

indaTinagh 1300Jantho

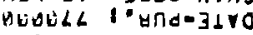

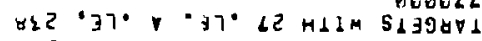

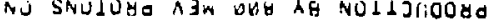

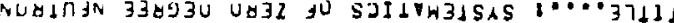

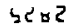

QS
SUE

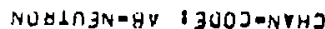

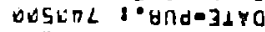
SnT

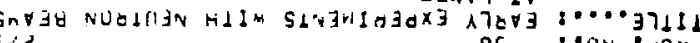
lic

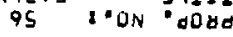

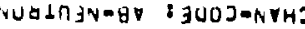
DovesL to

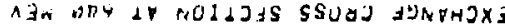

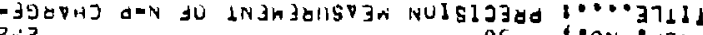


TITLE.... ACTIVATION STUOIES OF PION-INDUCED REACTIONS ON C, N, O, F, AL, AND CU

DATEAPUB, 759630

CHANACODE, P3, LEP, AB-NUCCHEM,

PROP. NO.I GT

TITLE,...I THE FFFFCT OF DEFORMATION

DATEAPUB, TRAIAK

CHAN=CODE, P3, LEP,

PROP. NO.1 67

TITLE.... DEEP INFL.ASTIC PIONE INDUCED NUCLEAR REACTIONS IN THE ISOBAF MODEL

DATE-PUB II 7712 ศI

CHANACODE, 3 ; LEP,

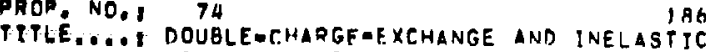

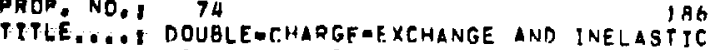
SCATTERTNG IN OIN + 3-HELIUM

OATEOPUB T T

CHANACODE: EPICS

PROP. NO.1 79

TITLE.... AN OOTITAI MODEL STUDY OF LOW ENERGY PION SCATTERING FROM I OOXYGEN

OATE-PUB. 77 GQAR

CHANACODE LED

PROP. NOE: OU
TITLE...E PIDNENUPLLIUS FORWARO SCATTERING AMLLITUAES NFAR THE 3/2, 3/? RESONANTE

DATEAPUB.: T6IOSA

CHANECODE P3 EAST

PAOP. NO.: BP DATEODUA, OR० 16 OXYGEN SCATTERING

DATEEDUA, I 75060

CHANMCONE: D $3-E$

PROP. NO.I SP TPLE.... TUNE-UP DF THE LAMPF hIGH ENEHGY 2 TMG

ION CHANNEL

DATE-OUS, 75 PATA

CHANACOOE, P $3 \rightarrow E$ 
PROP. NO.1 37

TITLE....I PRECISTON MFASURFMENT OF ISPSUR 160 MUONIUM GYPEPF INE ST

DATE-PUR, 75A4Z8

CHAN-CODE: SME

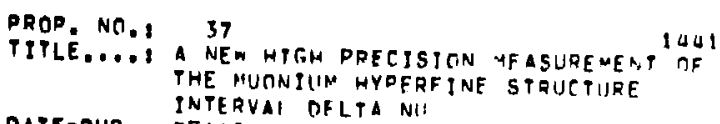

DATEOPUR INTERVAI OFLTA NII

CHANGCODE: SMEOS

PROP. NO, HO

TITLE....I ELASTIC AND INFLASTIT SCATIERINO ZRUG ELASTIC AND INFLASTIE SCATIERING OF BAA MEV PROTONS FRIM BQAVTTRIUM AND

OATE-PUB. TBOIGA

CHAN-COOE, HRS

PREP. NO.I UQ

TITLE...? ELASTIE B. TNELASTIS SEATTERTNG 2882 Bga MEY bi- groNe O9-ZIRCONIUM TARM BQOYTTRIUM ANR

DATE-PUB I TORUGA

CHAN-CODE: HRS

PROP. NO.I SH

TITLE...: DHOTON SPECTRLM IN PION CAPTURE ON3

TITL.... T PHETON

DATE-PUB:I TRAM19

CHAN-CODE! LEP

PROP. NO.I 50

TIPLE...I RADIATIVE OI. CAPTURE UM LIOUID 2845

DATE-pur TRITIUN

CHANGCODE 78 IIAO

PROP. NO.t

TITLE.... NEUTAOA RACKEROHIO STUDY FOQ LAMOE

EXDERIMENT NO 531 STIJOY OF LHE

NEUTRTNO CAPTURE CROSS SEETIONS

CHLORINE- 37 WITH MII OSECAY IONS IN

OATE-PUA, 75A3GH

CHANOCODE! NFUTRINA A

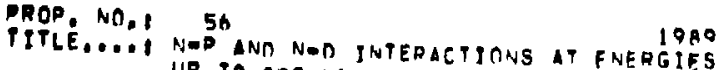

DATE-PUA IJP TO AOA MEV

CHAN-CODE TOIGZO

DROP. NR, 20

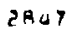

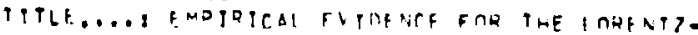

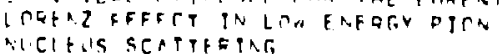

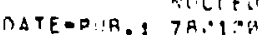

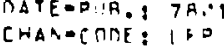

DERP. $A, 1$,

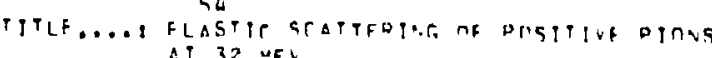
DATE-PU,A : TR:M

CHAM-CINCE: LEO

PROP. NT:: ?Q

$7254^{4}$

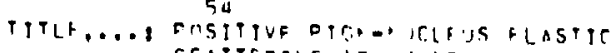

DATEDPHH.: TA, TAB

CHA DOCCOCE: IFO

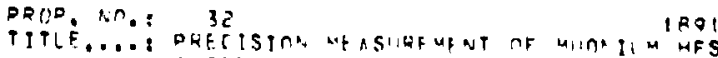

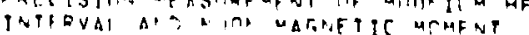

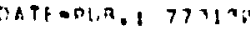

CHA UOCOAF: SAC-5

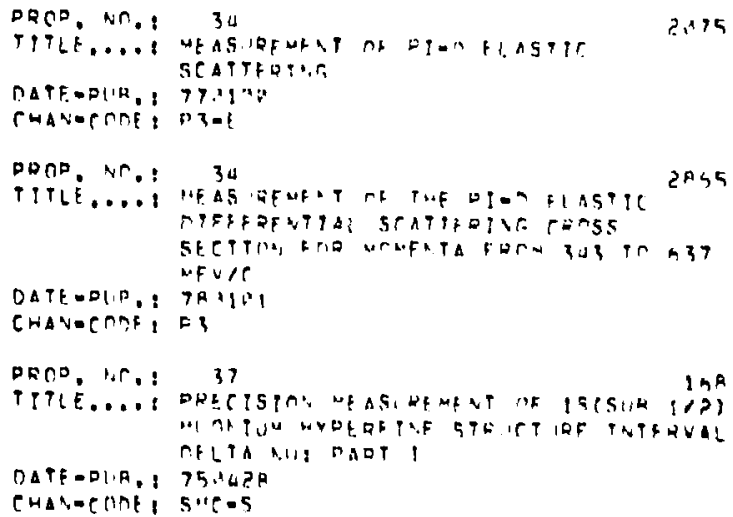


PROP. NO,! Q $2860^{\circ}$

TITLE...I PI+OR- SCATTERING FRTM LACA, LZCA, UUCA, AND $\angle B E A$

OATE-PUB, 78140 C

CHANECOOEI EPICS

PROP. NO, 12 2970

PROP: NO, I IZ RADIAL THAQGE PAQAMETERS ARID THE OUAORUP TLF MAMENT OF IAI-TANTALUM USING MIIONIS $X$ TRAYS

DATEAPUB I T TOGAR

CHAN-COOE: SMC.

PROP. NO,I 12 284? TITLE.... I IMPROVFO CASCAME PROGRAM FOR MIIOVIC

OATE-OUB. TROMS

CHAN-CDDE: SMC

PROP. NO, MUONTE XORAY STURY OF THE CHARGE

DISTRIALITION OF 1B7=KHENGUM

DATE-PUB. 78 OICA

CHAN-CDOE: SMC

PROP. NO. 12

TITLE... F. TOMENTS IN 109, 2Q1-MEQCURY

OATEAPUB. TOMIOA

CHANGCODE I SME

PROP. NO, I 14

TITLE, PIOA POAMUCTIOH USTHG POLARIZER DROTONS

DATEORUB, TAAI HA

CHANMCODE; EPICS

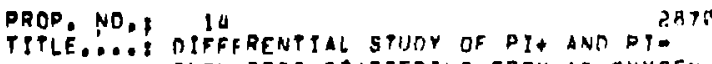
INELASTIC SCATTERTNG FROM IANOXYGEN

DATEOPUB TBALAR

CHANECONEI FPICS

PROP. ND,! IU ?RI

TITLE..... INELASTPC SCATTERING RE O Ja ANO FROP 1

OATEFOR I T790

CHANOCODE, LED

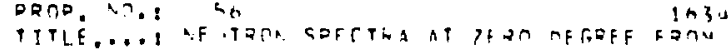

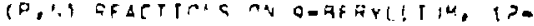

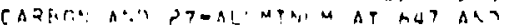
QCA NEY

OATE-DUA, 7GM7!h

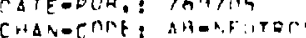

PQRO. NC.: 56

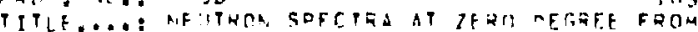

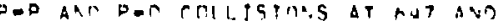

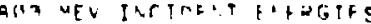

T) $A E=01: 0,70+7.15$

CHAB-ETIE: AHABE.HTHON

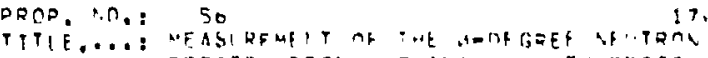
SPECTZA FEOM A.ST AND E:M MTV DOOPOS TGNHARANAT CF LIGHS WCIE!

CATE-DIHA TSOTSO

CHAGCOOCE: AH WE, ITRO

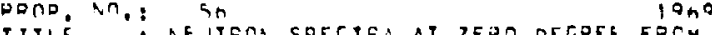

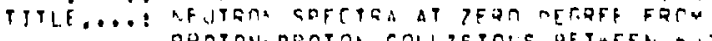

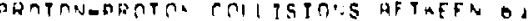
AND BAS NEV

NATE-DUA, : 77.11.31

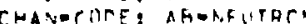

PQRP, A.ก.: 50 : 230

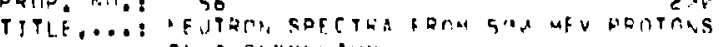

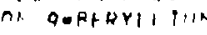

SATE $=0,174,14$

CMANECOAE: AGOAF,TTHA:

Quno. s..: 50

32

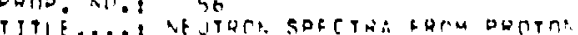

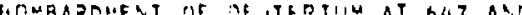
fis MF

TATE-PIA, T53015

CHANGCORE: AHEREITOOA

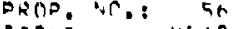

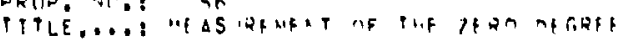

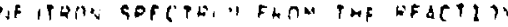

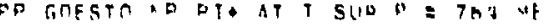

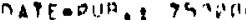

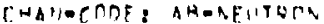



पर

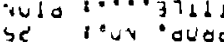

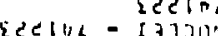

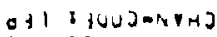$$
\text { MJ }
$$

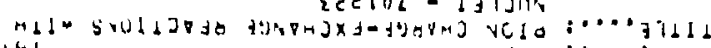

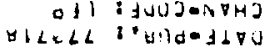

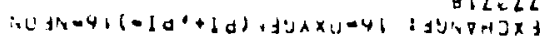

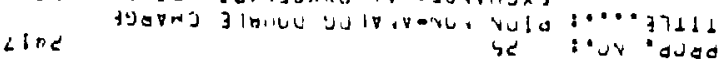

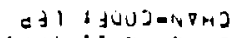

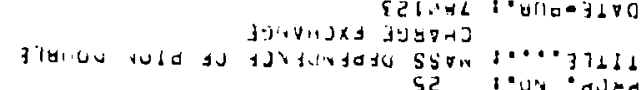

$$
\begin{aligned}
& \text { Sट run ojea } \\
& \text { (a) उUUJ-ivis }
\end{aligned}
$$

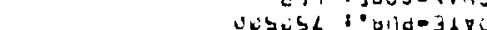

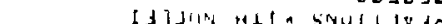

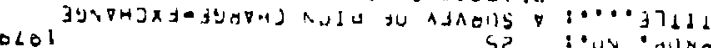

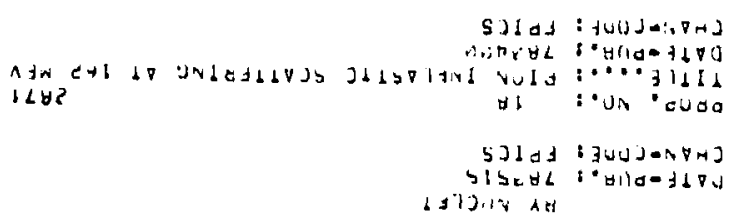

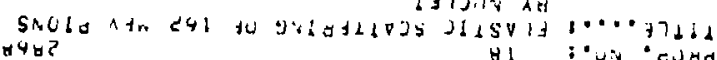

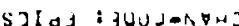

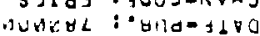

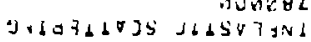

- 1 d $V i, \nabla+10$ gu

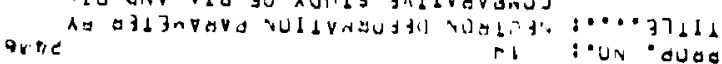

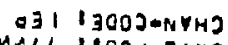

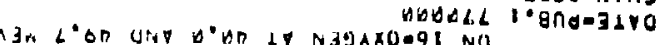
$\cos 10$

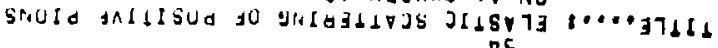
bouc

05
$050 h \cdot 9000$

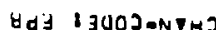

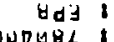

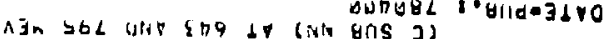
a

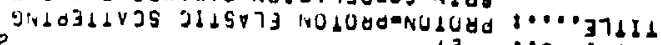

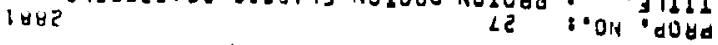

\section{HdJ :300JonYHz}

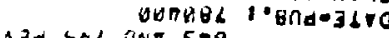

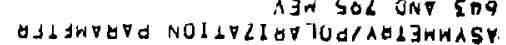

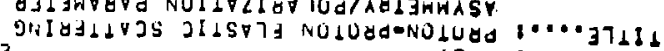
1482

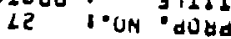

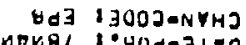

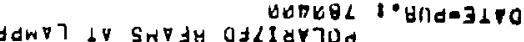

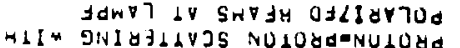

OSBC

Lotgd $\ldots 3711$

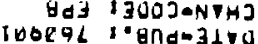

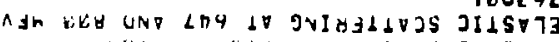

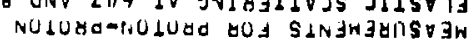

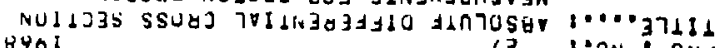
8401 LC I.ON COOAd

d3า IJ00J-NYHJ

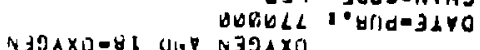
(a)

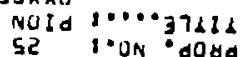
$\operatorname{con} 2$

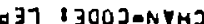

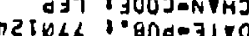

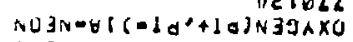

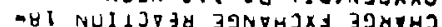


PROP. NO.?

TITLE.... PIONONIJLLEAR TOTAL CROSS SECTIONS 1626 DATEAPUB, 760614

CHANACODE, LEP

PROP. NO.: PIOENUCLEUS TOTAL CROSS-SECTICN ${ }^{2}$ DATA FROM LAMPF ANO RNL.

DATEMPUA, 769524

CHANOCODE, LEP

PROP. NO, I O
TIPLE....I PION CROSS SECTION MEASUREMERTS ON ILIGNED IBSHO IN THE $(3,3)$ RFSONANCE REGION

DATE-PUB, 77129A

CMANACODE! LEP

PROP. NO, 4 29?7

TIPLE..:? + $P$ + 4 HE ELASTIC SCATTERIAG AT AAT MEV DATEOPUA: 770829

CHANECODE, HRS

PROP. NO:! PA EIASTIC SCATTERING AT TSA MEV DATE-PUB, 7800 GA

CHAN-CODE: HAS

PAOP, NO,! 5 TILE...?I OUASI-EI ASTIC SCATTERING OF AAG MEV

OATE-PUA: PROTONS

CHANOCODEI HRS

PROP. NO.I MOLE...II MODEL-INDEPENDENT INTERPRETATION OG THE DYNAMTC HYPERFINE EFFECT IN MUONIC ATOMS WITH AN APPLICATION TO 192-OSM tllM

DATEAPUB, 7704A9

CHAN-CODE, SMC

PROP. NO,I TTLE. DS NUCLFI FROM MUONIC $X$ RAYS

DATEEPU \& 771121

PROP NO. I

PITLE.... MUONIC X-RAYS EqTM THE TRAMSITION NUCLEI 194,105,196-PLATINUM

DATEAPB., 75म42B

CHANOCODE: IMC

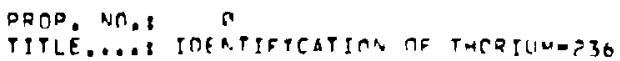

$23 n$

OATE-DUA. $73120 \mathrm{~A}$

CHANIOCONE: LINE A

DROP. NOA:

TITLE,...: NEJTOON WAOII OF CAICIIIM ISOTONES FROM PINA. TCTAL TOCSS SECTIOA. MEASURF MFATS

DATE=PUF, 77 FS? 3

CHAN-CODE: IED

PROP. NO.1 ?

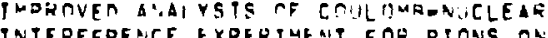
in OXYGFN

DATEOPUA, TOOSPU

CHANECOOE: LEO

PROD. NOA: DO TITLE..... D DONGN

DATE-DUA.1 75111

CHANECONE: IED

DPOS. NO.1 5 I I H

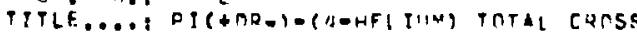

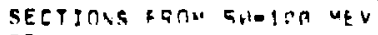

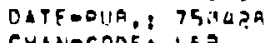

CMAN-CONE: LE

PSOP. H.:

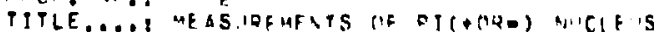
TOTAL CEOSE SFTTIOAS AT ENFRGITS

DATEODUA.: TSThOO TOTAL PEOSS SFTTIOUS AT ENFATGIS

CHANOCOAE: LEP

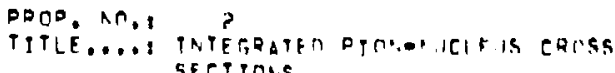

DATE-DUA, 7DOA

CHAW-CODE?

PROF. NOE: RE ?

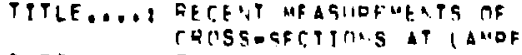

OATE-DUA: 7 ThO? 3

CHANGCCOEं, LEO 


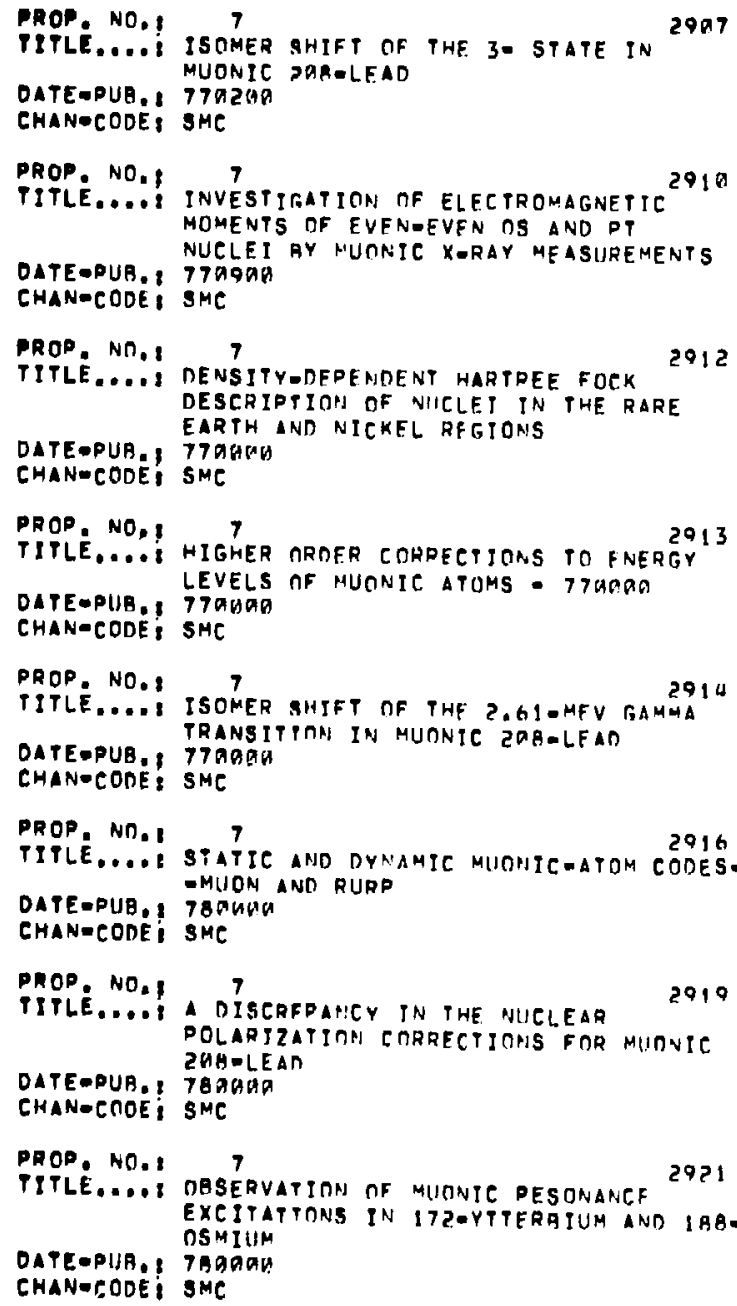

PROP. NO.1 7 3RTA

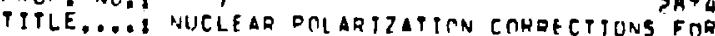
MUCATC JARAIFAD FROM A MONFL. I WAFPF ROENT COWATAFD ANAL YSIS RE ELASTIC FIFCTRON STATTERTAT, AND MUINATC $X-R A Y S$

DATE-PUA, 7 TSHAD

CHAN-CODE: $5 M C$

PRกP, Nก, 7

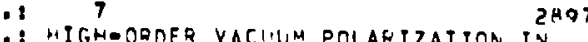

TITLE....: WIGHEOROFE VACLLM DOLANTZATIOA IV

DATF-DUR, 75AnOA

CHANGCONE: SHC

PROP. NO, : 7

inE $(2 A)>A B$ V VACIIIM

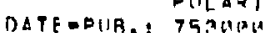
CHAN-COOE: SMC

PEOP. AM. T 7

TITLE.... COMGENT OA. THE VALTOTTY TIF TWF SI LTEA ADPROXJ MATION TA, FLECTRCA

DATE-PIAT, TSOBDil

CHAMECADE: $5 \mathrm{MC}$

PROP. A.ก. :

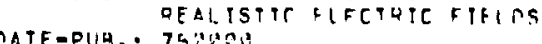

CHAN-COAE: SMC

PRחP. A I I :

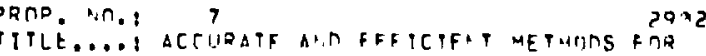

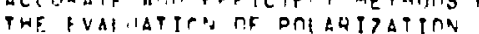

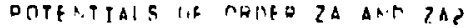

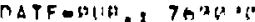

CHAN-CODE, SME

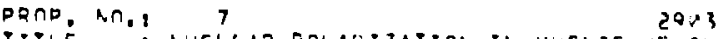

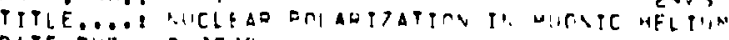

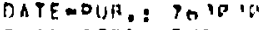

CHANECONF: SMC

WRCO. NO.1 1

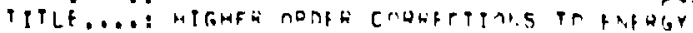

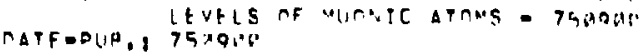

CHANECONE: SMC 